\title{
Radical Trifluoromethylation-of-Alkenes-Triggered Nitrile Insertion/Remote Functionalization Relay Processes: Diverse Synthesis of Trifluoromethylated Azaheterocycles Enabled by Copper Catalysis
}

\footnotetext{
Ji-Ming Xi, ${ }^{a}$ Yun-Hai Sun, ${ }^{a}$ Wen-Cheng Li, ${ }^{a}$ Yu-Heng Wu, ${ }^{a}$ Zhong-Lin Wei, ${ }^{a}$ Wei-Wei Liao ${ }^{a} b^{*}$

${ }^{a}$ Department of Organic Chemistry, College of Chemistry, Jilin University, 2699 Qianjin Street, Changchun 130012, P R China.

${ }^{b}$ State Key Laboratory of Organometallic Chemistry, Shanghai Institute of Organic Chemistry, Chinese Academy of Sciences, Shanghai 200032, P R China
}

\section{E-mail: wliao@j]lu.edu.cn}

Table of Contents

I. General Information...............................................................

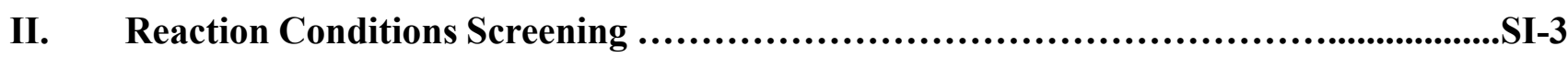

III. Selected Bioactive Molecules Including Bicyclic Amidine and N-Alkenyl Lactam Motifs............................................................................SI-8

IV. Preparation of Substrates.........................................................

V. General Procedure and Experimental Details.......................................

VI. Other Unsuccessful Examples....................................................SI-42

VII. Scale-up Reaction and Synthetic Transformations................................SI-43

VIII. Radical Trapping and Isotope Labeling Experiments...................................................................SI-45

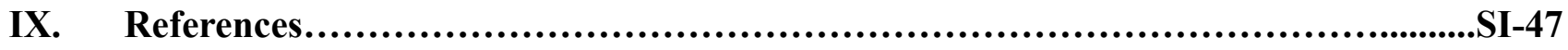

X. Crystal Data and Structure Refinement for Compounds 3a and 6a........................SI-48

XI. $\quad$ NMR Spectral Copies..........................................................SI-68 


\section{General Information}

All reactions were carried out under inert atmospheric condition unless otherwise noted, and solvents were dried according to established procedures. Reactions were monitored by thin layer chromatography (TLC) visualizing with ultraviolet light (UV), $\mathrm{KMnO}_{4}, p$-anisaldehyde stain, and phosphomolybdic acid (PMA) stain; column chromatography purifications were carried out using silica gel. For reactions that require heating, heating mantle was used. Proton nuclear magnetic resonance ( ${ }^{1} \mathrm{H}$ NMR) spectra were recorded on a 300, 400 or $500 \mathrm{MHz}$ spectrometer in $\mathrm{CDCl}_{3}$ or DMSO, fluorine nuclear magnetic resonance $\left({ }^{19} \mathrm{~F}\right.$ NMR) spectra were recorded on a 376 or $470 \mathrm{MHz}$ spectrometer in $\mathrm{CDCl}_{3}$ or DMSO, and carbon nuclear magnetic resonance $\left({ }^{13} \mathrm{C} \mathrm{NMR}\right)$ spectra were recorded on 125 or $100 \mathrm{MHz}$ spectrometer in $\mathrm{CDCl}_{3}$ or DMSO unless otherwise noted. Chemical shifts for protons are reported in parts per million downfield from tetramethylsilane (TMS) and are referenced to residual protium in the NMR solvent $\left(\mathrm{CHCl}_{3}=\delta 7.26 \mathrm{ppm}, \mathrm{DMSO}=\delta 2.50 \mathrm{ppm}\right)$. Chemical shifts for carbon are reported in parts per million downfield from tetramethylsilane (TMS) and are referenced to the carbon resonances of the solvent residual peak $\left(\mathrm{CDCl}_{3}=\delta\right.$ $77.16 \mathrm{ppm}, \mathrm{DMSO}=\delta 39.52 \mathrm{ppm})$. NMR data are presented as follows: chemical shift $(\delta \mathrm{ppm})$, multiplicity $(\mathrm{s}=$ singlet, $\mathrm{d}=$ doublet, $\mathrm{t}=$ triplet, $\mathrm{q}=$ quartet, $\mathrm{m}=$ multiplet, $\mathrm{br}=$ broad), coupling constant in Hertz $(\mathrm{Hz})$, integration. Mass spectra were recorded on the Bruker MicrOTOF Q II. 


\section{Reaction Conditions Screening}

\section{Optimized Reaction Conditions for Preparation of 3}

\section{Table S1. Selected Optimal Reaction Conditions ${ }^{a}$}

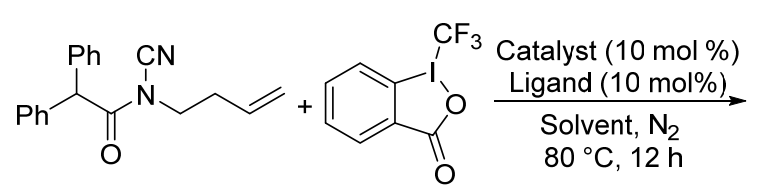

$1 \mathrm{a}$

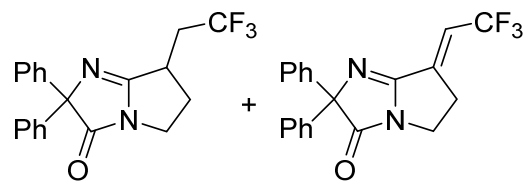

$3 a$
4

\begin{tabular}{ccccc}
\hline entry & cat. & ligand & solvent & yield of 3a $(\%)^{b}$ \\
\hline 1 & $\mathrm{Cu}_{2} \mathrm{O}$ & bpy & DCE & 68 \\
2 & $\mathrm{CuI}$ & bpy & DCE & 40 \\
3 & $\mathrm{Cu}(\mathrm{acac})_{2}$ & bpy & DCE & 65 \\
4 & $\mathrm{Cu}(\mathrm{OAc})_{2}$ & bpy & DCE & 67 \\
5 & $\mathrm{Fe}(\mathrm{acac})_{3}$ & bpy & DCE & nd \\
6 & $\mathrm{Cu}(\mathrm{OAc})_{2}$ & phen & DCE & 75 \\
$7^{c}$ & $\mathrm{Cu}(\mathrm{OAc})_{2}$ & phen & DCM & 86 \\
$8^{c}$ & $\mathrm{Cu}(\mathrm{OAc})_{2}$ & phen & DMSO & 17 \\
$9^{d, e}$ & $\mathrm{Cu}(\mathrm{OAc})_{2}$ & - & $\mathrm{DCM}$ & $91(90)$ \\
$11^{d, f}$ & $\mathrm{Cu}(\mathrm{OAc})_{2}$ & - & DCM & $94(92)$ \\
$12^{d, e, g}$ & $\mathrm{Cu}(\mathrm{OAc})_{2}$ & - & DCM & 65 \\
\hline
\end{tabular}

${ }^{a}$ Reaction conditions: 1a $(0.2 \mathrm{mmol}), \mathbf{2 a}$ (Togni II, $\left.0.3 \mathrm{mmol}\right)$, catalyst $(10 \mathrm{~mol} \%)$, ligand $(10 \mathrm{~mol} \%)$, in solvent $(2 \mathrm{~mL}), 80^{\circ} \mathrm{C}, 12$ $\mathrm{h}, \mathrm{N}_{2} .{ }^{b}{ }^{1} \mathrm{H}$ NMR yield using mesitylene as the internal standard. Isolated yield in parentheses. ${ }^{c} \mathbf{4}$ was obtained in $20 \%$ yield. ${ }^{d}$ Catalyst (5 mol \%). ${ }^{e} \mathbf{2 a}(0.24 \mathrm{mmol}) .{ }^{f}$ Togni I (2b). ${ }^{g}$ Under air.

To validate our hypothesis, our initial investigation commenced with $\mathrm{N}$-cyanamide alkene $\mathbf{1 a}$ and Togni-II reagent 2a as the trifluoroalkylating reagent in the presence of a catalytic amount of copper catalysts (Table S1). To our delight, the desired bicyclic amidine 3a was obtained in $68 \%$ of yield when the reaction was performed with $\mathrm{Cu}_{2} \mathrm{O}$ (10 mol \%)/bpy (2,2'-bipyridine, $10 \mathrm{~mol} \%$ ) in DCE (entry 1). The different copper salts were examined, and copper hal ides gave the inferior results, while $\mathrm{Cu}(\mathrm{acac})_{2}$ and $\mathrm{Cu}(\mathrm{OAc})_{2}$ afforded the comparable yields of product $3 \mathrm{a}$ (entries 2-4, also see Table S1). In contrast, no desired product was obtained by using Fe(acac) (entry 5), instead of small amount of benzophenone. The effects of ligand and solvent were evaluated in the presence of $\mathrm{Cu}(\mathrm{OAc})_{2}$ as the promising catalyst for further investigation. The increased production of 3a was obtained in DCM, while DMSO provided deteriorated production of desired product $\mathbf{3 a}$ in $17 \%$ yield along with the small amount of dehydrogenated product 4 (entries 6-8). Notably, ligand was not essential to this relay process, since slightly increased yield of 3a was obtained in the absence of ligand when DCM was chosen as a suitable solvent (entry 9). To our delight, this relay process proceeded smoothly in the presence of $\mathrm{Cu}(\mathrm{OAc})_{2}(5 \mathrm{~mol} \%)$ and the reduced amount of Togni-II reagent $\mathbf{2 a}$ under the ligand-free reaction conditions (entry 10). Togni-I reagent $\mathbf{2 b}$ can also serve as $\mathrm{CF}_{3}$ radical source, albeit with moderate yield (entry 11). In addition, control experiments indicated that catalyst was indispensable, and inert atmosphere was essential to the high production of the desired product (entry 12) (see Table S2-S5 for full detailed). 
Table S2. Optimized reaction conditions: effect of catalyst ${ }^{a}$

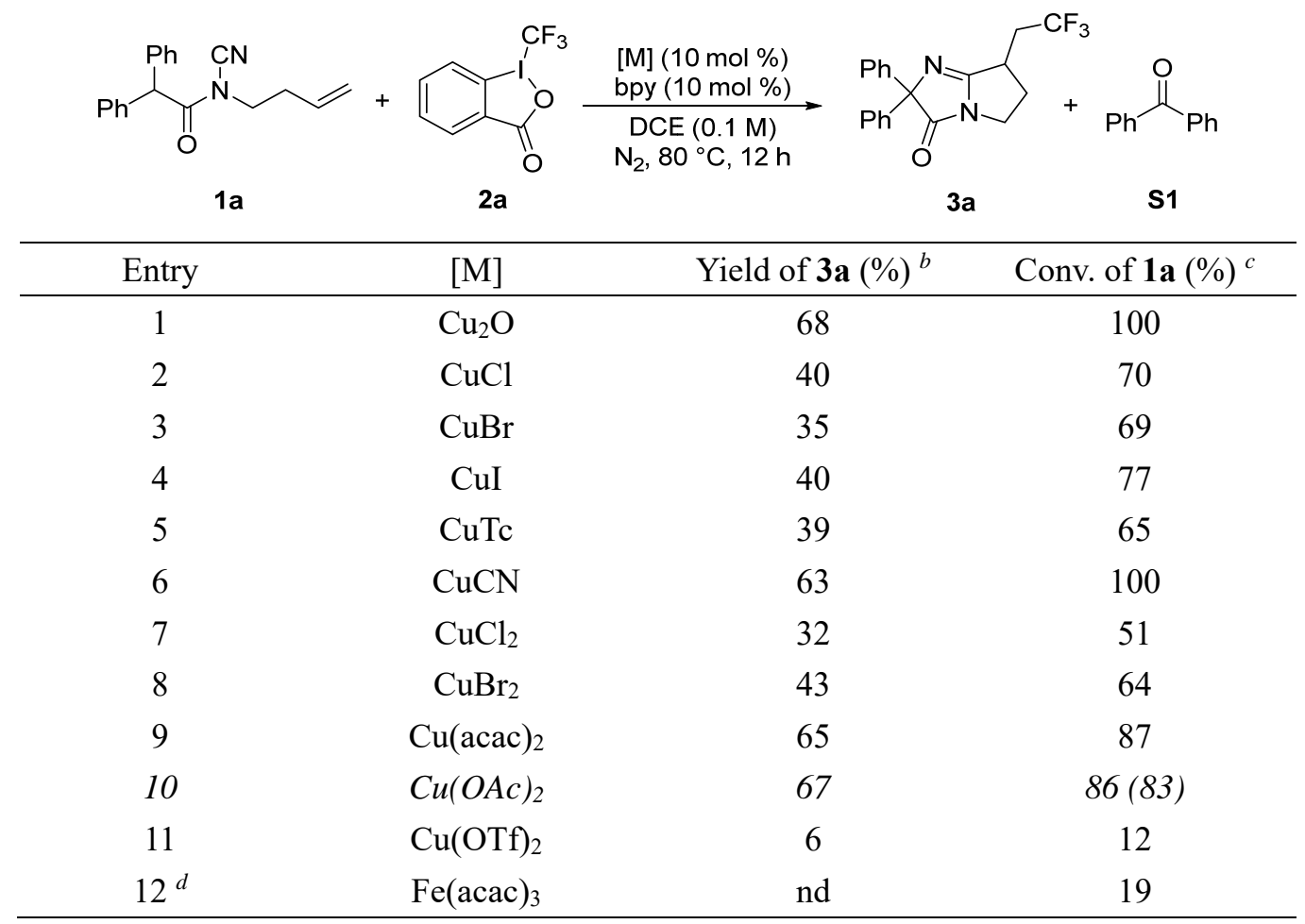

${ }^{a}$ Reaction conditions: 1a $(0.2 \mathrm{mmol}), \mathbf{2 a}$ (Togni II, $\left.0.3 \mathrm{mmol}\right)$, catalyst $(10 \mathrm{~mol} \%)$, bpy $(10 \mathrm{~mol} \%), \mathrm{DCE}(2 \mathrm{~mL}), 80^{\circ} \mathrm{C}, 12 \mathrm{~h}$, under $\mathrm{N}_{2}{ }^{b}{ }^{1} \mathrm{H}$ NMR yield using mesitylene as the internal standard. Isolated yield in parentheses. ${ }^{c}$ Determined by ${ }^{1} \mathrm{H}$ NMR analysis. ${ }^{d} \mathbf{S} 1$ $(8 \%)$ was obtained.

Table S3. Optimized reaction conditions: effect of ligand in DCE ${ }^{a}$

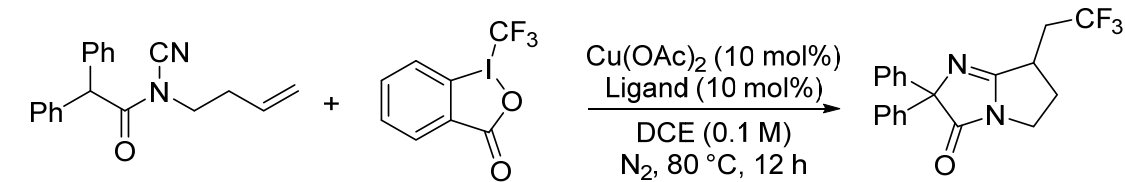<smiles>Cc1ccnc(-c2cc(C)ccn2)c1</smiles>

L1

L2 L3

L4 phen

\begin{tabular}{cccc}
\hline Entry & Ligand & ${\text { Yield of } \mathbf{3 a}(\%)^{b}}^{b}$ & ${\text { Conv. of } \mathbf{1 a}(\%)^{c}}^{c}$ \\
\hline 1 & bpy & 67 & 86 \\
2 & L1 & 38 & 56 \\
3 & L2 & 57 & 80 \\
4 & L3 & 56 & 78 \\
5 & L4 & 71 & 90 \\
6 & phen & 75 & 96 \\
7 & TMEDA & 70 & 90 \\
8 & PMDETA & 70 & 100 \\
9 & - & 68 & 97 \\
\hline
\end{tabular}

$\mathrm{SI}-4$ 
${ }^{a}$ Reaction conditions: 1a $(0.2 \mathrm{mmol}), \mathbf{2 a}$ (Togni II, $\left.0.3 \mathrm{mmol}\right), \mathrm{Cu}(\mathrm{OAc})_{2}(10 \mathrm{~mol} \%)$, ligand $(10 \mathrm{~mol} \%), \mathrm{DCE}(2 \mathrm{~mL}), 80{ }^{\circ} \mathrm{C}, 12 \mathrm{~h}$, under $\mathrm{N}_{2} .{ }^{b}{ }^{1} \mathrm{H}$ NMR yield using mesitylene as the internal standard. ${ }^{c}$ Determined by ${ }^{1} \mathrm{H}$ NMR analysis. bpy: $2,2^{\prime}$-bipyridine.

Table S4. Optimized reaction conditions: effect of solvent ${ }^{a}$<smiles>N#CC1CCN2C(=O)C3(c4ccccc4)N=C1C(=CC(F)(F)F)CC[C@H]23</smiles>

$1 \mathbf{a}$

$2 a$

$3 a$

4

\begin{tabular}{|c|c|c|c|c|}
\hline Entry & Solvent & Yield of 3a $(\%){ }^{b}$ & Yield of $4(\%)^{b}$ & Conv. of $1 \mathrm{a}(\%)^{C}$ \\
\hline 1 & DCE & 75 & nd & 96 \\
\hline 2 & $D C M$ & $86(87)$ & nd & 97 \\
\hline 3 & $\mathrm{CHCl}_{3}$ & 52 & nd & 70 \\
\hline 4 & $\mathrm{MeCN}$ & 66 & nd & 96 \\
\hline 5 & 1,4-dioxane & 52 & nd & 73 \\
\hline 6 & THF & 19 & nd & 30 \\
\hline 7 & acetone & 79 & nd & 95 \\
\hline 8 & EA & 78 & nd & 100 \\
\hline 9 & Toluene & 5 & nd & 12 \\
\hline 10 & $\mathrm{PhCF}_{3}$ & 12 & nd & 33 \\
\hline 11 & $\mathrm{PhCl}$ & 16 & nd & 43 \\
\hline 12 & $\mathrm{DMF}$ & nd & $25(21)$ & 89 \\
\hline 13 & DMSO & 17 & $20(17)$ & 100 \\
\hline
\end{tabular}

${ }^{a}$ Reaction conditions: 1a $(0.2 \mathrm{mmol}), \mathbf{2 a}$ (Togni II, $\left.0.3 \mathrm{mmol}\right), \mathrm{Cu}(\mathrm{OAc})_{2}(10 \mathrm{~mol} \%)$, phen $(10 \mathrm{~mol} \%)$, solvent $(2 \mathrm{~mL}), 80{ }^{\circ} \mathrm{C}, 12 \mathrm{~h}$, under $\mathrm{N}_{2} .{ }^{b}{ }^{1} \mathrm{H}$ NMR yield using mesitylene as the internal standard. Isolated yield in parentheses. ${ }^{c}$ Determined by ${ }^{1} \mathrm{H}$ NMR analysis.

Table S5. Optimized reaction conditions: effect of other reaction parameters ${ }^{a}$

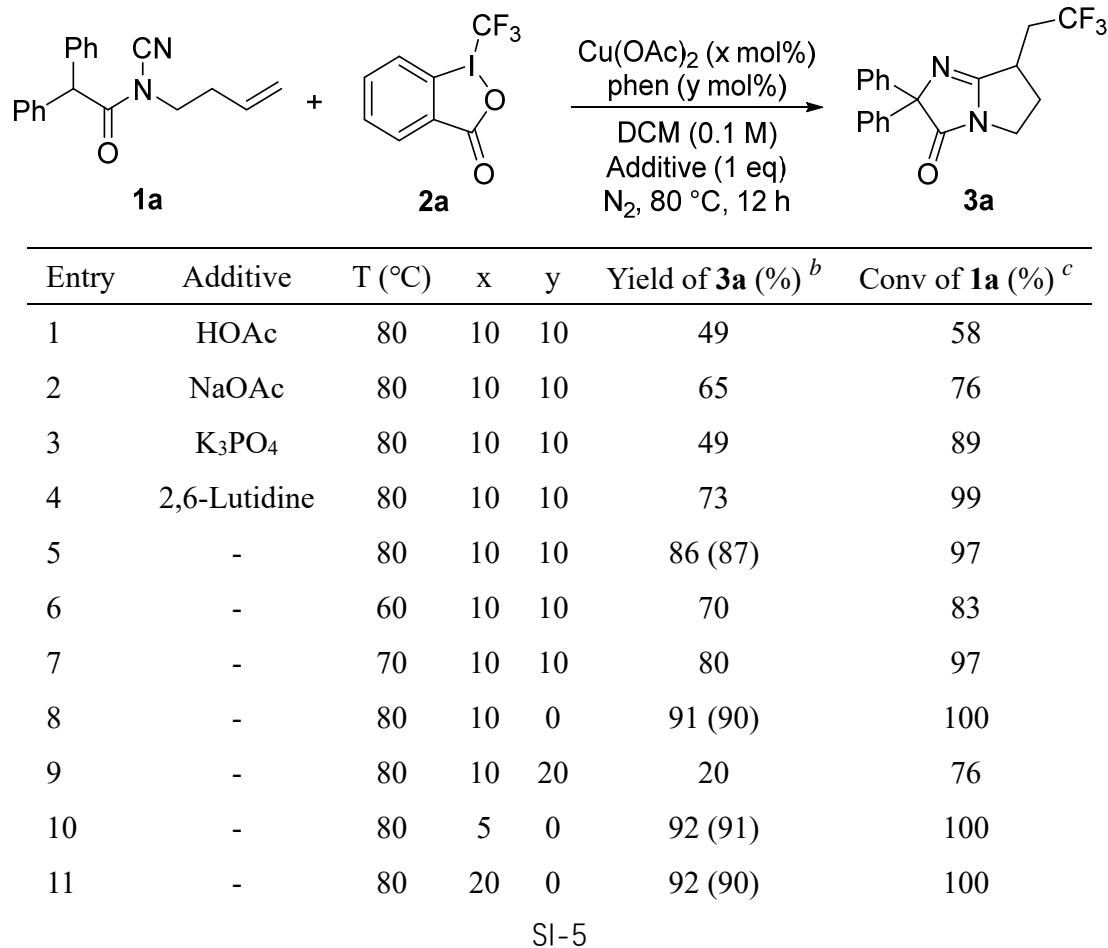




\begin{tabular}{lcccccc}
$12^{d}$ & - & 80 & 5 & 0 & $93(92)$ & 98 \\
$13^{e}$ & - & 80 & 5 & 0 & $94(92)$ & 100 \\
$14^{f}$ & - & 80 & 5 & 0 & 65 & 75 \\
$15^{d, g}$ & - & 80 & 5 & 0 & 42 & 49 \\
$16^{d, h}$ & $\mathrm{H}_{2} \mathrm{O}$ & 80 & 5 & 0 & 80 & 88 \\
$16^{d}$ & - & 80 & 0 & 0 & $\mathrm{nd}$ & 0 \\
\hline
\end{tabular}

${ }^{a}$ Reaction conditions: 1a $(0.2 \mathrm{mmol}), \mathbf{2 a}$ (Togni II, $\left.0.3 \mathrm{mmol}\right), \mathrm{Cu}(\mathrm{OAc}) 2\left(\mathrm{x} \mathrm{mol} \mathrm{\% ),} \mathrm{phen} \mathrm{(y} \mathrm{mol} \mathrm{\% ),} \mathrm{DCM}(2 \mathrm{~mL}), 12 \mathrm{~h}, \mathrm{under} \mathrm{N} 2 .{ }^{b}\right.$ ${ }^{1} \mathrm{H}$ NMR yield using mesitylene as the internal standard. Isolated yield in parentheses. ${ }^{c}$ Determined by ${ }^{1} \mathrm{H}$ NMR analysis. ${ }^{d} \mathbf{2 a}$ (Togni II, $0.24 \mathrm{mmol}$ ). ${ }^{e} \mathbf{2 a}$ (Togni II, $\left.0.36 \mathrm{mmol}\right) .{ }^{f} \mathbf{2 b}$ (Togni I, $\left.0.3 \mathrm{mmol}\right) .{ }^{g}$ Under air. ${ }^{h} \mathrm{H}_{2} \mathrm{O}$ (50 eq).<smiles>Cc1ccccc1C(C)(C)C</smiles>

\section{Optimized Reaction Conditions for Preparation of 6}

Table S6. Optimized reaction conditions: effect of catalyst ${ }^{a}$

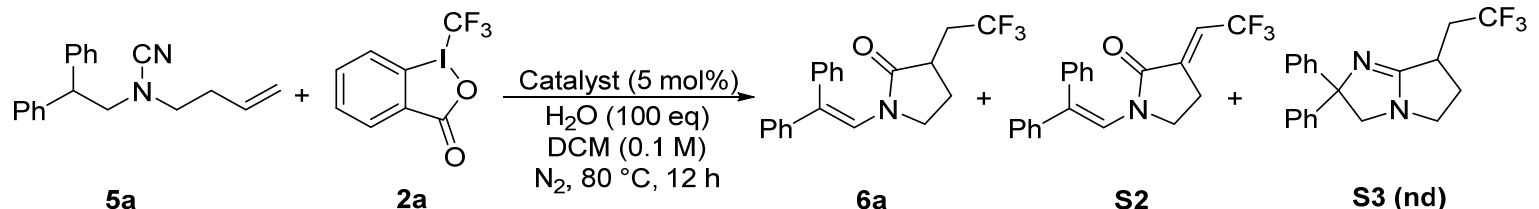

$5 a$ 2a $\mathrm{N}_{2}, 80^{\circ} \mathrm{C}, 12 \mathrm{~h}$

$6 a$ S2 S3 (nd)

\begin{tabular}{ccccc}
\hline Entry & Catalyst & Yield of 6a (\%) $^{b}$ & Yield of S2 (\%) $^{b}$ & Conv of 5a (\%) $^{c}$ \\
\hline $1^{d}$ & $\mathrm{Cu}(\mathrm{OAc})_{2}$ & $<1$ & - & 91 \\
2 & $\mathrm{Cu}(\mathrm{OAc})_{2}$ & 73 & 7 & 96 \\
3 & $\mathrm{Cu} 2$ & 69 & 4 & 95 \\
4 & $\mathrm{CuCN}$ & 67 & 4 & 94 \\
5 & $\mathrm{Cu}(\mathrm{acac})_{2}$ & 72 & 8 & 94 \\
6 & $\mathrm{Cu}\left(\mathrm{CH}_{3} \mathrm{CN}\right)_{4} \mathrm{BF}_{4}$ & 73 & 6 & 95
\end{tabular}

${ }^{a}$ Reaction conditions: 5a $(0.2 \mathrm{mmol}), \mathbf{2 a}(0.24 \mathrm{mmol})$, catalyst $(5 \mathrm{~mol} \%)$, DCM $(2 \mathrm{~mL})$ and $\mathrm{H}_{2} \mathrm{O}(100 \mathrm{eq}), 12 \mathrm{~h}$, under $_{2} .{ }^{b}$ Isolated yield. ${ }^{c}$ Determined by ${ }^{1} \mathrm{H}$ NMR analysis. ${ }^{d}$ Without $\mathrm{H}_{2} \mathrm{O}$.

Table S7. Optimized reaction conditions: effect of solvent ${ }^{a}$

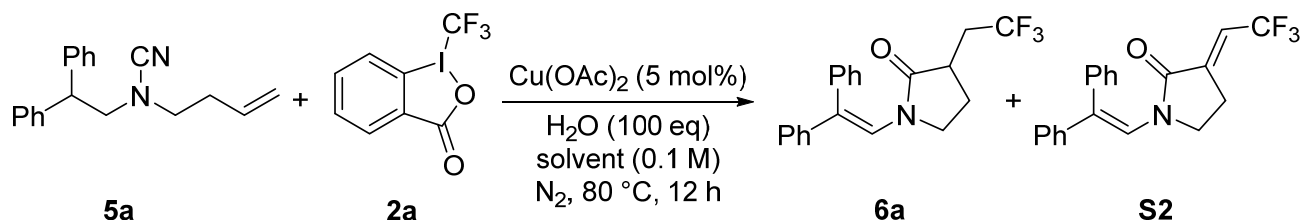

$5 a$

2a $\mathrm{N}_{2}, 80^{\circ} \mathrm{C}, 12 \mathrm{~h}$

$6 a$ S2

\begin{tabular}{ccccc}
\hline Entry & Solvent & Yield of 6a (\%) $^{b}$ & Yield of S2 (\%) $^{b}$ & Conv of 5a (\%) $^{c}$ \\
\hline 1 & DCM & 73 & 7 & 96 \\
2 & DCE & 66 & 9 & 93 \\
3 & MeCN & 42 & 6 & 93 \\
4 & EtOAc & 62 & 7 & 85 \\
5 & Acetone & 55 & 7 & 86 \\
\hline
\end{tabular}

${ }^{a}$ Reaction conditions: 5a $(0.2 \mathrm{mmol}), \mathbf{2 a}(0.24 \mathrm{mmol}), \mathrm{Cu}(\mathrm{OAc}) 2(5 \mathrm{~mol} \%)$, solvent $(2 \mathrm{~mL}), 12 \mathrm{~h}$, under $^{\mathrm{N}} .^{b}{ }^{b}$ Isolated yield. ${ }^{c}$ Determined by ${ }^{1} \mathrm{H}$ NMR analysis. 
Table S8. Optimized reaction conditions: effect of other reaction parameters ${ }^{a}$

\begin{tabular}{|c|c|c|c|c|c|}
\hline f' & $\mathrm{I}_{1}^{\mathrm{CN}}$ & $\prod_{0}^{C_{1}^{1}} \frac{\mathrm{Cu}}{A}$ & $\begin{array}{l}(\mathrm{OAc})_{2}(5 \mathrm{~mol} \%) \\
\stackrel{\text { Additive }(\mathrm{x} \mathrm{eq})}{\longrightarrow} \\
\mathrm{H}_{2} \mathrm{O}(\mathrm{y} \text { eq }) \\
\mathrm{DCM}(0.1 \mathrm{M}) \\
\mathrm{v}_{2}, 80^{\circ} \mathrm{C}, 12 \mathrm{~h}\end{array}$ & $\sum^{\mathrm{N}}{ }^{\mathrm{CF}_{3}}$ & $\overbrace{\mathrm{N}}^{\mathrm{CF}_{3}}$ \\
\hline Entry & Additive (x eq) & $\mathrm{H}_{2} \mathrm{O}$ (y eq) & Yield of $6 a(\%)^{b}$ & Yield of S2 (\%) ${ }^{b}$ & Conv. of $5 \mathbf{a}(\%)^{c}$ \\
\hline 1 & - & 100 & 73 & 7 & 96 \\
\hline 2 & HOAc (1.0 eq) & 100 & 71 & 6 & 100 \\
\hline 3 & $\operatorname{PTSA}(0.2$ eq $)$ & 100 & - & - & $\mathrm{nr}$ \\
\hline 4 & $\mathrm{MeOH}(50$ eq) & 0 & 13 & nd & 80 \\
\hline 5 & 2,6-Lutidine (1.0 eq) & 100 & 71 & 7 & 92 \\
\hline 6 & ${ }^{t} \mathrm{BuONa}(1.0 \mathrm{eq})$ & 50 & 56 & 9 & 100 \\
\hline 7 & $\mathrm{~K}_{3} \mathrm{PO}_{4}(1.0 \mathrm{eq})$ & 100 & 54 & 4 & 88 \\
\hline 8 & HOAc (3.0 eq) & 100 & nd & nd & $<5$ \\
\hline $9^{d}$ & HOAc (1.0 eq) & 100 & - & - & $\mathrm{nr}$ \\
\hline $10^{e}$ & HOAc (1.0 eq) & 100 & 68 & 3 & 82 \\
\hline 11 & - & 10 & 61 & 4 & 94 \\
\hline 12 & - & 50 & 75 & 6 & 95 \\
\hline 13 & - & 200 & 70 & 5 & 94 \\
\hline $14^{f}$ & - & 50 & 76 & 5 & 95 \\
\hline $15^{g}$ & - & 50 & 74 & 11 & 99 \\
\hline
\end{tabular}

${ }^{a}$ Reaction conditions: 5a $(0.2 \mathrm{mmol}), \mathbf{2 a}(0.24 \mathrm{mmol}), \mathrm{Cu}(\mathrm{OAc})_{2}(5 \mathrm{~mol} \%), \mathrm{DCM}(2 \mathrm{~mL}), 12 \mathrm{~h}$, under N$_{2} .{ }^{b}$ Isolated yield. ${ }^{c}$ Determined by ${ }^{1} \mathrm{H}$ NMR analysis. ${ }^{d}$ Bpy $(5 \mathrm{~mol} \%)$ was added. ${ }^{e} \mathbf{2 a}(0.2 \mathrm{mmol})$ was used. ${ }^{f} \mathrm{Cu}(\mathrm{OAc})_{2}(10 \mathrm{~mol} \%)$ was used. ${ }^{g} \mathbf{2 a}(0.3 \mathrm{mmol})$ was used.

\section{Optimized Reaction Conditions for Preparation of 7d}

\section{Table S9. Optimized Reaction Conditions for Preparation of $7 \mathrm{~d}^{a}$}<smiles>C=CCC(C)(C)C(=O)N(C)CC(c1ccccc1)c1ccccc1</smiles><smiles>CC1(C)CC(CC(F)(F)F)C2=NC(c3ccccc3)(c3ccccc3)CN2C1=O</smiles>

\begin{tabular}{lccc}
\hline Entry & Additive & Yield of $\mathbf{7 d ~ ( \% )}^{b}$ & Conv of $\mathbf{5 m}(\%)^{c}$ \\
\hline 1 & - & 52 & 94 \\
2 & $\mathrm{H}_{2} \mathrm{O}(50 \mathrm{eq})$ & 89 & 96 \\
3 & $\mathrm{MeOH}(50 \mathrm{eq})$ & 78 & 93 \\
4 & $\mathrm{HOAc}(1.0 \mathrm{eq})$ & 24 & 29 \\
5 & TFA (1.0 eq) & nd & $\mathrm{nr}$ \\
\hline
\end{tabular}

${ }^{a}$ Reaction conditions: $\mathbf{5 m}(0.2 \mathrm{mmol}), \mathbf{2 a}(0.24 \mathrm{mmol}), \mathrm{Cu}(\mathrm{OAc})_{2}(5 \mathrm{~mol} \%)$, DCM $(2 \mathrm{~mL})$ and additive, $12 \mathrm{~h}$, under $_{2} .{ }^{b}$ Isolated yield.

${ }^{c}$ Determined by ${ }^{1} \mathrm{H}$ NMR analysis. 


\section{Selected Bioactive Molecules Including Bicyclic Amidine and $N$-Alkenyl Lactam Motifs}

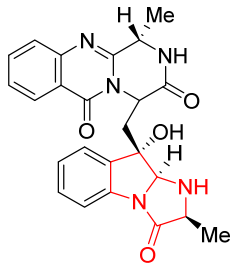

Fumiquinazoline $\mathrm{A}^{\mathrm{a}}$

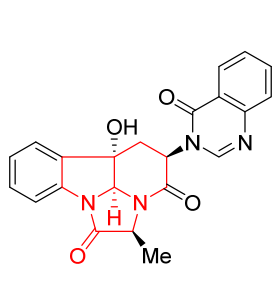

(-)-Chaetominine ${ }^{b}$

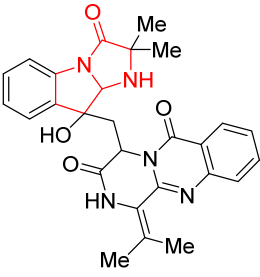

Quinadoline A ${ }^{c}$
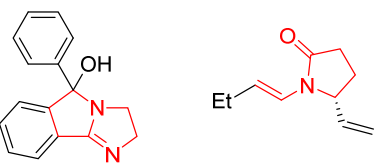

Anorectic agent $^{d} \quad$ Anticonvulsant reagent ${ }^{e}$

Figure S1. Selected bioactive molecules including bicyclic amidine and $N$-alkenyl lactam motifs ${ }^{1}$

\section{Preparation of Substrates}

$$
\begin{aligned}
& \mathrm{NH}_{2} \mathrm{CN}+\mathrm{R}^{1}-\mathrm{Br} \underset{{ }^{\circ} \mathrm{C} \text { to rt, } 24 \mathrm{~h}}{\stackrel{\mathrm{Cs}_{2} \mathrm{CO}_{3}, \mathrm{DMF}}{\longrightarrow}} \mathrm{NC}-\mathrm{NHR}^{1} \quad \text { eq-1 } \\
& \begin{array}{lll}
\text { S4 } & \text { S5 } & \text { S6 }
\end{array} \\
& \underset{\mathrm{S7}}{\mathrm{BrCN}}+\underset{\mathbf{S 8}}{\mathrm{H}_{2} \mathrm{~N}-\mathrm{R}^{2}} \frac{\mathrm{NaOAc, \textrm {MeOH }}}{0^{\circ} \mathrm{C} \text { to rt, } 24 \mathrm{~h}} \mathrm{NC}-\mathrm{NHR}^{2} \\
& \text { S6 or S6' }
\end{aligned}
$$

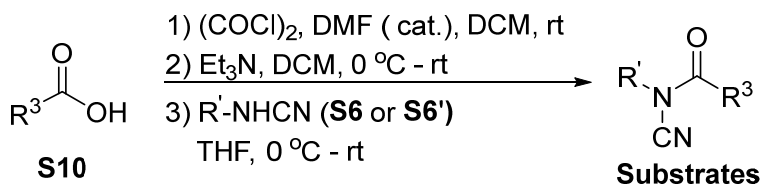

\section{Preparation of Substrates 1a-1p, 1w and 9g-9i (eq-1 and eq-4)}

Eq-1: ${ }^{2}$ To a flame-dried Schlenk tube equipped with a magnetic stir bar were added $\mathrm{NH}_{2} \mathrm{CN}$ (S4, 3 equiv.) and $\mathrm{Cs}_{2} \mathrm{CO}_{3}$ (1 equiv). The tube was evacuated and backfilled with nitrogen for three times, and DMF $(8.0 \mathrm{ml}, 0.25 \mathrm{M})$ was added via syringe. Then $\mathbf{S 5}$ ( $2 \mathrm{mmol}, 1$ equiv.) was added dropwise to the solution at $0{ }^{\circ} \mathrm{C}$. The tube was stirred at room temperature for $24 \mathrm{~h}$. The tube was stirred at room temperature for $24 \mathrm{~h}$. After completion, $\mathrm{H}_{2} \mathrm{O}$ was added to the solution. Then the aqueous phase was extracted with EtOAc. The combined organic layers were dried over $\mathrm{Na}_{2} \mathrm{SO}_{4}$, then filtered and concentrated under reduced pressure. The residue was purified by flash chromatography (silica gel, EtOAc/Petroleum ether $\left(60-90^{\circ} \mathrm{C}\right)$ ) to give the desired compounds $\mathbf{S 6}$.

Eq-4: ${ }^{3}$ To a suspension of acid S10 (1.2 equiv.) in DCM (1 ml, $1.2 \mathrm{M}$ ) was added DMF ( 2 drops), followed by dropwise addition of $(\mathrm{COCl})_{2}(1.2$ equiv. $)$ under nitrogen atmosphere at $0{ }^{\circ} \mathrm{C}$. The mixture was stirred for 1 hour, whereupon gas evolution ceased and all acid dissolved. The reaction flask was cooled to $-12{ }^{\circ} \mathrm{C}$ and a solution of $\mathrm{Et}_{3} \mathrm{~N}$ (2.4 equiv.) in DCM (3.0 M) was dropwise added. Thereafter, a solution of cyanamide $\mathbf{S 6}$ (1.0 mmol, 1 equiv.) in $\mathrm{CH}_{2} \mathrm{Cl}_{2} / \mathrm{THF}(3 / 1,0.8 \mathrm{ml}, 1.2 \mathrm{M})$ was slowly added at the same temperature. The resulting mixture was allowed to warm up to room temperature and stirred for 20-24 h. Upon completion, the reaction mixture was quenched with $\mathrm{H}_{2} \mathrm{O}$ and the aqueous phase was extracted with DCM. The combined organic phases were washed with brine, dried over $\mathrm{Na}_{2} \mathrm{SO}_{4}$, filtered, and concentrated in vacuo. The residue was purified by flash chromatography (silica gel, 
EtOAc/Petroleum ether $\left.\left(60-90{ }^{\circ} \mathrm{C}\right)\right)$ to give the desired substrates.

\section{Preparation of Substrates 5a-5i, 5p, 9a-9f and 9j (eq-2 and eq-3)}

Eq-2: ${ }^{3}$ To a stirring solution of $\mathbf{S 8}\left(2 \mathrm{mmol}, 1.0\right.$ equiv.) and $\mathrm{NaOAc}\left(3.0\right.$ equiv.) in $\mathrm{MeOH}(6.7 \mathrm{ml}, 0.3 \mathrm{M})$ at $0{ }^{\circ} \mathrm{C}$, BrCN S7 (1.2 equiv.) was added. Then the reaction was warmed to room temperature and stirred for $12 \mathrm{~h}$. Upon completion, the solvent was removed in vacuo. To the residue was added water and extracted with ethyl acetate, and the combined organic layers were washed by brine and dried over $\mathrm{Na}_{2} \mathrm{SO}_{4}$, then filtered and concentrated under reduced pressure. The residue was purified by flash column chromatography (silica gel, EtOAc/Petroleum ether $\left.\left(60-90^{\circ} \mathrm{C}\right)\right)$ to give compound $\mathbf{S 6}$ '.

Eq-3: ${ }^{4}$ Under a $\mathrm{N}_{2}$ atmosphere, to a two neck round bottom flask was added $\mathbf{S 6}$ ' (1 mmol, 1 equiv.), $\mathrm{K}_{2} \mathrm{CO}_{3}(2.0$ equiv.) and DMF (3.3 ml, $0.3 \mathrm{M})$ at $0{ }^{\circ} \mathrm{C}$. The reaction mixture was stirred for half an hour. Then, bromide $\mathbf{S 9}$ (1.1 equiv.) was added dropwise at $0{ }^{\circ} \mathrm{C}$, and the resulting reaction mixture was warmed to $110{ }^{\circ} \mathrm{C}$ and stirred for $14 \mathrm{~h}$ (for substrates $\mathbf{5 h - 5 i}, 9 \mathbf{e}-9 \mathbf{f}$, the resulting reaction mixture was warmed to room temperature and stirred for 2 days). Upon completion, $\mathrm{H}_{2} \mathrm{O}$ was added and the mixture was extracted with EtOAc, and the combined organic layers were washed by brine and dried over $\mathrm{Na}_{2} \mathrm{SO}_{4}$, then filtered and concentrated under reduced pressure. The residue was purified by flash column chromatography (silica gel, EtOAc/Petroleum ether $\left(60-90{ }^{\circ} \mathrm{C}\right)$ ) to give the desired substrates.

The NMR spectra data of compound $9 \mathbf{a}$ were in accordance with the reported data of the known literatures. ${ }^{3}$

\section{Preparation of Substrates $1 \mathrm{q}-1 \mathrm{v}, 5 \mathrm{j}-50$ and $9 \mathrm{k}-9 \mathrm{n}$ (eq-2 and eq-4)}

Substrates1q-1v, 5j-5o and 9k-9n were prepared according to the same procedures of eq-2 and eq-4.

\section{Spectral data for N-(but-3-en-1-yl)-N-cyano-2,2-diphenylacetamide (1a)}<smiles>C=CCCN(C)C(=O)C(c1ccccc1)c1ccccc1</smiles>

Purified by column chromatography (silica gel, EtOAc/Petroleum ether: 1/20) to afford the title compound (62\% yield, $180 \mathrm{mg}$ ) as white solid, mp: $48-49^{\circ} \mathrm{C} .{ }^{1} \mathrm{H}$ NMR $\left(400 \mathrm{MHz}, \mathrm{CDCl}_{3}\right) \delta 7.39-7.27(\mathrm{~m}, 10 \mathrm{H}), 5.68$ (ddt, $J=$ 17.1, 10.2, $6.9 \mathrm{~Hz}, 1 \mathrm{H}), 5.60(\mathrm{~s}, 1 \mathrm{H}), 5.12-5.01(\mathrm{~m}, 2 \mathrm{H}), 3.70(\mathrm{t}, J=6.9 \mathrm{~Hz}, 2 \mathrm{H}), 2.50-2.34(\mathrm{~m}, 2 \mathrm{H}) .{ }^{13} \mathrm{C} \mathrm{NMR}$ $\left(125 \mathrm{MHz}, \mathrm{CDCl}_{3}\right) \delta 171.5,136.9,132.7,128.9,128.8,127.9,118.9,110.7,56.0,46.0,31.9$. HRMS (ESI) m/z: $[\mathrm{M}+\mathrm{Na}]^{+}$calcd for $\mathrm{C}_{19} \mathrm{H}_{18} \mathrm{~N}_{2} \mathrm{NaO} 313.1311$; found: 313.1317 .

\section{Spectral data for N-(but-3-en-1-yl)-2,2-bis(4-chlorophenyl)-N-cyanoacetamide (1b)}<smiles>C=CCCN(C)C(=O)C(c1ccc(Cl)cc1)c1ccc(Cl)cc1</smiles>

Purified by column chromatography (silica gel, EtOAc/Petroleum ether: $1 / 20)$ to afford the title compound (30\% yield, $108 \mathrm{mg}$ ) as yellowish oil. ${ }^{1} \mathrm{H}$ NMR $\left(400 \mathrm{MHz}, \mathrm{CDCl}_{3}\right) \delta 7.36-7.30(\mathrm{~m}, 4 \mathrm{H}), 7.23-7.18(\mathrm{~m}, 4 \mathrm{H}), 5.67$ (ddt, $J=17.2,10.3,6.9 \mathrm{~Hz}, 1 \mathrm{H}), 5.53(\mathrm{~s}, 1 \mathrm{H}), 5.15-5.02(\mathrm{~m}, 2 \mathrm{H}), 3.70(\mathrm{t}, J=6.9 \mathrm{~Hz}, 2 \mathrm{H}), 2.52-2.30(\mathrm{~m}, 2 \mathrm{H}) .{ }^{13} \mathrm{C} \mathrm{NMR}$ $\left(125 \mathrm{MHz}, \mathrm{CDCl}_{3}\right) \delta 170.9,134.9,134.2,132.5,130.1,129.2,119.1,110.1,54.6,46.1,31.9 . \mathrm{HRMS}(\mathrm{ESI}) \mathrm{m} / \mathrm{z}$ : [M $+\mathrm{H}]^{+}$calcd for $\mathrm{C}_{19} \mathrm{H}_{17} \mathrm{Cl}_{2} \mathrm{~N}_{2} \mathrm{O} 359.0712$; found: 359.0725 . 
Me

Purified by column chromatography (silica gel, EtOAc/Petroleum ether: 1/20) to afford the title compound (70\% yield, $160 \mathrm{mg}$ ) as colorless oil. ${ }^{1} \mathrm{H}$ NMR $\left(400 \mathrm{MHz}, \mathrm{CDCl}_{3}\right) \delta 7.42-7.30(\mathrm{~m}, 5 \mathrm{H}), 5.73-5.58(\mathrm{~m}, 1 \mathrm{H}), 5.10-4.96$ $(\mathrm{m}, 2 \mathrm{H}), 4.30$ (q, $J=6.9 \mathrm{~Hz}, 1 \mathrm{H}), 3.78-3.49(\mathrm{~m}, 2 \mathrm{H}), 2.50-2.24(\mathrm{~m}, 2 \mathrm{H}), 1.55(\mathrm{~d}, J=6.9 \mathrm{~Hz}, 3 \mathrm{H}) .{ }^{13} \mathrm{C}$ NMR $(125$ $\left.\mathrm{MHz}, \mathrm{CDCl}_{3}\right) \delta 173.3,138.6,132.6,129.0,127.8,118.7,110.5,45.7,44.9,31.9,19.4$. HRMS (ESI) m/z: [M+Na] ${ }^{+}$ calcd for $\mathrm{C}_{14} \mathrm{H}_{16} \mathrm{~N}_{2} \mathrm{NaO} 251.1155$; found: 251.1160 .

\section{Spectral data for N-(but-3-en-1-yl)-N-cyano-2-phenylbutanamide (1d)}<smiles>C=CCCN(C)C(=O)C(CC)c1ccccc1</smiles>

Purified by column chromatography (silica gel, EtOAc/Petroleum ether: 1/20) to afford the title compound (47\% yield, $114 \mathrm{mg}$ ) as yellowish oil. ${ }^{1} \mathrm{H}$ NMR $\left(400 \mathrm{MHz}, \mathrm{CDCl}_{3}\right) \delta 7.38-7.27(\mathrm{~m}, 5 \mathrm{H}), 5.82-5.33(\mathrm{~m}, 1 \mathrm{H}), 5.16-4.62$ $(\mathrm{m}, 2 \mathrm{H}), 4.01(\mathrm{t}, J=7.4 \mathrm{~Hz}, 1 \mathrm{H}), 3.72-3.38(\mathrm{~m}, 2 \mathrm{H}), 2.47-2.26(\mathrm{~m}, 2 \mathrm{H}), 2.19-2.05(\mathrm{~m}, 1 \mathrm{H}), 1.89-1.75(\mathrm{~m}, 1 \mathrm{H})$, $0.89(\mathrm{t}, J=7.4 \mathrm{~Hz}, 3 \mathrm{H}) .{ }^{13} \mathrm{C} \mathrm{NMR}\left(125 \mathrm{MHz}, \mathrm{CDCl}_{3}\right) \delta 172.8,137.1,132.6,128.9,128.3,127.9,118.8,110.6,52.4$, 45.7, 31.9, 27.2, 11.9. HRMS (ESI) m/z: $[\mathrm{M}+\mathrm{H}]^{+}$calcd for $\mathrm{C}_{15} \mathrm{H}_{19} \mathrm{~N}_{2} \mathrm{O} 243.1492$; found: 243.1484 .

\section{Spectral data for N-(but-3-en-1-yl)-N-cyano-2-(p-tolyl)propanamide (1e)}<smiles>C=CCCN(C#N)C(=O)C(c1ccccc1)c1ccc(C)cc1</smiles>

Purified by column chromatography (silica gel, EtOAc/Petroleum ether: $1 / 20)$ to afford the title compound (52\% yield, $126 \mathrm{mg}$ ) as colorless oil. ${ }^{1} \mathrm{H}$ NMR $\left(500 \mathrm{MHz}, \mathrm{CDCl}_{3}\right) \delta 7.21(\mathrm{~d}, J=7.8 \mathrm{~Hz}, 2 \mathrm{H}), 7.15(\mathrm{~d}, J=7.8 \mathrm{~Hz}, 2 \mathrm{H}), 5.64$ (ddt, $J=16.9,10.8,6.9 \mathrm{~Hz}, 1 \mathrm{H}), 5.13-4.85(\mathrm{~m}, 2 \mathrm{H}), 4.22(\mathrm{q}, J=6.9 \mathrm{~Hz}, 1 \mathrm{H}), 3.60(\mathrm{td}, J=7.0,2.0 \mathrm{~Hz}, 2 \mathrm{H}), 2.38-$ $2.34(\mathrm{~m}, 2 \mathrm{H}), 2.33(\mathrm{~s}, 3 \mathrm{H}), 1.49(\mathrm{~d}, J=6.8 \mathrm{~Hz}, 3 \mathrm{H}) .{ }^{13} \mathrm{C} \mathrm{NMR}\left(125 \mathrm{MHz}, \mathrm{CDCl}_{3}\right) \delta 173.5,137.6,135.6,132.7,129.6$, 127.7, 118.7, 110.5, 45.7, 44.6, 31.9, 21.1, 19.4. HRMS (ESI) m/z: $[\mathrm{M}+\mathrm{H}]^{+}$calcd for $\mathrm{C}_{15} \mathrm{H}_{19} \mathrm{~N}_{2} \mathrm{O} 243.1492$; found: 243.1483.

\section{Spectral data for N-(but-3-en-1-yl)-N-cyanoisobutyramide (1f)}<smiles>C=CCCN(C)C(=O)C(C)C</smiles>

Purified by column chromatography (silica gel, EtOAc/Petroleum ether: 1/30) to afford the title compound (72\% yield, $120 \mathrm{mg}$ ) as colorless oil. ${ }^{1} \mathrm{H} \mathrm{NMR}\left(400 \mathrm{MHz}, \mathrm{CDCl}_{3}\right) \delta 5.76(\mathrm{ddt}, J=17.1,10.2,6.9 \mathrm{~Hz}, 1 \mathrm{H}), 5.35-4.93(\mathrm{~m}$, $2 \mathrm{H}), 3.69(\mathrm{t}, J=6.9 \mathrm{~Hz}, 2 \mathrm{H}), 3.16$ (hept, $J=6.8 \mathrm{~Hz}, 1 \mathrm{H}), 2.47$ (qt, $J=6.9,1.3 \mathrm{~Hz}, 2 \mathrm{H}), 1.25$ (d, $J=6.8 \mathrm{~Hz}, 6 \mathrm{H}) .{ }^{13} \mathrm{C}$ NMR $\left(125 \mathrm{MHz}, \mathrm{CDCl}_{3}\right) \delta 176.4,133.0,118.7,110.7,45.3,33.5,32.1,19.0$. HRMS (ESI) m/z: $[\mathrm{M}+\mathrm{H}]^{+}$calcd for $\mathrm{C}_{9} \mathrm{H}_{15} \mathrm{~N}_{2} \mathrm{O} 167.1179$; found: 167.1176 .

\section{Spectral data for N-(but-3-en-1-yl)-N-cyano-2-propylpentanamide (1g)}<smiles>C=CCCN(C)C(=O)C(C(C)C)C(F)(F)F</smiles> 
Purified by column chromatography (silica gel, EtOAc/Petroleum ether: 1/20) to afford the title compound (95\% yield, $211 \mathrm{mg}$ ) as colorless oil. ${ }^{1} \mathrm{H}$ NMR $\left(400 \mathrm{MHz}, \mathrm{CDCl}_{3}\right) \delta 5.74$ (ddt, $\left.J=17.1,10.2,6.9 \mathrm{~Hz}, 1 \mathrm{H}\right), 5.27-5.02(\mathrm{~m}$, 2H), $3.68(\mathrm{t}, J=6.9 \mathrm{~Hz}, 2 \mathrm{H}), 3.03(\mathrm{tt}, J=8.5,5.3 \mathrm{~Hz}, 1 \mathrm{H}), 2.54-2.26(\mathrm{~m}, 2 \mathrm{H}), 1.71-1.59(\mathrm{~m}, 2 \mathrm{H}), 1.53-1.41(\mathrm{~m}$, 2H), $1.38-1.23(\mathrm{~m}, 4 \mathrm{H}), 0.91(\mathrm{t}, J=7.3 \mathrm{~Hz}, 6 \mathrm{H}) .{ }^{13} \mathrm{C} \mathrm{NMR}\left(125 \mathrm{MHz}, \mathrm{CDCl}_{3}\right) \delta$ 175.7, 133.0, 118.6, 110.7, 45.3, 44.2, 34.7, 32.0, 20.4, 13.9. HRMS (ESI) $\mathrm{m} / \mathrm{z}:[\mathrm{M}+\mathrm{H}]^{+}$calcd for $\mathrm{C}_{13} \mathrm{H}_{23} \mathrm{~N}_{2} \mathrm{O} 223.1805$; found: 223.1802 .

\section{Spectral data for N-(but-3-en-1-yl)-N-cyano-2-methylbutanamide (1h)}<smiles>C=CCCN(C#N)C(=O)C(CC)[N+](=O)[O-]</smiles>

Purified by column chromatography (silica gel, EtOAc/Petroleum ether: 1/30) to afford the title compound (33\% yield, $60 \mathrm{mg}$ ) as colorless oil. ${ }^{1} \mathrm{H}$ NMR (400 MHz, $\left.\mathrm{CDCl}_{3}\right) \delta 5.74$ (ddt, $\left.J=17.0,10.1,6.9 \mathrm{~Hz}, 1 \mathrm{H}\right), 5.31-4.97(\mathrm{~m}$, $2 \mathrm{H}), 3.80-3.49(\mathrm{~m}, 2 \mathrm{H}), 3.09-2.80(\mathrm{~m}, 1 \mathrm{H}), 2.59-2.29(\mathrm{~m}, 2 \mathrm{H}), 1.84-1.67(\mathrm{~m}, 1 \mathrm{H}), 1.57-1.44(\mathrm{~m}, 1 \mathrm{H}), 1.20$ $(\mathrm{d}, J=6.7 \mathrm{~Hz}, 3 \mathrm{H}), 0.94$ (t, $J=7.4 \mathrm{~Hz}, 3 \mathrm{H}) .{ }^{13} \mathrm{C} \mathrm{NMR}\left(125 \mathrm{MHz}, \mathrm{CDCl}_{3}\right) \delta 176.0,132.9,118.7,110.7,45.3,40.3$, 32.1, 26.8, 16.8, 11.4. HRMS (ESI) m/z: [M+Na] $]^{+}$calcd for $\mathrm{C}_{10} \mathrm{H}_{16} \mathrm{~N}_{2} \mathrm{NaO}$ 203.1155; found: 203.1157.

\section{Spectral data for N-(but-3-en-1-yl)-N-cyano-2-phenylacetamide (1i)}<smiles>C=CCCN(C)C(=O)Cc1ccccc1</smiles>

Purified by column chromatography (silica gel, EtOAc/Petroleum ether: $1 / 20)$ to afford the title compound (75\% yield, $161 \mathrm{mg}$ ) as colorless oil. ${ }^{1} \mathrm{H}$ NMR $\left(500 \mathrm{MHz}, \mathrm{CDCl}_{3}\right) \delta 7.38-7.33(\mathrm{~m}, 2 \mathrm{H}), 7.33-7.28(\mathrm{~m}, 3 \mathrm{H}), 5.69$ (ddt, $J$ $=17.1,10.2,6.9 \mathrm{~Hz}, 1 \mathrm{H}), 5.23-4.90(\mathrm{~m}, 2 \mathrm{H}), 3.97(\mathrm{~s}, 2 \mathrm{H}), 3.65(\mathrm{t}, J=7.0 \mathrm{~Hz}, 2 \mathrm{H}), 2.55-2.19(\mathrm{~m}, 2 \mathrm{H}) .{ }^{13} \mathrm{C} \mathrm{NMR}$ $\left(125 \mathrm{MHz}, \mathrm{CDCl}_{3}\right) \delta 170.2,132.7,132.1,129.4,128.9,127.8,118.8,110.7,45.7,41.3,31.9$. HRMS (ESI) m/z: [M $+\mathrm{H}]^{+}$calcd for $\mathrm{C}_{13} \mathrm{H}_{15} \mathrm{~N}_{2} \mathrm{O} 215.1179$; found: 215.1172 .

\section{Spectral data for N-(but-3-en-1-yl)-N-cyanocyclobutanecarboxamide (1j)}<smiles>C=CCCN(C)C(=O)C1CCC1</smiles>

Purified by column chromatography (silica gel, EtOAc/Petroleum ether: 1/30) to afford the title compound (92\% yield, $164 \mathrm{mg}$ ) as colorless oil. ${ }^{1} \mathrm{H} \mathrm{NMR}\left(400 \mathrm{MHz}, \mathrm{CDCl}_{3}\right) \delta 5.73(\mathrm{ddt}, J=17.1,10.2,6.9 \mathrm{~Hz}, 1 \mathrm{H}), 5.32-4.98(\mathrm{~m}$, 2H), $3.79-3.25(\mathrm{~m}, 3 \mathrm{H}), 2.43(\mathrm{q}, J=6.7 \mathrm{~Hz}, 2 \mathrm{H}), 2.39-2.27(\mathrm{~m}, 4 \mathrm{H}), 2.13-1.98(\mathrm{~m}, 1 \mathrm{H}), 1.97-1.85(\mathrm{~m}, 1 \mathrm{H})$. ${ }^{13} \mathrm{C}$ NMR $\left(125 \mathrm{MHz}, \mathrm{CDCl}_{3}\right) \delta 174.1,132.9,118.7,110.5,45.1,38.3,32.0,24.9,17.8$. HRMS (ESI) m/z: $[\mathrm{M}+\mathrm{H}]^{+}$ calcd for $\mathrm{C}_{10} \mathrm{H}_{15} \mathrm{~N}_{2} \mathrm{O}$ 179.1179; found: 179.1173 .

\section{Spectral data for N-(but-3-en-1-yl)-N-cyanocyclopentanecarboxamide (1k)}<smiles>C=CCCN(C)C(=O)C1CCCC1</smiles>

Purified by column chromatography (silica gel, EtOAc/Petroleum ether: 1/30) to afford the title compound (61\% yield, $117 \mathrm{mg})$ as colorless oil. ${ }^{1} \mathrm{H}$ NMR $\left(400 \mathrm{MHz}, \mathrm{CDCl}_{3}\right) \delta 5.74(\mathrm{ddt}, J=17.1,10.2,6.9 \mathrm{~Hz}, 1 \mathrm{H}), 5.22-5.08(\mathrm{~m}$, 2H), $3.65(\mathrm{t}, J=6.9 \mathrm{~Hz}, 2 \mathrm{H}), 3.29-3.21(\mathrm{~m}, 1 \mathrm{H}), 2.51-2.36(\mathrm{~m}, 2 \mathrm{H}), 2.08-1.95(\mathrm{~m}, 2 \mathrm{H}), 1.87-1.79(\mathrm{~m}, 2 \mathrm{H}), 1.79$ $-1.71(\mathrm{~m}, 2 \mathrm{H}), 1.68-1.59$ (m, 2H). ${ }^{13} \mathrm{C} \mathrm{NMR}\left(125 \mathrm{MHz}, \mathrm{CDCl}_{3}\right) \delta 175.7,133.0,118.6,110.8,45.3,43.4,32.0,30.1$, 
26.0. HRMS (ESI) m/z: [M+ H] $]^{+}$calcd for $\mathrm{C}_{11} \mathrm{H}_{17} \mathrm{~N}_{2} \mathrm{O}$ 193.1335; found: 193.1331 .

Spectral data for N-(but-3-en-1-yl)-N-cyanotetrahydrofuran-3-carboxamide (11)<smiles>C=CCCN(C)C(=O)C1CCOC1</smiles>

Purified by column chromatography (silica gel, EtOAc/Petroleum ether: $1 / 5$ ) to afford the title compound (74\% yield, $144 \mathrm{mg}$ ) as yellowish oil. ${ }^{1} \mathrm{H}$ NMR $\left(500 \mathrm{MHz}, \mathrm{CDCl}_{3}\right) \delta 5.73$ (ddt, $\left.J=17.0,10.1,6.9 \mathrm{~Hz}, 1 \mathrm{H}\right), 5.23-5.10(\mathrm{~m}, 2 \mathrm{H})$, $4.09-4.03(\mathrm{~m}, 1 \mathrm{H}), 3.99-3.95(\mathrm{~m}, 1 \mathrm{H}), 3.95-3.89(\mathrm{~m}, 1 \mathrm{H}), 3.87-3.81(\mathrm{~m}, 1 \mathrm{H}), 3.68(\mathrm{t}, J=6.9 \mathrm{~Hz}, 2 \mathrm{H}), 3.62-$ $3.54(\mathrm{~m}, 1 \mathrm{H}), 2.50-2.39(\mathrm{~m}, 2 \mathrm{H}), 2.32-2.14(\mathrm{~m}, 2 \mathrm{H}) .{ }^{13} \mathrm{C} \mathrm{NMR}\left(125 \mathrm{MHz}, \mathrm{CDCl}_{3}\right) \delta 172.9,132.8,118.9,110.3$, 70.0, 68.4, 45.5, 43.4, 32.0, 29.9. HRMS (ESI) m/z: $[\mathrm{M}+\mathrm{H}]^{+}$calcd for $\mathrm{C}_{10} \mathrm{H}_{15} \mathrm{~N}_{2} \mathrm{O}_{2}$ 195.1128; found: 195.1127 .

\section{Spectral data for N-(but-3-en-1-yl)-N-cyanocyclohexanecarboxamide (1m)}<smiles>C=CCCN(C)C(=O)C1CCCCC1</smiles>

Purified by column chromatography (silica gel, EtOAc/Petroleum ether: 1/30) to afford the title compound (73\% yield, $151 \mathrm{mg}$ ) as colorless oil. ${ }^{1} \mathrm{H}$ NMR $\left(500 \mathrm{MHz}, \mathrm{CDCl}_{3}\right) \delta 5.72(\mathrm{ddt}, J=17.1,10.2,6.9 \mathrm{~Hz}, 1 \mathrm{H}), 5.34-4.92(\mathrm{~m}$, 2H), 3.64 (t, $J=6.9 \mathrm{~Hz}, 2 \mathrm{H}), 3.04-2.75(\mathrm{~m}, 1 \mathrm{H}), 2.58-2.27(\mathrm{~m}, 2 \mathrm{H}), 1.95-1.87(\mathrm{~m}, 2 \mathrm{H}), 1.86-1.76(\mathrm{~m}, 2 \mathrm{H})$, $1.75-1.66(\mathrm{~m}, 1 \mathrm{H}), 1.54-1.41(\mathrm{~m}, 2 \mathrm{H}), 1.40-1.16(\mathrm{~m}, 3 \mathrm{H}) .{ }^{13} \mathrm{C} \mathrm{NMR}\left(125 \mathrm{MHz}, \mathrm{CDCl}_{3}\right) \delta$ 175.2, 132.9, 118.6, 110.7, 45.1, 42.9, 32.0, 28.9, 25.4, 25.1. HRMS (ESI) m/z: $[\mathrm{M}+\mathrm{H}]^{+}$calcd for $\mathrm{C}_{12} \mathrm{H}_{19} \mathrm{~N}_{2} \mathrm{O} 207.1492$; found: 207.1490 .

\section{Spectral data for N-(but-3-en-1-yl)-N-cyanotetrahydro-2H-pyran-4-carboxamide (1n)}<smiles>C=CCCN(C#N)C(=O)C1CCOCC1</smiles>

Purified by column chromatography (silica gel, EtOAc/Petroleum ether: 1/10) to afford the title compound (32\% yield, $67 \mathrm{mg}$ ) as yellow oil. ${ }^{1} \mathrm{H}$ NMR $\left(400 \mathrm{MHz}, \mathrm{CDCl}_{3}\right) \delta 5.73(\mathrm{ddt}, J=17.1,10.2,6.9 \mathrm{~Hz}, 1 \mathrm{H}), 5.22-5.08(\mathrm{~m}, 2 \mathrm{H})$, $4.11-3.94(\mathrm{~m}, 2 \mathrm{H}), 3.67(\mathrm{t}, J=6.9 \mathrm{~Hz}, 2 \mathrm{H}), 3.57-3.38(\mathrm{~m}, 2 \mathrm{H}), 3.18-3.01(\mathrm{~m}, 1 \mathrm{H}), 2.54-2.35(\mathrm{~m}, 2 \mathrm{H}), 1.93-$ $1.75(\mathrm{~m}, 4 \mathrm{H}) .{ }^{13} \mathrm{C} \mathrm{NMR}\left(125 \mathrm{MHz}, \mathrm{CDCl}_{3}\right) \delta 173.6,132.8,118.8,110.5,66.6,45.3,40.0,32.0,28.4$. HRMS (ESI) $\mathrm{m} / \mathrm{z}:[\mathrm{M}+\mathrm{H}]^{+}$calcd for $\mathrm{C}_{11} \mathrm{H}_{17} \mathrm{~N}_{2} \mathrm{O}_{2} 209.1285$; found: 209.1272 .

\section{Spectral data for tert-butyl 4-(but-3-en-1-yl(cyano)carbamoyl)piperidine-1-carboxylate (10)}<smiles>C=CCCN(C)C(=O)C1CCN(C(=O)OC(C)(C)C)CC1</smiles>

Purified by column chromatography (silica gel, EtOAc/Petroleum ether: $1 / 5$ ) to afford the title compound (19\% yield, $58 \mathrm{mg}$ ) as colorless oil. ${ }^{1} \mathrm{H}$ NMR $\left(400 \mathrm{MHz}, \mathrm{CDCl}_{3}\right) \delta 5.72(\mathrm{ddt}, J=17.1,10.2,6.9 \mathrm{~Hz}, 1 \mathrm{H}), 5.31-5.01(\mathrm{~m}, 2 \mathrm{H})$, 4.15 (brs, 2H), 3.67 (t, $J=6.8 \mathrm{~Hz}, 2 \mathrm{H}), 3.11-2.93(\mathrm{~m}, 1 \mathrm{H}), 2.88-2.74(\mathrm{~m}, 2 \mathrm{H}), 2.51-2.36(\mathrm{~m}, 2 \mathrm{H}), 1.92-1.81$ (m, 2H), $1.74-1.61(\mathrm{~m}, 2 \mathrm{H}), 1.46(\mathrm{~s}, 9 \mathrm{H}) .{ }^{13} \mathrm{C} \mathrm{NMR}\left(125 \mathrm{MHz}, \mathrm{CDCl}_{3}\right) \delta 173.8,154.5,132.8,118.9,110.4,79.9$, 45.3, 41.0, 32.0, 28.4, 27.9. HRMS (ESI) m/z: $[\mathrm{M}+\mathrm{Na}]^{+}$calcd for $\mathrm{C}_{16} \mathrm{H}_{25} \mathrm{~N}_{3} \mathrm{NaO}_{3} 330.1788$; found: 330.1777 . 
<smiles>C=C(C)CCN(C)C(=O)C(c1ccccc1)c1ccccc1</smiles>

Purified by column chromatography (silica gel, EtOAc/Petroleum ether: 1/20) to afford the title compound (75\% yield, $228 \mathrm{mg}$ ) as colorless oil. ${ }^{1} \mathrm{H}$ NMR $\left(300 \mathrm{MHz}, \mathrm{CDCl}_{3}\right) \delta 7.40-7.26(\mathrm{~m}, 10 \mathrm{H}), 5.60(\mathrm{~s}, 1 \mathrm{H}), 4.78-4.75(\mathrm{~m}$, $1 \mathrm{H}), 4.67(\mathrm{dq}, J=2.0,1.0 \mathrm{~Hz}, 1 \mathrm{H}), 3.75(\mathrm{t}, J=7.1 \mathrm{~Hz}, 2 \mathrm{H}), 2.35(\mathrm{t}, J=7.0,2 \mathrm{H}), 1.72(\mathrm{dd}, J=1.5,0.9 \mathrm{~Hz}, 3 \mathrm{H}) .{ }^{13} \mathrm{C}$ $\operatorname{NMR}\left(125 \mathrm{MHz}, \mathrm{CDCl}_{3}\right) \delta 171.5,140.2,136.9,128.9,128.8,127.9,114.1,110.3,55.9,44.8,35.6,21.9$. HRMS (ESI) $\mathrm{m} / \mathrm{z}:[\mathrm{M}+\mathrm{H}]^{+}$calcd for $\mathrm{C}_{20} \mathrm{H}_{21} \mathrm{~N}_{2} \mathrm{O} 305.1648$; found: 305.1647 .

\section{Spectral data for N-cyano-2,2-diphenyl-N-(3-phenylbut-3-en-1-yl)acetamide (1q)}<smiles>C=C(CCN(C#N)C(=O)C(c1ccccc1)c1ccccc1)c1ccccc1</smiles>

Purified by column chromatography (silica gel, EtOAc/Petroleum ether: $1 / 20)$ to afford the title compound (88\% yield, $322 \mathrm{mg}$ ) as yellowish oil. ${ }^{1} \mathrm{H}$ NMR $\left(400 \mathrm{MHz} \mathrm{CDCl}_{3}\right) \delta 7.37-7.27(\mathrm{~m}, 15 \mathrm{H}), 5.58(\mathrm{~s}, 1 \mathrm{H}), 5.30(\mathrm{~s}, 1 \mathrm{H}), 5.03$ (s, $1 \mathrm{H}), 3.70(\mathrm{t}, J=7.2 \mathrm{~Hz}, 2 \mathrm{H}), 2.85(\mathrm{t}, J=7.3 \mathrm{~Hz}, 2 \mathrm{H}) .{ }^{13} \mathrm{C} \mathrm{NMR}\left(125 \mathrm{MHz}, \mathrm{CDCl}_{3}\right) \delta 171.5,143.4,139.5,137.0$, 129.0, 128.9, 128.7, 128.1, 128.0, 126.2, 115.9, 110.4, 56.0, 46.0, 33.6. HRMS (ESI) m/z: $[\mathrm{M}+\mathrm{H}]^{+}$calcd for $\mathrm{C}_{25} \mathrm{H}_{23} \mathrm{~N}_{2} \mathrm{O} 367.1805$; found: 367.1794 .

\section{Spectral data for N-cyano-N-(2-(cyclopent-1-en-1-yl)ethyl)-2,2-diphenylacetamide (1r)}<smiles>CN(CCC1=CCCC1)C(C(=O)C#N)c1ccccc1</smiles>

Purified by column chromatography (silica gel, EtOAc/Petroleum ether: $1 / 20)$ to afford the title compound (74\% yield, $245 \mathrm{mg}$ ) as yellowish oil. ${ }^{1} \mathrm{H}$ NMR $\left(500 \mathrm{MHz}, \mathrm{CDCl}_{3}\right) \delta 7.37-7.32(\mathrm{~m}, 4 \mathrm{H}), 7.32-7.27(\mathrm{~m}, 6 \mathrm{H}), 5.60(\mathrm{~s}$, $1 \mathrm{H}), 5.34(\mathrm{~s}, 1 \mathrm{H}), 3.74(\mathrm{t}, J=6.9 \mathrm{~Hz}, 2 \mathrm{H}), 2.44(\mathrm{t}, J=6.9 \mathrm{~Hz}, 2 \mathrm{H}), 2.21(\mathrm{t}, J=7.5 \mathrm{~Hz}, 4 \mathrm{H}), 1.84-1.78(\mathrm{~m}, 2 \mathrm{H}) .{ }^{13} \mathrm{C}$ $\operatorname{NMR}\left(125 \mathrm{MHz}, \mathrm{CDCl}_{3}\right) \delta 171.5,139.0,137.1,129.0,128.9,128.0,110.4,56.0,45.0,34.7,32.6,29.2,23.5 . \mathrm{HRMS}$ (ESI) $\mathrm{m} / \mathrm{z}:[\mathrm{M}+\mathrm{H}]^{+}$calcd for $\mathrm{C}_{22} \mathrm{H}_{23} \mathrm{~N}_{2} \mathrm{O} 331.1805$; found: 331.1818 .

\section{Spectral data for N-cyano-N-(2-(cyclohex-1-en-1-yl)ethyl)-2,2-diphenylacetamide (1s)}<smiles>N#CC(=O)N(CCC1=CCCCC1)c1ccccc1</smiles>

Purified by column chromatography (silica gel, EtOAc/Petroleum ether: 1/50) to afford the title compound (45\% yield, $155 \mathrm{mg}$ ) as colorless oil. ${ }^{1} \mathrm{H}$ NMR $\left(500 \mathrm{MHz}, \mathrm{CDCl}_{3}\right) \delta 7.39-7.26(\mathrm{~m}, 10 \mathrm{H}), 5.61(\mathrm{~s}, 1 \mathrm{H}), 5.44-5.24(\mathrm{~m}$, $1 \mathrm{H}), 3.70(\mathrm{t}, J=6.7 \mathrm{~Hz}, 2 \mathrm{H}), 2.25(\mathrm{t}, J=6.8 \mathrm{~Hz}, 2 \mathrm{H}), 1.94-1.80(\mathrm{~m}, 4 \mathrm{H}), 1.61-1.52(\mathrm{~m}, 2 \mathrm{H}), 1.50-1.42(\mathrm{~m}, 2 \mathrm{H})$. ${ }^{13} \mathrm{C}$ NMR $\left(125 \mathrm{MHz}, \mathrm{CDCl}_{3}\right) \delta 171.5,137.1,132.4,128.9,128.0,125.7,110.6,56.0,45.0,36.1,27.8,25.3,22.8$, 22.1. HRMS (ESI) m/z: [M+ H] calcd for $\mathrm{C}_{23} \mathrm{H}_{25} \mathrm{~N}_{2} \mathrm{O} 345.1961$; found: 345.1958 .

\section{Spectral data for N-cyano-2,2-diphenyl-N-(1-phenylbut-3-en-1-yl)acetamide (1t)}<smiles>C=CCC(c1ccccc1)N(C)C(=O)C(c1ccccc1)c1ccccc1</smiles>

Purified by column chromatography (silica gel, EtOAc/Petroleum ether: 1/60) to afford the title compound (78\% 
yield, $286 \mathrm{mg}$ ) as yellowish oil. ${ }^{1} \mathrm{H}$ NMR $\left(400 \mathrm{MHz}, \mathrm{CDCl}_{3}\right) \delta 7.38-7.30(\mathrm{~m}, 7 \mathrm{H}), 7.30-7.22(\mathrm{~m}, 6 \mathrm{H}), 7.16-7.11$ $(\mathrm{m}, 2 \mathrm{H}), 5.71-5.58(\mathrm{~m}, 1 \mathrm{H}), 5.55(\mathrm{~s}, 1 \mathrm{H}), 5.52-5.44(\mathrm{~m}, 1 \mathrm{H}), 5.17(\mathrm{~d}, J=17.1 \mathrm{~Hz}, 1 \mathrm{H}), 5.03(\mathrm{~d}, J=10.1 \mathrm{~Hz}, 1 \mathrm{H})$, $2.91-2.78(\mathrm{~m}, 1 \mathrm{H}), 2.77-2.64(\mathrm{~m}, 1 \mathrm{H}) .{ }^{13} \mathrm{C} \mathrm{NMR}\left(125 \mathrm{MHz}, \mathrm{CDCl}_{3}\right) \delta 171.5,137.4,137.1,136.7,132.4,129.1$, 129.0, 128.9, 128.8, 128.0, 127.9, 127.4, 119.5, 109.1, 57.8, 56.6, 36.6. HRMS (ESI) m/z: $[\mathrm{M}+\mathrm{H}]^{+}$calcd for $\mathrm{C}_{25} \mathrm{H}_{23} \mathrm{~N}_{2} \mathrm{O} 367.1805$; found: 367.1784 .

\section{Spectral data for N-cyano-N-(2-methylpent-4-en-2-yl)-2,2-diphenylacetamide (1u)}<smiles>C=CCC(C)(C)N(C)C(=O)C(c1ccccc1)c1ccccc1</smiles>

Purified by column chromatography (silica gel, EtOAc/Petroleum ether: 1/40) to afford the title compound (17\% yield, $54 \mathrm{mg}$ ) as yellowish oil. ${ }^{1} \mathrm{H}$ NMR $\left(400 \mathrm{MHz}, \mathrm{CDCl}_{3}\right) \delta 7.40-7.26(\mathrm{~m}, 10 \mathrm{H}), 5.66(\mathrm{~s}, 1 \mathrm{H}), 5.70-5.58(\mathrm{~m}$, $1 \mathrm{H}), 5.14-4.95(\mathrm{~m}, 2 \mathrm{H}), 2.63(\mathrm{~d}, J=7.4 \mathrm{~Hz}, 2 \mathrm{H}), 1.49(\mathrm{~s}, 6 \mathrm{H}) .{ }^{13} \mathrm{C}$ NMR $\left(125 \mathrm{MHz}, \mathrm{CDCl}_{3}\right) \delta 172.4,137.3,132.0$, 129.0, 128.9, 127.9, 120.1, 110.2, 63.5, 57.4, 43.0, 26.7. HRMS (ESI) m/z: $[\mathrm{M}+\mathrm{H}]^{+}$calcd for $\mathrm{C}_{21} \mathrm{H}_{23} \mathrm{~N}_{2} \mathrm{O} 319.1805$; found: 319.1803 .

\section{Spectral data for N-((1-allylcyclopentyl)methyl)-N-cyano-2,2-diphenylacetamide (1v)}<smiles>C=CCC1(CN(C)C(=O)C(c2ccccc2)c2ccccc2)CCCC1</smiles>

Purified by column chromatography (silica gel, EtOAc/Petroleum ether: 1/30) to afford the title compound (67\% yield, $240 \mathrm{mg}$ ) as yellowish oil. ${ }^{1} \mathrm{H}$ NMR $\left(500 \mathrm{MHz}, \mathrm{CDCl}_{3}\right) \delta 7.38-7.27(\mathrm{~m}, 10 \mathrm{H}), 5.76(\mathrm{ddt}, J=17.4,10.4,7.3$ $\mathrm{Hz}, 1 \mathrm{H}), 5.69(\mathrm{~s}, 1 \mathrm{H}), 5.11-4.99(\mathrm{~m}, 2 \mathrm{H}), 3.60(\mathrm{~s}, 2 \mathrm{H}), 2.06(\mathrm{~d}, J=7.3 \mathrm{~Hz}, 2 \mathrm{H}), 1.64-1.56(\mathrm{~m}, 4 \mathrm{H}), 1.50-1.39$ (m, 4H). ${ }^{13} \mathrm{C} \mathrm{NMR}\left(125 \mathrm{MHz}, \mathrm{CDCl}_{3}\right) \delta 172.3,137.0,134.2,129.0,128.0,118.6,112.0,55.8,53.1,47.6,41.4,35.1$, 24.4. HRMS (ESI) m/z: $[\mathrm{M}+\mathrm{H}]^{+}$calcd for $\mathrm{C}_{24} \mathrm{H}_{27} \mathrm{~N}_{2} \mathrm{O} 359.2118$; found: 359.2120 .

\section{Spectral data for N-cyano-N-(pent-4-en-1-yl)-2,2-diphenylacetamide (1w)}<smiles>C=CCCN(C)C(=O)C(c1ccccc1)c1ccccc1</smiles>

Purified by column chromatography (silica gel, EtOAc/Petroleum ether: 1/20) to afford the title compound (53\% yield, $161 \mathrm{mg}$ ) as colorless oil. ${ }^{1} \mathrm{H}$ NMR $\left(400 \mathrm{MHz}, \mathrm{CDCl}_{3}\right) \delta 7.39-7.27(\mathrm{~m}, 10 \mathrm{H}), 5.73(\mathrm{ddt}, J=17.0,10.3,6.6 \mathrm{~Hz}$, $1 \mathrm{H}), 5.60(\mathrm{~s}, 1 \mathrm{H}), 5.06-4.95(\mathrm{~m}, 2 \mathrm{H}), 3.63(\mathrm{t}, J=7.3 \mathrm{~Hz}, 2 \mathrm{H}), 2.05(\mathrm{q}, J=7.1 \mathrm{~Hz}, 2 \mathrm{H}), 1.80-1.73(\mathrm{~m}, 2 \mathrm{H}) .{ }^{13} \mathrm{C}$ NMR $\left(125 \mathrm{MHz}, \mathrm{CDCl}_{3}\right) \delta 171.6,137.0,136.5,129.0,128.9,128.0,116.3,110.5,56.1,46.5,30.3,26.7$. HRMS (ESI) m/z: $[\mathrm{M}+\mathrm{H}]^{+}$calcd for $\mathrm{C}_{20} \mathrm{H}_{21} \mathrm{~N}_{2} \mathrm{O} 305.1648$; found: 305.1639 .

\section{Spectral data for N-(but-3-en-1-yl)-N-(2,2-diphenylethyl)cyanamide (5a)}<smiles>C=CCCN(C#N)CC(c1ccccc1)c1ccccc1</smiles>

Purified by column chromatography (silica gel, EtOAc/Petroleum ether: $1 / 5$ ) to afford the title compound ( $82 \%$ yield, $227 \mathrm{mg}$ ) as white solid, mp: $72-73{ }^{\circ} \mathrm{C} .{ }^{1} \mathrm{H}$ NMR $\left(500 \mathrm{MHz}, \mathrm{CDCl}_{3}\right) \delta 7.38-7.33(\mathrm{~m}, 4 \mathrm{H}), 7.31-7.24(\mathrm{~m}, 6 \mathrm{H}), 5.65$ (ddt, $J=17.1,10.3,6.8 \mathrm{~Hz}, 1 \mathrm{H}), 5.22-4.86(\mathrm{~m}, 2 \mathrm{H}), 4.36(\mathrm{t}, J=8.1 \mathrm{~Hz}, 1 \mathrm{H}), 3.66(\mathrm{~d}, J=8.2 \mathrm{~Hz}, 2 \mathrm{H}), 2.93(\mathrm{t}, J=$ $7.3 \mathrm{~Hz}, 2 \mathrm{H}), 2.38-2.17(\mathrm{~m}, 2 \mathrm{H}) .{ }^{13} \mathrm{C} \mathrm{NMR}\left(125 \mathrm{MHz}, \mathrm{CDCl}_{3}\right) \delta 141.0,133.7,128.9,128.0,127.2,117.9,117.2$, 56.4, 51.7, 49.8, 32.1. HRMS (ESI) m/z: [M + H] calcd for $\mathrm{C}_{19} \mathrm{H}_{21} \mathrm{~N}_{2} 277.1699$; found: 277.1696. 
<smiles>C=CCCN(C#N)CCc1ccccc1</smiles>

Purified by column chromatography (silica gel, EtOAc/Petroleum ether: $1 / 5)$ to afford the title compound (72\% yield, $144 \mathrm{mg}$ ) as colorless oil. ${ }^{1} \mathrm{H}$ NMR $\left(500 \mathrm{MHz}, \mathrm{CDCl}_{3}\right) \delta 7.37-7.29(\mathrm{~m}, 2 \mathrm{H}), 7.28-7.15(\mathrm{~m}, 3 \mathrm{H}), 5.72$ (ddt, $J=17.1$, $10.2,6.8 \mathrm{~Hz}, 1 \mathrm{H}), 5.21-4.92(\mathrm{~m}, 2 \mathrm{H}), 3.29-3.17(\mathrm{~m}, 2 \mathrm{H}), 3.01(\mathrm{t}, J=7.2 \mathrm{~Hz}, 2 \mathrm{H}), 2.95(\mathrm{t}, J=7.2 \mathrm{~Hz}, 2 \mathrm{H}), 2.44-$ $2.20(\mathrm{~m}, 2 \mathrm{H}) .{ }^{13} \mathrm{C} \mathrm{NMR}\left(100 \mathrm{MHz}, \mathrm{CDCl}_{3}\right) \delta 137.7,133.8,128.9,128.8,126.9,118.1,117.4,53.2,51.5,34.4,32.2$. HRMS (ESI) m/z: [M+ H] calcd for $\mathrm{C}_{13} \mathrm{H}_{17} \mathrm{~N}_{2} 201.1386$; found: 201.1380 .

\section{Spectral data for N-(but-3-en-1-yl)-N-butylcyanamide (5c)}<smiles>C=CCCN(C#N)CCCC</smiles>

Purified by column chromatography (silica gel, EtOAc/Petroleum ether: 1/10) to afford the title compound (44\% yield, $67 \mathrm{mg}$ ) as a colorless oil. ${ }^{1} \mathrm{H}$ NMR $\left(300 \mathrm{MHz}, \mathrm{CDCl}_{3}\right) \delta 5.90-5.67(\mathrm{~m}, 1 \mathrm{H}), 5.34-5.01(\mathrm{~m}, 2 \mathrm{H}), 3.06(\mathrm{t}, J=$ $7.2 \mathrm{~Hz}, 2 \mathrm{H}), 3.00(\mathrm{t}, J=6.0 \mathrm{~Hz}, 2 \mathrm{H}), 2.50-2.33(\mathrm{~m}, 2 \mathrm{H}), 1.75-1.54(\mathrm{~m}, 2 \mathrm{H}), 1.51-1.29(\mathrm{~m}, 2 \mathrm{H}), 0.95(\mathrm{t}, J=7.3$ $\mathrm{Hz}, 3 \mathrm{H}) .{ }^{13} \mathrm{C} \mathrm{NMR}\left(125 \mathrm{MHz}, \mathrm{CDCl}_{3}\right) \delta 133.8,117.8,117.6,51.3,51.0,32.1,29.7,19.7,13.6 . \mathrm{HRMS}(\mathrm{ESI}) \mathrm{m} / \mathrm{z}$ : [M $+\mathrm{H}]^{+}$calcd for $\mathrm{C}_{9} \mathrm{H}_{17} \mathrm{~N}_{2}$ 153.1386; Found: 153.1380 .

\section{Spectral data for N-(but-3-en-1-yl)-N-isobutylcyanamide (5d)}<smiles>C=CCCN(C)CC(C)C</smiles>

Purified by column chromatography (silica gel, EtOAc/Petroleum ether: $1 / 8$ ) to afford the title compound (61\% yield, $93 \mathrm{mg}$ ) as colorless oil. ${ }^{1} \mathrm{H} \mathrm{NMR}\left(500 \mathrm{MHz}, \mathrm{CDCl}_{3}\right) \delta 5.79$ (ddt, $\left.J=17.0,10.1,6.8 \mathrm{~Hz}, 1 \mathrm{H}\right), 5.22-5.07(\mathrm{~m}, 2 \mathrm{H})$, 3.07 (t, $J=7.3 \mathrm{~Hz}, 2 \mathrm{H}), 2.80$ (d, $J=7.4 \mathrm{~Hz}, 2 \mathrm{H}), 2.41$ (q, $J=7.0 \mathrm{~Hz}, 2 \mathrm{H}), 2.01-1.89$ (m, 1H), $0.97(\mathrm{~d}, J=6.6 \mathrm{~Hz}$, $6 \mathrm{H}) .{ }^{13} \mathrm{C}$ NMR $\left(125 \mathrm{MHz}, \mathrm{CDCl}_{3}\right) \delta 133.9,118.0,59.6,51.5,32.2,27.4,20.0$. HRMS (ESI) $\mathrm{m} / \mathrm{z}:[\mathrm{M}+\mathrm{H}]^{+}$calcd for $\mathrm{C}_{9} \mathrm{H}_{17} \mathrm{~N}_{2}$ 153.1386; found: 153.1381 .

\section{Spectral data for methyl N-(but-3-en-1-yl)-N-cyano-L-phenylalaninate (5e)}<smiles>C=CCCN(C)C(Cc1ccccc1)C(C)=O</smiles>

Purified by column chromatography (silica gel, EtOAc/Petroleum ether: $1 / 5)$ to afford the title compound (80\% yield, $207 \mathrm{mg}$ ) as colorless oil. ${ }^{1} \mathrm{H}$ NMR $\left(500 \mathrm{MHz}, \mathrm{CDCl}_{3}\right) \delta 7.37-7.27(\mathrm{~m}, 5 \mathrm{H}), 5.52(\mathrm{ddt}, J=16.8,9.8,6.7 \mathrm{~Hz}, 1 \mathrm{H})$, $5.08-4.79(\mathrm{~m}, 2 \mathrm{H}), 3.80(\mathrm{~s}, 3 \mathrm{H}), 3.67(\mathrm{dd}, J=10.5,5.0 \mathrm{~Hz}, 1 \mathrm{H}), 3.39-3.22(\mathrm{~m}, 1 \mathrm{H}), 3.18-3.06(\mathrm{~m}, 2 \mathrm{H}), 2.92-$ $2.79(\mathrm{~m}, 1 \mathrm{H}), 2.28-2.07(\mathrm{~m}, 2 \mathrm{H}) .{ }^{13} \mathrm{C}$ NMR $\left(125 \mathrm{MHz}, \mathrm{CDCl}_{3}\right) \delta 170.3,136.0,133.3,129.1,128.8,127.3,117.8$, 115.0, 64.9, 52.8, 52.0, 36.6, 31.9. HRMS (ESI) m/z: $[\mathrm{M}+\mathrm{H}]^{+}$calcd for $\mathrm{C}_{15} \mathrm{H}_{19} \mathrm{~N}_{2} \mathrm{O}_{2}$ 259.1441; found: 259.1446 .

\section{Spectral data for methyl N-(but-3-en-1-yl)-N-cyano-L-valinate (5f)}<smiles>C=CCCN(C)C(C(C)=O)C(C)C</smiles>

Purified by column chromatography (silica gel, EtOAc/Petroleum ether: $1 / 8)$ to afford the title compound (55\% yield, $116 \mathrm{mg})$ as yellowish oil. ${ }^{1} \mathrm{H}$ NMR $\left(300 \mathrm{MHz}, \mathrm{CDCl}_{3}\right) \delta 5.85-5.61(\mathrm{~m}, 1 \mathrm{H}), 5.28-5.02(\mathrm{~m}, 2 \mathrm{H}), 3.80(\mathrm{~s}, 3 \mathrm{H}), 3.25$ $-3.11(\mathrm{~m}, 1 \mathrm{H}), 3.09-2.94(\mathrm{~m}, 2 \mathrm{H}), 2.52-2.33(\mathrm{~m}, 2 \mathrm{H}), 2.31-2.14(\mathrm{~m}, 1 \mathrm{H}), 1.11(\mathrm{~d}, J=6.6 \mathrm{~Hz}, 3 \mathrm{H}), 0.97(\mathrm{~d}, J=$ 
$6.7 \mathrm{~Hz}, 3 \mathrm{H}) .{ }^{13} \mathrm{C} \mathrm{NMR}\left(125 \mathrm{MHz}, \mathrm{CDCl}_{3}\right) \delta 170.8,133.4,118.1,115.1,69.5,52.3,51.7,32.0,29.5,19.8,19.0 . \mathrm{HRMS}$ (ESI) $\mathrm{m} / \mathrm{z}:[\mathrm{M}+\mathrm{H}]^{+}$calcd for $\mathrm{C}_{11} \mathrm{H}_{19} \mathrm{~N}_{2} \mathrm{O}_{2} 211.1441$; found: 211.1433 .

\section{Spectral data for N-(but-3-en-1-yl)-N-cyclopentylcyanamide (5g)}<smiles>C=CCCN(C)C1CCCC1</smiles>

Purified by column chromatography (silica gel, EtOAc/Petroleum ether: $1 / 8)$ to afford the title compound (70\% yield, 115 $\mathrm{mg}$ ) as colorless oil. ${ }^{1} \mathrm{H}$ NMR $\left(400 \mathrm{MHz}, \mathrm{CDCl}_{3}\right) \delta 5.78(\mathrm{ddt}, J=17.0,10.2,6.8 \mathrm{~Hz}, 1 \mathrm{H}), 5.25-4.98(\mathrm{~m}, 2 \mathrm{H}), 3.42-3.24$ (m, $1 \mathrm{H}), 3.06(\mathrm{t}, J=7.3 \mathrm{~Hz}, 2 \mathrm{H}), 2.55-2.33(\mathrm{~m}, 2 \mathrm{H}), 2.00-1.86(\mathrm{~m}, 2 \mathrm{H}), 1.83-1.66(\mathrm{~m}, 4 \mathrm{H}), 1.66-1.52(\mathrm{~m}, 2 \mathrm{H}) .{ }^{13} \mathrm{C}$ NMR $\left(125 \mathrm{MHz}, \mathrm{CDCl}_{3}\right) \delta 1339,117.7,116.5,61.7,50.4,32.3,31.0,23.4$. HRMS (ESI) m/z: $[\mathrm{M}+\mathrm{H}]^{+}$calcd for $\mathrm{C}_{10} \mathrm{H}_{17} \mathrm{~N}_{2}$ 165.1386; found: 165.1380 .

\section{Spectral data for N-(2,2-diphenylethyl)-N-(3-methylbut-3-en-1-yl)cyanamide (5h)}<smiles>C=C(C)CCN(C)CC(c1ccccc1)c1ccccc1</smiles>

Purified by column chromatography (silica gel, EtOAc/Petroleum ether: $1 / 20)$ to afford the title compound (49\% yield, $142 \mathrm{mg}$ ) as white solid, $\mathrm{mp}: 63-64{ }^{\circ} \mathrm{C} .{ }^{1} \mathrm{H}$ NMR $\left(400 \mathrm{MHz}, \mathrm{CDCl}_{3}\right) \delta 7.35-7.29(\mathrm{~m}, 4 \mathrm{H}), 7.29-7.21(\mathrm{~m}$, $6 \mathrm{H}), 4.77(\mathrm{~s}, 1 \mathrm{H}), 4.65(\mathrm{~s}, 1 \mathrm{H}), 4.33(\mathrm{t}, J=8.1 \mathrm{~Hz}, 1 \mathrm{H}), 3.63(\mathrm{~d}, J=8.1 \mathrm{~Hz}, 2 \mathrm{H}), 3.01-2.85(\mathrm{~m}, 2 \mathrm{H}), 2.20$ (t, $J=7.5$ $\mathrm{Hz}, 2 \mathrm{H}), 1.65(\mathrm{~s}, 3 \mathrm{H}) .{ }^{13} \mathrm{C} \mathrm{NMR}\left(100 \mathrm{MHz}, \mathrm{CDCl}_{3}\right) \delta 141.2,141.0,128.9,128.1,127.3,117.3,112.9,56.4,50.6$, 49.8, 35.7, 22.4. HRMS (ESI) m/z: [M+ H] calcd for $\mathrm{C}_{20} \mathrm{H}_{23} \mathrm{~N}_{2}$ 291.1856; found: 291.1861.

\section{Spectral data for N-(2,2-diphenylethyl)-N-(3-phenylbut-3-en-1-yl)cyanamide (5i)}<smiles>C=C(CCN(C)CC(c1ccccc1)c1ccccc1)c1ccccc1</smiles>

Purified by column chromatography (silica gel, EtOAc/Petroleum ether: 1/10) to afford the title compound (28\% yield, $99 \mathrm{mg}$ ) as yellowish oil. ${ }^{1} \mathrm{H}$ NMR $\left(400 \mathrm{MHz}, \mathrm{CDCl}_{3}\right) \delta 7.38-7.27(\mathrm{~m}, 10 \mathrm{H}), 7.24-7.13(\mathrm{~m}, 5 \mathrm{H}), 5.32(\mathrm{~s}$, 1H), 5.06 (s, 1H), 4.26 (t, $J=8.1 \mathrm{~Hz}, 1 \mathrm{H}), 3.57$ (d, $J=8.1 \mathrm{~Hz}, 2 \mathrm{H}), 2.91$ (t, $J=7.3 \mathrm{~Hz}, 2 \mathrm{H}), 2.69$ (t, $J=7.4 \mathrm{~Hz}, 2 \mathrm{H}$ ). ${ }^{13} \mathrm{C} \mathrm{NMR}\left(125 \mathrm{MHz}, \mathrm{CDCl}_{3}\right) \delta 144.2,141.0,139.9,128.9,128.7,128.1,128.0,127.2,126.2,117.2,115.3,56.7,51.0$, 49.9, 33.9. HRMS (ESI) $\mathrm{m} / \mathrm{z}$ : $[\mathrm{M}+\mathrm{H}]^{+}$calcd for $\mathrm{C}_{25} \mathrm{H}_{25} \mathrm{~N}_{2}$ 353.2012; found: 353.2017 .

\section{Spectral data for N-cyano-N-(2,2-diphenylethyl)-2,2-dimethylbut-3-enamide (5j)}<smiles>C=CC(C)(C)C(=O)N(C)CC(c1ccccc1)c1ccccc1</smiles>

Purified by column chromatography (silica gel, EtOAc/Petroleum ether: 1/40) to afford the title compound (80\% yield, $254 \mathrm{mg}$ ) as colorless oil. ${ }^{1} \mathrm{H}$ NMR $\left(500 \mathrm{MHz}, \mathrm{CDCl}_{3}\right) \delta 7.37-7.31(\mathrm{~m}, 8 \mathrm{H}), 7.30-7.25(\mathrm{~m}, 2 \mathrm{H}), 5.82(\mathrm{dd}, J$ $=17.5,10.7 \mathrm{~Hz}, 1 \mathrm{H}), 5.12(\mathrm{~d}, J=10.7 \mathrm{~Hz}, 1 \mathrm{H}), 4.89(\mathrm{~d}, J=17.6 \mathrm{~Hz}, 1 \mathrm{H}), 4.50(\mathrm{t}, J=8.6 \mathrm{~Hz}, 1 \mathrm{H}), 4.27(\mathrm{~d}, J=8.6$ $\mathrm{Hz}, 2 \mathrm{H}), 1.27$ (s, 6H). ${ }^{13} \mathrm{C}$ NMR (125 MHz, $\left.\mathrm{CDCl}_{3}\right) \delta 175.3,140.2,139.9,128.9,128.3,127.5,116.6,110.6,51.2$, 49.0, 46.9, 25.5. HRMS (ESI) m/z: [M+ H] $]^{+}$calcd for $\mathrm{C}_{21} \mathrm{H}_{23} \mathrm{~N}_{2} \mathrm{O} 319.1805$; found: 319.1799 . 
<smiles>C=CC(C)(C)C(=O)N(C)CCc1ccccc1</smiles>

Purified by column chromatography (silica gel, EtOAc/Petroleum ether: 1/80) to afford the title compound (34\% yield, $82 \mathrm{mg}$ ) as colorless oil. ${ }^{1} \mathrm{H}$ NMR (500 MHz, $\left.\mathrm{CDCl}_{3}\right) \delta 7.34-7.29(\mathrm{~m}, 2 \mathrm{H}), 7.27-7.24(\mathrm{~m}, 1 \mathrm{H}), 7.23-7.21$ $(\mathrm{m}, 2 \mathrm{H}), 5.97(\mathrm{dd}, J=17.5,10.6 \mathrm{~Hz}, 1 \mathrm{H}), 5.25(\mathrm{~d}, J=10.7 \mathrm{~Hz}, 1 \mathrm{H}), 5.09(\mathrm{~d}, J=17.5 \mathrm{~Hz}, 1 \mathrm{H}), 3.84(\mathrm{t}, J=7.5 \mathrm{~Hz}$, $2 \mathrm{H}), 2.97(\mathrm{t}, J=7.5 \mathrm{~Hz}, 2 \mathrm{H}), 1.38(\mathrm{~s}, 6 \mathrm{H}) .{ }^{13} \mathrm{C} \mathrm{NMR}\left(125 \mathrm{MHz}, \mathrm{CDCl}_{3}\right) \delta 175.2,140.4,136.6,129.1,128.8,127.2$, 116.8, 110.8, 49.3, 47.0, 33.7, 25.6. HRMS (ESI) m/z: $[\mathrm{M}+\mathrm{H}]^{+}$calcd for $\mathrm{C}_{15} \mathrm{H}_{19} \mathrm{~N}_{2} \mathrm{O}$ 243.1492; found: 243.1480 .

\section{Spectral data for N-cyano-N-(2,2-diphenylethyl)-2,2-dimethylpent-4-enamide (5I)}<smiles>C=CCC(C)(C)C(=O)N(C)Cc1ccccc1</smiles>

Purified by column chromatography (silica gel, EtOAc/Petroleum ether: 1/30) to afford the title compound (89\% yield, $296 \mathrm{mg}$ ) as colorless oil. ${ }^{1} \mathrm{H}$ NMR $\left(400 \mathrm{MHz}, \mathrm{CDCl}_{3}\right) \delta 7.37-7.22(\mathrm{~m}, 10 \mathrm{H}), 5.42(\mathrm{ddt}, J=17.3,10.1,7.3 \mathrm{~Hz}$, 1H), $5.11-4.88(\mathrm{~m}, 2 \mathrm{H}), 4.46(\mathrm{t}, J=8.6 \mathrm{~Hz}, 1 \mathrm{H}), 4.24(\mathrm{~d}, J=8.6 \mathrm{~Hz}, 2 \mathrm{H}), 2.37$ (d, $J=7.3 \mathrm{~Hz}, 2 \mathrm{H}), 1.17(\mathrm{~s}, 6 \mathrm{H})$. ${ }^{13} \mathrm{C}$ NMR $\left(100 \mathrm{MHz}, \mathrm{CDCl}_{3}\right) \delta 175.7,139.9,132.7,128.8,128.2,127.4,118.8,111.1,51.5,48.9,44.7,43.4,24.5$. HRMS (ESI) m/z: $[\mathrm{M}+\mathrm{H}]^{+}$calcd for $\mathrm{C}_{22} \mathrm{H}_{25} \mathrm{~N}_{2} \mathrm{O} 333.1961$; found: 333.1962 .

\section{Spectral data for N-cyano-N-isobutyl-2,2-dimethylpent-4-enamide (5m)}<smiles>C=CCC(C)(C)C(=O)N(C)CC(C)C</smiles>

Purified by column chromatography (silica gel, EtOAc/Petroleum ether: $1 / 30)$ to afford the title compound (67\% yield, $140 \mathrm{mg}$ ) as colorless oil. ${ }^{1} \mathrm{H}$ NMR $\left(400 \mathrm{MHz}, \mathrm{CDCl}_{3}\right) \delta 5.73$ (ddt, $\left.J=17.2,10.0,7.3 \mathrm{~Hz}, 1 \mathrm{H}\right), 5.25-5.03(\mathrm{~m}$, 2H), $3.41(\mathrm{~d}, J=7.4 \mathrm{~Hz}, 2 \mathrm{H}), 2.58(\mathrm{~d}, J=7.3 \mathrm{~Hz}, 2 \mathrm{H}), 2.13-1.94(\mathrm{~m}, 1 \mathrm{H}), 1.40(\mathrm{~s}, 6 \mathrm{H}), 0.96(\mathrm{~d}, J=6.8 \mathrm{~Hz}, 6 \mathrm{H})$. ${ }^{13} \mathrm{C}$ NMR $\left(125 \mathrm{MHz}, \mathrm{CDCl}_{3}\right) \delta 175.9,133.0,119.0,111.9,55.7,44.9,43.5,27.3,24.9,19.7$. HRMS (ESI) m/z: [M + $\mathrm{H}]^{+}$calcd for $\mathrm{C}_{12} \mathrm{H}_{21} \mathrm{~N}_{2} \mathrm{O} 209.1648$; found: 209.1638 .

\section{Spectral data for N-cyano-2,2-dimethyl-N-phenethylpent-4-enamide (5n)}<smiles>C=CCC(C)(C)C(=O)N(C)CCc1ccccc1</smiles>

Purified by column chromatography (silica gel, EtOAc/Petroleum ether: 1/30) to afford the title compound (81\% yield, $208 \mathrm{mg}$ ) as colorless oil. ${ }^{1} \mathrm{H}$ NMR (400 MHz, $\left.\mathrm{CDCl}_{3}\right) \delta 7.36-7.29(\mathrm{~m}, 2 \mathrm{H}), 7.27-7.18(\mathrm{~m}, 3 \mathrm{H}), 5.62$ (ddt, $J$ $=17.3,10.1,7.3 \mathrm{~Hz}, 1 \mathrm{H}), 5.22-4.97(\mathrm{~m}, 2 \mathrm{H}), 3.83(\mathrm{t}, J=7.5 \mathrm{~Hz}, 2 \mathrm{H}), 2.96(\mathrm{t}, J=7.3 \mathrm{~Hz}, 2 \mathrm{H}), 2.50$ (d, $J=7.3 \mathrm{~Hz}$, 2H), $1.32(\mathrm{~s}, 6 \mathrm{H}) .{ }^{13} \mathrm{C} \mathrm{NMR}\left(125 \mathrm{MHz}, \mathrm{CDCl}_{3}\right) \delta 175.8,136.7,133.0,129.1,128.9,127.2,119.0,111.4,49.6,44.9$, 43.6, 33.8, 24.9. HRMS (ESI) m/z: [M+ H] calcd for $\mathrm{C}_{16} \mathrm{H}_{21} \mathrm{~N}_{2} \mathrm{O} 257.1648$; found: 257.1649.

\section{Spectral data for N-butyl-N-cyano-2,2-dimethylpent-4-enamide (5o)}<smiles>C=CCC(C)(C)C(=O)N(C)CCCC</smiles>

Purified by column chromatography (silica gel, EtOAc/Petroleum ether: $1 / 30)$ to afford the title compound (68\% 
yield, $142 \mathrm{mg})$ as colorless oil. ${ }^{1} \mathrm{H}$ NMR $\left(500 \mathrm{MHz}, \mathrm{CDCl}_{3}\right) \delta 5.79-5.65(\mathrm{~m}, 1 \mathrm{H}), 5.23-5.03(\mathrm{~m}, 2 \mathrm{H}), 3.59(\mathrm{t}, J=$ $7.3 \mathrm{~Hz}, 2 \mathrm{H}), 2.57$ (d, J = 7.4 Hz, 2H), $1.69-1.61(\mathrm{~m}, 2 \mathrm{H}), 1.39(\mathrm{~s}, 6 \mathrm{H}), 1.37-1.34(\mathrm{~m}, 2 \mathrm{H}), 0.95(\mathrm{t}, J=7.3 \mathrm{~Hz}, 3 \mathrm{H})$. ${ }^{13} \mathrm{C}$ NMR $\left(125 \mathrm{MHz}, \mathrm{CDCl}_{3}\right) \delta 175.9,133.0,119.0,111.7,48.6,45.0,43.6,29.6,25.0,19.6,13.7$. HRMS (ESI) m/z: $[\mathrm{M}+\mathrm{H}]^{+}$calcd for $\mathrm{C}_{12} \mathrm{H}_{21} \mathrm{~N}_{2} \mathrm{O} 209.1648$; found: 209.1645 .

\section{Spectral data for N-(but-3-en-1-yl)-N-(2,2-diphenylethyl)cyanamide (5p)}<smiles>C=CCCCN(C#N)CC(c1ccccc1)c1ccccc1</smiles>

Purified by column chromatography (silica gel, EtOAc/Petroleum ether: $1 / 5$ ) to afford the title compound (77\% yield, $224 \mathrm{mg}$ ) as colorless oil. ${ }^{1} \mathrm{H}$ NMR $\left(400 \mathrm{MHz}, \mathrm{CDCl}_{3}\right) \delta 7.35-7.29(\mathrm{~m}, 4 \mathrm{H}), 7.28-7.21(\mathrm{~m}, 6 \mathrm{H}), 5.66$ (ddt, $J=18.1$, 9.4, $6.7 \mathrm{~Hz}, 1 \mathrm{H}), 4.98-4.92(\mathrm{~m}, 2 \mathrm{H}), 4.33(\mathrm{t}, J=8.1 \mathrm{~Hz}, 1 \mathrm{H}), 3.61(\mathrm{~d}, J=8.1 \mathrm{~Hz}, 2 \mathrm{H}), 2.84(\mathrm{t}, J=7.1 \mathrm{~Hz}, 2 \mathrm{H}), 1.95$ $(\mathrm{q}, J=7.1 \mathrm{~Hz}, 2 \mathrm{H}), 1.65-1.55(\mathrm{~m}, 2 \mathrm{H}) .{ }^{13} \mathrm{C}$ NMR $\left(125 \mathrm{MHz}, \mathrm{CDCl}_{3}\right) \delta 141.9,137.0,128.9,128.1,127.3,117.4$, 115.9, 56.4, 51.5, 49.8, 30.3, 26.8. HRMS (ESI) m/z: $[\mathrm{M}+\mathrm{H}]^{+}$calcd for $\mathrm{C}_{20} \mathrm{H}_{23} \mathrm{~N}_{2} 291.1856$; found: 291.1865 .

\section{Spectral data for N-(but-3-en-1-yl)-N-(o-tolyl)cyanamide (9a)}<smiles>C=CCCN(C)c1ccccc1C</smiles>

Purified by column chromatography (silica gel, EtOAc/Petroleum ether: 1/12) to afford the title compound ( $90 \%$ yield, $168 \mathrm{mg}$ ) as a colorless oil. ${ }^{1} \mathrm{H}$ NMR $\left(400 \mathrm{MHz}, \mathrm{CDCl}_{3}\right) \delta 7.26-7.16(\mathrm{~m}, 4 \mathrm{H}), 5.88-5.70(\mathrm{~m}, 1 \mathrm{H}), 5.24-5.10$ $(\mathrm{m}, 2 \mathrm{H}), 3.49(\mathrm{t}, J=7.3 \mathrm{~Hz}, 2 \mathrm{H}), 2.56-2.44(\mathrm{~m}, 2 \mathrm{H}), 2.39(\mathrm{~s}, 3 \mathrm{H}) .{ }^{13} \mathrm{C} \mathrm{NMR}\left(100 \mathrm{MHz}, \mathrm{CDCl}_{3}\right) \delta 139.1,134.0$, $133.5,131.8,127.7,127.2,124.7,118.3,115.5,53.1,32.1,17.9$.

\section{Spectral data for N-(but-3-en-1-yl)-N-(2-ethylphenyl)cyanamide (9b)}<smiles>C=CCCN(C#N)c1ccccc1CC</smiles>

Purified by column chromatography (silica gel, EtOAc/Petroleum ether: $1 / 30)$ to afford the title compound (70\% yield, $140 \mathrm{mg}$ ) as yellowish oil. ${ }^{1} \mathrm{H}$ NMR $\left(400 \mathrm{MHz}, \mathrm{CDCl}_{3}\right) \delta$ 7.33-7.22 (m, 4H), 5.81 (ddt, $J=17.0,10.2,6.7 \mathrm{~Hz}$, 1H), $5.34-4.95$ (m, 2H), 3.46 (t, $J=7.4 \mathrm{~Hz}, 2 \mathrm{H}), 2.76$ (q, $J=7.5 \mathrm{~Hz}, 2 \mathrm{H}), 2.57-2.44$ (m, 2H), 1.29 (t, $J=7.6 \mathrm{~Hz}$, $3 \mathrm{H}) .{ }^{13} \mathrm{C}$ NMR $\left(100 \mathrm{MHz}, \mathrm{CDCl}_{3}\right) \delta 140.2,138.7,133.5,129.9,128.2,127.2,125.5,118.3,116.0,53.7,32.2,23.8$, 14.4. HRMS (ESI) m/z: $[\mathrm{M}+\mathrm{H}]^{+}$calcd for $\mathrm{C}_{13} \mathrm{H}_{17} \mathrm{~N}_{2} 201.1386$; found: 201.1384 .

\section{Spectral data for N-(2-benzylphenyl)-N-(but-3-en-1-yl)cyanamide (9c)}<smiles>C=CCCN(C#N)c1ccccc1Br</smiles>

Purified by column chromatography (silica gel, EtOAc/Petroleum ether: $1 / 20)$ to afford the title compound (71\% yield, $186 \mathrm{mg}$ ) as yellow oil. ${ }^{1} \mathrm{H}$ NMR (400 MHz, $\left.\mathrm{CDCl}_{3}\right) \delta 7.32-7.27(\mathrm{~m}, 4 \mathrm{H}), 7.25-7.21(\mathrm{~m}, 2 \mathrm{H}), 7.20-7.13$ (m, 3H), $5.66(\mathrm{ddt}, J=17.0,10.2,6.8 \mathrm{~Hz}, 1 \mathrm{H}), 5.26-4.91(\mathrm{~m}, 2 \mathrm{H}), 4.13(\mathrm{~s}, 2 \mathrm{H}), 3.34-3.05(\mathrm{~m}, 2 \mathrm{H}), 2.47-2.07$ $(\mathrm{m}, 2 \mathrm{H}) .{ }^{13} \mathrm{C}$ NMR $\left(100 \mathrm{MHz}, \mathrm{CDCl}_{3}\right) \delta 139.7,139.2,137.4,133.5,131.9,129.0,128.7,128.2,128.0,126.5,125.8$, 118.3, 115.6, 53.4, 37.3, 32.0. HRMS (ESI) $\mathrm{m} / \mathrm{z}$ : $[\mathrm{M}+\mathrm{H}]^{+}$calcd for $\mathrm{C}_{18} \mathrm{H}_{19} \mathrm{~N}_{2} 263.1543$; found: 263.1551 . 


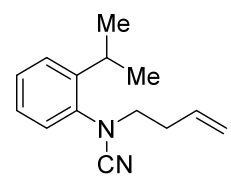

Purified by column chromatography (silica gel, EtOAc/Petroleum ether: 1/20) to afford the title compound (79\% yield, $169 \mathrm{mg}$ ) as yellowish oil. ${ }^{1} \mathrm{H}$ NMR $\left(300 \mathrm{MHz}, \mathrm{CDCl}_{3}\right) \delta 7.37-7.30(\mathrm{~m}, 2 \mathrm{H}), 7.25-7.17(\mathrm{~m}, 2 \mathrm{H}), 5.81$ (ddt, $J=17.0,10.2,6.7 \mathrm{~Hz}, 1 \mathrm{H}), 5.27-5.04(\mathrm{~m}, 2 \mathrm{H}), 3.48-3.41(\mathrm{~m}, 2 \mathrm{H}), 3.41-3.28(\mathrm{~m}, 1 \mathrm{H}), 2.63-2.36(\mathrm{~m}, 2 \mathrm{H}), 1.26$ (d, 6H). ${ }^{13} \mathrm{C}$ NMR $\left(125 \mathrm{MHz}, \mathrm{CDCl}_{3}\right) \delta 145.4,137.9,133.5,128.7,127.4,127.0,126.1,118.2,116.3,54.2,32.1$, 27.6, 23.8. HRMS (ESI) $\mathrm{m} / \mathrm{z}$ : $[\mathrm{M}+\mathrm{H}]^{+}$calcd for $\mathrm{C}_{14} \mathrm{H}_{19} \mathrm{~N}_{2} 215.1543$; found: 215.1541 .

\section{Spectral data for N-(3-methylbut-3-en-1-yl)-N-(o-tolyl)cyanamide (9e)}<smiles>C=C(C)CCN(C)c1ccccc1C</smiles>

Purified by column chromatography (silica gel, EtOAc/Petroleum ether: 1/20) to afford the title compound (57\% yield, $114 \mathrm{mg}$ ) as yellow oil. ${ }^{1} \mathrm{H}$ NMR $\left(500 \mathrm{MHz}, \mathrm{CDCl}_{3}\right) \delta 7.38-7.10(\mathrm{~m}, 4 \mathrm{H}), 4.87(\mathrm{~s}, 1 \mathrm{H}), 4.79(\mathrm{~s}, 1 \mathrm{H}), 3.55(\mathrm{t}$, $J=7.5 \mathrm{~Hz}, 2 \mathrm{H}), 2.46$ (t, $J=7.5 \mathrm{~Hz}, 2 \mathrm{H}), 2.39$ (s, 3H), 1.75 (s, 3H). ${ }^{13} \mathrm{C}$ NMR $\left(125 \mathrm{MHz}, \mathrm{CDCl}_{3}\right) \delta 141.1,139.2$, 134.1, 131.9, 127.8, 127.3, 124.7, 115.6, 113.2, 52.1, 35.9, 22.5, 18.0. HRMS (ESI) m/z: $[\mathrm{M}+\mathrm{H}]^{+}$calcd for $\mathrm{C}_{13} \mathrm{H}_{17} \mathrm{~N}_{2}$ 201.1386; found: 201.1378.

\section{Spectral data for N-(3-phenylbut-3-en-1-yl)-N-(o-tolyl)cyanamide (9f)}<smiles>C=C(CCNc1ccccc1C(=O)c1ccccc1)CCC(=C)c1ccccc1</smiles>

$\mathrm{CN}$

Purified by column chromatography (silica gel, EtOAc/Petroleum ether: 1/20) to afford the title compound (52\% yield, $136 \mathrm{mg})$ as colorless oil. ${ }^{1} \mathrm{H}$ NMR $\left(500 \mathrm{MHz}, \mathrm{CDCl}_{3}\right) \delta 7.36-7.27(\mathrm{~m}, 5 \mathrm{H}), 7.23-7.15(\mathrm{~m}, 4 \mathrm{H}), 5.40(\mathrm{~s}, 1 \mathrm{H})$, $5.20(\mathrm{~d}, J=1.3 \mathrm{~Hz}, 1 \mathrm{H}), 3.51(\mathrm{t}, J=7.5 \mathrm{~Hz}, 2 \mathrm{H}), 2.97(\mathrm{td}, J=7.5,1.2 \mathrm{~Hz}, 2 \mathrm{H}), 2.34(\mathrm{~s}, 3 \mathrm{H}) .{ }^{13} \mathrm{C} \mathrm{NMR}(125 \mathrm{MHz}$, $\left.\mathrm{CDCl}_{3}\right) \delta 144.1,139.9,139.2,134.0,131.9,128.7,128.0,127.8,127.3,126.2,124.8,115.6,115.5,52.5,34.0,18.0$. HRMS (ESI) m/z: [M+ H] calcd for $\mathrm{C}_{18} \mathrm{H}_{19} \mathrm{~N}_{2} 263.1543$; found: 263.1550 .

\section{Spectral data for N-(but-3-en-1-yl)-N-cyano-2,2-dimethylbutanamide (9g)}<smiles>C=CCCN(CC)C(=O)C(C)(C)CC</smiles>

Purified by column chromatography (silica gel, EtOAc/Petroleum ether: 1/20) to afford the title compound (80\% yield, $155 \mathrm{mg}$ ) as colorless oil. ${ }^{1} \mathrm{H}$ NMR $\left(400 \mathrm{MHz}, \mathrm{CDCl}_{3}\right) \delta 5.74(\mathrm{ddt}, J=17.1,10.2,6.9 \mathrm{~Hz}, 1 \mathrm{H}), 5.25-4.87(\mathrm{~m}$, $2 \mathrm{H}), 3.68(\mathrm{t}, J=6.9 \mathrm{~Hz}, 2 \mathrm{H}), 2.52-2.32(\mathrm{~m}, 2 \mathrm{H}), 1.86(\mathrm{q}, J=7.5 \mathrm{~Hz}, 2 \mathrm{H}), 1.34(\mathrm{~s}, 6 \mathrm{H}), 0.88(\mathrm{t}, J=7.5 \mathrm{~Hz}, 3 \mathrm{H}) .{ }^{13} \mathrm{C}$ NMR $\left(125 \mathrm{MHz}, \mathrm{CDCl}_{3}\right) \delta 176.2,133.1,118.6,111.4,47.7,45.2,32.0,31.9,24.6,9.1$. HRMS (ESI) m/z: [M + H] ${ }^{+}$ calcd for $\mathrm{C}_{11} \mathrm{H}_{19} \mathrm{~N}_{2} \mathrm{O}$ 195.1492; found: 195.1491 .

\section{Spectral data for N-(but-3-en-1-yl)-N-cyano-3,3-diphenylpropanamide (9h)}<smiles>C=CCCN(CCC(=O)O)CCc1ccccc1</smiles> 
Purified by column chromatography (silica gel, EtOAc/Petroleum ether: $1 / 20)$ to afford the title compound (83\% yield, $253 \mathrm{mg}$ ) as colorless oil. ${ }^{1} \mathrm{H}$ NMR $\left(500 \mathrm{MHz}, \mathrm{CDCl}_{3}\right) \delta 7.32-7.21(\mathrm{~m}, 10 \mathrm{H}), 5.57$ (ddt, $J=17.1,10.3,6.9 \mathrm{~Hz}$, 1H), $5.08-4.99(\mathrm{~m}, 2 \mathrm{H}), 4.63(\mathrm{t}, J=7.7 \mathrm{~Hz}, 1 \mathrm{H}), 3.54$ (t, $J=7.0 \mathrm{~Hz}, 2 \mathrm{H}), 3.41$ (d, $J=7.7 \mathrm{~Hz}, 2 \mathrm{H}), 2.33-2.19$ (m, 2H). ${ }^{13} \mathrm{C}$ NMR (125 MHz, $\left.\mathrm{CDCl}_{3}\right) \delta 170.5,142.6,132.8,128.9,127.8,127.0,118.8,110.7,47.0,45.6,40.6,31.9$. HRMS (ESI) m/z: [M+Na] calcd for $\mathrm{C}_{20} \mathrm{H}_{20} \mathrm{~N}_{2} \mathrm{NaO} 327.1468$; found: 327.1461 .

\section{Spectral data for N-(but-3-en-1-yl)-N-cyano-3-phenylpropanamide (9i)}<smiles>C=CCCN(C#N)C(=O)CCc1ccccc1</smiles>

Purified by column chromatography (silica gel, EtOAc/Petroleum ether: $1 / 20)$ to afford the title compound (76\% yield, $174 \mathrm{mg}$ ) as colorless oil. ${ }^{1} \mathrm{H}$ NMR $\left(500 \mathrm{MHz}, \mathrm{CDCl}_{3}\right) \delta 7.33-7.27(\mathrm{~m}, 2 \mathrm{H}), 7.25-7.19(\mathrm{~m}, 3 \mathrm{H}), 5.70$ (ddt, $J$ $=17.0,10.2,6.9 \mathrm{~Hz}, 1 \mathrm{H}), 5.18-5.06(\mathrm{~m}, 2 \mathrm{H}), 3.63(\mathrm{t}, J=7.0 \mathrm{~Hz}, 2 \mathrm{H}), 3.03-2.94(\mathrm{~m}, 4 \mathrm{H}), 2.43-2.36(\mathrm{~m}, 2 \mathrm{H})$. ${ }^{13} \mathrm{C}$ NMR $\left(125 \mathrm{MHz}, \mathrm{CDCl}_{3}\right) \delta 171.5,139.5,132.9,128.8,128.5,126.8,118.9,110.6,45.5,36.4,32.1,30.6$. HRMS (ESI) $\mathrm{m} / \mathrm{z}:[\mathrm{M}+\mathrm{H}]^{+}$calcd for $\mathrm{C}_{14} \mathrm{H}_{17} \mathrm{~N}_{2} \mathrm{O} 229.1335$; found: 229.1324 .

\section{Spectral data for N-(but-3-en-1-yl)-N-(2,2-dimethyl-3-phenylpropyl)cyanamide (9j)}<smiles>C=CCCN(C#N)CC(C)(C)Cc1ccccc1</smiles>

Purified by column chromatography (silica gel, EtOAc/Petroleum ether: $1 / 8)$ to afford the title compound (61\% yield, $148 \mathrm{mg}$ ) as yellowish oil. ${ }^{1} \mathrm{H} \mathrm{NMR}\left(500 \mathrm{MHz}, \mathrm{CDCl}_{3}\right) \delta 7.30-7.25(\mathrm{~m}, 2 \mathrm{H}), 7.25-7.20(\mathrm{~m}, 1 \mathrm{H}), 7.14-7.10(\mathrm{~m}$, 2H), 5.79 (ddt, $J=17.1,10.2,6.8 \mathrm{~Hz}, 1 \mathrm{H}), 5.23-5.10$ (m, 2H), 3.10 (t, $J=7.3 \mathrm{~Hz}, 2 \mathrm{H}), 2.89(\mathrm{~s}, 2 \mathrm{H}), 2.58(\mathrm{~s}, 2 \mathrm{H})$, $2.48-2.42(\mathrm{~m}, 2 \mathrm{H}), 0.96(\mathrm{~s}, 6 \mathrm{H}) .{ }^{13} \mathrm{C} \mathrm{NMR}\left(125 \mathrm{MHz}, \mathrm{CDCl}_{3}\right) \delta 137.6,133.8,130.7,128.1,126.4,119.0,118.3$, 63.9, 53.9, 46.4, 37.9, 32.5, 24.9. HRMS (ESI) m/z: $[\mathrm{M}+\mathrm{H}]^{+}$calcd for $\mathrm{C}_{16} \mathrm{H}_{23} \mathrm{~N}_{2} 243.1856$; found: 243.1867 .

\section{Spectral data for N-cyano-N-(3,3-diphenylpropyl)-2,2-dimethylbut-3-enamide (9k)}<smiles>C=CC(C)(C)C(=O)N(C)CCC(c1ccccc1)c1ccccc1</smiles>

Purified by column chromatography (silica gel, EtOAc/Petroleum ether: $1 / 50$ ) to afford the title compound (35\% yield, $116 \mathrm{mg}$ ) as colorless oil. ${ }^{1} \mathrm{H}$ NMR $\left(500 \mathrm{MHz}, \mathrm{CDCl}_{3}\right) \delta 7.29(\mathrm{t}, J=7.6 \mathrm{~Hz}, 4 \mathrm{H}), 7.26-7.23(\mathrm{~m}, 4 \mathrm{H}), 7.19$ (t, $J=7.1 \mathrm{~Hz}, 2 \mathrm{H}), 6.02(\mathrm{dd}, J=17.5,10.6 \mathrm{~Hz}, 1 \mathrm{H}), 5.29(\mathrm{~d}, J=10.6 \mathrm{~Hz}, 1 \mathrm{H}), 5.17(\mathrm{~d}, J=17.5 \mathrm{~Hz}, 1 \mathrm{H}), 3.97(\mathrm{t}, J=$ $8.0 \mathrm{~Hz}, 1 \mathrm{H}), 3.54(\mathrm{t}, J=7.7 \mathrm{~Hz}, 2 \mathrm{H}), 2.46-2.36(\mathrm{~m}, 2 \mathrm{H}), 1.42(\mathrm{~s}, 6 \mathrm{H}) .{ }^{13} \mathrm{C} \mathrm{NMR}\left(125 \mathrm{MHz}, \mathrm{CDCl}_{3}\right) \delta 175.3,143.3$, 140.5, 128.9, 127.8, 126.8, 116.8, 111.0, 48.7, 47.8, 47.0, 33.4, 25.6. HRMS (ESI) m/z: $[\mathrm{M}+\mathrm{H}]^{+}$calcd for $\mathrm{C}_{22} \mathrm{H}_{25} \mathrm{~N}_{2} \mathrm{O}$ 333.1961; found: 333.1946 .

\section{Spectral data for N-cyano-N-(2,2-dimethyl-3-phenylpropyl)-2,2-dimethylbut-3-enamide (9l)}<smiles>C=CC(C)(C)C(=O)N(C)CC(C)(C)Cc1ccccc1</smiles>

Purified by column chromatography (silica gel, EtOAc/Petroleum ether: 1/50) to afford the title compound (71\% yield, $202 \mathrm{mg}$ ) as colorless oil. ${ }^{1} \mathrm{H}$ NMR $\left(400 \mathrm{MHz}, \mathrm{CDCl}_{3}\right) \delta 7.31-7.22(\mathrm{~m}, 3 \mathrm{H}), 7.14-7.10(\mathrm{~m}, 2 \mathrm{H}), 6.11(\mathrm{dd}, J$ $=17.5,10.6 \mathrm{~Hz}, 1 \mathrm{H}), 5.33(\mathrm{~d}, J=10.7 \mathrm{~Hz}, 1 \mathrm{H}), 5.22(\mathrm{~d}, J=17.5 \mathrm{~Hz}, 1 \mathrm{H}), 3.56(\mathrm{~s}, 2 \mathrm{H}), 2.57(\mathrm{~s}, 2 \mathrm{H}), 1.48(\mathrm{~s}, 6 \mathrm{H})$, $0.93(\mathrm{~s}, 6 \mathrm{H}) .{ }^{13} \mathrm{C} \mathrm{NMR}\left(125 \mathrm{MHz}, \mathrm{CDCl}_{3}\right) \delta 175.9,140.7,137.3,130.7,128.1,126.5,116.9,112.5,58.3,47.1,46.8$, 
37.7, 25.9, 24.9. HRMS (ESI) m/z: $[\mathrm{M}+\mathrm{H}]^{+}$calcd for $\mathrm{C}_{18} \mathrm{H}_{25} \mathrm{~N}_{2} \mathrm{O} 285.1961$; found: 285.1944 .

Spectral data for N-((12-azaneylidene)-13-methyl)-N-(3,3-diphenylpropyl)-2,2-dimethylpent-4-enamide (9m)<smiles>C=CCC(C)(C)C(=O)N(C)CCC(c1ccccc1)c1ccccc1</smiles>

Purified by column chromatography (silica gel, EtOAc/Petroleum ether: 1/20) to afford the title compound (55\% yield, $191 \mathrm{mg}$ ) as colorless oil. ${ }^{1} \mathrm{H}$ NMR $\left(500 \mathrm{MHz}, \mathrm{CDCl}_{3}\right) \delta 7.29$ (t, J=7.6 Hz, 4H), $7.24(\mathrm{~d}, J=7.2 \mathrm{~Hz}, 4 \mathrm{H}), 7.19$ (t, $J=7.2 \mathrm{~Hz}, 2 \mathrm{H}), 5.70$ (ddt, $J=17.3,10.1,7.3 \mathrm{~Hz}, 1 \mathrm{H}), 5.25-5.00(\mathrm{~m}, 2 \mathrm{H}), 3.97$ (t, $J=7.9 \mathrm{~Hz}, 1 \mathrm{H}), 3.57-3.50$ (m, 2H), $2.54(\mathrm{~d}, J=7.4 \mathrm{~Hz}, 2 \mathrm{H}), 2.44-2.35(\mathrm{~m}, 2 \mathrm{H}), 1.36(\mathrm{~s}, 6 \mathrm{H}) .{ }^{13} \mathrm{C}$ NMR $\left(125 \mathrm{MHz}, \mathrm{CDCl}_{3}\right) \delta 175.8,143.4$, 133.0, 128.9, 127.8, 126.8, 119.1, 111.6, 48.7, 48.1, 45.0, 43.6, 33.4, 25.0. HRMS (ESI) m/z: $[\mathrm{M}+\mathrm{H}]^{+}$calcd for $\mathrm{C}_{23} \mathrm{H}_{27} \mathrm{~N}_{2} \mathrm{O} 347.2118$; found: 347.2117 .

\section{Spectral data for N-((1-allylcyclopentyl)methyl)-N-cyano-3,3-diphenylpropanamide (9n)}<smiles>C=CCC1(CN(C)C(=O)CC(c2ccccc2)c2ccccc2)CCCC1</smiles>

Purified by column chromatography (silica gel, EtOAc/Petroleum ether: 1/80) to afford the title compound (78\% yield, $291 \mathrm{mg}$ ) as white solid, mp: $99-101{ }^{\circ} \mathrm{C} .{ }^{1} \mathrm{H}$ NMR (400 MHz, $\left.\mathrm{CDCl}_{3}\right) \delta 7.33-7.24(\mathrm{~m}, 8 \mathrm{H}), 7.24-7.16(\mathrm{~m}$, 2H), 5.69 (ddt, $J=17.4,10.3,7.3 \mathrm{~Hz}, 1 \mathrm{H}), 5.11-4.91(\mathrm{~m}, 2 \mathrm{H}), 4.64(\mathrm{t}, J=7.8 \mathrm{~Hz}, 1 \mathrm{H}), 3.46(\mathrm{~d}, J=7.2 \mathrm{~Hz}, 2 \mathrm{H}), \delta$ $3.45(\mathrm{~s}, 2 \mathrm{H}), 1.93(\mathrm{~d}, J=7.3 \mathrm{~Hz}, 2 \mathrm{H}), 1.59-1.49(\mathrm{~m}, 4 \mathrm{H}), 1.39-1.30(\mathrm{~m}, 2 \mathrm{H}), 1.30-1.20(\mathrm{~m}, 2 \mathrm{H}) .{ }^{13} \mathrm{C}$ NMR $(100$ $\left.\mathrm{MHz}, \mathrm{CDCl}_{3}\right) \delta 171.2,142.5,134.2,128.9,127.8,127.0,118.4,112.2,52.5,47.3,47.2,41.1,40.5,34.8,24.3$. HRMS (ESI) $\mathrm{m} / \mathrm{z}:[\mathrm{M}+\mathrm{H}]^{+}$calcd for $\mathrm{C}_{25} \mathrm{H}_{29} \mathrm{~N}_{2} \mathrm{O} 373.2274$; found: 373.2269 .

\section{Spectral data for N-(but-3-en-1-yl)-N-cyanotetrahydrofuran-3-carboxamide (S11)}<smiles>C=CCCN(C)C(=O)C1CCCO1</smiles>

Purified by column chromatography (silica gel, EtOAc/Petroleum ether: $1 / 8)$ to afford the title compound (70\% yield, $136 \mathrm{mg}$ ) as yellowish oil. ${ }^{1} \mathrm{H} \mathrm{NMR}\left(500 \mathrm{MHz}, \mathrm{CDCl}_{3}\right) \delta 5.74(\mathrm{ddt}, J=17.0,10.0,6.9 \mathrm{~Hz}, 1 \mathrm{H}), 5.25-5.08(\mathrm{~m}, 2 \mathrm{H})$, $4.93-4.77(\mathrm{~m}, 1 \mathrm{H}), 4.12-4.01(\mathrm{~m}, 1 \mathrm{H}), 4.01-3.90(\mathrm{~m}, 1 \mathrm{H}), 3.80-3.57(\mathrm{~m}, 2 \mathrm{H}), 2.50-2.41(\mathrm{~m}, 2 \mathrm{H}), 2.40-2.29$ $(\mathrm{m}, 1 \mathrm{H}), 2.14-2.04(\mathrm{~m}, 1 \mathrm{H}), 2.03-1.90(\mathrm{~m}, 2 \mathrm{H}) .{ }^{13} \mathrm{C} \mathrm{NMR}\left(125 \mathrm{MHz}, \mathrm{CDCl}_{3}\right) \delta 172.6,132.8,118.8,109.8,76.9$, 69.7, 45.9, 31.8, 30.1, 25.5. HRMS (ESI) $\mathrm{m} / \mathrm{z}$ : $[\mathrm{M}+\mathrm{H}]^{+}$calcd for $\mathrm{C}_{10} \mathrm{H}_{15} \mathrm{~N}_{2} \mathrm{O}_{2}$ 195.1128; found: 195.1130 .

\section{Spectral data for N-(but-3-en-1-yl)-N-cyanopropionamide (S12)}<smiles>C=CCCN(C)C(=O)COC</smiles>

Purified by column chromatography (silica gel, EtOAc/Petroleum ether: 1/30) to afford the title compound (61\% yield, $93 \mathrm{mg}$ ) as colorless oil. ${ }^{1} \mathrm{H}$ NMR $\left(500 \mathrm{MHz}, \mathrm{CDCl}_{3}\right) \delta 5.74(\mathrm{ddt}, J=17.1,10.2,6.9 \mathrm{~Hz}, 1 \mathrm{H}), 5.22-5.09(\mathrm{~m}$, 2H), $3.65(\mathrm{t}, J=7.0 \mathrm{~Hz}, 2 \mathrm{H}), 2.69$ (q, $J=7.4 \mathrm{~Hz}, 2 \mathrm{H}), 2.47-2.40(\mathrm{~m}, 2 \mathrm{H}), 1.21(\mathrm{t}, J=7.4 \mathrm{~Hz}, 3 \mathrm{H}) .{ }^{13} \mathrm{C}$ NMR $(125$ $\left.\mathrm{MHz}, \mathrm{CDCl}_{3}\right) \delta 173.1,133.0,118.8,110.8,45.4,32.1,28.2,8.7$. HRMS (ESI) m/z: $[\mathrm{M}+\mathrm{H}]^{+}$calcd for $\mathrm{C}_{8} \mathrm{H}_{13} \mathrm{~N}_{2} \mathrm{O}$ 153.1022; found: 153.1016 . 
<smiles>C=CCCN(C#N)OCc1ccccc1</smiles>

Purified by column chromatography (silica gel, EtOAc/Petroleum ether: 1/10) to afford the title compound (86\% yield, $174 \mathrm{mg}$ ) as colorless oil. ${ }^{1} \mathrm{H}$ NMR $\left(400 \mathrm{MHz}, \mathrm{CDCl}_{3}\right) \delta 7.43-7.32(\mathrm{~m}, 5 \mathrm{H}), 5.71$ (ddt, $J=17.0,10.2,6.7 \mathrm{~Hz}$, $1 \mathrm{H}), 5.28-5.01(\mathrm{~m}, 2 \mathrm{H}), 4.87(\mathrm{~s}, 2 \mathrm{H}), 3.26(\mathrm{t}, J=7.2 \mathrm{~Hz}, 2 \mathrm{H}), 2.61-2.23(\mathrm{~m}, 2 \mathrm{H}) .{ }^{13} \mathrm{C} \mathrm{NMR}\left(125 \mathrm{MHz}, \mathrm{CDCl}_{3}\right) \delta$ 134.7, 133.5, 129.4, 129.0, 128.6, 117.9, 115.0, 77.4, 55.7, 30.4. HRMS (ESI) m/z: $[\mathrm{M}+\mathrm{H}]^{+}$calcd for $\mathrm{C}_{12} \mathrm{H}_{15} \mathrm{~N}_{2} \mathrm{O}$ 203.1179; found: 203.1175 .

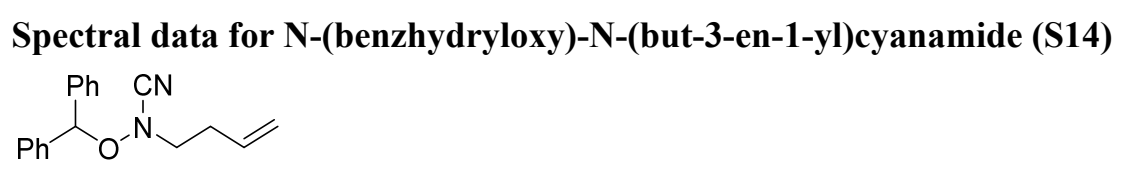

Purified by column chromatography (silica gel, EtOAc/Petroleum ether: 1/10) to afford the title compound (80\% yield, $223 \mathrm{mg}$ ) as white solid, mp: $132-133{ }^{\circ} \mathrm{C} .{ }^{1} \mathrm{H}$ NMR $\left(300 \mathrm{MHz}, \mathrm{CDCl}_{3}\right) \delta 7.42-7.28(\mathrm{~m}, 11 \mathrm{H}), 5.66$ (ddt, $J=$ $17.2,10.4,6.7 \mathrm{~Hz}, 1 \mathrm{H}), 5.25-4.83(\mathrm{~m}, 2 \mathrm{H}), 3.35-3.06(\mathrm{~m}, 2 \mathrm{H}), 2.53-2.21(\mathrm{~m}, 2 \mathrm{H}) .{ }^{13} \mathrm{C} \mathrm{NMR}\left(125 \mathrm{MHz}, \mathrm{CDCl}_{3}\right)$ $\delta 138.9,133.5,128.6,128.6,127.9,118.0,115.0,88.5,56.0,30.4$. HRMS (ESI) m/z: $[\mathrm{M}+\mathrm{H}]^{+}$calcd for $\mathrm{C}_{18} \mathrm{H}_{19} \mathrm{~N}_{2} \mathrm{O}$ 279.1492; found: 279.1484 .

\section{Spectral data for N-(but-3-en-1-yl)-N-cyclohexylcyanamide (S15)}<smiles>C=CCCN(C)C1CCCCC1</smiles>

Purified by column chromatography (silica gel, EtOAc/Petroleum ether: $1 / 8)$ to afford the title compound (72\% yield, $128 \mathrm{mg}$ ) as yellowish oil. ${ }^{1} \mathrm{H}$ NMR $\left(300 \mathrm{MHz}, \mathrm{CDCl}_{3}\right) \delta 5.78$ (ddt, $\left.J=17.0,10.2,6.8 \mathrm{~Hz}, 1 \mathrm{H}\right), 5.22-5.06(\mathrm{~m}, 2 \mathrm{H})$, $3.07(\mathrm{t}, J=7.5 \mathrm{~Hz}, 2 \mathrm{H}), 2.79-2.63(\mathrm{~m}, 1 \mathrm{H}), 2.47-2.31(\mathrm{~m}, 2 \mathrm{H}), 2.02-1.90(\mathrm{~m}, 2 \mathrm{H}), 1.90-1.77(\mathrm{~m}, 2 \mathrm{H}), 1.68-$ $1.61(\mathrm{~m}, 1 \mathrm{H}), 1.51-1.09(\mathrm{~m}, 5 \mathrm{H}) .{ }^{13} \mathrm{C} \mathrm{NMR}\left(125 \mathrm{MHz}, \mathrm{CDCl}_{3}\right) \delta 134.0,117.7,116.5,59.4,49.4,32.6,31.1,25.2$, 25.1. HRMS (ESI) $\mathrm{m} / \mathrm{z}:[\mathrm{M}+\mathrm{H}]^{+}$calcd for $\mathrm{C}_{11} \mathrm{H}_{19} \mathrm{~N}_{2}$ 179.1543; found: 179.1540 .

\section{Spectral data for N-(but-3-en-1-yl)-N-(2-phenylpropyl)cyanamide (S16)}

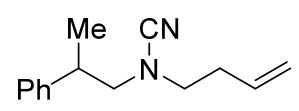

Purified by column chromatography (silica gel, EtOAc/Petroleum ether: 1/10) to afford the title compound (70\% yield, $150 \mathrm{mg}$ ) as colorless oil. ${ }^{1} \mathrm{H}$ NMR (400 MHz, $\left.\mathrm{CDCl}_{3}\right) \delta 7.37-7.30(\mathrm{~m}, 2 \mathrm{H}), 7.28-7.20(\mathrm{~m}, 3 \mathrm{H}), 5.67$ (ddt, $J$ $=17.1,10.3,6.8 \mathrm{~Hz}, 1 \mathrm{H}), 5.20-5.01(\mathrm{~m}, 2 \mathrm{H}), 3.23-3.05(\mathrm{~m}, 3 \mathrm{H}), 2.91(\mathrm{t}, J=7.2 \mathrm{~Hz}, 2 \mathrm{H}), 2.33-2.25(\mathrm{~m}, 2 \mathrm{H})$, $1.34(\mathrm{~d}, J=6.1 \mathrm{~Hz}, 3 \mathrm{H}) .{ }^{13} \mathrm{C}$ NMR $\left(125 \mathrm{MHz}, \mathrm{CDCl}_{3}\right) \delta 143.2,133.8,128.8,127.3,127.1,118.0,117.7,59.1,51.7$, 38.9, 32.2, 18.9. HRMS (ESI) m/z: [M + H] $]^{+}$calcd for $\mathrm{C}_{14} \mathrm{H}_{19} \mathrm{~N}_{2} 215.1543$; found: 215.1539 .

\section{Spectral data for N-(but-3-en-1-yl)-N-(cyclohexylmethyl)cyanamide (S17)}<smiles>C=CCCN(C)CC1CCCCC1</smiles>

Purified by column chromatography (silica gel, EtOAc/Petroleum ether: $1 / 8)$ to afford the title compound (53\% yield, $102 \mathrm{mg}$ ) as colorless oil. ${ }^{1} \mathrm{H}$ NMR $\left(400 \mathrm{MHz}, \mathrm{CDCl}_{3}\right) \delta 5.78$ (ddt, $\left.J=17.0,10.2,6.8 \mathrm{~Hz}, 1 \mathrm{H}\right), 5.42-4.83(\mathrm{~m}, 2 \mathrm{H})$, $3.06(\mathrm{t}, J=7.2 \mathrm{~Hz}, 2 \mathrm{H}), 2.82(\mathrm{~d}, J=7.3 \mathrm{~Hz}, 2 \mathrm{H}), 2.48-2.29(\mathrm{~m}, 2 \mathrm{H}), 1.91-1.64(\mathrm{~m}, 6 \mathrm{H}), 1.36-1.10(\mathrm{~m}, 3 \mathrm{H}), 1.02$ $-0.79(\mathrm{~m}, 2 \mathrm{H}) .{ }^{13} \mathrm{C}$ NMR $\left(125 \mathrm{MHz}, \mathrm{CDCl}_{3}\right) \delta 133.8,118.0,117.9,58.3,51.5,36.5,32.2,30.7,26.3,25.6$. HRMS 
(ESI) $\mathrm{m} / \mathrm{z}:[\mathrm{M}+\mathrm{Na}]^{+}$calcd for $\mathrm{C}_{12} \mathrm{H}_{20} \mathrm{~N}_{2} \mathrm{Na} 215.1519$; found: 215.1524 .

\section{Spectral data for N-(but-3-en-1-yl)-N-(sec-butyl)cyanamide (S18)}<smiles>C=CCCN(C)C(C)CC</smiles>

Purified by column chromatography (silica gel, EtOAc/Petroleum ether: $1 / 5$ ) to afford the title compound (32\% yield, $49 \mathrm{mg}$ ) as colorless oil. ${ }^{1} \mathrm{H}$ NMR $\left(400 \mathrm{MHz}, \mathrm{CDCl}_{3}\right) \delta 5.87-5.69(\mathrm{~m}, 1 \mathrm{H}), 5.24-5.03(\mathrm{~m}, 2 \mathrm{H}), 3.15-2.96(\mathrm{~m}, 2 \mathrm{H})$, $2.94-2.76(\mathrm{~m}, 1 \mathrm{H}), 2.51-2.23(\mathrm{~m}, 2 \mathrm{H}), 1.78-1.61(\mathrm{~m}, 1 \mathrm{H}), 1.59-1.42(\mathrm{~m}, 1 \mathrm{H}), 1.24(\mathrm{~d}, J=6.5 \mathrm{~Hz}, 3 \mathrm{H}), 0.96(\mathrm{t}$, $J=7.2 \mathrm{~Hz}, 3 \mathrm{H}) .{ }^{13} \mathrm{C}$ NMR $\left(125 \mathrm{MHz}, \mathrm{CDCl}_{3}\right) \delta 133.9,117.7,116.2,58.6,49.7,32.5,27.8,18.3$ 10.6. HRMS (ESI) $\mathrm{m} / \mathrm{z}:[\mathrm{M}+\mathrm{Na}]^{+}$calcd for $\mathrm{C}_{9} \mathrm{H}_{16} \mathrm{~N}_{2} \mathrm{Na}$ 175.1206; found: 175.1201 .

\section{Spectral data for N-(but-3-en-1-yl)-N-(3,3-diphenylpropyl)cyanamide (S21)}<smiles>C=CCCN(C#N)CCc1ccccc1</smiles>

Purified by column chromatography (silica gel, EtOAc/Petroleum ether: $1 / 5)$ to afford the title compound ( $85 \%$ yield, $247 \mathrm{mg}$ ) as colorless oil. ${ }^{1} \mathrm{H}$ NMR $\left(400 \mathrm{MHz}, \mathrm{CDCl}_{3}\right) \delta 7.32-7.25(\mathrm{~m}, 5 \mathrm{H}), 7.25-7.17(\mathrm{~m}, 5 \mathrm{H}), 5.73$ (ddt, $J=17.0$, 10.2, $6.8 \mathrm{~Hz}, 1 \mathrm{H}), 5.18-5.07(\mathrm{~m}, 2 \mathrm{H}), 4.02(\mathrm{t}, J=7.9 \mathrm{~Hz}, 1 \mathrm{H}), 2.99(\mathrm{t}, J=7.2 \mathrm{~Hz}, 2 \mathrm{H}), 2.93(\mathrm{t}, J=7.2 \mathrm{~Hz}, 2 \mathrm{H})$, $2.45-2.30(\mathrm{~m}, 4 \mathrm{H}) .{ }^{13} \mathrm{C} \mathrm{NMR}\left(125 \mathrm{MHz}, \mathrm{CDCl}_{3}\right) \delta 143.6,133.8,128.8,127.8,126.8,118.1,117.5,51.3,50.1,48.2$, 33.6, 32.1. HRMS (ESI) $\mathrm{m} / \mathrm{z}:[\mathrm{M}+\mathrm{H}]^{+}$calcd for $\mathrm{C}_{20} \mathrm{H}_{23} \mathrm{~N}_{2}$ 291.1856; found: 291.1858.

\section{General Procedure and Experimental Details}

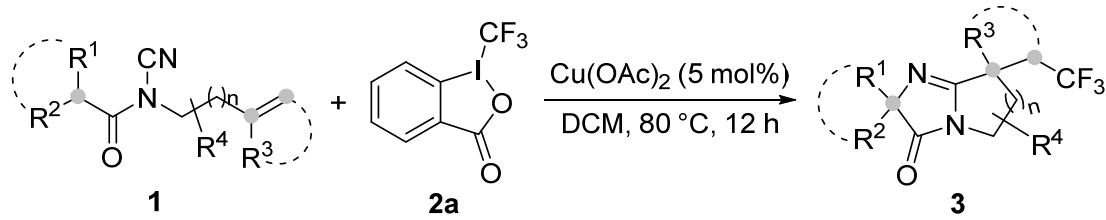

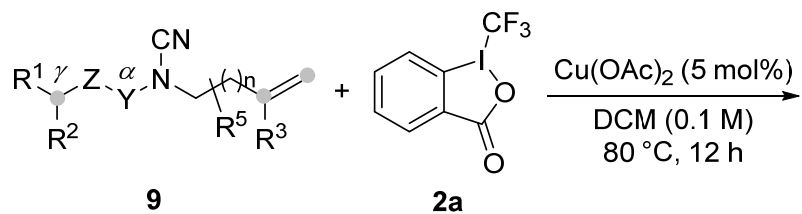

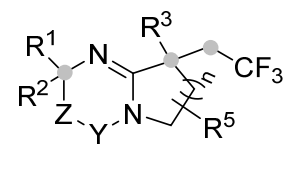

10

\section{General procedure for preparation of products 3 and 10}

To a flame-dried sealed tube equipped with a magnetic stir bar were added 1 or 9 ( 0.2 mmol, 1 equiv.), Togni's reagent $2 \mathrm{a}(0.24 \mathrm{mmol}, 1.2$ equiv. $)$ and $\mathrm{Cu}(\mathrm{OAc})_{2}(5 \mathrm{~mol} \%, 0.01 \mathrm{mmol})$. The tube was evacuated and backfilled with nitrogen for three times, and dichloromethane $(2.0 \mathrm{~mL})$ was added via syringe. The tube was stirred at $80{ }^{\circ} \mathrm{C}$ for 12 h. After completion, the mixture was cooled to room temperature, and sat. aq. soln. of $\mathrm{NaHCO}_{3}$ was added. Then the aqueous phase was extracted with DCM, washed with brine, dried over $\mathrm{Na}_{2} \mathrm{SO}_{4}$. The filtrate was concentrated under reduced pressure and the crude was purified by flash chromatography (silica gel, EtOAc/Petroleum ether $\left(60-90{ }^{\circ} \mathrm{C}\right)$ ) to give the desired products $\mathbf{3}$ and $\mathbf{1 0}$. 


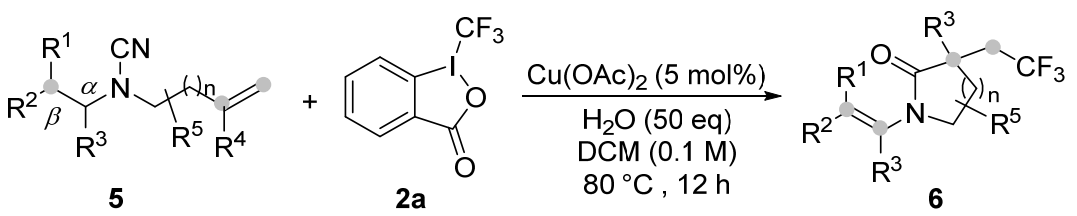

\section{General procedure for preparation of products 6}

To a flame-dried sealed tube equipped with a magnetic stir bar were added 5 ( 0.2 mmol, 1 equiv. $)$, Togni's reagent 2a $(0.24 \mathrm{mmol}, 1.2$ equiv. $)$ and $\mathrm{Cu}(\mathrm{OAc})_{2}(5 \mathrm{~mol} \%, 0.01 \mathrm{mmol})$. The tube was evacuated and backfilled with nitrogen for three times. Then dichloromethane $(2.0 \mathrm{~mL})$ and $\mathrm{H}_{2} \mathrm{O}(10 \mathrm{mmol})$ were added via syringe. The tube was stirred at $80{ }^{\circ} \mathrm{C}$ for $12 \mathrm{~h}$. After completion, the mixture was cooled to room temperature, and sat. aq. soln. of $\mathrm{NaHCO}_{3}$ was added. Then the aqueous phase was extracted with DCM, washed with brine, dried over $\mathrm{Na}_{2} \mathrm{SO}_{4}$. The filtrate was concentrated under reduced pressure and the crude was purified by flash chromatography (silica gel, EtOAc/Petroleum ether $\left.\left(60-90{ }^{\circ} \mathrm{C}\right)\right)$ to give the desired compounds 6.

Spectral data for 2,2-diphenyl-7-(2,2,2-trifluoroethyl)-2,5,6,7-tetrahydro-3H-pyrrolo[1,2-a]imidazol-3-one (3a)<smiles>O=C1N2CCC(CC(F)(F)F)C2=NC1(c1ccccc1)c1ccccc1</smiles>

Purified by column chromatography (silica gel, EtOAc/Petroleum ether: $1 / 5)$ to afford the title compound ( $92 \%$ yield, $66 \mathrm{mg}$ ) as white solid, mp: $120-122{ }^{\circ} \mathrm{C} .{ }^{1} \mathrm{H} \mathrm{NMR}\left(500 \mathrm{MHz}, \mathrm{CDCl}_{3}\right) \delta 7.54-7.48(\mathrm{~m}, 4 \mathrm{H}), 7.35-7.30(\mathrm{~m}, 4 \mathrm{H})$, $7.29-7.26(\mathrm{~m}, 2 \mathrm{H}), 3.79-3.69(\mathrm{~m}, 1 \mathrm{H}), 3.54-3.43(\mathrm{~m}, 1 \mathrm{H}), 3.30-3.19(\mathrm{~m}, 1 \mathrm{H}), 3.17-3.03(\mathrm{~m}, 1 \mathrm{H}), 2.82-2.68$ $(\mathrm{m}, 1 \mathrm{H}), 2.38-2.18(\mathrm{~m}, 2 \mathrm{H}) .{ }^{13} \mathrm{C} \mathrm{NMR}\left(125 \mathrm{MHz}, \mathrm{CDCl}_{3}\right) \delta 178.6,169.3,140.3,140.1,128.6,128.5,127.9,127.8$, $127.1,126.8,126.4$ (q, $J=276.6 \mathrm{~Hz}), 86.6,39.2,35.0$ (q, $J=29.4 \mathrm{~Hz}), 32.4,31.4 .{ }^{19} \mathrm{~F} \mathrm{NMR}\left(470 \mathrm{MHz} \mathrm{CDCl}_{3}\right) \delta-$ $64.90(\mathrm{t}, J=10.7 \mathrm{~Hz})$. HRMS (ESI) m/z: $[\mathrm{M}+\mathrm{H}]^{+}$calcd for $\mathrm{C}_{20} \mathrm{H}_{18} \mathrm{~F}_{3} \mathrm{~N}_{2} \mathrm{O}$ 359.1366; found: 359.1381 .

Spectral data for 2,2-bis(4-chlorophenyl)-7-(2,2,2-trifluoroethyl)-2,5,6,7-tetrahydro-3H-pyrrolo[1,2a]imidazol-3-one (3b)<smiles>O=C1N2CCC(CC(F)(F)F)C2=NC1(c1ccc(Cl)cc1)c1ccc(Cl)cc1</smiles>

Purified by column chromatography (silica gel, EtOAc/Petroleum ether: 1/8) to afford the title compound (91\% yield, $78 \mathrm{mg}$ ) as white solid, mp: $145-147^{\circ} \mathrm{C} .{ }^{1} \mathrm{H}$ NMR (500 MHz, $\left.\mathrm{CDCl}_{3}\right) \delta 7.49-7.41$ (m, 4H), $7.32-7.27$ (m, $\left.4 \mathrm{H}\right)$, $3.79-3.65(\mathrm{~m}, 1 \mathrm{H}), 3.55-3.44(\mathrm{~m}, 1 \mathrm{H}), 3.31-3.17(\mathrm{~m}, 1 \mathrm{H}), 3.14-2.99(\mathrm{~m}, 1 \mathrm{H}), 2.83-2.69(\mathrm{~m}, 1 \mathrm{H}), 2.37-2.19$ $(\mathrm{m}, 2 \mathrm{H}) .{ }^{13} \mathrm{C}$ NMR $\left(125 \mathrm{MHz}, \mathrm{CDCl}_{3}\right) \delta 177.9,169.9,138.5,138.3,134.1,128.8,128.7,128.3,128.1,126.2(\mathrm{q}, J=$ 276.3, 275.9 Hz), 85.2, 39.4, 34.9 (q, $J=29.6 \mathrm{~Hz}), 32.4,31.4 .{ }^{19} \mathrm{~F}$ NMR $\left(470 \mathrm{MHz}, \mathrm{CDCl}_{3}\right) \delta-60.47$ (t, $\left.J=11.0 \mathrm{~Hz}\right)$. HRMS (ESI) m/z: $[\mathrm{M}+\mathrm{H}]^{+}$calcd for $\mathrm{C}_{20} \mathrm{H}_{16} \mathrm{Cl}_{2} \mathrm{~F}_{3} \mathrm{~N}_{2} \mathrm{O} 427.0586$; found: 427.0593 .

Spectral data for 2-methyl-2-phenyl-7-(2,2,2-trifluoroethyl)-2,5,6,7-tetrahydro-3H-pyrrolo[1,2-a]imidazol-3one (3c) 
<smiles>C[C@]1(c2ccccc2)N=C2C(CC(F)(F)F)CCN2C1=O</smiles>

Purified by column chromatography (silica gel, EtOAc/Petroleum ether: 1/2) to afford the title compound (an inseparable mixture, $97 \%$ yield, $57 \mathrm{mg}$, $\mathrm{dr}=1.2 / 1)$ as yellowish oil. The major isomer: ${ }^{1} \mathrm{H}$ NMR $\left(400 \mathrm{MHz}, \mathrm{CDCl}_{3}\right)$ $\delta 7.59(\mathrm{~m}, 2 \mathrm{H}), 7.40-7.32(\mathrm{~m}, 2 \mathrm{H}), 7.30(\mathrm{~m}, 1 \mathrm{H}), 3.70-3.62(\mathrm{~m}, 1 \mathrm{H}), 3.46-3.37(\mathrm{~m}, 1 \mathrm{H}), 3.27-3.15(\mathrm{~m}, 1 \mathrm{H})$, $3.14-2.95(\mathrm{~m}, 1 \mathrm{H}), 2.84-2.67(\mathrm{~m}, 1 \mathrm{H}), 2.38-2.14(\mathrm{~m}, 2 \mathrm{H}), 1.73(\mathrm{~s}, 3 \mathrm{H})$. The minor isomer: ${ }^{1} \mathrm{H}$ NMR $(400 \mathrm{MHz}$, $\left.\mathrm{CDCl}_{3}\right) \delta 7.59(\mathrm{~m}, 2 \mathrm{H}), 7.40-7.32(\mathrm{~m}, 2 \mathrm{H}), 7.30(\mathrm{~m}, 1 \mathrm{H}), 3.78-3.71(\mathrm{~m}, 1 \mathrm{H}), 3.53-3.46(\mathrm{~m}, 1 \mathrm{H}), 3.27-3.15(\mathrm{~m}$, $1 \mathrm{H}), 3.14-2.95(\mathrm{~m}, 1 \mathrm{H}), 2.84-2.67(\mathrm{~m}, 1 \mathrm{H}), 2.38-2.14(\mathrm{~m}, 2 \mathrm{H}), 1.72(\mathrm{~s}, 3 \mathrm{H}) .{ }^{13} \mathrm{C}$ NMR $\left(125 \mathrm{MHz}, \mathrm{CDCl}_{3}\right) \delta$ $180.7,168.9,168.8,139.9,139.7,128.5,128.4,127.8,127.7,126.3$ (q, $J=276.8 \mathrm{~Hz}), 126.2$ (q, $J=276.7 \mathrm{~Hz}), 125.5$, 125.4, 81.1, 80.6, 39.1, 39.0, $35.1(\mathrm{q}, J=29.2 \mathrm{~Hz}), 34.9(\mathrm{q}, J=29.4 \mathrm{~Hz}), 32.4,31.3,31.1,26.5$, 26.4. The major isomer: ${ }^{19} \mathrm{~F}$ NMR $\left(376 \mathrm{MHz}, \mathrm{CDCl}_{3}\right) \delta-64.94$. The minor isomer: ${ }^{19} \mathrm{~F}$ NMR $\left(376 \mathrm{MHz}, \mathrm{CDCl}_{3}\right) \delta-64.83$. HRMS (ESI) $\mathrm{m} / \mathrm{z}:[\mathrm{M}+\mathrm{H}]^{+}$calcd for $\mathrm{C}_{15} \mathrm{H}_{16} \mathrm{~F}_{3} \mathrm{~N}_{2} \mathrm{O}$ 297.1209; found: 297.1217.

\section{Spectral data for 2-ethyl-2-phenyl-7-(2,2,2-trifluoroethyl)-2,5,6,7-tetrahydro-3H-pyrrolo[1,2-a]imidazol-3-} one (3d)<smiles>O=C1C2CCN2C2(CC(F)(F)F)CCN1c1ccccc12</smiles>

Purified by column chromatography (silica gel, EtOAc/Petroleum ether: 1/5) to afford the title compound (an inseparable mixture, $71 \%$ yield, $44 \mathrm{mg}, \mathrm{dr}=1.3: 1)$ as yellowish oil. The major isomer: ${ }^{1} \mathrm{H} \mathrm{NMR}\left(500 \mathrm{MHz}, \mathrm{CDCl}_{3}\right)$ $\delta 7.70-7.48(\mathrm{~m}, 2 \mathrm{H}), 7.41-7.30(\mathrm{~m}, 2 \mathrm{H}), 7.30-7.21(\mathrm{~m}, 1 \mathrm{H}), 3.66-3.57(\mathrm{~m}, 1 \mathrm{H}), 3.52-3.43(\mathrm{~m}, 1 \mathrm{H}), 3.28-$ $3.14(\mathrm{~m}, 1 \mathrm{H}), 3.14-2.98(\mathrm{~m}, 1 \mathrm{H}), 2.81-2.65(\mathrm{~m}, 1 \mathrm{H}), 2.38-2.15(\mathrm{~m}, 2 \mathrm{H}), 2.15-1.97(\mathrm{~m}, 2 \mathrm{H}), 0.93-0.68(\mathrm{~m}$, $3 \mathrm{H})$. The minor isomer: ${ }^{1} \mathrm{H}$ NMR $\left(500 \mathrm{MHz}, \mathrm{CDCl}_{3}\right) \delta 7.70-7.48(\mathrm{~m}, 2 \mathrm{H}), 7.41-7.30(\mathrm{~m}, 2 \mathrm{H}), 7.30-7.21(\mathrm{~m}$, $1 \mathrm{H}), 3.77-3.68(\mathrm{~m}, 1 \mathrm{H}), 3.42-3.32(\mathrm{~m}, 1 \mathrm{H}), 3.28-3.14(\mathrm{~m}, 1 \mathrm{H}), 3.14-2.98(\mathrm{~m}, 1 \mathrm{H}), 2.81-2.65(\mathrm{~m}, 1 \mathrm{H}), 2.38-$ $2.15(\mathrm{~m}, 2 \mathrm{H}), 2.15-1.97(\mathrm{~m}, 2 \mathrm{H}), 0.93-0.68(\mathrm{~m}, 3 \mathrm{H}) .{ }^{13} \mathrm{C} \mathrm{NMR}\left(125 \mathrm{MHz}, \mathrm{CDCl}_{3}\right) \delta 180.2,180.1,169.0,139.4$, 139.2, 128.4, 127.7, 127.6, 126.3 (q, $J=276.7 \mathrm{~Hz}), 126.2$ (q, $J=276.3 \mathrm{~Hz}), 125.8,125.7,84.8,84.6,38.9,38.8,35.1$ (q, $J=29.5 \mathrm{~Hz}), 35.0$ (q, $J=29.3 \mathrm{~Hz}), 33.6,33.4,32.7,32.3,31.2,31.1$. The major isomer: ${ }^{19} \mathrm{~F}$ NMR $(470 \mathrm{MHz}$, $\left.\mathrm{CDCl}_{3}\right) \delta-64.94(\mathrm{t}, J=10.6 \mathrm{~Hz})$. The minor isomer: ${ }^{19} \mathrm{~F} \mathrm{NMR}\left(470 \mathrm{MHz}, \mathrm{CDCl}_{3}\right) \delta-64.85(\mathrm{t}, J=10.7 \mathrm{~Hz})$. HRMS (ESI) $\mathrm{m} / \mathrm{z}$ : $[\mathrm{M}+\mathrm{H}]^{+}$calcd for $\mathrm{C}_{16} \mathrm{H}_{18} \mathrm{~F}_{3} \mathrm{~N}_{2} \mathrm{O}$ 311.1366; found: 311.1371 .

Spectral data for 2-methyl-2-(p-tolyl)-7-(2,2,2-trifluoroethyl)-2,5,6,7-tetrahydro-3H-pyrrolo[1,2-a]imidazol-3one (3e)<smiles>Cc1ccc(C2(C)N=C3C(CC(F)(F)F)CCN3C2=O)cc1</smiles>

Purified by column chromatography (silica gel, EtOAc/Petroleum ether: 1/5) to afford the title compound (an inseparable mixture, $91 \%$ yield, $57 \mathrm{mg}$, $\mathrm{dr}=1.3: 1)$ as yellowish oil. The major isomer: ${ }^{1} \mathrm{H}$ NMR $\left(500 \mathrm{MHz}, \mathrm{CDCl}_{3}\right)$ $\delta 7.50-7.40(\mathrm{~m}, 2 \mathrm{H}), 7.19-7.10(\mathrm{~m}, 2 \mathrm{H}), 3.67-3.57(\mathrm{~m}, 1 \mathrm{H}), 3.53-3.44(\mathrm{~m}, 1 \mathrm{H}), 3.30-3.13(\mathrm{~m}, 1 \mathrm{H}), 3.12-$ $2.94(\mathrm{~m}, 1 \mathrm{H}), 2.82-2.64(\mathrm{~m}, 1 \mathrm{H}), 2.33(\mathrm{~s}, 3 \mathrm{H}), 2.30-2.16(\mathrm{~m}, 2 \mathrm{H}), 1.71(\mathrm{~s}, 3 \mathrm{H})$. The minor isomer: ${ }^{1} \mathrm{H}$ NMR $(500$ $\left.\mathrm{MHz}, \mathrm{CDCl}_{3}\right) \delta 7.50-7.40(\mathrm{~m}, 2 \mathrm{H}), 7.19-7.10(\mathrm{~m}, 2 \mathrm{H}), 3.77-3.69(\mathrm{~m}, 1 \mathrm{H}), 3.44-3.34(\mathrm{~m}, 1 \mathrm{H}), 3.30-3.13(\mathrm{~m}$, 
$1 \mathrm{H}), 3.12-2.94(\mathrm{~m}, 1 \mathrm{H}), 2.82-2.64(\mathrm{~m}, 1 \mathrm{H}), 2.32(\mathrm{~s}, 3 \mathrm{H}), 2.30-2.16(\mathrm{~m}, 2 \mathrm{H}), 1.69(\mathrm{~s}, 3 \mathrm{H}) .{ }^{13} \mathrm{C} \mathrm{NMR}(125 \mathrm{MHz}$, $\left.\mathrm{CDCl}_{3}\right) \delta 180.9,168.8,168.7,137.5,137.0,136.7,129.2,126.3(\mathrm{q}, J=276.9 \mathrm{~Hz}), 126.2(\mathrm{q}, J=276.7 \mathrm{~Hz}), 125.4$, $125.3,81.0,80.6,39.1,39.0,35.0(\mathrm{q}, J=29.3 \mathrm{~Hz}), 34.9(\mathrm{q}, J=29.6 \mathrm{~Hz}), 32.4,31.2,31.1,26.3,26.2$, 21.0. The major isomer: ${ }^{19} \mathrm{~F}$ NMR $\left(470 \mathrm{MHz}, \mathrm{CDCl}_{3}\right) \delta-64.85(\mathrm{t}, J=10.7 \mathrm{~Hz}),-64.95(\mathrm{t}, J=10.7 \mathrm{~Hz})$. The minor isomer: ${ }^{19} \mathrm{~F}$ NMR $\left(470 \mathrm{MHz}, \mathrm{CDCl}_{3}\right) \delta-64.85(\mathrm{t}, J=10.7 \mathrm{~Hz}),-64.95(\mathrm{t}, J=10.7 \mathrm{~Hz})$. HRMS (ESI) m/z: $[\mathrm{M}+\mathrm{H}]^{+}$calcd for $\mathrm{C}_{16} \mathrm{H}_{18} \mathrm{~F}_{3} \mathrm{~N}_{2} \mathrm{O} 311.1366$; found: 311.1374 .

Spectral data for 2,2-dimethyl-7-(2,2,2-trifluoroethyl)-2,5,6,7-tetrahydro-3H-pyrrolo[1,2-a]imidazol-3-one (3f)<smiles>CC1(C)N=C2C(CC(F)(F)F)CCN2C1=O</smiles>

Purified by column chromatography (silica gel, EtOAc/Petroleum ether: $1 / 5)$ to afford the title compound (71\% yield, $33 \mathrm{mg}$ ) as white solid, mp: $63-65^{\circ} \mathrm{C} .{ }^{1} \mathrm{H}$ NMR $\left(400 \mathrm{MHz}, \mathrm{CDCl}_{3}\right) \delta 3.78-3.64(\mathrm{~m}, 1 \mathrm{H}), 3.53-3.37(\mathrm{~m}, 1 \mathrm{H}), 3.19$ $-3.06(\mathrm{~m}, 1 \mathrm{H}), 3.01-2.84(\mathrm{~m}, 1 \mathrm{H}), 2.81-2.67(\mathrm{~m}, 1 \mathrm{H}), 2.32-2.13(\mathrm{~m}, 2 \mathrm{H}), 1.38(\mathrm{~s}, 3 \mathrm{H}), 1.37(\mathrm{~s}, 3 \mathrm{H}) .{ }^{13} \mathrm{C} \mathrm{NMR}$ $\left(100 \mathrm{MHz}, \mathrm{CDCl}_{3}\right) \delta 182.8,167.9,126.3(\mathrm{q}, J=276.7 \mathrm{~Hz}), 76.7,38.9,34.8(\mathrm{q}, J=29.5 \mathrm{~Hz}), 32.4,31.0(\mathrm{q}, J=2.8$ $\mathrm{Hz}), 24.1,24.0 .{ }^{19} \mathrm{~F}$ NMR $\left(376 \mathrm{MHz}, \mathrm{CDCl}_{3}\right) \delta$-65.01. HRMS (ESI) $\mathrm{m} / \mathrm{z}$ : $[\mathrm{M}+\mathrm{H}]^{+}$calcd for $\mathrm{C}_{10} \mathrm{H}_{14} \mathrm{~F}_{3} \mathrm{~N}_{2} \mathrm{O} 235.1053$; found: 235.1059 .

Spectral data for 2,2-dipropyl-7-(2,2,2-trifluoroethyl)-2,5,6,7-tetrahydro-3H-pyrrolo[1,2-a]imidazol-3-one (3g)<smiles>O=C1N2CCC(CC(F)(F)F)C2=NC1(C(F)(F)F)C(F)(F)F</smiles>

Purified by column chromatography (silica gel, EtOAc/Petroleum ether: $1 / 5)$ to afford the title compound ( $82 \%$ yield, $48 \mathrm{mg}$ ) as colorless oil. ${ }^{1} \mathrm{H}$ NMR $\left(500 \mathrm{MHz}, \mathrm{CDCl}_{3}\right) \delta 3.72-3.60(\mathrm{~m}, 1 \mathrm{H}), 3.47-3.32(\mathrm{~m}, 1 \mathrm{H}), 3.19-3.08(\mathrm{~m}, 1 \mathrm{H})$, $3.03-2.88(\mathrm{~m}, 1 \mathrm{H}), 2.81-2.62(\mathrm{~m}, 1 \mathrm{H}), 2.30-2.06(\mathrm{~m}, 2 \mathrm{H}), 1.83-1.57(\mathrm{~m}, 4 \mathrm{H}), 1.24-0.95(\mathrm{~m}, 4 \mathrm{H}), 0.87(\mathrm{t}, J=$ $7.3 \mathrm{~Hz}, 6 \mathrm{H}) .{ }^{13} \mathrm{C}$ NMR $\left(125 \mathrm{MHz}, \mathrm{CDCl}_{3}\right) \delta 181.9,168.3,126.3$ (q, $\left.J=276.6 \mathrm{~Hz}\right), 83.7,39.3,39.1,38.6,35.1$ (q, $J$ $=29.4 \mathrm{~Hz}), 32.7,30.9,16.9,16.8,14.0 .{ }^{19} \mathrm{~F}$ NMR $\left(470 \mathrm{MHz}, \mathrm{CDCl}_{3}\right) \delta-64.96(\mathrm{t}, \mathrm{J}=10.6 \mathrm{~Hz}) . \mathrm{HRMS}(\mathrm{ESI}) \mathrm{m} / \mathrm{z}:[\mathrm{M}$ $+\mathrm{H}]^{+}$calcd for $\mathrm{C}_{14} \mathrm{H}_{22} \mathrm{~F}_{3} \mathrm{~N}_{2} \mathrm{O} 291.1679$; found: 291.1685 .

Spectral data for 2-ethyl-2-methyl-7-(2,2,2-trifluoroethyl)-2,5,6,7-tetrahydro-3H-pyrrolo[1,2-a]imidazol-3one (3h)<smiles>CCC1(C)N=C2C(CC(F)(F)F)CCN2C1=O</smiles>

Purified by column chromatography (silica gel, EtOAc/Petroleum ether: 1/2) to afford the title compound (an inseparable mixture, $81 \%$ yield, $41 \mathrm{mg}$, $\mathrm{dr}=1 / 1)$ as colorless oil. The mixture: ${ }^{1} \mathrm{H}$ NMR $\left(500 \mathrm{MHz}, \mathrm{CDCl}_{3}\right) \delta 3.80-$ $3.64(\mathrm{~m}, 2 \mathrm{H}), 3.50-3.34(\mathrm{~m}, 2 \mathrm{H}), 3.22-3.04(\mathrm{~m}, 2 \mathrm{H}), 3.03-2.89(\mathrm{~m}, 2 \mathrm{H}), 2.82-2.66(\mathrm{~m}, 2 \mathrm{H}), 2.34-2.08(\mathrm{~m}$, 4H), $1.89-1.73(\mathrm{~m}, 4 \mathrm{H}), 1.36(\mathrm{~s}, 3 \mathrm{H}), 1.34(\mathrm{~s}, 3 \mathrm{H}), 0.79(\mathrm{t}, J=7.4 \mathrm{~Hz}, 3 \mathrm{H}), 0.74(\mathrm{t}, J=7.4 \mathrm{~Hz}, 3 \mathrm{H})$. The minor isomer: ${ }^{1} \mathrm{H}$ NMR $\left(500 \mathrm{MHz}, \mathrm{CDCl}_{3}\right) \delta 3.80-3.64(\mathrm{~m}, 2 \mathrm{H}), 3.50-3.34(\mathrm{~m}, 2 \mathrm{H}), 3.22-3.04(\mathrm{~m}, 2 \mathrm{H}), 3.03-2.89$ $(\mathrm{m}, 2 \mathrm{H}), 2.82-2.66(\mathrm{~m}, 2 \mathrm{H}), 2.34-2.08(\mathrm{~m}, 4 \mathrm{H}), 1.89-1.73(\mathrm{~m}, 4 \mathrm{H}), 1.36(\mathrm{~s}, 3 \mathrm{H}), 1.34(\mathrm{~s}, 3 \mathrm{H}), 0.79(\mathrm{t}, J=7.4 \mathrm{~Hz}$, $3 \mathrm{H}), 0.74$ (t, $J=7.4 \mathrm{~Hz}, 3 \mathrm{H}) .{ }^{13} \mathrm{C}$ NMR $\left(125 \mathrm{MHz}, \mathrm{CDCl}_{3}\right) \delta 182.3,182.2,168.4,168.2,126.3$ (q, $\left.J=276.9 \mathrm{~Hz}\right)$, 
$126.2(\mathrm{q}, J=276.9 \mathrm{~Hz}), 80.4,80.3,38.8,38.7,35.0(\mathrm{q}, J=29.5 \mathrm{~Hz}), 34.9$ (q, $J=29.3 \mathrm{~Hz}), 32.6,32.4,31.1,30.7$, 30.6, 22.9, 22.8, 8.1, 8.0. ${ }^{19} \mathrm{~F}$ NMR $\left(470 \mathrm{MHz}, \mathrm{CDCl}_{3}\right) \delta-64.92$ (t, $\left.J=10.4 \mathrm{~Hz}\right), \delta-64.94(\mathrm{t}, J=10.7 \mathrm{~Hz}) . \mathrm{HRMS}$ (ESI) $\mathrm{m} / \mathrm{z}:[\mathrm{M}+\mathrm{H}]^{+}$calcd for $\mathrm{C}_{11} \mathrm{H}_{16} \mathrm{~F}_{3} \mathrm{~N}_{2} \mathrm{O} 249.1209$; found: 249.1205 .

Spectral data for 2-phenyl-7-(2,2,2-trifluoroethyl)-5,6-dihydro-3H-pyrrolo[1,2-a]imidazol-3-one (3i)<smiles>O=C1C2=C(CC(F)(F)F)CCC2=NC1c1ccccc1</smiles>

Purified by column chromatography (silica gel, EtOAc/Petroleum ether: $1 / 5)$ to afford the title compound (22\% yield, $12 \mathrm{mg}$ ) as brown solid, mp: $150-152{ }^{\circ} \mathrm{C} .{ }^{1} \mathrm{H}$ NMR $\left(500 \mathrm{MHz}, \mathrm{CDCl}_{3}\right) \delta 8.53(\mathrm{~d}, J=7.7 \mathrm{~Hz}, 2 \mathrm{H}), 7.54(\mathrm{t}, J=7.3 \mathrm{~Hz}$, $1 \mathrm{H}), 7.48(\mathrm{t}, J=7.5 \mathrm{~Hz}, 2 \mathrm{H}), 3.98(\mathrm{t}, J=6.5 \mathrm{~Hz}, 2 \mathrm{H}), 3.47(\mathrm{q}, J=10.5 \mathrm{~Hz}, 2 \mathrm{H}), 3.31(\mathrm{t}, J=6.6 \mathrm{~Hz}, 2 \mathrm{H}) .{ }^{13} \mathrm{C} \mathrm{NMR}$ $\left(125 \mathrm{MHz}, \mathrm{CDCl}_{3}\right) \delta 167.5,159.8,151.7,125.1(\mathrm{q}, \mathrm{J}=278 \mathrm{~Hz}) 132.5,131.0,129.1,128.7,113.3,39.6,36.9,31.9$ $(\mathrm{q}, J=31.6 \mathrm{~Hz}) .{ }^{19} \mathrm{~F}$ NMR $\left(470 \mathrm{MHz}, \mathrm{CDCl}_{3}\right) \delta-63.64(\mathrm{t}, J=10.7 \mathrm{~Hz})$. HRMS (ESI) m/z: $[\mathrm{M}+\mathrm{H}]^{+}$calcd for $\mathrm{C}_{14} \mathrm{H}_{12} \mathrm{~F}_{3} \mathrm{~N}_{2} \mathrm{O} 281.0896$; found: 281.0898 .

Spectral data for 7'-(2,2,2-trifluoroethyl)-6',7'-dihydro-3'H,5'H-spiro[cyclobutane-1,2'-pyrrolo[1,2a]imidazol]-3'-one (3j)<smiles>O=C1N2CCC(CC(F)(F)F)C2=NC12CCC2</smiles>

Purified by column chromatography (silica gel, EtOAc/Petroleum ether: $1 / 2)$ to afford the title compound (88\% yield, $44 \mathrm{mg})$ as yellowish solid, mp: $79-80{ }^{\circ} \mathrm{C} .{ }^{1} \mathrm{H}$ NMR $\left(500 \mathrm{MHz}, \mathrm{CDCl}_{3}\right) \delta 3.72-3.61(\mathrm{~m}, 1 \mathrm{H}), 3.45-3.36(\mathrm{~m}, 1 \mathrm{H})$, $3.17-3.05(\mathrm{~m}, 1 \mathrm{H}), 3.03-2.87(\mathrm{~m}, 1 \mathrm{H}), 2.78-2.66(\mathrm{~m}, 1 \mathrm{H}), 2.57-2.46(\mathrm{~m}, 2 \mathrm{H}), 2.43-2.31(\mathrm{~m}, 2 \mathrm{H}), 2.28-2.01$ (m, 4H). ${ }^{13} \mathrm{C}$ NMR $\left(125 \mathrm{MHz}, \mathrm{CDCl}_{3}\right) \delta 181.1,168.5,126.3$ (q, $\left.J=276.6 \mathrm{~Hz}\right), 78.7,38.8,34.9$ (q, $\left.J=29.5 \mathrm{~Hz}\right), 32.5$, 30.9, 30.8, 15.4. ${ }^{19} \mathrm{~F}$ NMR (470 MHz, $\left.\mathrm{CDCl}_{3}\right) \delta-65.04(\mathrm{t}, J=10.7 \mathrm{~Hz})$. HRMS (ESI) m/z: $[\mathrm{M}+\mathrm{H}]^{+}$calcd for $\mathrm{C}_{11} \mathrm{H}_{14} \mathrm{~F}_{3} \mathrm{~N}_{2} \mathrm{O} 247.1053$; found: 247.1062 .

Spectral data for 7'-(2,2,2-trifluoroethyl)-6',7'-dihydro-3'H,5'H-spiro[cyclopentane-1,2'-pyrrolo[1,2a]imidazol]-3'-one (3k)

5-(2,2,2-trifluoroethyl)-1,2,3,3a,5,6,7,9a-octahydro-9H-cyclopenta[d]pyrrolo[1,2-a]pyrimidin-9-one (3k')<smiles>O=C1C2CCCC2N=C2C(CC(F)(F)F)CCN12</smiles>

Purified by column chromatography (silica gel, EtOAc/Petroleum ether: 1/5) to afford the title compound (an inseparable mixture, 52\% yield, $27 \mathrm{mg}, \mathbf{3 k} / \mathbf{3} \mathbf{k}^{\prime}=1.7 / 1 ; \mathbf{3 k}$ ': $\left.\mathrm{dr}=1.2 / 1\right)$ colorless oil. $\mathbf{3 k}:{ }^{1} \mathrm{H}$ NMR $\left(400 \mathrm{MHz}, \mathrm{CDCl}_{3}\right)$ $\delta 3.79-3.68(\mathrm{~m}, 1 \mathrm{H}), 3.54-3.42(\mathrm{~m}, 1 \mathrm{H}), 3.20-3.08(\mathrm{~m}, 1 \mathrm{H}), 3.05-2.89(\mathrm{~m}, 1 \mathrm{H}), 2.82-2.65(\mathrm{~m}, 1 \mathrm{H}), 2.33-$ $2.12(\mathrm{~m}, 2 \mathrm{H}), 2.08-1.58(\mathrm{~m}, 8 \mathrm{H}) .{ }^{19} \mathrm{~F}$ NMR $\left(470 \mathrm{MHz}, \mathrm{CDCl}_{3}\right) \delta-64.98(\mathrm{t}, J=10.9 \mathrm{~Hz})$. HRMS (ESI) m/z: [M + $\mathrm{H}]^{+}$calcd for $\mathrm{C}_{12} \mathrm{H}_{16} \mathrm{~F}_{3} \mathrm{~N}_{2} \mathrm{O} 261.1209$; found: 261.1215 . 3k' $(\mathrm{dr}=1.2 / 1)$ : The major isomer: ${ }^{1} \mathrm{H}$ NMR (400 MHz, $\left.\mathrm{CDCl}_{3}\right) \delta 4.19-4.10(\mathrm{~m}, 1 \mathrm{H}), 4.05-3.95(\mathrm{~m}, 1 \mathrm{H}), 3.54-3.42(\mathrm{~m}, 1 \mathrm{H}), 3.05-2.91(\mathrm{~m}, 1 \mathrm{H}), 2.51-2.39(\mathrm{~m}, 1 \mathrm{H})$, $2.31-2.14(\mathrm{~m}, 2 \mathrm{H}), 2.08-1.58(\mathrm{~m}, 8 \mathrm{H})$. The minor isomer: ${ }^{1} \mathrm{H}$ NMR $\left(400 \mathrm{MHz}, \mathrm{CDCl}_{3}\right) \delta 4.19-4.10(\mathrm{~m}, 1 \mathrm{H})$, 
$4.05-3.95(\mathrm{~m}, 1 \mathrm{H}), 3.56-3.40(\mathrm{~m}, 1 \mathrm{H}), 3.07-2.86(\mathrm{~m}, 1 \mathrm{H}), 2.51-2.39(\mathrm{~m}, 1 \mathrm{H}), 2.31-2.14(\mathrm{~m}, 2 \mathrm{H}), 2.08-1.58$ $(\mathrm{m}, 8 \mathrm{H})$. The major isomer: ${ }^{19} \mathrm{~F}$ NMR $\left(470 \mathrm{MHz}, \mathrm{CDCl}_{3}\right) \delta-64.65(\mathrm{t}, J=11.0 \mathrm{~Hz})$. The minor isomer: ${ }^{19} \mathrm{~F}$ NMR $(470$ $\left.\mathrm{MHz}, \mathrm{CDCl}_{3}\right) \delta-64.54\left(\mathrm{t}, J=11.0 \mathrm{~Hz}\right.$ ). HRMS (ESI) $\mathrm{m} / \mathrm{z}$ : $[\mathrm{M}+\mathrm{H}]^{+}$calcd for $\mathrm{C}_{12} \mathrm{H}_{16} \mathrm{~F}_{3} \mathrm{~N}_{2} \mathrm{O} 261.1209$; found: 261.1218. The mixture of 3k/3k': ${ }^{13} \mathrm{C}$ NMR $\left(100 \mathrm{MHz}, \mathrm{CDCl}_{3}\right) \delta 182.9,170.8,170.7,167.9,155.7,155.2,126.8(\mathrm{q}$, $J=276.7 \mathrm{~Hz}), 126.7(\mathrm{q}, J=276.7 \mathrm{~Hz}), 126.3(\mathrm{q}, J=276.8 \mathrm{~Hz}), 86.1,61.9,61.7,43.0,42.9,42.2,41.9,38.9,37.4$, $37.3,35.8(\mathrm{q}, J=28.9 \mathrm{~Hz}), 35.6(\mathrm{q}, J=28.9 \mathrm{~Hz}), 35.3,35.2,35.0(\mathrm{q}, J=29.5 \mathrm{~Hz}), 32.5,31.0(\mathrm{q}, J=2.9 \mathrm{~Hz}), 30.0$, $26.9,26.8,25.9,25.8,22.7,22.6$.

Spectral data for $7^{\prime}$-(2,2,2-trifluoroethyl)-4,5,6',7'-tetrahydro-2H,3'H,5'H-spiro[furan-3,2'-pyrrolo[1,2a]imidazol]-3'-one (3I)<smiles>O=C1N2CCC(CC(F)(F)F)C2=NC12CCOC2</smiles>

Purified by column chromatography (silica gel, EtOAc/Petroleum ether: 1/2) to afford the title compound (an inseparable mixture, $61 \%$ yield, $32 \mathrm{mg}$, $\mathrm{dr}=1: 1)$ as yellowish oil. The mixture: ${ }^{1} \mathrm{H}$ NMR $\left(400 \mathrm{MHz}, \mathrm{CDCl}_{3}\right) \delta 4.23$ $-4.07(\mathrm{~m}, 4 \mathrm{H}), 4.01-3.89(\mathrm{~m}, 4 \mathrm{H}), 3.82-3.69(\mathrm{~m}, 2 \mathrm{H}), 3.57-3.42(\mathrm{~m}, 2 \mathrm{H}), 3.25-3.11(\mathrm{~m}, 2 \mathrm{H}), 3.07-2.89(\mathrm{~m}$, $2 \mathrm{H}), 2.85-2.72(\mathrm{~m}, 2 \mathrm{H}), 2.45-2.36(\mathrm{~m}, 2 \mathrm{H}), 2.34-2.21(\mathrm{~m}, 4 \mathrm{H}), 2.20-2.13(\mathrm{~m}, 2 \mathrm{H}) .{ }^{13} \mathrm{C} \mathrm{NMR}\left(125 \mathrm{MHz}, \mathrm{CDCl}_{3}\right)$ $\delta 179.6,169.7,126.3(\mathrm{q}, J=276.1), 126.2(\mathrm{q}, J=276.8), 85.1,85.0,68.9,68.8,39.2,37.8,37.7,34.8(\mathrm{q}, J=29.6$ $\mathrm{Hz}), 34.7(\mathrm{q}, J=29.5 \mathrm{~Hz}), 32.4,31.1 .{ }^{19} \mathrm{~F} \mathrm{NMR}\left(470 \mathrm{MHz}, \mathrm{CDCl}_{3}\right) \delta-64.95(\mathrm{t}, J=10.4 \mathrm{~Hz}), \delta-64.93(\mathrm{t}, J=10.2$ $\mathrm{Hz}$ ). HRMS (ESI) m/z: [M+H] $]^{+}$calcd for $\mathrm{C}_{11} \mathrm{H}_{14} \mathrm{~F}_{3} \mathrm{~N}_{2} \mathrm{O}_{2} 263.1002$; found: 263.1011 .

\section{Spectral data for 7'-(2,2,2-trifluoroethyl)-6',7'-dihydro-3'H,5'H-spiro[cyclohexane-1,2'-pyrrolo[1,2-} a]imidazol]-3'-one (3m)<smiles>O=C1N2CCC(CC(F)(F)F)C2=NC12CCCCC2</smiles>

Purified by column chromatography (silica gel, EtOAc/Petroleum ether: $1 / 2)$ to afford the title compound (71\% yield, $39 \mathrm{mg}$ ) as white solid, mp: $98-99^{\circ} \mathrm{C} .{ }^{1} \mathrm{H}$ NMR $\left(500 \mathrm{MHz}, \mathrm{CDCl}_{3}\right) \delta 3.73-3.63(\mathrm{~m}, 1 \mathrm{H}), 3.49-3.38(\mathrm{~m}, 1 \mathrm{H}), 3.17$ $-3.07(\mathrm{~m}, 1 \mathrm{H}), 3.03-2.89(\mathrm{~m}, 1 \mathrm{H}), 2.79-2.65(\mathrm{~m}, 1 \mathrm{H}), 2.29-2.14(\mathrm{~m}, 2 \mathrm{H}), 1.87-1.64(\mathrm{~m}, 7 \mathrm{H}), 1.58-1.35(\mathrm{~m}$, $3 \mathrm{H}) .{ }^{13} \mathrm{C}$ NMR $\left(100 \mathrm{MHz}, \mathrm{CDCl}_{3}\right) \delta 182.8,167.7,126.4$ (q, $\left.J=276.8 \mathrm{~Hz}\right), 79.7,38.7,35.1$ (q, $\left.J=29.3 \mathrm{~Hz}\right), 33.4$, 33.2, 32.4, 31.0 (q, $J=2.9 \mathrm{~Hz}), 25.1,21.7,21.6 .{ }^{19} \mathrm{~F}$ NMR $\left(376 \mathrm{MHz}, \mathrm{CDCl}_{3}\right) \delta-64.89 . \mathrm{HRMS}(\mathrm{ESI}) \mathrm{m} / \mathrm{z}:[\mathrm{M}+\mathrm{H}]^{+}$ calcd for $\mathrm{C}_{13} \mathrm{H}_{18} \mathrm{~F}_{3} \mathrm{~N}_{2} \mathrm{O} 275.1366$; found: 275.1363 .

Spectral data for $7^{\prime}-(2,2,2$-trifluoroethyl)-2,3,5,6,6',7'-hexahydro-3'H,5'H-spiro[pyran-4,2'-pyrrolo[1,2a]imidazol]-3'-one (3n)<smiles>O=C1N2CCC(CC(F)(F)F)C2=NC12CCOCC2</smiles>

Purified by column chromatography (silica gel, EtOAc/Petroleum ether: $1 / 1)$ to afford the title compound (78\% yield, $43 \mathrm{mg}$ ) as brown solid, $\mathrm{mp}: 85-87^{\circ} \mathrm{C} .{ }^{1} \mathrm{H}$ NMR $\left(500 \mathrm{MHz}, \mathrm{CDCl}_{3}\right) \delta 4.03-3.94(\mathrm{~m}, 2 \mathrm{H}), 3.94-3.81(\mathrm{~m}, 2 \mathrm{H}), 3.76$ $-3.62(\mathrm{~m}, 1 \mathrm{H}), 3.52-3.39(\mathrm{~m}, 1 \mathrm{H}), 3.21-3.09(\mathrm{~m}, 1 \mathrm{H}), 3.05-2.89(\mathrm{~m}, 1 \mathrm{H}), 2.83-2.69(\mathrm{~m}, 1 \mathrm{H}), 2.31-2.17(\mathrm{~m}$, 
2H), $2.10-1.97(\mathrm{~m}, 2 \mathrm{H}), 1.52-1.38(\mathrm{~m}, 2 \mathrm{H}) .{ }^{13} \mathrm{C} \mathrm{NMR}\left(125 \mathrm{MHz}, \mathrm{CDCl}_{3}\right) \delta 181.3,168.7,126.3(\mathrm{q}, J=276.6 \mathrm{~Hz})$, 76.7, 63.6, 63.5, 38.9, 35.0 (q, $J=29.6 \mathrm{~Hz}), 33.0,32.9,32.3,31.1 .{ }^{19} \mathrm{~F}$ NMR $\left(470 \mathrm{MHz}, \mathrm{CDCl}_{3}\right) \delta-64.83(\mathrm{t}, J=10.7$ $\mathrm{Hz}$ ). HRMS (ESI) m/z: [M+H] $]^{+}$calcd for $\mathrm{C}_{12} \mathrm{H}_{16} \mathrm{~F}_{3} \mathrm{~N}_{2} \mathrm{O}_{2} 277.1158$; found: 277.1162 .

Spectral data for tert-butyl 3'-oxo-7'-(2,2,2-trifluoroethyl)-6',7'-dihydro-3'H,5'H-spiro[piperidine-4,2'pyrrolo[1,2-a]imidazole]-1-carboxylate (3o)<smiles></smiles>

Purified by column chromatography (silica gel, EtOAc/Petroleum ether: $1 / 1)$ to afford the title compound (75\% yield, $57 \mathrm{mg}$ ) as colorless oil. ${ }^{1} \mathrm{H}$ NMR (400 MHz, $\left.\mathrm{CDCl}_{3}\right) \delta$ 4.11-3.90 (m, 2H), $3.76-3.61(\mathrm{~m}, 1 \mathrm{H}), 3.54-3.41(\mathrm{~m}, 1 \mathrm{H})$, 3.31 (brs, $2 \mathrm{H}), 3.23-3.07(\mathrm{~m}, 1 \mathrm{H}), 3.05-2.87(\mathrm{~m}, 1 \mathrm{H}), 2.83-2.68(\mathrm{~m}, 1 \mathrm{H}), 2.37-2.15(\mathrm{~m}, 2 \mathrm{H}), 1.96-1.83(\mathrm{~m}$, 2H), $1.47(\mathrm{~s}, 11 \mathrm{H}) .{ }^{13} \mathrm{C}$ NMR $\left(125 \mathrm{MHz}, \mathrm{CDCl}_{3}\right) \delta 181.4,168.8,154.7,126.3$ (q, $\left.J=277.4 \mathrm{~Hz}\right), 79.7,77.6,38.9$, 35.0 (q, $J=29.5 \mathrm{~Hz}), 32.6,32.5,32.4,31.1,28.5 .{ }^{19} \mathrm{~F}$ NMR $\left(470 \mathrm{MHz}, \mathrm{CDCl}_{3}\right) \delta-64.84 . \mathrm{HRMS}(\mathrm{ESI}) \mathrm{m} / \mathrm{z}$ : [M + $\mathrm{H}]^{+}$calcd for $\mathrm{C}_{17} \mathrm{H}_{25} \mathrm{~F}_{3} \mathrm{~N}_{3} \mathrm{O}_{3} 376.1843$; found: 376.1837 .

\section{Spectral data for 7-methyl-2,2-diphenyl-7-(2,2,2-trifluoroethyl)-2,5,6,7-tetrahydro-3H-pyrrolo[1,2- a]imidazol-3-one (3p)}<smiles>CC1(CC(F)(F)F)CCN2C(=O)C(c3ccccc3)(c3ccccc3)N=C21</smiles>

Purified by column chromatography (silica gel, EtOAc/Petroleum ether: $1 / 8$ ) to afford the title compound (74\% yield, $55 \mathrm{mg})$ as colorless oil. ${ }^{1} \mathrm{H}$ NMR $\left(400 \mathrm{MHz}, \mathrm{CDCl}_{3}\right) \delta 7.53-7.48(\mathrm{~m}, 2 \mathrm{H}), 7.48-7.42(\mathrm{~m}, 2 \mathrm{H}), 7.36-7.26(\mathrm{~m}, 6 \mathrm{H})$, $3.74-3.63(\mathrm{~m}, 1 \mathrm{H}), 3.61-3.44(\mathrm{~m}, 1 \mathrm{H}), 2.92-2.70(\mathrm{~m}, 1 \mathrm{H}), 2.66-2.44(\mathrm{~m}, 2 \mathrm{H}), 2.42-2.28(\mathrm{~m}, 1 \mathrm{H}), 1.51(\mathrm{~s}, 3 \mathrm{H})$. ${ }^{13} \mathrm{C}$ NMR (125 MHz, $\left.\mathrm{CDCl}_{3}\right) \delta 179.0,172.9,140.3,140.1,128.5,128.4,127.8,126.2(\mathrm{q}, J=277.7 \mathrm{~Hz}), 86.4,40.4$ (q, $J=28.2 \mathrm{~Hz}), 38.5,38.1,37.5,23.3 .{ }^{19} \mathrm{~F}$ NMR $\left(470 \mathrm{MHz}, \mathrm{CDCl}_{3}\right) \delta-60.47(\mathrm{t}, J=11.0 \mathrm{~Hz}) . \mathrm{HRMS}(\mathrm{ESI}) \mathrm{m} / \mathrm{z}:[\mathrm{M}$ $+\mathrm{H}]^{+}$calcd for $\mathrm{C}_{21} \mathrm{H}_{20} \mathrm{~F}_{3} \mathrm{~N}_{2} \mathrm{O} 373.1522$; found: 373.1519 .

\section{Spectral data for 2,2,7-triphenyl-7-(2,2,2-trifluoroethyl)-2,5,6,7-tetrahydro-3H-pyrrolo[1,2-a]imidazol-3-one} (3q)<smiles>O=C1C(c2ccccc2)N=C2N1CCC2(CC(F)(F)F)c1ccccc1</smiles>

Purified by column chromatography (silica gel, EtOAc/Petroleum ether: $1 / 10)$ to afford the title compound (60\% yield, $52 \mathrm{mg}$ ) as white solid, mp: $104-105^{\circ} \mathrm{C} .{ }^{1} \mathrm{H}$ NMR $\left(500 \mathrm{MHz}, \mathrm{CDCl}_{3}\right) \delta 7.58(\mathrm{~d}, J=7.6 \mathrm{~Hz}, 4 \mathrm{H}), 7.50-7.42$ (m, 2H), $7.37-7.32(\mathrm{~m}, 4 \mathrm{H}), 7.32-7.22(\mathrm{~m}, 5 \mathrm{H}), 3.77-3.68(\mathrm{~m}, 1 \mathrm{H}), 3.40-3.28(\mathrm{~m}, 1 \mathrm{H}), 3.22-3.06(\mathrm{~m}, 2 \mathrm{H})$, $2.95-2.81(\mathrm{~m}, 1 \mathrm{H}), 2.77-2.64(\mathrm{~m}, 1 \mathrm{H}) .{ }^{13} \mathrm{C} \mathrm{NMR}\left(125 \mathrm{MHz}, \mathrm{CDCl}_{3}\right) \delta 178.8,170.9,140.2,140.1,137.1,129.2$, 128.6, 128.5, 127.9, 127.2, 126.7, 125.8 (q, $J=278.3 \mathrm{~Hz}), 86.4,45.3,42.5$ (q, $J=27.9 \mathrm{~Hz}), 38.6,36.4 .{ }^{19} \mathrm{~F}$ NMR $\left(470 \mathrm{MHz}, \mathrm{CDCl}_{3}\right) \delta-60.35\left(\mathrm{t}, J=10.8 \mathrm{~Hz}\right.$ ). HRMS (ESI) $\mathrm{m} / \mathrm{z}$ : $[\mathrm{M}+\mathrm{H}]^{+}$calcd for $\mathrm{C}_{26} \mathrm{H}_{22} \mathrm{~F}_{3} \mathrm{~N}_{2} \mathrm{O}$ 435.1679; found: 435.1675 . 
Spectral data for 2',2'-diphenyl-2-(trifluoromethyl)-5',6'-dihydrospiro[cyclopentane-1,7'-pyrrolo[1,2a]imidazol]-3'(2'H)-one (3r)<smiles>O=C1C(c2ccccc2)=NC2N1CCC21CCCC1C(F)(F)F</smiles>

Purified by column chromatography (silica gel, EtOAc/Petroleum ether: 1/10) to afford the title compound (69\% yield, $55 \mathrm{mg}, \mathrm{dr}>19: 1)$ as white solid, mp: $118-120^{\circ} \mathrm{C} .{ }^{1} \mathrm{H}$ NMR (500 MHz, $\left.\mathrm{CDCl}_{3}\right) \delta 7.54-7.49(\mathrm{~m}, 2 \mathrm{H}), 7.47-$ $7.41(\mathrm{~m}, 2 \mathrm{H}), 7.35-7.22(\mathrm{~m}, 6 \mathrm{H}), 3.71-3.63(\mathrm{~m}, 1 \mathrm{H}), 3.53-3.44(\mathrm{~m}, 1 \mathrm{H}), 3.35-3.24(\mathrm{~m}, 1 \mathrm{H}), 2.78-2.68(\mathrm{~m}$, $1 \mathrm{H}), 2.34-2.24(\mathrm{~m}, 1 \mathrm{H}), 2.23-2.10(\mathrm{~m}, 2 \mathrm{H}), 2.01-1.81(\mathrm{~m}, 4 \mathrm{H}) .{ }^{13} \mathrm{C} \mathrm{NMR}\left(125 \mathrm{MHz}, \mathrm{CDCl}_{3}\right) \delta 179.1,172.9$, 140.4, 140.3, 128.5, 128.4, 127.8, 127.4 (q, $J=278.5 \mathrm{~Hz}$ ), 127.2, 127.1, 86.3, 48.0 (q, $J=28.2 \mathrm{~Hz}$ ), 47.9, 39.1, 38.8, 34.7, 26.0, 22.9. ${ }^{19} \mathrm{~F}$ NMR $\left(470 \mathrm{MHz}, \mathrm{CDCl}_{3}\right) \delta-65.75(\mathrm{~d}, J=9.7 \mathrm{~Hz})$. HRMS (ESI) m/z: $[\mathrm{M}+\mathrm{H}]^{+}$calcd for $\mathrm{C}_{23} \mathrm{H}_{22} \mathrm{~F}_{3} \mathrm{~N}_{2} \mathrm{O} 399.1679$; found: 399.1678 .

\section{Spectral data for 2',2'-diphenyl-2-(trifluoromethyl)-5',6'-dihydrospiro[cyclohexane-1,7'-pyrrolo[1,2-} a]imidazol]-3'(2'H)-one (3s)<smiles>O=C1C(c2ccccc2)NC2N1CCC21CCCCC1C(F)(F)F</smiles>

Purified by column chromatography (silica gel, EtOAc/Petroleum ether: $1 / 8)$ to afford the title compound (62\% yield, $51 \mathrm{mg}, \mathrm{dr}>19 / 1)$ as colorless oil. ${ }^{1} \mathrm{H}$ NMR $\left(500 \mathrm{MHz}, \mathrm{CDCl}_{3}\right) \delta 7.50-7.46(\mathrm{~m}, 2 \mathrm{H}), 7.44-7.40(\mathrm{~m}, 2 \mathrm{H}), 7.35-$ $7.22(\mathrm{~m}, 6 \mathrm{H}), 3.58(\mathrm{t}, J=7.2 \mathrm{~Hz}, 2 \mathrm{H}), 2.87-2.75(\mathrm{~m}, 1 \mathrm{H}), 2.74-2.63(\mathrm{~m}, 1 \mathrm{H}), 2.32-2.22(\mathrm{~m}, 1 \mathrm{H}), 2.11-2.03(\mathrm{~m}$, $1 \mathrm{H}), 2.03-1.94(\mathrm{~m}, 1 \mathrm{H}), 1.92-1.85(\mathrm{~m}, 1 \mathrm{H}), 1.83-1.76(\mathrm{~m}, 2 \mathrm{H}), 1.58-1.31(\mathrm{~m}, 3 \mathrm{H}) .{ }^{13} \mathrm{C} \mathrm{NMR}\left(125 \mathrm{MHz}, \mathrm{CDCl}_{3}\right)$ $\delta 179.3,173.7,140.9,140.1,128.5,128.4,127.8,127.7,127.4,127.2(q, J=281.7 \mathrm{~Hz}), 127.1,86.6,45.9$ (q, $J=24.8$ $\mathrm{Hz}), 41.3,38.1,37.2,31.9,24.1,22.7(\mathrm{q}, J=2.7 \mathrm{~Hz}), 21.6 .{ }^{19} \mathrm{~F}$ NMR $\left(470 \mathrm{MHz}, \mathrm{CDCl}_{3}\right) \delta-66.55(\mathrm{~d}, J=9.2 \mathrm{~Hz})$. HRMS (ESI) m/z: [M + H] calcd for $\mathrm{C}_{24} \mathrm{H}_{24} \mathrm{~F}_{3} \mathrm{~N}_{2} \mathrm{O} 413.1835$; found: 413.1831 .

\section{Spectral data for (5)-2,2,5-triphenyl-7-(2,2,2-trifluoroethyl)-2,5,6,7-tetrahydro-3H-pyrrolo[1,2-a]imidazol-3-} one (3t)<smiles>O=C1C(CC(F)(F)F)CC2=NC(c3ccccc3)(c3ccccc3)CN12</smiles>

Purified by column chromatography (silica gel, EtOAc/Petroleum ether: 1/15) to afford the title compound (an inseparable mixture, $82 \%$ yield, $72 \mathrm{mg}, \mathrm{dr}=2: 1)$ as colorless oil. The major isomer: ${ }^{1} \mathrm{H} \mathrm{NMR}\left(400 \mathrm{MHz}, \mathrm{CDCl}_{3}\right) \delta$ $7.56-7.13(\mathrm{~m}, 15 \mathrm{H}), 5.12(\mathrm{dd}, J=7.3,2.6 \mathrm{~Hz}, 1 \mathrm{H}), 3.42-3.26(\mathrm{~m}, 1 \mathrm{H}), 3.24-3.05(\mathrm{~m}, 2 \mathrm{H}), 2.79-2.59(\mathrm{~m}, 1 \mathrm{H})$, $2.44-2.24(\mathrm{~m}, 1 \mathrm{H})$. The minor isomer: ${ }^{1} \mathrm{H}$ NMR $\left(400 \mathrm{MHz}, \mathrm{CDCl}_{3}\right) \delta 7.56-7.13(\mathrm{~m}, 15 \mathrm{H}), 4.92(\mathrm{t}, J=7.8 \mathrm{~Hz}, 1 \mathrm{H})$, $3.42-3.26(\mathrm{~m}, 1 \mathrm{H}), 2.79-2.59(\mathrm{~m}, 2 \mathrm{H}), 2.44-2.24(\mathrm{~m}, 1 \mathrm{H}), 2.25-2.11(\mathrm{~m}, 1 \mathrm{H}) .{ }^{13} \mathrm{C} \mathrm{NMR}\left(125 \mathrm{MHz}, \mathrm{CDCl}_{3}\right) \delta$ 178.3, 178.0, 169.6, 169.4, 140.5, 140.4, 140.3, 140.2, 138.8, 137.3, 129.3, 129.1, 128.8, 128.7, 128.5, 128.3, 128.0, $127.9,127.2,127.0,126.9,126.3(\mathrm{q}, J=277.2 \mathrm{~Hz}), 126.0,125.3,86.7,57.2,54.8,43.7,41.9,35.2(\mathrm{q}, J=29.6 \mathrm{~Hz})$, 35.0 (q, $J=29.5 \mathrm{~Hz}), 31.2,29.9$. The major isomer: ${ }^{19} \mathrm{~F} \mathrm{NMR}\left(470 \mathrm{MHz}, \mathrm{CDCl}_{3}\right) \delta-64.83(\mathrm{t}, J=10.7 \mathrm{~Hz})$. The minor isomer: ${ }^{19} \mathrm{~F}$ NMR (470 MHz, $\left.\mathrm{CDCl}_{3}\right) \delta-64.91$ (t, $J=10.6 \mathrm{~Hz}$ ). HRMS (ESI) m/z: $[\mathrm{M}+\mathrm{H}]^{+}$calcd for $\mathrm{C}_{26} \mathrm{H}_{22} \mathrm{~F}_{3} \mathrm{~N}_{2} \mathrm{O}$ 435.1679; found: 435.1682 . 
Spectral data for 5,5-dimethyl-2,2-diphenyl-7-(2,2,2-trifluoroethyl)-2,5,6,7-tetrahydro-3H-pyrrolo[1,2a]imidazol-3-one (3u)<smiles>CC1(C)CC(CC(F)(F)F)C2=NC(c3ccccc3)(c3ccccc3)C(=O)N21</smiles>

Purified by column chromatography (silica gel, EtOAc/Petroleum ether: $1 / 20)$ to afford the title compound (82\% yield, $64 \mathrm{mg}$,) as white solid, mp: $119-120{ }^{\circ} \mathrm{C} .{ }^{1} \mathrm{H} \mathrm{NMR}\left(500 \mathrm{MHz}, \mathrm{CDCl}_{3}\right) \delta 7.51-7.47(\mathrm{~m}, 2 \mathrm{H}), 7.44-7.40(\mathrm{~m}$, $2 \mathrm{H}), 7.35-7.25(\mathrm{~m}, 6 \mathrm{H}), 3.30-3.19(\mathrm{~m}, 1 \mathrm{H}), 3.17-3.02(\mathrm{~m}, 1 \mathrm{H}), 2.59-2.51(\mathrm{~m}, 1 \mathrm{H}), 2.35-2.20(\mathrm{~m}, 1 \mathrm{H}), 2.11-$ $2.02(\mathrm{~m}, 1 \mathrm{H}), 1.60(\mathrm{~s}, 3 \mathrm{H}), 1.46(\mathrm{~s}, 3 \mathrm{H}) .{ }^{13} \mathrm{C} \mathrm{NMR}\left(125 \mathrm{MHz}, \mathrm{CDCl}_{3}\right) \delta 178.7,169.3,140.4,128.7,128.6,127.9$, 127.2, 126.9, 126.5 (q, $J=276.7 \mathrm{~Hz}), 86.6,58.1,48.0,35.4$ (q, $J=29.4 \mathrm{~Hz}), 30.0,26.4,26.1 .{ }^{19} \mathrm{~F} \mathrm{NMR}(470 \mathrm{MHz}$, $\left.\mathrm{CDCl}_{3}\right) \delta-64.94(\mathrm{t}, J=10.7 \mathrm{~Hz})$. HRMS (ESI) $\mathrm{m} / \mathrm{z}:[\mathrm{M}+\mathrm{H}]^{+}$calcd for $\mathrm{C}_{22} \mathrm{H}_{22} \mathrm{~F}_{3} \mathrm{~N}_{2} \mathrm{O} 387.1679$; found: 387.1677 .

Spectral data for 2',2'-diphenyl-8'-(2,2,2-trifluoroethyl)-7',8'-dihydro-5'H-spiro[cyclopentane-1,6'imidazo[1,2-a]pyridin]-3'(2'H)-one (3v)<smiles>O=C1N2CC3(CCCC3)CC(CC(F)(F)F)C2=NC1(c1ccccc1)c1ccccc1</smiles>

Purified by column chromatography (silica gel, EtOAc/Petroleum ether: $1 / 30)$ to afford the title compound (81\% yield, $69 \mathrm{mg})$ as white solid, mp: $128-130{ }^{\circ} \mathrm{C} .{ }^{1} \mathrm{H} \mathrm{NMR}\left(500 \mathrm{MHz}, \mathrm{CDCl}_{3}\right) \delta 7.58-7.55(\mathrm{~m}, 2 \mathrm{H}), 7.45-7.41(\mathrm{~m}$, $2 \mathrm{H}), 7.34-7.26(\mathrm{~m}, 6 \mathrm{H}), 3.60-3.53(\mathrm{~m}, 1 \mathrm{H}), 3.46-3.33(\mathrm{~m}, 1 \mathrm{H}), 3.15-3.08(\mathrm{~m}, 1 \mathrm{H}), 3.01-2.98(\mathrm{~m}, 1 \mathrm{H}), 2.40-$ $2.26(\mathrm{~m}, 1 \mathrm{H}), 2.11-2.01(\mathrm{~m}, 1 \mathrm{H}), 1.80-1.51(\mathrm{~m}, 8 \mathrm{H}), 1.47-1.38(\mathrm{~m}, 1 \mathrm{H}) .{ }^{13} \mathrm{C} \mathrm{NMR}\left(125 \mathrm{MHz}, \mathrm{CDCl}_{3}\right) \delta 182.7$, $160.8,140.5,140.4,128.7,128.5,127.8,127.2,127.1,126.9$ (q, $J=276.0 \mathrm{~Hz}), 78.4,50.0,41.1,38.7,38.6,35.9$ (q, $J=28.7 \mathrm{~Hz}), 34.7,30.2,24.9,24.2 .{ }^{19} \mathrm{~F} \mathrm{NMR}\left(470 \mathrm{MHz}, \mathrm{CDCl}_{3}\right) \delta-63.12(\mathrm{t}, J=11.3 \mathrm{~Hz}) . \mathrm{HRMS}(\mathrm{ESI}) \mathrm{m} / \mathrm{z}:[\mathrm{M}+$ $\mathrm{H}]^{+}$calcd for $\mathrm{C}_{25} \mathrm{H}_{26} \mathrm{~F}_{3} \mathrm{~N}_{2} \mathrm{O} 427.1992$; found: 427.1997.

Spectral data for (E)-2,2-diphenyl-7-(2,2,2-trifluoroethylidene)-2,5,6,7-tetrahydro-3H-pyrrolo[1,2-a]imidazol3-one (4)<smiles>O=C1N2CC/C(=C\C(F)(F)F)C2=NC1(c1ccccc1)c1ccccc1</smiles>

Purified by column chromatography (silica gel, EtOAc/Petroleum ether: 1/30) to afford the title compound (21\% yield, $15 \mathrm{mg}$ ) as white solid, mp: $145-146{ }^{\circ} \mathrm{C}$ (Table S3, entry 12). ${ }^{1} \mathrm{H}$ NMR $\left(500 \mathrm{MHz}, \mathrm{CDCl}_{3}\right) \delta 7.55-7.49(\mathrm{~m}$, $4 \mathrm{H}), 7.38-7.26(\mathrm{~m}, 6 \mathrm{H}), 6.85(\mathrm{qt}, J=8.2,3.1 \mathrm{~Hz}, 1 \mathrm{H}), 3.72(\mathrm{t}, J=7.1 \mathrm{~Hz}, 2 \mathrm{H}), 3.43-3.31(\mathrm{~m}, 2 \mathrm{H}) .{ }^{13} \mathrm{C} \mathrm{NMR}(125$ $\left.\mathrm{MHz}_{2} \mathrm{CDCl}_{3}\right) \delta 179.1,164.0,140.0,136.4,128.7,128.1,127.2,122.8(\mathrm{q}, J=270.9 \mathrm{~Hz}), 118.1(\mathrm{q}, J=36.3 \mathrm{~Hz}), 87.6$, 38.4, 29.8. ${ }^{19} \mathrm{~F}$ NMR (470 MHz, $\left.\mathrm{CDCl}_{3}\right) \delta-60.99$ - -61.02 (m). HRMS (ESI) m/z: $[\mathrm{M}+\mathrm{H}]^{+}$calcd for $\mathrm{C}_{20} \mathrm{H}_{16} \mathrm{~F}_{3} \mathrm{~N}_{2} \mathrm{O}$ 357.1209; found: 357.1219 .

Spectral data for 1-(2,2-diphenylvinyl)-3-(2,2,2-trifluoroethyl)pyrrolidin-2-one (6a) 


Purified by column chromatography (silica gel, EtOAc/Petroleum ether: $1 / 20)$ to afford the title compound (75\% yield, $52 \mathrm{mg}$ ) as white solid, $\mathrm{mp}: 97-99^{\circ} \mathrm{C} .{ }^{1} \mathrm{H}$ NMR $\left(400 \mathrm{MHz}, \mathrm{CDCl}_{3}\right) \delta 7.42-7.32(\mathrm{~m}, 3 \mathrm{H}), 7.30-7.26(\mathrm{~m}, 1 \mathrm{H})$, $7.24-7.14(\mathrm{~m}, 7 \mathrm{H}), 3.05-2.84(\mathrm{~m}, 3 \mathrm{H}), 2.78-2.65(\mathrm{~m}, 1 \mathrm{H}), 2.31-2.19(\mathrm{~m}, 1 \mathrm{H}), 2.12-1.92(\mathrm{~m}, 1 \mathrm{H}), 1.73-1.60$ $(\mathrm{m}, 1 \mathrm{H}) .{ }^{13} \mathrm{C}$ NMR $\left(125 \mathrm{MHz}, \mathrm{CDCl}_{3}\right) \delta 174.2,141.2,138.7,130.7,128.2,128.1,127.9,127.4,127.2,126.8(\mathrm{q}, J=$ $276.9 \mathrm{~Hz}), 122.2,46.6,36.3,35.3(\mathrm{q}, J=29.2 \mathrm{~Hz}), 26.8 .{ }^{19} \mathrm{~F}$ NMR $\left(470 \mathrm{MHz}, \mathrm{CDCl}_{3}\right) \delta-64.98(\mathrm{t}, J=10.9 \mathrm{~Hz})$. HRMS (ESI) $\mathrm{m} / \mathrm{z}$ : $[\mathrm{M}+\mathrm{H}]^{+}$calcd for $\mathrm{C}_{20} \mathrm{H}_{19} \mathrm{~F}_{3} \mathrm{NO} 346.1413$; found: 346.1416 .

\section{Spectral data for (E)-1-(2,2-diphenylvinyl)-3-(2,2,2-trifluoroethylidene)pyrrolidin-2-one (S2)}<smiles>O=C1/C(=C/C(F)(F)F)CCN1/C=C(\c1ccccc1)C(F)(F)F</smiles>

Purified by column chromatography (silica gel, EtOAc/Petroleum ether: $1 / 20)$ to afford the title compound (11\% yield, $8 \mathrm{mg}$ ) as yellow solid, $\mathrm{mp}: 83-85^{\circ} \mathrm{C}$ (Table S7, entry 15). ${ }^{1} \mathrm{H}$ NMR (500 MHz, $\left.\mathrm{CDCl}_{3}\right) \delta 7.45-7.39(\mathrm{~m}, 4 \mathrm{H})$, $7.34-7.31(\mathrm{~m}, 2 \mathrm{H}), 7.28-7.25(\mathrm{~m}, 2 \mathrm{H}), 7.25-7.21(\mathrm{~m}, 2 \mathrm{H}), 6.59$ (qt, $J=8.3,3.0 \mathrm{~Hz}, 1 \mathrm{H}), 3.10(\mathrm{t}, J=6.5 \mathrm{~Hz}, 2 \mathrm{H})$, 2.86-2.82 (m, 2H). ${ }^{13} \mathrm{C}$ NMR (125 MHz, $\left.\mathrm{CDCl}_{3}\right) \delta 166.1,140.9,138.3,130.7,130.1,128.3,128.1,127.6,127.5$, 123.3 (q, $J=270.3 \mathrm{~Hz}), 122.2,118.6$ (q, $J=35.5 \mathrm{~Hz}), 45.4,22.9 .{ }^{19} \mathrm{~F}$ NMR $\left(376 \mathrm{MHz}, \mathrm{CDCl}_{3}\right) \delta-60.90 . \mathrm{HRMS}(\mathrm{ESI})$ $\mathrm{m} / \mathrm{z}:[\mathrm{M}+\mathrm{H}]^{+}$calcd for $\mathrm{C}_{20} \mathrm{H}_{17} \mathrm{~F}_{3} \mathrm{NO} 344.1257$; found: 344.1269 .

\section{Spectral data for 1-styryl-3-(2,2,2-trifluoroethyl)pyrrolidin-2-one (6b)}<smiles>O=C1C(CC(F)(F)F)CCN1C=Cc1ccccc1</smiles>

Purified by column chromatography (silica gel, EtOAc/Petroleum ether: 1/20) to afford the title compound (an inseparable mixture, $53 \%$ yield, $29 \mathrm{mg}, \mathrm{E} / \mathrm{Z}=1 / 4)$ as yellowish oil. (Z)-Isomer: ${ }^{1} \mathrm{H} \mathrm{NMR}\left(400 \mathrm{MHz}, \mathrm{CDCl}_{3}\right) \delta 7.42$ $-7.31(\mathrm{~m}, 3 \mathrm{H}), 7.25-7.18(\mathrm{~m}, 2 \mathrm{H}), 6.81(\mathrm{~d}, J=9.9 \mathrm{~Hz}, 1 \mathrm{H}), 6.09(\mathrm{~d}, J=9.8 \mathrm{~Hz}, 1 \mathrm{H}), 3.31-3.20(\mathrm{~m}, 1 \mathrm{H}), 3.20$ $3.11(\mathrm{~m}, 1 \mathrm{H}), 3.06-2.86(\mathrm{~m}, 1 \mathrm{H}), 2.83-2.71(\mathrm{~m}, 1 \mathrm{H}), 2.43-2.29(\mathrm{~m}, 1 \mathrm{H}), 2.18-1.91(\mathrm{~m}, 1 \mathrm{H}), 1.83-1.68(\mathrm{~m}$, 1H). (E)-Isomer: ${ }^{1} \mathrm{H}$ NMR $\left(400 \mathrm{MHz}, \mathrm{CDCl}_{3}\right) \delta 7.63(\mathrm{~d}, J=15.0 \mathrm{~Hz}, 1 \mathrm{H}), 7.42-7.31(\mathrm{~m}, 3 \mathrm{H}), 7.25-7.18(\mathrm{~m}, 2 \mathrm{H})$, $6.00(\mathrm{~d}, J=14.9 \mathrm{~Hz}, 1 \mathrm{H}), 3.84-3.72(\mathrm{~m}, 1 \mathrm{H}), 3.68-3.52(\mathrm{~m}, 1 \mathrm{H}), 3.06-2.86(\mathrm{~m}, 2 \mathrm{H}), 2.67-2.55(\mathrm{~m}, 1 \mathrm{H}), 2.18$ $-1.91(\mathrm{~m}, 2 \mathrm{H}) .{ }^{13} \mathrm{C} \mathrm{NMR}\left(126 \mathrm{MHz}, \mathrm{CDCl}_{3}\right) \delta 174.2,172.1,136.0,129.1,128.8,128.0,127.2,126.9,126.8$ (q, $J=$ $276.7 \mathrm{~Hz}$ ), 126.7 (q, $J=276.5 \mathrm{~Hz}), 125.8,123.6,123.4,114.8,112.7,46.4,43.6,37.3,36.3,35.2$ (q, $J=29.0), 35.1$ (q, $J=28.3 \mathrm{~Hz}), 26.8,25.5$. (Z)-Isomer: ${ }^{19} \mathrm{~F} \mathrm{NMR}\left(470 \mathrm{MHz}, \mathrm{CDCl}_{3}\right) \delta$-64.99 (t, $\left.J=10.9 \mathrm{~Hz}\right)$. (E)-Isomer: ${ }^{19} \mathrm{~F} \mathrm{NMR}$ $\left(470 \mathrm{MHz}, \mathrm{CDCl}_{3}\right) \delta-64.93\left(\mathrm{t}, J=10.7 \mathrm{~Hz}\right.$ ). HRMS (ESI) $\mathrm{m} / \mathrm{z}$ : $[\mathrm{M}+\mathrm{H}]^{+}$calcd for $\mathrm{C}_{14} \mathrm{H}_{15} \mathrm{~F}_{3} \mathrm{NO} 270.1100$; found: 270.1103.

\section{Spectral data for 1-(4,4,4-trifluoro-2-methylenebutyl)-3-(2,2,2-trifluoroethyl)pyrrolidin-2-one (6d)}<smiles>C=C(CN1CCC(CC(F)(F)F)C1=O)CC(F)(F)F</smiles>

Purified by column chromatography (silica gel, EtOAc/Petroleum ether: 1/20) to afford the title compound (15\% yield, $9 \mathrm{mg}$ ) as colorless oil. ${ }^{1} \mathrm{H}$ NMR $\left(400 \mathrm{MHz}, \mathrm{CDCl}_{3}\right) \delta 5.27(\mathrm{~s}, 1 \mathrm{H}), 5.19(\mathrm{~s}, 1 \mathrm{H}), 4.02(\mathrm{~d}, J=15.4 \mathrm{~Hz}, 1 \mathrm{H}), 3.85$ $(\mathrm{d}, J=15.4 \mathrm{~Hz}, 1 \mathrm{H}), 3.36-3.23(\mathrm{~m}, 2 \mathrm{H}), 2.97-2.86(\mathrm{~m}, 1 \mathrm{H}), 2.86-2.67(\mathrm{~m}, 3 \mathrm{H}), 2.50-2.37(\mathrm{~m}, 1 \mathrm{H}), 2.13-1.95$ 
$(\mathrm{m}, 1 \mathrm{H}), 1.90-1.76(\mathrm{~m}, 1 \mathrm{H}) .{ }^{13} \mathrm{C}$ NMR $\left(125 \mathrm{MHz}, \mathrm{CDCl}_{3}\right) \delta 174.1,133.1,127.0(\mathrm{q}, J=277.7 \mathrm{~Hz}), 125.7(\mathrm{q}, J=$ $277.3 \mathrm{~Hz}), 119.8,48.4,45.1,37.9(\mathrm{q}, J=29.6 \mathrm{~Hz}), 36.9,35.7$ (q, $J=28.9 \mathrm{~Hz}), 26.0 .{ }^{19} \mathrm{~F}$ NMR $\left(470 \mathrm{MHz}, \mathrm{CDCl}_{3}\right) \delta$ $-64.78(\mathrm{t}, J=10.8 \mathrm{~Hz}),-64.96(\mathrm{t}, J=11.0 \mathrm{~Hz})$. HRMS (ESI) $\mathrm{m} / \mathrm{z}$ : $[\mathrm{M}+\mathrm{H}]^{+}$calcd for $\mathrm{C}_{11} \mathrm{H}_{14} \mathrm{~F}_{6} \mathrm{NO} 290.0974$; found: 290.0978 .

\section{Spectral data for methyl (E)-2-(2-oxo-3-(2,2,2-trifluoroethyl)pyrrolidin-1-yl)-3-phenylacrylate (6e)}<smiles>COC(=O)N1CCC(CC(F)(F)F)C1=O</smiles>

Purified by column chromatography (silica gel, EtOAc/Petroleum ether: $1 / 5)$ to afford the title compound (55\% yield, $36 \mathrm{mg})$ as yellowish oil. ${ }^{1} \mathrm{H}$ NMR $\left(400 \mathrm{MHz}, \mathrm{CDCl}_{3}\right) \delta 7.71(\mathrm{~s}, 1 \mathrm{H}), 7.52-7.34(\mathrm{~m}, 5 \mathrm{H}), 3.85(\mathrm{~s}, 3 \mathrm{H}), 3.58-3.47$ $(\mathrm{m}, 1 \mathrm{H}), 3.45-3.32(\mathrm{~m}, 1 \mathrm{H}), 3.01-2.77(\mathrm{~m}, 2 \mathrm{H}), 2.59-2.40(\mathrm{~m}, 1 \mathrm{H}), 2.24-2.08(\mathrm{~m}, 1 \mathrm{H}), 2.06-1.91(\mathrm{~m}, 1 \mathrm{H})$. ${ }^{13} \mathrm{C}$ NMR $\left(125 \mathrm{MHz}, \mathrm{CDCl}_{3}\right) \delta 175.0,164.4,139.1,132.7,130.4,129.4,128.9,126.8$ (q, $\left.J=276.1 \mathrm{~Hz}\right), 126.6,52.7$, 46.4, 36.6, 35.1 (q, $J=28.8 \mathrm{~Hz}), 26.9 .{ }^{19} \mathrm{~F} \mathrm{NMR}\left(470 \mathrm{MHz}, \mathrm{CDCl}_{3}\right) \delta-64.86(\mathrm{t}, J=10.9 \mathrm{~Hz})$. HRMS (ESI) m/z: [M $+\mathrm{H}]^{+}$calcd for $\mathrm{C}_{16} \mathrm{H}_{17} \mathrm{~F}_{3} \mathrm{NO}_{3} 328.1155$; found: 328.1151 .

\section{Spectral data for methyl 3-methyl-2-(2-oxo-3-(2,2,2-trifluoroethyl)pyrrolidin-1-yl)but-2-enoate (6f)}<smiles>CC(=O)OC(C)=C(C)C</smiles>

Purified by column chromatography (silica gel, EtOAc/Petroleum ether: $1 / 8$ ) to afford the title compound (53\% yield, $30 \mathrm{mg})$ as yellowish oil. ${ }^{1} \mathrm{H}$ NMR $\left(500 \mathrm{MHz}, \mathrm{CDCl}_{3}\right) \delta 3.73(\mathrm{~s}, 3 \mathrm{H}), 3.56-3.47(\mathrm{~m}, 1 \mathrm{H}), 3.40-3.31(\mathrm{~m}, 1 \mathrm{H}), 2.96$ $-2.82(\mathrm{~m}, 1 \mathrm{H}), 2.81-2.72(\mathrm{~m}, 1 \mathrm{H}), 2.51-2.42(\mathrm{~m}, 1 \mathrm{H}), 2.26(\mathrm{~s}, 3 \mathrm{H}), 2.16-2.02(\mathrm{~m}, 1 \mathrm{H}), 2.00-1.89(\mathrm{~m}, 1 \mathrm{H})$, $1.83(\mathrm{~s}, 3 \mathrm{H}) .{ }^{13} \mathrm{C} \mathrm{NMR}\left(125 \mathrm{MHz}, \mathrm{CDCl}_{3}\right) \delta 174.1,164.3,153.3,127.0$ (q, $\left.J=276.6 \mathrm{~Hz}\right), 122.9,52.0,47.0,36.6$, 35.4 (q, $J=29.2 \mathrm{~Hz}), 26.7,22.8,21.6 .{ }^{19} \mathrm{~F} \mathrm{NMR}\left(470 \mathrm{MHz}, \mathrm{CDCl}_{3}\right) \delta-64.90$ (t, $\left.J=10.9 \mathrm{~Hz}\right) . \mathrm{HRMS}(\mathrm{ESI}) \mathrm{m} / \mathrm{z}$ : [M $+\mathrm{H}]^{+}$calcd for $\mathrm{C}_{12} \mathrm{H}_{17} \mathrm{~F}_{3} \mathrm{NO}_{3} 280.1155$; found: 280.1149 .

\section{Spectral data for 3-(2,2,2-trifluoroethyl)-1-(5-(trifluoromethyl)cyclopent-1-en-1-yl)pyrrolidin-2-one (6g)}<smiles>O=C1C(CC(F)(F)F)CCN1C1=CCCC1C(F)(F)F</smiles>

Purified by column chromatography (silica gel, EtOAc/Petroleum ether: 1/20) to afford the title compound (an inseparable mixture, $22 \%$ yield, $13 \mathrm{mg}$, $\mathrm{dr}=1.4 / 1)$ as colorless oil. The major isomer: ${ }^{1} \mathrm{H} \mathrm{NMR}\left(500 \mathrm{MHz}, \mathrm{CDCl}_{3}\right)$ $\delta 5.50(\mathrm{~s}, 1 \mathrm{H}), 4.53-4.40(\mathrm{~m}, 1 \mathrm{H}), 3.70-3.63(\mathrm{~m}, 1 \mathrm{H}), 3.53-3.43(\mathrm{~m}, 1 \mathrm{H}), 2.97-2.67(\mathrm{~m}, 2 \mathrm{H}), 2.57-2.41(\mathrm{~m}$, 2H), $2.39-2.27(\mathrm{~m}, 1 \mathrm{H}), 2.22-2.12(\mathrm{~m}, 2 \mathrm{H}), 2.11-1.99(\mathrm{~m}, 1 \mathrm{H}), 1.97-1.83(\mathrm{~m}, 1 \mathrm{H})$. The minor isomer: ${ }^{1} \mathrm{H}$ NMR $\left(500 \mathrm{MHz}, \mathrm{CDCl}_{3}\right) \delta 5.69(\mathrm{~s}, 1 \mathrm{H}), 4.39-4.24(\mathrm{~m}, 1 \mathrm{H}), 3.76-3.70(\mathrm{~m}, 1 \mathrm{H}), 3.53-3.43(\mathrm{~m}, 1 \mathrm{H}), 2.97-2.67(\mathrm{~m}$, 2H), $2.57-2.41(\mathrm{~m}, 2 \mathrm{H}), 2.39-2.27(\mathrm{~m}, 2 \mathrm{H}), 2.22-2.12(\mathrm{~m}, 2 \mathrm{H}), 2.11-1.99(\mathrm{~m}, 1 \mathrm{H}), 1.97-1.83(\mathrm{~m}, 1 \mathrm{H}) .{ }^{13} \mathrm{C}$ $\operatorname{NMR}\left(100 \mathrm{MHz}, \mathrm{CDCl}_{3}\right) \delta 172.5,172.3,135.2(\mathrm{q}, J=2.3 \mathrm{~Hz}), 134.6(\mathrm{q}, J=2.7 \mathrm{~Hz}), 127.4(\mathrm{q}, J=281.4 \mathrm{~Hz}), 126.8$ (q, $J=276.3 \mathrm{~Hz}), 126.7$ (q, $J=276.5 \mathrm{~Hz}), 122.3,119.9,47.8,47.2,46.1$ (q, $J=27.5 \mathrm{~Hz}), 45.6(\mathrm{q}, J=27.4 \mathrm{~Hz}), 37.8$ $(\mathrm{q}, J=2.7 \mathrm{~Hz}), 37.4(\mathrm{q}, J=2.7 \mathrm{~Hz}), 35.4(\mathrm{q}, J=29.1 \mathrm{~Hz}), 35.3(\mathrm{q}, J=29.1 \mathrm{~Hz}), 29.7,28.4,28.2,26.4,25.5,24.4-$ $24.0(\mathrm{~m})$. The major isomer: ${ }^{19} \mathrm{~F} \mathrm{NMR}\left(470 \mathrm{MHz}, \mathrm{CDCl}_{3}\right) \delta-65.02(\mathrm{t}, J=11.0 \mathrm{~Hz}),-71.27(\mathrm{~d}, J=9.3 \mathrm{~Hz})$. The minor isomer: ${ }^{9} \mathrm{~F}$ NMR $\left(470 \mathrm{MHz}, \mathrm{CDCl}_{3}\right) \delta-64.91(\mathrm{t}, J=10.9 \mathrm{~Hz}),-71.27(\mathrm{~d}, J=9.3 \mathrm{~Hz})$. HRMS (ESI) m/z: $[\mathrm{M}+\mathrm{H}]^{+}$ 
calcd for $\mathrm{C}_{12} \mathrm{H}_{14} \mathrm{~F}_{6} \mathrm{NO} 302.0974$; found: 302.0966 .

Spectral data for 1-(2,2-diphenylvinyl)-3-methyl-3-(2,2,2-trifluoroethyl)pyrrolidin-2-one (6h)<smiles>CC(F)(F)CC1(C)CCN(C=C(c2ccccc2)c2ccccc2)C1=O</smiles>

Purified by column chromatography (silica gel, EtOAc/Petroleum ether: 1/30) to afford the title compound (60\% yield, $43 \mathrm{mg}$ ) as white solid, mp: $107-109{ }^{\circ} \mathrm{C} .{ }^{1} \mathrm{H}$ NMR (400 MHz, $\left.\mathrm{CDCl}_{3}\right) \delta 7.40-7.35(\mathrm{~m}, 3 \mathrm{H}), 7.28-7.18(\mathrm{~m}$, $8 \mathrm{H}), 3.02-2.83(\mathrm{~m}, 2 \mathrm{H}), 2.66-2.43(\mathrm{~m}, 1 \mathrm{H}), 2.38-2.22(\mathrm{~m}, 1 \mathrm{H}), 2.07-1.95(\mathrm{~m}, 1 \mathrm{H}), 1.89-1.76(\mathrm{~m}, 1 \mathrm{H}), 1.24$ (s, 3H). ${ }^{13} \mathrm{C}$ NMR $\left(125 \mathrm{MHz}, \mathrm{CDCl}_{3}\right) \delta 177.4,141.1,138.7,130.7,128.6,128.2,127.8,127.4,127.2,126.6(\mathrm{q}, J=$ $278.1 \mathrm{~Hz}), 122.4,45.0,40.9,40.4(\mathrm{q}, J=27.7 \mathrm{~Hz}), 31.8,22.0 .{ }^{19} \mathrm{~F}$ NMR $\left(470 \mathrm{MHz}, \mathrm{CDCl}_{3}\right) \delta-60.45(\mathrm{t}, J=11.4 \mathrm{~Hz})$. HRMS (ESI) m/z: $[\mathrm{M}+\mathrm{H}]^{+}$calcd for $\mathrm{C}_{21} \mathrm{H}_{21} \mathrm{~F}_{3} \mathrm{NO} 360.1570$; found: 360.1569 .

\section{Spectral data for 1-(2,2-diphenylvinyl)-3-phenyl-3-(2,2,2-trifluoroethyl)pyrrolidin-2-one (6i)}<smiles>O=C1N(C=C(c2ccccc2)c2ccccc2)CCC1(CC(F)(F)F)c1ccccc1</smiles>

Purified by column chromatography (silica gel, EtOAc/Petroleum ether: 1/50) to afford the title compound (25\% yield, $21 \mathrm{mg}$ ) as white solid, $\mathrm{mp}: 114-116{ }^{\circ} \mathrm{C} .{ }^{1} \mathrm{H}$ NMR $\left(500 \mathrm{MHz}, \mathrm{CDCl}_{3}\right) \delta 7.47-7.41(\mathrm{~m}, 2 \mathrm{H}), 7.40-7.34(\mathrm{~m}$, 2H), $7.34-7.19(\mathrm{~m}, 8 \mathrm{H}), 7.18-7.13(\mathrm{~m}, 2 \mathrm{H}), 7.09-7.03(\mathrm{~m}, 2 \mathrm{H}), 3.04-2.91(\mathrm{~m}, 1 \mathrm{H}), 2.91-2.77(\mathrm{~m}, 2 \mathrm{H}), 2.74-$ $2.60(\mathrm{~m}, 2 \mathrm{H}), 2.28-2.15(\mathrm{~m}, 1 \mathrm{H}) .{ }^{13} \mathrm{C} \mathrm{NMR}\left(125 \mathrm{MHz}, \mathrm{CDCl}_{3}\right) \delta$ 175.0, 141.1, 138.6, 137.1, 130.7, 129.1, 129.0, 128.3, 128.2, 128.0, 127.9, 127.6, 127.4, 126.4, 126.2 (q, $J=278.2 \mathrm{~Hz}), 122.4,48.5,45.3,42.3$ (q, J=27.3 Hz), 30.6. ${ }^{19} \mathrm{~F} \mathrm{NMR}\left(470 \mathrm{MHz}, \mathrm{CDCl}_{3}\right) \delta-60.39(\mathrm{t}, J=10.9 \mathrm{~Hz})$. HRMS (ESI) $\mathrm{m} / \mathrm{z}:[\mathrm{M}+\mathrm{H}]^{+}$calcd for $\mathrm{C}_{26} \mathrm{H}_{23} \mathrm{~F}_{3} \mathrm{NO} 422.1726$; found: 422.1733 .

\section{Spectral data for 6,6-dimethyl-2,2-diphenyl-7-(2,2,2-trifluoroethyl)-2,3,6,7-tetrahydro-5H-pyrrolo[1,2-} a]imidazol-5-one (7a)<smiles>CC1(C)C(=O)N2CC(c3ccccc3)N=C2C1CC(F)(F)F</smiles>

Purified by column chromatography (silica gel, EtOAc/Petroleum ether: 1/10) to afford the title compound (67\% yield, $52 \mathrm{mg}$ ) as colorless oil. ${ }^{1} \mathrm{H}$ NMR $\left(500 \mathrm{MHz}, \mathrm{CDCl}_{3}\right) \delta 7.36-7.24(\mathrm{~m}, 10 \mathrm{H}), 4.31(\mathrm{~s}, 2 \mathrm{H}), 3.16-3.11(\mathrm{~m}, 1 \mathrm{H})$, $3.10-2.97(\mathrm{~m}, 1 \mathrm{H}), 2.54-2.42(\mathrm{~m}, 1 \mathrm{H}), 1.41(\mathrm{~s}, 3 \mathrm{H}), 1.23(\mathrm{~s}, 3 \mathrm{H}) .{ }^{13} \mathrm{C}$ NMR $\left(125 \mathrm{MHz}, \mathrm{CDCl}_{3}\right) \delta 175.5,164.0$, 146.2, 145.9, 128.8, 127.6, 127.5, 126.5, 126.3 (q, $J=276.9 \mathrm{~Hz}), 126.2,86.0,55.1,49.9,39.7,31.0$ (q, $J=30.2 \mathrm{~Hz})$, 24.3, 21.9. ${ }^{19} \mathrm{~F} \mathrm{NMR}\left(470 \mathrm{MHz}, \mathrm{CDCl}_{3}\right) \delta-64.32$ (t, $J=10.9 \mathrm{~Hz}$ ). HRMS (ESI) $\mathrm{m} / \mathrm{z}:[\mathrm{M}+\mathrm{H}]^{+}$calcd for $\mathrm{C}_{22} \mathrm{H}_{22} \mathrm{~F}_{3} \mathrm{~N}_{2} \mathrm{O}$ 387.1679; found: 387.1697 .

Spectral data for 1-(2,2-diphenylvinyl)-5-imino-3,3-dimethyl-4-(2,2,2-trifluoroethyl)pyrrolidin-2-one (6j)<smiles>CC1(C)C(=O)N(/C=C/c2ccccc2)C(=N)C1CC(F)(F)F</smiles>

Purified by column chromatography (silica gel, EtOAc/Petroleum ether: $1 / 8)$ to afford the title compound (12\% yield, $9 \mathrm{mg}$ ) as unstable colorless oil. ${ }^{1} \mathrm{H}$ NMR $\left(400 \mathrm{MHz}, \mathrm{CDCl}_{3}\right) \delta 7.39-7.28(\mathrm{~m}, 8 \mathrm{H}), 7.18-7.05(\mathrm{~m}, 2 \mathrm{H}), 6.40(\mathrm{~s}, 1 \mathrm{H})$, 
2.90 (brs, 1H), $2.75(\mathrm{dd}, J=9.3,3.2 \mathrm{~Hz}, 1 \mathrm{H}), 2.27-2.11(\mathrm{~m}, 1 \mathrm{H}), 1.25(\mathrm{~s}, 3 \mathrm{H}), 0.89$ (s, 3H). ${ }^{13} \mathrm{C}$ NMR $(125 \mathrm{MHz}$, $\left.\mathrm{CDCl}_{3}\right) \delta 179.2,147.8,138.6,137.3,129.2,128.8,128.7,128.6,128.2,126.6$ (q, $\left.J=275.3,274.6 \mathrm{~Hz}\right), 115.1,44.5$, 43.4, 30.7 (q, $J=30.1 \mathrm{~Hz}), 23.4,21.0 .{ }^{19} \mathrm{~F}$ NMR $\left(376 \mathrm{MHz}, \mathrm{CDCl}_{3}\right) \delta-64.04(\mathrm{t}, J=11.1 \mathrm{~Hz}) . \mathrm{HRMS}(\mathrm{ESI}) \mathrm{m} / \mathrm{z}:[\mathrm{M}$ $+\mathrm{H}]^{+}$calcd for $\mathrm{C}_{22} \mathrm{H}_{22} \mathrm{~F}_{3} \mathrm{~N}_{2} \mathrm{O} 387.1679$; found: 387.1689 .

Spectral data for 6,6-dimethyl-2-phenyl-7-(2,2,2-trifluoroethyl)-2,3,6,7-tetrahydro-5H-pyrrolo[1,2a]imidazol-5-one (7b)<smiles>CC1(C)C(=O)N2CC(c3ccccc3)N=C2C1CC(F)(F)F</smiles>

Purified by column chromatography (silica gel, EtOAc/Petroleum ether: 1/5) to afford the title compound (an inseparable mixture, $69 \%$ yield, $43 \mathrm{mg}$, $\mathrm{dr}=1.3: 1)$ as colorless oil. The major isomer: ${ }^{1} \mathrm{H} \mathrm{NMR}\left(400 \mathrm{MHz}, \mathrm{CDCl}_{3}\right)$ $\delta 7.41-7.34(\mathrm{~m}, 2 \mathrm{H}), 7.33-7.28(\mathrm{~m}, 1 \mathrm{H}), 7.27-7.23(\mathrm{~m}, 1 \mathrm{H}), 7.21-7.16(\mathrm{~m}, 1 \mathrm{H}), 5.66-5.52(\mathrm{~m}, 1 \mathrm{H}), 4.16-$ $3.99(\mathrm{~m}, 1 \mathrm{H}), 3.67-3.49(\mathrm{~m}, 1 \mathrm{H}), 3.17-3.03(\mathrm{~m}, 1 \mathrm{H}), 3.02-2.88(\mathrm{~m}, 1 \mathrm{H}), 2.57-2.41(\mathrm{~m}, 1 \mathrm{H}), 1.42(\mathrm{~s}, 3 \mathrm{H}), 1.26$ (s, 3H). The minor isomer: ${ }^{1} \mathrm{H}$ NMR $\left(400 \mathrm{MHz}, \mathrm{CDCl}_{3}\right) \delta 7.41-7.34(\mathrm{~m}, 2 \mathrm{H}), 7.33-7.28(\mathrm{~m}, 1 \mathrm{H}), 7.27-7.23(\mathrm{~m}$, 1H), $7.21-7.16(\mathrm{~m}, 1 \mathrm{H}), 5.66-5.52(\mathrm{~m}, 1 \mathrm{H}), 4.16-3.99(\mathrm{~m}, 1 \mathrm{H}), 3.67-3.49(\mathrm{~m}, 1 \mathrm{H}), 3.17-3.03(\mathrm{~m}, 1 \mathrm{H}), 3.02-$ $2.88(\mathrm{~m}, 1 \mathrm{H}), 2.57-2.41(\mathrm{~m}, 1 \mathrm{H}), 1.42(\mathrm{~s}, 3 \mathrm{H}), 1.27(\mathrm{~s}, 3 \mathrm{H}) .{ }^{13} \mathrm{C} \mathrm{NMR}\left(125 \mathrm{MHz}, \mathrm{CDCl}_{3}\right) \delta 175.5,175.3,166.3$, 166.2, 141.8, 141.7, 129.1, 128.1, 128.1, 126.6, 126.3, 126.2 (q, $J=276.8 \mathrm{~Hz}), 75.9,75.5,50.2,49.8,49.0,48.9$, 39.5, $30.9(\mathrm{q}, J=30.5 \mathrm{~Hz}), 24.2,24.1,21.8,21.7$. The major isomer: ${ }^{19} \mathrm{~F}$ NMR $\left(470 \mathrm{MHz}, \mathrm{CDCl}_{3}\right) \delta-64.50(\mathrm{t}, J=$ $10.9 \mathrm{~Hz})$. The minor isomer: ${ }^{19} \mathrm{~F} \mathrm{NMR}\left(470 \mathrm{MHz}, \mathrm{CDCl}_{3}\right) \delta-64.43(\mathrm{t}, J=10.9 \mathrm{~Hz})$. HRMS (ESI) $\mathrm{m} / \mathrm{z}:[\mathrm{M}+\mathrm{H}]^{+}$ calcd for $\mathrm{C}_{16} \mathrm{H}_{18} \mathrm{~F}_{3} \mathrm{~N}_{2} \mathrm{O} 311.1366$; found: 311.1367 .

Spectral data for 6,6-dimethyl-2,2-diphenyl-8-(2,2,2-trifluoroethyl)-2,6,7,8-tetrahydroimidazo[1,2-a]pyridin5(3H)-one (7c)<smiles>CC1(C)CC(CC(F)(F)F)C2=NC(c3ccccc3)(c3ccccc3)CN2C1=O</smiles>

Purified by column chromatography (silica gel, EtOAc/Petroleum ether: 1/20) to afford the title compound (89\% yield, $71 \mathrm{mg}$ ) as yellow solid, $\mathrm{mp}: 44-46{ }^{\circ} \mathrm{C} .{ }^{1} \mathrm{H}$ NMR $\left(400 \mathrm{MHz}, \mathrm{CDCl}_{3}\right) \delta 7.38-7.28(\mathrm{~m}, 8 \mathrm{H}), 7.25-7.20(\mathrm{~m}$, 2H), $4.47(\mathrm{~d}, J=11.8 \mathrm{~Hz}, 1 \mathrm{H}), 4.44(\mathrm{~d}, J=11.8 \mathrm{~Hz}, 1 \mathrm{H}), 3.57-3.36(\mathrm{~m}, 1 \mathrm{H}), 3.20-3.07(\mathrm{~m}, 1 \mathrm{H}), 2.40-2.22(\mathrm{~m}$, 1H), $2.12-2.00(\mathrm{~m}, 1 \mathrm{H}), 1.80-1.66(\mathrm{~m}, 1 \mathrm{H}), 1.30(\mathrm{~s}, 3 \mathrm{H}), 1.29(\mathrm{~s}, 3 \mathrm{H}) .{ }^{13} \mathrm{C}$ NMR $\left(125 \mathrm{MHz}, \mathrm{CDCl}_{3}\right) \delta 173.4,157.1$, 146.6, 146.5, 128.7, 127.3, 126.8 (q, $J=276.7 \mathrm{~Hz}), 126.4,57.5,39.5,39.0,35.6$ (q, $J=28.9,28.4 \mathrm{~Hz}), 28.3,26.1$, 25.3. ${ }^{19} \mathrm{~F}$ NMR (470 MHz, $\left.\mathrm{CDCl}_{3}\right) \delta-63.37$ (t, $J=11.3 \mathrm{~Hz}$ ). HRMS (ESI) $\mathrm{m} / \mathrm{z}$ : $[\mathrm{M}+\mathrm{H}]^{+}$calcd for $\mathrm{C}_{23} \mathrm{H}_{24} \mathrm{~F}_{3} \mathrm{~N}_{2} \mathrm{O}$ 401.1835; found: 401.1834 .

Spectral data for 2,2,6,6-tetramethyl-8-(2,2,2-trifluoroethyl)-2,6,7,8-tetrahydroimidazo[1,2-a]pyridin-5(3H)one (7d)<smiles>CC(F)(F)CC1CC(C)(C)C(=O)N2CC(C)(C)N=C12</smiles>

Purified by column chromatography (silica gel, EtOAc/Petroleum ether: $1 / 10)$ to afford the title compound (61\% 
yield, $34 \mathrm{mg})$ as white solid, mp: $72-74{ }^{\circ} \mathrm{C} .{ }^{1} \mathrm{H}$ NMR $\left(500 \mathrm{MHz}, \mathrm{CDCl}_{3}\right) \delta 3.55(\mathrm{~d}, J=11.4 \mathrm{~Hz}, 1 \mathrm{H}), 3.49(\mathrm{~d}, J=$ $11.4 \mathrm{~Hz}, 1 \mathrm{H}), 3.27-3.13(\mathrm{~m}, 1 \mathrm{H}), 3.03-2.94(\mathrm{~m}, 1 \mathrm{H}), 2.26-2.14(\mathrm{~m}, 1 \mathrm{H}), 2.06-1.98(\mathrm{~m}, 1 \mathrm{H}), 1.72-1.62(\mathrm{~m}$, $1 \mathrm{H}), 1.30(\mathrm{~s}, 3 \mathrm{H}), 1.29(\mathrm{~s}, 3 \mathrm{H}), 1.28(\mathrm{~s}, 3 \mathrm{H}), 1.27(\mathrm{~s}, 3 \mathrm{H}) \cdot{ }^{13} \mathrm{C} \mathrm{NMR}\left(125 \mathrm{MHz}, \mathrm{CDCl}_{3}\right) \delta 173.7,156.2,126.8(\mathrm{q}, J=$ $277.0 \mathrm{~Hz}), 66.0,56.4,39.5,38.9,35.6(\mathrm{q}, J=28.6 \mathrm{~Hz}), 29.3,29.2,28.1,26.3,25.3 .{ }^{19} \mathrm{~F} \mathrm{NMR}\left(470 \mathrm{MHz}, \mathrm{CDCl}_{3}\right) \delta$ $63.42\left(\mathrm{t}, J=11.2 \mathrm{~Hz}\right.$ ). HRMS (ESI) $\mathrm{m} / \mathrm{z}$ : $[\mathrm{M}+\mathrm{H}]^{+}$calcd for $\mathrm{C}_{13} \mathrm{H}_{20} \mathrm{~F}_{3} \mathrm{~N}_{2} \mathrm{O} 277.1522$; found: 277.1524.

Spectral data for 6,6-dimethyl-2-phenyl-8-(2,2,2-trifluoroethyl)-2,6,7,8-tetrahydroimidazo[1,2-a]pyridin5(3H)-one (7e)<smiles>CC(F)(F)CC1CC(C)(C)C(=O)N2CC(c3ccccc3)N=C12</smiles>

Purified by column chromatography (silica gel, EtOAc/Petroleum ether: 1/10) to afford the title compound (an inseparable mixture, $77 \%$ yield, $50 \mathrm{mg}$, $\mathrm{dr}=1: 1)$ as colorless oil. The mixture: ${ }^{1} \mathrm{H}$ NMR $\left(400 \mathrm{MHz}, \mathrm{CDCl}_{3}\right) \delta 7.41-$ $7.33(\mathrm{~m}, 4 \mathrm{H}), 7.32-7.28(\mathrm{~m}, 2 \mathrm{H}), 7.25-7.19(\mathrm{~m}, 4 \mathrm{H}), 5.25-5.13(\mathrm{~m}, 2 \mathrm{H}), 4.32-4.14(\mathrm{~m}, 2 \mathrm{H}), 3.78-3.63(\mathrm{~m}$, 2H), $3.40-3.23(\mathrm{~m}, 2 \mathrm{H}), 3.17-3.04(\mathrm{~m}, 2 \mathrm{H}), 2.38-2.18(\mathrm{~m}, 2 \mathrm{H}), 2.13-2.04(\mathrm{~m}, 2 \mathrm{H}), 1.81-1.69(\mathrm{~m}, 2 \mathrm{H}), 1.32$ (s, 12H). ${ }^{13} \mathrm{C} \mathrm{NMR}\left(125 \mathrm{MHz}, \mathrm{CDCl}_{3}\right) \delta 173.5,173.4,159.3,159.0,142.2,142.1,129.0,128.9,127.9,127.8,126.7$ (q, $J=277.1 \mathrm{~Hz}), 126.6,126.4,67.8,67.6,51.9,51.8,39.6,39.5,39.1,39.0,35.6$ (q, $J=28.8 \mathrm{~Hz}), 35.5$ (q, $J=28.9$ $\mathrm{Hz}), 28.3,28.2,26.2,26.1,25.3,25.2 .{ }^{19} \mathrm{~F} \mathrm{NMR}\left(470 \mathrm{MHz}, \mathrm{CDCl}_{3}\right) \delta-63.49(\mathrm{t}, J=11.1 \mathrm{~Hz}),-63.49(\mathrm{t}, \mathrm{J}=11.2 \mathrm{~Hz})$. HRMS (ESI) m/z: $[\mathrm{M}+\mathrm{H}]^{+}$calcd for $\mathrm{C}_{17} \mathrm{H}_{20} \mathrm{~F}_{3} \mathrm{~N}_{2} \mathrm{O} 325.1522$; found: 325.1536 .

Spectral data for 2-ethyl-6,6-dimethyl-8-(2,2,2-trifluoroethyl)-2,6,7,8-tetrahydroimidazo[1,2-a]pyridin-5(3H)one (7f)

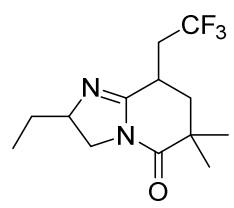

Purified by column chromatography (silica gel, EtOAc/Petroleum ether: 1/8) to afford the title compound (an inseparable mixture, $42 \%$ yield, $23 \mathrm{mg}, \mathrm{dr}=1: 1)$ as yellowish solid. The mixture: ${ }^{1} \mathrm{H}$ NMR $\left(500 \mathrm{MHz}, \mathrm{CDCl}_{3}\right) \delta 4.05$ $-3.96(\mathrm{~m}, 2 \mathrm{H}), 3.91-3.78(\mathrm{~m}, 2 \mathrm{H}), 3.48-3.42(\mathrm{~m}, 1 \mathrm{H}), 3.41-3.35(\mathrm{~m}, 1 \mathrm{H}), 3.28-3.16(\mathrm{~m}, 2 \mathrm{H}), 3.03-2.95(\mathrm{~m}$, $2 \mathrm{H}), 2.25-2.14(\mathrm{~m}, 2 \mathrm{H}), 2.06-1.99(\mathrm{~m}, 2 \mathrm{H}), 1.73-1.62(\mathrm{~m}, 4 \mathrm{H}), 1.57-1.47(\mathrm{~m}, 2 \mathrm{H}), 1.29(\mathrm{~s}, 6 \mathrm{H}), 1.28(\mathrm{~s}, 3 \mathrm{H})$, $1.27(\mathrm{~s}, 3 \mathrm{H}), 0.96(\mathrm{t}, J=7.4 \mathrm{~Hz}, 3 \mathrm{H}), 0.96(\mathrm{t}, J=7.4 \mathrm{~Hz}, 3 \mathrm{H}) .{ }^{13} \mathrm{C} \mathrm{NMR}\left(125 \mathrm{MHz}, \mathrm{CDCl}_{3}\right) \delta 173.5,158.0,157.8$, $126.8(\mathrm{q}, J=276.8 \mathrm{~Hz}), 66.3,66.2,49.0,48.9,35.6(\mathrm{q}, J=28.6 \mathrm{~Hz}), 35.5(\mathrm{q}, J=28.6 \mathrm{~Hz}), 29.2,29.1,28.1,26.3$, 26.2, 25.3, 25.2, 10.0, 9.9. ${ }^{19} \mathrm{~F}$ NMR (470 MHz, $\left.\mathrm{CDCl}_{3}\right) \delta-63.52(\mathrm{t}, J=11.3 \mathrm{~Hz}),-63.58(\mathrm{~d}, J=11.2 \mathrm{~Hz})$. HRMS (ESI) m/z: $[\mathrm{M}+\mathrm{H}]^{+}$calcd for $\mathrm{C}_{13} \mathrm{H}_{20} \mathrm{~F}_{3} \mathrm{~N}_{2} \mathrm{O} 277.1522$; found: 277.1528 .

\section{Spectral data for (E)-N-(2,2-diphenylethyl)-N-(6,6,6-trifluorohex-3-en-1-yl)cyanamide (8)}<smiles>N#CCN(CC/C=C/CC(F)(F)F)CC(c1ccccc1)c1ccccc1</smiles>

Purified by column chromatography (silica gel, EtOAc/Petroleum ether: 1/12) to afford the title compound (an inseparable mixture, $44 \%$ yield, $32 \mathrm{mg}, E / Z=4 / 1)$ as yellow oil. (E)-Isomer: ${ }^{1} \mathrm{H} \mathrm{NMR}\left(500 \mathrm{MHz}, \mathrm{CDCl}_{3}\right) \delta 7.33(\mathrm{t}$, $J=7.5 \mathrm{~Hz}, 4 \mathrm{H}), 7.28-7.25(\mathrm{~m}, 6 \mathrm{H}), 5.52-5.35(\mathrm{~m}, 2 \mathrm{H}), 4.32(\mathrm{t}, J=8.1 \mathrm{~Hz}, 1 \mathrm{H}), 3.62(\mathrm{~d}, J=8.2 \mathrm{~Hz}, 2 \mathrm{H}), 2.89(\mathrm{t}$, $J=7.0 \mathrm{~Hz}, 2 \mathrm{H}), 2.76-2.66(\mathrm{~m}, 2 \mathrm{H}), 2.28-2.21(\mathrm{~m}, 2 \mathrm{H})$. (Z)-Isomer: ${ }^{1} \mathrm{H}$ NMR $\left(500 \mathrm{MHz}, \mathrm{CDCl}_{3}\right) \delta 7.33(\mathrm{t}, J=7.5$ $\mathrm{Hz}, 4 \mathrm{H}), 7.28-7.25(\mathrm{~m}, 6 \mathrm{H}), 5.52-5.35(\mathrm{~m}, 2 \mathrm{H}), 4.32(\mathrm{t}, J=8.1 \mathrm{~Hz}, 1 \mathrm{H}), 3.64(\mathrm{~d}, J=8.2 \mathrm{~Hz}, 2 \mathrm{H}), 2.86(\mathrm{t}, J=7.0$ 
$\mathrm{Hz}, 2 \mathrm{H}), 2.76-2.66(\mathrm{~m}, 2 \mathrm{H}), 2.28-2.21(\mathrm{~m}, 2 \mathrm{H}) .{ }^{13} \mathrm{C} \mathrm{NMR}\left(125 \mathrm{MHz}, \mathrm{CDCl}_{3}\right) \delta 141.0,133.1,131.2,129.0,128.1$ (q, $J=277.7 \mathrm{~Hz}), 128.0,127.3,121.4,120.4,117.1,56.9,56.7,51.7,51.6,49.9,37.4$ (q, $J=29.8 \mathrm{~Hz}), 31.0,26.2$. (E)-Isomer: ${ }^{19} \mathrm{~F}$ NMR $\left(470 \mathrm{MHz}, \mathrm{CDCl}_{3}\right) \delta-66.47(\mathrm{t}, J=10.6 \mathrm{~Hz})$. (Z)-Isomer: ${ }^{19} \mathrm{~F}$ NMR $\left(470 \mathrm{MHz}, \mathrm{CDCl}_{3}\right) \delta-66.05$ (t, $J=10.8 \mathrm{~Hz}$ ). HRMS (ESI) m/z: [M+ H] $]^{+}$calcd for $\mathrm{C}_{21} \mathrm{H}_{22} \mathrm{~F}_{3} \mathrm{~N}_{2} 359.1730$; found: 359.1747 .

\section{Spectral data for 3-(2,2,2-trifluoroethyl)-1,2,3,5-tetrahydropyrrolo[1,2-a]quinazoline (10a)}<smiles>FC(F)(F)CC1CCN2C1=NCc1ccccc12</smiles>

Purified by column chromatography (silica gel, EtOAc/Petroleum ether: $1 / 5)$ to afford the title compound (47\% yield, $24 \mathrm{mg}$ ) as yellowsih oil. ${ }^{1} \mathrm{H}$ NMR $\left(500 \mathrm{MHz}, \mathrm{CDCl}_{3}\right) \delta 7.22-7.13(\mathrm{~m}, 1 \mathrm{H}), 7.08-6.93(\mathrm{~m}, 2 \mathrm{H}), 6.61(\mathrm{~d}, J=7.9 \mathrm{~Hz}$, 1H), $4.72(\mathrm{~d}, J=17.5 \mathrm{~Hz}, 1 \mathrm{H}), 4.59(\mathrm{~d}, J=16.9 \mathrm{~Hz}, 1 \mathrm{H}), 3.80-3.63(\mathrm{~m}, 1 \mathrm{H}), 3.48-3.31(\mathrm{~m}, 1 \mathrm{H}), 3.02-2.77(\mathrm{~m}$, 2H), $2.58-2.42(\mathrm{~m}, 1 \mathrm{H}), 2.24-2.04(\mathrm{~m}, 1 \mathrm{H}), 2.04-1.84(\mathrm{~m}, 1 \mathrm{H}) .{ }^{13} \mathrm{C} \mathrm{NMR}\left(125 \mathrm{MHz}, \mathrm{CDCl}_{3}\right) \delta 159.5,138.1$, 127.6, 126.9 (q, $J=275.3 \mathrm{~Hz}), 125.9,123.5,119.8,110.7,49.4,44.9,36.5,36.4(\mathrm{q}, J=28.5 \mathrm{~Hz}), 27.7 .{ }^{19} \mathrm{~F}$ NMR $\left(470 \mathrm{MHz}, \mathrm{CDCl}_{3}\right) \delta$-64.74. HRMS (ESI) m/z: $[\mathrm{M}+\mathrm{H}]^{+}$calcd for $\mathrm{C}_{13} \mathrm{H}_{14} \mathrm{~F}_{3} \mathrm{~N}_{2} 255.1104$; found: 255.1112 .

\section{Spectral data for 5-methyl-3-(2,2,2-trifluoroethyl)-1,2,3,5-tetrahydropyrrolo[1,2-a]quinazoline (10b)}

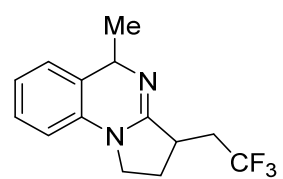

Purified by column chromatography (silica gel, EtOAc/Petroleum ether: 1/2) to afford the title compound (an inseparable mixture, $47 \%$ yield, $25 \mathrm{mg}, \mathrm{dr}=2.2: 1)$ as white solid. The major isomer: ${ }^{1} \mathrm{H} \mathrm{NMR}\left(400 \mathrm{MHz}, \mathrm{CDCl}_{3}\right) \delta$ $7.23-7.15(\mathrm{~m}, 1 \mathrm{H}), 7.12-6.98(\mathrm{~m}, 2 \mathrm{H}), 6.70-6.56(\mathrm{~m}, 1 \mathrm{H}), 4.74(\mathrm{q}, J=6.8 \mathrm{~Hz}, 1 \mathrm{H}), 3.80-3.73(\mathrm{~m}, 1 \mathrm{H}), 3.44-$ $3.31(\mathrm{~m}, 1 \mathrm{H}), 3.09-2.83(\mathrm{~m}, 3 \mathrm{H}), 2.24-2.03(\mathrm{~m}, 1 \mathrm{H}), 2.02-1.82(\mathrm{~m}, 1 \mathrm{H}), 1.30(\mathrm{~d}, J=6.8 \mathrm{~Hz}, 3 \mathrm{H})$. The minor isomer: ${ }^{1} \mathrm{H}$ NMR $\left(400 \mathrm{MHz}, \mathrm{CDCl}_{3}\right) \delta 7.23-7.15(\mathrm{~m}, 1 \mathrm{H}), 7.12-6.98(\mathrm{~m}, 2 \mathrm{H}), 6.70-6.56(\mathrm{~m}, 1 \mathrm{H}), 4.60(\mathrm{q}, J=$ $6.8 \mathrm{~Hz}, 1 \mathrm{H}), 3.73-3.67(\mathrm{~m}, 1 \mathrm{H}), 3.56-3.45(\mathrm{~m}, 1 \mathrm{H}), 2.65-2.39(\mathrm{~m}, 3 \mathrm{H}), 2.24-2.03(\mathrm{~m}, 1 \mathrm{H}), 2.02-1.82(\mathrm{~m}, 1 \mathrm{H})$, $1.55(\mathrm{~d}, J=6.8 \mathrm{~Hz}, 3 \mathrm{H}) .{ }^{13} \mathrm{C} \mathrm{NMR}\left(125 \mathrm{MHz}, \mathrm{CDCl}_{3}\right) \delta 158.0,157.6,137.4,136.8,127.4,127.3,127.0$ (q, $J=276.3$ $\mathrm{Hz}), 126.4(\mathrm{q}, J=276.6 \mathrm{~Hz}), 126.1,125.6,125.2,124.5,123.4,110.8,110.7,54.1,52.7,45.0(\mathrm{q}, J=6.1 \mathrm{~Hz}), 36.8$ $(\mathrm{q}, J=28.5 \mathrm{~Hz}), 36.5(\mathrm{q}, J=28.9 \mathrm{~Hz}), 27.7,27.2,25.2,23.8$. The major isomer: ${ }^{19} \mathrm{~F}$ NMR $\left(470 \mathrm{MHz}, \mathrm{CDCl}_{3}\right) \delta-$ $64.68(\mathrm{t}, J=11.0 \mathrm{~Hz})$. The minor isomer: ${ }^{19} \mathrm{~F} \mathrm{NMR}\left(470 \mathrm{MHz}, \mathrm{CDCl}_{3}\right) \delta-64.55(\mathrm{t}, J=11.0 \mathrm{~Hz})$. HRMS (ESI) m/z: $[\mathrm{M}+\mathrm{H}]^{+}$calcd for $\mathrm{C}_{14} \mathrm{H}_{16} \mathrm{~F}_{3} \mathrm{~N}_{2} 269.1260$; found: 269.1272 .

Spectral data for 3-phenyl-3-(2,2,2-trifluoroethyl)-1,2,3,5-tetrahydropyrrolo[1,2-a]quinazoline (10c)<smiles>FC(F)(F)CC1CCN2C1=NC(c1ccccc1)c1ccccc12</smiles>

Purified by column chromatography (silica gel, EtOAc/Petroleum ether: 1/2) to afford the title compound (an inseparable mixture, $62 \%$ yield, $41 \mathrm{mg}, \mathrm{dr}=1.4: 1)$ as colorless oil. The major isomer: ${ }^{1} \mathrm{H} \mathrm{NMR}\left(500 \mathrm{MHz}, \mathrm{CDCl}_{3}\right)$ $\delta 7.40-7.13(\mathrm{~m}, 7 \mathrm{H}), 7.09-6.99(\mathrm{~m}, 2 \mathrm{H}), 5.82(\mathrm{~s}, 1 \mathrm{H}), 3.85-3.75(\mathrm{~m}, 1 \mathrm{H}), 3.51-3.40(\mathrm{~m}, 1 \mathrm{H}), 3.14-2.89(\mathrm{~m}$, 2H), $2.65-2.47(\mathrm{~m}, 1 \mathrm{H}), 2.22-2.08(\mathrm{~m}, 1 \mathrm{H}), 2.05-1.89(\mathrm{~m}, 1 \mathrm{H})$. The minor isomer: ${ }^{1} \mathrm{H}$ NMR $\left(500 \mathrm{MHz}, \mathrm{CDCl}_{3}\right)$ $\delta 7.40-7.13(\mathrm{~m}, 5 \mathrm{H}), 6.93(\mathrm{t}, J=7.5 \mathrm{~Hz}, 1 \mathrm{H}), 6.70(\mathrm{t}, J=8.7 \mathrm{~Hz}, 3 \mathrm{H}), 5.62(\mathrm{~s}, 1 \mathrm{H}), 3.85-3.75(\mathrm{~m}, 1 \mathrm{H}), 3.60-3.52$ $(\mathrm{m}, 1 \mathrm{H}), 3.14-2.89(\mathrm{~m}, 2 \mathrm{H}), 2.65-2.47(\mathrm{~m}, 1 \mathrm{H}), 2.22-2.08(\mathrm{~m}, 1 \mathrm{H}), 2.05-1.89(\mathrm{~m}, 1 \mathrm{H}) .{ }^{13} \mathrm{C}$ NMR $(125 \mathrm{MHz}$, $\left.\mathrm{CDCl}_{3}\right) \delta 158.2,158.0,145.5,144.9,137.0,136.7,128.6,128.5,128.2,127.8,127.7,127.6,127.5,127.2,127.0$, 
$126.9(\mathrm{q}, J=276.3 \mathrm{~Hz}), 126.8(\mathrm{q}, J=276.7 \mathrm{~Hz}), 126.7,123.7,123.4,123.0,122.6,111.3,110.8,62.2,62.1,45.2$, $36.7(\mathrm{q}, J=28.9 \mathrm{~Hz}), 36.6,36.5(\mathrm{q}, J=28.3 \mathrm{~Hz}), 27.6,27.3$. The major isomer: ${ }^{19} \mathrm{~F} \mathrm{NMR}\left(470 \mathrm{MHz}, \mathrm{CDCl}_{3}\right) \delta-64.68$ $(\mathrm{t}, J=11.1 \mathrm{~Hz})$. The minor isomer: ${ }^{19} \mathrm{~F}$ NMR $\left(470 \mathrm{MHz}, \mathrm{CDCl}_{3}\right) \delta \delta-64.51(\mathrm{t}, J=11.0 \mathrm{~Hz})$. HRMS (ESI) m/z: [M + $\mathrm{H}]^{+}$calcd for $\mathrm{C}_{19} \mathrm{H}_{18} \mathrm{~F}_{3} \mathrm{~N}_{2} 331.1417$; found: 331.1429 .

Spectral data for 1-(2-(4,4,4-trifluorobut-1-en-2-yl)phenyl)-3-(2,2,2-trifluoroethyl)pyrrolidin-2-imine (10d) and 1-(2-(prop-1-en-2-yl)phenyl)-3-(2,2,2-trifluoroethyl)pyrrolidin-2-imine (10d')<smiles>C=C(CC(F)(F)F)c1ccccc1N1CCC(CC(F)(F)F)C1=N</smiles>

10d<smiles>C=C(C)c1ccccc1N1CCC(CC(F)(F)F)C1=N</smiles>

10d'

Purified by column chromatography (silica gel, EtOAc/Petroleum ether: 1/10) to afford the title compound (an inseparable mixture, $37 \%$ yield, $\left.24 \mathrm{mg}, \mathbf{1 0 d} / \mathbf{1 0 d}{ }^{\prime}=1.2 / 1\right)$ as colorless oil. 10d: ${ }^{1} \mathrm{H}$ NMR $\left(500 \mathrm{MHz}, \mathrm{CDCl}_{3}\right) \delta 7.48$ - $7.29(\mathrm{~m}, 3 \mathrm{H}), 7.17$ (t, $J=7.6 \mathrm{~Hz}, 1 \mathrm{H}), 5.47$ (s, 1H), $5.36(\mathrm{~s}, 1 \mathrm{H}), 3.69-3.59$ (m, 2H), 3.09 (q, $J=10.7 \mathrm{~Hz}, 2 \mathrm{H})$, $3.05-2.94(\mathrm{~m}, 2 \mathrm{H}), 2.53-2.36(\mathrm{~m}, 1 \mathrm{H}), 2.24-2.10(\mathrm{~m}, 1 \mathrm{H}), 1.94-1.81(\mathrm{~m}, 1 \mathrm{H}) .{ }^{19} \mathrm{~F}$ NMR $\left(470 \mathrm{MHz}, \mathrm{CDCl}_{3}\right) \delta-$ $64.30(\mathrm{t}, J=10.6 \mathrm{~Hz}),-64.63(\mathrm{t}, J=11.0 \mathrm{~Hz})$. HRMS (ESI) $\mathrm{m} / \mathrm{z}:[\mathrm{M}+\mathrm{H}]^{+}$calcd for $\mathrm{C}_{16} \mathrm{H}_{17} \mathrm{~F}_{6} \mathrm{~N}_{2} 351.1290$; found: 351.1301. 10d': ${ }^{1} \mathrm{H}$ NMR (500 MHz, $\left.\mathrm{CDCl}_{3}\right) \delta 7.45-7.29$ (m, 3H), 7.17 (t, J = 7.6 Hz, 1H), 5.15 (s, 1H), 4.96 (s, $1 \mathrm{H}), 3.59-3.50(\mathrm{~m}, 2 \mathrm{H}), 3.15-3.05(\mathrm{~m}, 2 \mathrm{H}), 3.04-2.92(\mathrm{~m}, 1 \mathrm{H}), 2.49-2.37(\mathrm{~m}, 1 \mathrm{H}), 2.24-2.09(\mathrm{~m}, 1 \mathrm{H}), 2.00$ (s, 3H), $1.95-1.79(\mathrm{~m}, 1 \mathrm{H}) .{ }^{19} \mathrm{~F}$ NMR $\left(470 \mathrm{MHz}, \mathrm{CDCl}_{3}\right) \delta-64.63(\mathrm{t}, J=11.0 \mathrm{~Hz}) . \mathrm{HRMS}(\mathrm{ESI}) \mathrm{m} / \mathrm{z}:[\mathrm{M}+\mathrm{H}]^{+}$ calcd for $\mathrm{C}_{15} \mathrm{H}_{18} \mathrm{~F}_{3} \mathrm{~N}_{2} 283.1417$; found: 283.1413. The mixture of $\mathbf{1 0 d}$ and $10 d$ ': ${ }^{13} \mathrm{C}$ NMR (125 MHz, $\left.\mathrm{CDCl}_{3}\right) \delta 167.4$, $167.3,143.4,142.7,140.3,137.4,136.9,136.2,130.9,130.2,129.7,128.8,128.4,128.3,128.2,128.1,127.1$ (q, $J=$ $267.1 \mathrm{~Hz}), 125.6(\mathrm{q}, J=277.5 \mathrm{~Hz}), 122.1,115.8,50.8,50.7,40.3(\mathrm{q}, J=29.2 \mathrm{~Hz}), 37.4,36.7(\mathrm{q}, J=28.1 \mathrm{~Hz}), 29.8$, $28.3,28.2,23.2$.

\section{Spectral data for 3-methyl-3-(2,2,2-trifluoroethyl)-1,2,3,5-tetrahydropyrrolo[1,2-a]quinazoline (10e)}

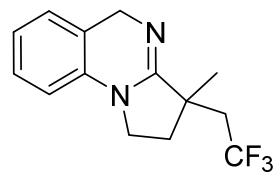

Purified by column chromatography (silica gel, EtOAc/Petroleum ether: $1 / 5$ ) to afford the title compound (70\% yield, $36 \mathrm{mg}$ ) as yellow solid, mp: $126-128{ }^{\circ} \mathrm{C} .{ }^{1} \mathrm{H}$ NMR $\left(400 \mathrm{MHz}, \mathrm{CDCl}_{3}\right) \delta 7.21-7.14(\mathrm{~m}, 1 \mathrm{H}), 7.05-6.93(\mathrm{~m}, 2 \mathrm{H})$, $6.62(\mathrm{~d}, J=7.9 \mathrm{~Hz}, 1 \mathrm{H}), 4.70(\mathrm{~d}, J=17.5 \mathrm{~Hz}, 1 \mathrm{H}), 4.60(\mathrm{~d}, J=17.5 \mathrm{~Hz}, 1 \mathrm{H}), 3.71-3.61(\mathrm{~m}, 1 \mathrm{H}), 3.51-3.40(\mathrm{~m}$, $1 \mathrm{H}), 2.74-2.57(\mathrm{~m}, 1 \mathrm{H}), 2.42-2.21(\mathrm{~m}, 2 \mathrm{H}), 2.18-2.05(\mathrm{~m}, 1 \mathrm{H}), 1.26(\mathrm{~s}, 3 \mathrm{H}) .{ }^{13} \mathrm{C} \mathrm{NMR}\left(125 \mathrm{MHz}, \mathrm{CDCl}_{3}\right) \delta$ $163.5,138.4,127.7,127.0$ (q, $J=278.2 \mathrm{~Hz}), 126.0,123.5,120.0,110.9,49.3,43.7,41.9$ (q, $J=27.3 \mathrm{~Hz}), 41.5,33.3$, 23.1. ${ }^{19} \mathrm{~F}$ NMR (470 MHz, $\left.\mathrm{CDCl}_{3}\right) \delta-60.33\left(\mathrm{t}, J=11.6 \mathrm{~Hz}\right.$ ). HRMS (ESI) m/z: $[\mathrm{M}+\mathrm{H}]^{+}$calcd for $\mathrm{C}_{14} \mathrm{H}_{16} \mathrm{~F}_{3} \mathrm{~N}_{2}$ 269.1260; found: 269.1251 .

Spectral data for 3-phenyl-3-(2,2,2-trifluoroethyl)-1,2,3,5-tetrahydropyrrolo[1,2-a]quinazoline (10f)<smiles>FC(F)(F)CC1(c2ccccc2)CCN2C1=NCc1ccccc12</smiles> 
Purified by column chromatography (silica gel, EtOAc/Petroleum ether: $1 / 5$ ) to afford the title compound (35\% yield, $23 \mathrm{mg}$ ) as yellow oil. ${ }^{1} \mathrm{H}$ NMR (500 MHz, $\left.\mathrm{CDCl}_{3}\right) \delta 7.57-7.50(\mathrm{~m}, 2 \mathrm{H}), 7.30(\mathrm{t}, J=7.6 \mathrm{~Hz}, 2 \mathrm{H}), 7.26-7.19(\mathrm{~m}$, 1H), $7.14-7.07(\mathrm{~m}, 1 \mathrm{H}), 7.03-6.94(\mathrm{~m}, 2 \mathrm{H}), 6.49$ (d, $J=7.9 \mathrm{~Hz}, 1 \mathrm{H}), 4.83(\mathrm{~d}, J=17.6 \mathrm{~Hz}, 1 \mathrm{H}), 4.69$ (d, $J=17.6$ $\mathrm{Hz}, 1 \mathrm{H}), 3.66(\mathrm{t}, J=8.4 \mathrm{~Hz}, 1 \mathrm{H}), 3.33-3.21(\mathrm{~m}, 1 \mathrm{H}), 3.09-2.88(\mathrm{~m}, 2 \mathrm{H}), 2.84-2.69(\mathrm{~m}, 1 \mathrm{H}), 2.54-2.40(\mathrm{~m}, 1 \mathrm{H})$. ${ }^{13} \mathrm{C}$ NMR $\left(125 \mathrm{MHz}, \mathrm{CDCl}_{3}\right) \delta 161.1,139.0,138.3,128.7,127.7,127.6,126.8,126.0,125.8$ (q, $\left.J=278.0 \mathrm{~Hz}\right), 123.4$, 120.1, 110.6, 49.5, 48.8, 43.5 (q, $J=26.7 \mathrm{~Hz}), 43.4,31.8 .{ }^{19} \mathrm{~F}$ NMR $\left(470 \mathrm{MHz}, \mathrm{CDCl}_{3}\right) \delta-60.12(\mathrm{t}, J=11.1 \mathrm{~Hz})$. HRMS (ESI) $\mathrm{m} / \mathrm{z}$ : $[\mathrm{M}+\mathrm{H}]^{+}$calcd for $\mathrm{C}_{19} \mathrm{H}_{18} \mathrm{~F}_{3} \mathrm{~N}_{2} 331.1417$; found: 331.1433 .

Spectral data for 2,3,3-trimethyl-8-(2,2,2-trifluoroethyl)-2,6,7,8-tetrahydropyrrolo[1,2-a]pyrimidin-4(3H)-one $(10 \mathrm{~g})$<smiles></smiles>

Purified by column chromatography (silica gel, EtOAc/Petroleum ether: 1/5) to afford the title compound (an inseparable mixture, $29 \%$ yield, $15 \mathrm{mg}$, $\mathrm{dr}=1.1: 1)$ as colorless oil. The major isomer: ${ }^{1} \mathrm{H}$ NMR $\left(500 \mathrm{MHz}, \mathrm{CDCl}_{3}\right)$ $\delta 4.10-3.77(\mathrm{~m}, 1 \mathrm{H}), 3.62-3.29(\mathrm{~m}, 1 \mathrm{H}), 3.11-2.78(\mathrm{~m}, 2 \mathrm{H}), 2.24-2.00(\mathrm{~m}, 2 \mathrm{H}), 1.88-1.71(\mathrm{~m}, 2 \mathrm{H}), 1.24(\mathrm{~d}, J$ $=7.0 \mathrm{~Hz}, 3 \mathrm{H}), 1.21(\mathrm{~s}, 3 \mathrm{H}), 1.00(\mathrm{~s}, 3 \mathrm{H})$. The minor isomer: ${ }^{1} \mathrm{H}$ NMR $\left(500 \mathrm{MHz}, \mathrm{CDCl}_{3}\right) \delta 4.10-3.77(\mathrm{~m}, 1 \mathrm{H}), 3.62$ $-3.29(\mathrm{~m}, 3 \mathrm{H}), 3.11-2.78(\mathrm{~m}, 2 \mathrm{H}), 2.54-2.34(\mathrm{~m}, 2 \mathrm{H}), 1.19(\mathrm{~s}, 3 \mathrm{H}), 1.15(\mathrm{~d}, J=7.0 \mathrm{~Hz}, 3 \mathrm{H}), 1.07(\mathrm{~s}, 3 \mathrm{H}) .{ }^{13} \mathrm{C}$ $\operatorname{NMR}\left(125 \mathrm{MHz}, \mathrm{CDCl}_{3}\right) \delta 175.0,174.4,156.5,156.4,126.7(\mathrm{q}, J=276.1 \mathrm{~Hz}), 126.6(\mathrm{q}, J=277.2 \mathrm{~Hz}), 126.5(\mathrm{q}, J$ $=276.9 \mathrm{~Hz}), 60.5,59.7,42.9,42.8,40.3,40.1,36.4,35.7$ (q, $J=29.0 \mathrm{~Hz}), 35.6$ (q, $J=29.2 \mathrm{~Hz}), 29.7,27.2,23.5$, 22.4, 18.6, 17.7, 15.8. The major isomer: ${ }^{19} \mathrm{~F} \mathrm{NMR}\left(470 \mathrm{MHz}, \mathrm{CDCl}_{3}\right) \delta-64.68(\mathrm{t}, J=10.9 \mathrm{~Hz})$. The minor isomer: ${ }^{19} \mathrm{~F} \mathrm{NMR}\left(470 \mathrm{MHz}, \mathrm{CDCl}_{3}\right) \delta-64.62(\mathrm{t}, J=11.0 \mathrm{~Hz})$. HRMS $(\mathrm{ESI}) \mathrm{m} / \mathrm{z}:[\mathrm{M}+\mathrm{H}]^{+}$calcd for $\mathrm{C}_{12} \mathrm{H}_{18} \mathrm{~F}_{3} \mathrm{~N}_{2} \mathrm{O} 263.1366$; found: 263.1363 .

Spectral data for 2,2-diphenyl-8-(2,2,2-trifluoroethyl)-2,6,7,8-tetrahydropyrrolo[1,2-a]pyrimidin-4(3H)-one (10h)<smiles>O=C1CC(c2ccccc2)(c2ccccc2)N=C1CC(F)(F)F</smiles>

Purified by column chromatography (silica gel, EtOAc/Petroleum ether: 1/10) to afford the title compound (17\% yield, $13 \mathrm{mg}$ ) as white solid, mp: $148-149{ }^{\circ} \mathrm{C} .{ }^{1} \mathrm{H}$ NMR (400 MHz, $\left.\mathrm{CDCl}_{3}\right) \delta 7.39-7.32(\mathrm{~m}, 4 \mathrm{H}), 7.32-7.25(\mathrm{~m}$, 4H), $7.25-7.18(\mathrm{~m}, 2 \mathrm{H}), 3.95-3.78(\mathrm{~m}, 1 \mathrm{H}), 3.50-3.37(\mathrm{~m}, 1 \mathrm{H}), 3.24-3.04(\mathrm{~m}, 3 \mathrm{H}), 2.99$ (d, J=16.9 Hz, 1H), $2.47-2.36(\mathrm{~m}, 1 \mathrm{H}), 2.21-2.04(\mathrm{~m}, 1 \mathrm{H}), 1.77-1.63(\mathrm{~m}, 1 \mathrm{H}) .{ }^{13} \mathrm{C} \mathrm{NMR}\left(100 \mathrm{MHz}, \mathrm{CDCl}_{3}\right) \delta 167.6,157.1,146.3$, 145.7, 128.5, 127.1, 127.1, 126.9 (q, $J=276.6 \mathrm{~Hz}), 126.5,126.4,65.8,42.7,41.9,37.1$ (q, $J=2.6 \mathrm{~Hz}), 35.9$ (q, $J=$ $29.0 \mathrm{~Hz}), 27.2 .{ }^{19} \mathrm{~F}$ NMR $\left(470 \mathrm{MHz}, \mathrm{CDCl}_{3}\right) \delta-64.57(\mathrm{t}, J=11.1 \mathrm{~Hz})$. HRMS (ESI) $\mathrm{m} / \mathrm{z}:[\mathrm{M}+\mathrm{H}]^{+}$calcd for $\mathrm{C}_{21} \mathrm{H}_{20} \mathrm{~F}_{3} \mathrm{~N}_{2} \mathrm{O} 373.1522$; found: 373.1536 .

Spectral data for 7,7-dimethyl-2,2-diphenyl-8-(2,2,2-trifluoroethyl)-3,4,7,8-tetrahydropyrrolo[1,2a]pyrimidin-6(2H)-one (10k) 
<smiles>CC1(C)C(=O)N2CCC(c3ccccc3)N=C2C1CC(F)(F)F</smiles>

Purified by column chromatography (silica gel, EtOAc/Petroleum ether: $1 / 8)$ to afford the title compound (26\% yield, $21 \mathrm{mg}$ ) as colorless oil. ${ }^{1} \mathrm{H}$ NMR $\left(500 \mathrm{MHz}, \mathrm{CDCl}_{3}\right) \delta 7.43-7.39(\mathrm{~m}, 2 \mathrm{H}), 7.38-7.34(\mathrm{~m}, 2 \mathrm{H}), 7.33-7.27(\mathrm{~m}, 4 \mathrm{H})$, $7.22-7.17(\mathrm{~m}, 2 \mathrm{H}), 3.49-3.39(\mathrm{~m}, 2 \mathrm{H}), 3.38-3.27(\mathrm{~m}, 1 \mathrm{H}), 3.08(\mathrm{dd}, J=10.5,2.4 \mathrm{~Hz}, 1 \mathrm{H}), 2.47(\mathrm{t}, J=6.1 \mathrm{~Hz}$, $2 \mathrm{H}), 2.44-2.33(\mathrm{~m}, 1 \mathrm{H}), 1.38(\mathrm{~s}, 3 \mathrm{H}), 1.15(\mathrm{~s}, 3 \mathrm{H}) .{ }^{13} \mathrm{C} \mathrm{NMR}\left(125 \mathrm{MHz}, \mathrm{CDCl}_{3}\right) \delta 179.9,154.0,147.0,146.7,128.6$, 127.0 (q, $J=276.7 \mathrm{~Hz}), 126.9,126.6,126.4,63.5,44.5,43.2,35.7,31.4,30.7$ (q, $J=30.0 \mathrm{~Hz}$ ), 23.4, 21.8. ${ }^{19} \mathrm{~F}$ NMR $\left(470 \mathrm{MHz}, \mathrm{CDCl}_{3}\right) \delta-63.77\left(\mathrm{t}, J=11.2 \mathrm{~Hz}\right.$ ). HRMS (ESI) $\mathrm{m} / \mathrm{z}$ : $[\mathrm{M}+\mathrm{H}]^{+}$calcd for $\mathrm{C}_{23} \mathrm{H}_{24} \mathrm{~F}_{3} \mathrm{~N}_{2} \mathrm{O} 401.1835$; found: 401.1838 .

Spectral data for 3,3,7,7-tetramethyl-2-phenyl-8-(2,2,2-trifluoroethyl)-3,4,7,8-tetrahydropyrrolo[1,2a]pyrimidin-6(2H)-one (101)<smiles>CC1(C)CN2C(=O)C(C)(C)C(CC(F)(F)F)C2=NC1c1ccccc1</smiles>

Purified by column chromatography (silica gel, EtOAc/Petroleum ether: 1/12) to afford the title compound (an inseparable mixture, $71 \%$ yield, $50 \mathrm{mg}, \mathrm{dr}=1.2: 1)$ as colorless oil. The major isomer: ${ }^{1} \mathrm{H} \mathrm{NMR}\left(500 \mathrm{MHz}, \mathrm{CDCl}_{3}\right)$ $\delta 7.36-7.27(\mathrm{~m}, 3 \mathrm{H}), 7.14-7.09(\mathrm{~m}, 2 \mathrm{H}), 4.34(\mathrm{~d}, J=2.6 \mathrm{~Hz}, 1 \mathrm{H}), 3.30-3.10(\mathrm{~m}, 3 \mathrm{H}), 3.08-2.98(\mathrm{~m}, 1 \mathrm{H}), 2.43$ $-2.23(\mathrm{~m}, 1 \mathrm{H}), 1.42(\mathrm{~s}, 3 \mathrm{H}), 1.25(\mathrm{~s}, 3 \mathrm{H}), 1.03(\mathrm{~s}, 3 \mathrm{H}), 0.61(\mathrm{~s}, 3 \mathrm{H})$. The minor isomer: ${ }^{1} \mathrm{H}$ NMR $\left(500 \mathrm{MHz}, \mathrm{CDCl}_{3}\right)$ $\delta 7.36-7.27(\mathrm{~m}, 3 \mathrm{H}), 7.14-7.09(\mathrm{~m}, 2 \mathrm{H}), 4.32(\mathrm{~d}, J=2.2 \mathrm{~Hz}, 1 \mathrm{H}), 3.41(\mathrm{~d}, J=12.9 \mathrm{~Hz}, 1 \mathrm{H}), 3.30-3.10(\mathrm{~m}, 2 \mathrm{H})$, $3.08-2.98(\mathrm{~m}, 1 \mathrm{H}), 2.43-2.23(\mathrm{~m}, 1 \mathrm{H}), 1.43(\mathrm{~s}, 3 \mathrm{H}), 1.21(\mathrm{~s}, 3 \mathrm{H}), 0.99(\mathrm{~s}, 3 \mathrm{H}), 0.68(\mathrm{~s}, 3 \mathrm{H}) .{ }^{13} \mathrm{C}$ NMR $(125 \mathrm{MHz}$, $\left.\mathrm{CDCl}_{3}\right) \delta 180.1,180.0,154.7,154.5,139.9,139.8,128.6,128.5,128.0,128.0,127.5,127.4,126.9$ (q, J = 273.8 Hz), 68.7, 68.5, 48.7, 47.7, 44.0, 43.5, 43.1, 30.9, 30.6 (q, $J=30.0 \mathrm{~Hz}), 30.5$ (q, $J=32.3 \mathrm{~Hz}$ ), 30.4, 26.2, 26.1, 23.7, 23.5, 22.1, 21.9, 20.6. The mixture: ${ }^{19} \mathrm{~F}$ NMR $\left(470 \mathrm{MHz}, \mathrm{CDCl}_{3}\right) \delta-63.88-63.94(\mathrm{~m}) . \mathrm{HRMS}(\mathrm{ESI}) \mathrm{m} / \mathrm{z}:[\mathrm{M}+\mathrm{H}]^{+}$calcd for $\mathrm{C}_{19} \mathrm{H}_{24} \mathrm{~F}_{3} \mathrm{~N}_{2} \mathrm{O} 353.1835$; found: 353.1846 .

Spectral data for 7,7-dimethyl-2,2-diphenyl-9-(2,2,2-trifluoroethyl)-2,3,4,7,8,9-hexahydro-6H-pyrido[1,2a]pyrimidin-6-one (10m)<smiles>CC1(C)CC(CC(F)(F)F)C2=NC(c3ccccc3)(c3ccccc3)CCN2C1=O</smiles>

Purified by column chromatography (silica gel, EtOAc/Petroleum ether: 1/20) to afford the title compound (64\% yield, $53 \mathrm{mg}$ ) as colorless oil. ${ }^{1} \mathrm{H}$ NMR $\left(500 \mathrm{MHz}, \mathrm{CDCl}_{3}\right) \delta 7.43-7.33(\mathrm{~m}, 4 \mathrm{H}), 7.33-7.26(\mathrm{~m}, 4 \mathrm{H}), 7.23-7.14$ (m, 2H), $3.72-3.47(\mathrm{~m}, 3 \mathrm{H}), 3.15-3.03(\mathrm{~m}, 1 \mathrm{H}), 2.60-2.50(\mathrm{~m}, 1 \mathrm{H}), 2.50-2.39(\mathrm{~m}, 1 \mathrm{H}), 2.32-2.18(\mathrm{~m}, 1 \mathrm{H})$, $2.10-2.01(\mathrm{~m}, 1 \mathrm{H}), 1.79-1.66(\mathrm{~m}, 1 \mathrm{H}), 1.31(\mathrm{~s}, 3 \mathrm{H}), 1.27(\mathrm{~s}, 3 \mathrm{H}) .{ }^{13} \mathrm{C} \mathrm{NMR}\left(100 \mathrm{MHz}, \mathrm{CDCl}_{3}\right) \delta 175.7,149.9$, $147.5,147.0,128.5,128.4,127.5(\mathrm{q}, J=276.8 \mathrm{~Hz}), 126.7,126.6,126.5,126.4,61.9,38.9,38.7,37.7,35.4(\mathrm{q}, J=$ $28.1 \mathrm{~Hz}$ ), 32.7 (q, $J=2.7 \mathrm{~Hz}$ ), 32.1, 27.7, 25.5. ${ }^{19} \mathrm{~F}$ NMR (470 MHz, $\left.\mathrm{CDCl}_{3}\right) \delta-62.89$ (t, $\left.J=11.4 \mathrm{~Hz}\right)$. HRMS (ESI) $\mathrm{m} / \mathrm{z}:[\mathrm{M}+\mathrm{H}]^{+}$calcd for $\mathrm{C}_{24} \mathrm{H}_{26} \mathrm{~F}_{3} \mathrm{~N}_{2} \mathrm{O}$ 415.1992; found: 415.1997 .

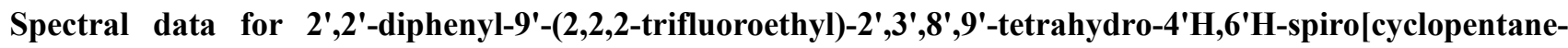


Spectral data for $1,7^{\prime}$-pyrido[1,2-a]pyrimidin]-4'-one (10n)

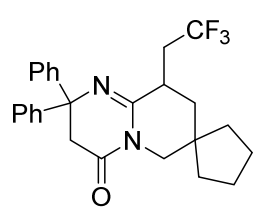

Purified by column chromatography (silica gel, EtOAc/Petroleum ether: $1 / 20)$ to afford the title compound (47\% yield, $42 \mathrm{mg}$ ) as white solid, mp: $134-136{ }^{\circ} \mathrm{C} .{ }^{1} \mathrm{H}$ NMR $\left(400 \mathrm{MHz}, \mathrm{CDCl}_{3}\right) \delta 7.43-7.38(\mathrm{~m}, 2 \mathrm{H}), 7.37-7.32(\mathrm{~m}$, 2H), $7.32-7.23(\mathrm{~m}, 4 \mathrm{H}), 7.22-7.15(\mathrm{~m}, 2 \mathrm{H}), 3.50-3.36(\mathrm{~m}, 3 \mathrm{H}), 3.30(\mathrm{~d}, J=16.4 \mathrm{~Hz}, 1 \mathrm{H}), 3.00-2.91(\mathrm{~m}, 1 \mathrm{H})$, $2.87(\mathrm{~d}, J=16.4 \mathrm{~Hz}, 1 \mathrm{H}), 2.33-2.16(\mathrm{~m}, 1 \mathrm{H}), 2.10-2.01(\mathrm{~m}, 1 \mathrm{H}), 1.66-1.32(\mathrm{~m}, 7 \mathrm{H}), 1.03-0.85(\mathrm{~m}, 2 \mathrm{H}) .{ }^{13} \mathrm{C}$ NMR $\left(125 \mathrm{MHz}, \mathrm{CDCl}_{3}\right) \delta 168.8,153.6,146.9,144.8,128.4,128.3,127.3$ (q, $\left.J=276.9 \mathrm{~Hz}\right), 127.1,126.9,126.6$, $126.4,63.0,48.4,42.1,41.6,40.6,38.4,36.9,36.0$ (q, $J=28.3 \mathrm{~Hz}), 33.9,24.5,24.1 .{ }^{19} \mathrm{~F} \mathrm{NMR}\left(470 \mathrm{MHz}, \mathrm{CDCl}_{3}\right) \delta$ $-63.16\left(\mathrm{t}, J=11.4 \mathrm{~Hz}\right.$ ). HRMS (ESI) m/z: $[\mathrm{M}+\mathrm{H}]^{+}$calcd for $\mathrm{C}_{26} \mathrm{H}_{28} \mathrm{~F}_{3} \mathrm{~N}_{2} \mathrm{O} 441.2148$; found: 441.2157 . 


\section{Other Unsuccessful Substrates}

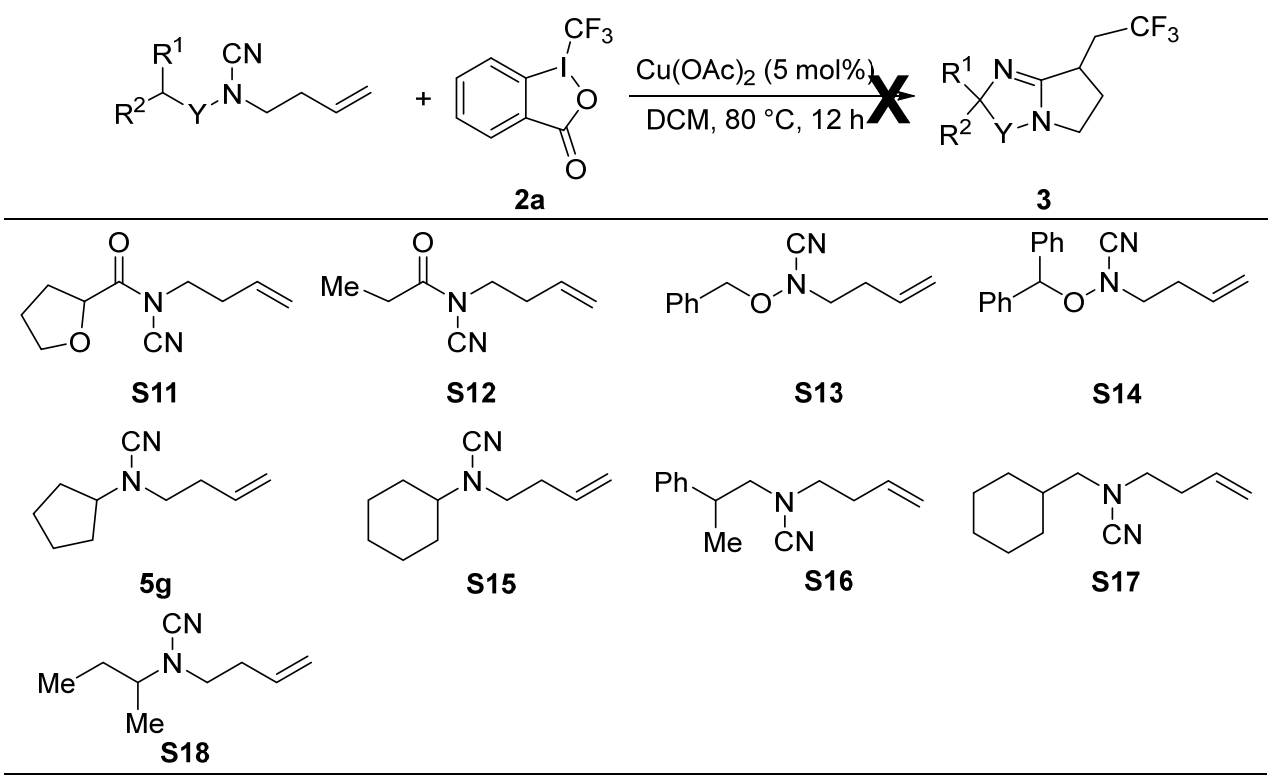

The reactions gave the unidentified complex mixture.

Scheme S1. Unsuccessful Substrates for preparation of $\mathbf{3}$
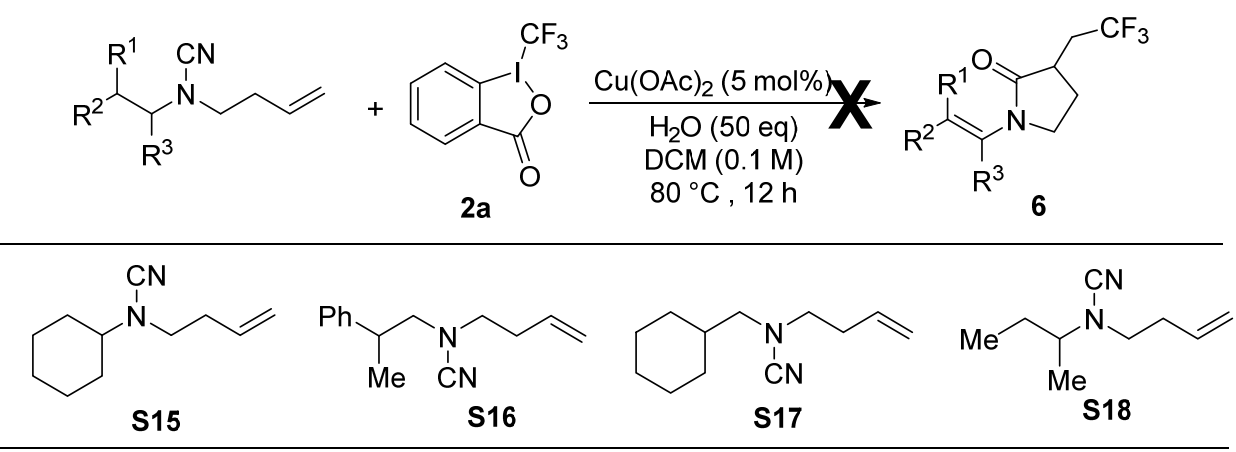

The reactions gave the unidentified complex mixture.

Scheme S2. Unsuccessful Substrates for preparation of 6
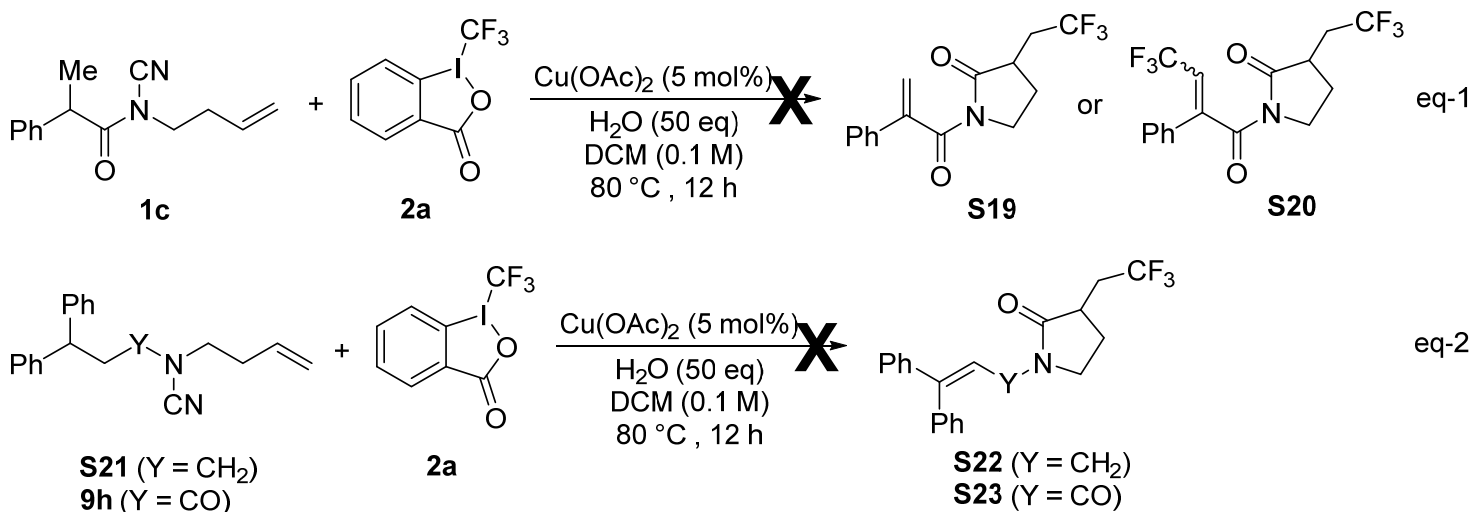

Scheme S3. Unsuccessful Substrates for preparation of S19-S20, S22-S23 


\section{Scale-up Reaction and Synthetic Transformations}

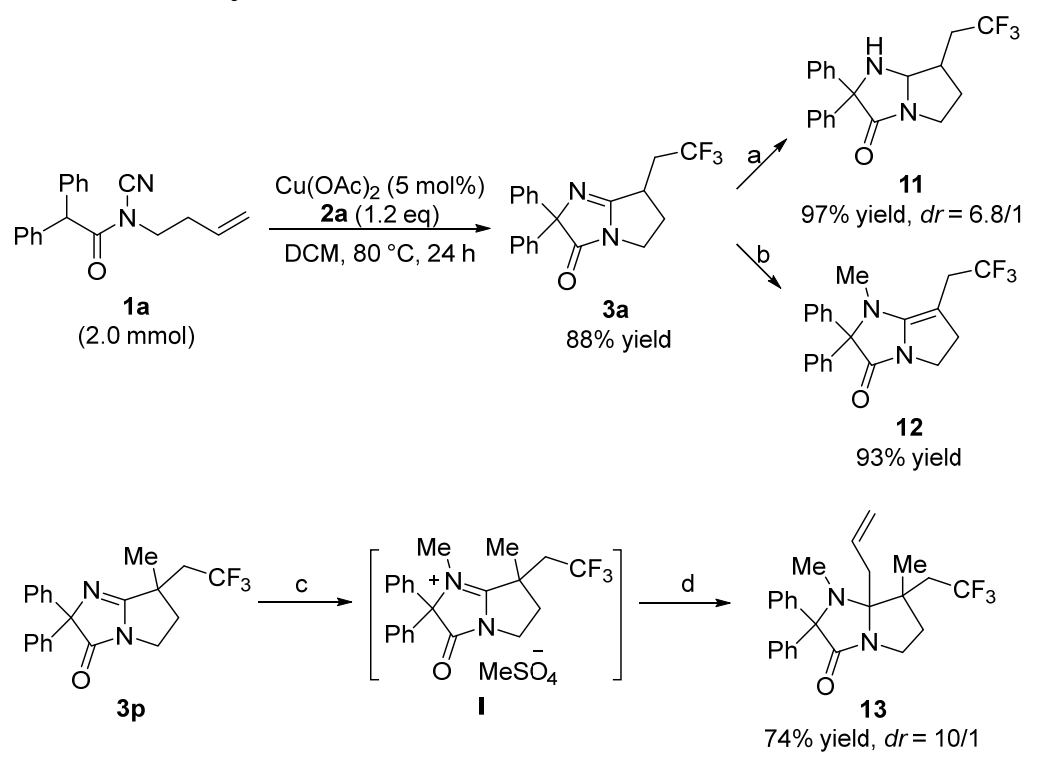

Reaction conditions: (a) $\mathrm{NaBH}_{3} \mathrm{CN}, \mathrm{DCM} / \mathrm{HOAc}=10 / 1,-78^{\circ} \mathrm{C}, 1 \mathrm{~h}$; (b) $\mathrm{Me}_{2} \mathrm{SO}_{4}, \mathrm{CH}_{3} \mathrm{CN}, 60^{\circ} \mathrm{C}, 14 \mathrm{~h}$; then, $0.2 \% \mathrm{~K}_{2} \mathrm{CO} 3$ (aq.), rt, 1 h; c) $\mathrm{Me}_{2} \mathrm{SO}_{4}, \mathrm{CH}_{3} \mathrm{CN}, 60{ }^{\circ} \mathrm{C}, 12 \mathrm{~h}$; d) Allylmagnesium chloride, THF, $-40{ }^{\circ} \mathrm{C}, 30 \mathrm{~min}$.

\section{Scale-up Reaction for Preparation of Product 3a}

To a flame-dried sealed tube equipped with a magnetic stir bar were added 1a ( 2 mmol, 1 equiv.), Togni's reagent 2a $(2.4 \mathrm{mmol}, 1.2$ equiv. $)$ and $\mathrm{Cu}(\mathrm{OAc})_{2}(5 \mathrm{~mol} \%, 0.1 \mathrm{mmol})$. The tube was evacuated and backfilled with nitrogen for three times, and dichloromethane $(15 \mathrm{~mL})$ was added via syringe. The tube was stirred at $80{ }^{\circ} \mathrm{C}$ for $24 \mathrm{~h}$. After completion, the mixture was cooled to room temperature, and the sat. aq. $\mathrm{NaHCO}_{3}(40 \mathrm{~mL})$ was added. Then the aqueous phase was extracted with DCM, washed with brine, dried over $\mathrm{Na}_{2} \mathrm{SO}_{4}$. The filtrate was concentrated under reduced pressure and the crude was purified by flash chromatography (silica gel, EtOAc/Petroleum ether $\left(60-90^{\circ} \mathrm{C}\right)$ ) to give the desired compounds $3 \mathbf{a}$ (630 mg, $88 \%$ yield).

\section{Preparation of Product 11}<smiles>O=C1N2CCC(CC(F)(F)F)C2NC1(c1ccccc1)c1ccccc1</smiles>

To solution of $3 \mathbf{a}\left(0.2 \mathrm{mmol}, 1\right.$ equiv.) in $\mathrm{HOAc} / \mathrm{DCM}(1 / 10,0.1 \mathrm{M})$ in a dried tube was added $\mathrm{NaBH}_{3} \mathrm{CN}(3.0$ equiv.) at $-78^{\circ} \mathrm{C}$. The mixture was stirred for 1 hour. Upon completion, the reaction was quenched with saturated $\mathrm{NaHCO}_{3}$ at $-78^{\circ} \mathrm{C}$, and the mixture was extracted with DCM. The combined organic phases were washed with brine before being dried over $\mathrm{Na}_{2} \mathrm{SO}_{4}$ and concentrated under reduced pressure.

\section{Spectral data for 2,2-diphenyl-7-(2,2,2-trifluoroethyl)hexahydro-3H-pyrrolo[1,2-a]imidazol-3-one (11)}

The major isomer: purified by column chromatography (silica gel, EtOAc/Petroleum ether: 1/5) to afford the title compound (84\% yield, $61 \mathrm{mg}, d r=6.8 / 1)$ as white solid, mp: $147-148{ }^{\circ} \mathrm{C} .{ }^{1} \mathrm{H}$ NMR $\left(500 \mathrm{MHz}, \mathrm{CDCl}_{3}\right) \delta 7.64-$ $7.60(\mathrm{~m}, 2 \mathrm{H}), 7.34-7.25(\mathrm{~m}, 8 \mathrm{H}), 4.51(\mathrm{~d}, J=8.2 \mathrm{~Hz}, 1 \mathrm{H}), 3.69-3.57(\mathrm{~m}, 1 \mathrm{H}), 3.34-3.26(\mathrm{~m}, 1 \mathrm{H}), 2.70(\mathrm{brs}, 1 \mathrm{H})$, $2.51-2.30(\mathrm{~m}, 2 \mathrm{H}), 2.21-2.07(\mathrm{~m}, 1 \mathrm{H}), 2.06-1.94(\mathrm{~m}, 1 \mathrm{H}), 1.86-1.75(\mathrm{~m}, 1 \mathrm{H}) .{ }^{13} \mathrm{C} \mathrm{NMR}\left(125 \mathrm{MHz} \mathrm{CDCl}_{3}\right) \delta$ 174.5, 144.0, 142.6, 128.9, 127.9, 127.6, 126.8, 126.6 (q, J=276.9 Hz), 78.7, 75.2, 41.2, 40.1, 35.2 (q, J=29.1 Hz), 31.2. ${ }^{19} \mathrm{~F} \mathrm{NMR}\left(470 \mathrm{MHz}, \mathrm{CDCl}_{3}\right) \delta-64.21\left(\mathrm{t}, J=10.9 \mathrm{~Hz}\right.$ ). HRMS (ESI) m/z: $[\mathrm{M}+\mathrm{H}]^{+}$calcd for $\mathrm{C}_{20} \mathrm{H}_{20} \mathrm{~F}_{3} \mathrm{~N}_{2} \mathrm{O}$ 
361.1522; found: 361.1517 .

The minor isomer: purified by column chromatography (silica gel, EtOAc/Petroleum ether: 1/8) to afford the title compound (13\% yield, $9 \mathrm{mg}$ ) as white solid, mp: $202-204{ }^{\circ} \mathrm{C} .{ }^{1} \mathrm{H}$ NMR (400 MHz, $\left.\mathrm{CDCl}_{3}\right) \delta 7.53-7.47(\mathrm{~m}, 2 \mathrm{H})$, $7.39-7.25(\mathrm{~m}, 8 \mathrm{H}), 4.91(\mathrm{t}, J=5.7 \mathrm{~Hz}, 1 \mathrm{H}), 3.88-3.78(\mathrm{~m}, 1 \mathrm{H}), 3.16-3.05(\mathrm{~m}, 1 \mathrm{H}), 2.90-2.72(\mathrm{~m}, 1 \mathrm{H}), 2.56-$ $2.37(\mathrm{~m}, 2 \mathrm{H}), 2.20-2.09(\mathrm{~m}, 1 \mathrm{H}), 2.07-1.94(\mathrm{~m}, 1 \mathrm{H}), 1.87-1.73(\mathrm{~m}, 1 \mathrm{H}) .{ }^{13} \mathrm{C}$ NMR $\left(125 \mathrm{MHz}, \mathrm{CDCl}_{3}\right) \delta 174.1$, 143.3, 142.2, 129.0, 128.0, 127.7, 127.3 (q, $J=276.0 \mathrm{~Hz}), 126.6,74.9,73.3,42.3,35.9,32.0$ (q, $J=29.1 \mathrm{~Hz}), 31.4$. ${ }^{19} \mathrm{~F}$ NMR (470 MHz, $\left.\mathrm{CDCl}_{3}\right) \delta-64.19\left(\mathrm{t}, J=11.2 \mathrm{~Hz}\right.$ ). HRMS (ESI) $\mathrm{m} / \mathrm{z}$ : $[\mathrm{M}+\mathrm{H}]^{+}$calcd for $\mathrm{C}_{20} \mathrm{H}_{20} \mathrm{~F}_{3} \mathrm{~N}_{2} \mathrm{O} 361.1522$; found: 361.1528 .

\section{Preparation of Product 12}

To a solution of $3 \mathbf{a}(0.15 \mathrm{mmol})$ in acetonitrile $(0.15 \mathrm{~mL})$ dimethyl sulfate $(43 \mu 1,0.45 \mathrm{mmol})$ was added. The resulting mixture was stirred at $60{ }^{\circ} \mathrm{C}$ for $14 \mathrm{~h}$. Then $0.2 \%$ aqueous solution $\mathrm{K}_{2} \mathrm{CO}_{3}(10 \mathrm{~mL})$ was added at room temperature. After $1 \mathrm{~h}, \mathrm{DCM}(10 \mathrm{~mL})$ were added. The organic layer was separated. Then the aqueous phase was extracted with DCM, washed with brine, dried over $\mathrm{Na}_{2} \mathrm{SO}_{4}$. The filtrate was concentrated under reduced pressure and the crude was purified by flash chromatography.

Spectral data for 1-methyl-2,2-diphenyl-7-(2,2,2-trifluoroethyl)-1,2,5,6-tetrahydro-3H-pyrrolo[1,2a]imidazol-3-one (12)

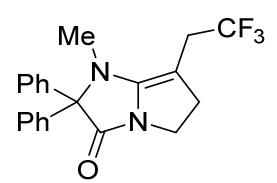

Purified by column chromatography (silica gel, EtOAc/Petroleum ether: 1/8) to afford the title compound ( $93 \%$ yield, $52 \mathrm{mg}$ ) as colorless oil. ${ }^{1} \mathrm{H}$ NMR (500 MHz, $\left.\mathrm{CDCl}_{3}\right) \delta 7.40-7.32(\mathrm{~m}, 6 \mathrm{H}), 7.31-7.27(\mathrm{~m}, 4 \mathrm{H}), 3.76-3.69(\mathrm{~m}, 2 \mathrm{H})$, $3.05-2.98(\mathrm{~m}, 2 \mathrm{H}), 2.96(\mathrm{q}, J=10.6 \mathrm{~Hz}, 2 \mathrm{H}), 2.66(\mathrm{~s}, 3 \mathrm{H}) .{ }^{13} \mathrm{C} \mathrm{NMR}\left(125 \mathrm{MHz}, \mathrm{CDCl}_{3}\right) \delta 167.3,144.9,138.2$, $128.5,128.4,126.4(\mathrm{q}, J=278.2 \mathrm{~Hz}), 82.7,71.0,39.2,35.9,31.6(\mathrm{q}, J=30.6 \mathrm{~Hz}), 30.7 .{ }^{19} \mathrm{~F} \mathrm{NMR}\left(470 \mathrm{MHz}, \mathrm{CDCl}_{3}\right)$ $\delta$-65.77 (t, $J=10.4 \mathrm{~Hz}$ ). HRMS (ESI) m/z: $[\mathrm{M}+\mathrm{H}]^{+}$calcd for $\mathrm{C}_{21} \mathrm{H}_{20} \mathrm{~F}_{3} \mathrm{~N}_{2} \mathrm{O} 373.1522$; found: 373.1531 .

\section{Preparation of Product 13}

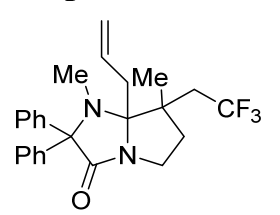

To a solution of $3 \mathbf{p}(0.15 \mathrm{mmol})$ in acetonitrile $(0.15 \mathrm{~mL})$ dimethyl sulfate $(43 \mu 1,0.45 \mathrm{mmol})$ was added. The resulting mixture was stirred at $60{ }^{\circ} \mathrm{C}$ for $12 \mathrm{~h}$. Then $0.2 \%$ aqueous solution $\mathrm{K}_{2} \mathrm{CO}_{3}(10 \mathrm{~mL})$ was added at room temperature. After $1 \mathrm{~h}, \mathrm{DCM}(10 \mathrm{~mL})$ were added. The solvent was removed under reduced pressure and the crude was washed by $\mathrm{Et}_{2} \mathrm{O}$ to give salt $\mathbf{I}$ without the need for further purification. To solution of salt $\mathbf{I}$ in THF in a dried tube was added allylmagnesium chloride ( 1.2 equiv., $2 \mathrm{M}$ in THF) at $-40{ }^{\circ} \mathrm{C}$. The mixture was stirred for $30 \mathrm{~min}$. Upon completion, the reaction was quenched with saturated ammonium chloride solution at $-40{ }^{\circ} \mathrm{C}$, and the mixture was extracted with DCM. The combined organic phases were washed with brine, dried over anhydrous $\mathrm{Na}_{2} \mathrm{SO}_{4}$. The organic layer was then concentrated under vacuum.

Spectral data for 7a-allyl-1,7-dimethyl-2,2-diphenyl-7-(2,2,2-trifluoroethyl)hexahydro-3H-pyrrolo[1,2a]imidazol-3-one (13)

Purified by column chromatography (silica gel, EtOAc/Petroleum ether: 1/10) to afford the title compound (74\% yield, $47 \mathrm{mg}, d r=10 / 1)$ as colorless oil. ${ }^{1} \mathrm{H}$ NMR (400 MHz, $\left.\mathrm{CDCl}_{3}\right) \delta 7.42-7.37(\mathrm{~m}, 2 \mathrm{H}), 7.34-7.26(\mathrm{~m}, 6 \mathrm{H})$, 
$7.16-7.10(\mathrm{~m}, 2 \mathrm{H}), 5.87-5.74(\mathrm{~m}, 1 \mathrm{H}), 5.17-5.06(\mathrm{~m}, 2 \mathrm{H}), 3.65-3.55(\mathrm{~m}, 1 \mathrm{H}), 3.35-3.26(\mathrm{~m}, 1 \mathrm{H}), 2.57-2.37$ (m, 3H), $2.33(\mathrm{~s}, 3 \mathrm{H}), 2.33-2.26(\mathrm{~m}, 2 \mathrm{H}), 2.13-2.03(\mathrm{~m}, 1 \mathrm{H}), 1.16(\mathrm{~s}, 3 \mathrm{H}) .{ }^{13} \mathrm{C}$ NMR $\left(100 \mathrm{MHz}, \mathrm{CDCl}_{3}\right) \delta 172.9$, 140.9, 139.3, 133.1, 129.9, 128.6, 127.8, 127.8, 127.6, 127.4, 126.9 (q, $J=278.7 \mathrm{~Hz}), 119.2,89.0,79.7,45.6,40.3$, $40.2(\mathrm{q}, J=27.4 \mathrm{~Hz}), 38.1,38.0,31.3,23.1$. The major isomer: ${ }^{19} \mathrm{~F}$ NMR $\left(376 \mathrm{MHz}, \mathrm{CDCl}_{3}\right) \delta-59.63(\mathrm{t}, J=11.4$ $\mathrm{Hz}$ ). The minor isomer: ${ }^{19} \mathrm{~F}$ NMR $\left(376 \mathrm{MHz}, \mathrm{CDCl}_{3}\right) \delta-58.17$ (t, $J=11.8 \mathrm{~Hz}$ ). HRMS (ESI) $\mathrm{m} / \mathrm{z}:[\mathrm{M}+\mathrm{H}]^{+}$calcd for $\mathrm{C}_{25} \mathrm{H}_{28} \mathrm{~F}_{3} \mathrm{~N}_{2} \mathrm{O}$ 429.2148; found: 429.2161.

\section{Radical Trapping and Isotope Labeling Experiments}

\section{Radical Trapping Experiment}<smiles>C=CCCN(C#N)C(=O)C(c1ccccc1)c1ccccc1</smiles>

1a<smiles>CCC1CCN2C(=O)C(c3ccccc3)(c3ccccc3)N=C12</smiles>

$3 a$<smiles>CC1(C)CCCC(C)(C)N1OC(F)(F)F</smiles>

TEMPO- $\mathrm{CF}_{3}$

3a: trace, $<5 \%$ conv (1a);

TEMPO-CF 3 : $35 \%$ (based on ${ }^{19} \mathrm{~F} \mathrm{NMR}$ using $\mathrm{PhCF}_{3}$ as the internal standard)

TEMPO-CF $3:{ }^{19} \mathrm{~F}$ NMR $\left(376 \mathrm{MHz}, \mathrm{CDCl}_{3}\right) \delta-55.71$. HRMS m/z (ESI) calcd for $\mathrm{C}_{10} \mathrm{H}_{19} \mathrm{~F}_{3} \mathrm{~N}_{2} \mathrm{O}[\mathrm{M}+\mathrm{H}]^{+} 226.1413$, found 226.1416. The ${ }^{19} \mathrm{~F}$ NMR spectra data were in accordance with the reported data of the known literatures. ${ }^{5}$

\section{Isotope Labeling Experiments}

1) Intermolecular KIE

a) One-pot KIE<smiles>C=CCC(C)(C)C(=O)N(C#N)CCc1ccccc1</smiles><smiles>[2H]C([2H])(CN(C)C(=O)C(C)(C)C/C=C\CC(=O)O[SnH3])c1ccccc1</smiles>

(0.11 mmol, 91\% D)

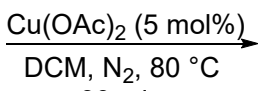

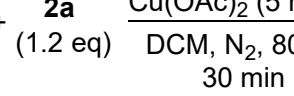

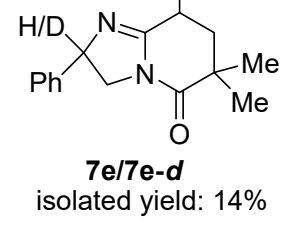

$$
\mathrm{K}_{\mathrm{H}} / \mathrm{K}_{\mathrm{D}}=1
$$

b) Parallel KIE

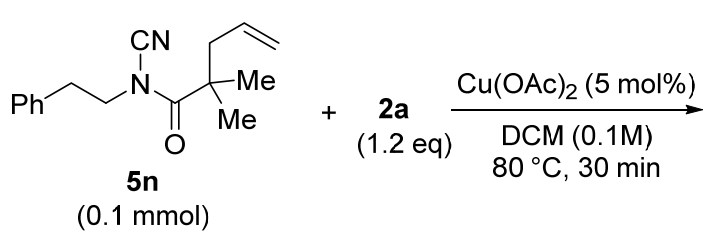<smiles>CC1(C)CC(CC(F)(F)F)C2=NC(c3ccccc3)CN2C1=O</smiles>
isolated yield: $15 \%$<smiles>[2H]C([2H])(CN(C#N)C(=O)C(C)(C)CC=C)c1ccccc1</smiles>
(0.1 mmol, $91 \% \mathrm{D}$ ) $\underset{\mathrm{Cu}(\mathrm{OAc})_{2}(5 \mathrm{~mol} \%)}{\stackrel{\mathrm{DCM}(0.1 \mathrm{M})}{\longrightarrow}}$
$80^{\circ} \mathrm{C}, 30 \mathrm{~min}$<smiles>[2H]C1(c2ccccc2)CN2C(=O)C(C)(C)CC(CC(F)(F)F)C2=N1</smiles>
isolated yield: $10 \%,(99 \% \mathrm{D})$ eq-6 eq-7 eq-8

$$
\begin{array}{r}
\mathrm{K}_{\mathrm{H}} / \mathrm{K}_{\mathrm{D}}=1.37 \\
\mathrm{SI}-45
\end{array}
$$




\section{2) Intramolecular KIE}

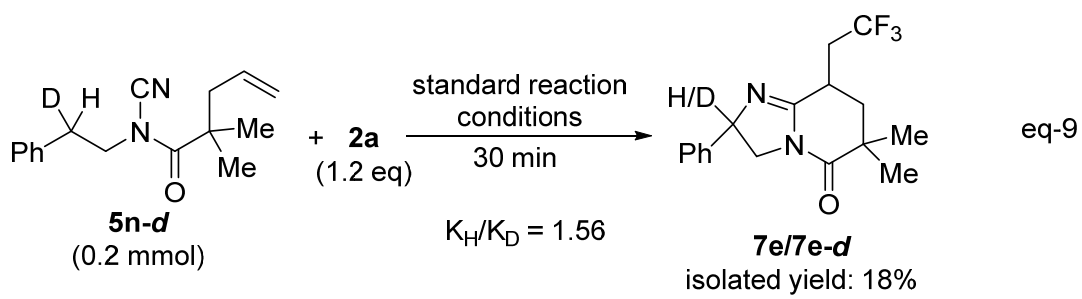

Calculate result is shown as follows: ${ }^{6}$

In the starting material, $\mathrm{H} / \mathrm{D}=0.90 / 1.10 ; \mathrm{D} \%=71 \%$ at product's benzyl position $(\mathrm{H} / \mathrm{D}=0.29 / 0.71)$. From this information, we can deduce the first equation.

$\left(0.90-\mathrm{K}_{\mathrm{H}} * \mathrm{t}\right) /\left(1.10-\mathrm{K}_{\mathrm{D}} * \mathrm{t}\right)=0.29 / 0.71($ Eq. 1$)$

There is another equation about the proton conservation.

$(0.90+1.10)-\left(\mathrm{K}_{\mathrm{H}}+\mathrm{K}_{\mathrm{D}}\right) * \mathrm{t}=0.29+0.71($ Eq. 2$)$

Combine Eq. 1 and 2, we can get the ratio $\mathrm{K}_{\mathrm{H}} / \mathrm{K}_{\mathrm{D}}=1.56$.

To gain some insights into the mechanism of this relay process, several control experiments were carried out (eq5 to eq-9). At first, treatment of $\mathbf{1} \mathbf{a}$ and $\mathbf{2 a}$ with TEMPO afforded no desired product $\mathbf{3} \mathbf{a}$ under the standard reaction conditions. Instead, the corresponding coupling product (TEMPO- $\mathrm{CF}_{3}$ ) could be detected by ${ }^{19} \mathrm{~F}$ NMR analysis with $35 \%$ yield (eq-5). These results indicated that this reaction may undergo a radical pathway and a free $\mathrm{CF}_{3}$ radical was generated to initiate this radical relay transformation. Then, a series of deuterium labeling investigations were conducted. However, whether intermolecular (one-pot KIE: $\mathrm{K}_{\mathrm{H}} / \mathrm{K}_{\mathrm{D}}=1$; parallel KIE: $\mathrm{K}_{\mathrm{H}} / \mathrm{K}_{\mathrm{D}}=1.37$ ) or intramolecular kinetic isotope effect $(\mathrm{KIE}=1.56)$ experimental results did not indicate that the HAT process was involved in the rate determining step (eq-6 to eq-9).

\section{Spectral data for N-cyano-2,2-dimethyl-N-(2-phenylethyl-2-d)pent-4-enamide (5n-d)}<smiles>[2H]C(CN(C)C(=O)C(C)(C)CC=C)c1ccccc1</smiles>

Purified by column chromatography (silica gel, EtOAc/Petroleum ether: 1/60) to afford the title compound (65\% yield, $167 \mathrm{mg}$ ) as colorless oil. ${ }^{1} \mathrm{H} \mathrm{NMR}\left(400 \mathrm{MHz}, \mathrm{CDCl}_{3}\right) \delta 7.38-7.33(\mathrm{~m}, 2 \mathrm{H}), 7.32-7.29(\mathrm{~m}, 1 \mathrm{H}), 7.29-7.25$ $(\mathrm{m}, 2 \mathrm{H}), 5.73-5.60(\mathrm{~m}, 1 \mathrm{H}), 5.22-5.08(\mathrm{~m}, 2 \mathrm{H}), 3.91-3.83(\mathrm{~m}, 2 \mathrm{H}), 3.03-2.94(\mathrm{~m}, 1 \mathrm{H}), 2.55(\mathrm{~d}, J=7.8 \mathrm{~Hz}, 2 \mathrm{H})$, 1.37 (s, 6H). ${ }^{13} \mathrm{C}$ NMR (100 MHz, $\left.\mathrm{CDCl}_{3}\right) \delta$ 175.8, 133.0, 129.1, 128.9, 127.2, 119.0, 111.4, 49.6, 44.9, 43.6, 33.8, 24.9. HRMS (ESI) m/z: $[\mathrm{M}+\mathrm{H}]^{+}$calcd for $\mathrm{C}_{16} \mathrm{H}_{20} \mathrm{DN}_{2} \mathrm{O} 258.1711$, found 258.1703 .

\section{Spectral data for N-cyano-2,2-dimethyl-N-(2-phenylethyl-2,2-d2)pent-4-enamide (5n-d $)$}<smiles>[2H]C([2H])(CN(C)C(=O)C(C)(C)CC=C)c1ccccc1</smiles>

Purified by column chromatography (silica gel, EtOAc/Petroleum ether: 1/40) to afford the title compound (42\% yield, $109 \mathrm{mg} .91 \% \mathrm{D})$ as yellowish oil. ${ }^{1} \mathrm{H} \mathrm{NMR}\left(400 \mathrm{MHz}, \mathrm{CDCl}_{3}\right) \delta 7.35-7.29(\mathrm{~m}, 2 \mathrm{H}), 7.28-7.20(\mathrm{~m}, 3 \mathrm{H})$, $5.62(\mathrm{ddt}, J=17.3,10.1,7.3 \mathrm{~Hz}, 1 \mathrm{H}), 5.19-5.03(\mathrm{~m}, 2 \mathrm{H}), 3.82(\mathrm{~s}, 2 \mathrm{H}), 2.50(\mathrm{~d}, J=7.4 \mathrm{~Hz}, 2 \mathrm{H}), 1.32(\mathrm{~s}, 6 \mathrm{H}) .{ }^{13} \mathrm{C}$ NMR $\left(125 \mathrm{MHz}, \mathrm{CDCl}_{3}\right) \delta 175.7,136.5,132.9,129.0,128.8,127.2,119.0,111.4,49.5,44.9,43.5,24.9$. HRMS (ESI) $\mathrm{m} / \mathrm{z}:[\mathrm{M}+\mathrm{H}]^{+}$calcd for $\mathrm{C}_{16} \mathrm{H}_{19} \mathrm{D}_{2} \mathrm{~N}_{2} \mathrm{O} 259.1774$, found 259.1770 . 


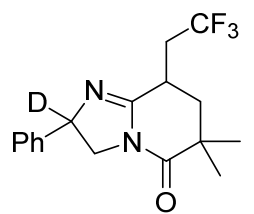

Purified by column chromatography (silica gel, EtOAc/Petroleum ether: $1 / 8$ ) to afford the title compound (11\% yield, $4 \mathrm{mg}, \mathrm{dr}=1: 1)$ as colorless oil. ${ }^{1} \mathrm{H}$ NMR $\left(500 \mathrm{MHz}, \mathrm{CDCl}_{3}\right) \delta 7.39-7.34(\mathrm{~m}, 4 \mathrm{H}), 7.31-7.27(\mathrm{~m}, 2 \mathrm{H}), 7.25-7.20$ $(\mathrm{m}, 4 \mathrm{H}), 4.25(\mathrm{~d}, J=11.7 \mathrm{~Hz}, 1 \mathrm{H}), 4.20(\mathrm{~d}, J=11.7 \mathrm{~Hz}, 1 \mathrm{H}), 3.73(\mathrm{~d}, J=11.6 \mathrm{~Hz}, 1 \mathrm{H}), 3.70(\mathrm{~d}, J=11.7 \mathrm{~Hz}, 1 \mathrm{H})$, $3.38-3.25(\mathrm{~m}, 2 \mathrm{H}), 3.16-3.06(\mathrm{~m}, 2 \mathrm{H}), 2.37-2.20(\mathrm{~m}, 2 \mathrm{H}), 2.13-2.04(\mathrm{~m}, 2 \mathrm{H}), 1.75(\mathrm{t}, J=13.5 \mathrm{~Hz}, 2 \mathrm{H}), 1.32$ $(\mathrm{s}, 9 \mathrm{H}), 1.31(\mathrm{~s}, 3 \mathrm{H}) .{ }^{13} \mathrm{C}$ NMR $\left(100 \mathrm{MHz}, \mathrm{CDCl}_{3}\right) \delta 173.5,173.4,159.4,159.0,142.2,142.0,129.0,128.9,127.9$, $127.8,126.7$ (q, $J=276.9 \mathrm{~Hz}), 126.6,126.5,51.8,51.7,39.7,39.5,39.1,39.0,35.7$ (q, $J=28.9 \mathrm{~Hz}), 35.5$ (q, $J=29.1$ $\mathrm{Hz}), 29.9,28.3(\mathrm{q}, J=5.2 \mathrm{~Hz}), 26.3,26.2,25.3,25.2 .{ }^{19} \mathrm{~F} \mathrm{NMR}\left(376 \mathrm{MHz}, \mathrm{CDCl}_{3}\right) \delta-63.47(\mathrm{t}, J=11.5 \mathrm{~Hz}) . \mathrm{HRMS}$ (ESI) $\mathrm{m} / \mathrm{z}:[\mathrm{M}+\mathrm{H}]^{+}$calcd for $\mathrm{C}_{17} \mathrm{H}_{19} \mathrm{DF}_{3} \mathrm{~N}_{2} \mathrm{O} 326.1585$, found 326.1578 .

\section{References}

(1) (a) Snider, B. B.; Zeng, H., Total Synthesis of (-)-Fumiquinazolines A, B, C, E, H, and I. Approaches to the Synthesis of Fiscalin A. J. Org. Chem. 2003, 68, 545-563. (b) Toumi, M.; Couty, F.; Marrot, J.; Evano, G., Total Synthesis of Chaetominine. Org. Lett. 2008, 10, 5027-5030. (c) Koyama, N.; Inoue, Y.; Sekine, M.; Hayakawa, Y.; Homma, H.; Ōmura, S.; Tomoda, H., Relative and Absolute Stereochemistry of Quinadoline B, an Inhibitor of Lipid Droplet Synthesis in Macrophages. Org. Lett. 2008, 10, 5273-5276. (d) Aeberli, P.; Eden, P.; Gogerty, J. H.; Houlihan, W. J.; Penberthy, C., 5-Aryl-2,3-dihydro-5H-imidazo[2,1-a]isoindol-5-ols. Novel class of anorectic agents. J. Med. Chem. 1975, 18, 177-182. (e) Kwon, T.-W.; Kwon, P.-S.; Song, S.-J.; Park, M.-S.; Choi, J.-W., Synthesis and Anticonvulsant Evaluation of 5-Vinyl-pyrrolidin-2-ones: A Potential GABA-AT Inhibitor. Bull. Korean Chem. Soc. 2002, $23,917-921$.

(2) Castillo, J.-C.; Orrego-Hernández, J.; Portilla, J., $\mathrm{Cs}_{2} \mathrm{CO}_{3}$-Promoted Direct N-Alkylation: Highly Chemoselective Synthesis of NAlkylated Benzylamines and Anilines. Eur. J. Org. Chem. 2016, 2016, 3824-3835.

(3) Pan, Z.; Wang, S.; Brethorst, J. T.; Douglas, C. J., Palladium and Lewis-Acid-Catalyzed Intramolecular Aminocyanation of Alkenes: Scope, Mechanism, and Stereoselective Alkene Difunctionalizations. J. Am. Chem. Soc. 2018, 140, 3331-3338.

(4) Li, Z.; Jiao, L.; Sun, Y.; He, Z.; Wei, Z.; Liao, W. W., $\mathrm{CF}_{3} \mathrm{SO}_{2} \mathrm{Na}$ as a Bifunctional Reagent: Electrochemical Trifluoromethylation of Alkenes Accompanied by $\mathrm{SO}_{2}$ Insertion to Access Trifluoromethylated Cyclic N-Sulfonylimines. Angew. Chem. Int. Ed. 2020, 59, 7266-7270.

(5) Choi, G.; Lee, G. S.; Park, B.; Kim, D.; Hong, S. H., Direct C(sp3)-H Trifluoromethylation of Unactivated Alkanes Enabled by Multifunctional Trifluoromethyl Copper Complexes. Angew. Chem. Int. Ed. 2021, 60, 5467-5474.

(6) Yang, M.; Su, B.; Wang, Y.; Chen, K.; Jiang, X.; Zhang, Y.-F.; Zhang, X.-S.; Chen, G.; Cheng, Y.; Cao, Z.; Guo, Q.-Y.; Wang, L.; Shi, Z.-J. Silver-catalysed direct amination of unactivated C-H bonds of functionalized molecules. Nat. Commun. 2014, 5, 4707-4712. 


\section{Crystal Data and Structure Refinement for Compounds 3a and 6a}

1)

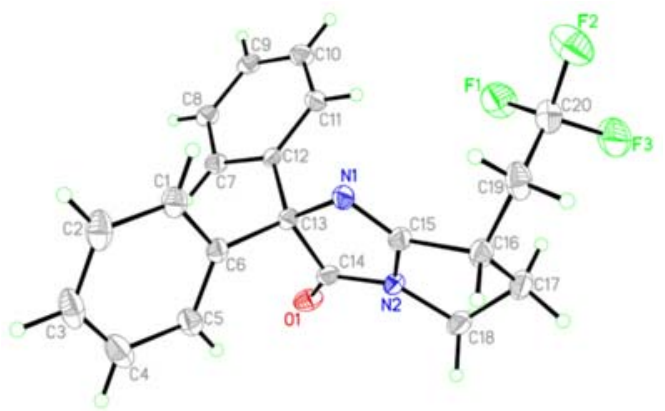<smiles>O=C1N2CCC(CC(F)(F)F)C2=NC1(c1ccccc1)c1ccccc1</smiles>

3a

X-ray crystal structure of compound 3a. Single crystal of 3a was obtained by slow evaporation of Petroleum ether /EtOAc solution. Crystal measurement of 3a was measured on R-Axis RAPID of Rigaku Corporation plus $291.2 \mathrm{k}$. Thermal ellipsoids are set at $30 \%$ probability.

CCDC number: 2122208

Table S-crystal-1. Crystal data and structure refinement for 3a.

Identification code

Empirical formula

Formula weight

Temperature

Wavelength

Crystal system

Space group

Unit cell dimensions

Volume

Z

Density (calculated)

Absorption coefficient

$\mathrm{F}(000)$

Crystal size

Theta range for data collection

Index ranges

Reflections collected

Independent reflections

Completeness to theta $=25.242^{\circ}$

Absorption correction 3a

C20 H17 F3 N2 O

358.35

293(2) K

$0.71073 \AA$

Monoclinic

Pc

$\mathrm{a}=7.6253(3) \AA$ $\alpha=90^{\circ}$.

$\mathrm{b}=12.8963(4) \AA$ $\beta=101.2540(10)^{\circ}$.

$\mathrm{c}=17.8365(6) \AA$ $\gamma=90^{\circ}$.
1720.28(10) $\AA^{3}$

4

$1.384 \mathrm{Mg} / \mathrm{m}^{3}$

$0.109 \mathrm{~mm}^{-1}$

744

$0.190 \times 0.170 \times 0.160 \mathrm{~mm}^{3}$

2.724 to $26.145^{\circ}$.

$-9<=\mathrm{h}<=9,-15<=\mathrm{k}<=15,-22<=1<=22$

57759

$6860[\mathrm{R}(\mathrm{int})=0.0352]$

$99.8 \%$

None 
Refinement method

Data / restraints / parameters

Goodness-of-fit on $\mathrm{F}^{2}$

Final $\mathrm{R}$ indices $[\mathrm{I}>2 \operatorname{sigma}(\mathrm{I})]$

$\mathrm{R}$ indices (all data)

Absolute structure parameter

Extinction coefficient

Largest diff. peak and hole
Full-matrix least-squares on $\mathrm{F}^{2}$

6860 / 878 / 469

1.083

$\mathrm{R} 1=0.0814, \mathrm{wR} 2=0.2192$

$\mathrm{R} 1=0.0925, \mathrm{wR} 2=0.2358$

$-0.16(17)$

$\mathrm{n} / \mathrm{a}$

0.959 and -0.437 e. $\AA^{-3}$

Table S-crystal-2. Atomic coordinates $(\mathrm{x} \mathrm{104})$ and equivalent isotropic displacement parameters $\left(\AA^{2} \mathrm{x} 10^{3}\right)$ for $Y$. $U(e q)$ is defined as one third of the trace of the orthogonalized $U^{i j}$ tensor.

\begin{tabular}{|c|c|c|c|c|}
\hline & $\mathrm{x}$ & $\mathrm{y}$ & z & $\mathrm{U}(\mathrm{eq})$ \\
\hline $\mathrm{F}(1)$ & $11080(7)$ & $9730(4)$ & 1291(3) & $92(2)$ \\
\hline $\mathrm{F}(2)$ & $12872(8)$ & $10988(5)$ & $1681(4)$ & $106(2)$ \\
\hline $\mathrm{F}(3)$ & $11960(8)$ & $10020(6)$ & $2465(3)$ & $108(2)$ \\
\hline $\mathrm{O}(1)$ & $3949(7)$ & $8443(4)$ & $342(3)$ & $60(1)$ \\
\hline $\mathrm{N}(1)$ & $7131(6)$ & 10496(4) & $351(3)$ & $41(1)$ \\
\hline $\mathrm{N}(2)$ & $5985(6)$ & $9459(4)$ & $1155(2)$ & $46(1)$ \\
\hline $\mathrm{C}(1)$ & $4885(9)$ & $11295(5)$ & $-1032(4)$ & $53(1)$ \\
\hline$C(2)$ & $3652(12)$ & $11988(6)$ & $-1436(4)$ & $68(2)$ \\
\hline$C(3)$ & $1879(12)$ & $11935(6)$ & $-1376(5)$ & $74(2)$ \\
\hline$C(4)$ & $1350(11)$ & $11233(7)$ & $-918(5)$ & $74(2)$ \\
\hline$C(5)$ & $2552(8)$ & $10522(5)$ & $-502(4)$ & $53(1)$ \\
\hline$C(6)$ & $4329(7)$ & $10556(4)$ & $-559(3)$ & $39(1)$ \\
\hline$C(7)$ & $5387(8)$ & $8644(4)$ & $-1267(3)$ & $43(1)$ \\
\hline$C(8)$ & $6074(8)$ & $7937(5)$ & $-1726(3)$ & $48(1)$ \\
\hline $\mathrm{C}(9)$ & $7875(9)$ & $7695(5)$ & $-1575(3)$ & $49(1)$ \\
\hline$C(10)$ & 8994(9) & $8169(5)$ & $-973(4)$ & $55(1)$ \\
\hline $\mathrm{C}(11)$ & $8334(7)$ & $8869(5)$ & $-512(3)$ & $45(1)$ \\
\hline $\mathrm{C}(12)$ & $6538(6)$ & $9107(4)$ & $-653(3)$ & $34(1)$ \\
\hline $\mathrm{C}(13)$ & $5748(7)$ & $9833(4)$ & $-124(3)$ & $36(1)$ \\
\hline$C(14)$ & $5031(7)$ & $9135(4)$ & $465(3)$ & $42(1)$ \\
\hline$C(15)$ & $7125(8)$ & $10262(5)$ & $1026(3)$ & $44(1)$ \\
\hline$C(16)$ & $8080(9)$ & $10671(5)$ & 1798(3) & $53(1)$ \\
\hline $\mathrm{C}(17)$ & $7924(10)$ & $9737(6)$ & $2322(4)$ & $62(2)$ \\
\hline $\mathrm{C}(18)$ & $6143(10)$ & $9179(6)$ & $1957(4)$ & $60(2)$ \\
\hline
\end{tabular}




\begin{tabular}{|c|c|c|c|c|}
\hline$C(19)$ & $9877(11)$ & 11191(6) & $1794(5)$ & $67(2)$ \\
\hline$C(20)$ & $11403(10)$ & $10502(6)$ & 1795(4) & $64(2)$ \\
\hline $\mathrm{F}(4)$ & $8019(12)$ & $3552(10)$ & $8668(5)$ & $164(4)$ \\
\hline $\mathrm{F}(5)$ & $10509(9)$ & $4140(7)$ & $9353(5)$ & $121(2)$ \\
\hline $\mathrm{F}(6)$ & $8725(10)$ & $3278(6)$ & $9868(5)$ & $120(2)$ \\
\hline $\mathrm{O}(2)$ & $1179(6)$ & 6542(4) & 7781(3) & $56(1)$ \\
\hline $\mathrm{N}(3)$ & $4610(6)$ & 4599(4) & $7955(3)$ & $47(1)$ \\
\hline $\mathrm{N}(4)$ & $3384(7)$ & 5733(4) & $8663(3)$ & $46(1)$ \\
\hline $\mathrm{C}(21)$ & 5773(9) & $5877(5)$ & 6901(3) & $49(1)$ \\
\hline$C(22)$ & $6467(11)$ & $6495(6)$ & $6403(4)$ & $64(2)$ \\
\hline $\mathrm{C}(23)$ & $5347(11)$ & $7057(6)$ & $5845(4)$ & $65(2)$ \\
\hline$C(24)$ & $3567(12)$ & 6981(6) & $5788(4)$ & $68(2)$ \\
\hline$C(25)$ & $2805(10)$ & $6352(5)$ & $6293(4)$ & $58(1)$ \\
\hline$C(26)$ & $3934(8)$ & 5794(4) & $6850(3)$ & $42(1)$ \\
\hline $\mathrm{C}(27)$ & $2317(9)$ & $3633(5)$ & $6563(4)$ & $55(1)$ \\
\hline$C(28)$ & $1082(10)$ & $2898(5)$ & $6189(4)$ & $63(2)$ \\
\hline$C(29)$ & $-654(10)$ & $2900(5)$ & $6304(5)$ & $62(2)$ \\
\hline $\mathrm{C}(30)$ & $-1132(10)$ & $3591(6)$ & $6795(5)$ & $64(2)$ \\
\hline$C(31)$ & $27(9)$ & $4332(5)$ & $7152(4)$ & $53(1)$ \\
\hline$C(32)$ & $1772(8)$ & $4369(4)$ & $7038(3)$ & $41(1)$ \\
\hline$C(33)$ & $3145(7)$ & $5152(4)$ & $7422(3)$ & $40(1)$ \\
\hline $\mathrm{C}(34)$ & $2369(7)$ & 5912(4) & 7951(3) & $43(1)$ \\
\hline$C(35)$ & $4619(9)$ & $4975(5)$ & $8608(3)$ & $50(1)$ \\
\hline$C(36)$ & $5798(11)$ & $4858(7)$ & $9390(4)$ & $73(2)$ \\
\hline$C(37)$ & $5273(16)$ & $5754(9)$ & $9844(6)$ & $96(2)$ \\
\hline $\mathrm{C}(38)$ & $3492(12)$ & $6169(6)$ & $9422(4)$ & $66(2)$ \\
\hline $\mathrm{C}(39)$ & $7732(14)$ & 4942(8) & $9448(7)$ & $93(2)$ \\
\hline $\mathrm{C}(40)$ & 8761(14) & $4058(8)$ & $9370(7)$ & $88(2)$ \\
\hline
\end{tabular}

Table S-crystal-3. Bond lengths $[\AA]$ and angles $\left[{ }^{\circ}\right]$ for $\mathrm{Y}$.

\begin{tabular}{ll}
\hline $\mathrm{F}(1)-\mathrm{C}(20)$ & $1.331(9)$ \\
$\mathrm{F}(2)-\mathrm{C}(20)$ & $1.333(10)$ \\
$\mathrm{F}(3)-\mathrm{C}(20)$ & $1.341(9)$ \\
$\mathrm{O}(1)-\mathrm{C}(14)$ & $1.206(7)$ \\
$\mathrm{N}(1)-\mathrm{C}(15)$ & $1.243(7)$ \\
$\mathrm{N}(1)-\mathrm{C}(13)$ & $1.486(7)$
\end{tabular}




\begin{tabular}{|c|c|}
\hline $\mathrm{N}(2)-\mathrm{C}(14)$ & $1.368(7)$ \\
\hline $\mathrm{N}(2)-\mathrm{C}(15)$ & $1.400(8)$ \\
\hline $\mathrm{N}(2)-\mathrm{C}(18)$ & $1.457(8)$ \\
\hline $\mathrm{C}(1)-\mathrm{C}(2)$ & $1.392(10)$ \\
\hline$C(1)-C(6)$ & $1.393(9)$ \\
\hline $\mathrm{C}(1)-\mathrm{H}(1)$ & 0.9300 \\
\hline$C(2)-C(3)$ & $1.378(13)$ \\
\hline $\mathrm{C}(2)-\mathrm{H}(2)$ & 0.9300 \\
\hline$C(3)-C(4)$ & $1.333(14)$ \\
\hline $\mathrm{C}(3)-\mathrm{H}(3)$ & 0.9300 \\
\hline$C(4)-C(5)$ & $1.402(10)$ \\
\hline $\mathrm{C}(4)-\mathrm{H}(4)$ & 0.9300 \\
\hline$C(5)-C(6)$ & $1.379(9)$ \\
\hline $\mathrm{C}(5)-\mathrm{H}(5)$ & 0.9300 \\
\hline$C(6)-C(13)$ & $1.521(7)$ \\
\hline$C(7)-C(8)$ & $1.395(8)$ \\
\hline$C(7)-C(12)$ & $1.396(8)$ \\
\hline $\mathrm{C}(7)-\mathrm{H}(7)$ & 0.9300 \\
\hline$C(8)-C(9)$ & $1.383(9)$ \\
\hline $\mathrm{C}(8)-\mathrm{H}(8)$ & 0.9300 \\
\hline$C(9)-C(10)$ & $1.378(10)$ \\
\hline $\mathrm{C}(9)-\mathrm{H}(9)$ & 0.9300 \\
\hline$C(10)-C(11)$ & $1.381(9)$ \\
\hline $\mathrm{C}(10)-\mathrm{H}(10)$ & 0.9300 \\
\hline$C(11)-C(12)$ & $1.378(7)$ \\
\hline $\mathrm{C}(11)-\mathrm{H}(11)$ & 0.9300 \\
\hline$C(12)-C(13)$ & $1.535(7)$ \\
\hline $\mathrm{C}(13)-\mathrm{C}(14)$ & $1.561(7)$ \\
\hline$C(15)-C(16)$ & $1.520(8)$ \\
\hline$C(16)-C(19)$ & $1.527(11)$ \\
\hline$C(16)-C(17)$ & $1.544(10)$ \\
\hline $\mathrm{C}(16)-\mathrm{H}(16)$ & 0.9800 \\
\hline$C(17)-C(18)$ & $1.562(11)$ \\
\hline $\mathrm{C}(17)-\mathrm{H}(17 \mathrm{~A})$ & 0.9700 \\
\hline $\mathrm{C}(17)-\mathrm{H}(17 \mathrm{~B})$ & 0.9700 \\
\hline $\mathrm{C}(18)-\mathrm{H}(18 \mathrm{~A})$ & 0.9700 \\
\hline $\mathrm{C}(18)-\mathrm{H}(18 \mathrm{~B})$ & 0.9700 \\
\hline $\mathrm{C}(19)-\mathrm{C}(20)$ & $1.464(11)$ \\
\hline
\end{tabular}




\begin{tabular}{|c|c|}
\hline $\mathrm{C}(19)-\mathrm{H}(19 \mathrm{~A})$ & 0.9700 \\
\hline $\mathrm{C}(19)-\mathrm{H}(19 \mathrm{~B})$ & 0.9700 \\
\hline $\mathrm{F}(4)-\mathrm{C}(40)$ & $1.428(15)$ \\
\hline $\mathrm{F}(5)-\mathrm{C}(40)$ & $1.344(12)$ \\
\hline $\mathrm{F}(6)-\mathrm{C}(40)$ & $1.346(12)$ \\
\hline $\mathrm{O}(2)-\mathrm{C}(34)$ & $1.211(7)$ \\
\hline $\mathrm{N}(3)-\mathrm{C}(35)$ & $1.260(8)$ \\
\hline $\mathrm{N}(3)-\mathrm{C}(33)$ & $1.499(7)$ \\
\hline N(4)-C(34) & $1.371(7)$ \\
\hline $\mathrm{N}(4)-\mathrm{C}(35)$ & $1.374(8)$ \\
\hline $\mathrm{N}(4)-\mathrm{C}(38)$ & $1.453(8)$ \\
\hline$C(21)-C(22)$ & $1.374(9)$ \\
\hline$C(21)-C(26)$ & $1.392(9)$ \\
\hline $\mathrm{C}(21)-\mathrm{H}(21)$ & 0.9300 \\
\hline$C(22)-C(23)$ & $1.384(12)$ \\
\hline $\mathrm{C}(22)-\mathrm{H}(22)$ & 0.9300 \\
\hline $\mathrm{C}(23)-\mathrm{C}(24)$ & $1.345(12)$ \\
\hline $\mathrm{C}(23)-\mathrm{H}(23)$ & 0.9300 \\
\hline$C(24)-C(25)$ & $1.419(10)$ \\
\hline $\mathrm{C}(24)-\mathrm{H}(24)$ & 0.9300 \\
\hline$C(25)-C(26)$ & $1.382(9)$ \\
\hline $\mathrm{C}(25)-\mathrm{H}(25)$ & 0.9300 \\
\hline$C(26)-C(33)$ & $1.525(7)$ \\
\hline $\mathrm{C}(27)-\mathrm{C}(32)$ & $1.389(8)$ \\
\hline $\mathrm{C}(27)-\mathrm{C}(28)$ & $1.409(10)$ \\
\hline $\mathrm{C}(27)-\mathrm{H}(27)$ & 0.9300 \\
\hline $\mathrm{C}(28)-\mathrm{C}(29)$ & $1.379(11)$ \\
\hline $\mathrm{C}(28)-\mathrm{H}(28)$ & 0.9300 \\
\hline C(29)-C(30) & $1.349(10)$ \\
\hline $\mathrm{C}(29)-\mathrm{H}(29)$ & 0.9300 \\
\hline$C(30)-C(31)$ & $1.370(10)$ \\
\hline $\mathrm{C}(30)-\mathrm{H}(30)$ & 0.9300 \\
\hline$C(31)-C(32)$ & $1.385(9)$ \\
\hline $\mathrm{C}(31)-\mathrm{H}(31)$ & 0.9300 \\
\hline$C(32)-C(33)$ & $1.517(8)$ \\
\hline C(33)-C(34) & $1.556(7)$ \\
\hline$C(35)-C(36)$ & $1.513(9)$ \\
\hline$C(36)-C(39)$ & $1.462(14)$ \\
\hline
\end{tabular}




\begin{tabular}{|c|c|}
\hline$C(36)-C(37)$ & $1.509(15)$ \\
\hline $\mathrm{C}(36)-\mathrm{H}(36)$ & 0.9800 \\
\hline $\mathrm{C}(37)-\mathrm{C}(38)$ & $1.516(14)$ \\
\hline $\mathrm{C}(37)-\mathrm{H}(37 \mathrm{~A})$ & 0.9700 \\
\hline $\mathrm{C}(37)-\mathrm{H}(37 \mathrm{~B})$ & 0.9700 \\
\hline $\mathrm{C}(38)-\mathrm{H}(38 \mathrm{~A})$ & 0.9700 \\
\hline $\mathrm{C}(38)-\mathrm{H}(38 \mathrm{~B})$ & 0.9700 \\
\hline$C(39)-C(40)$ & $1.406(16)$ \\
\hline $\mathrm{C}(39)-\mathrm{H}(39 \mathrm{~A})$ & 0.9700 \\
\hline C(39)-H(39B) & 0.9700 \\
\hline $\mathrm{C}(15)-\mathrm{N}(1)-\mathrm{C}(13)$ & $106.0(5)$ \\
\hline $\mathrm{C}(14)-\mathrm{N}(2)-\mathrm{C}(15)$ & $108.4(4)$ \\
\hline $\mathrm{C}(14)-\mathrm{N}(2)-\mathrm{C}(18)$ & $137.4(6)$ \\
\hline$C(15)-N(2)-C(18)$ & $114.2(5)$ \\
\hline $\mathrm{C}(2)-\mathrm{C}(1)-\mathrm{C}(6)$ & $119.9(7)$ \\
\hline $\mathrm{C}(2)-\mathrm{C}(1)-\mathrm{H}(1)$ & 120.0 \\
\hline $\mathrm{C}(6)-\mathrm{C}(1)-\mathrm{H}(1)$ & 120.0 \\
\hline$C(3)-C(2)-C(1)$ & $120.1(8)$ \\
\hline $\mathrm{C}(3)-\mathrm{C}(2)-\mathrm{H}(2)$ & 120.0 \\
\hline $\mathrm{C}(1)-\mathrm{C}(2)-\mathrm{H}(2)$ & 120.0 \\
\hline $\mathrm{C}(4)-\mathrm{C}(3)-\mathrm{C}(2)$ & $120.0(7)$ \\
\hline $\mathrm{C}(4)-\mathrm{C}(3)-\mathrm{H}(3)$ & 120.0 \\
\hline $\mathrm{C}(2)-\mathrm{C}(3)-\mathrm{H}(3)$ & 120.0 \\
\hline C(3)-C(4)-C(5) & $121.6(8)$ \\
\hline $\mathrm{C}(3)-\mathrm{C}(4)-\mathrm{H}(4)$ & 119.2 \\
\hline $\mathrm{C}(5)-\mathrm{C}(4)-\mathrm{H}(4)$ & 119.2 \\
\hline$C(6)-C(5)-C(4)$ & $119.4(7)$ \\
\hline $\mathrm{C}(6)-\mathrm{C}(5)-\mathrm{H}(5)$ & 120.3 \\
\hline $\mathrm{C}(4)-\mathrm{C}(5)-\mathrm{H}(5)$ & 120.3 \\
\hline$C(5)-C(6)-C(1)$ & $119.0(5)$ \\
\hline$C(5)-C(6)-C(13)$ & $123.7(5)$ \\
\hline$C(1)-C(6)-C(13)$ & $117.3(5)$ \\
\hline$C(8)-C(7)-C(12)$ & $119.3(5)$ \\
\hline $\mathrm{C}(8)-\mathrm{C}(7)-\mathrm{H}(7)$ & 120.4 \\
\hline $\mathrm{C}(12)-\mathrm{C}(7)-\mathrm{H}(7)$ & 120.4 \\
\hline$C(9)-C(8)-C(7)$ & $120.4(5)$ \\
\hline $\mathrm{C}(9)-\mathrm{C}(8)-\mathrm{H}(8)$ & 119.8 \\
\hline
\end{tabular}




\begin{tabular}{|c|c|}
\hline $\mathrm{C}(7)-\mathrm{C}(8)-\mathrm{H}(8)$ & 119.8 \\
\hline$C(10)-C(9)-C(8)$ & $119.5(5)$ \\
\hline $\mathrm{C}(10)-\mathrm{C}(9)-\mathrm{H}(9)$ & 120.3 \\
\hline C(8)-C(9)-H(9) & 120.3 \\
\hline$C(9)-C(10)-C(11)$ & $120.8(5)$ \\
\hline $\mathrm{C}(9)-\mathrm{C}(10)-\mathrm{H}(10)$ & 119.6 \\
\hline $\mathrm{C}(11)-\mathrm{C}(10)-\mathrm{H}(10)$ & 119.6 \\
\hline$C(12)-C(11)-C(10)$ & $120.1(5)$ \\
\hline $\mathrm{C}(12)-\mathrm{C}(11)-\mathrm{H}(11)$ & 120.0 \\
\hline $\mathrm{C}(10)-\mathrm{C}(11)-\mathrm{H}(11)$ & 120.0 \\
\hline $\mathrm{C}(11)-\mathrm{C}(12)-\mathrm{C}(7)$ & $119.9(5)$ \\
\hline $\mathrm{C}(11)-\mathrm{C}(12)-\mathrm{C}(13)$ & $121.3(4)$ \\
\hline$C(7)-C(12)-C(13)$ & $118.8(4)$ \\
\hline $\mathrm{N}(1)-\mathrm{C}(13)-\mathrm{C}(6)$ & $107.1(4)$ \\
\hline $\mathrm{N}(1)-\mathrm{C}(13)-\mathrm{C}(12)$ & $112.4(4)$ \\
\hline $\mathrm{C}(6)-\mathrm{C}(13)-\mathrm{C}(12)$ & $112.6(4)$ \\
\hline $\mathrm{N}(1)-\mathrm{C}(13)-\mathrm{C}(14)$ & $104.6(4)$ \\
\hline $\mathrm{C}(6)-\mathrm{C}(13)-\mathrm{C}(14)$ & $112.9(4)$ \\
\hline$C(12)-C(13)-C(14)$ & $107.1(4)$ \\
\hline $\mathrm{O}(1)-\mathrm{C}(14)-\mathrm{N}(2)$ & $127.9(5)$ \\
\hline $\mathrm{O}(1)-\mathrm{C}(14)-\mathrm{C}(13)$ & $128.4(5)$ \\
\hline $\mathrm{N}(2)-\mathrm{C}(14)-\mathrm{C}(13)$ & $103.6(5)$ \\
\hline $\mathrm{N}(1)-\mathrm{C}(15)-\mathrm{N}(2)$ & $117.3(5)$ \\
\hline $\mathrm{N}(1)-\mathrm{C}(15)-\mathrm{C}(16)$ & $134.6(6)$ \\
\hline $\mathrm{N}(2)-\mathrm{C}(15)-\mathrm{C}(16)$ & $108.1(5)$ \\
\hline$C(15)-C(16)-C(19)$ & $114.8(6)$ \\
\hline$C(15)-C(16)-C(17)$ & $101.4(5)$ \\
\hline$C(19)-C(16)-C(17)$ & $121.4(6)$ \\
\hline $\mathrm{C}(15)-\mathrm{C}(16)-\mathrm{H}(16)$ & 106.0 \\
\hline$C(19)-C(16)-H(16)$ & 106.0 \\
\hline $\mathrm{C}(17)-\mathrm{C}(16)-\mathrm{H}(16)$ & 106.0 \\
\hline$C(16)-C(17)-C(18)$ & $105.7(5)$ \\
\hline $\mathrm{C}(16)-\mathrm{C}(17)-\mathrm{H}(17 \mathrm{~A})$ & 110.6 \\
\hline $\mathrm{C}(18)-\mathrm{C}(17)-\mathrm{H}(17 \mathrm{~A})$ & 110.6 \\
\hline $\mathrm{C}(16)-\mathrm{C}(17)-\mathrm{H}(17 \mathrm{~B})$ & 110.6 \\
\hline $\mathrm{C}(18)-\mathrm{C}(17)-\mathrm{H}(17 \mathrm{~B})$ & 110.6 \\
\hline $\mathrm{H}(17 \mathrm{~A})-\mathrm{C}(17)-\mathrm{H}(17 \mathrm{~B})$ & 108.7 \\
\hline $\mathrm{N}(2)-\mathrm{C}(18)-\mathrm{C}(17)$ & $101.2(6)$ \\
\hline
\end{tabular}




\begin{tabular}{|c|c|}
\hline $\mathrm{N}(2)-\mathrm{C}(18)-\mathrm{H}(18 \mathrm{~A})$ & 111.5 \\
\hline $\mathrm{C}(17)-\mathrm{C}(18)-\mathrm{H}(18 \mathrm{~A})$ & 111.5 \\
\hline $\mathrm{N}(2)-\mathrm{C}(18)-\mathrm{H}(18 \mathrm{~B})$ & 111.5 \\
\hline $\mathrm{C}(17)-\mathrm{C}(18)-\mathrm{H}(18 \mathrm{~B})$ & 111.5 \\
\hline $\mathrm{H}(18 \mathrm{~A})-\mathrm{C}(18)-\mathrm{H}(18 \mathrm{~B})$ & 109.4 \\
\hline$C(20)-C(19)-C(16)$ & $116.6(6)$ \\
\hline $\mathrm{C}(20)-\mathrm{C}(19)-\mathrm{H}(19 \mathrm{~A})$ & 108.1 \\
\hline $\mathrm{C}(16)-\mathrm{C}(19)-\mathrm{H}(19 \mathrm{~A})$ & 108.1 \\
\hline $\mathrm{C}(20)-\mathrm{C}(19)-\mathrm{H}(19 \mathrm{~B})$ & 108.1 \\
\hline $\mathrm{C}(16)-\mathrm{C}(19)-\mathrm{H}(19 \mathrm{~B})$ & 108.1 \\
\hline $\mathrm{H}(19 \mathrm{~A})-\mathrm{C}(19)-\mathrm{H}(19 \mathrm{~B})$ & 107.3 \\
\hline $\mathrm{F}(1)-\mathrm{C}(20)-\mathrm{F}(2)$ & $107.4(7)$ \\
\hline$F(1)-C(20)-F(3)$ & $103.9(7)$ \\
\hline$F(2)-C(20)-F(3)$ & $103.0(6)$ \\
\hline $\mathrm{F}(1)-\mathrm{C}(20)-\mathrm{C}(19)$ & $114.3(6)$ \\
\hline $\mathrm{F}(2)-\mathrm{C}(20)-\mathrm{C}(19)$ & $114.0(7)$ \\
\hline $\mathrm{F}(3)-\mathrm{C}(20)-\mathrm{C}(19)$ & $113.3(7)$ \\
\hline $\mathrm{C}(35)-\mathrm{N}(3)-\mathrm{C}(33)$ & $105.8(5)$ \\
\hline$C(34)-N(4)-C(35)$ & $108.9(5)$ \\
\hline $\mathrm{C}(34)-\mathrm{N}(4)-\mathrm{C}(38)$ & $135.8(6)$ \\
\hline$C(35)-N(4)-C(38)$ & $115.2(6)$ \\
\hline$C(22)-C(21)-C(26)$ & $120.9(6)$ \\
\hline $\mathrm{C}(22)-\mathrm{C}(21)-\mathrm{H}(21)$ & 119.6 \\
\hline $\mathrm{C}(26)-\mathrm{C}(21)-\mathrm{H}(21)$ & 119.6 \\
\hline$C(21)-C(22)-C(23)$ & $120.5(7)$ \\
\hline $\mathrm{C}(21)-\mathrm{C}(22)-\mathrm{H}(22)$ & 119.8 \\
\hline $\mathrm{C}(23)-\mathrm{C}(22)-\mathrm{H}(22)$ & 119.8 \\
\hline $\mathrm{C}(24)-\mathrm{C}(23)-\mathrm{C}(22)$ & $119.2(6)$ \\
\hline $\mathrm{C}(24)-\mathrm{C}(23)-\mathrm{H}(23)$ & 120.4 \\
\hline $\mathrm{C}(22)-\mathrm{C}(23)-\mathrm{H}(23)$ & 120.4 \\
\hline$C(23)-C(24)-C(25)$ & $121.7(7)$ \\
\hline $\mathrm{C}(23)-\mathrm{C}(24)-\mathrm{H}(24)$ & 119.1 \\
\hline $\mathrm{C}(25)-\mathrm{C}(24)-\mathrm{H}(24)$ & 119.1 \\
\hline$C(26)-C(25)-C(24)$ & $118.7(7)$ \\
\hline $\mathrm{C}(26)-\mathrm{C}(25)-\mathrm{H}(25)$ & 120.7 \\
\hline $\mathrm{C}(24)-\mathrm{C}(25)-\mathrm{H}(25)$ & 120.7 \\
\hline$C(25)-C(26)-C(21)$ & $119.0(6)$ \\
\hline$C(25)-C(26)-C(33)$ & $119.4(5)$ \\
\hline
\end{tabular}




\begin{tabular}{|c|c|}
\hline $\mathrm{C}(21)-\mathrm{C}(26)-\mathrm{C}(33)$ & $121.5(5)$ \\
\hline $\mathrm{C}(32)-\mathrm{C}(27)-\mathrm{C}(28)$ & $119.8(6)$ \\
\hline $\mathrm{C}(32)-\mathrm{C}(27)-\mathrm{H}(27)$ & 120.1 \\
\hline $\mathrm{C}(28)-\mathrm{C}(27)-\mathrm{H}(27)$ & 120.1 \\
\hline$C(29)-C(28)-C(27)$ & $120.1(6)$ \\
\hline $\mathrm{C}(29)-\mathrm{C}(28)-\mathrm{H}(28)$ & 120.0 \\
\hline $\mathrm{C}(27)-\mathrm{C}(28)-\mathrm{H}(28)$ & 120.0 \\
\hline $\mathrm{C}(30)-\mathrm{C}(29)-\mathrm{C}(28)$ & 119.1(7) \\
\hline $\mathrm{C}(30)-\mathrm{C}(29)-\mathrm{H}(29)$ & 120.5 \\
\hline $\mathrm{C}(28)-\mathrm{C}(29)-\mathrm{H}(29)$ & 120.5 \\
\hline $\mathrm{C}(29)-\mathrm{C}(30)-\mathrm{C}(31)$ & $122.0(7)$ \\
\hline $\mathrm{C}(29)-\mathrm{C}(30)-\mathrm{H}(30)$ & 119.0 \\
\hline $\mathrm{C}(31)-\mathrm{C}(30)-\mathrm{H}(30)$ & 119.0 \\
\hline $\mathrm{C}(30)-\mathrm{C}(31)-\mathrm{C}(32)$ & $120.5(6)$ \\
\hline $\mathrm{C}(30)-\mathrm{C}(31)-\mathrm{H}(31)$ & 119.8 \\
\hline $\mathrm{C}(32)-\mathrm{C}(31)-\mathrm{H}(31)$ & 119.8 \\
\hline $\mathrm{C}(31)-\mathrm{C}(32)-\mathrm{C}(27)$ & $118.4(6)$ \\
\hline$C(31)-C(32)-C(33)$ & $123.7(5)$ \\
\hline $\mathrm{C}(27)-\mathrm{C}(32)-\mathrm{C}(33)$ & $117.9(5)$ \\
\hline$N(3)-C(33)-C(32)$ & $109.4(4)$ \\
\hline$N(3)-C(33)-C(26)$ & $109.9(4)$ \\
\hline$C(32)-C(33)-C(26)$ & $112.8(5)$ \\
\hline$N(3)-C(33)-C(34)$ & $103.8(4)$ \\
\hline $\mathrm{C}(32)-\mathrm{C}(33)-\mathrm{C}(34)$ & $112.5(4)$ \\
\hline$C(26)-C(33)-C(34)$ & 108.1(4) \\
\hline $\mathrm{O}(2)-\mathrm{C}(34)-\mathrm{N}(4)$ & $127.2(5)$ \\
\hline $\mathrm{O}(2)-\mathrm{C}(34)-\mathrm{C}(33)$ & $128.7(5)$ \\
\hline$N(4)-C(34)-C(33)$ & 104.1(4) \\
\hline $\mathrm{N}(3)-\mathrm{C}(35)-\mathrm{N}(4)$ & $117.4(5)$ \\
\hline $\mathrm{N}(3)-\mathrm{C}(35)-\mathrm{C}(36)$ & $135.1(7)$ \\
\hline $\mathrm{N}(4)-\mathrm{C}(35)-\mathrm{C}(36)$ & $107.3(6)$ \\
\hline $\mathrm{C}(39)-\mathrm{C}(36)-\mathrm{C}(37)$ & $105.9(9)$ \\
\hline $\mathrm{C}(39)-\mathrm{C}(36)-\mathrm{C}(35)$ & $117.9(8)$ \\
\hline $\mathrm{C}(37)-\mathrm{C}(36)-\mathrm{C}(35)$ & $104.3(6)$ \\
\hline $\mathrm{C}(39)-\mathrm{C}(36)-\mathrm{H}(36)$ & 109.5 \\
\hline $\mathrm{C}(37)-\mathrm{C}(36)-\mathrm{H}(36)$ & 109.5 \\
\hline $\mathrm{C}(35)-\mathrm{C}(36)-\mathrm{H}(36)$ & 109.5 \\
\hline $\mathrm{C}(36)-\mathrm{C}(37)-\mathrm{C}(38)$ & $108.0(7)$ \\
\hline
\end{tabular}




$\begin{array}{ll}\mathrm{C}(36)-\mathrm{C}(37)-\mathrm{H}(37 \mathrm{~A}) & 110.1 \\ \mathrm{C}(38)-\mathrm{C}(37)-\mathrm{H}(37 \mathrm{~A}) & 110.1 \\ \mathrm{C}(36)-\mathrm{C}(37)-\mathrm{H}(37 \mathrm{~B}) & 110.1 \\ \mathrm{C}(38)-\mathrm{C}(37)-\mathrm{H}(37 \mathrm{~B}) & 110.1 \\ \mathrm{H}(37 \mathrm{~A})-\mathrm{C}(37)-\mathrm{H}(37 \mathrm{~B}) & 108.4 \\ \mathrm{~N}(4)-\mathrm{C}(38)-\mathrm{C}(37) & 102.1(7) \\ \mathrm{N}(4)-\mathrm{C}(38)-\mathrm{H}(38 \mathrm{~A}) & 111.4 \\ \mathrm{C}(37)-\mathrm{C}(38)-\mathrm{H}(38 \mathrm{~A}) & 111.4 \\ \mathrm{~N}(4)-\mathrm{C}(38)-\mathrm{H}(38 \mathrm{~B}) & 111.4 \\ \mathrm{C}(37)-\mathrm{C}(38)-\mathrm{H}(38 \mathrm{~B}) & 111.4 \\ \mathrm{H}(38 \mathrm{~A})-\mathrm{C}(38)-\mathrm{H}(38 \mathrm{~B}) & 109.2 \\ \mathrm{C}(40)-\mathrm{C}(39)-\mathrm{C}(36) & 120.5(9) \\ \mathrm{C}(40)-\mathrm{C}(39)-\mathrm{H}(39 \mathrm{~A}) & 107.2 \\ \mathrm{C}(36)-\mathrm{C}(39)-\mathrm{H}(39 \mathrm{~A}) & 107.2 \\ \mathrm{C}(40)-\mathrm{C}(39)-\mathrm{H}(39 B) & 107.2 \\ \mathrm{C}(36)-\mathrm{C}(39)-\mathrm{H}(39 \mathrm{~B}) & 107.2 \\ \mathrm{H}(39 \mathrm{~A})-\mathrm{C}(39)-\mathrm{H}(39 B) & 106.8 \\ \mathrm{~F}(5)-\mathrm{C}(40)-\mathrm{F}(6) & 102.8(8) \\ \mathrm{F}(5)-\mathrm{C}(40)-\mathrm{C}(39) & 120.9(10) \\ \mathrm{F}(6)-\mathrm{C}(40)-\mathrm{C}(39) & 117.2(9) \\ \mathrm{F}(5)-\mathrm{C}(40)-\mathrm{F}(4) & 104.0(9) \\ \mathrm{F}(6)-\mathrm{C}(40)-\mathrm{F}(4) & 100.4(10) \\ \mathrm{C}(39)-\mathrm{C}(40)-\mathrm{F}(4) & 108.9(9) \\ & \end{array}$

Symmetry transformations used to generate equivalent atoms:

Table S-crystal-4. Anisotropic displacement parameters $\left(\AA^{2} \times 10^{3}\right)$ for Y. The anisotropic displacement factor exponent takes the form: $\quad-2 \pi^{2}\left[\mathrm{~h}^{2} \mathrm{a}^{* 2} \mathrm{U}^{11}+\ldots+2 \mathrm{~h} \mathrm{k} \mathrm{a} \mathrm{a}^{*} \mathrm{U}^{12}\right]$

\begin{tabular}{lcccccc}
\hline & $\mathrm{U}^{11}$ & $\mathrm{U}^{22}$ & $\mathrm{U}^{33}$ & $\mathrm{U}^{23}$ & $\mathrm{U}^{13}$ & $\mathrm{U}^{12}$ \\
\hline $\mathrm{F}(1)$ & $83(3)$ & $85(3)$ & $107(4)$ & $-29(3)$ & $19(3)$ & $18(3)$ \\
$\mathrm{F}(2)$ & $71(3)$ & $92(4)$ & $150(6)$ & $27(4)$ & $10(3)$ & $-7(3)$ \\
$\mathrm{F}(3)$ & $83(3)$ & $145(6)$ & $88(4)$ & $46(4)$ & $-4(3)$ & $26(3)$ \\
$\mathrm{O}(1)$ & $68(3)$ & $55(3)$ & $64(3)$ & $-6(2)$ & $33(2)$ & $-17(2)$ \\
$\mathrm{N}(1)$ & $39(2)$ & $46(2)$ & $36(2)$ & $-6(2)$ & $4(2)$ & $0(2)$ \\
$\mathrm{N}(2)$ & $46(2)$ & $63(3)$ & $32(2)$ & $6(2)$ & $11(2)$ & $10(2)$ \\
$\mathrm{C}(1)$ & $62(3)$ & $45(3)$ & $50(3)$ & $3(2)$ & $4(2)$ & $5(2)$ \\
& & & & $\mathrm{SI}-57$ &
\end{tabular}




\begin{tabular}{|c|c|c|c|c|c|c|}
\hline$C(2)$ & $91(4)$ & $48(3)$ & $57(3)$ & $0(3)$ & $-8(3)$ & $12(3)$ \\
\hline$C(3)$ & $85(4)$ & $61(4)$ & $63(3)$ & $-11(3)$ & $-18(3)$ & $32(3)$ \\
\hline$C(4)$ & $60(4)$ & $74(4)$ & $79(4)$ & $-17(4)$ & $-8(3)$ & $24(3)$ \\
\hline$C(5)$ & $52(3)$ & $49(3)$ & $54(3)$ & $-10(2)$ & $2(2)$ & $8(2)$ \\
\hline$C(6)$ & $46(2)$ & $36(2)$ & $33(2)$ & $-8(2)$ & $3(2)$ & $3(2)$ \\
\hline$C(7)$ & $46(2)$ & $43(2)$ & $42(2)$ & $-6(2)$ & $13(2)$ & $1(2)$ \\
\hline$C(8)$ & $59(3)$ & $44(3)$ & $43(3)$ & $-8(2)$ & $17(2)$ & $4(2)$ \\
\hline$C(9)$ & $63(3)$ & $43(3)$ & $46(3)$ & $-1(2)$ & $27(2)$ & $11(2)$ \\
\hline$C(10)$ & $50(3)$ & $59(3)$ & $60(3)$ & $5(3)$ & $20(2)$ & $13(3)$ \\
\hline $\mathrm{C}(11)$ & $37(2)$ & $53(3)$ & $44(2)$ & $-4(2)$ & $8(2)$ & $2(2)$ \\
\hline$C(12)$ & $39(2)$ & $37(2)$ & $27(2)$ & $2(2)$ & $10(2)$ & $4(2)$ \\
\hline$C(13)$ & $42(2)$ & $36(2)$ & $30(2)$ & $-4(2)$ & $8(2)$ & $1(2)$ \\
\hline$C(14)$ & $46(2)$ & $43(2)$ & $40(2)$ & $-1(2)$ & $17(2)$ & $7(2)$ \\
\hline$C(15)$ & $47(2)$ & $48(3)$ & $37(2)$ & $-5(2)$ & $10(2)$ & $8(2)$ \\
\hline$C(16)$ & $57(3)$ & $58(3)$ & $41(3)$ & $-9(2)$ & $3(2)$ & 11(3) \\
\hline$C(17)$ & $69(3)$ & $75(4)$ & $39(3)$ & $0(3)$ & $4(3)$ & 11(3) \\
\hline $\mathrm{C}(18)$ & $66(3)$ & $75(4)$ & $42(3)$ & $10(3)$ & $18(2)$ & $16(3)$ \\
\hline$C(19)$ & $70(4)$ & $60(3)$ & $61(3)$ & $-17(3)$ & $-11(3)$ & $2(3)$ \\
\hline $\mathrm{C}(20)$ & $65(4)$ & $63(4)$ & $57(3)$ & $2(3)$ & $-1(3)$ & $-4(3)$ \\
\hline $\mathrm{F}(4)$ & $124(6)$ & $223(11)$ & $136(7)$ & $-49(7)$ & $5(5)$ & $69(6)$ \\
\hline$F(5)$ & $81(4)$ & $141(6)$ & $146(6)$ & $10(5)$ & $31(4)$ & $3(4)$ \\
\hline$F(6)$ & $127(5)$ & $110(5)$ & $133(5)$ & $46(4)$ & $50(4)$ & $39(4)$ \\
\hline $\mathrm{O}(2)$ & $57(2)$ & $51(2)$ & 61(3) & $-5(2)$ & $12(2)$ & $24(2)$ \\
\hline $\mathrm{N}(3)$ & $43(2)$ & $47(3)$ & $51(3)$ & $18(2)$ & $10(2)$ & $14(2)$ \\
\hline $\mathrm{N}(4)$ & $51(3)$ & $48(3)$ & $40(2)$ & $1(2)$ & $12(2)$ & $4(2)$ \\
\hline $\mathrm{C}(21)$ & $60(3)$ & $44(3)$ & $46(2)$ & $-5(2)$ & $20(2)$ & $-2(2)$ \\
\hline $\mathrm{C}(22)$ & $79(4)$ & $59(3)$ & $59(3)$ & $-12(3)$ & $32(3)$ & $-17(3)$ \\
\hline$C(23)$ & $98(4)$ & $49(3)$ & $53(3)$ & $-3(3)$ & $29(3)$ & $-19(3)$ \\
\hline$C(24)$ & 101(4) & $51(3)$ & $49(3)$ & $12(3)$ & $11(3)$ & $4(3)$ \\
\hline$C(25)$ & $71(3)$ & $48(3)$ & $54(3)$ & $12(2)$ & $12(3)$ & $6(3)$ \\
\hline$C(26)$ & $55(3)$ & $34(2)$ & $39(2)$ & $1(2)$ & $18(2)$ & $3(2)$ \\
\hline$C(27)$ & $62(3)$ & $47(3)$ & $64(3)$ & $-7(2)$ & $31(3)$ & $3(2)$ \\
\hline$C(28)$ & $75(4)$ & $48(3)$ & $71(4)$ & $-12(3)$ & $28(3)$ & $-1(3)$ \\
\hline$C(29)$ & $72(4)$ & $44(3)$ & $73(4)$ & $-7(3)$ & $20(3)$ & $-9(3)$ \\
\hline$C(30)$ & $56(3)$ & $57(3)$ & $80(4)$ & $-10(3)$ & $19(3)$ & $-5(3)$ \\
\hline $\mathrm{C}(31)$ & $53(3)$ & $48(3)$ & $62(3)$ & $-8(2)$ & $21(2)$ & $6(2)$ \\
\hline$C(32)$ & $49(2)$ & $33(2)$ & $44(2)$ & $3(2)$ & $16(2)$ & $6(2)$ \\
\hline$C(33)$ & $44(2)$ & $36(2)$ & $42(2)$ & $6(2)$ & $14(2)$ & $12(2)$ \\
\hline
\end{tabular}




$\begin{array}{lcllccc}\mathrm{C}(34) & 50(3) & 40(2) & 41(2) & 1(2) & 15(2) & 3(2) \\ \mathrm{C}(35) & 56(3) & 49(3) & 46(3) & 19(2) & 9(2) & 4(2) \\ \mathrm{C}(36) & 73(4) & 75(4) & 61(3) & 25(3) & -9(3) & 5(3) \\ \mathrm{C}(37) & 108(5) & 97(5) & 71(4) & 4(4) & -13(4) & 8(4) \\ \mathrm{C}(38) & 85(4) & 66(4) & 46(3) & -6(3) & 12(3) & -8(3) \\ \mathrm{C}(39) & 90(5) & 85(5) & 94(5) & 23(4) & -5(4) & -8(4) \\ \mathrm{C}(40) & 83(5) & 90(5) & 96(5) & 12(4) & 30(4) & 13(4)\end{array}$

Table S-crystal-5. Hydrogen coordinates ( $\left.\mathrm{x} 10^{4}\right)$ and isotropic displacement parameters $\left(\AA^{2} \times 10^{3}\right)$ for Y.

\begin{tabular}{|c|c|c|c|c|}
\hline & $\mathrm{x}$ & $\mathrm{y}$ & z & $\mathrm{U}(\mathrm{eq})$ \\
\hline $\mathrm{H}(1)$ & 6078 & 11326 & -1077 & 64 \\
\hline $\mathrm{H}(2)$ & 4025 & 12487 & -1748 & 82 \\
\hline $\mathrm{H}(3)$ & 1053 & 12389 & -1654 & 89 \\
\hline $\mathrm{H}(4)$ & 155 & 11214 & -874 & 89 \\
\hline $\mathrm{H}(5)$ & 2155 & 10033 & -190 & 63 \\
\hline $\mathrm{H}(7)$ & 4175 & 8806 & -1368 & 52 \\
\hline $\mathrm{H}(8)$ & 5316 & 7626 & -2136 & 57 \\
\hline $\mathrm{H}(9)$ & 8328 & 7216 & -1878 & 58 \\
\hline $\mathrm{H}(10)$ & 10209 & 8015 & -876 & 66 \\
\hline $\mathrm{H}(11)$ & 9103 & 9181 & -105 & 54 \\
\hline $\mathrm{H}(16)$ & 7305 & 11212 & 1941 & 64 \\
\hline $\mathrm{H}(17 \mathrm{~A})$ & 7885 & 9969 & 2836 & 74 \\
\hline $\mathrm{H}(17 \mathrm{~B})$ & 8934 & 9273 & 2345 & 74 \\
\hline $\mathrm{H}(18 \mathrm{~A})$ & 6234 & 8434 & 2030 & 72 \\
\hline $\mathrm{H}(18 \mathrm{~B})$ & 5142 & 9439 & 2164 & 72 \\
\hline $\mathrm{H}(19 \mathrm{~A})$ & 9744 & 11632 & 1346 & 80 \\
\hline $\mathrm{H}(19 \mathrm{~B})$ & 10157 & 11637 & 2239 & 80 \\
\hline $\mathrm{H}(21)$ & 6542 & 5509 & 7277 & 59 \\
\hline $\mathrm{H}(22)$ & 7700 & 6536 & 6442 & 76 \\
\hline $\mathrm{H}(23)$ & 5819 & 7482 & 5512 & 77 \\
\hline $\mathrm{H}(24)$ & 2815 & 7351 & 5408 & 81 \\
\hline $\mathrm{H}(25)$ & 1572 & 6315 & 6251 & 69 \\
\hline $\mathrm{H}(27)$ & 3494 & 3625 & 6493 & 66 \\
\hline
\end{tabular}




\begin{tabular}{lrrrr}
$\mathrm{H}(28)$ & 1439 & 2412 & 5864 & 76 \\
$\mathrm{H}(29)$ & -1485 & 2431 & 6046 & 75 \\
$\mathrm{H}(30)$ & -2284 & 3565 & 6894 & 76 \\
$\mathrm{H}(31)$ & -361 & 4813 & 7472 & 63 \\
$\mathrm{H}(36)$ & 5518 & 4203 & 9619 & 87 \\
$\mathrm{H}(37 \mathrm{~A})$ & 6175 & 6294 & 9896 & 116 \\
$\mathrm{H}(37 \mathrm{~B})$ & 5170 & 5523 & 10351 & 116 \\
$\mathrm{H}(38 \mathrm{~A})$ & 2515 & 5929 & 9653 & 79 \\
$\mathrm{H}(38 \mathrm{~B})$ & 3487 & 6921 & 9408 & 79 \\
$\mathrm{H}(39 \mathrm{~A})$ & 7934 & 5438 & 9065 & 112 \\
$\mathrm{H}(39 \mathrm{~B})$ & 8218 & 5246 & 9943 & 112 \\
& & & & \\
\hline
\end{tabular}

Table S-crystal-6. Hydrogen bonds for $\mathrm{Y} \quad\left[\AA\right.$ and $\left.{ }^{\circ}\right]$.

\begin{tabular}{lllll}
\hline D-H...A & d(D-H) & d(H...A & d(D...A & $<($ DHA $)$
\end{tabular}

2)

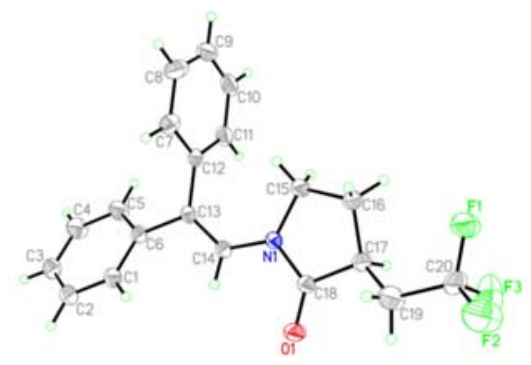<smiles>O=C1C(CC(F)(F)F)CCN1C=C(c1ccccc1)c1ccccc1</smiles>

X-ray crystal structure of compound 6a. Single crystal of $\mathbf{6 a}$ was obtained by slow evaporation of Petroleum ether /EtOAc solution. Crystal measurement of $\mathbf{6 a}$ was measured on R-Axis RAPID of Rigaku Corporation plus $291.2 \mathrm{k}$. Thermal ellipsoids are set at $30 \%$ probability.

CCDC number: 2122209

Table S-crystal-7. Crystal data and structure refinement for $\mathbf{6 a}$.

Identification code

Empirical formula

Formula weight

Temperature

Wavelength

Crystal system

Space group
$6 \mathrm{a}$

C20 H18 F3 N O

345.35

293(2) K

$0.71073 \AA$

Triclinic

P-1 


\begin{tabular}{|c|c|c|}
\hline \multirow[t]{3}{*}{ Unit cell dimensions } & $a=9.606(3) \AA$ & $\alpha=93.9$ \\
\hline & $\mathrm{b}=10.156(3) \AA$ & $\beta=115$ \\
\hline & $\mathrm{c}=10.923(4) \AA$ & $\gamma=109$ \\
\hline Volume & \multicolumn{2}{|l|}{$875.8(5) \AA^{3}$} \\
\hline $\mathrm{Z}$ & \multicolumn{2}{|c|}{2} \\
\hline Density (calculated) & \multicolumn{2}{|l|}{$1.310 \mathrm{Mg} / \mathrm{m}^{3}$} \\
\hline Absorption coefficient & \multicolumn{2}{|l|}{$0.102 \mathrm{~mm}^{-1}$} \\
\hline $\mathrm{F}(000)$ & \multicolumn{2}{|l|}{360} \\
\hline Crystal size & \multicolumn{2}{|c|}{$0.200 \times 0.180 \times 0.170 \mathrm{~mm}^{3}$} \\
\hline Theta range for data collection & \multicolumn{2}{|l|}{2.654 to $25.648^{\circ}$. } \\
\hline Index ranges & \multicolumn{2}{|c|}{$-11<=\mathrm{h}<=11,-12<=\mathrm{k}<=12,-13<=\mathrm{l}<=13$} \\
\hline Reflections collected & \multicolumn{2}{|l|}{34571} \\
\hline Independent reflections & \multicolumn{2}{|c|}{$3261[\mathrm{R}(\mathrm{int})=0.0403]$} \\
\hline Completeness to theta $=25.242^{\circ}$ & \multicolumn{2}{|l|}{$98.1 \%$} \\
\hline Max. and min. transmission & \multicolumn{2}{|c|}{0.9828 and 0.9798} \\
\hline Refinement method & \multicolumn{2}{|c|}{ Full-matrix least-squares on $\mathrm{F}^{2}$} \\
\hline Data / restraints / parameters & \multicolumn{2}{|l|}{$3261 / 218 / 227$} \\
\hline Goodness-of-fit on $\mathrm{F}^{2}$ & \multicolumn{2}{|l|}{1.039} \\
\hline Final $R$ indices $[\mathrm{I}>2 \operatorname{sigma}(\mathrm{I})]$ & \multicolumn{2}{|c|}{$\mathrm{R} 1=0.0861, \mathrm{wR} 2=0.2509$} \\
\hline $\mathrm{R}$ indices (all data) & \multicolumn{2}{|c|}{$\mathrm{R} 1=0.1059, \mathrm{wR} 2=0.2704$} \\
\hline Extinction coefficient & \multicolumn{2}{|c|}{$\mathrm{n} / \mathrm{a}$} \\
\hline Largest diff. peak and hole & \multicolumn{2}{|c|}{0.689 and -0.368 e. $\AA^{-3}$} \\
\hline
\end{tabular}

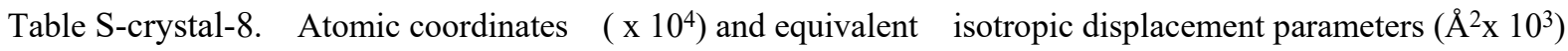
for $Y$. $U(e q)$ is defined as one third of the trace of the orthogonalized $U^{i j}$ tensor.

\begin{tabular}{lrrrr}
\hline & $\mathrm{x}$ & $\mathrm{y}$ & $\mathrm{z}$ & $\mathrm{U}(\mathrm{eq})$ \\
\hline $\mathrm{F}(1)$ & & & \\
$\mathrm{F}(2)$ & $8575(4)$ & $5336(5)$ & $2665(4)$ & $156(2)$ \\
$\mathrm{F}(3)$ & $6633(5)$ & $4921(6)$ & $647(3)$ & $163(2)$ \\
$\mathrm{O}(1)$ & $7159(7)$ & $6533(5)$ & $2186(6)$ & $174(2)$ \\
$\mathrm{N}(1)$ & $3355(3)$ & $3249(3)$ & $3323(3)$ & $77(1)$ \\
$\mathrm{C}(1)$ & $5506(3)$ & $2992(2)$ & $5180(2)$ & $45(1)$ \\
$\mathrm{C}(2)$ & $1894(4)$ & $-458(3)$ & $5734(4)$ & $60(1)$ \\
$\mathrm{C}(3)$ & $600(4)$ & $-1383(4)$ & $5913(4)$ & $72(1)$ \\
$\mathrm{C}(4)$ & $843(5)$ & $-1454(4)$ & $7240(5)$ & $77(1)$ \\
$\mathrm{C}(5)$ & $2368(5)$ & $-580(5)$ & $8374(5)$ & $87(1)$ \\
& $3668(5)$ & $349(4)$ & $8197(4)$ & $73(1)$
\end{tabular}




\begin{tabular}{lrrrr}
$\mathrm{C}(6)$ & $3445(4)$ & $439(3)$ & $6869(3)$ & $51(1)$ \\
$\mathrm{C}(7)$ & $7160(5)$ & $617(4)$ & $7599(4)$ & $62(1)$ \\
$\mathrm{C}(8)$ & $8779(6)$ & $789(6)$ & $8513(5)$ & $87(1)$ \\
$\mathrm{C}(9)$ & $9832(5)$ & $2001(6)$ & $9586(5)$ & $92(2)$ \\
$\mathrm{C}(10)$ & $9297(5)$ & $3039(5)$ & $9762(4)$ & $88(1)$ \\
$\mathrm{C}(11)$ & $7669(4)$ & $2890(3)$ & $8831(4)$ & $65(1)$ \\
$\mathrm{C}(12)$ & $6590(3)$ & $1676(3)$ & $7722(3)$ & $45(1)$ \\
$\mathrm{C}(13)$ & $4846(3)$ & $1466(3)$ & $6690(3)$ & $45(1)$ \\
$\mathrm{C}(14)$ & $4445(3)$ & $2087(3)$ & $5618(3)$ & $46(1)$ \\
$\mathrm{C}(15)$ & $7343(4)$ & $3533(5)$ & $5839(4)$ & $73(1)$ \\
$\mathrm{C}(16)$ & $7756(4)$ & $4174(5)$ & $4774(4)$ & $77(1)$ \\
$\mathrm{C}(17)$ & $6261(4)$ & $4394(4)$ & $3776(4)$ & $64(1)$ \\
$\mathrm{C}(18)$ & $4832(4)$ & $3476(3)$ & $4010(3)$ & $51(1)$ \\
$\mathrm{C}(19)$ & $5811(6)$ & $4257(7)$ & $2292(5)$ & $104(2)$ \\
$\mathrm{C}(20)$ & $7047(5)$ & $5237(6)$ & $1970(4)$ & $82(1)$ \\
\hline
\end{tabular}

Table S-crystal-9. Bond lengths $[\AA]$ and angles $\left[{ }^{\circ}\right]$ for $Y$.

\begin{tabular}{ll}
\hline $\mathrm{F}(1)-\mathrm{C}(20)$ & $1.292(5)$ \\
$\mathrm{F}(2)-\mathrm{C}(20)$ & $1.306(5)$ \\
$\mathrm{F}(3)-\mathrm{C}(20)$ & $1.280(6)$ \\
$\mathrm{O}(1)-\mathrm{C}(18)$ & $1.211(4)$ \\
$\mathrm{N}(1)-\mathrm{C}(18)$ & $1.371(4)$ \\
$\mathrm{N}(1)-\mathrm{C}(14)$ & $1.400(3)$ \\
$\mathrm{N}(1)-\mathrm{C}(15)$ & $1.459(4)$ \\
$\mathrm{C}(1)-\mathrm{C}(2)$ & $1.383(4)$ \\
$\mathrm{C}(1)-\mathrm{C}(6)$ & $1.386(5)$ \\
$\mathrm{C}(1)-\mathrm{H}(1)$ & 0.9300 \\
$\mathrm{C}(2)-\mathrm{C}(3)$ & $1.376(6)$ \\
$\mathrm{C}(2)-\mathrm{H}(2)$ & 0.9300 \\
$\mathrm{C}(3)-\mathrm{C}(4)$ & $1.370(6)$ \\
$\mathrm{C}(3)-\mathrm{H}(3)$ & 0.9300 \\
$\mathrm{C}(4)-\mathrm{C}(5)$ & $1.387(5)$ \\
$\mathrm{C}(4)-\mathrm{H}(4)$ & 0.9300 \\
$\mathrm{C}(5)-\mathrm{C}(6)$ & $1.387(5)$ \\
$\mathrm{C}(5)-\mathrm{H}(5)$ & 0.9300 \\
$\mathrm{C}(6)-\mathrm{C}(13)$ & $1.498(4)$ \\
&
\end{tabular}




\begin{tabular}{|c|c|}
\hline$C(7)-C(8)$ & $1.379(5)$ \\
\hline $\mathrm{C}(7)-\mathrm{C}(12)$ & $1.384(4)$ \\
\hline $\mathrm{C}(7)-\mathrm{H}(7)$ & 0.9300 \\
\hline $\mathrm{C}(8)-\mathrm{C}(9)$ & $1.361(7)$ \\
\hline $\mathrm{C}(8)-\mathrm{H}(8)$ & 0.9300 \\
\hline $\mathrm{C}(9)-\mathrm{C}(10)$ & $1.355(7)$ \\
\hline $\mathrm{C}(9)-\mathrm{H}(9)$ & 0.9300 \\
\hline $\mathrm{C}(10)-\mathrm{C}(11)$ & $1.396(6)$ \\
\hline $\mathrm{C}(10)-\mathrm{H}(10)$ & 0.9300 \\
\hline $\mathrm{C}(11)-\mathrm{C}(12)$ & $1.383(4)$ \\
\hline $\mathrm{C}(11)-\mathrm{H}(11)$ & 0.9300 \\
\hline$C(12)-C(13)$ & $1.490(4)$ \\
\hline$C(13)-C(14)$ & $1.340(4)$ \\
\hline $\mathrm{C}(14)-\mathrm{H}(14)$ & 0.9300 \\
\hline$C(15)-C(16)$ & $1.504(5)$ \\
\hline$C(15)-H(15 A)$ & 0.9700 \\
\hline $\mathrm{C}(15)-\mathrm{H}(15 \mathrm{~B})$ & 0.9700 \\
\hline$C(16)-C(17)$ & $1.483(5)$ \\
\hline $\mathrm{C}(16)-\mathrm{H}(16 \mathrm{~A})$ & 0.9700 \\
\hline $\mathrm{C}(16)-\mathrm{H}(16 \mathrm{~B})$ & 0.9700 \\
\hline$C(17)-C(19)$ & $1.470(5)$ \\
\hline $\mathrm{C}(17)-\mathrm{C}(18)$ & $1.513(4)$ \\
\hline $\mathrm{C}(17)-\mathrm{H}(17)$ & 0.9800 \\
\hline$C(19)-C(20)$ & $1.461(6)$ \\
\hline $\mathrm{C}(19)-\mathrm{H}(19 \mathrm{~A})$ & 0.9700 \\
\hline C(19)-H(19B) & 0.9700 \\
\hline $\mathrm{C}(18)-\mathrm{N}(1)-\mathrm{C}(14)$ & $120.0(2)$ \\
\hline $\mathrm{C}(18)-\mathrm{N}(1)-\mathrm{C}(15)$ & $112.6(2)$ \\
\hline$C(14)-N(1)-C(15)$ & $127.4(2)$ \\
\hline$C(2)-C(1)-C(6)$ & $121.6(3)$ \\
\hline $\mathrm{C}(2)-\mathrm{C}(1)-\mathrm{H}(1)$ & 119.2 \\
\hline $\mathrm{C}(6)-\mathrm{C}(1)-\mathrm{H}(1)$ & 119.2 \\
\hline$C(3)-C(2)-C(1)$ & $120.1(4)$ \\
\hline $\mathrm{C}(3)-\mathrm{C}(2)-\mathrm{H}(2)$ & 119.9 \\
\hline $\mathrm{C}(1)-\mathrm{C}(2)-\mathrm{H}(2)$ & 119.9 \\
\hline$C(4)-C(3)-C(2)$ & 119.2(3) \\
\hline $\mathrm{C}(4)-\mathrm{C}(3)-\mathrm{H}(3)$ & 120.4 \\
\hline
\end{tabular}




\begin{tabular}{|c|c|}
\hline $\mathrm{C}(2)-\mathrm{C}(3)-\mathrm{H}(3)$ & 120.4 \\
\hline$C(3)-C(4)-C(5)$ & $120.8(4)$ \\
\hline $\mathrm{C}(3)-\mathrm{C}(4)-\mathrm{H}(4)$ & 119.6 \\
\hline $\mathrm{C}(5)-\mathrm{C}(4)-\mathrm{H}(4)$ & 119.6 \\
\hline $\mathrm{C}(4)-\mathrm{C}(5)-\mathrm{C}(6)$ & $120.9(4)$ \\
\hline $\mathrm{C}(4)-\mathrm{C}(5)-\mathrm{H}(5)$ & 119.6 \\
\hline $\mathrm{C}(6)-\mathrm{C}(5)-\mathrm{H}(5)$ & 119.6 \\
\hline$C(1)-C(6)-C(5)$ & $117.4(3)$ \\
\hline$C(1)-C(6)-C(13)$ & $122.1(3)$ \\
\hline$C(5)-C(6)-C(13)$ & $120.4(3)$ \\
\hline $\mathrm{C}(8)-\mathrm{C}(7)-\mathrm{C}(12)$ & $121.3(4)$ \\
\hline $\mathrm{C}(8)-\mathrm{C}(7)-\mathrm{H}(7)$ & 119.3 \\
\hline $\mathrm{C}(12)-\mathrm{C}(7)-\mathrm{H}(7)$ & 119.3 \\
\hline $\mathrm{C}(9)-\mathrm{C}(8)-\mathrm{C}(7)$ & $119.8(4)$ \\
\hline $\mathrm{C}(9)-\mathrm{C}(8)-\mathrm{H}(8)$ & 120.1 \\
\hline $\mathrm{C}(7)-\mathrm{C}(8)-\mathrm{H}(8)$ & 120.1 \\
\hline $\mathrm{C}(10)-\mathrm{C}(9)-\mathrm{C}(8)$ & $120.4(4)$ \\
\hline $\mathrm{C}(10)-\mathrm{C}(9)-\mathrm{H}(9)$ & 119.8 \\
\hline $\mathrm{C}(8)-\mathrm{C}(9)-\mathrm{H}(9)$ & 119.8 \\
\hline$C(9)-C(10)-C(11)$ & $120.4(4)$ \\
\hline $\mathrm{C}(9)-\mathrm{C}(10)-\mathrm{H}(10)$ & 119.8 \\
\hline $\mathrm{C}(11)-\mathrm{C}(10)-\mathrm{H}(10)$ & 119.8 \\
\hline$C(12)-C(11)-C(10)$ & $120.0(4)$ \\
\hline $\mathrm{C}(12)-\mathrm{C}(11)-\mathrm{H}(11)$ & 120.0 \\
\hline $\mathrm{C}(10)-\mathrm{C}(11)-\mathrm{H}(11)$ & 120.0 \\
\hline$C(11)-C(12)-C(7)$ & $118.0(3)$ \\
\hline$C(11)-C(12)-C(13)$ & $122.3(3)$ \\
\hline$C(7)-C(12)-C(13)$ & $119.7(3)$ \\
\hline$C(14)-C(13)-C(12)$ & $125.5(2)$ \\
\hline $\mathrm{C}(14)-\mathrm{C}(13)-\mathrm{C}(6)$ & $118.4(3)$ \\
\hline$C(12)-C(13)-C(6)$ & $116.0(2)$ \\
\hline $\mathrm{C}(13)-\mathrm{C}(14)-\mathrm{N}(1)$ & $129.0(3)$ \\
\hline $\mathrm{C}(13)-\mathrm{C}(14)-\mathrm{H}(14)$ & 115.5 \\
\hline $\mathrm{N}(1)-\mathrm{C}(14)-\mathrm{H}(14)$ & 115.5 \\
\hline $\mathrm{N}(1)-\mathrm{C}(15)-\mathrm{C}(16)$ & $104.0(3)$ \\
\hline $\mathrm{N}(1)-\mathrm{C}(15)-\mathrm{H}(15 \mathrm{~A})$ & 111.0 \\
\hline $\mathrm{C}(16)-\mathrm{C}(15)-\mathrm{H}(15 \mathrm{~A})$ & 111.0 \\
\hline $\mathrm{N}(1)-\mathrm{C}(15)-\mathrm{H}(15 \mathrm{~B})$ & 111.0 \\
\hline
\end{tabular}




$\begin{array}{ll}\mathrm{C}(16)-\mathrm{C}(15)-\mathrm{H}(15 \mathrm{~B}) & 111.0 \\ \mathrm{H}(15 \mathrm{~A})-\mathrm{C}(15)-\mathrm{H}(15 \mathrm{~B}) & 109.0 \\ \mathrm{C}(17)-\mathrm{C}(16)-\mathrm{C}(15) & 107.3(3) \\ \mathrm{C}(17)-\mathrm{C}(16)-\mathrm{H}(16 \mathrm{~A}) & 110.3 \\ \mathrm{C}(15)-\mathrm{C}(16)-\mathrm{H}(16 \mathrm{~A}) & 110.3 \\ \mathrm{C}(17)-\mathrm{C}(16)-\mathrm{H}(16 \mathrm{~B}) & 110.3 \\ \mathrm{C}(15)-\mathrm{C}(16)-\mathrm{H}(16 \mathrm{~B}) & 110.3 \\ \mathrm{H}(16 \mathrm{~A})-\mathrm{C}(16)-\mathrm{H}(16 \mathrm{~B}) & 108.5 \\ \mathrm{C}(19)-\mathrm{C}(17)-\mathrm{C}(16) & 121.2(3) \\ \mathrm{C}(19)-\mathrm{C}(17)-\mathrm{C}(18) & 114.0(3) \\ \mathrm{C}(16)-\mathrm{C}(17)-\mathrm{C}(18) & 104.8(2) \\ \mathrm{C}(19)-\mathrm{C}(17)-\mathrm{H}(17) & 105.1 \\ \mathrm{C}(16)-\mathrm{C}(17)-\mathrm{H}(17) & 105.1 \\ \mathrm{C}(18)-\mathrm{C}(17)-\mathrm{H}(17) & 105.1 \\ \mathrm{O}(1)-\mathrm{C}(18)-\mathrm{N}(1) & 125.4(3) \\ \mathrm{O}(1)-\mathrm{C}(18)-\mathrm{C}(17) & 126.3(3) \\ \mathrm{N}(1)-\mathrm{C}(18)-\mathrm{C}(17) & 108.2(2) \\ \mathrm{C}(20)-\mathrm{C}(19)-\mathrm{C}(17) & 117.5(4) \\ \mathrm{C}(20)-\mathrm{C}(19)-\mathrm{H}(19 \mathrm{~A}) & 107.9 \\ \mathrm{C}(17)-\mathrm{C}(19)-\mathrm{H}(19 \mathrm{~A}) & 107.9 \\ \mathrm{C}(20)-\mathrm{C}(19)-\mathrm{H}(19 \mathrm{~B}) & 107.9 \\ \mathrm{C}(17)-\mathrm{C}(19)-\mathrm{H}(19 \mathrm{~B}) & 107.9 \\ \mathrm{H}(19 \mathrm{~A})-\mathrm{C}(19)-\mathrm{H}(19 \mathrm{~B}) & 107.2 \\ \mathrm{~F}(3)-\mathrm{C}(20)-\mathrm{F}(1) & 105.4(5) \\ \mathrm{F}(3)-\mathrm{C}(20)-\mathrm{F}(2) & 103.0(4) \\ \mathrm{F}(1)-\mathrm{C}(20)-\mathrm{F}(2) & 106.1(4) \\ \mathrm{F}(3)-\mathrm{C}(20)-\mathrm{C}(19) & 112.1(4) \\ \mathrm{F}(1)-\mathrm{C}(20)-\mathrm{C}(19) & 116.0(4) \\ \mathrm{F}(2)-\mathrm{C}(20)-\mathrm{C}(19) & 113.1(4) \\ & \end{array}$

Symmetry transformations used to generate equivalent atoms:

Table S-crystal-10. Anisotropic displacement parameters $\left(\AA^{2} \times 10^{3}\right)$ for Y. The anisotropic displacement factor exponent takes the form: $\quad-2 \pi^{2}\left[\mathrm{~h}^{2} \mathrm{a}^{* 2} \mathrm{U}^{11}+\ldots \quad+2 \mathrm{hk} \mathrm{a}^{*} \mathrm{~b}^{*} \mathrm{U}^{12}\right]$

\begin{tabular}{|c|c|c|c|c|c|c|}
\hline & $\mathrm{U}^{11}$ & $\mathrm{U}^{22}$ & $\mathrm{U}^{33}$ & $\mathrm{U}^{23}$ & $\mathrm{U}^{13}$ & $\mathrm{U}^{12}$ \\
\hline $\mathrm{F}(1)$ & $74(2)$ & $297(5)$ & $133(3)$ & $139(3)$ & $68(2)$ & $77(2)$ \\
\hline
\end{tabular}




\begin{tabular}{|c|c|c|c|c|c|c|}
\hline $\mathrm{F}(2)$ & $115(2)$ & $297(5)$ & $84(2)$ & $49(3)$ & $63(2)$ & $70(3)$ \\
\hline $\mathrm{F}(3)$ & $221(4)$ & $124(3)$ & $249(5)$ & $92(3)$ & $169(4)$ & $69(3)$ \\
\hline $\mathrm{O}(1)$ & $43(1)$ & $111(2)$ & $78(2)$ & $49(2)$ & $28(1)$ & $29(1)$ \\
\hline $\mathrm{N}(1)$ & $38(1)$ & $50(1)$ & $49(1)$ & $18(1)$ & $23(1)$ & $17(1)$ \\
\hline $\mathrm{C}(1)$ & $55(2)$ & $59(2)$ & $66(2)$ & $22(1)$ & $33(2)$ & $16(1)$ \\
\hline$C(2)$ & $53(2)$ & $68(2)$ & $91(3)$ & $28(2)$ & $36(2)$ & $17(2)$ \\
\hline$C(3)$ & $60(2)$ & $85(2)$ & 107(3) & $47(2)$ & $56(2)$ & $26(2)$ \\
\hline$C(4)$ & $71(2)$ & 121(3) & $83(3)$ & $51(2)$ & $53(2)$ & $29(2)$ \\
\hline$C(5)$ & $59(2)$ & $96(3)$ & $66(2)$ & $33(2)$ & $38(2)$ & $21(2)$ \\
\hline$C(6)$ & $47(2)$ & $55(2)$ & 61(2) & $24(1)$ & $33(1)$ & $21(1)$ \\
\hline$C(7)$ & $73(2)$ & $69(2)$ & $63(2)$ & $25(2)$ & $38(2)$ & $39(2)$ \\
\hline $\mathrm{C}(8)$ & $83(3)$ & $123(4)$ & $96(3)$ & $61(3)$ & $54(3)$ & $68(3)$ \\
\hline $\mathrm{C}(9)$ & $51(2)$ & $123(4)$ & $95(3)$ & $67(3)$ & $30(2)$ & $28(2)$ \\
\hline$C(10)$ & $62(2)$ & $78(2)$ & $64(2)$ & $24(2)$ & $9(2)$ & $-10(2)$ \\
\hline$C(11)$ & $64(2)$ & $51(2)$ & $61(2)$ & $12(1)$ & $25(2)$ & $10(1)$ \\
\hline$C(12)$ & $47(2)$ & $48(1)$ & $47(1)$ & $20(1)$ & $29(1)$ & $16(1)$ \\
\hline$C(13)$ & $44(1)$ & $45(1)$ & $51(2)$ & $12(1)$ & $28(1)$ & $15(1)$ \\
\hline$C(14)$ & $40(1)$ & $49(1)$ & $53(2)$ & $16(1)$ & $27(1)$ & $16(1)$ \\
\hline$C(15)$ & $45(2)$ & $106(3)$ & $80(2)$ & $55(2)$ & $36(2)$ & $31(2)$ \\
\hline$C(16)$ & $51(2)$ & $115(3)$ & $88(3)$ & $67(2)$ & $44(2)$ & $38(2)$ \\
\hline$C(17)$ & $50(2)$ & $88(2)$ & $64(2)$ & $40(2)$ & $33(2)$ & $30(2)$ \\
\hline$C(18)$ & $43(2)$ & $59(2)$ & $53(2)$ & $20(1)$ & $24(1)$ & $22(1)$ \\
\hline$C(19)$ & $68(3)$ & $166(5)$ & $70(2)$ & $48(3)$ & $37(2)$ & $31(3)$ \\
\hline$C(20)$ & $75(2)$ & $132(4)$ & $62(2)$ & $55(2)$ & $45(2)$ & $48(2)$ \\
\hline
\end{tabular}

Table S-crystal-11. Hydrogen coordinates ( x 104) and isotropic displacement parameters $\left(\AA^{2} \times 10^{3}\right)$ for $Y$.

\begin{tabular}{|c|c|c|c|c|}
\hline & $\mathrm{x}$ & $\mathrm{y}$ & z & $\mathrm{U}(\mathrm{eq})$ \\
\hline $\mathrm{H}(1)$ & 1720 & -437 & 4831 & 72 \\
\hline $\mathrm{H}(2)$ & -437 & -1959 & 5135 & 86 \\
\hline $\mathrm{H}(3)$ & -17 & -2088 & 7364 & 93 \\
\hline $\mathrm{H}(4)$ & 2533 & -609 & 9274 & 104 \\
\hline $\mathrm{H}(5)$ & 4702 & 919 & 8980 & 88 \\
\hline $\mathrm{H}(7)$ & 6436 & -227 & 6885 & 75 \\
\hline
\end{tabular}




\begin{tabular}{lrrrr}
$\mathrm{H}(8)$ & 9151 & 79 & 8397 & 104 \\
$\mathrm{H}(9)$ & 10924 & 2118 & 10202 & 110 \\
$\mathrm{H}(10)$ & 10019 & 3857 & 10507 & 105 \\
$\mathrm{H}(11)$ & 7311 & 3607 & 8957 & 78 \\
$\mathrm{H}(14)$ & 3304 & 1890 & 5091 & 55 \\
$\mathrm{H}(15 \mathrm{~A})$ & 7709 & 2753 & 6031 & 87 \\
$\mathrm{H}(15 \mathrm{~B})$ & 7877 & 4260 & 6709 & 87 \\
$\mathrm{H}(16 \mathrm{~A})$ & 8738 & 5088 & 5232 & 93 \\
$\mathrm{H}(16 \mathrm{~B})$ & 7999 & 3525 & 4283 & 93 \\
$\mathrm{H}(17)$ & 6486 & 5396 & 4137 & 76 \\
$\mathrm{H}(19 \mathrm{~A})$ & 5573 & 3274 & 1889 & 125 \\
$\mathrm{H}(19 B)$ & 4770 & 4391 & 1819 & 125 \\
& & & & \\
\hline
\end{tabular}


XI. NMR Spectral Copies 


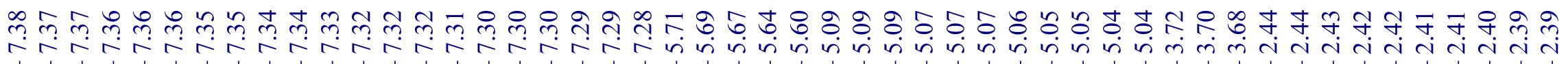

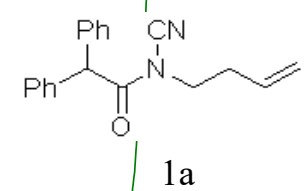

${ }^{1} \mathrm{H}$ NMR $\left(400 \mathrm{MHz}, \mathrm{CDCl}_{3}\right)$

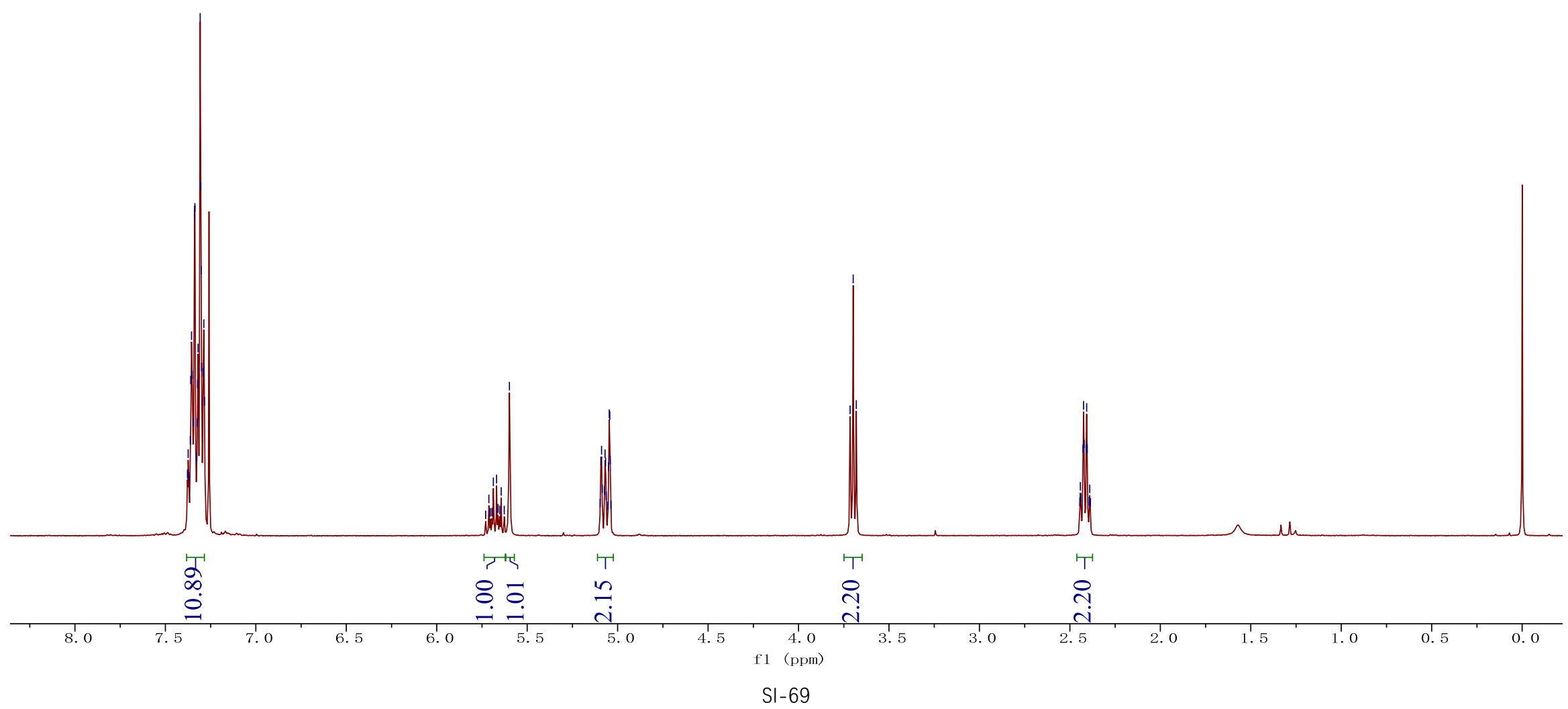



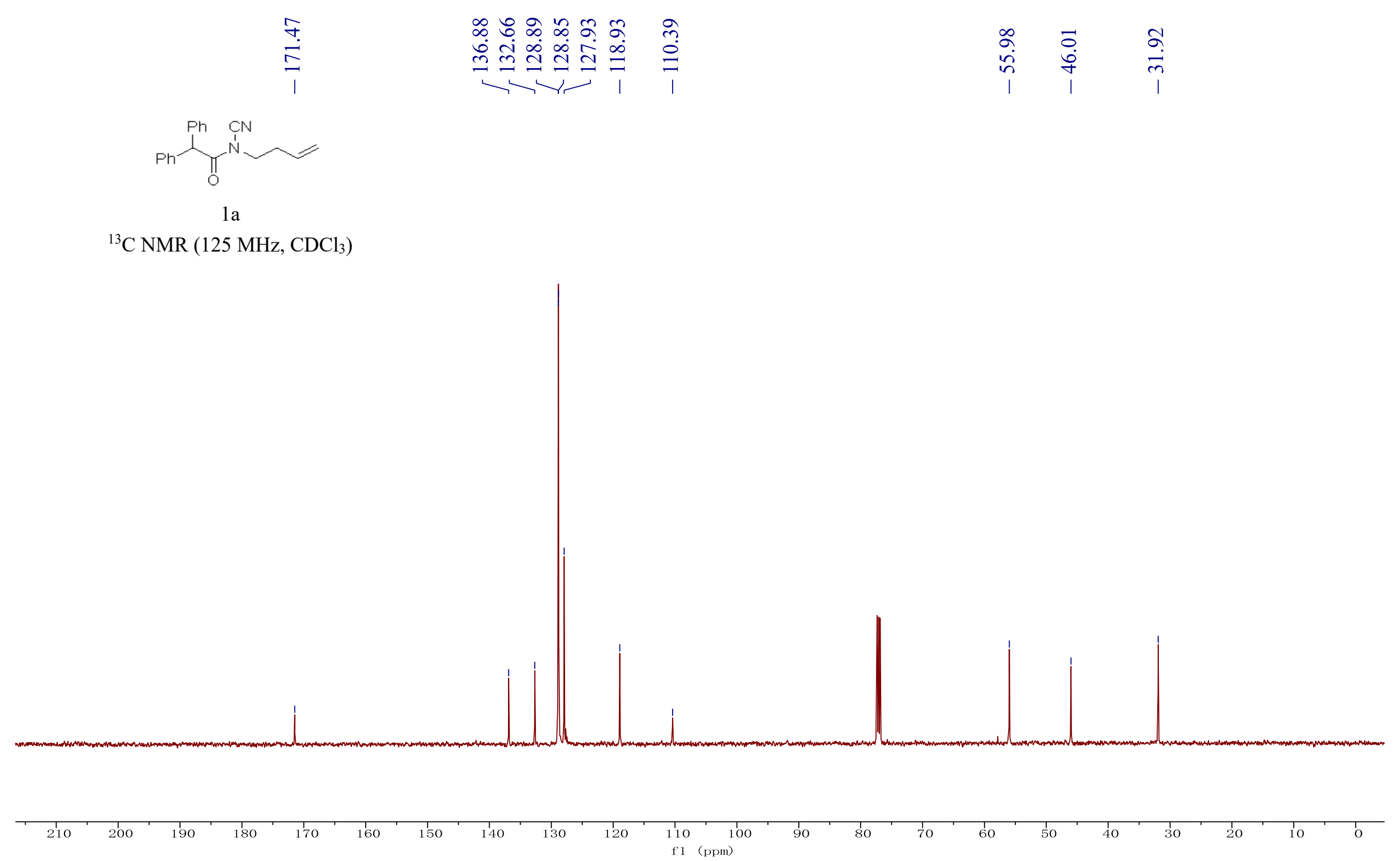


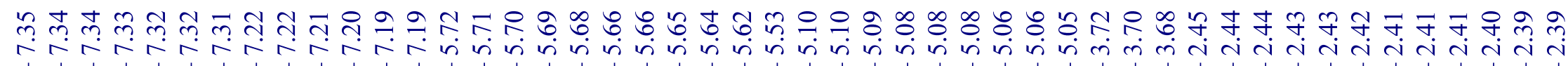

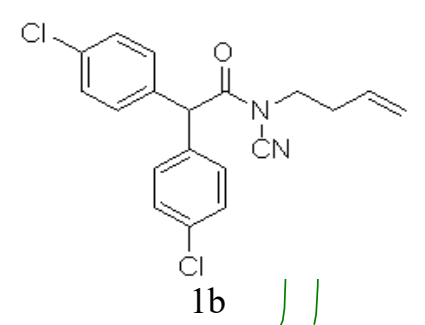

${ }^{1} \mathrm{H}$ NMR $\left(400 \mathrm{MHz}, \mathrm{CDCl}_{3}\right)$
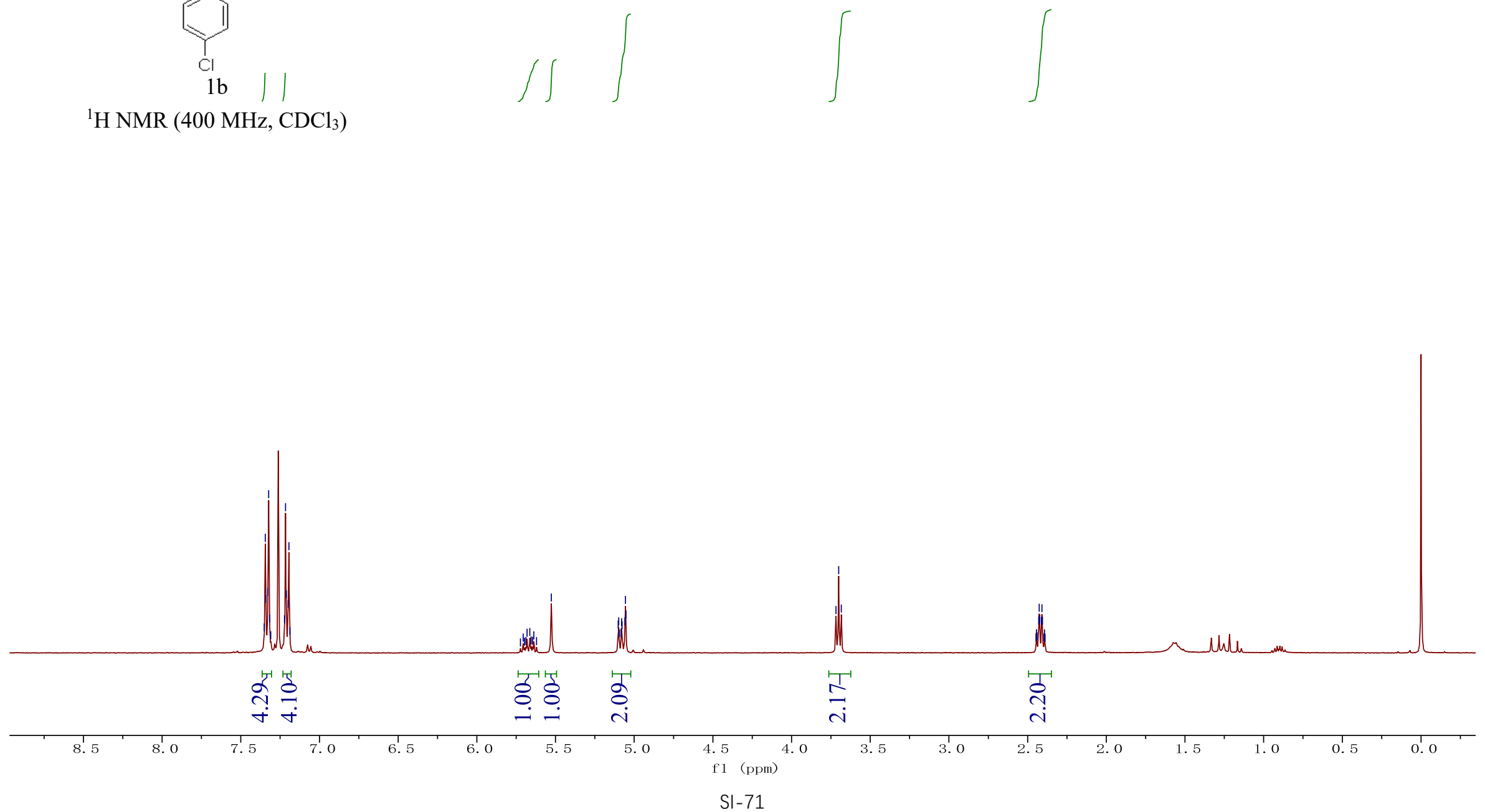


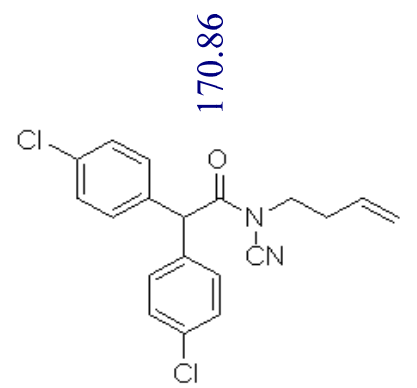

$1 \mathrm{~b}$

${ }^{13} \mathrm{C}$ NMR $\left(125 \mathrm{MHz}, \mathrm{CDCl}_{3}\right)$

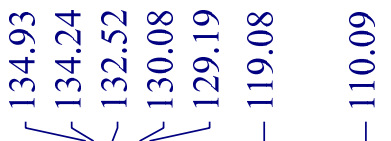

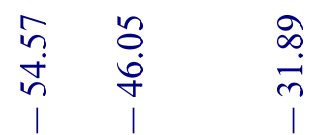

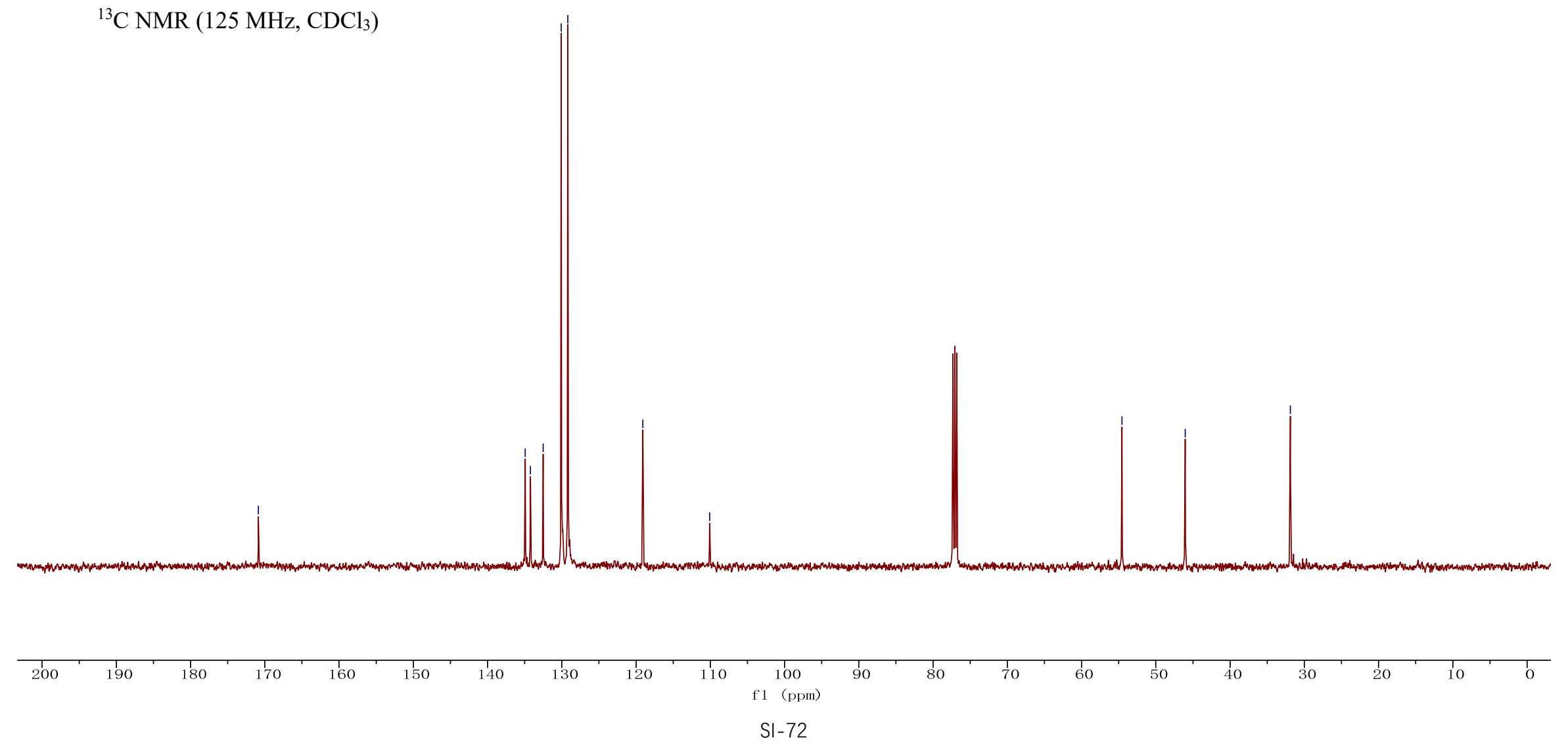




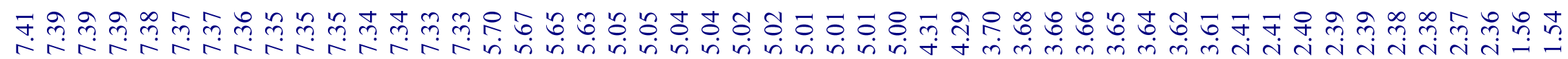

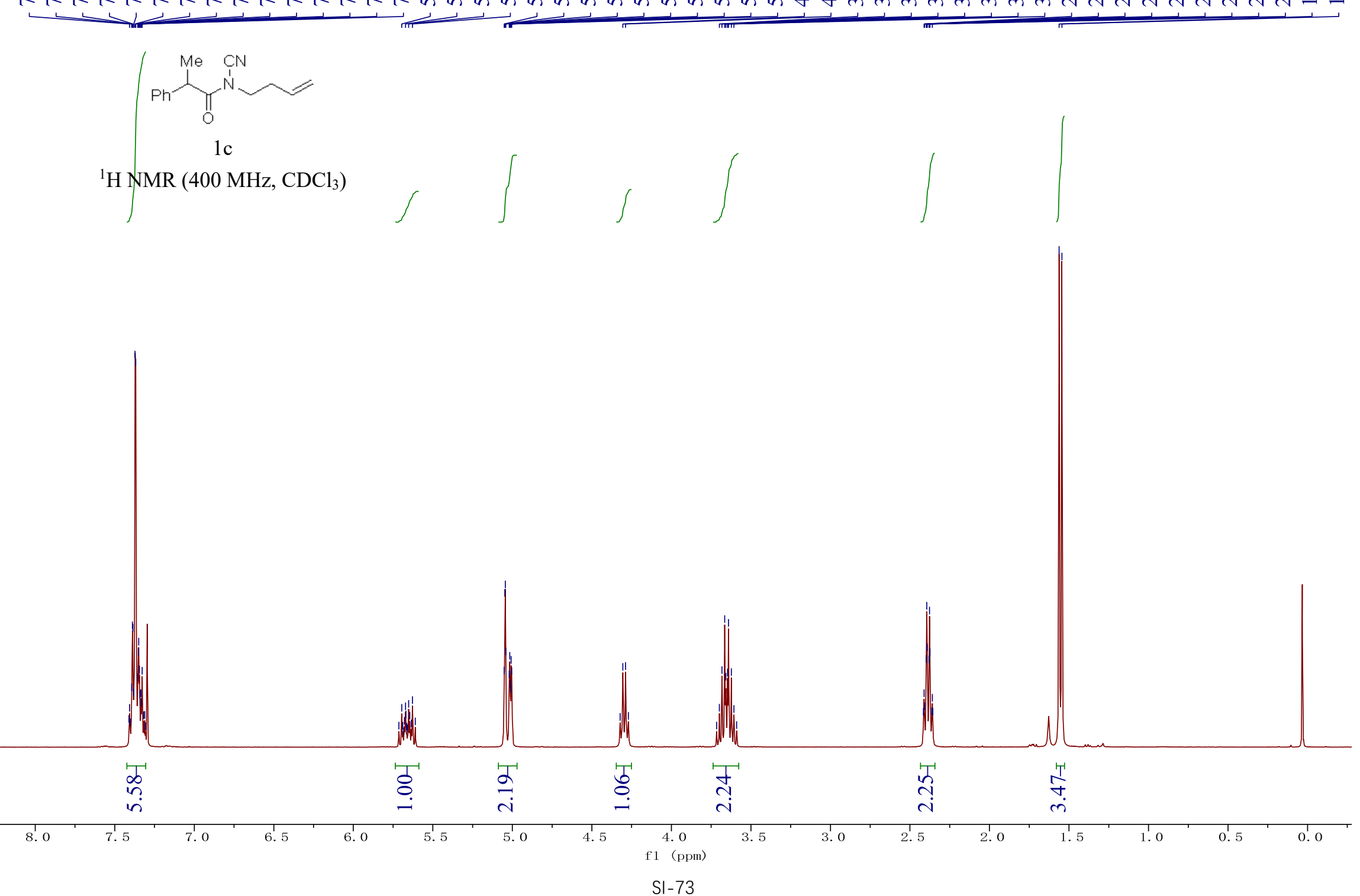




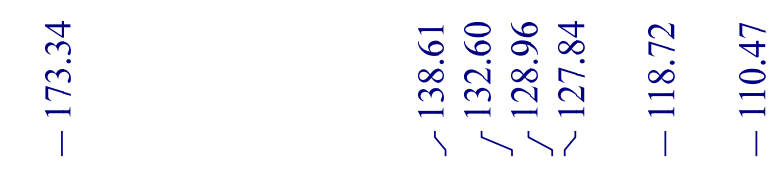

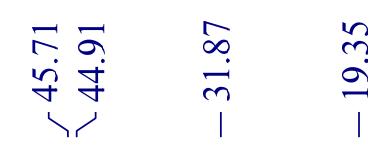
$\prod_{O}^{M e}$

1c

${ }^{13} \mathrm{C}$ NMR (125 MHz, $\left.\mathrm{CDCl}_{3}\right)$

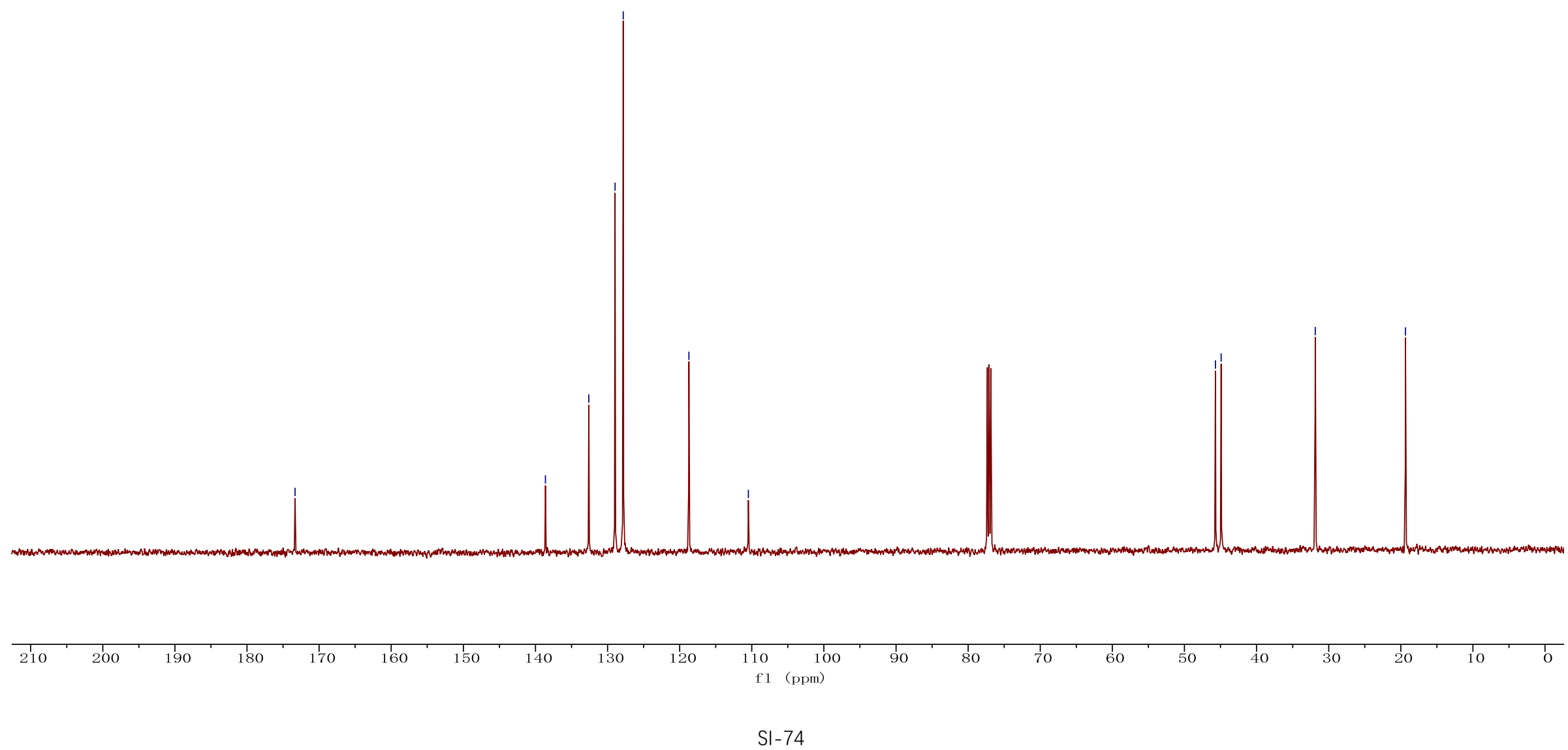




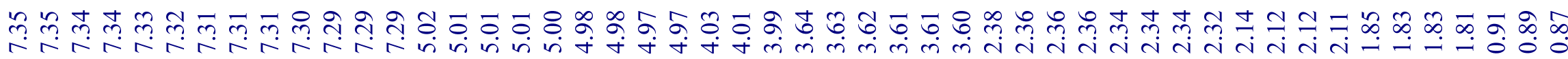

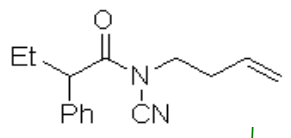

$1 \mathrm{~d}$

${ }^{1} \mathrm{H}$ NMR (400 MHz, $\mathrm{CDCl}_{3}$ )
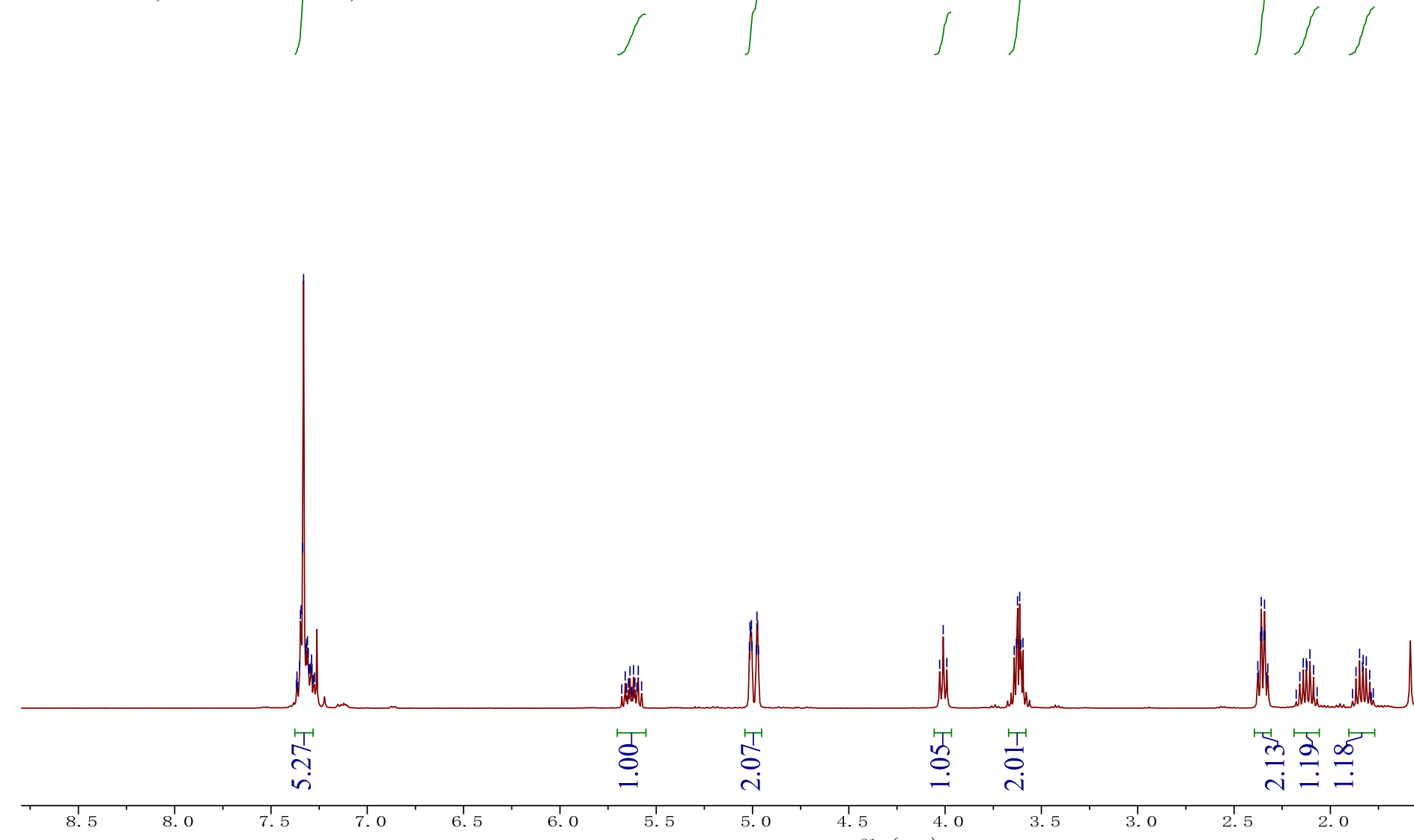

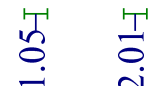

先定占

年

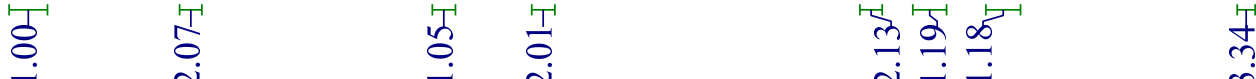




\section{$\stackrel{\infty}{\infty}$}

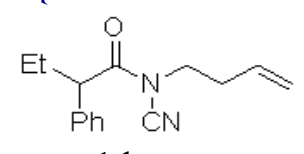

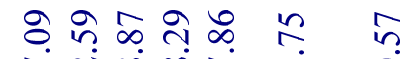

m.

$1 d$

${ }^{13} \mathrm{C}$ NMR (125 MHz, $\mathrm{CDCl}_{3}$ )

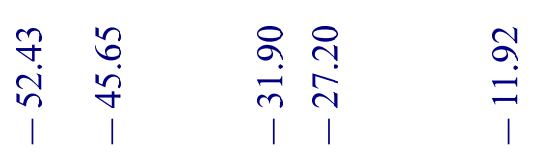
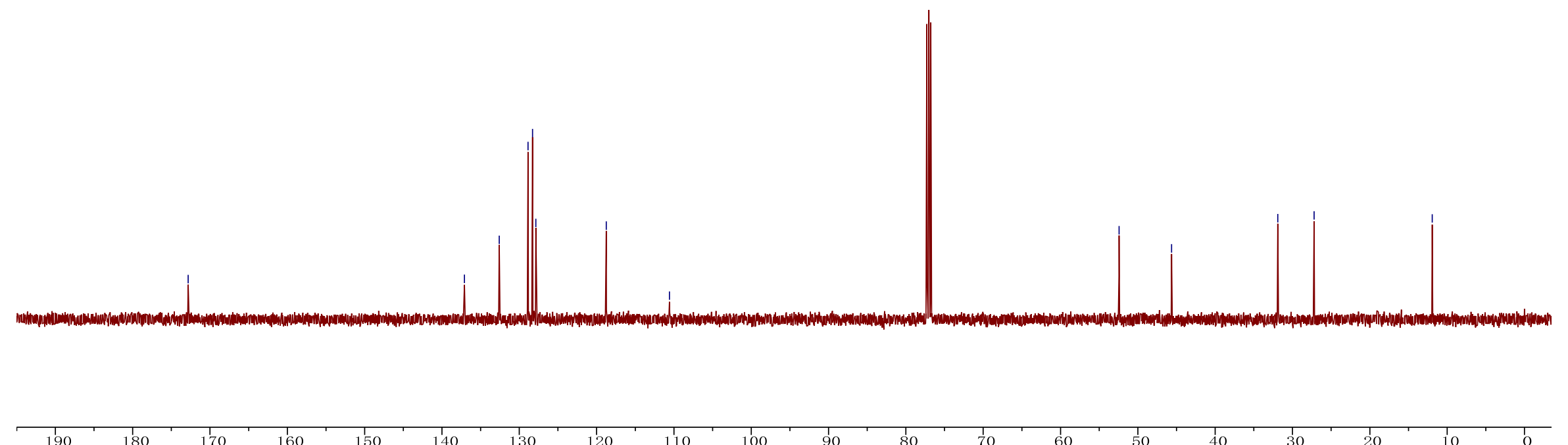

130

$100 \quad 90$

SI-76 

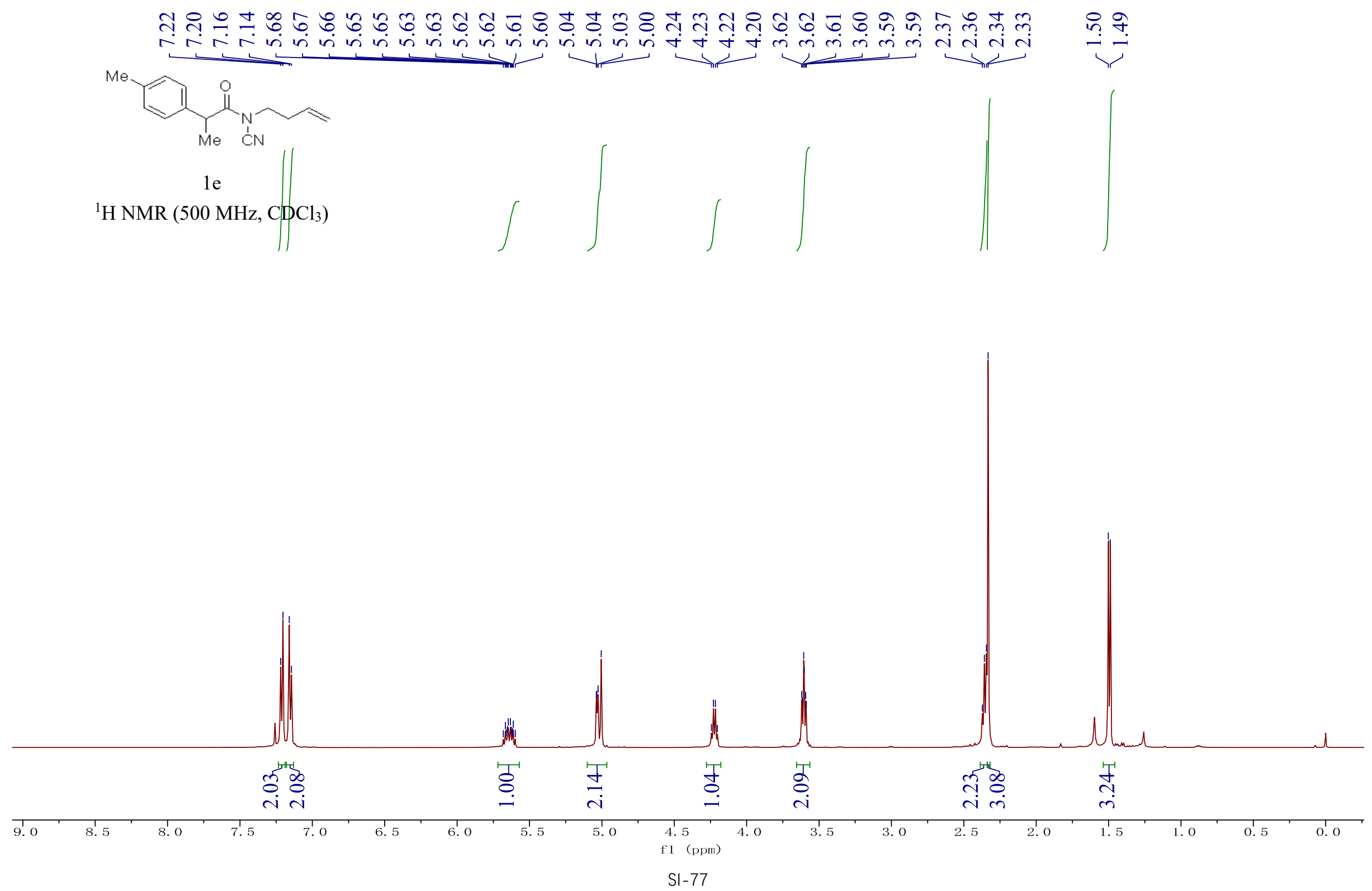


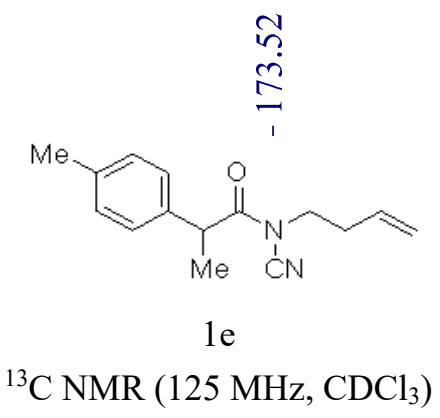

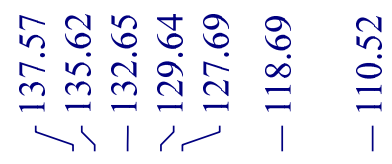

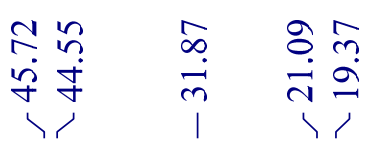

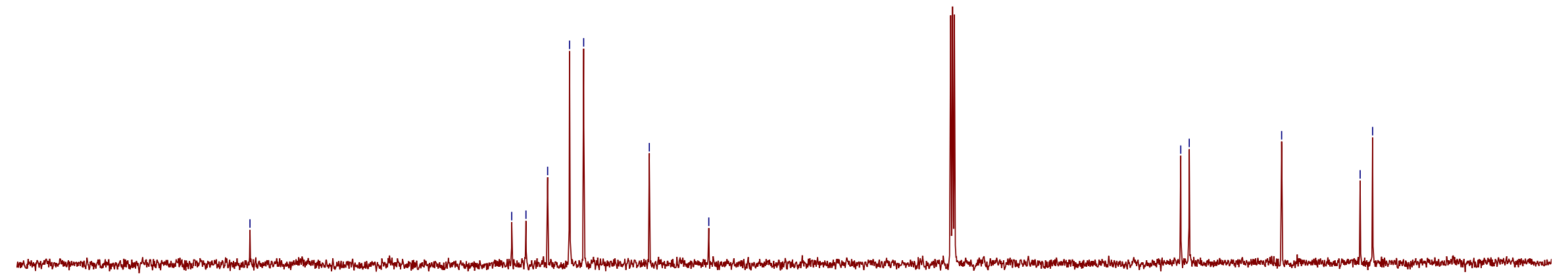




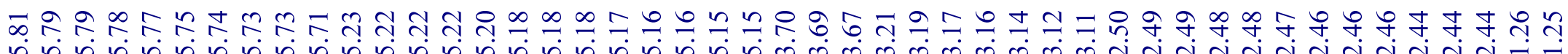

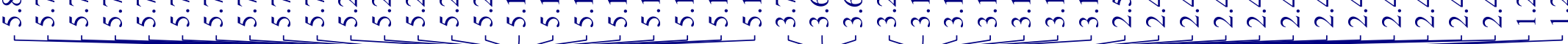

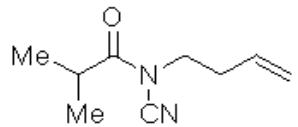

$1 \mathrm{f}$

${ }^{1} \mathrm{H}$ NMR $\left(400 \mathrm{MHz}, \mathrm{CDCl}_{3}\right)$

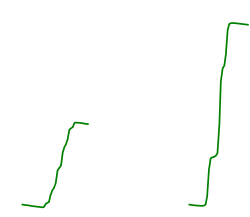

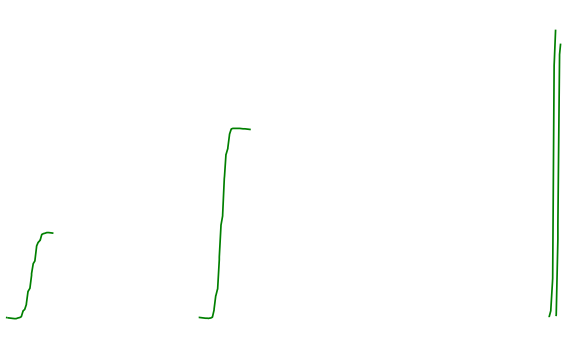

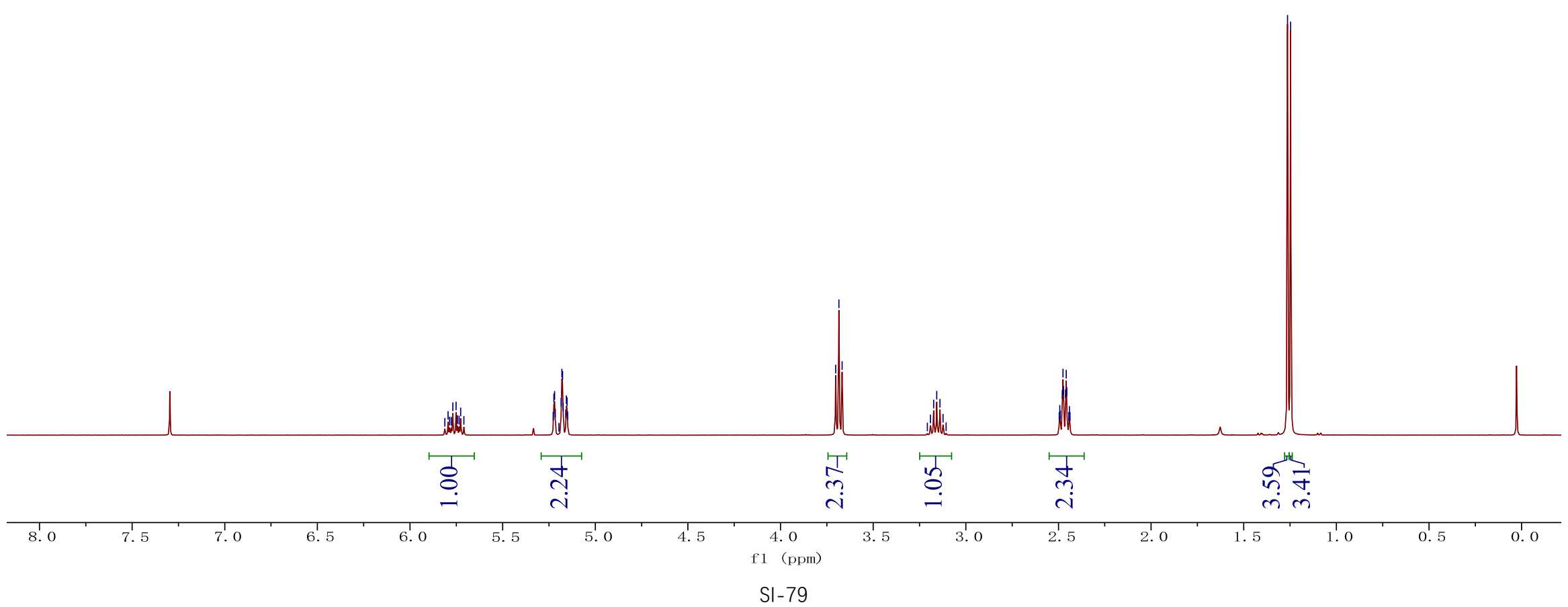



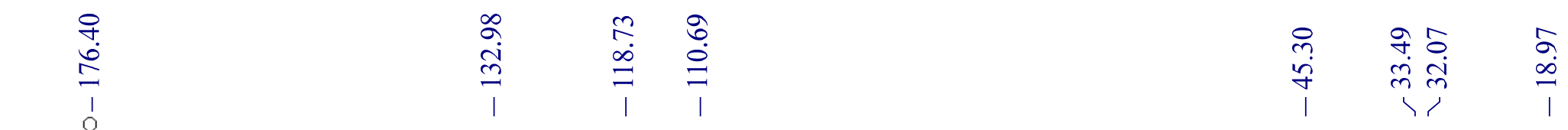

${ }^{13} \mathrm{C}$ NMR $\left(125 \mathrm{MHz}, \mathrm{CDCl}_{3}\right)$
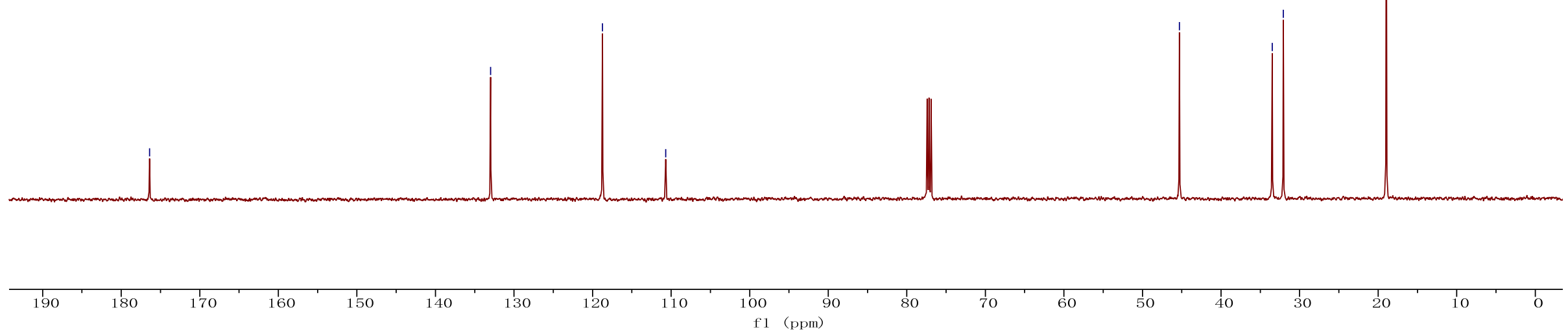

SI-80 


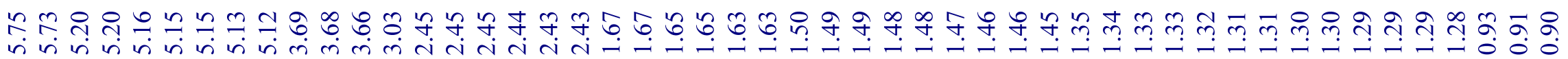

$$
\lim _{1 \mathrm{CN}}
$$

${ }^{1} \mathrm{H}$ NMR (400 MHz, $\mathrm{CDCl}_{3}$ )
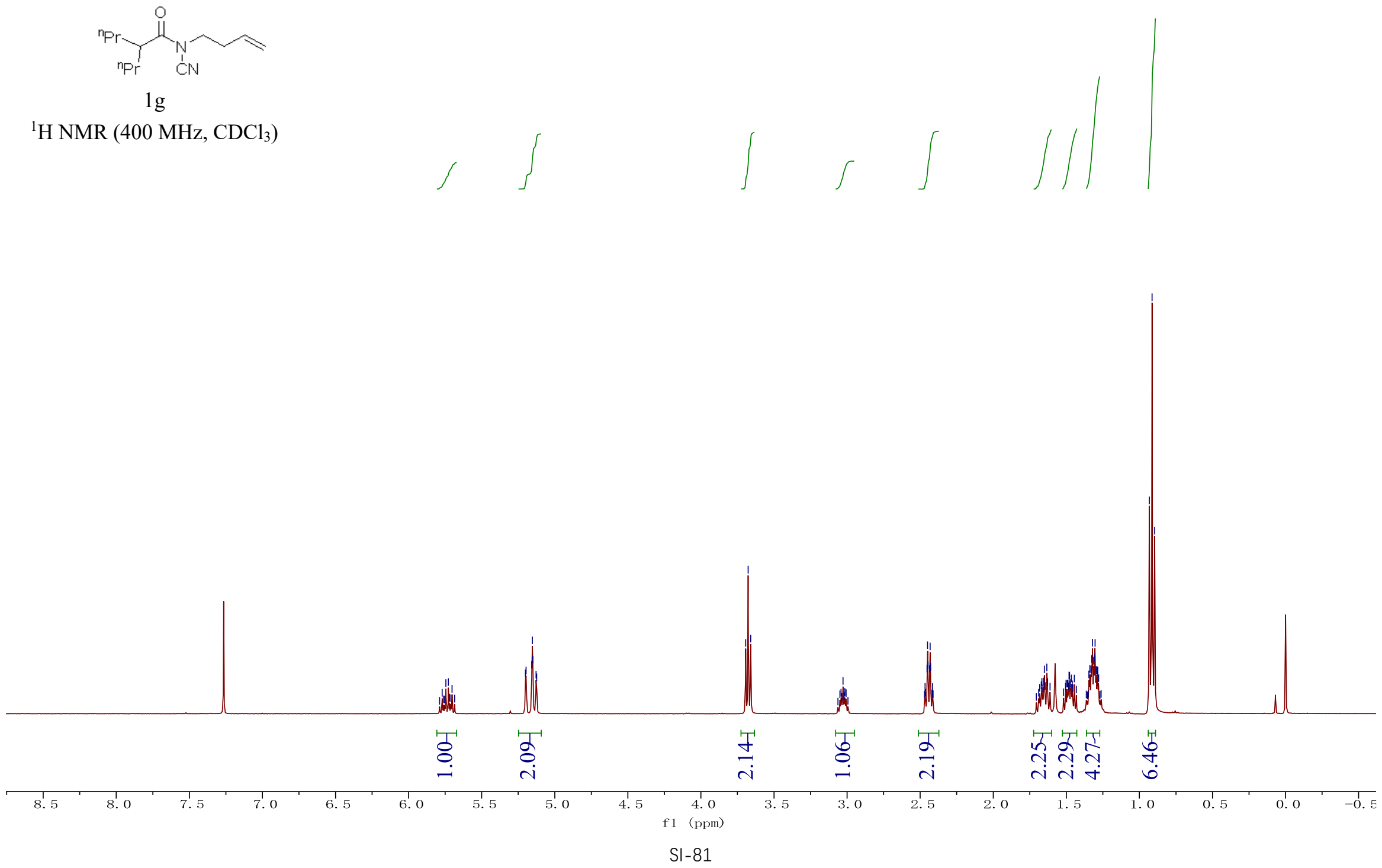

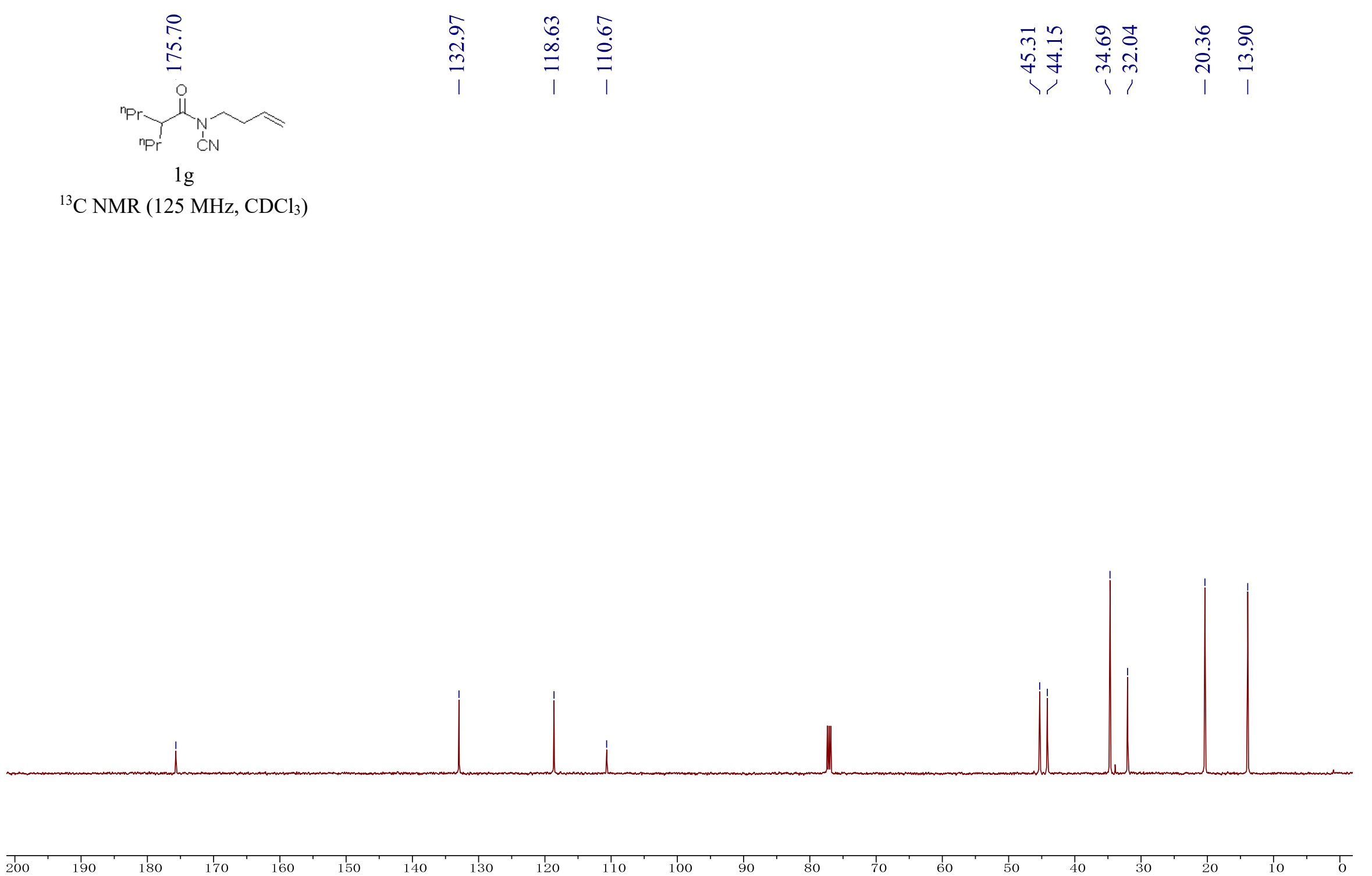


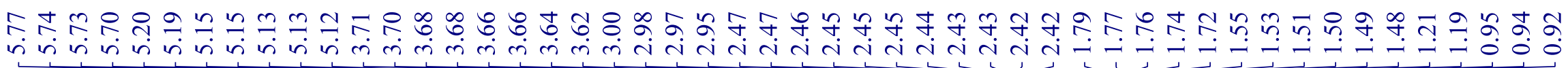

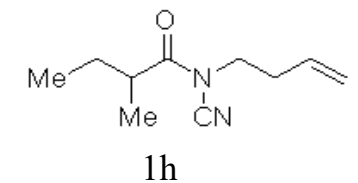

${ }^{1} \mathrm{H}$ NMR $\left(400 \mathrm{MHz}, \mathrm{CDCl}_{3}\right)$
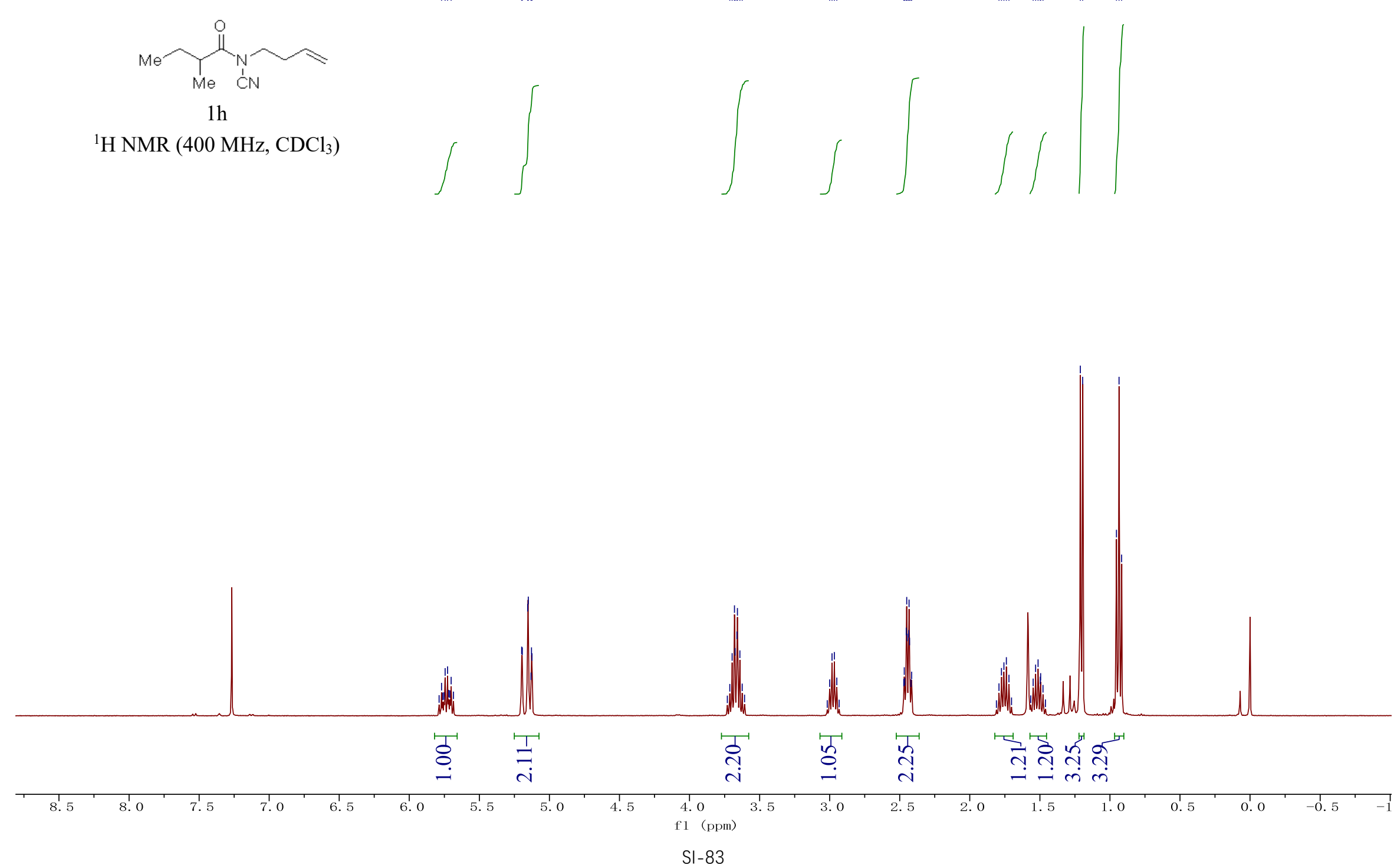
$\frac{2}{2}$

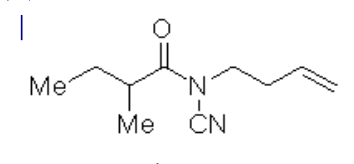

$1 \mathrm{~h}$

${ }^{13} \mathrm{C}$ NMR (125 MHz, $\left.\mathrm{CDCl}_{3}\right)$

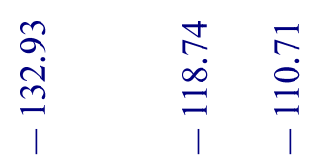

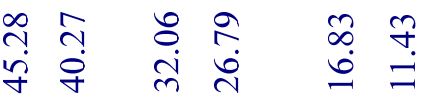

(1)

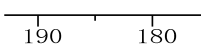




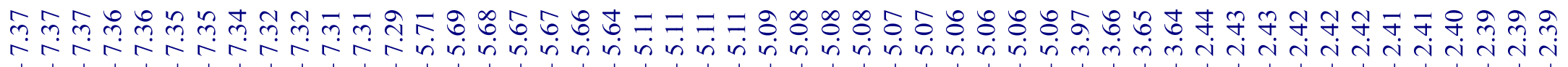
CN

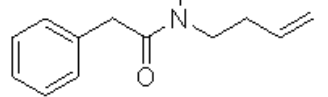

$1 \mathrm{i}$

${ }^{1} \mathrm{H}$ NMR (500 MHz, $\left.\mathrm{CDCl}_{3}\right)$
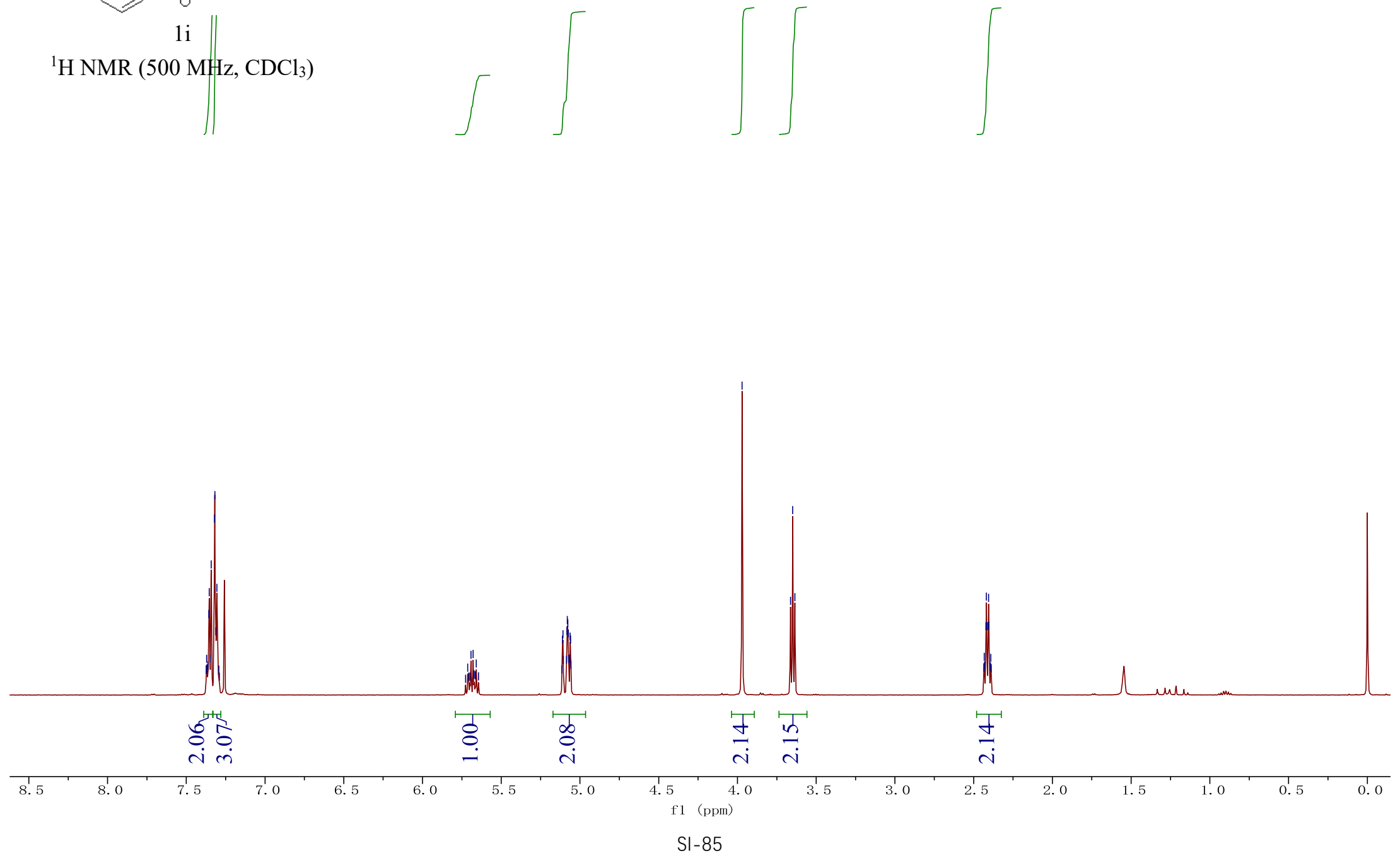

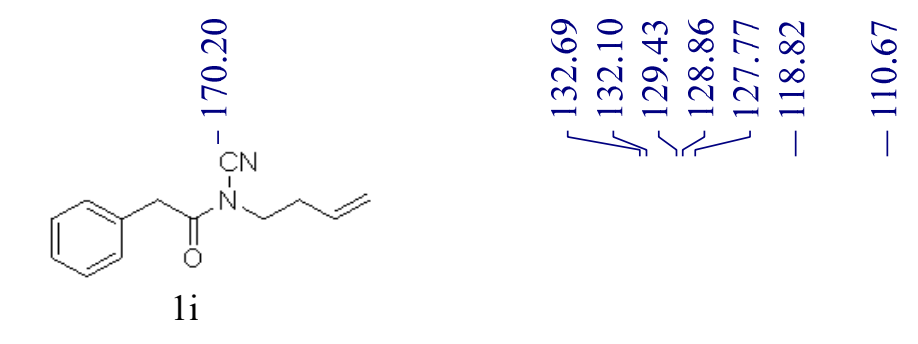

ஸैं ?

${ }^{13} \mathrm{C}$ NMR (125 MHz, $\left.\mathrm{CDCl}_{3}\right)$

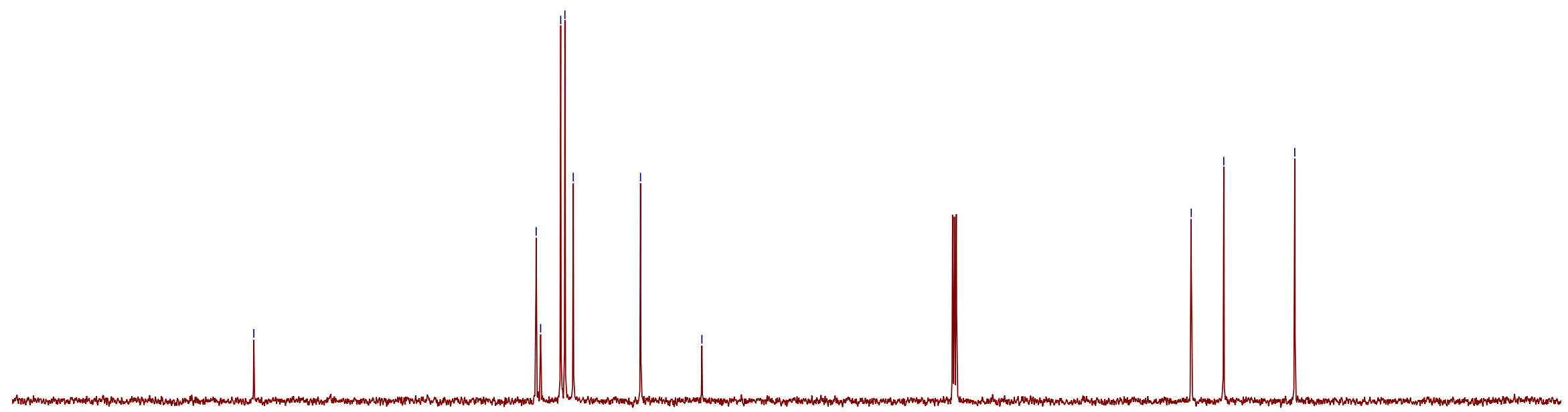




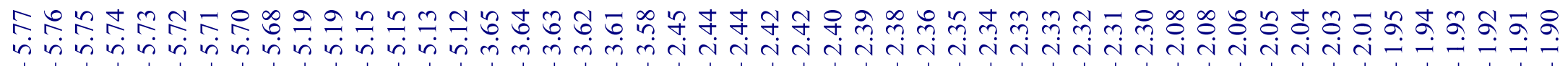<smiles>C=CCCN(C)C(=O)C1CC1</smiles>

${ }^{1} \mathrm{H} \mathrm{NMR}\left(400 \mathrm{MHz}, \mathrm{CDCl}_{3}\right)$
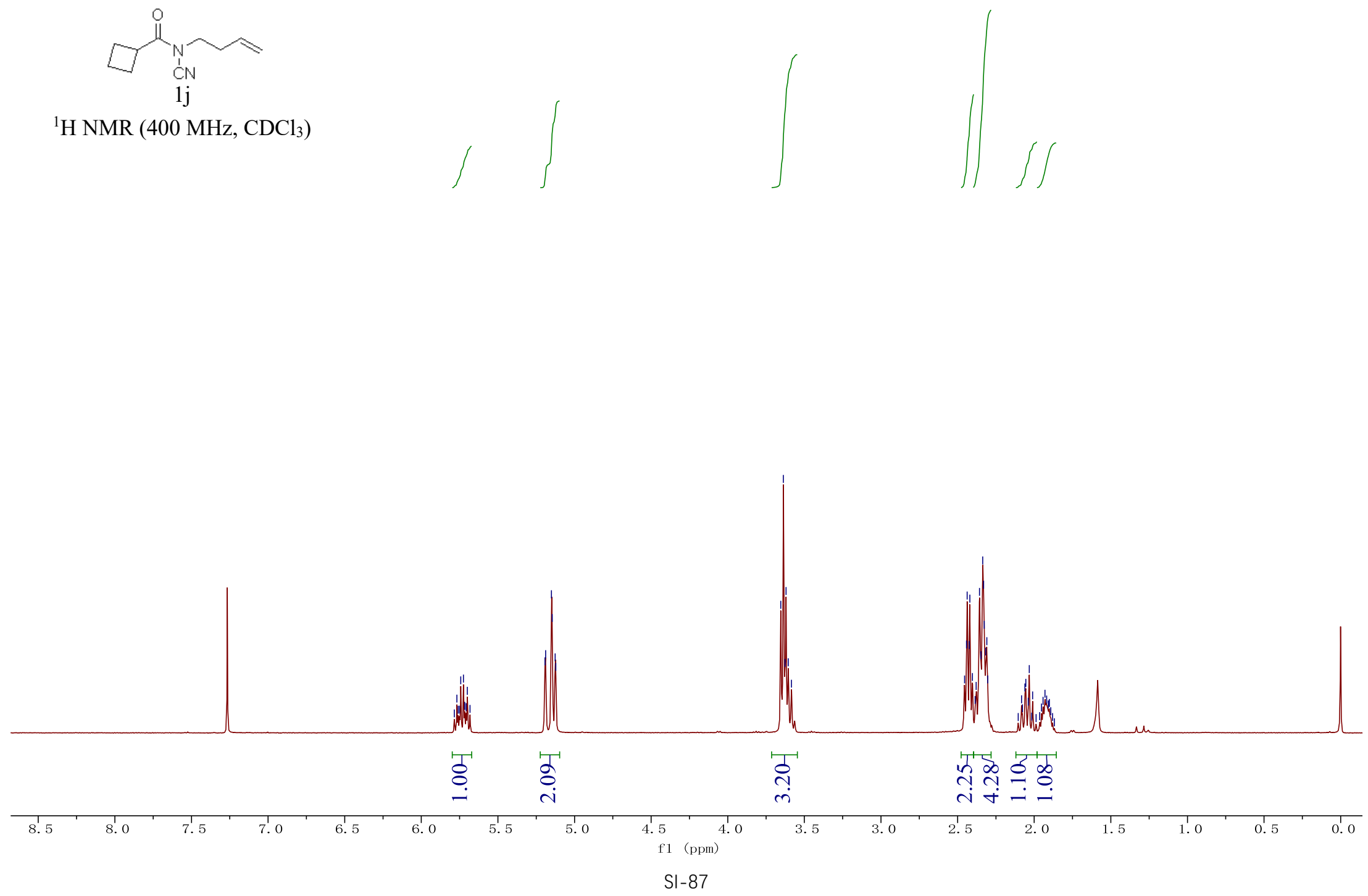

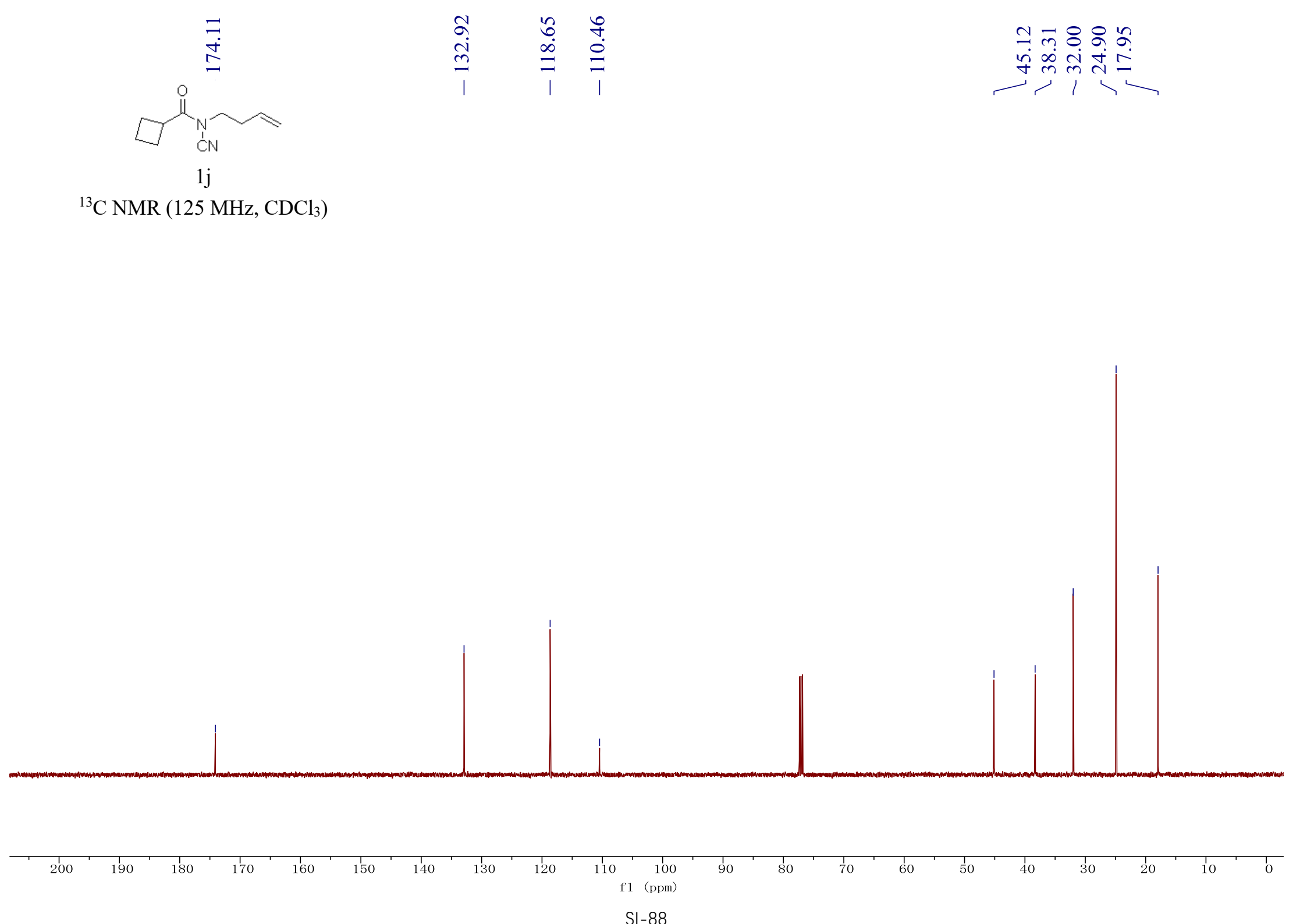


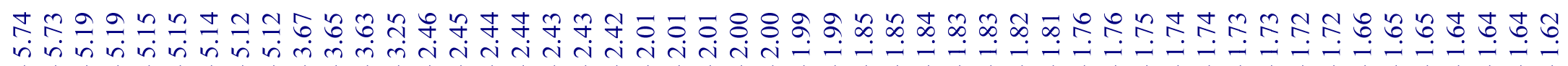
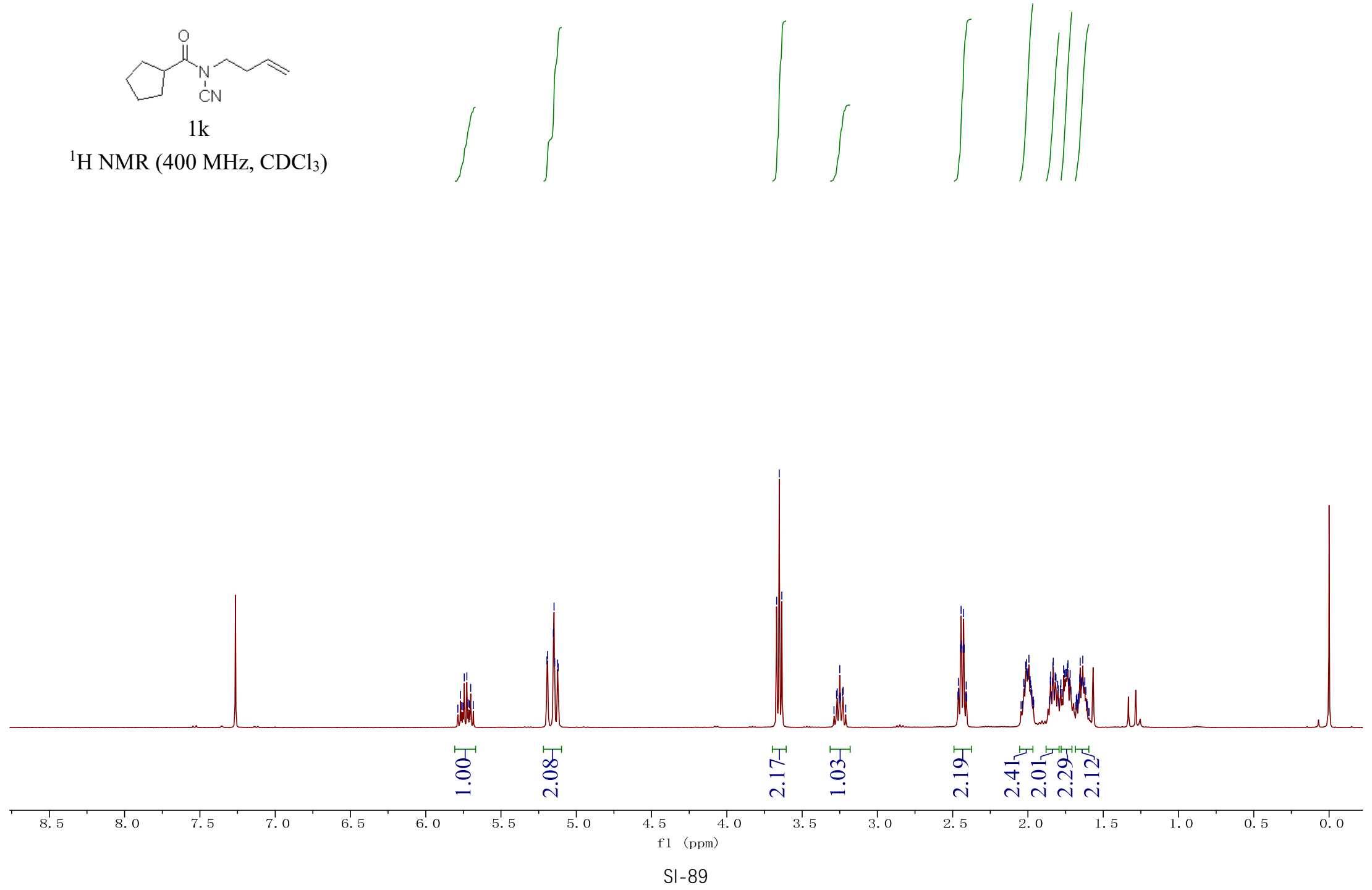


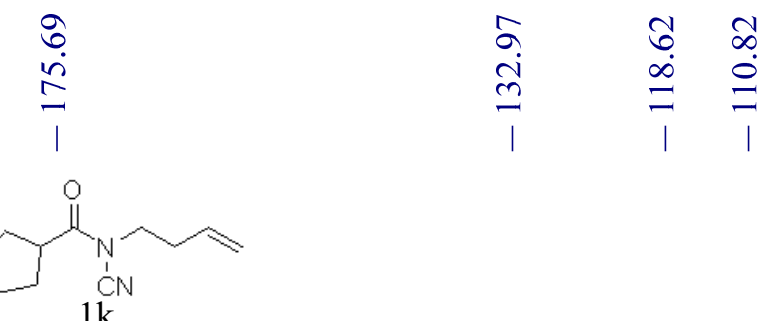

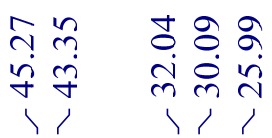

$1 \mathrm{k}$

${ }^{13} \mathrm{C}$ NMR (125 MHz, $\left.\mathrm{CDCl}_{3}\right)$

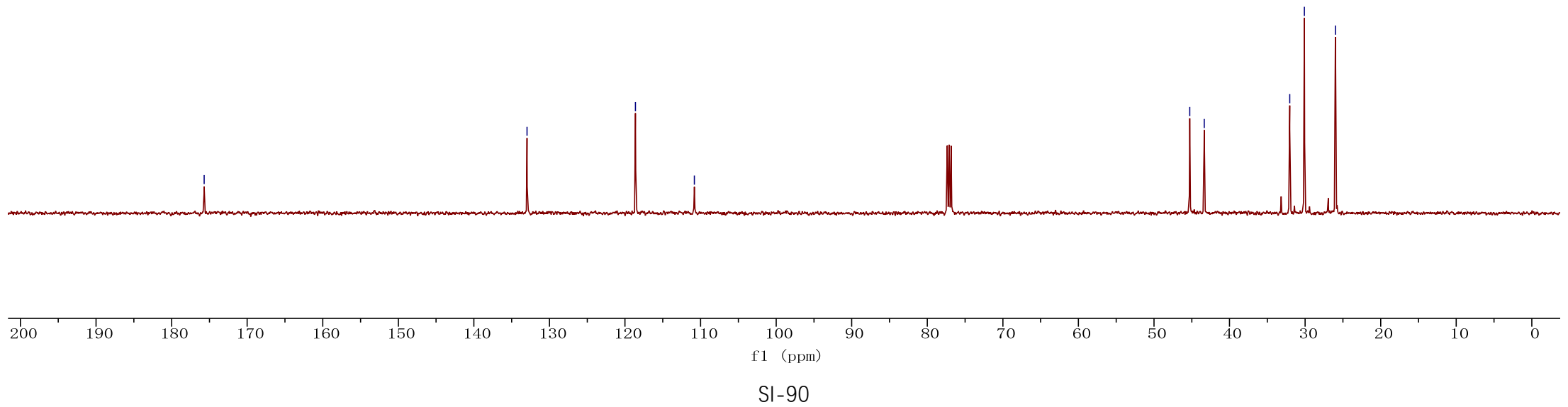




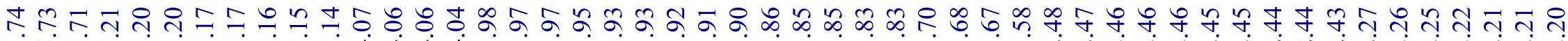

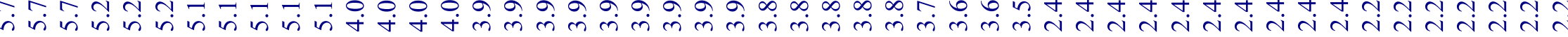

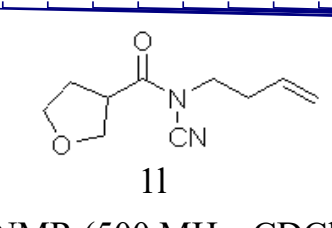

${ }^{1} \mathrm{H}$ NMR $\left(500 \mathrm{MHz}, \mathrm{CDCl}_{3}\right)$
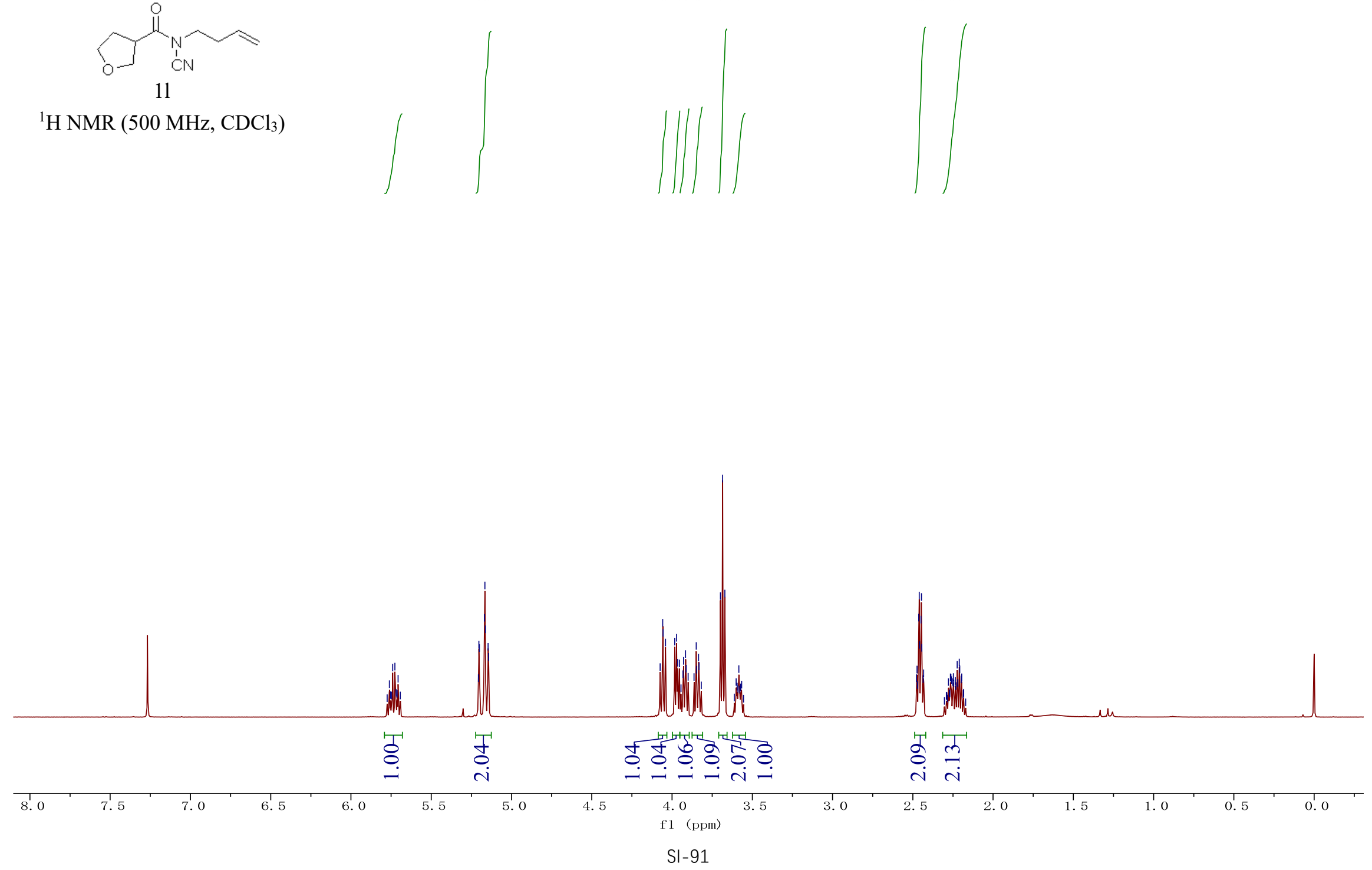


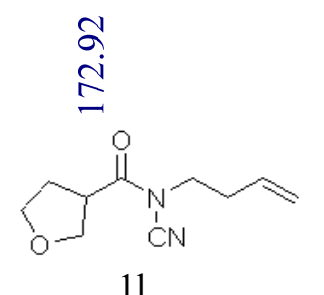

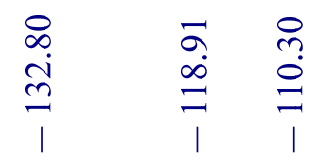

$\begin{array}{ll}+ & 0 \\ 0 & ? \\ 0 & 0\end{array}$

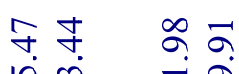

में कें

${ }^{13} \mathrm{C}$ NMR (125 MHz, $\mathrm{CDCl}_{3}$ )
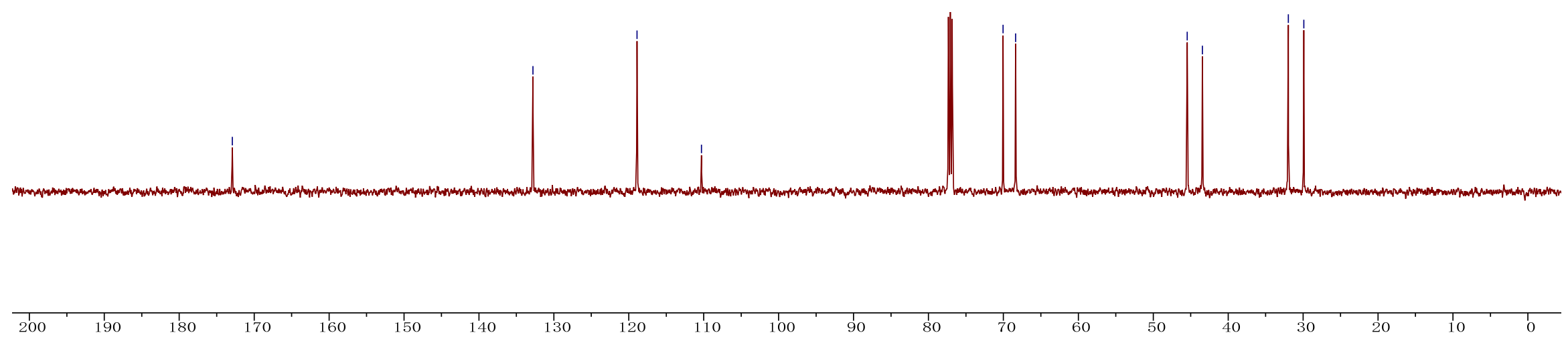

180

170

160

150

140

130

20

100

90

80

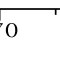

60

50

30

20 


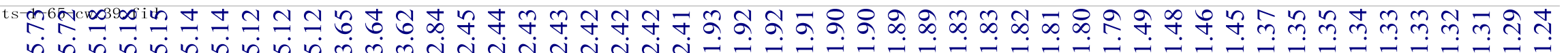

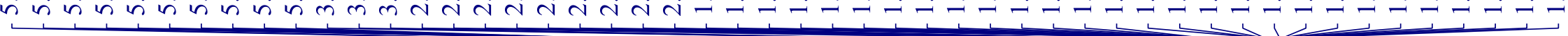

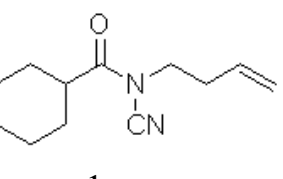

$1 \mathrm{~m}$

${ }^{1} \mathrm{H} \mathrm{NMR}\left(500 \mathrm{MHz}, \mathrm{CDCl}_{3}\right)$
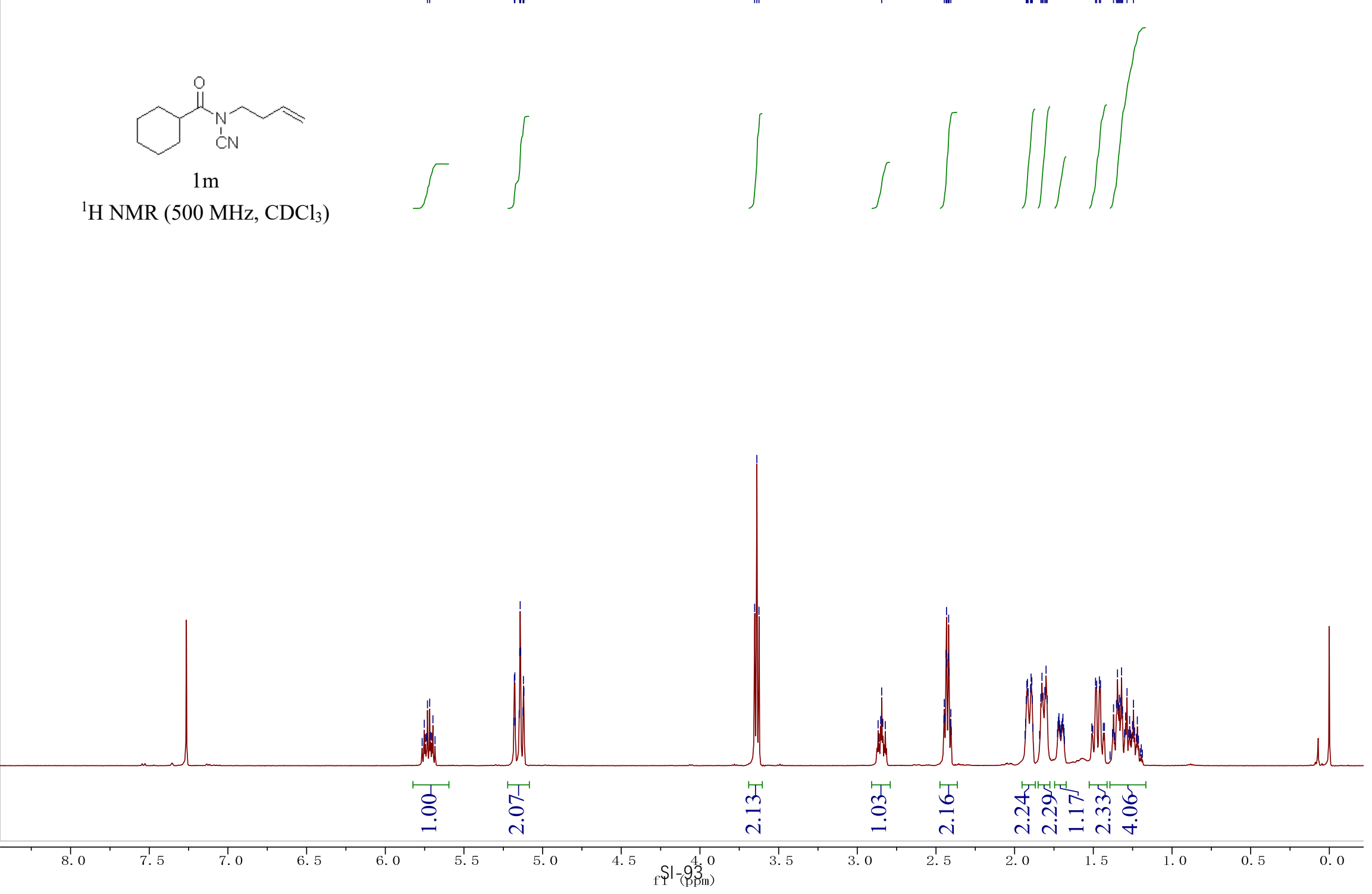


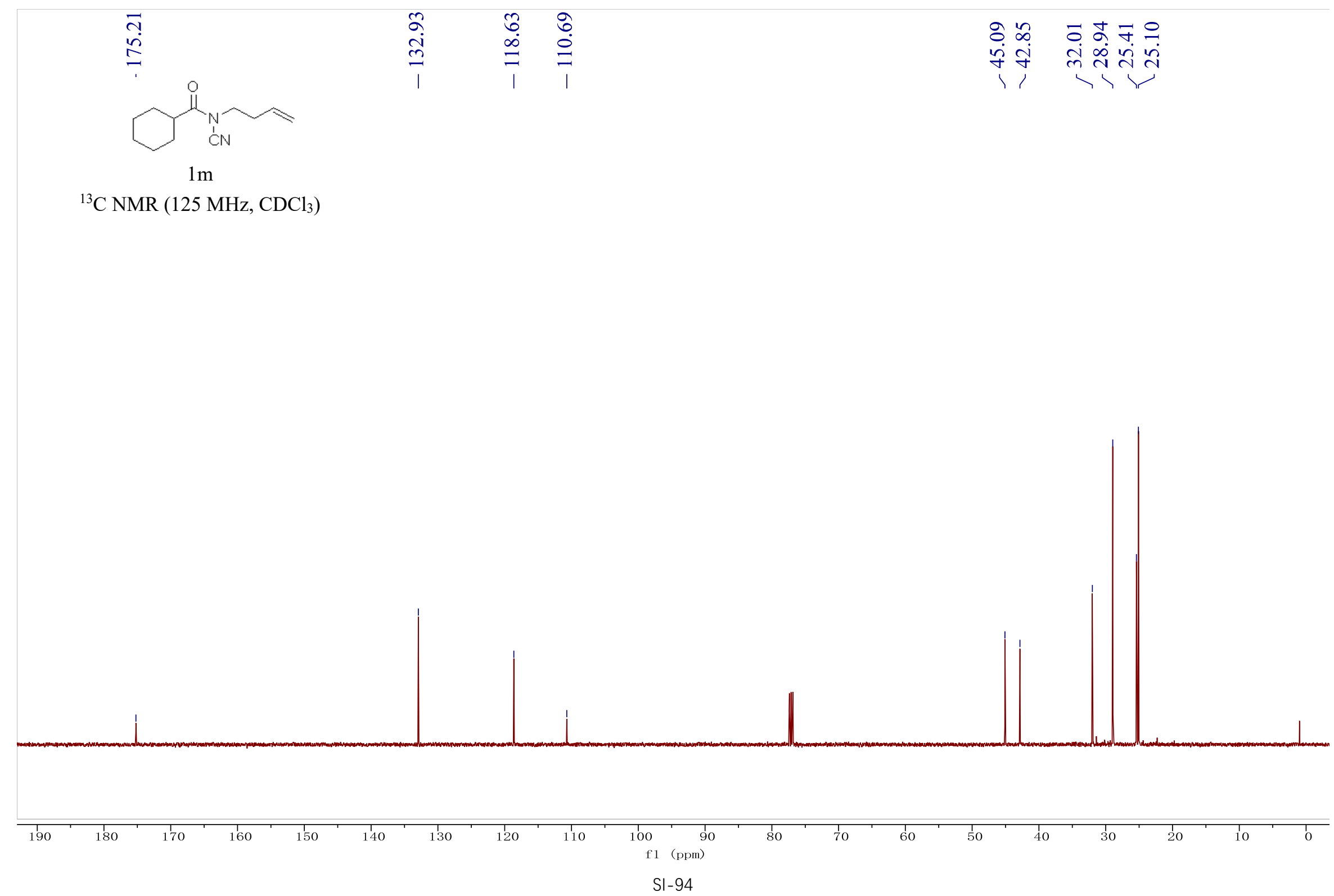




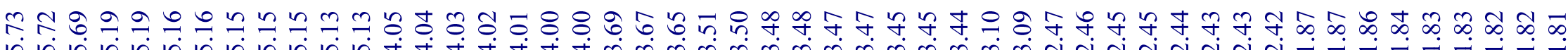
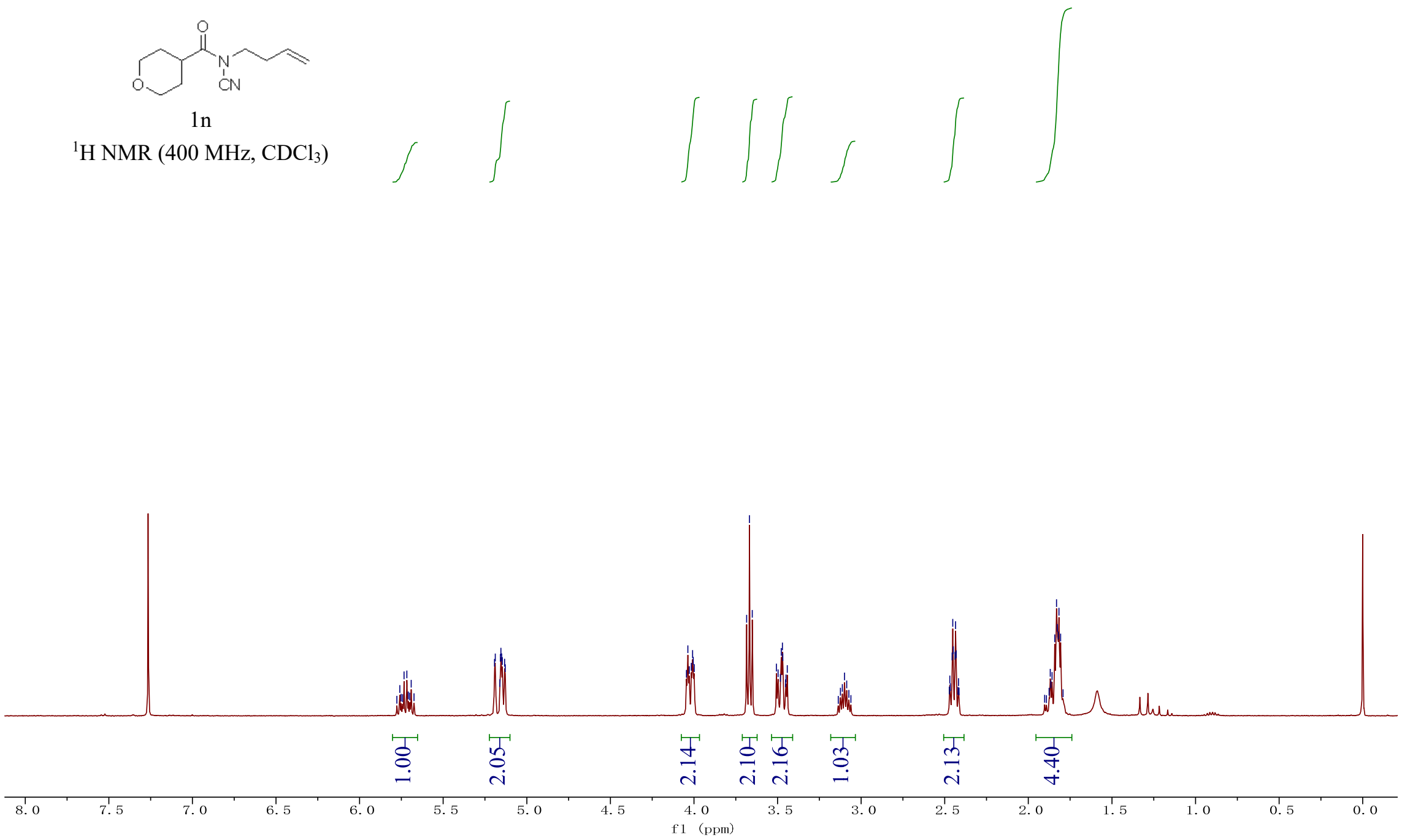


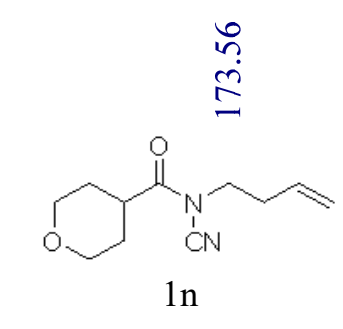

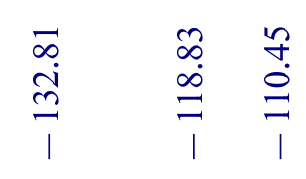

กิ

กิ

में क्षे

1,12

${ }^{13} \mathrm{C}$ NMR $\left(125 \mathrm{MHz}, \mathrm{CDCl}_{3}\right)$
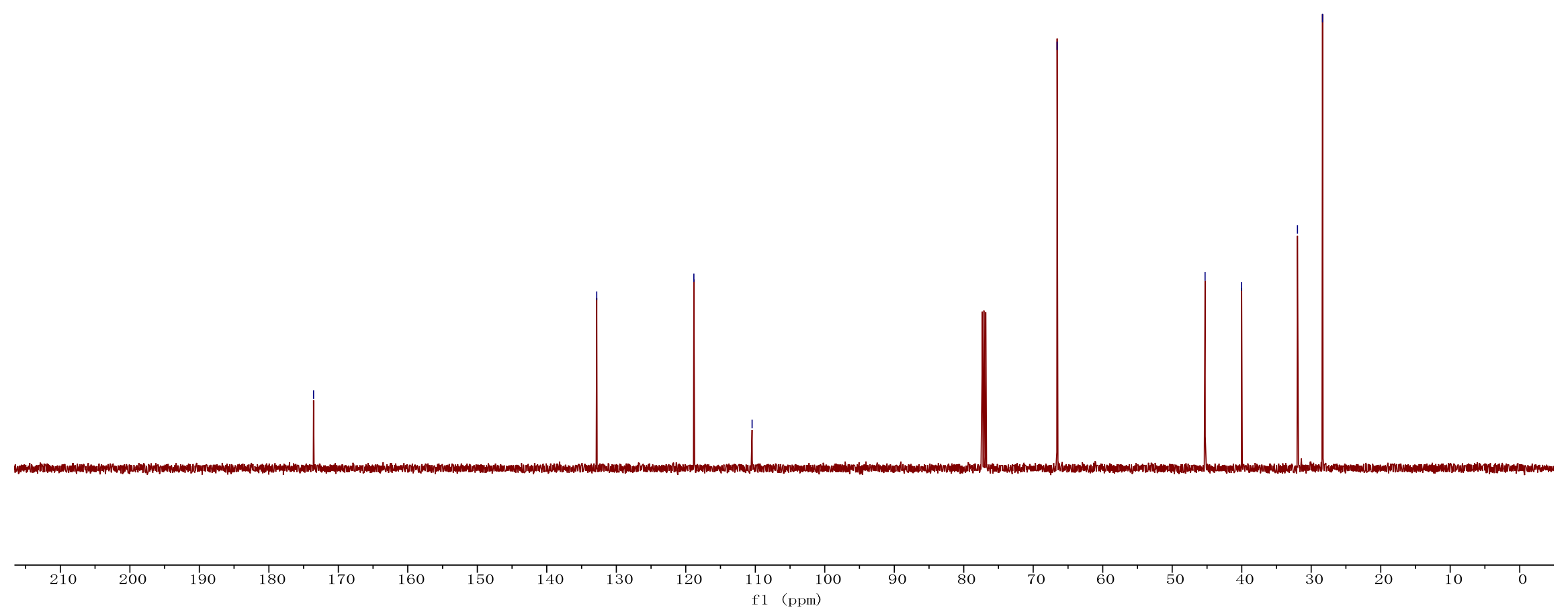


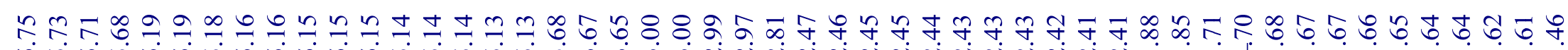

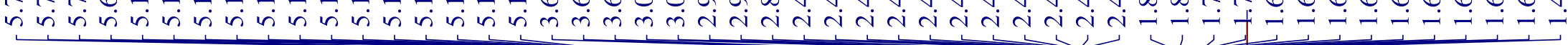

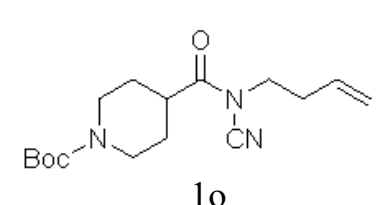

${ }^{1} \mathrm{H}$ NMR $\left(400 \mathrm{MHz}, \mathrm{CDCl}_{3}\right)$

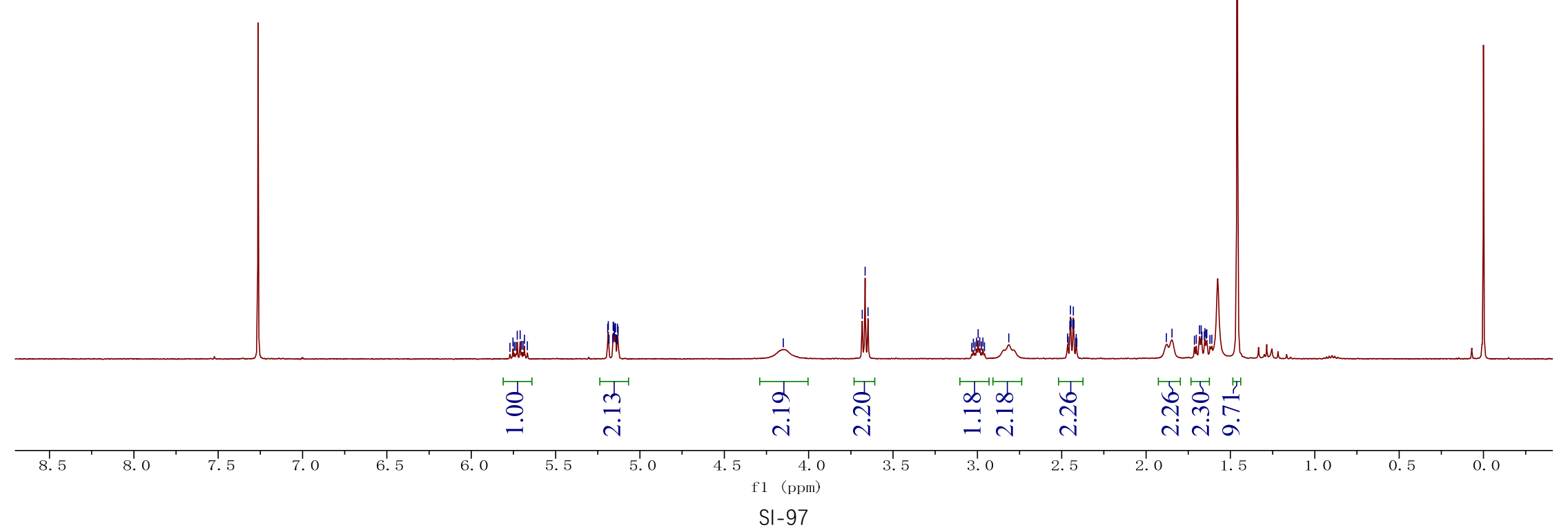



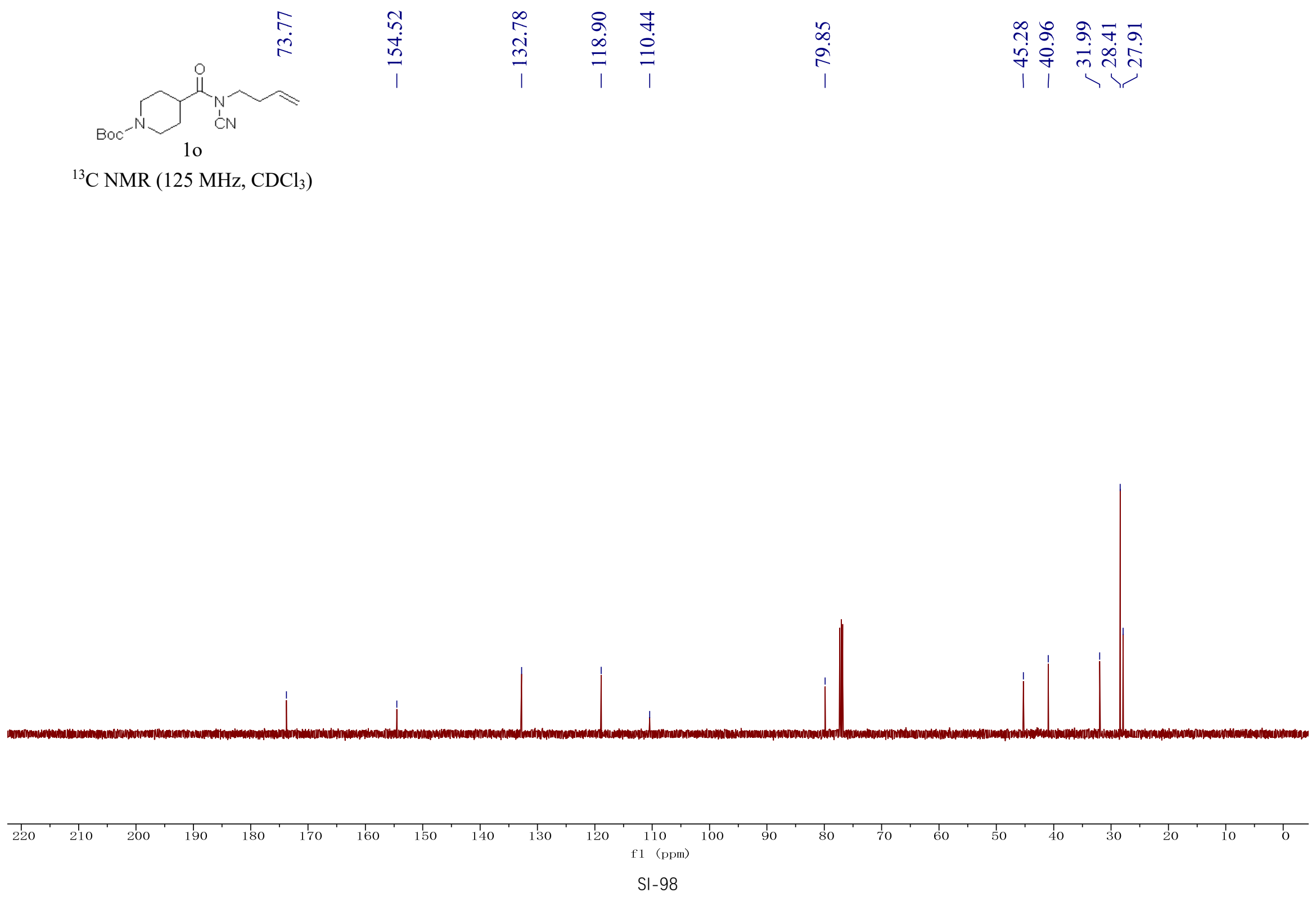


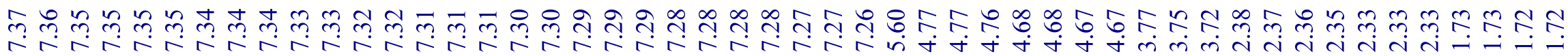

$\longrightarrow$

$$
\left.\right|_{1 \mathrm{p}} ^{\mathrm{C}}
$$

${ }^{1} \mathrm{H}$ NMR (300 MHz, $\left.\mathrm{CDCl}_{3}\right)$

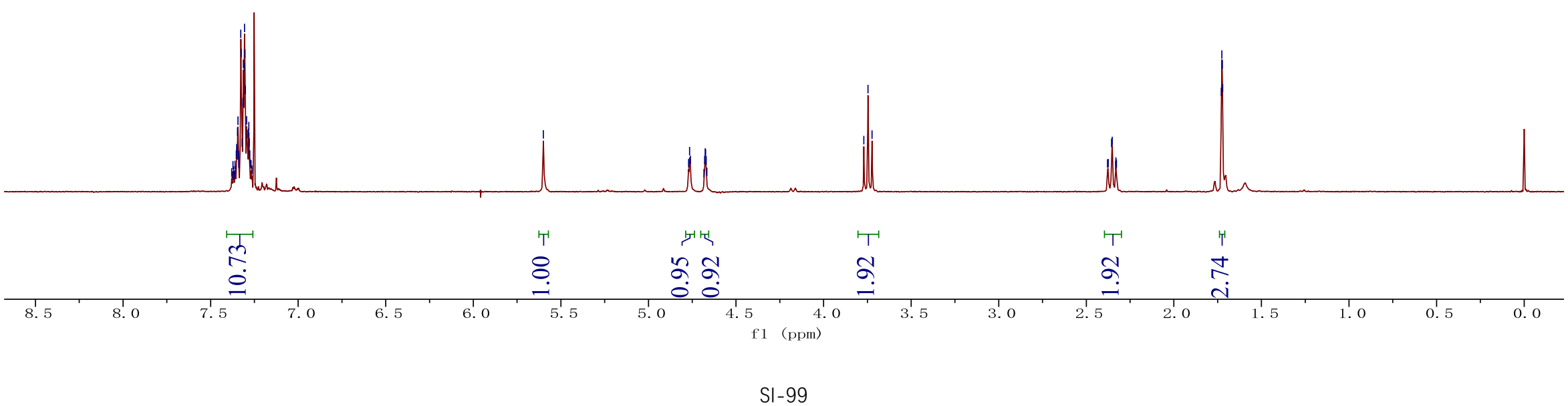




$$
\text { 1p }
$$

${ }^{13} \mathrm{C} \mathrm{NMR}\left(125 \mathrm{MHz}, \mathrm{CDCl}_{3}\right)$

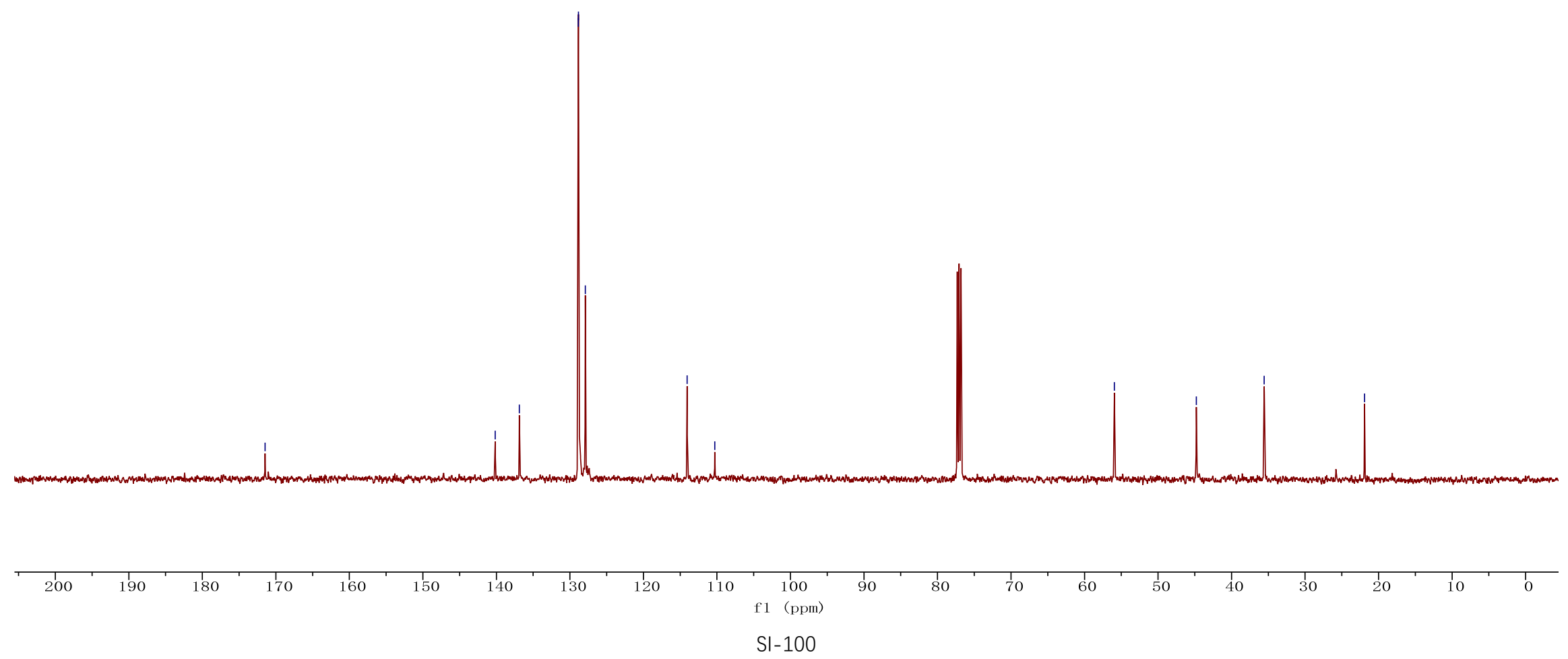




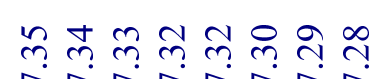
r. $\prod_{O}^{N}$

$1 \mathrm{q}$

${ }^{1} \mathrm{H}$ NMR $\left(400 \mathrm{MHz}, \mathrm{CDCl}_{3}\right)$

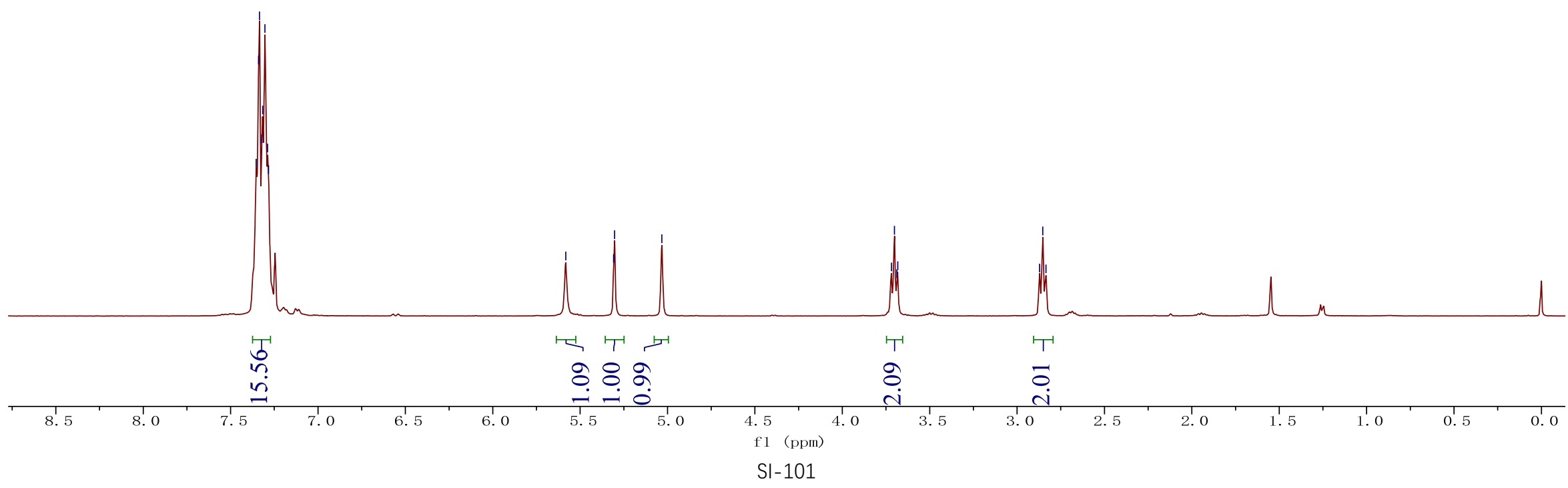

다융ㅇㅇ

mंmm Niri

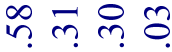

inin
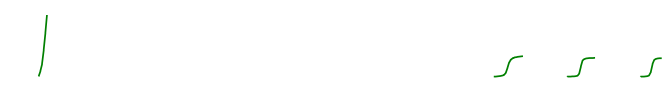


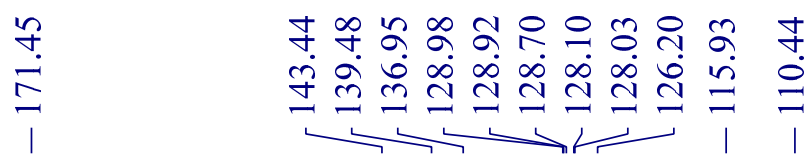

$\prod_{0}^{P N}$

$1 \mathrm{q}$

${ }^{13} \mathrm{C}$ NMR $\left(125 \mathrm{MHz}, \mathrm{CDCl}_{3}\right)$

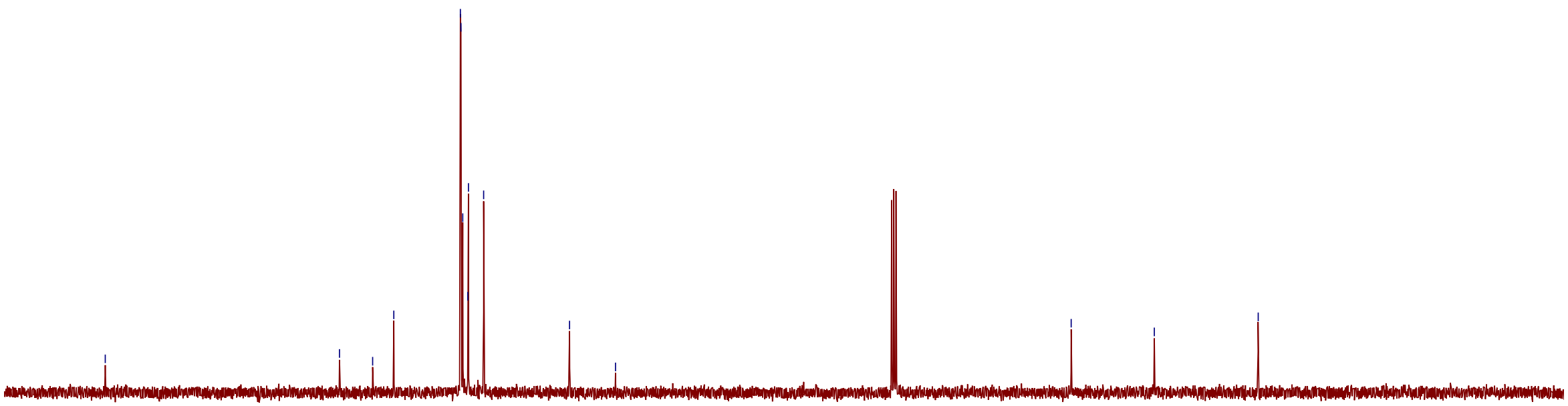




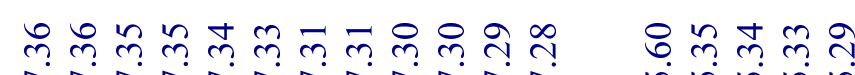

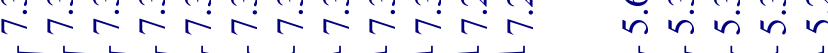

${ }^{1} \mathrm{NMR}\left(500 \mathrm{MHz}, \mathrm{CDCl}_{3}\right)$ $\left\{\begin{array}{c}\sqrt{2} \\ 5\end{array}\right.$

min

4

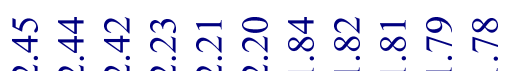

N N N
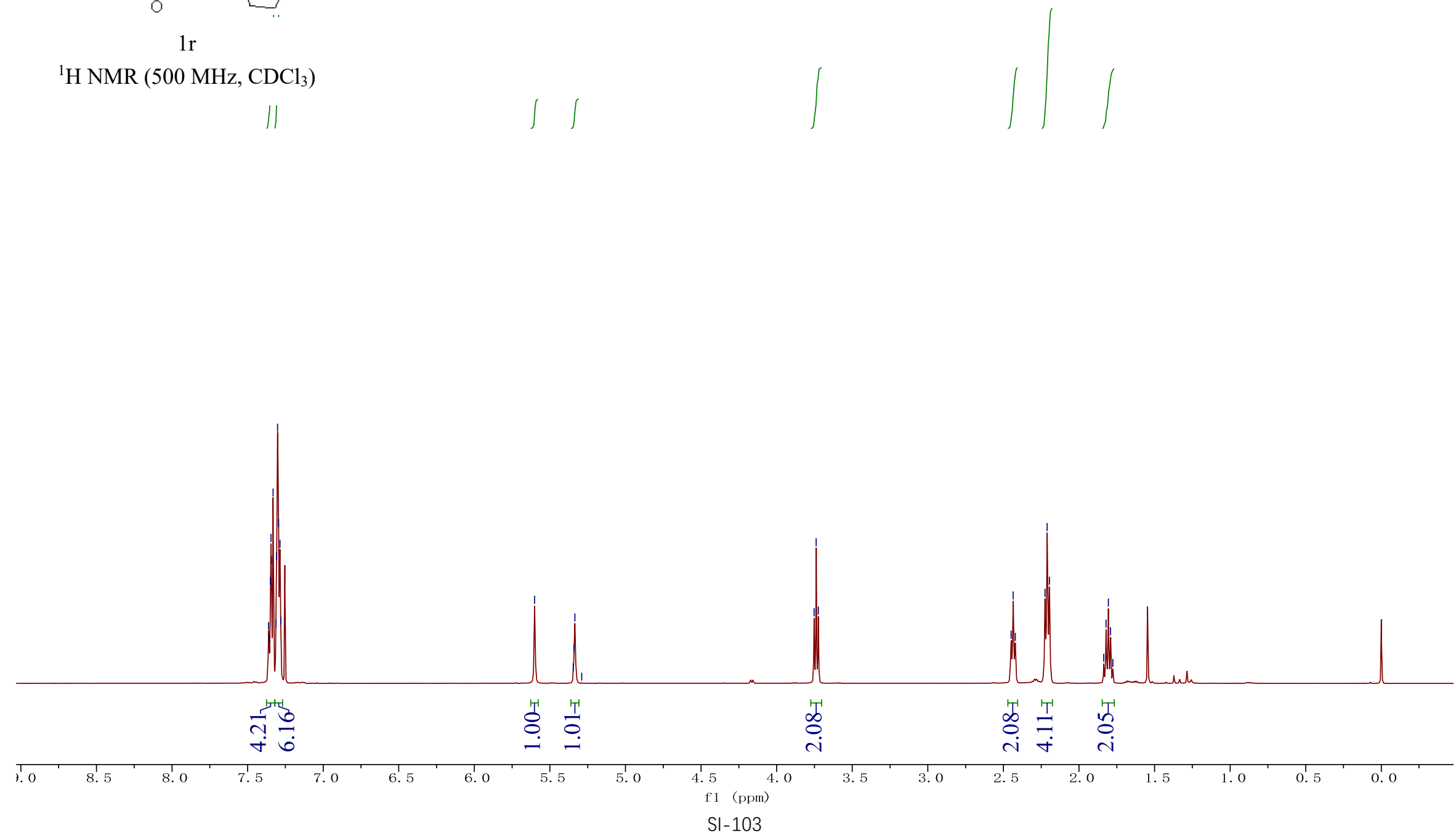


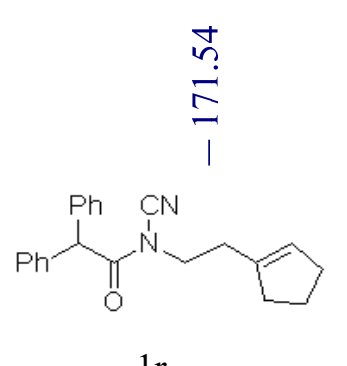

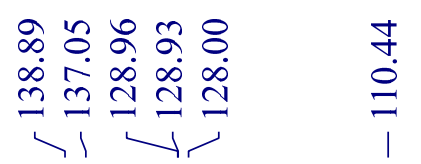

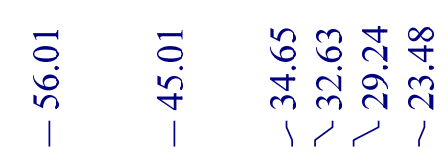

$1 \mathrm{r}$

${ }^{13} \mathrm{C} \mathrm{NMR}\left(125 \mathrm{MHz}, \mathrm{CDCl}_{3}\right)$

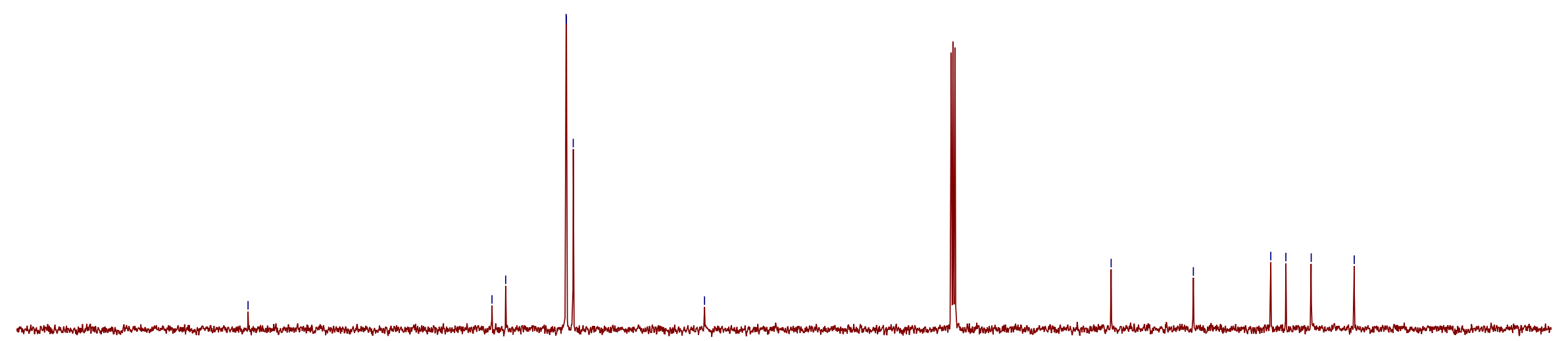

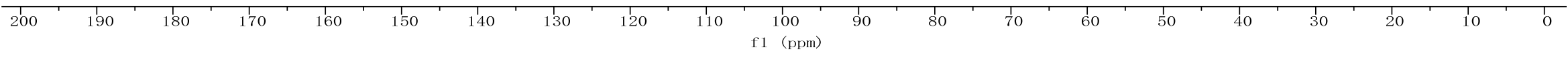
SI-104 


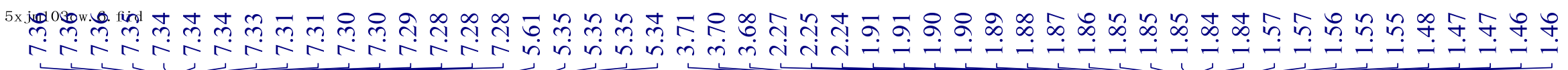

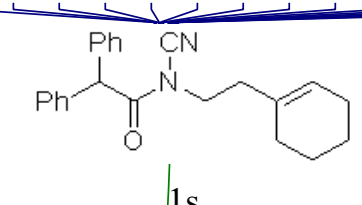

${ }^{1} \mathrm{H} \mathrm{NMR}\left(500 \mathrm{MHz}, \mathrm{CDCl}_{3}\right)$
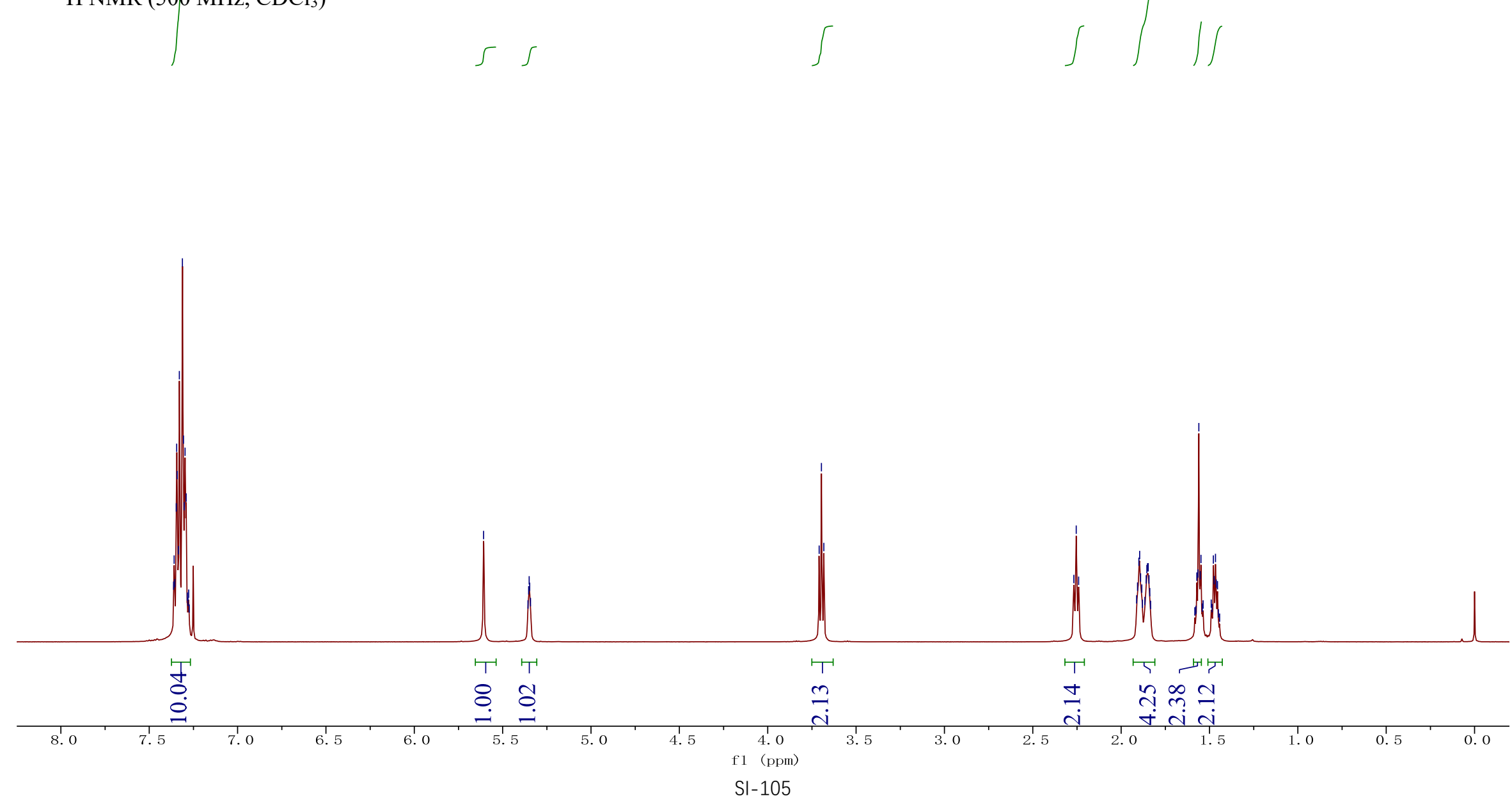

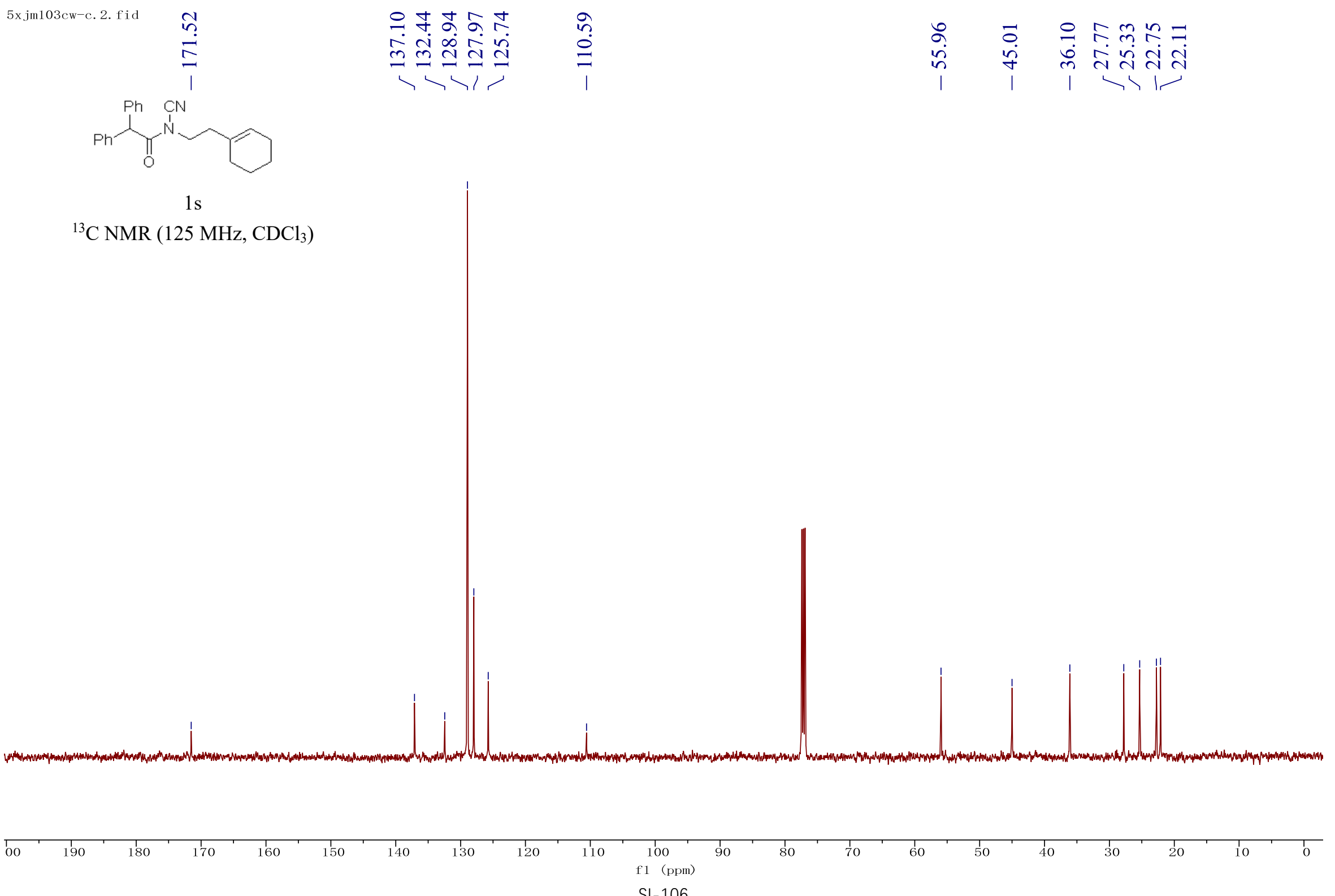


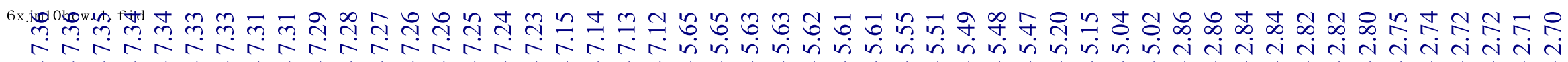
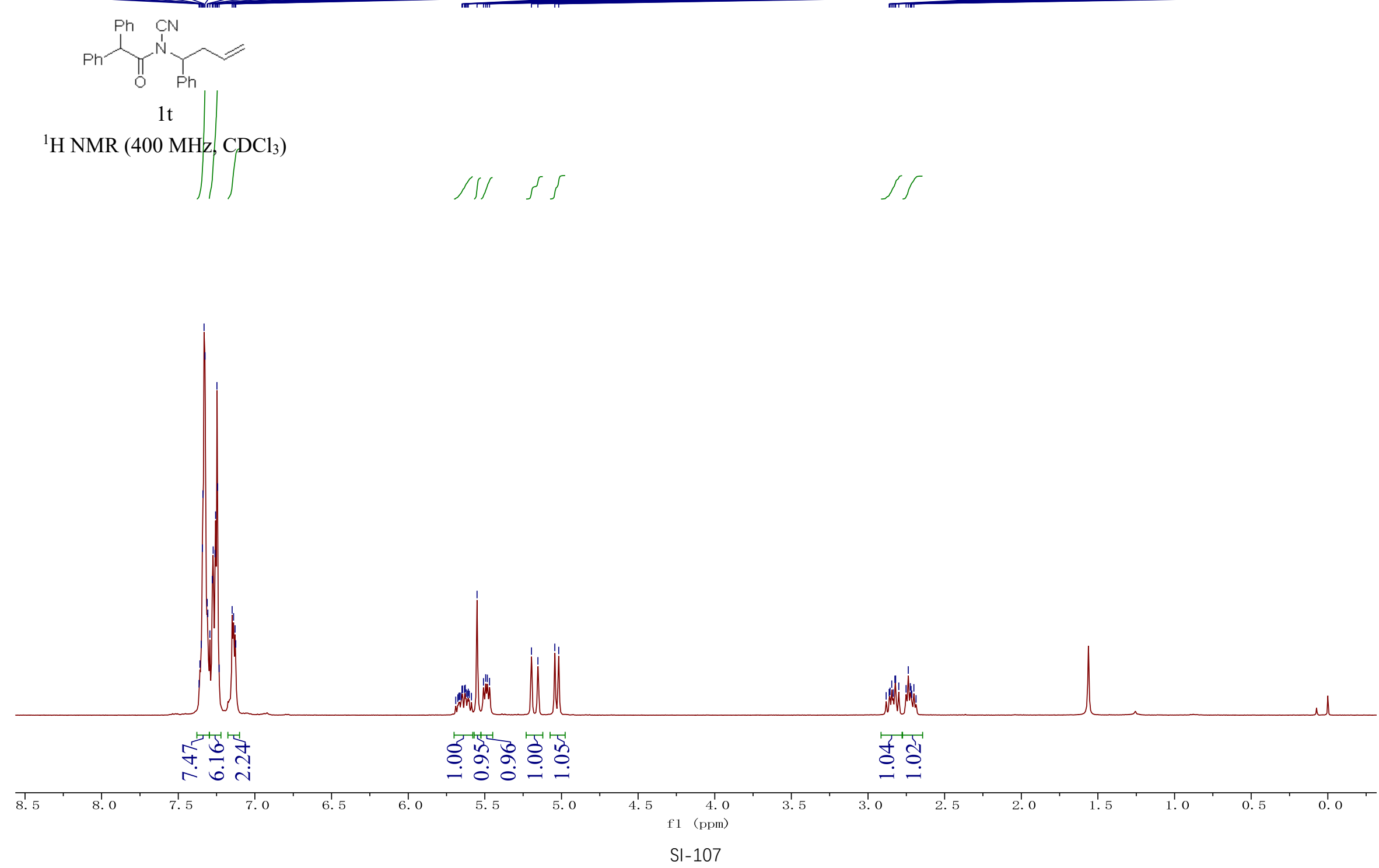


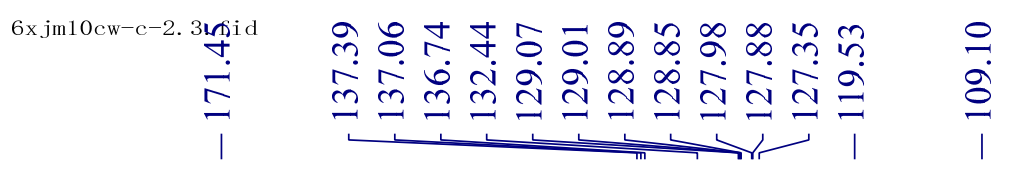

จุ

in in

$\stackrel{\infty}{n}$ $\prod_{0}^{\mathrm{Ph}} \mathrm{Ph}_{\mathrm{Ph}}^{\mathrm{CN}}$

$1 \mathrm{t}$

${ }^{13} \mathrm{C}$ NMR (125 MHz, $\left.\mathrm{CDCl}_{3}\right)$

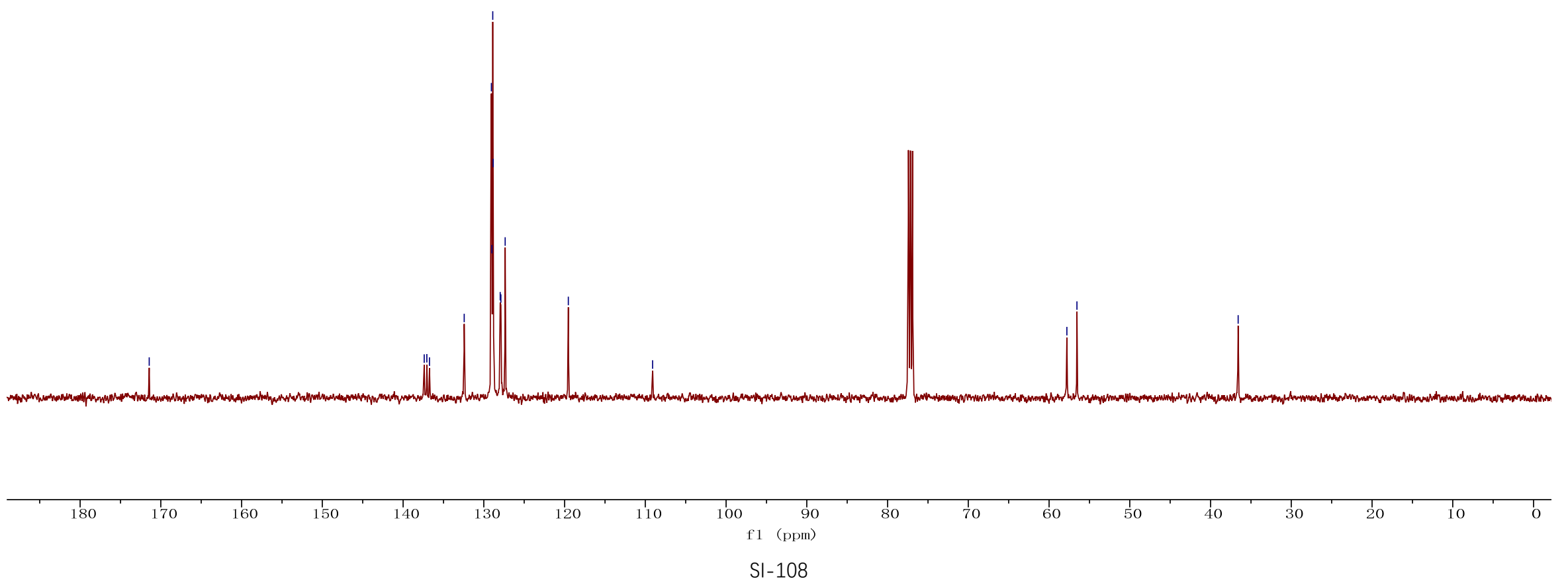




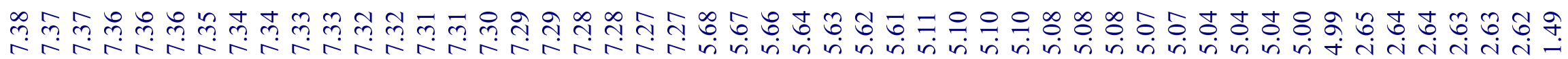
$\prod_{0}^{\mathrm{Ch}} \overbrace{1}^{\mathrm{C} N}$

$1 \mathrm{u}$

${ }^{1} \mathrm{H} \mathrm{NMR}\left(400 \mathrm{MHz}, \mathrm{CDCl}_{3}\right)$

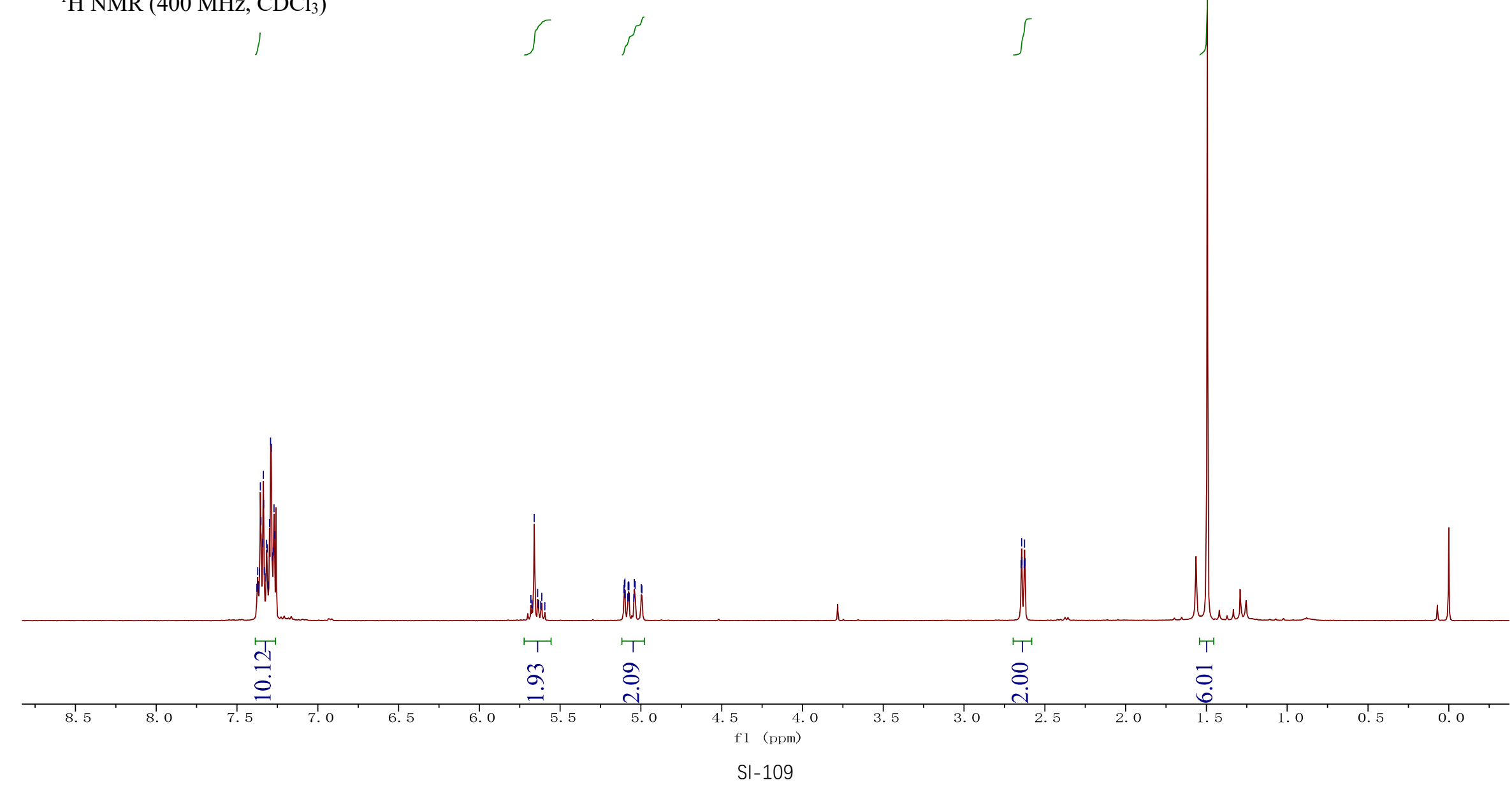




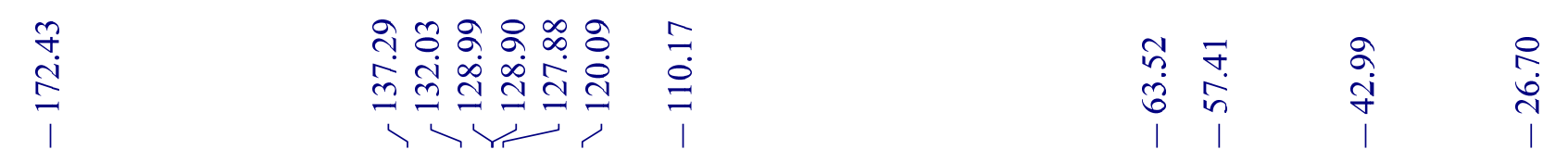

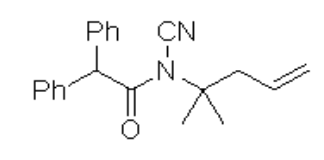

$1 \mathrm{u}$

${ }^{13} \mathrm{C}$ NMR (125 MHz, $\left.\mathrm{CDCl}_{3}\right)$
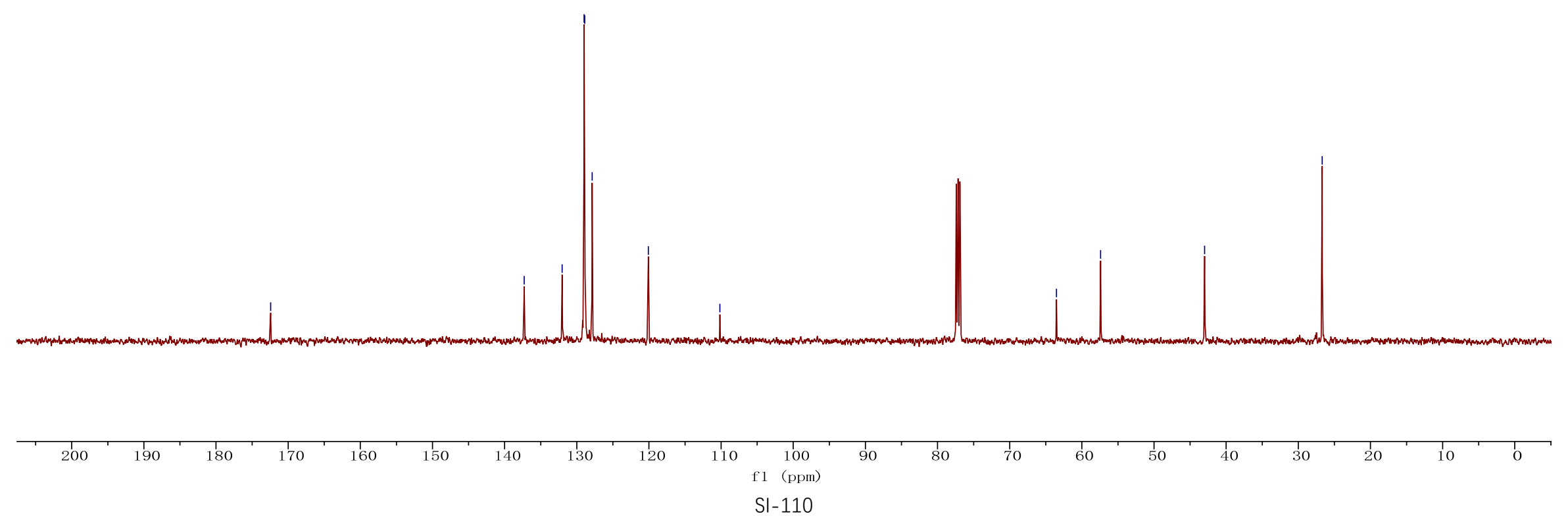


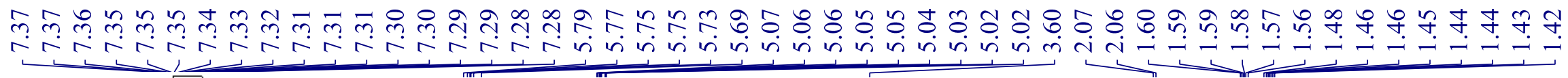
$\prod_{0}^{N}>$

1v

${ }^{1} \mathrm{H} \mathrm{NMR}\left(500 \mathrm{MHz}, \mathrm{CDCl}_{3}\right)$
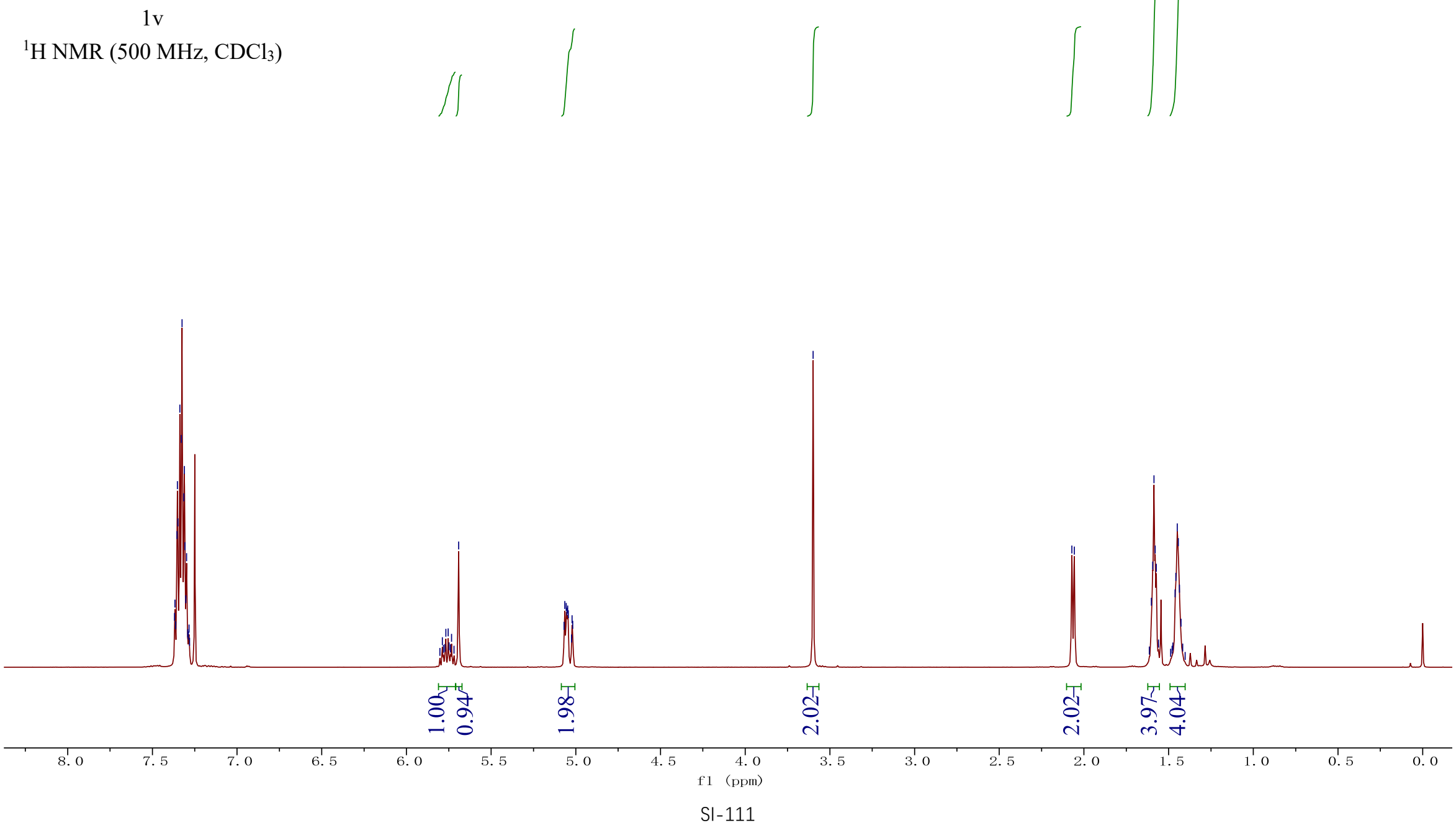


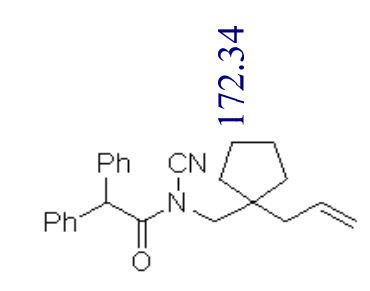

$1 \mathrm{v}$

${ }^{13} \mathrm{C}$ NMR (125 MHz, $\mathrm{CDCl}_{3}$ ) œ

nं

莳

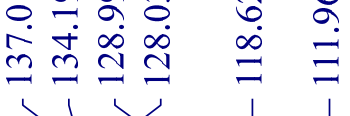

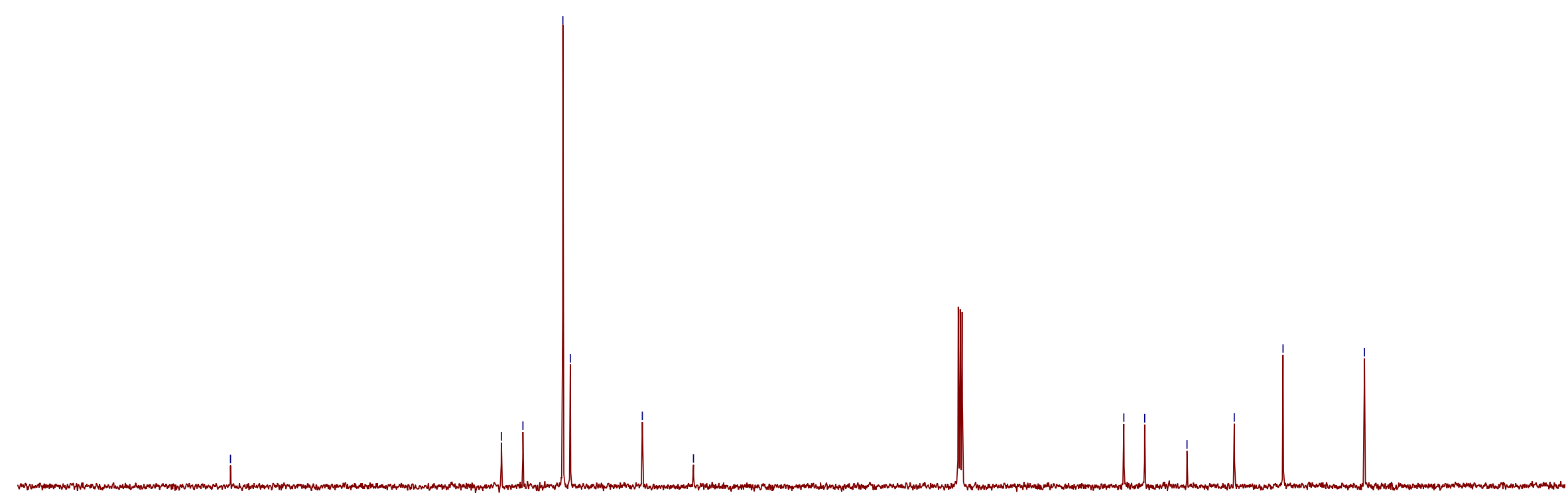

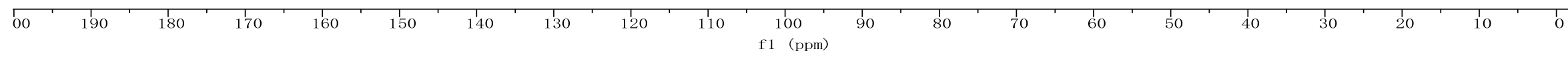
SI-112 
m

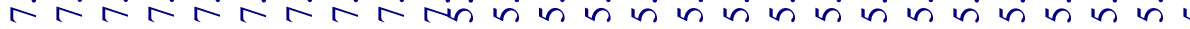

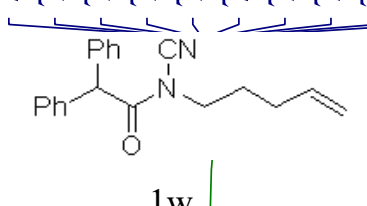

${ }^{1} \mathrm{H}$ NMR $\left(400 \mathrm{MHz}, \mathrm{CDCl}_{3}\right)$
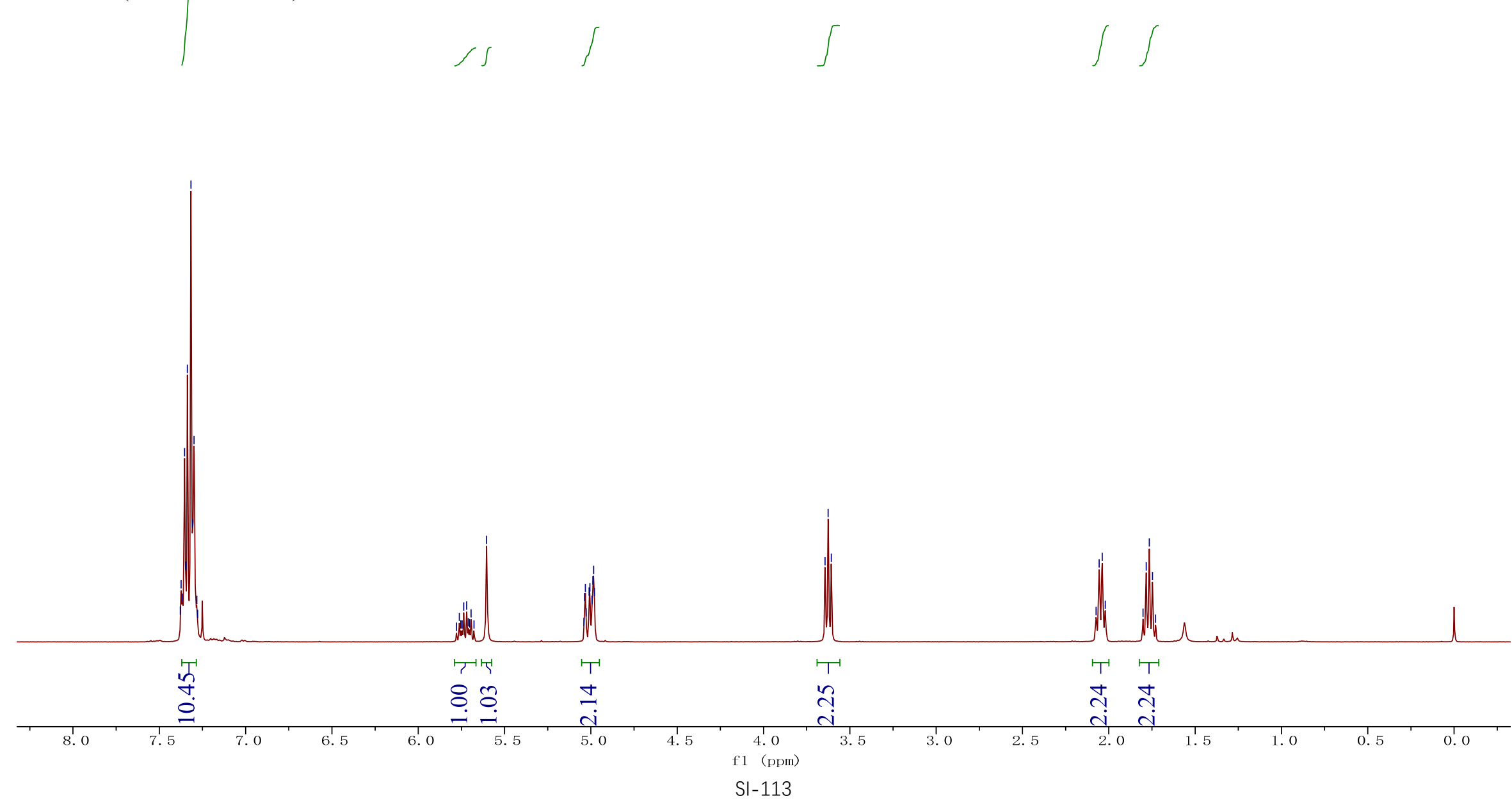


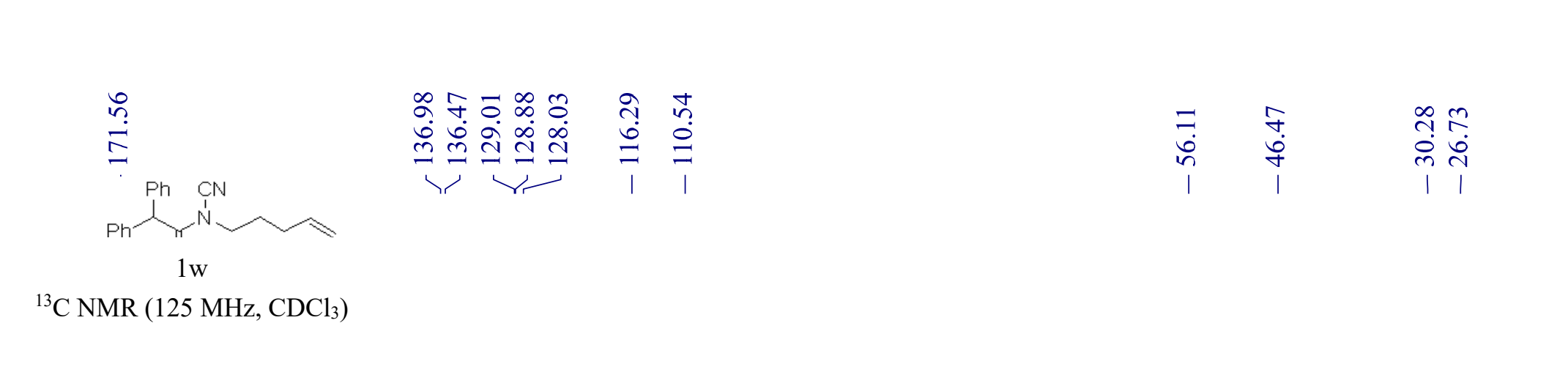

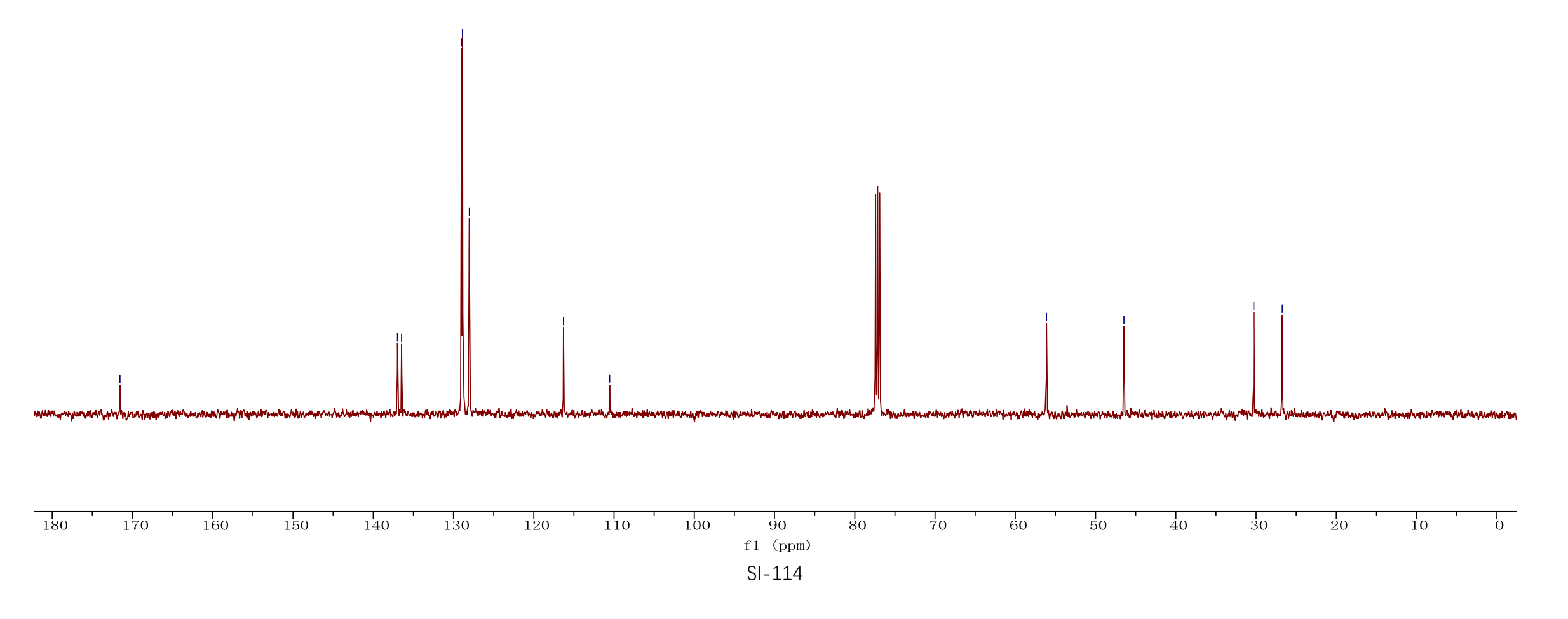




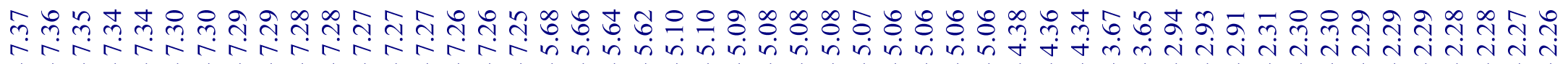
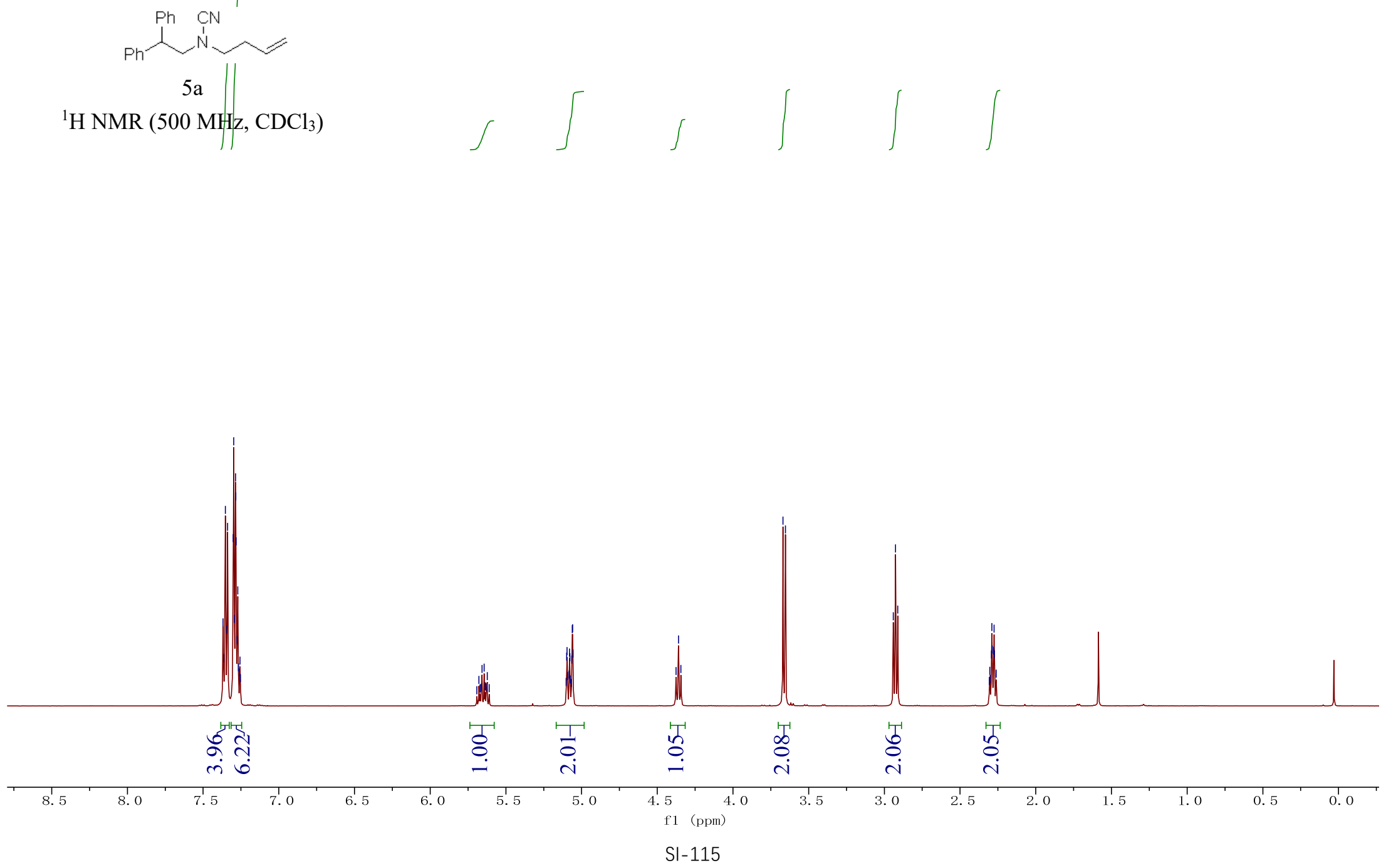


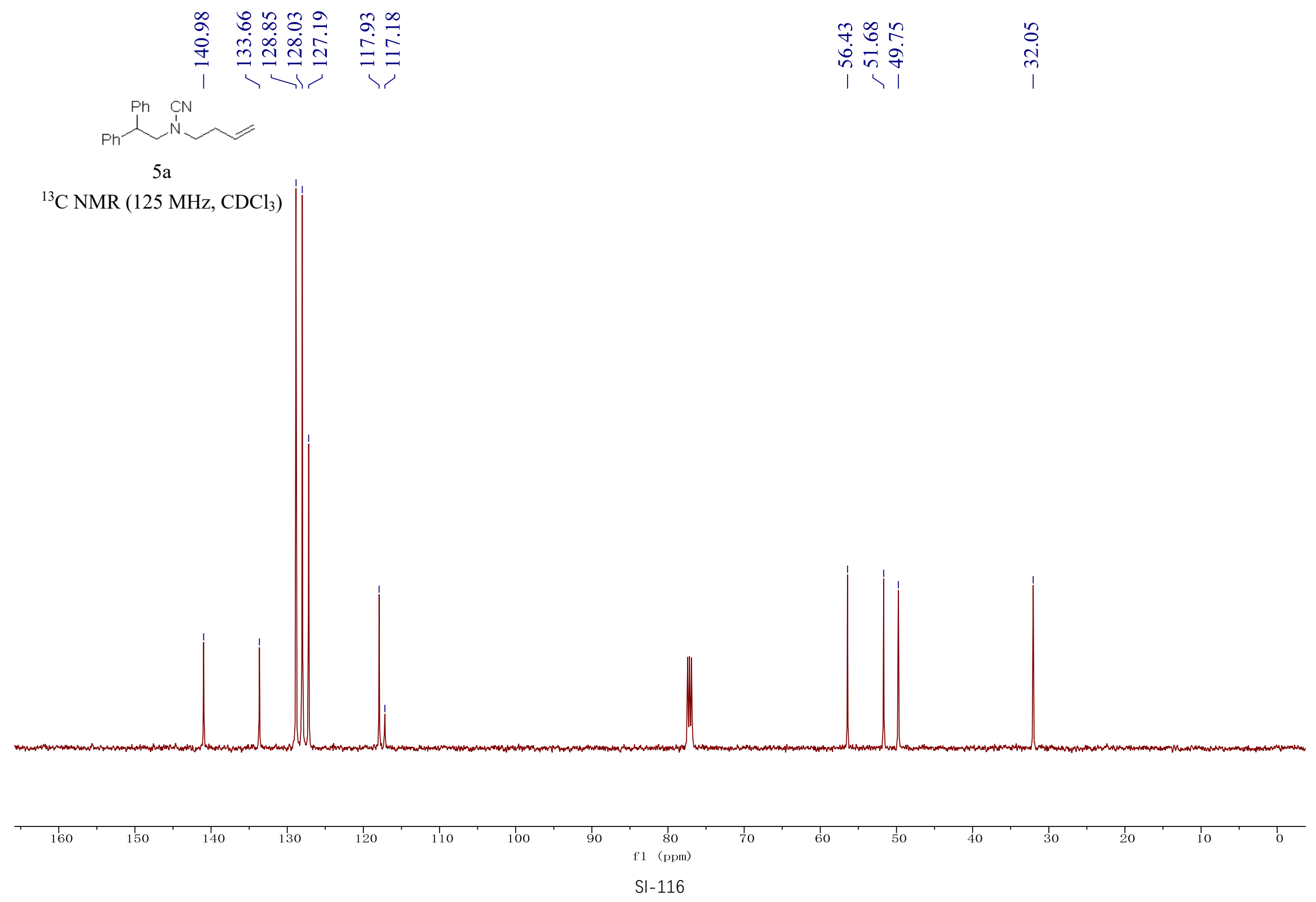




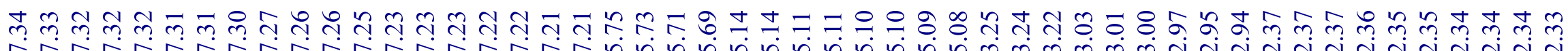

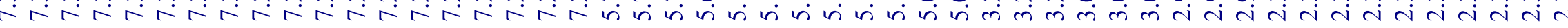
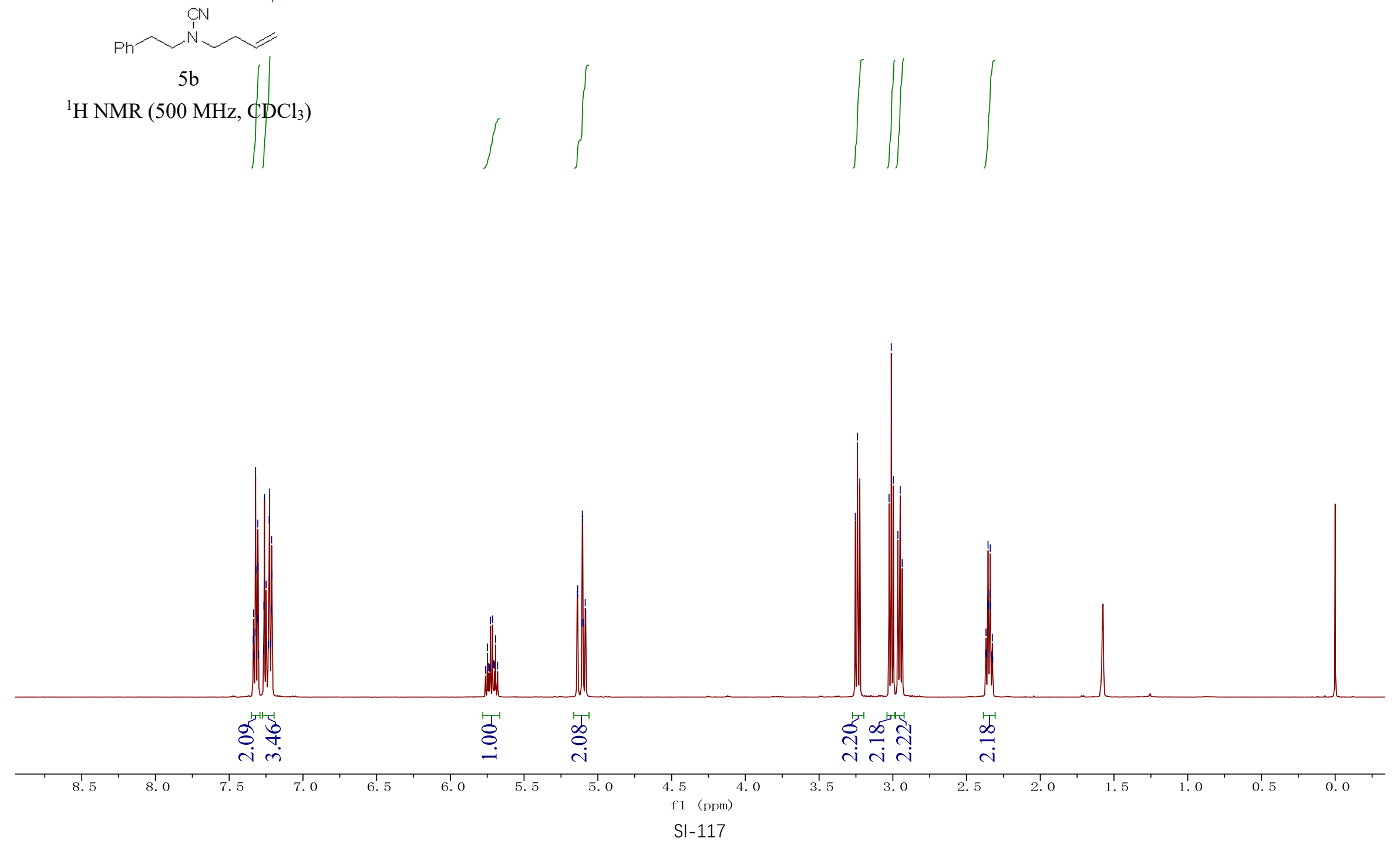
5xjm-ph-c. 10. fid

${ }^{13} \mathrm{C}$ NMR (100 MHz, $\left.\mathrm{CDCl}_{3}\right)$

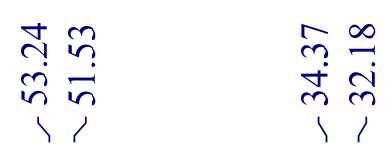

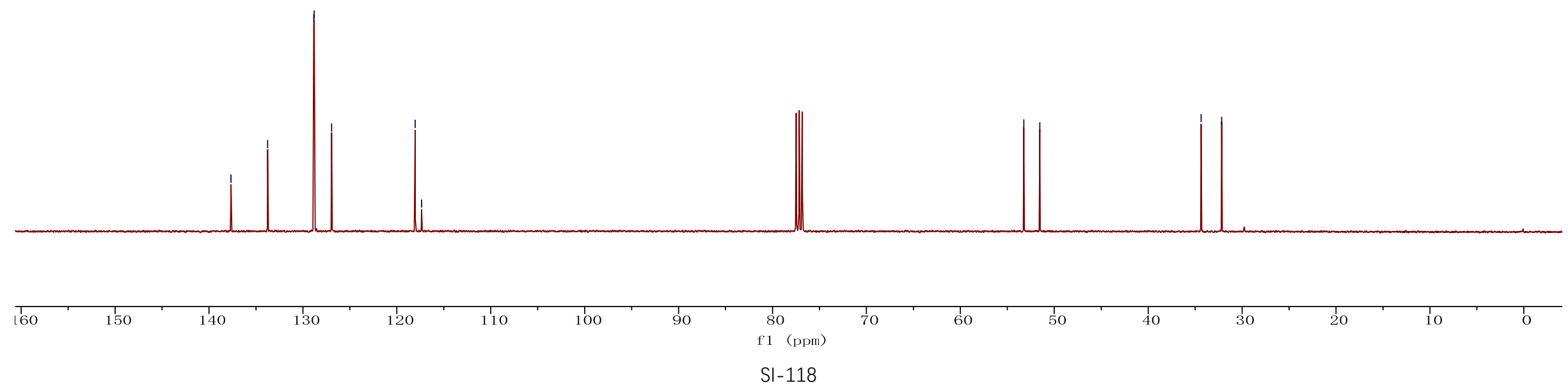




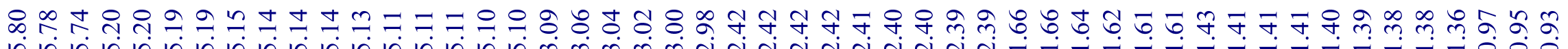

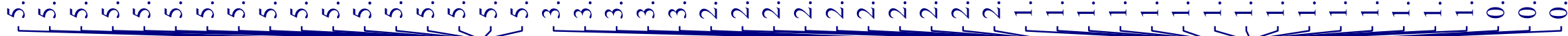

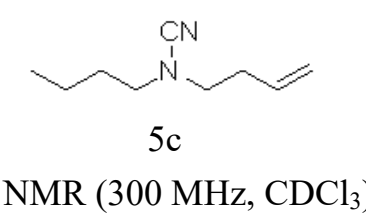

${ }^{1} \mathrm{H}$ NMR $\left(300 \mathrm{MHz}, \mathrm{CDCl}_{3}\right)$

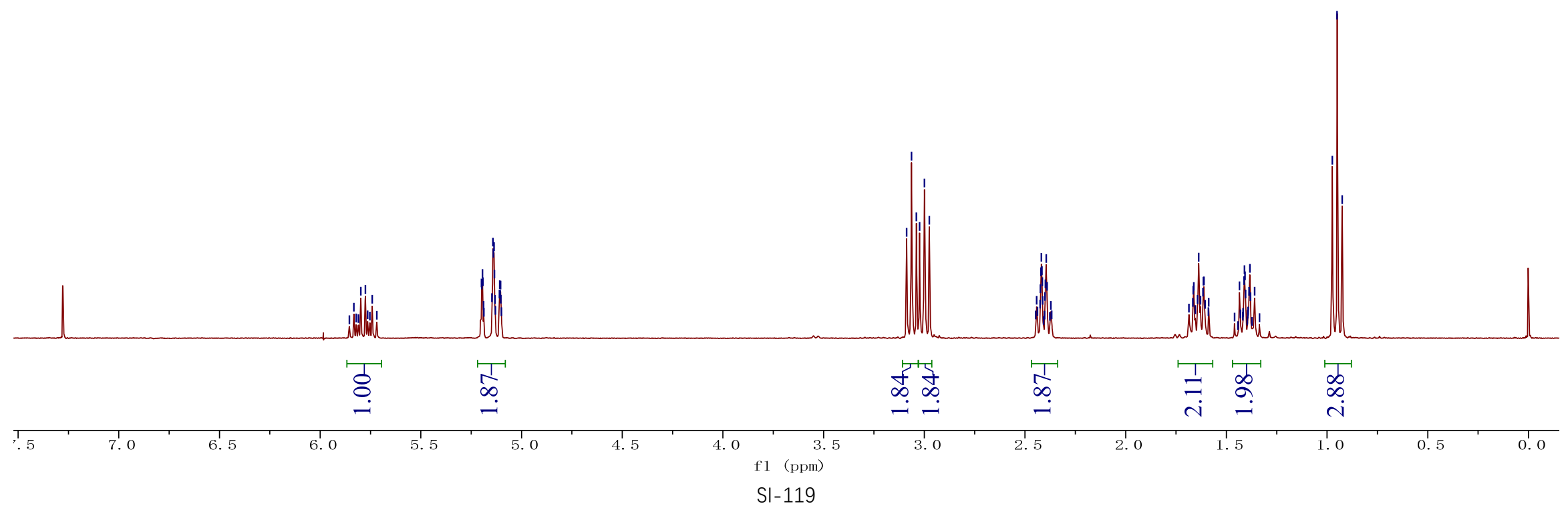




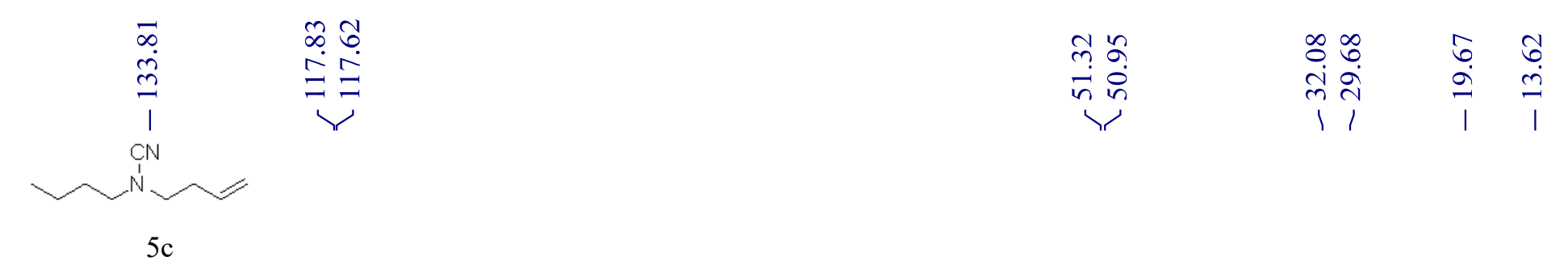

${ }^{13} \mathrm{C}$ NMR (125 MHz, $\mathrm{CDCl}_{3}$ )

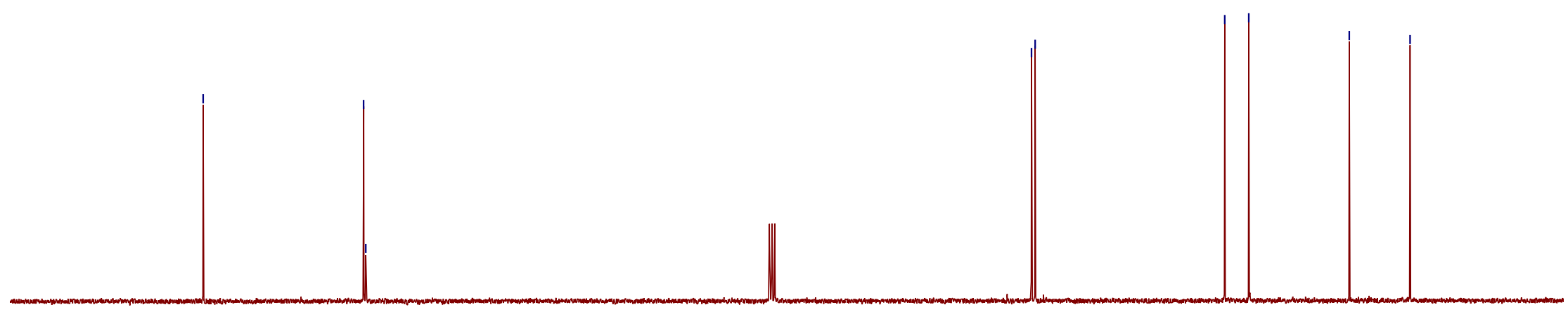

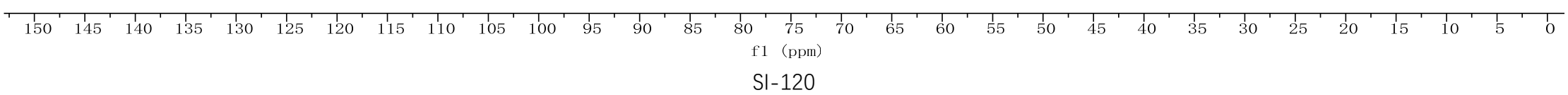




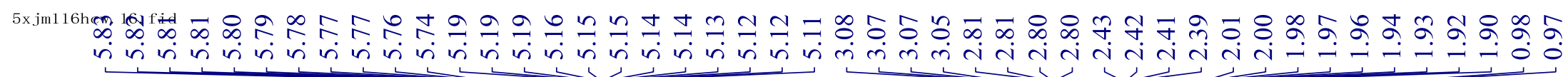

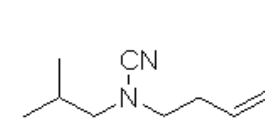

$5 \mathrm{~d}$

${ }^{1} \mathrm{H}$ NMR (500 MHz, $\mathrm{CDCl}_{3}$ )
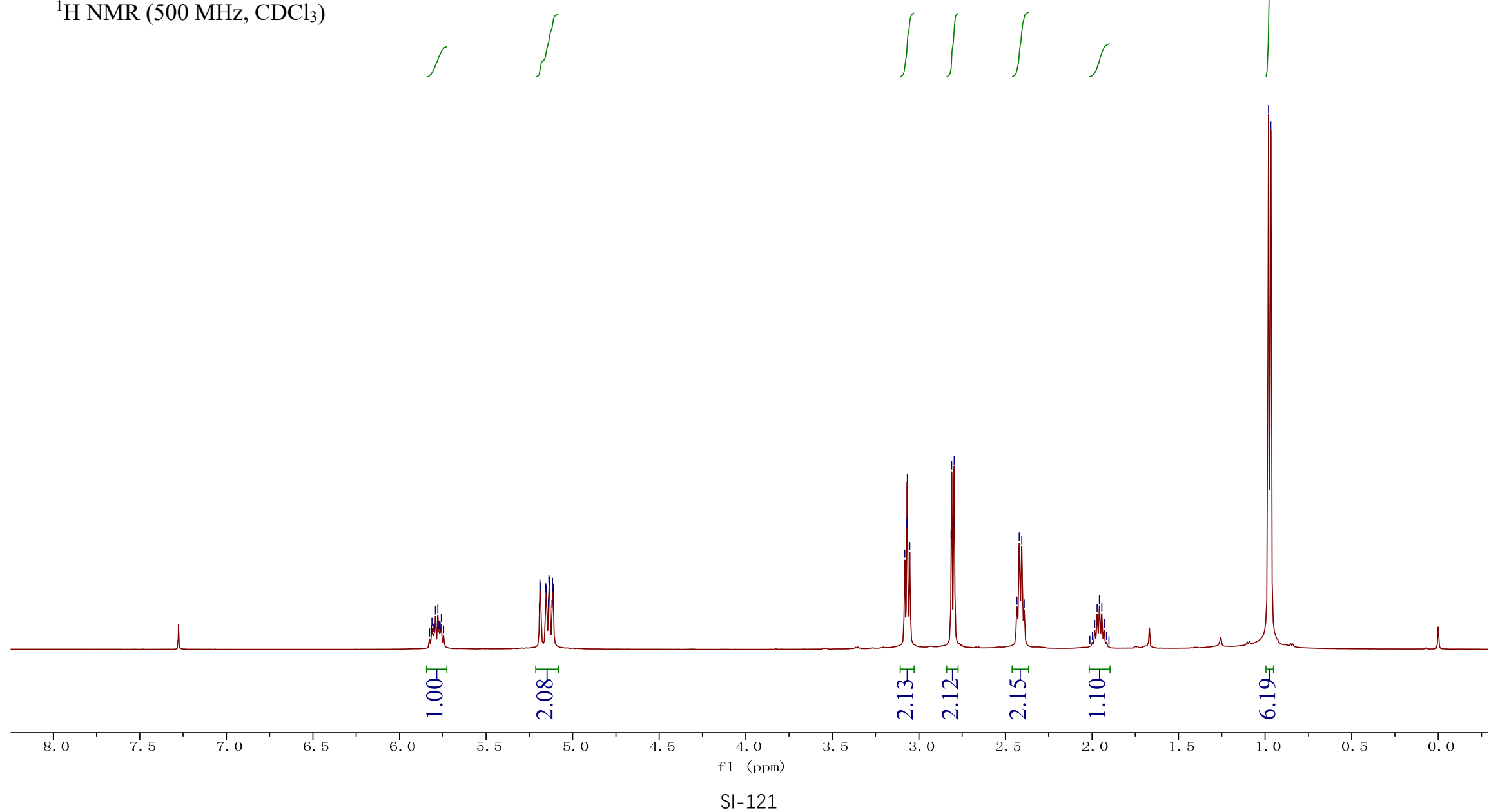
5xjm116cw-c.17.

$5 d$

${ }^{13} \mathrm{C}$ NMR (125 MHz, $\left.\mathrm{CDCl}_{3}\right)$

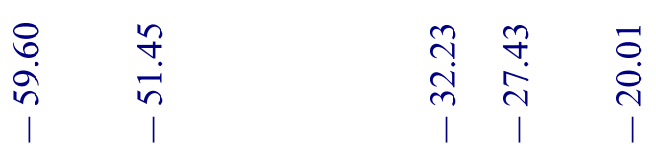

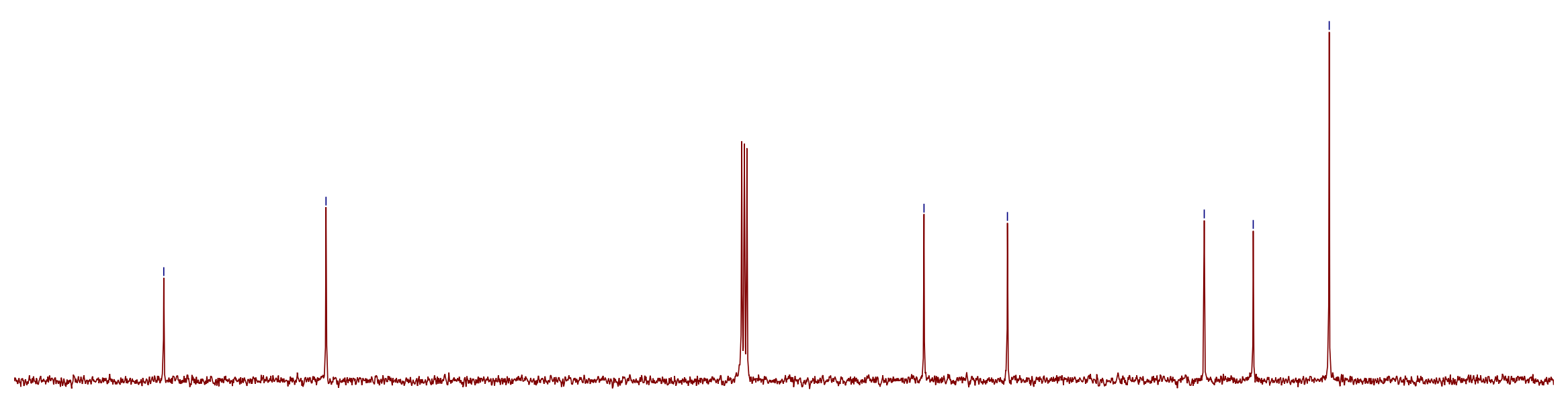

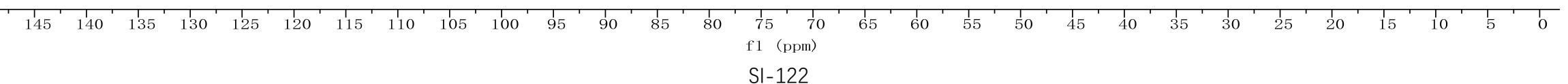




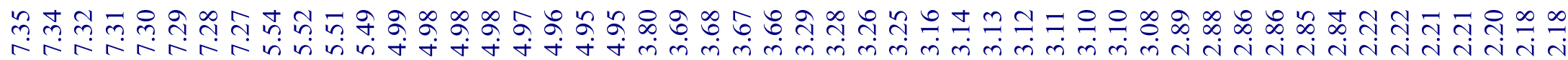<smiles>C=CCCN(C)C(Cc1ccccc1)C(=O)OCCN</smiles>

${ }^{1} \mathrm{H} \mathrm{NMR}\left(500 \mathrm{MHz}, \mathrm{CDCl}_{3}\right)$
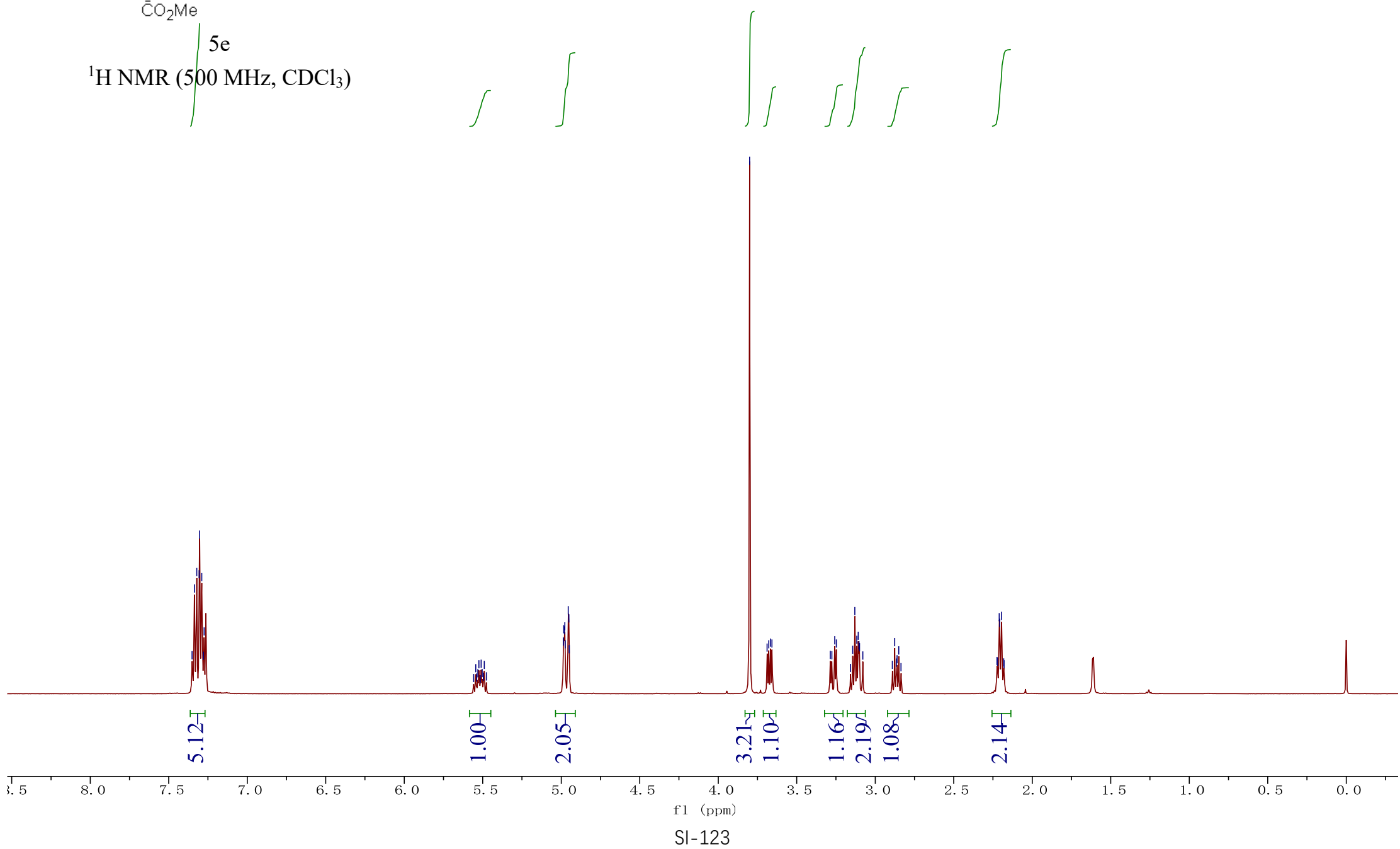


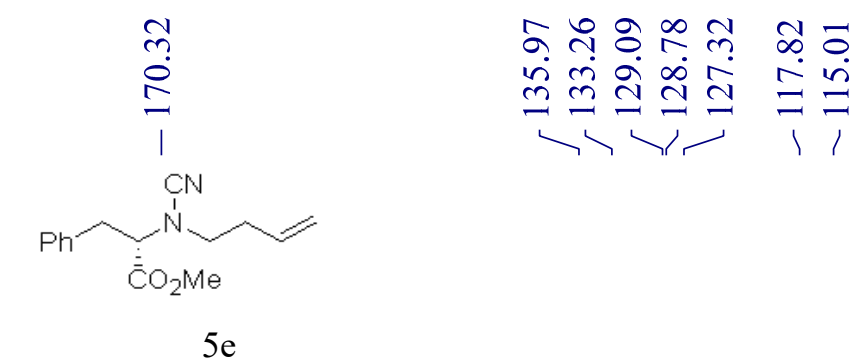

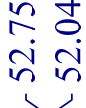

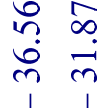

${ }^{13} \mathrm{C}$ NMR (125 MHz, $\mathrm{CDCl}_{3}$ )

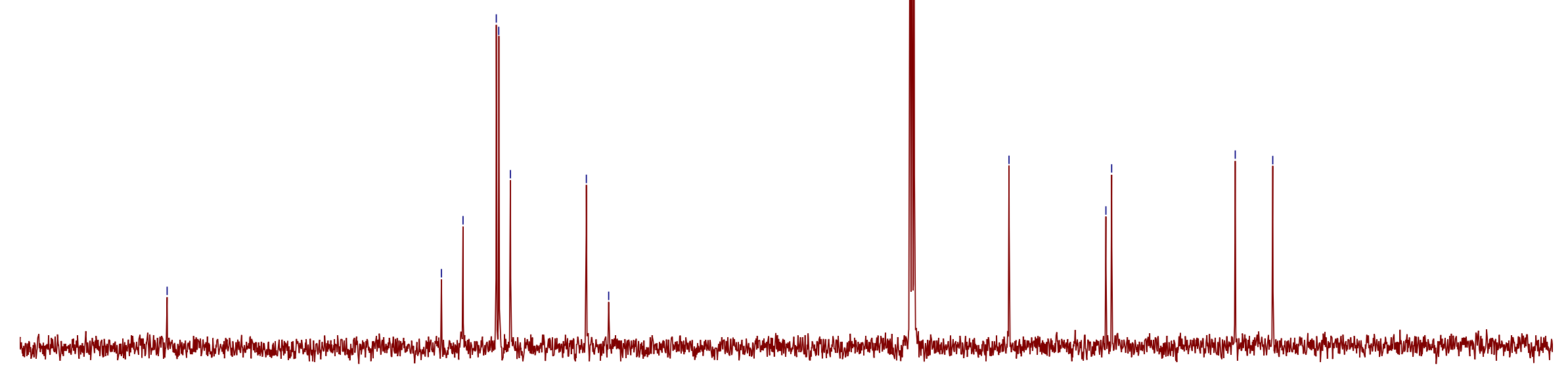




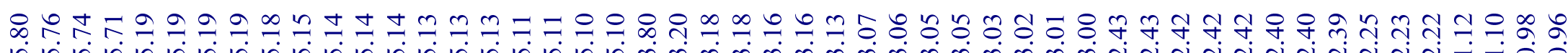

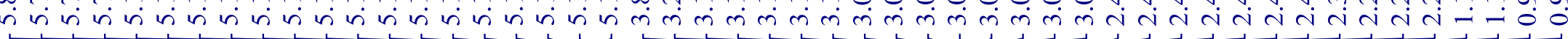

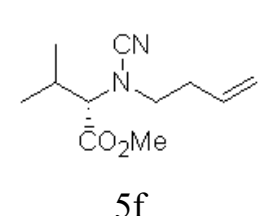

${ }^{1} \mathrm{H}$ NMR $\left(300 \mathrm{MHz}, \mathrm{CDCl}_{3}\right)$

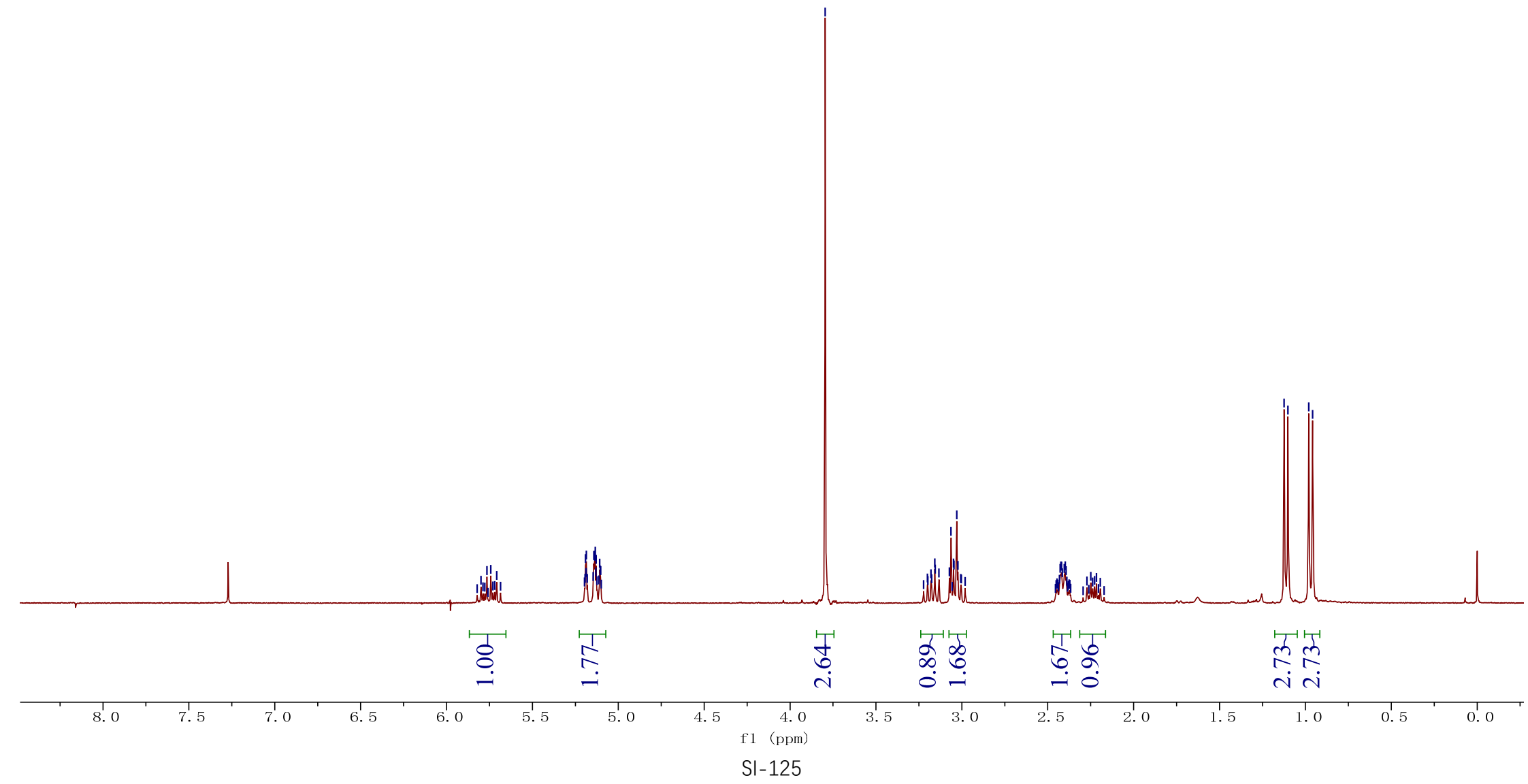




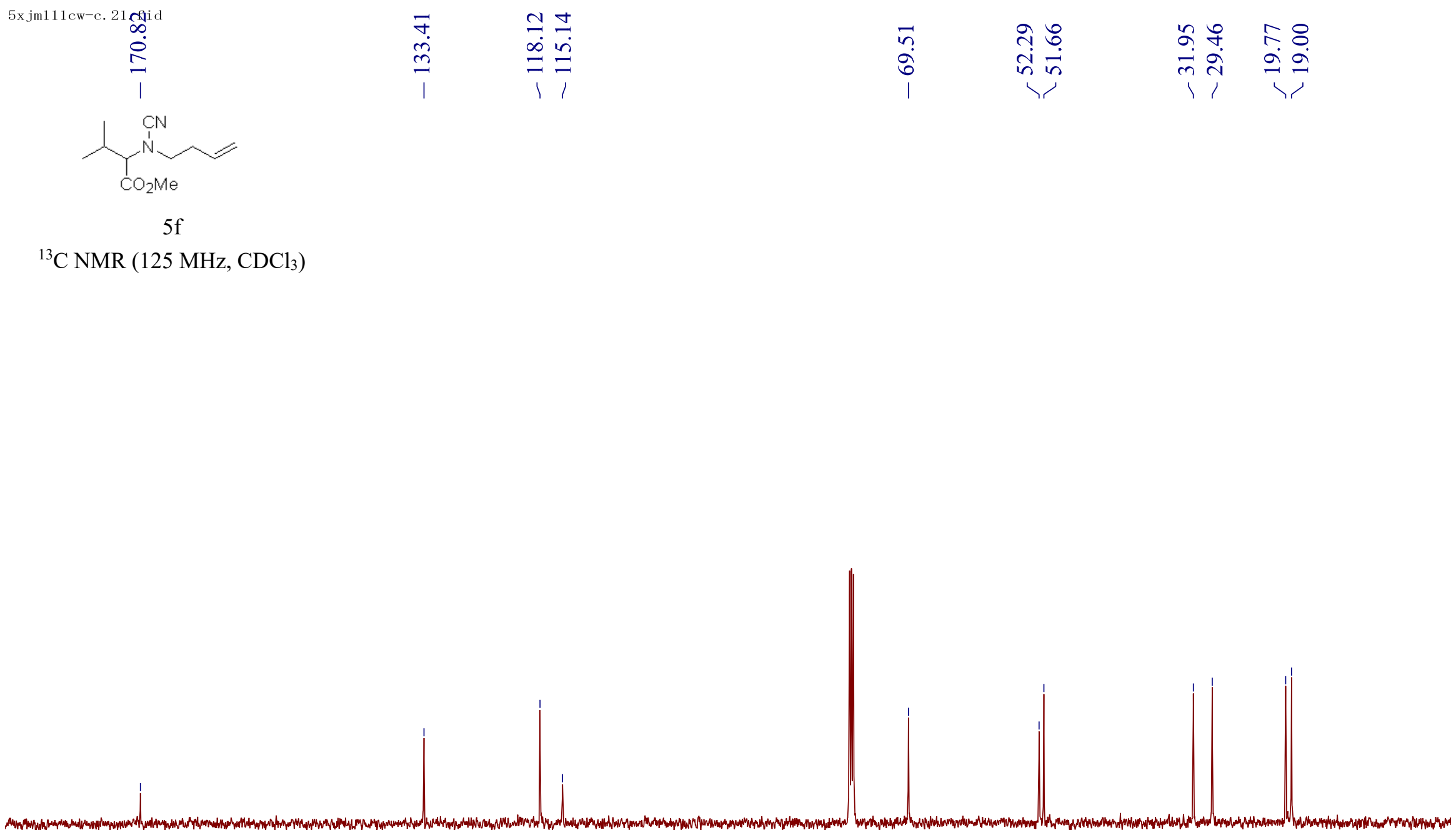

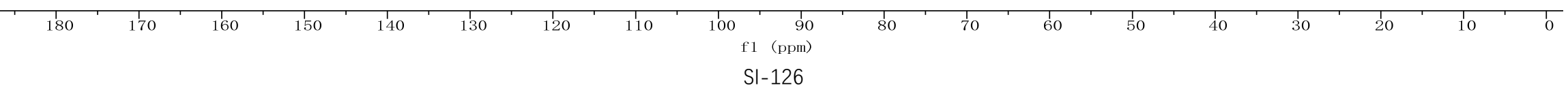


웅

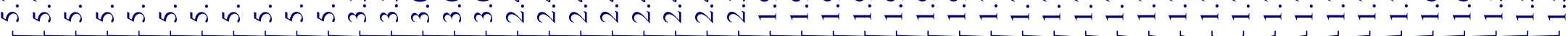

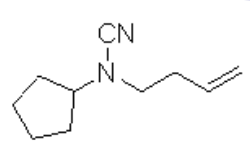

$5 \mathrm{~g}$

${ }^{1} \mathrm{H}$ NMR (400 MHz, $\left.\mathrm{CDCl}_{3}\right)$

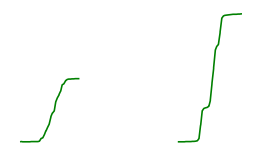

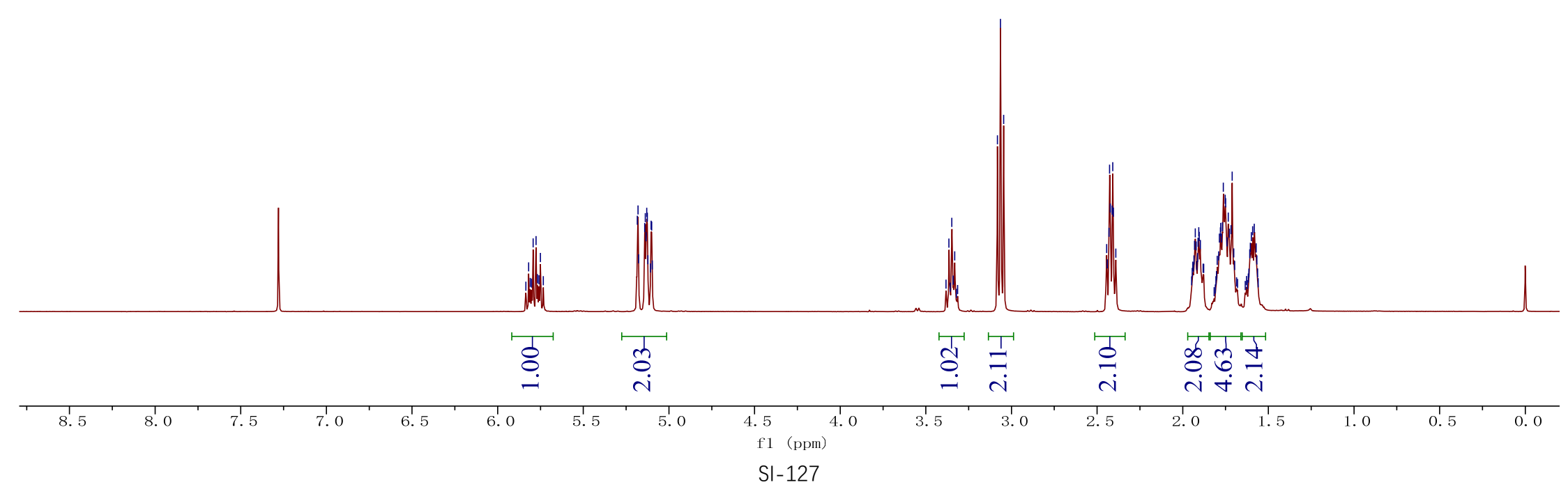




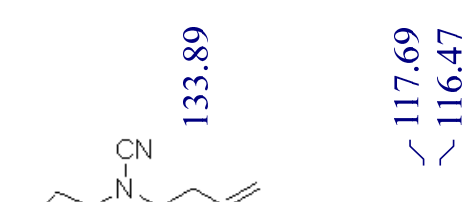

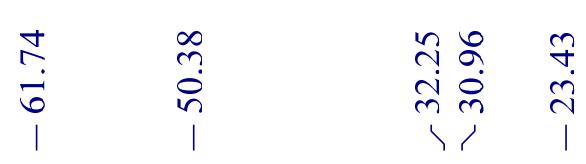

$5 \mathrm{~g}$

$\left.{ }^{13} \mathrm{C} \mathrm{NMR} \mathrm{(125} \mathrm{MHz,} \mathrm{CDCl}_{3}\right)$

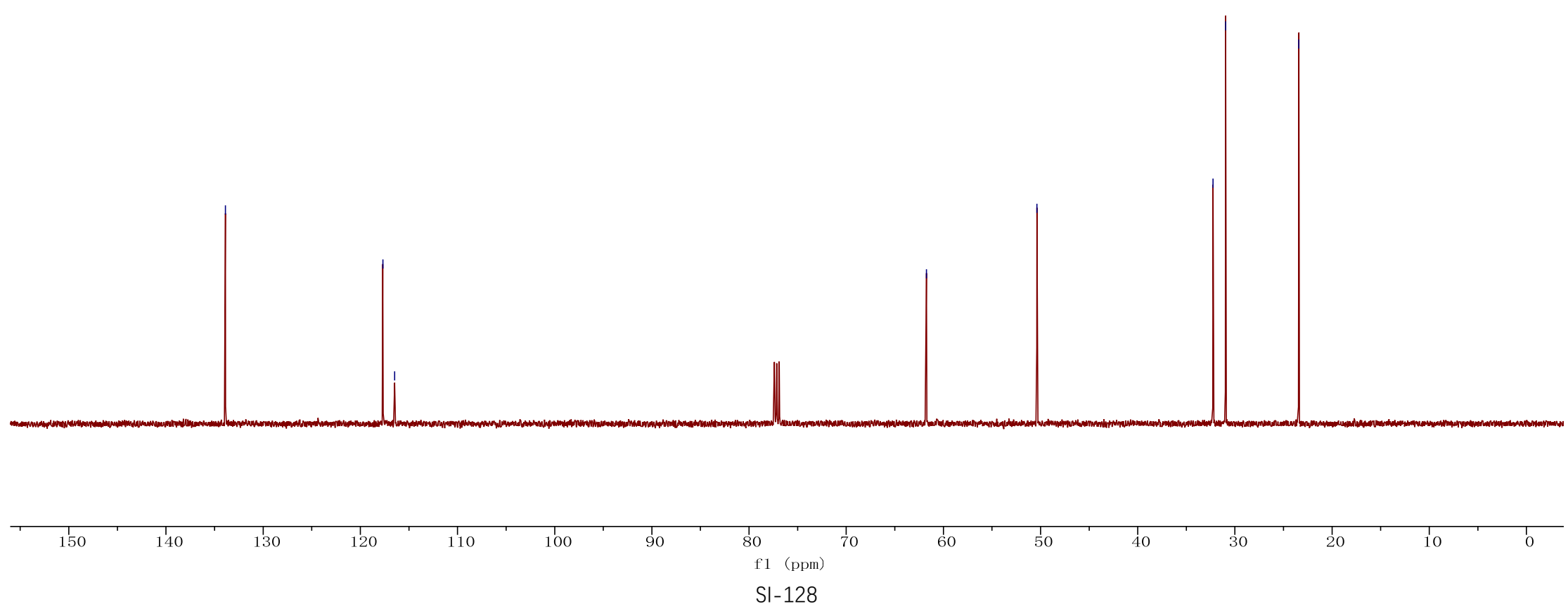




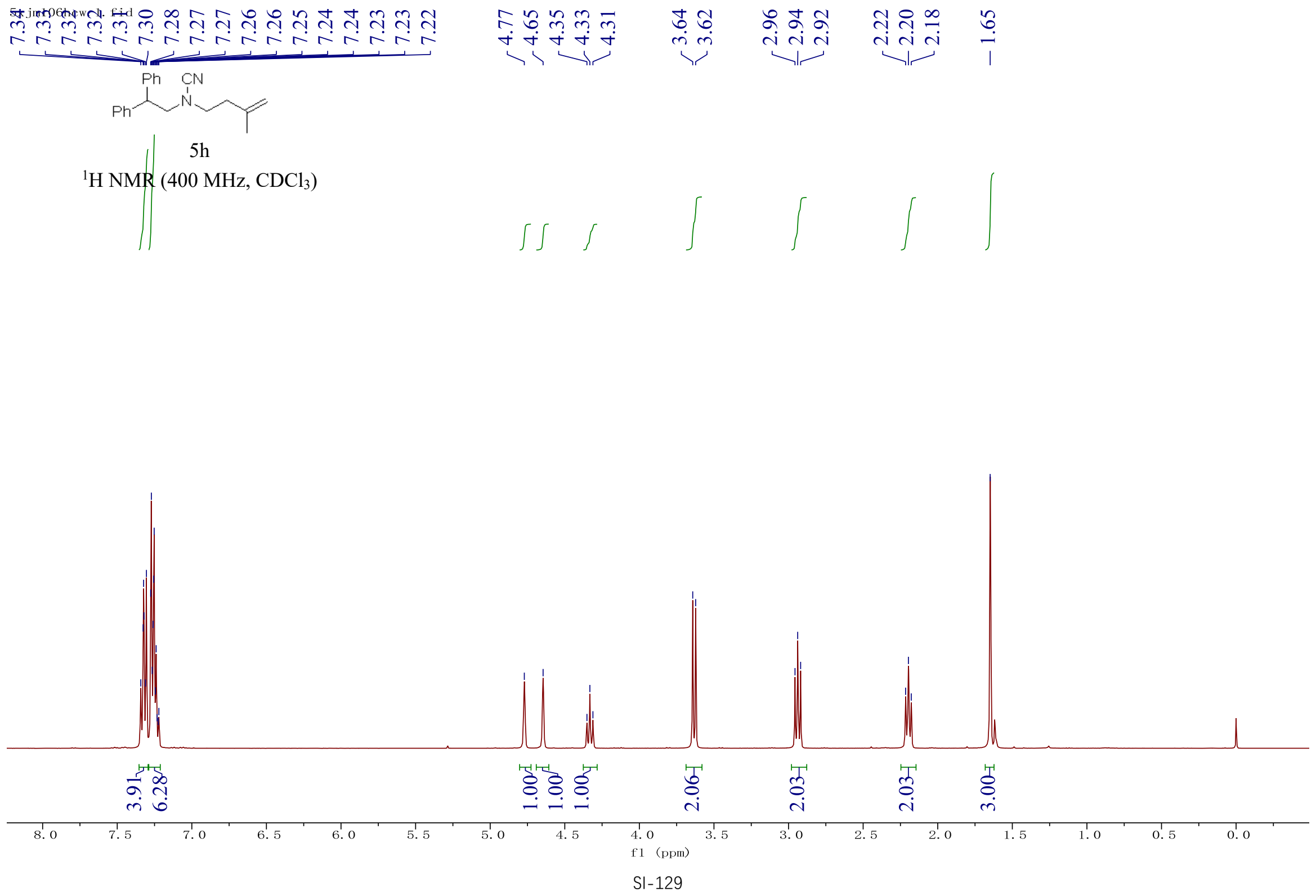




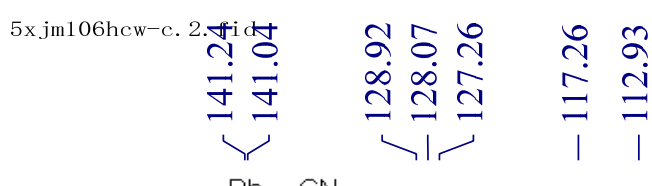

ले iñ

$\begin{array}{ll}n & 7 \\ \ddot{n} & \text { i } \\ 1 & 1\end{array}$

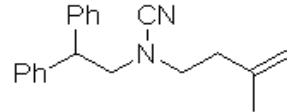

$5 \mathrm{~h}$

${ }^{13} \mathrm{C} \mathrm{NMR}\left(100 \mathrm{MHz}, \mathrm{CDCl}_{3}\right)$

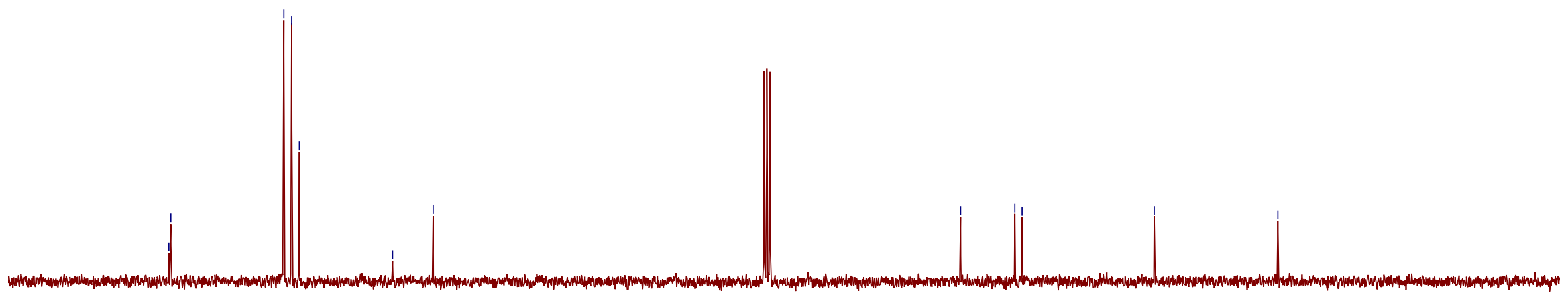

f1 (ppm)

50

30

20

SI-130 


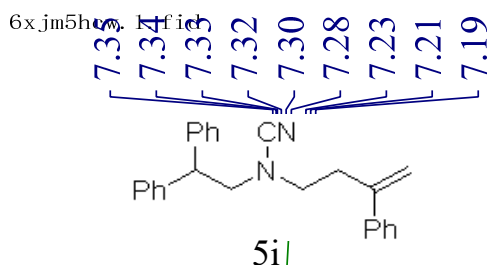

${ }^{1} \mathrm{H}$ NMR (400 MHz, $\mathrm{CDCl}_{3}$ )

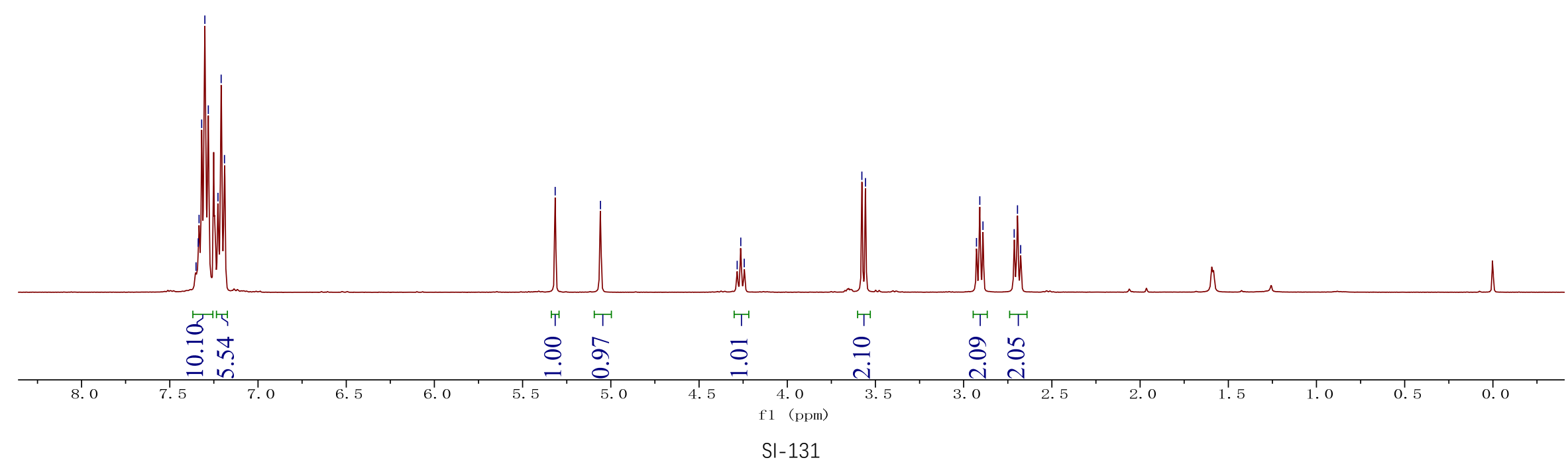

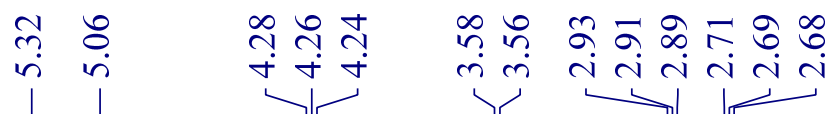

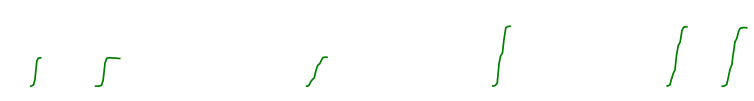




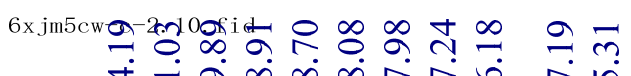

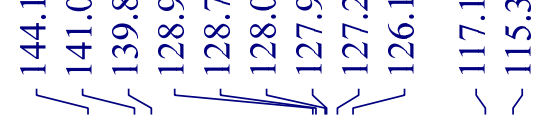

ดे

ம்

d

$\mathrm{Ph} \mathrm{CN}$

${ }_{5 \mathrm{i}}$

${ }^{13} \mathrm{C}$ NMR (125 MHz, $\mathrm{CDCl}_{3}$ )
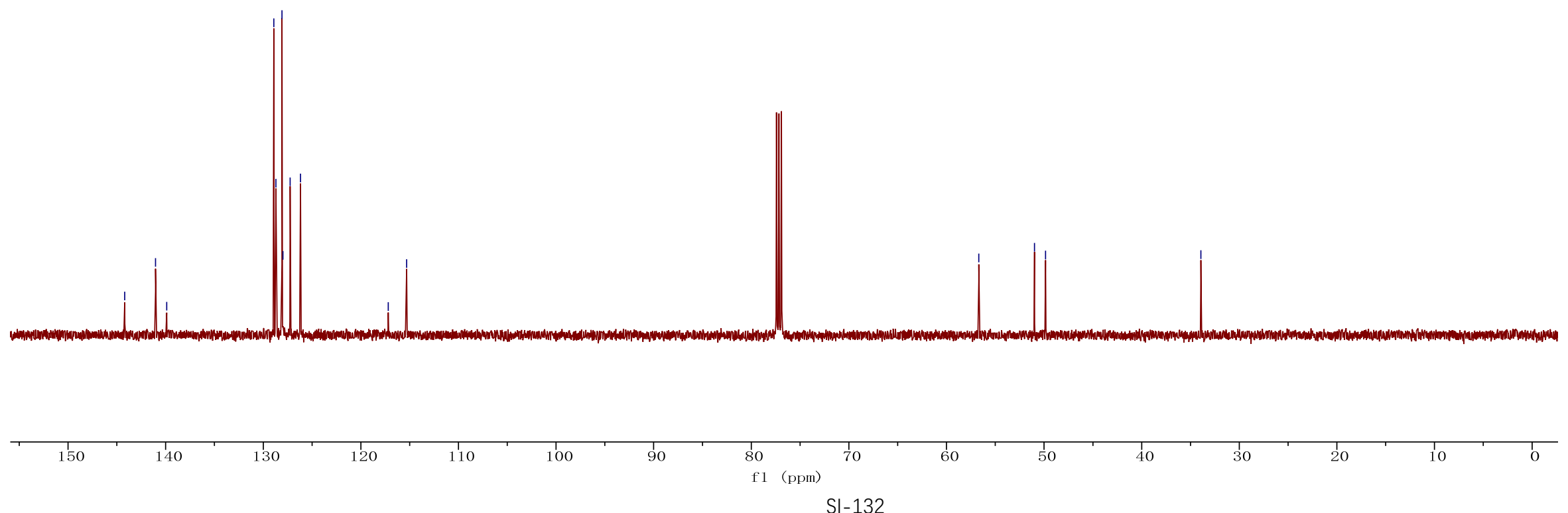


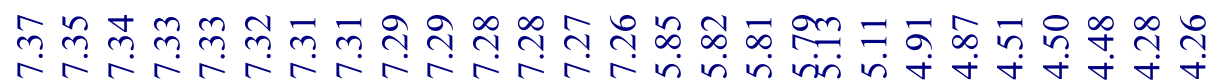

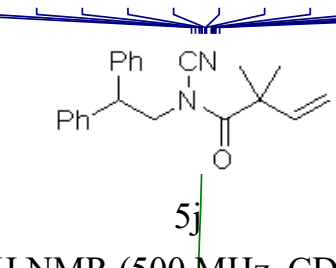

${ }^{1} \mathrm{H}$ NMR (500 MHz, $\left.\mathrm{CDCl}_{3}\right)$
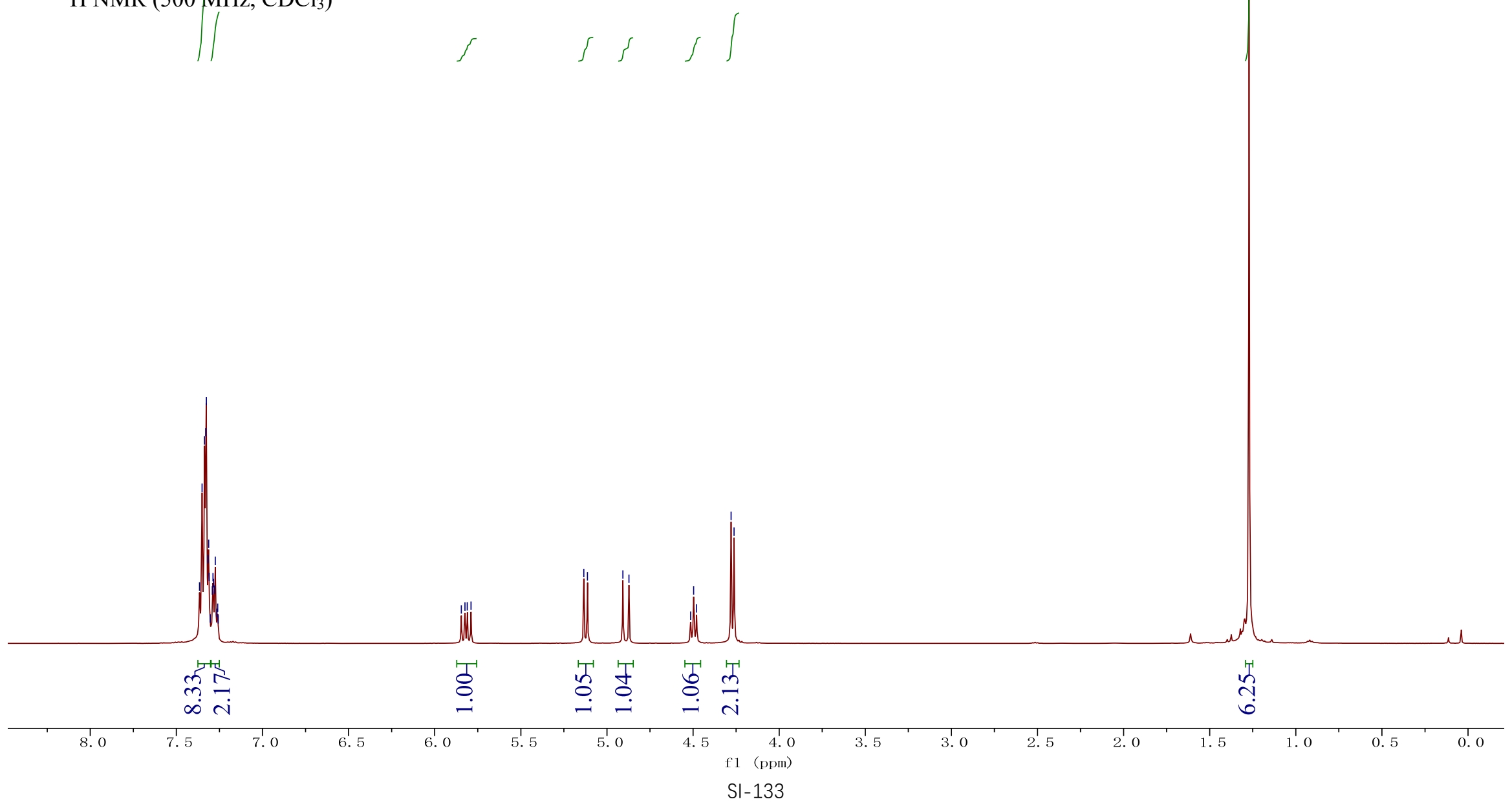


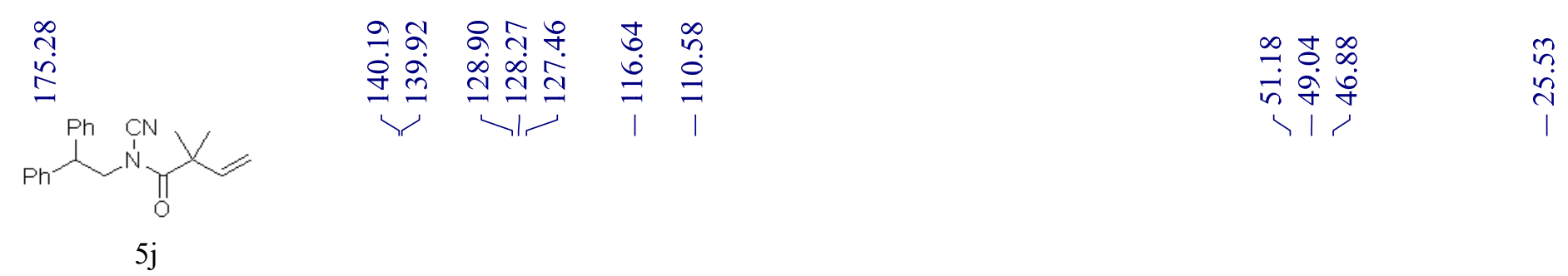

${ }^{13} \mathrm{C} \mathrm{NMR}\left(125 \mathrm{MHz}, \mathrm{CDCl}_{3}\right)$

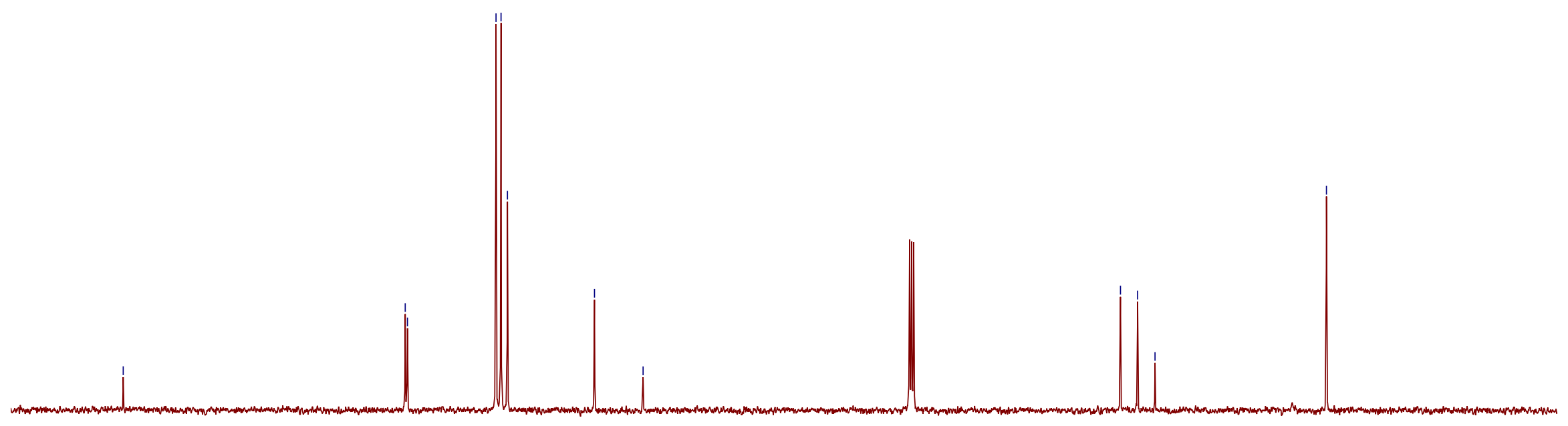

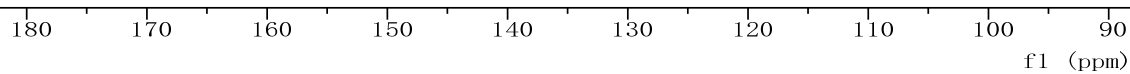
SI-134 


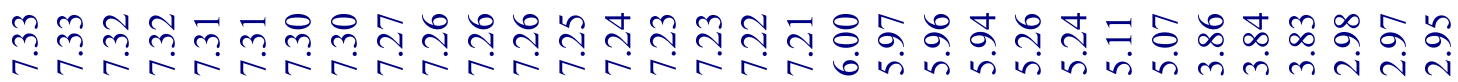

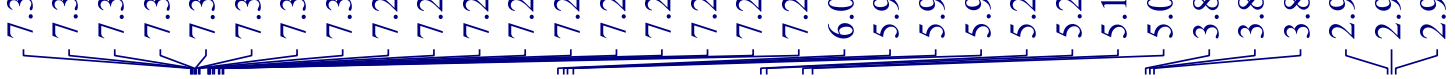

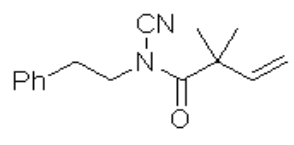

$5 \mathrm{k}$

${ }^{1} \mathrm{H}$ NMR $\left(500 \mathrm{MHz}, \mathrm{CDCl}_{3}\right)$ $\|$

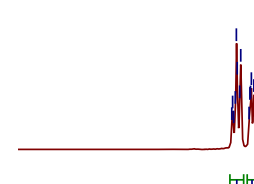

年

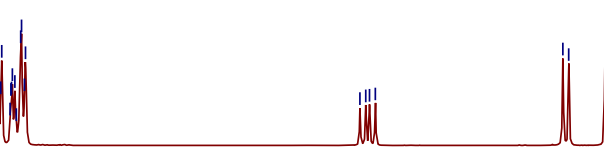

8. 0

7. 5

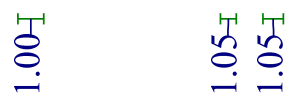

을

5. 0

f1 (ppm)

$\mathrm{SI}-135$ 


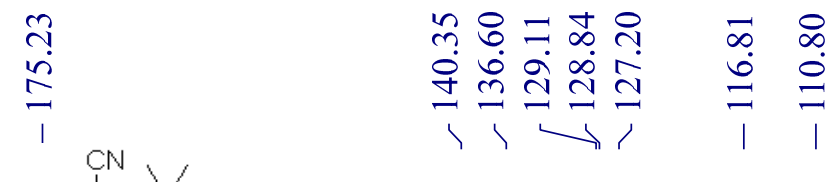

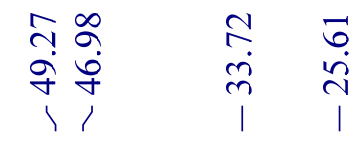

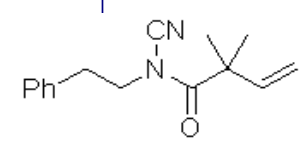

$5 \mathrm{k}$

${ }^{13} \mathrm{C}$ NMR (125 MHz, $\mathrm{CDCl}_{3}$ )

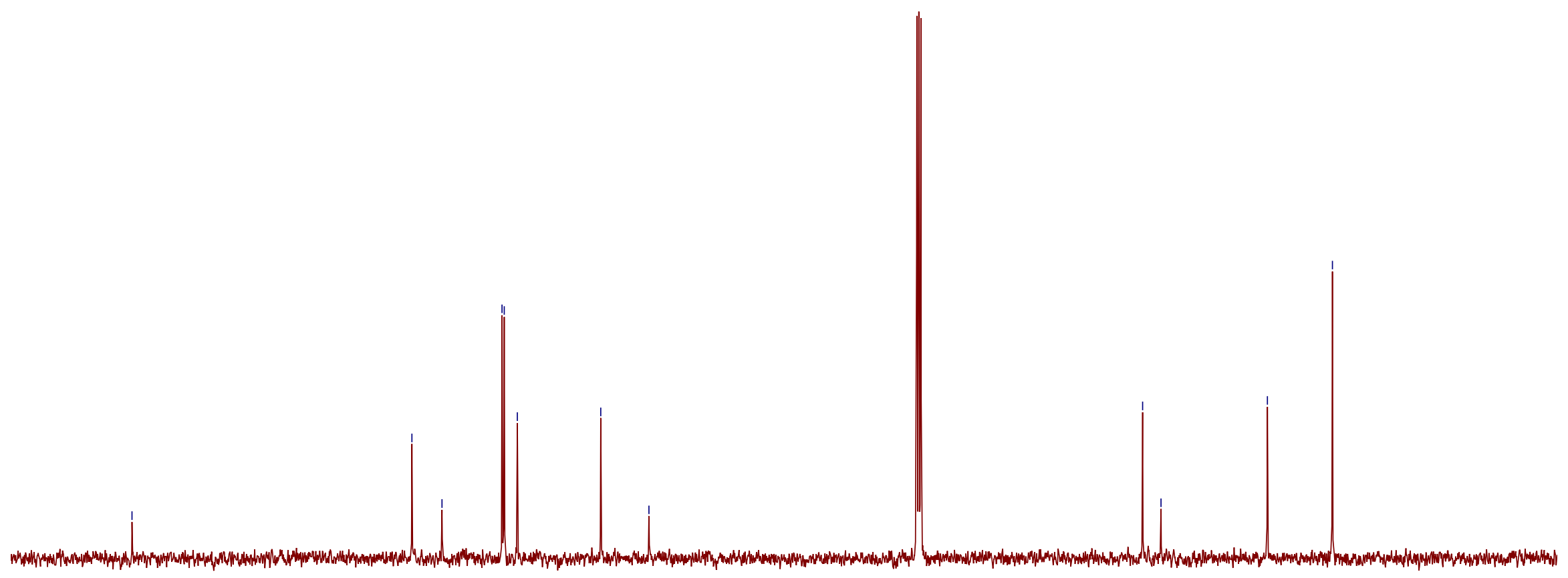




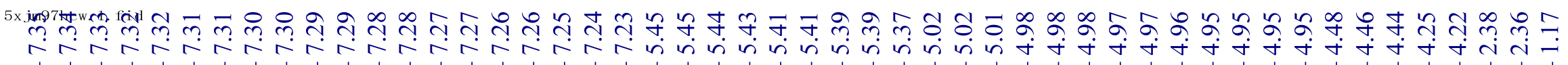

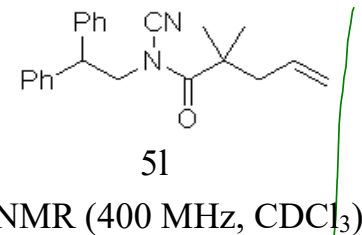

${ }^{1} \mathrm{H}$ NMR $\left(400 \mathrm{MHz}, \mathrm{CDCl}_{3}\right)$

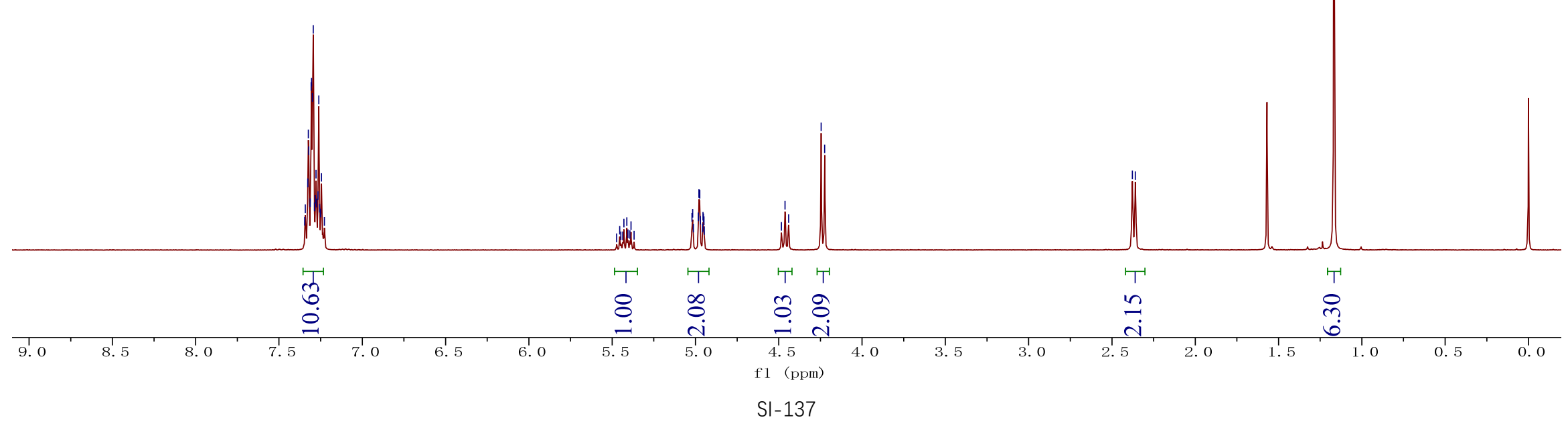


5x jm97cw-c. 10. fid

$\frac{\text { I }}{2}$

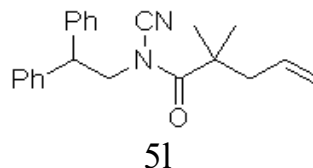

51

${ }^{13} \mathrm{C} \mathrm{NMR}\left(100 \mathrm{MHz}, \mathrm{CDCl}_{3}\right)$

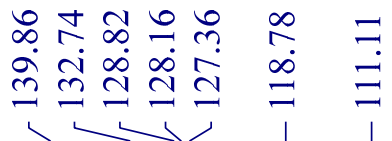

क्ष

的字我字

111

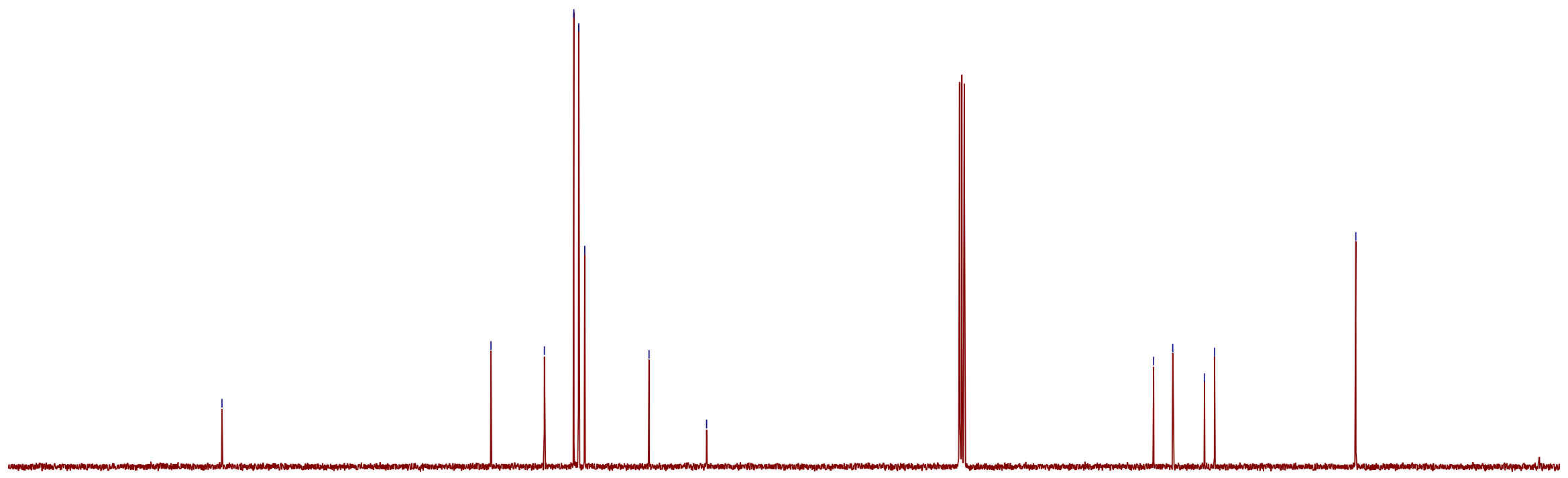

$\frac{1}{200}$

190

$180 \quad 170$

160

150

140

120

110

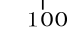

f1 (ppm) 


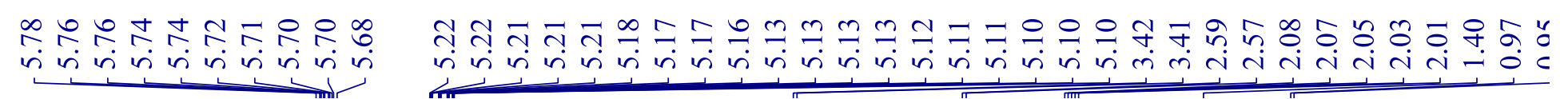
L $\pi_{0}^{N} \times$

$5 \mathrm{~m}$

${ }^{1} \mathrm{H}$ NMR (400 MHz, $\left.\mathrm{CDCl}_{3}\right)$

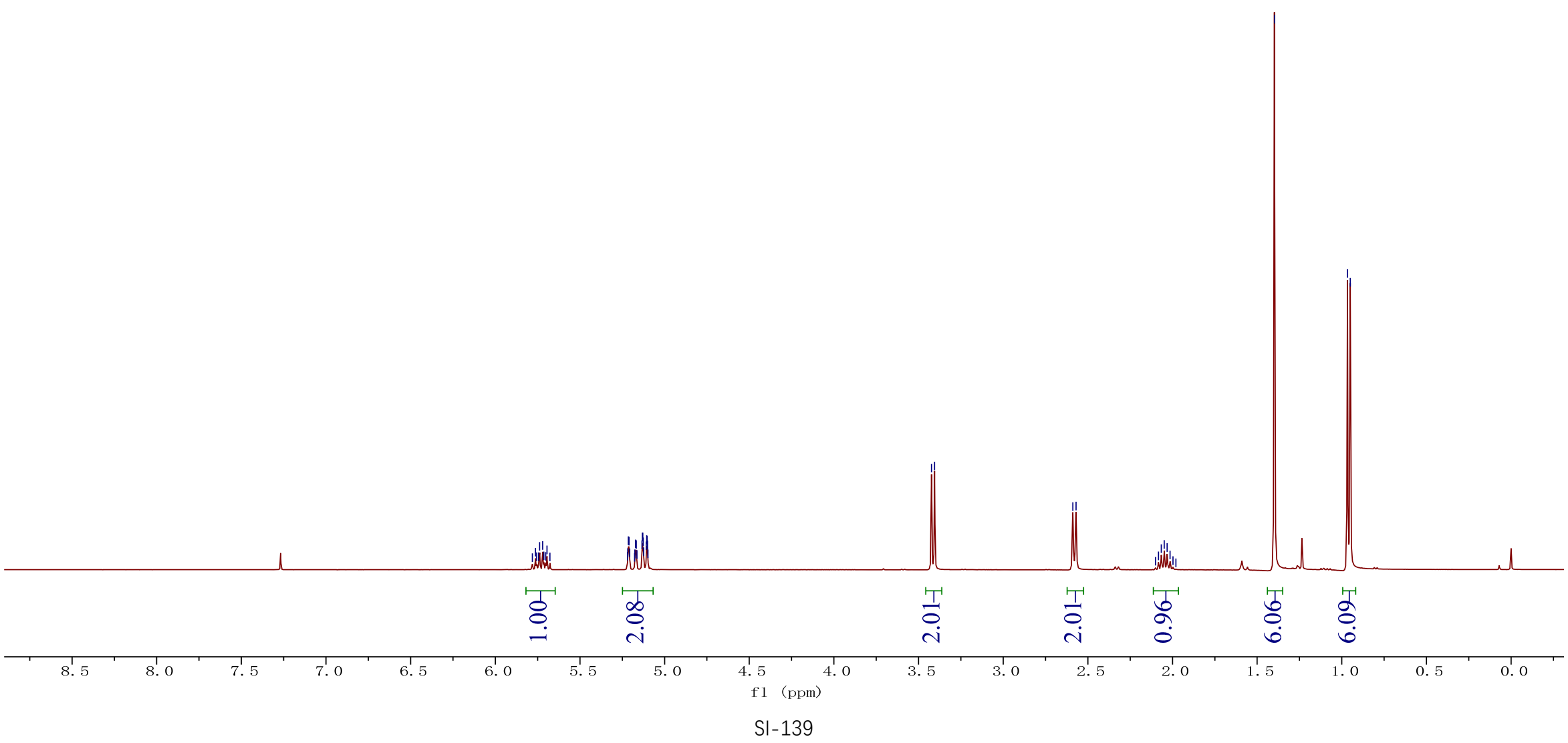




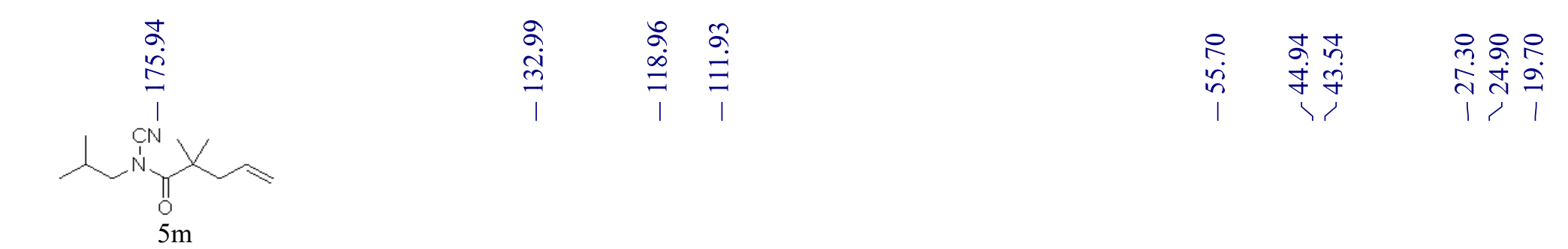

${ }^{13} \mathrm{C}$ NMR (125 MHz, $\left.\mathrm{CDCl}_{3}\right)$
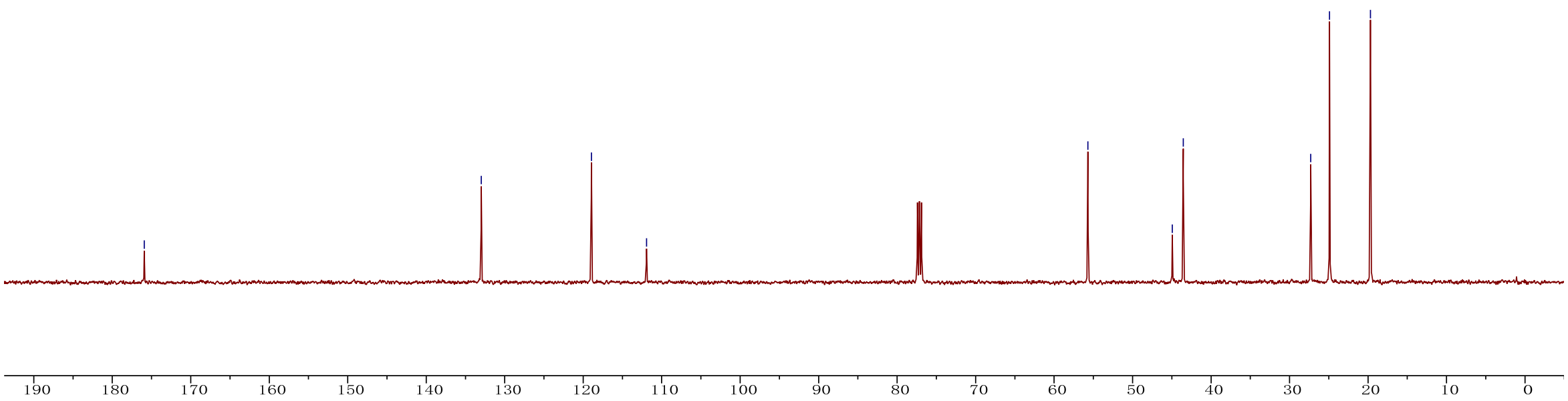


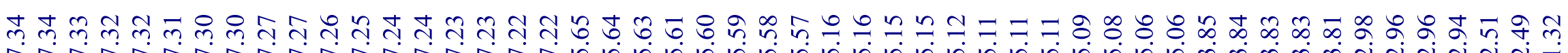

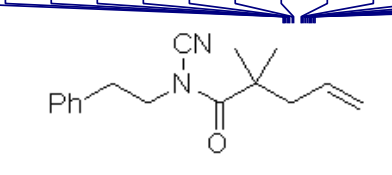

$5 \mathrm{n}$

${ }^{1} \mathrm{H}$ NMR $\left(400 \mathrm{MHz}, \mathrm{CDCl}_{3}\right)$
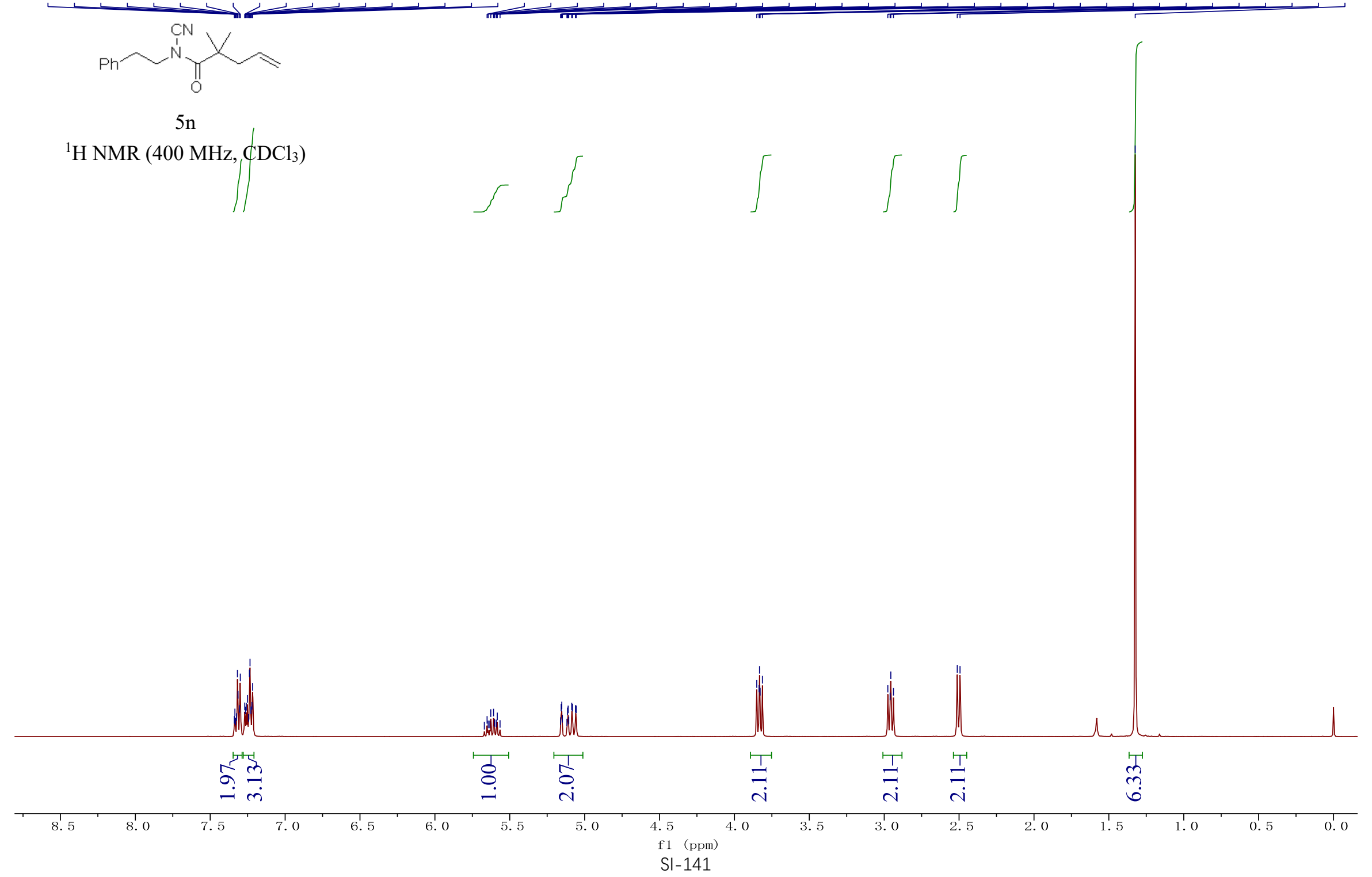

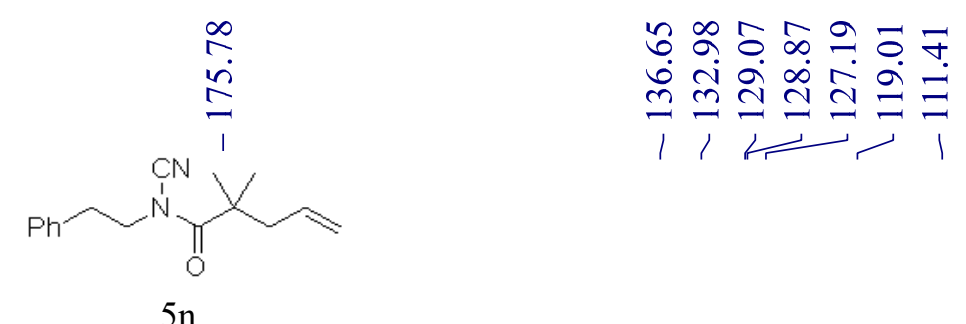

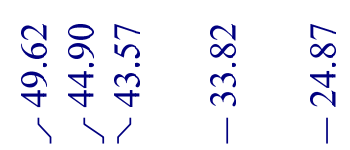

$5 \mathrm{n}$

${ }^{13} \mathrm{C}$ NMR $\left(125 \mathrm{MHz}, \mathrm{CDCl}_{3}\right)$

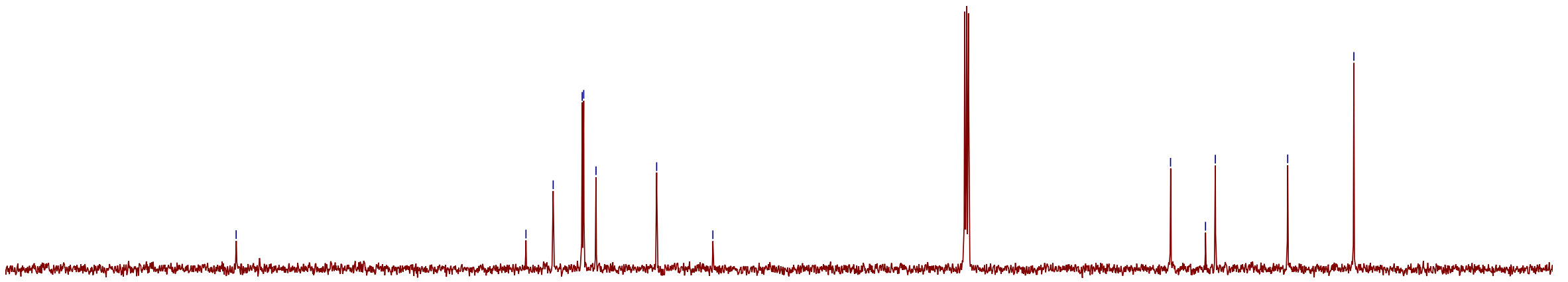

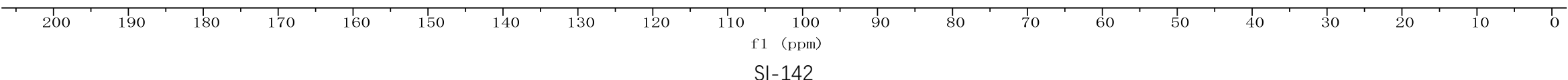




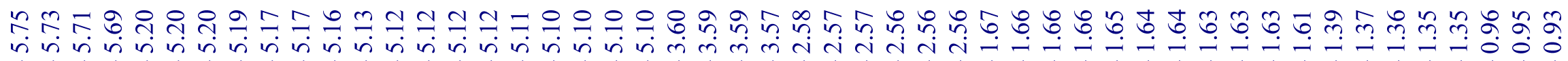

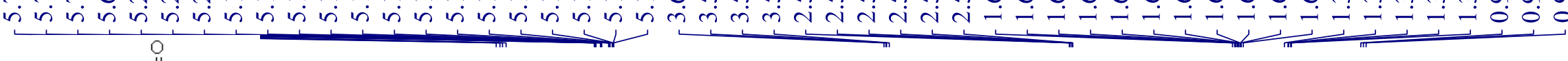

50

${ }^{1} \mathrm{H}$ NMR $\left(500 \mathrm{MHz}, \mathrm{CDCl}_{3}\right)$

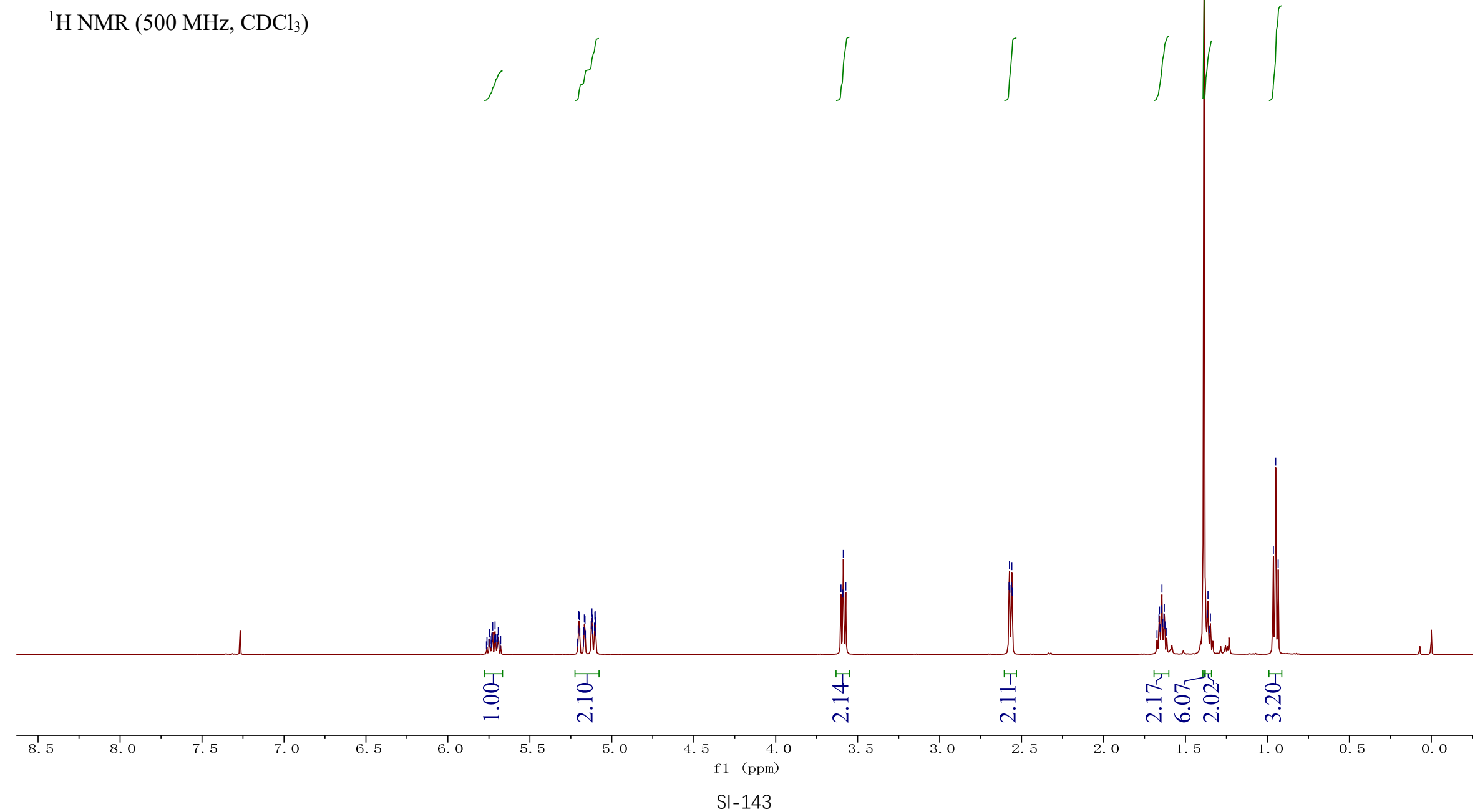




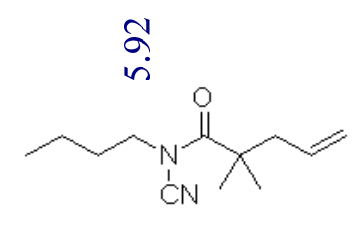

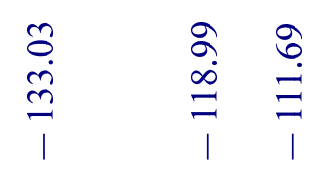

$\stackrel{\infty}{\infty} \infty \mathfrak{n}$

文导

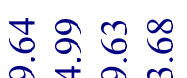

ते

50

${ }^{13} \mathrm{C} \mathrm{NMR}\left(125 \mathrm{MHz}, \mathrm{CDCl}_{3}\right)$

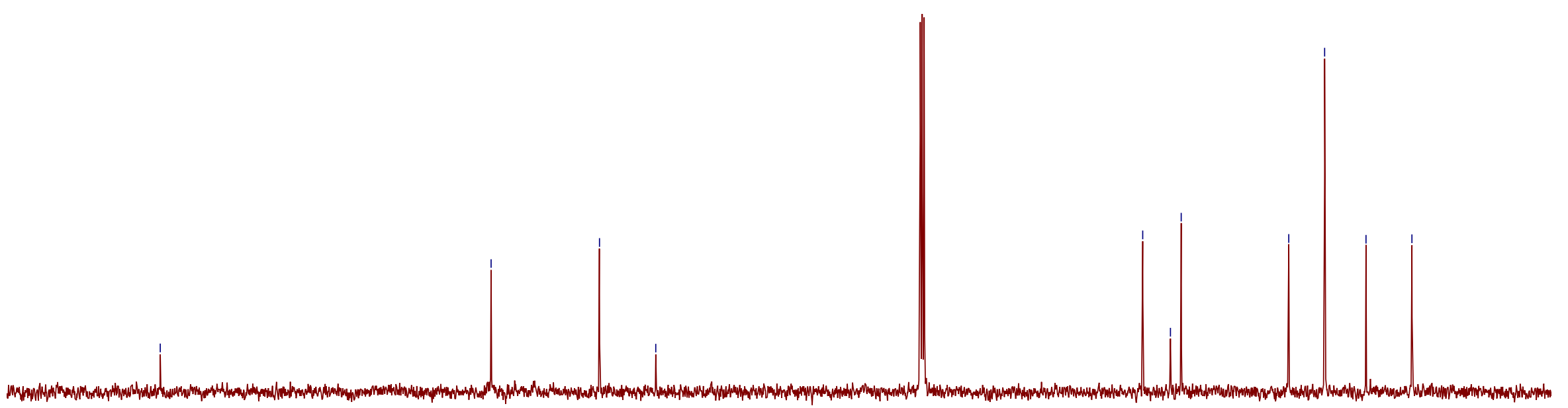

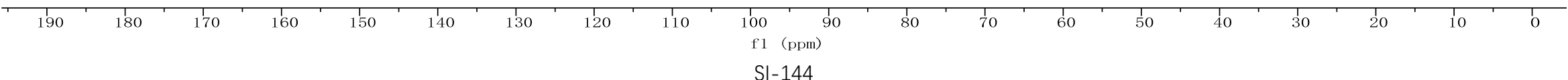




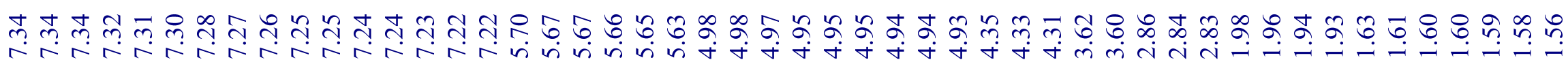

型

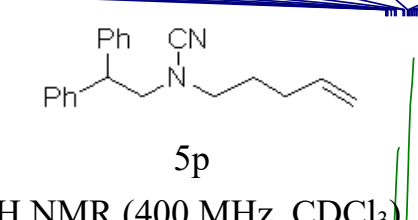

${ }^{1} \mathrm{H}$ NMR $\left(400 \mathrm{MHz}, \mathrm{CDCl}_{3}\right)$

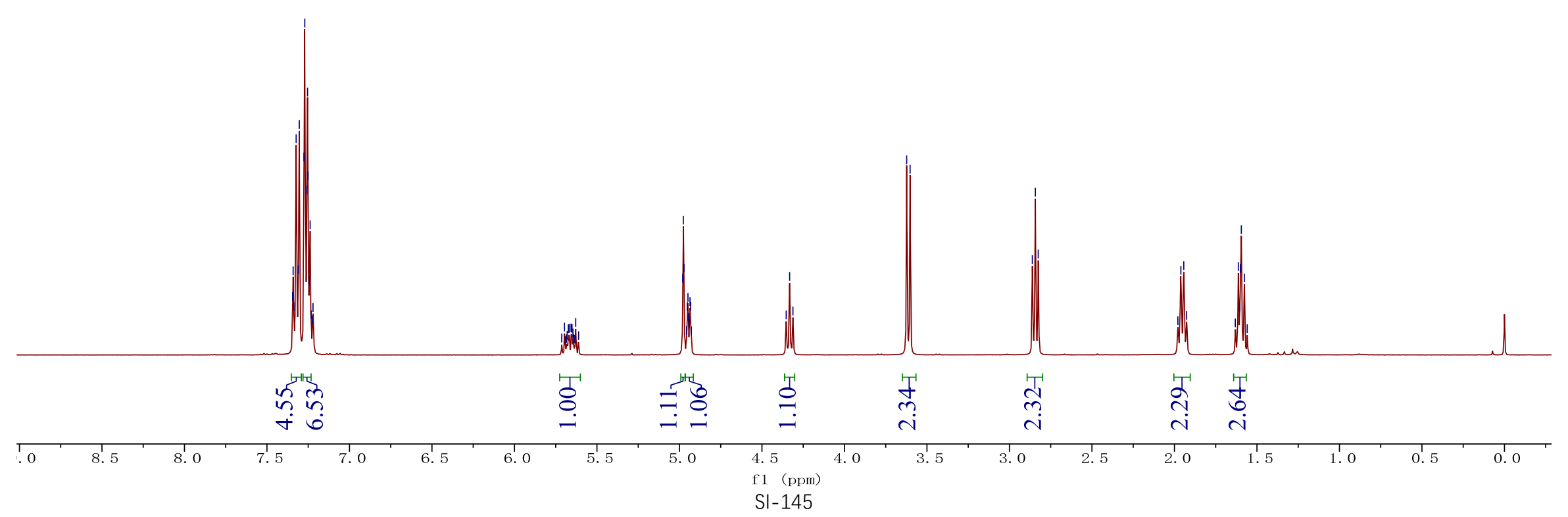




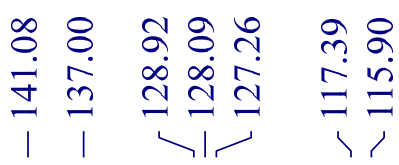

$$
\begin{aligned}
& \text { Ph }
\end{aligned}
$$$$
5 \mathrm{p}
$$

${ }^{13} \mathrm{C}$ NMR $\left(125 \mathrm{MHz}, \mathrm{CDCl}_{3}\right)$

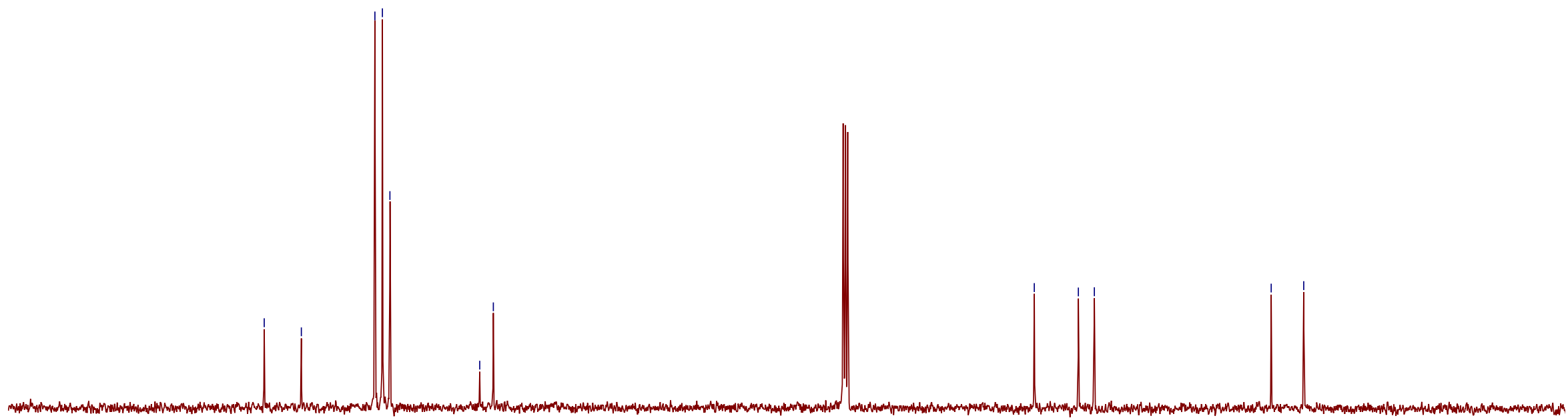

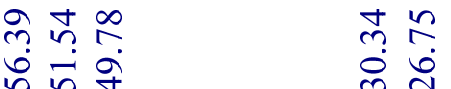

1 


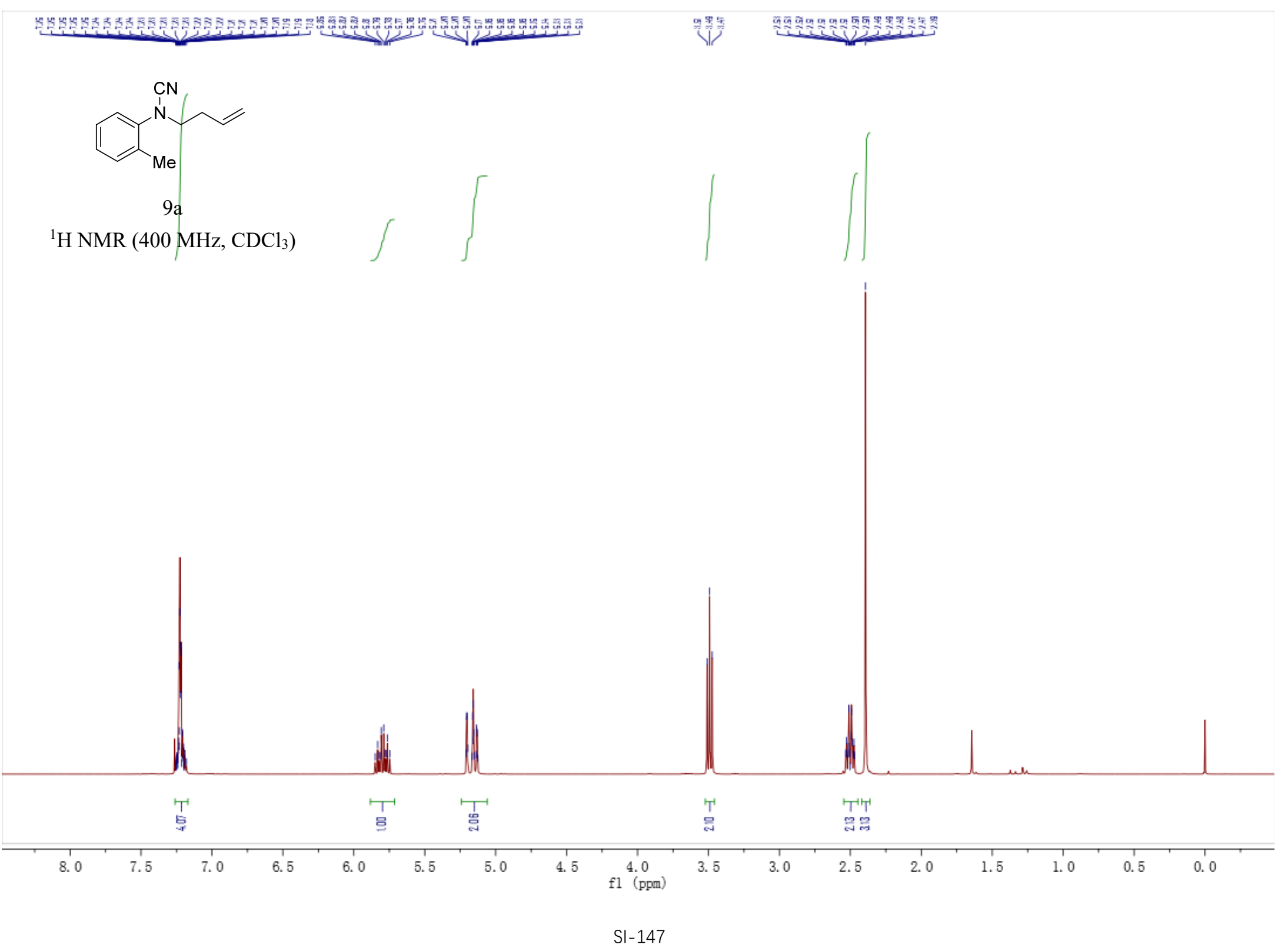




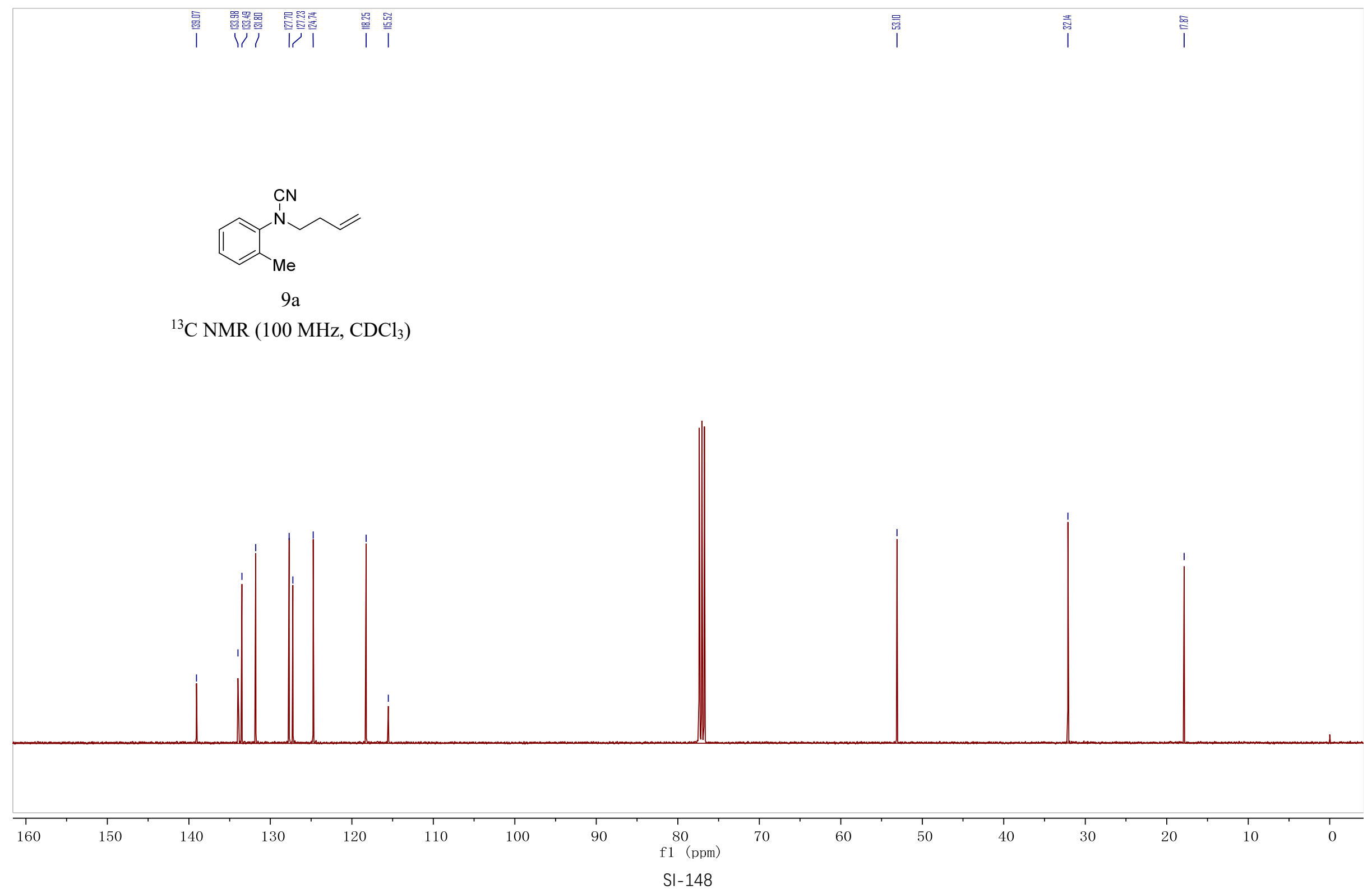




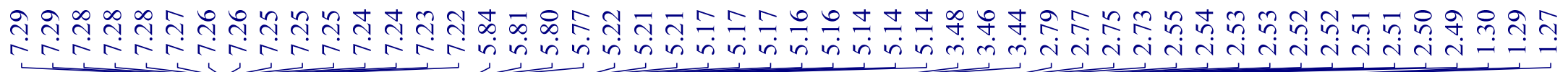
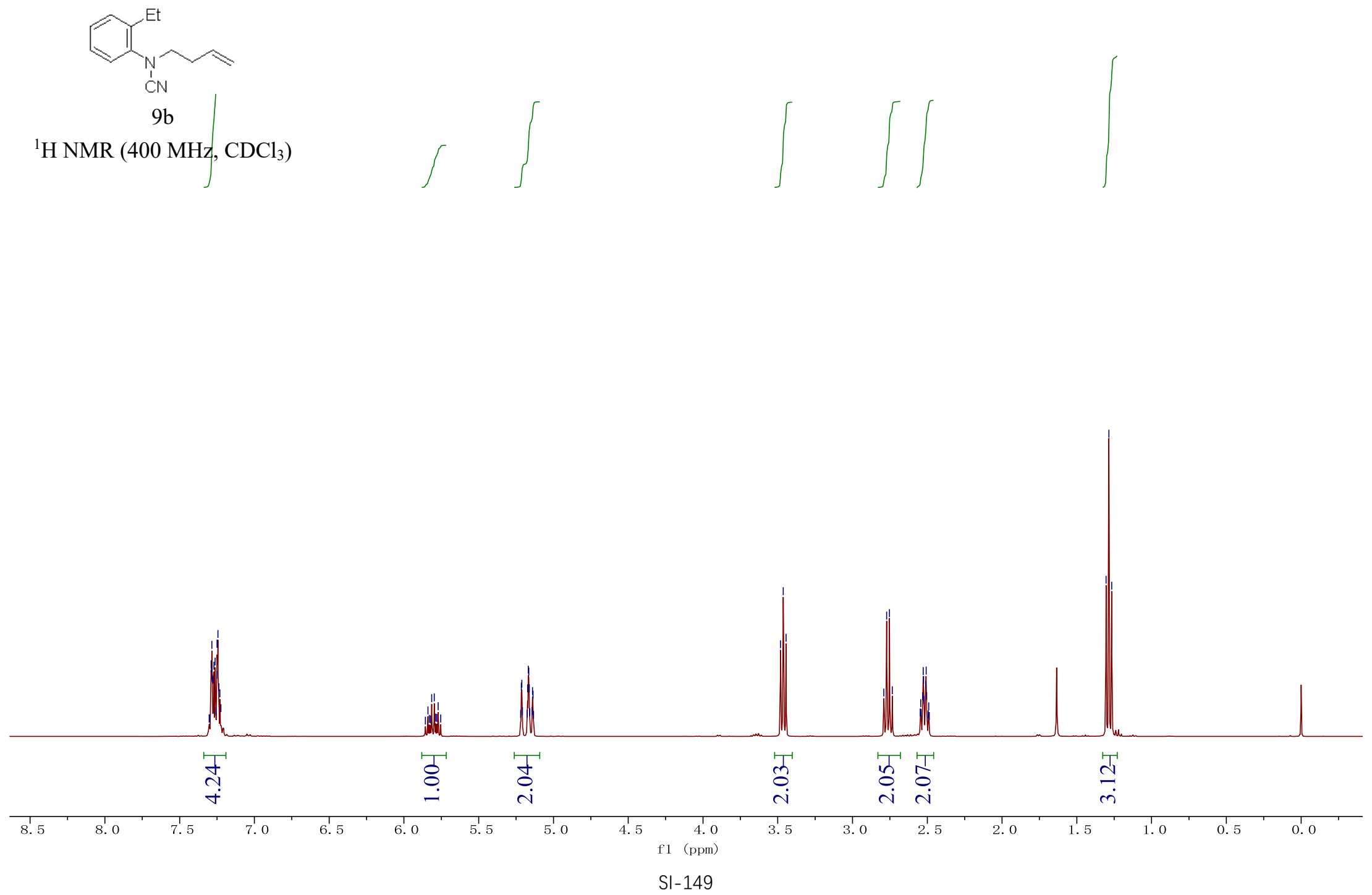


$$
\begin{aligned}
& \text { กุำ }
\end{aligned}
$$

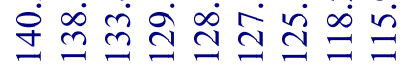

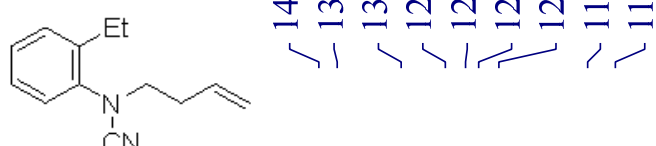

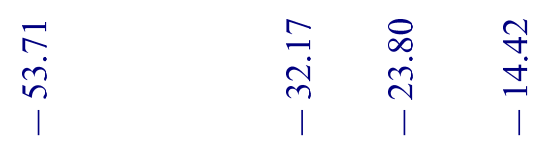

$9 \mathrm{~b}$

${ }^{13} \mathrm{C}$ NMR $\left(100 \mathrm{MHz}, \mathrm{CDCl}_{3}\right)$

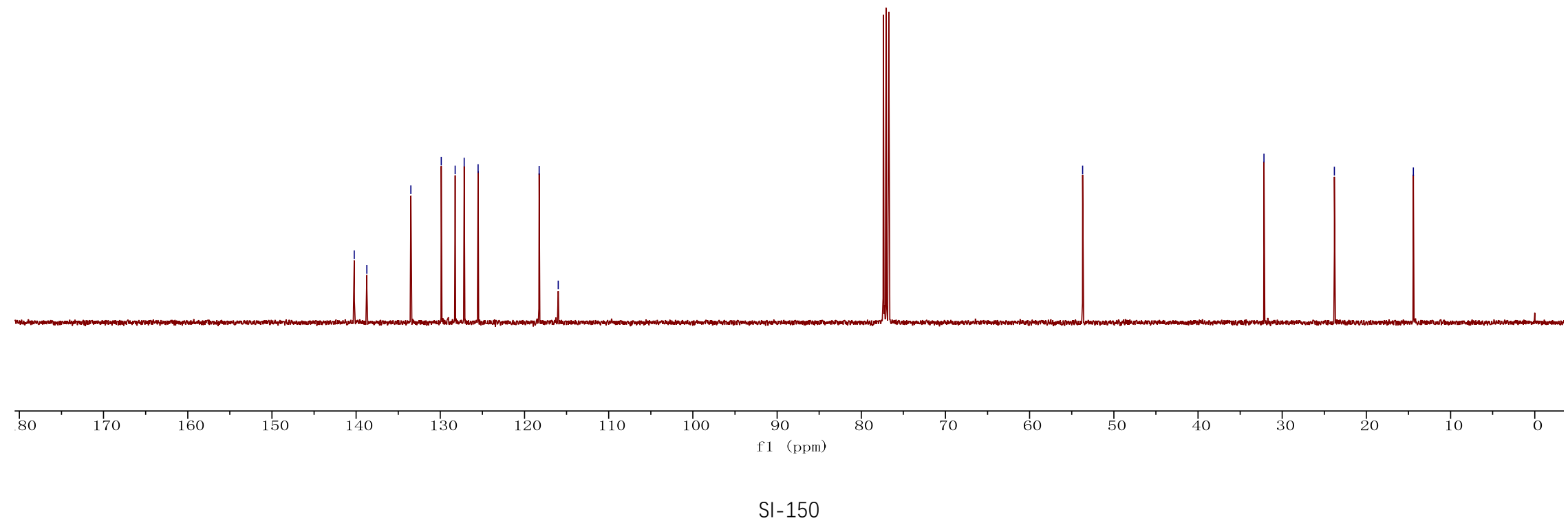




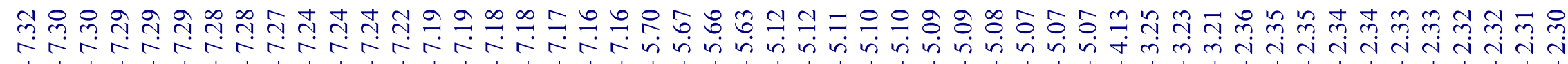

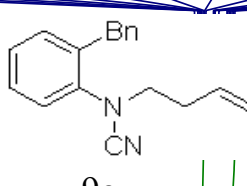

${ }^{1} \mathrm{H}$ NMR (400 MHz, $\left.\mathrm{CDCl}_{3}\right)$ $\|$
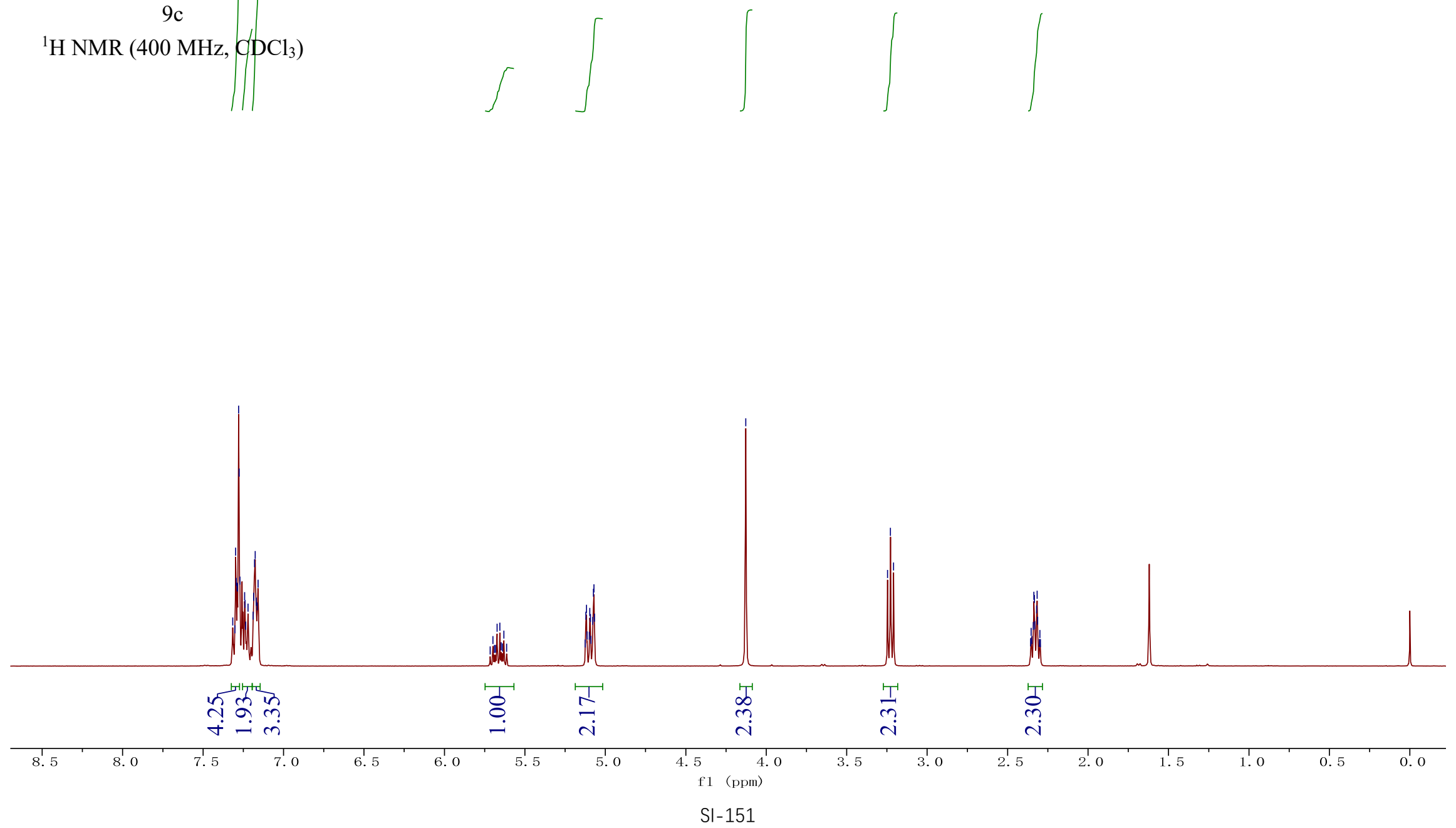
${ }^{13} \mathrm{C}$ NMR $\left(100 \mathrm{MHz}, \mathrm{CDCl}_{3}\right)$

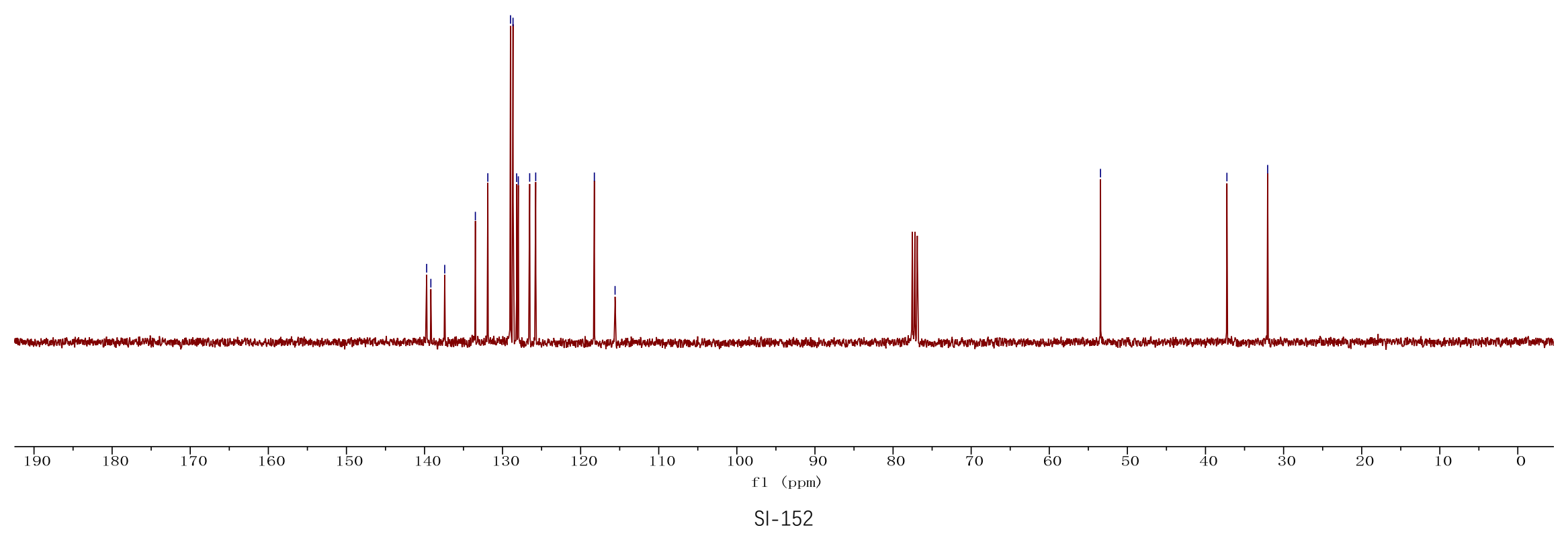




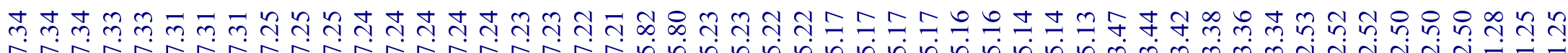

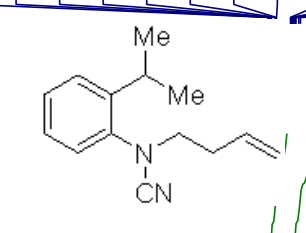

${ }^{1} \mathrm{H} \mathrm{NMR}\left(300 \mathrm{MHz}, \mathrm{CDCl}_{3}\right)$
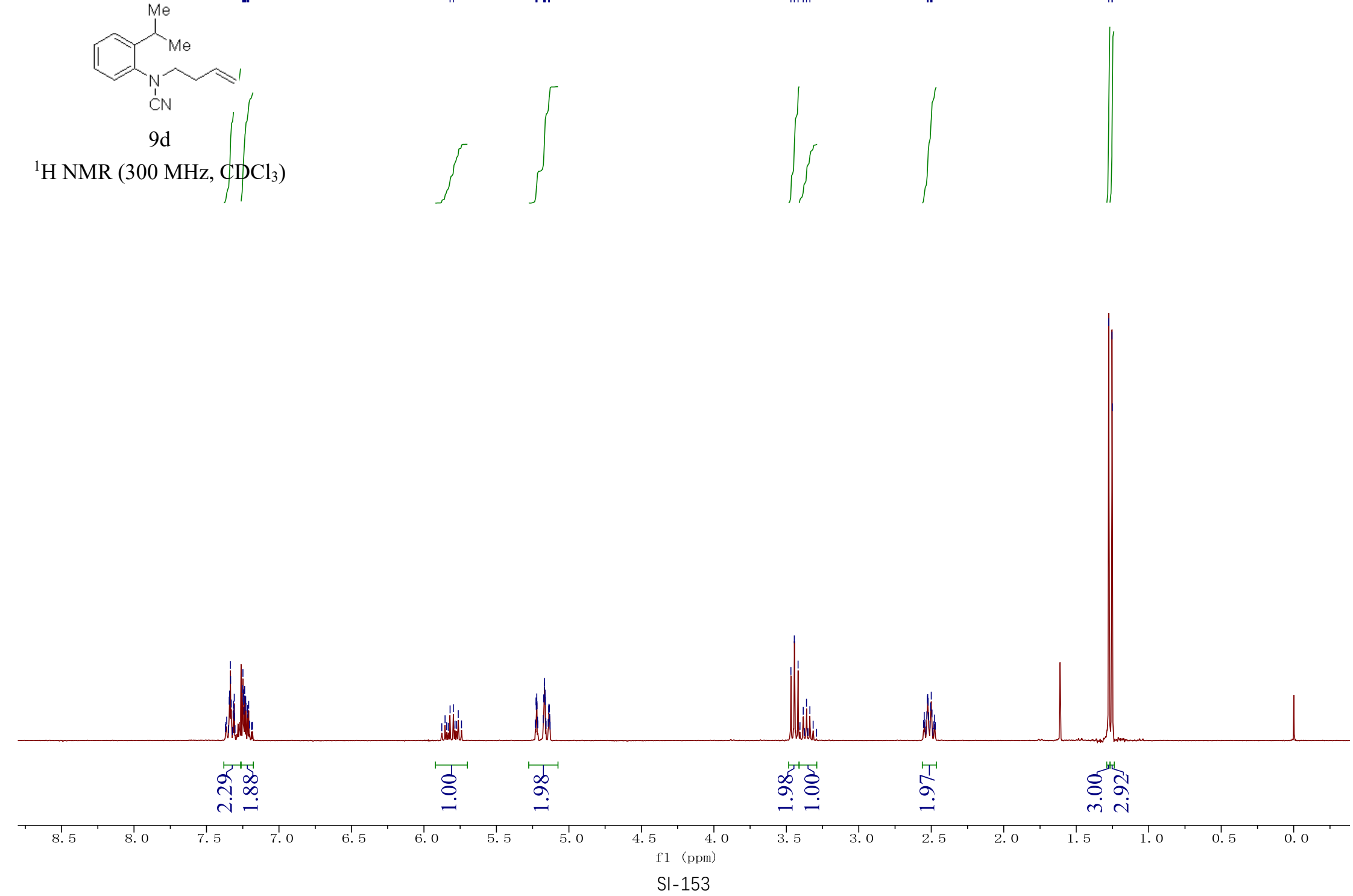


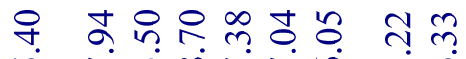

守

Mo

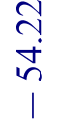

$\because$ 둥

लेत लें

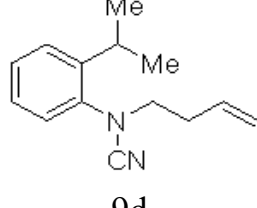

$9 \mathrm{~d}$

${ }^{13} \mathrm{C}$ NMR $\left(125 \mathrm{MHz}, \mathrm{CDCl}_{3}\right)$

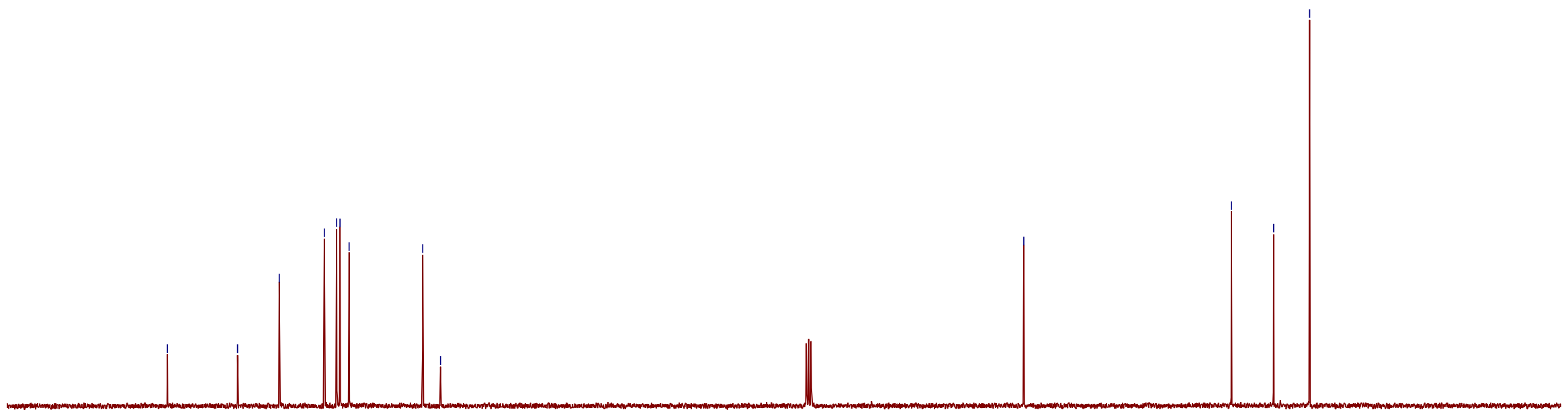

$\frac{1}{160}$

150

140

130

120

110

90

80

70

40 


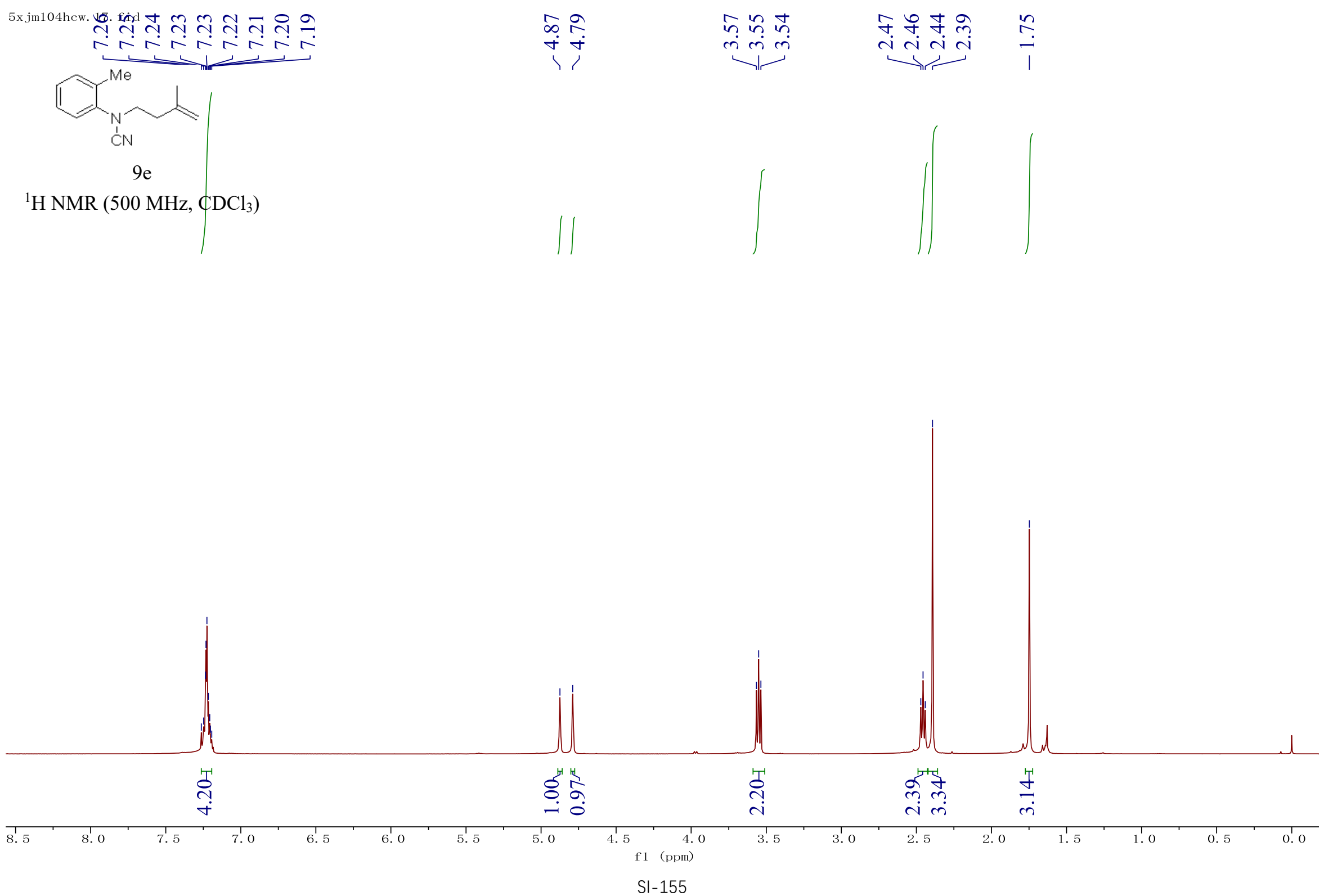



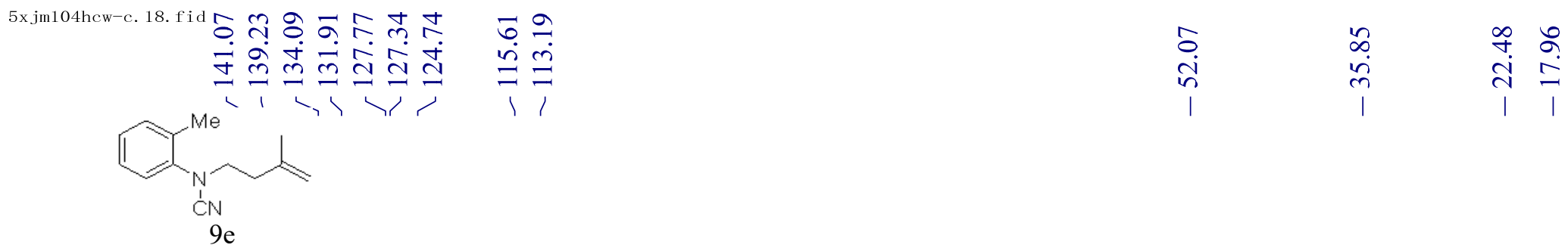

${ }^{13} \mathrm{C} \mathrm{NMR}\left(125 \mathrm{MHz}, \mathrm{CDCl}_{3}\right)$

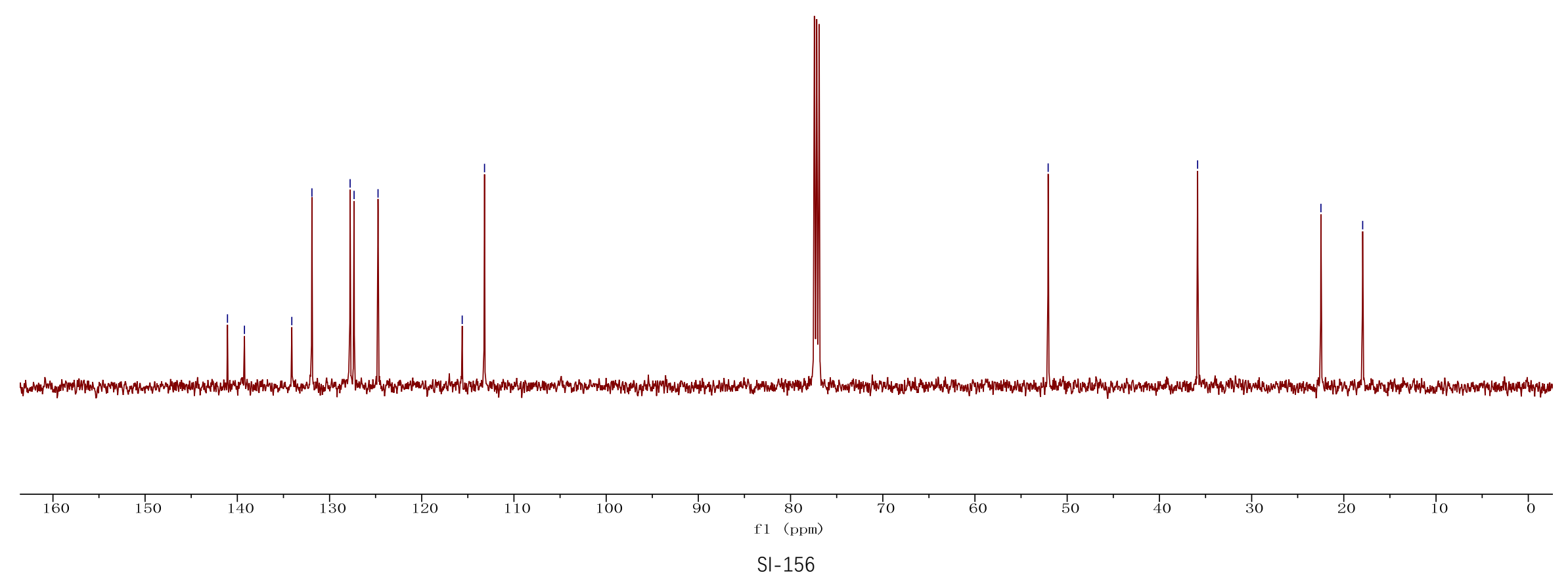




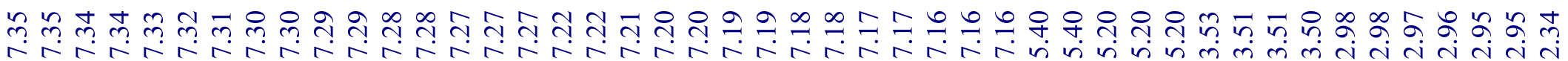
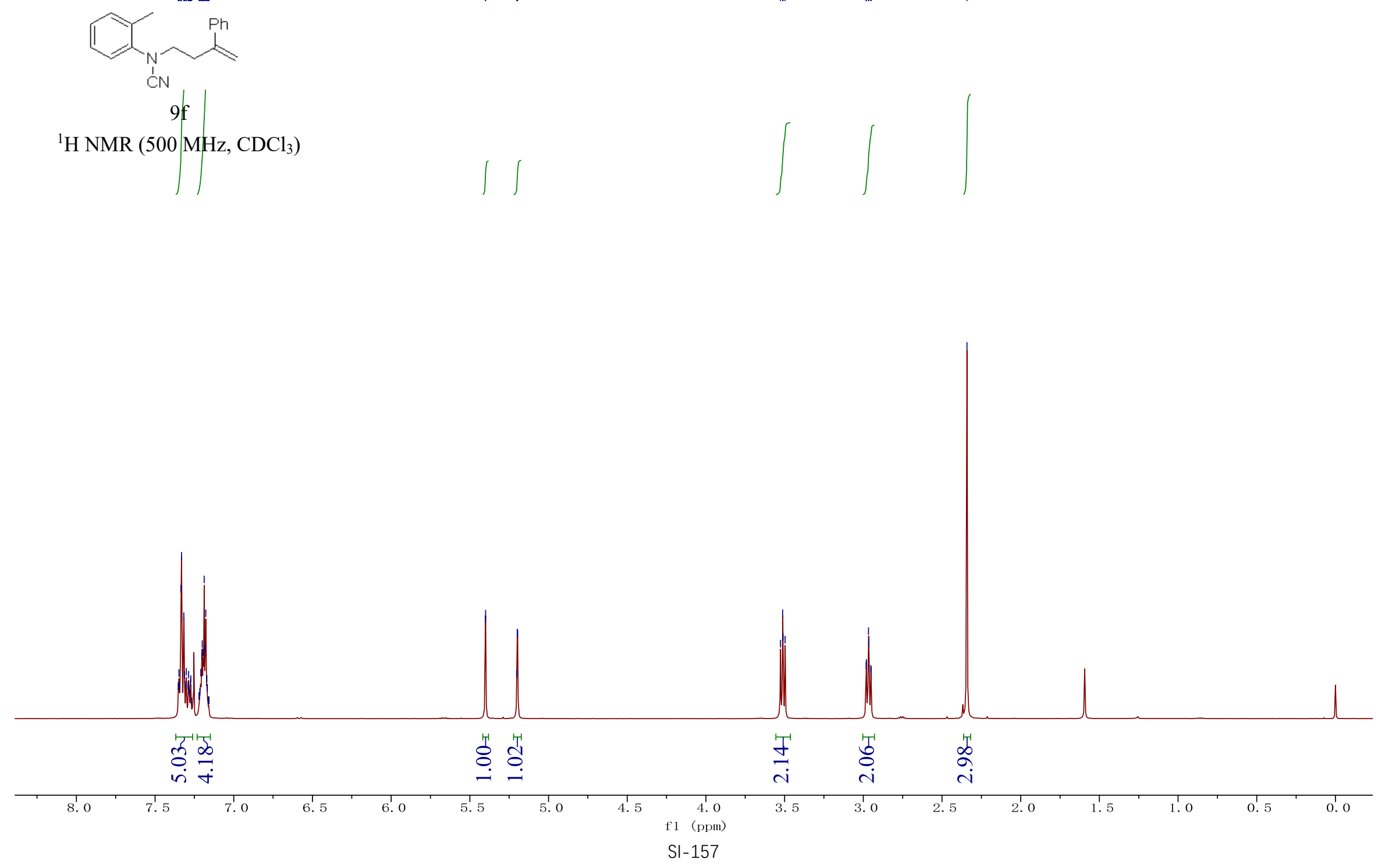


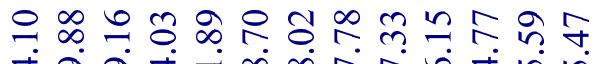

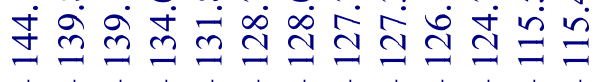

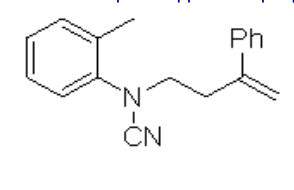

9f

${ }^{13} \mathrm{C}$ NMR (125 MHz, $\left.\mathrm{CDCl}_{3}\right)$

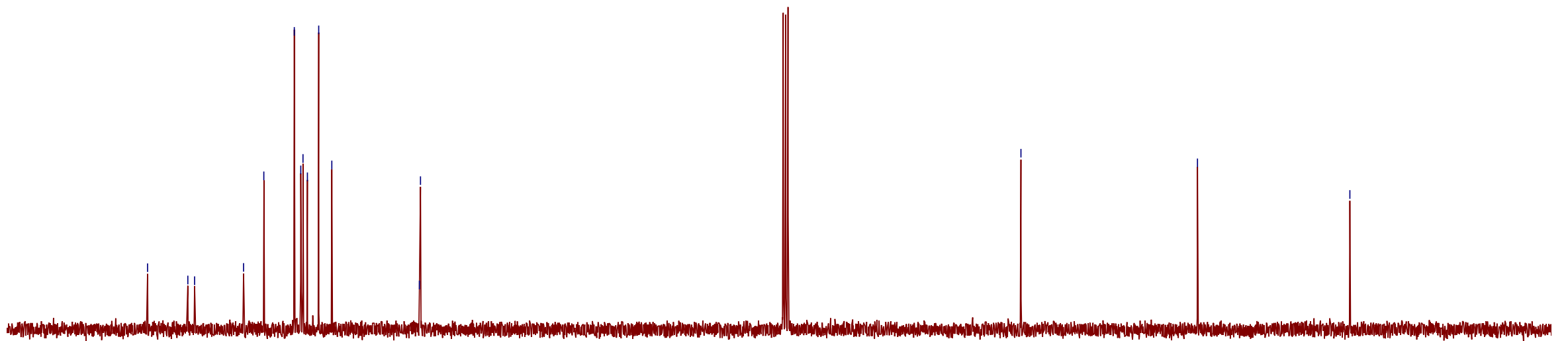

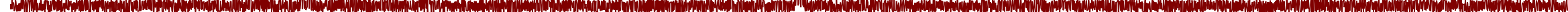

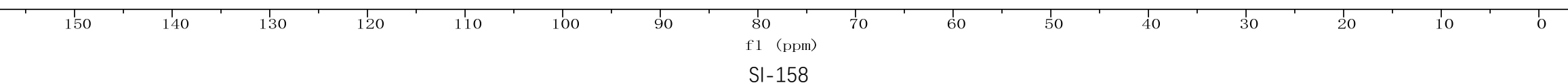




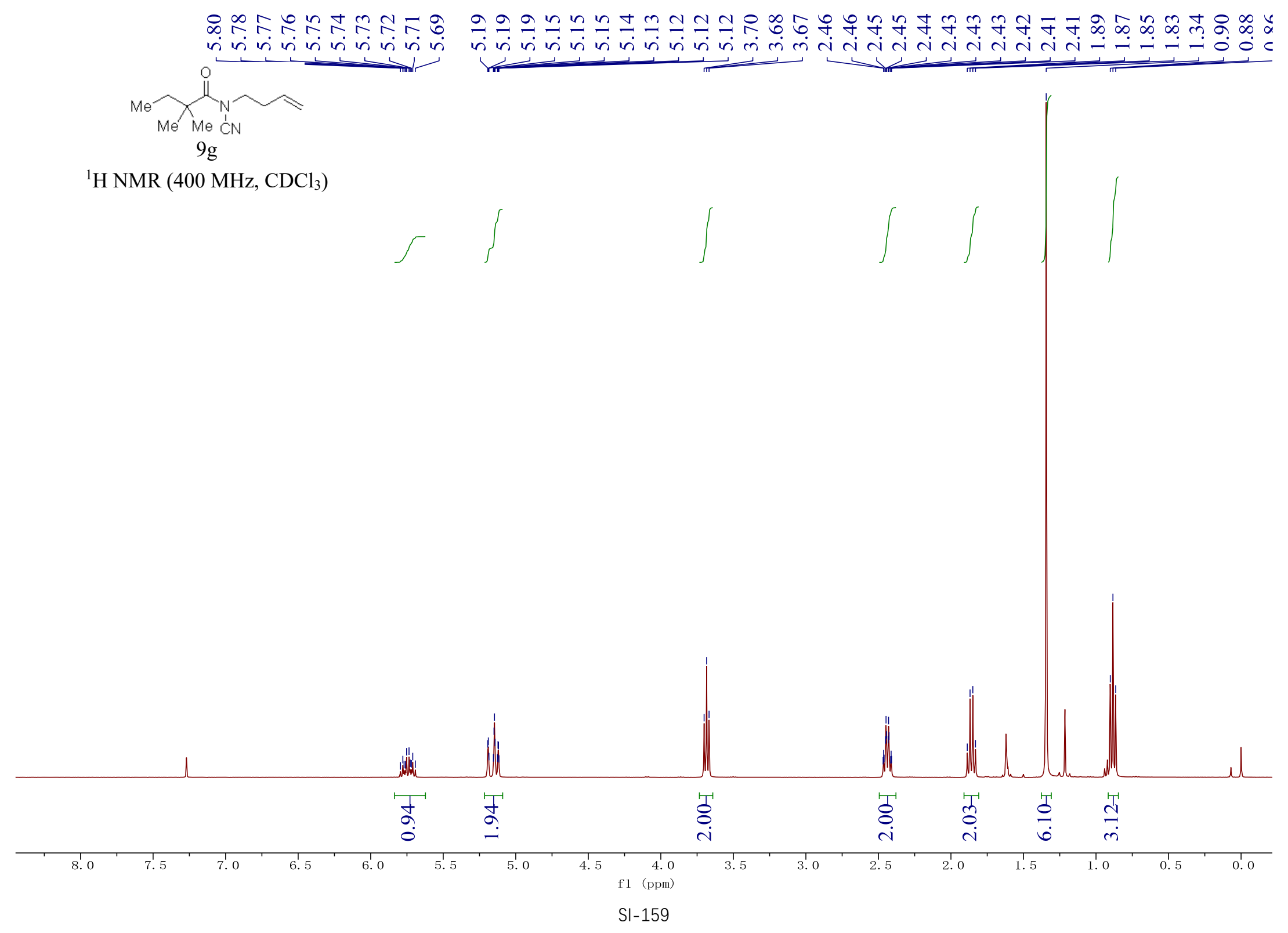




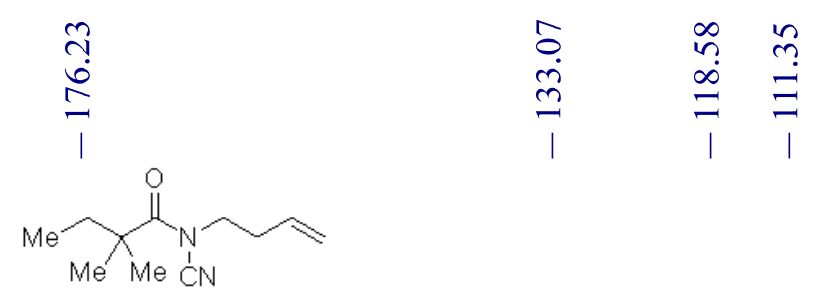

$\infty$

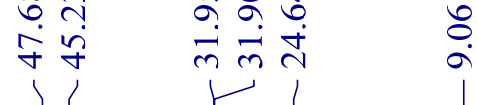

$9 \mathrm{~g}$

${ }^{13} \mathrm{C}$ NMR $\left(125 \mathrm{MHz}, \mathrm{CDCl}_{3}\right)$
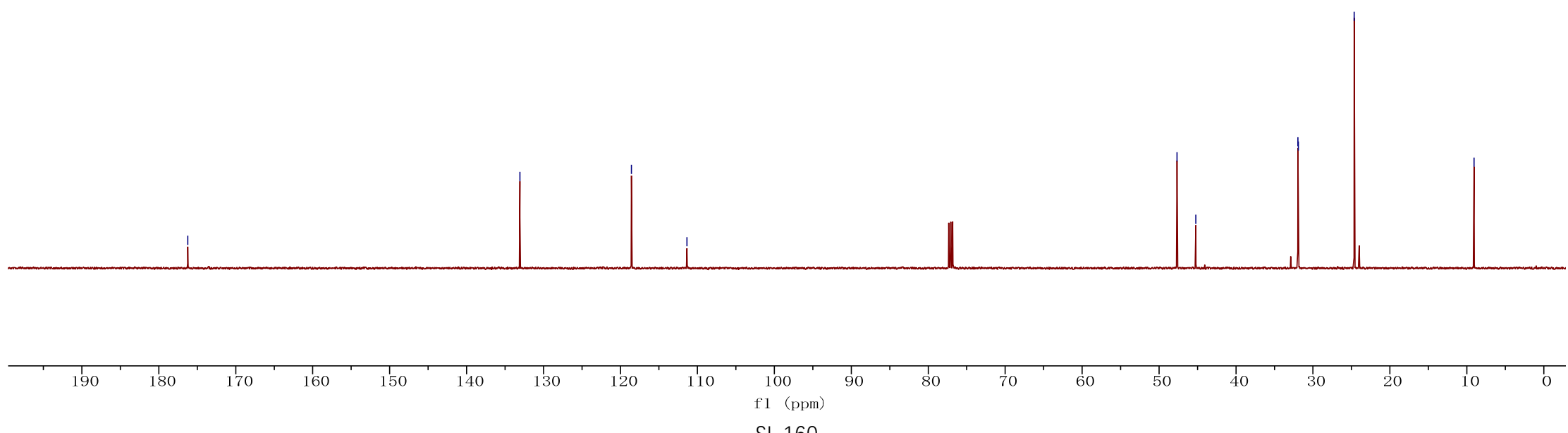

SI-160 


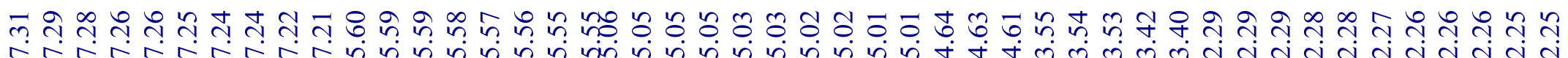

L

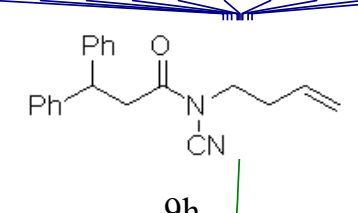

${ }^{1} \mathrm{H} \mathrm{NMR}\left(500 \mathrm{MHz}, \mathrm{CDCl}_{3}\right)$

(

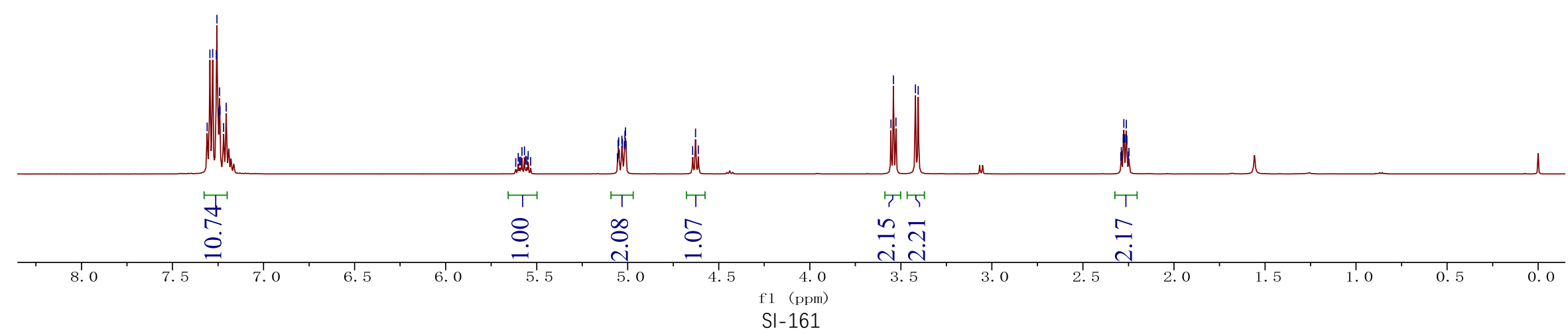




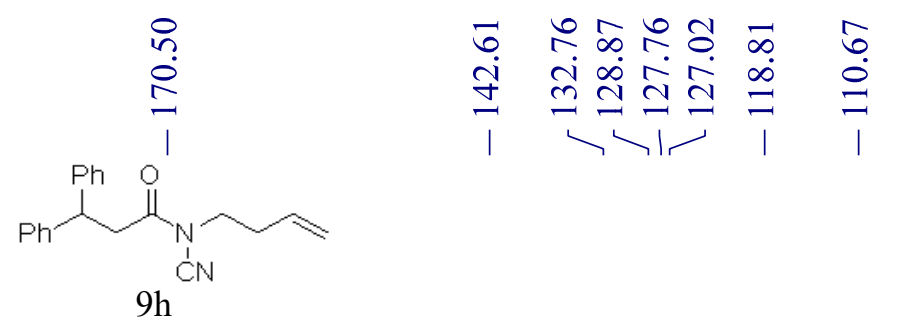

ఫิ กิ?

ํํㄱㅇ

${ }^{13} \mathrm{C}$ NMR $\left(125 \mathrm{MHz}, \mathrm{CDCl}_{3}\right)$

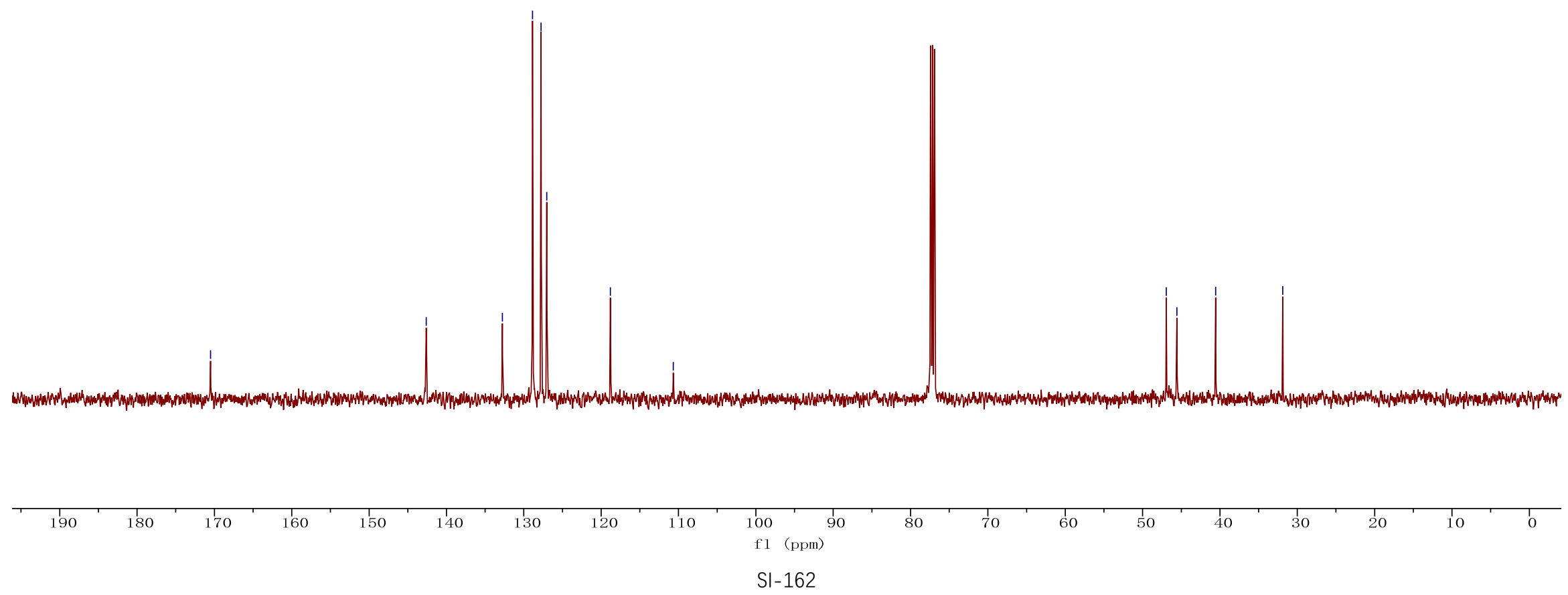




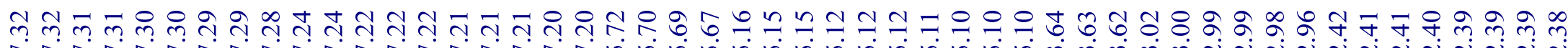

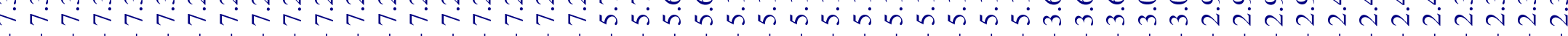

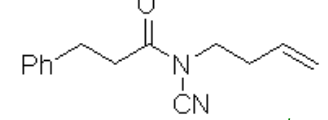

$9 \mathrm{i}$

${ }^{1} \mathrm{H}$ NMR $\left(500 \mathrm{MHz}, \mathrm{CDCl}_{3}\right)$
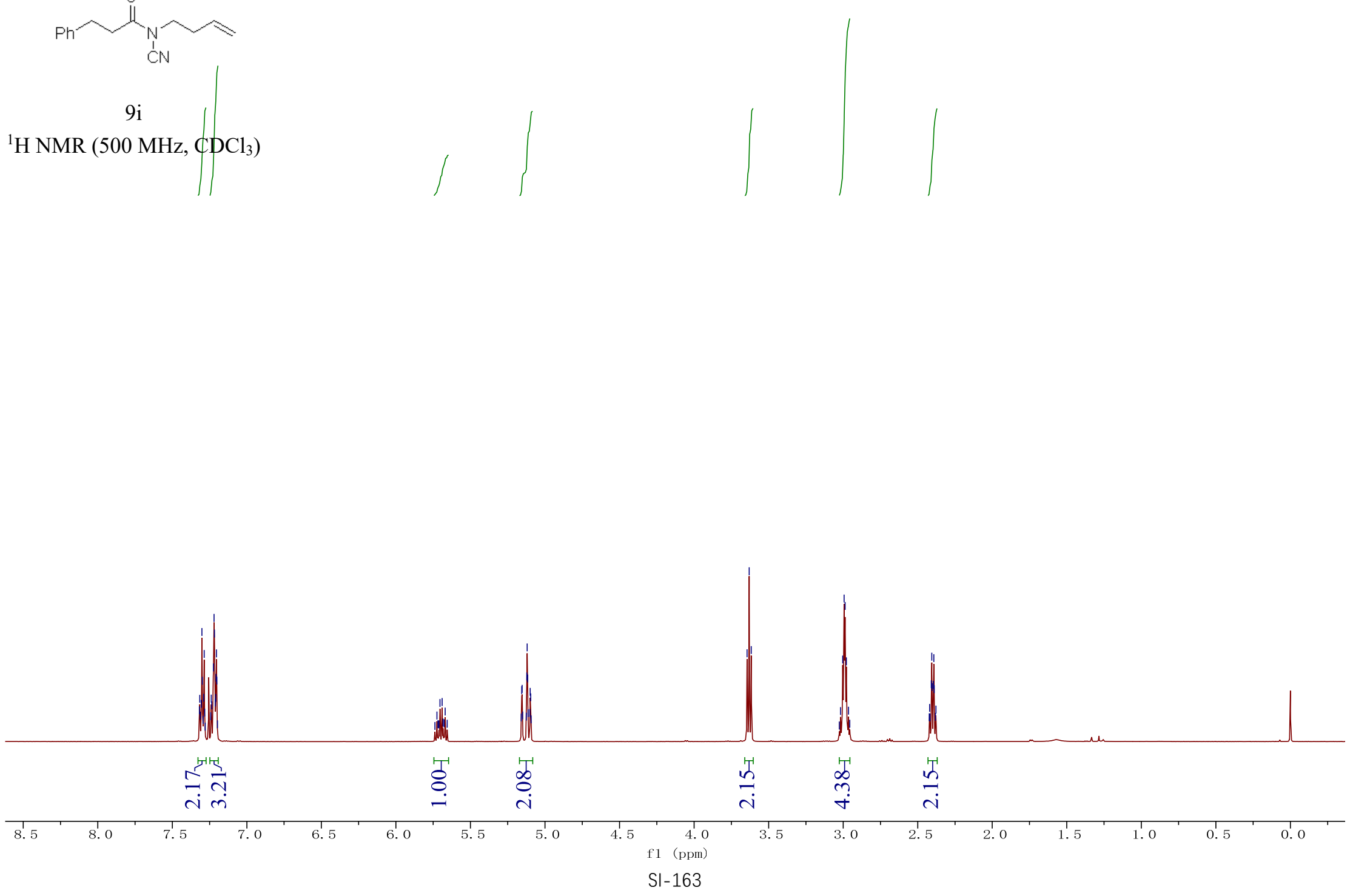


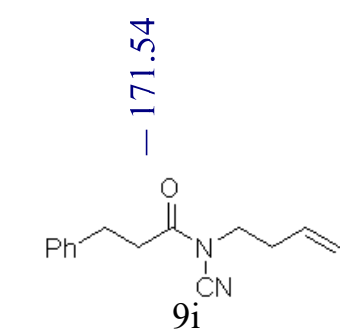

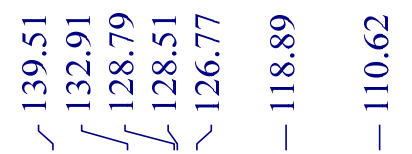

文

भ फंलें

${ }^{13} \mathrm{C} \mathrm{NMR}\left(125 \mathrm{MHz}, \mathrm{CDCl}_{3}\right)$

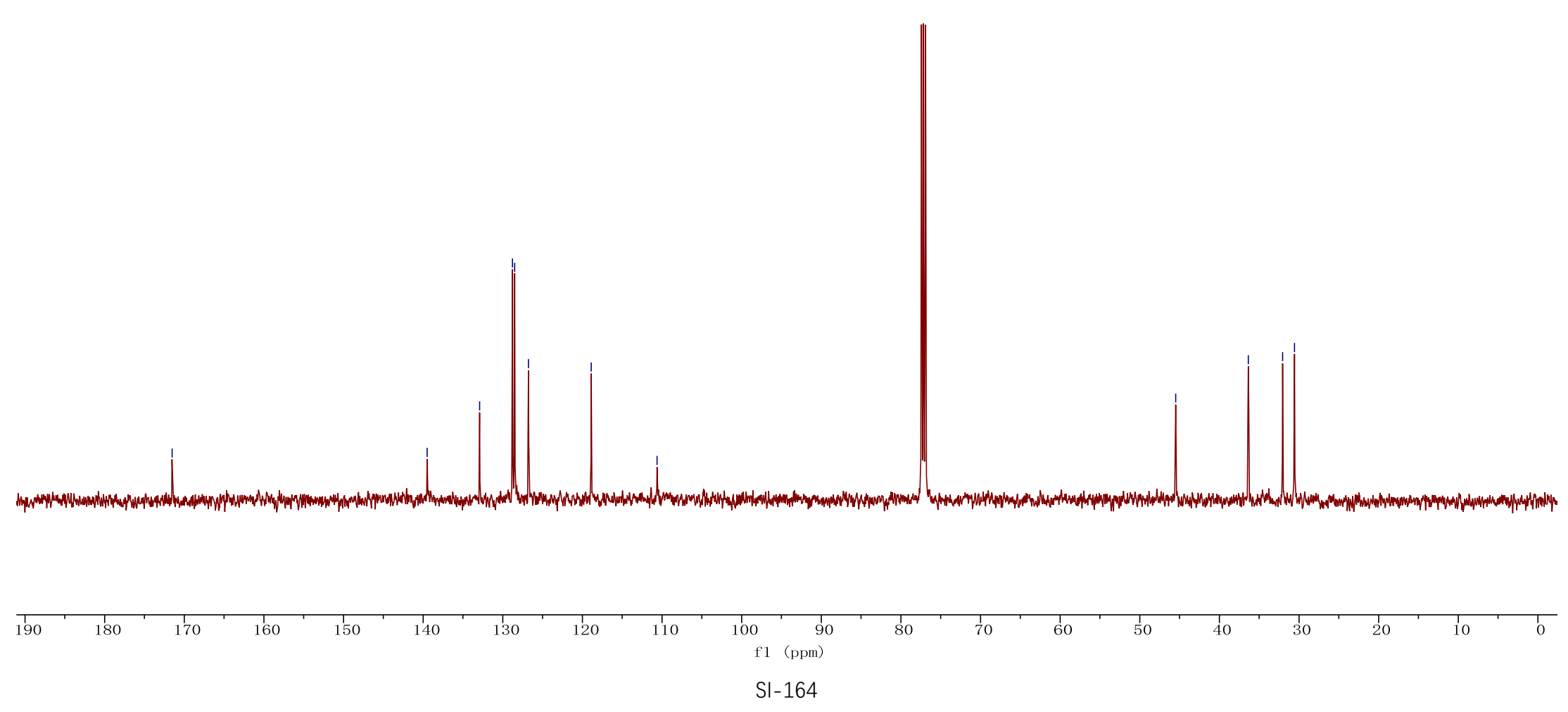




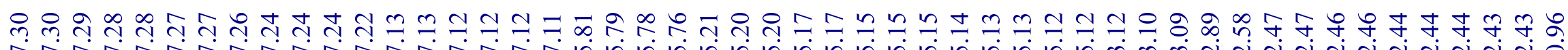

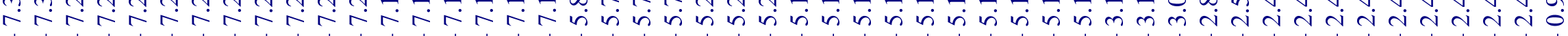

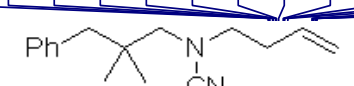

9j

${ }^{1} \mathrm{H}$ NMR (500 MHz, $\mathrm{CDCl}_{3}$ )
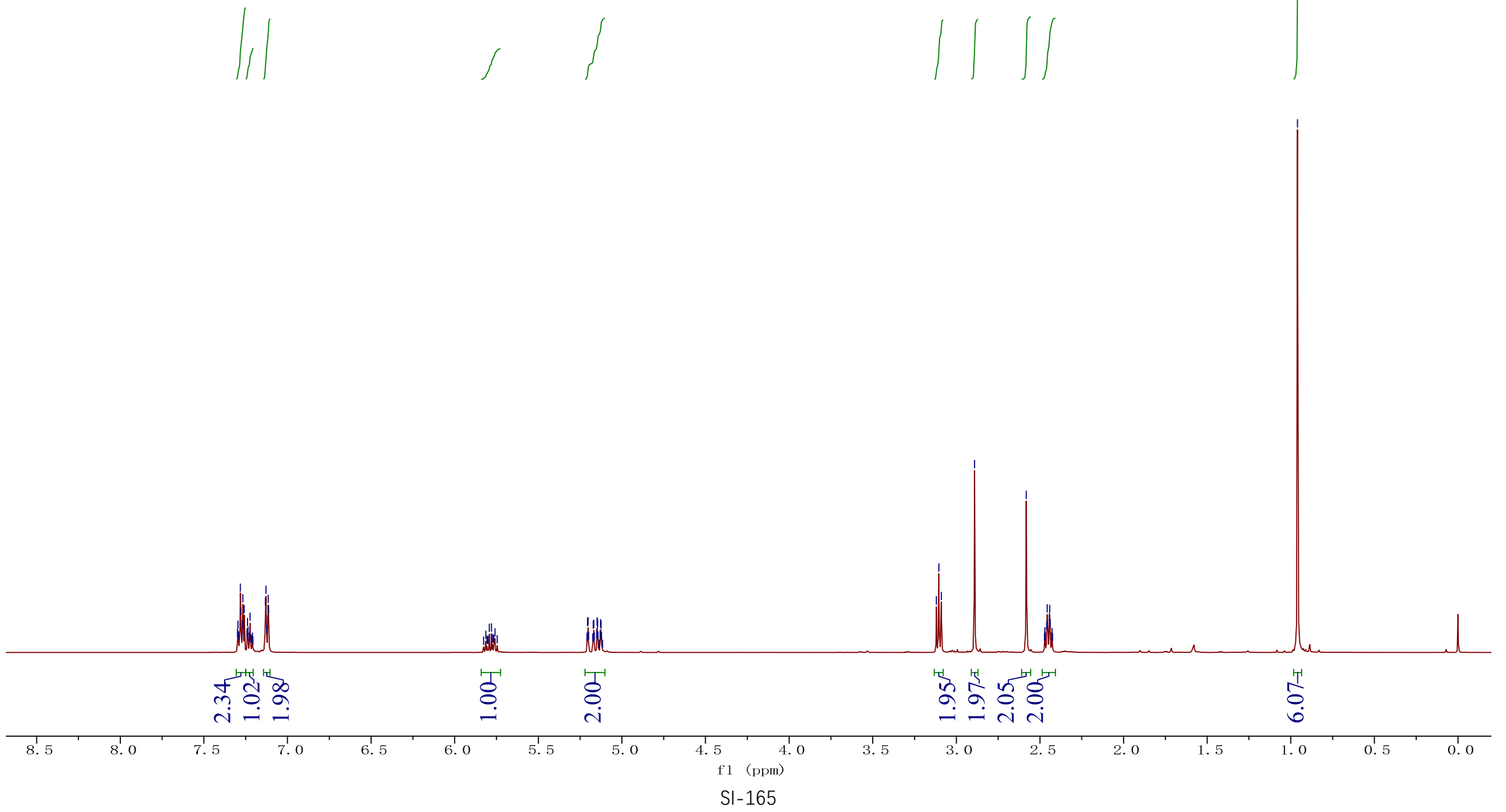


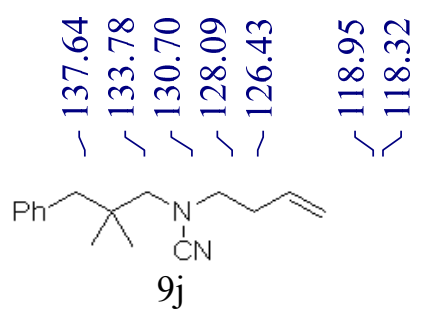

${ }^{13} \mathrm{C}$ NMR (125 MHz, $\mathrm{CDCl}_{3}$ )

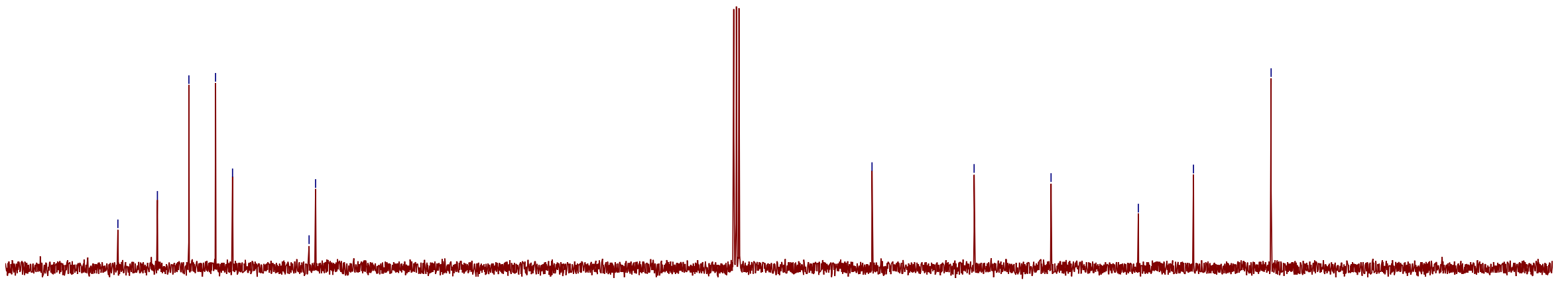

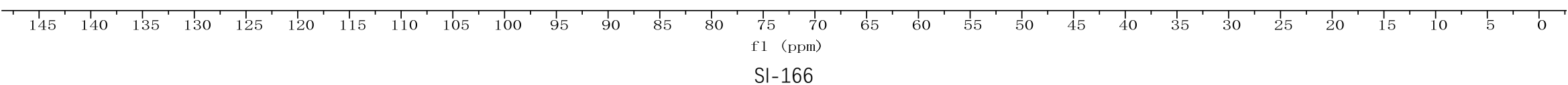




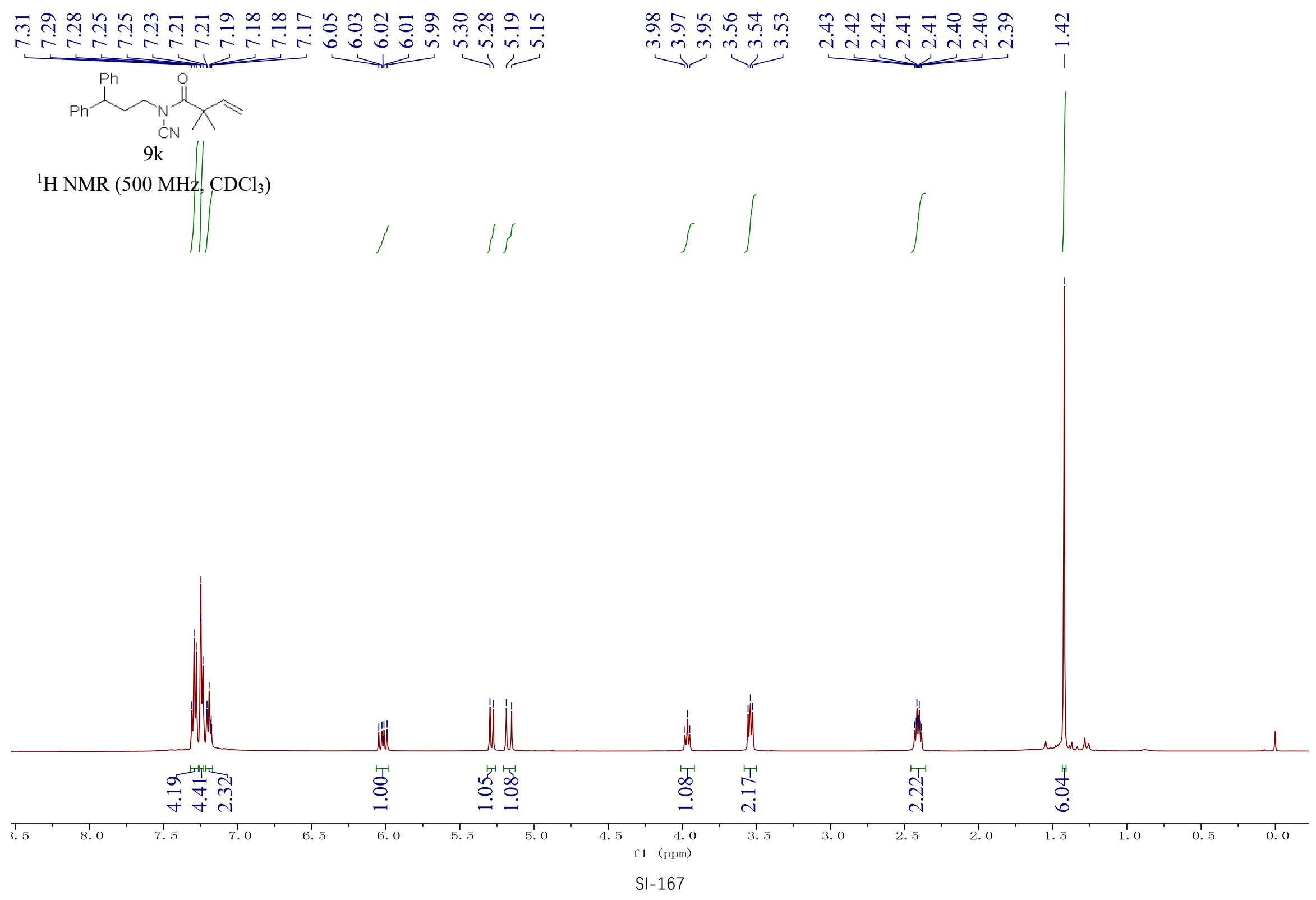



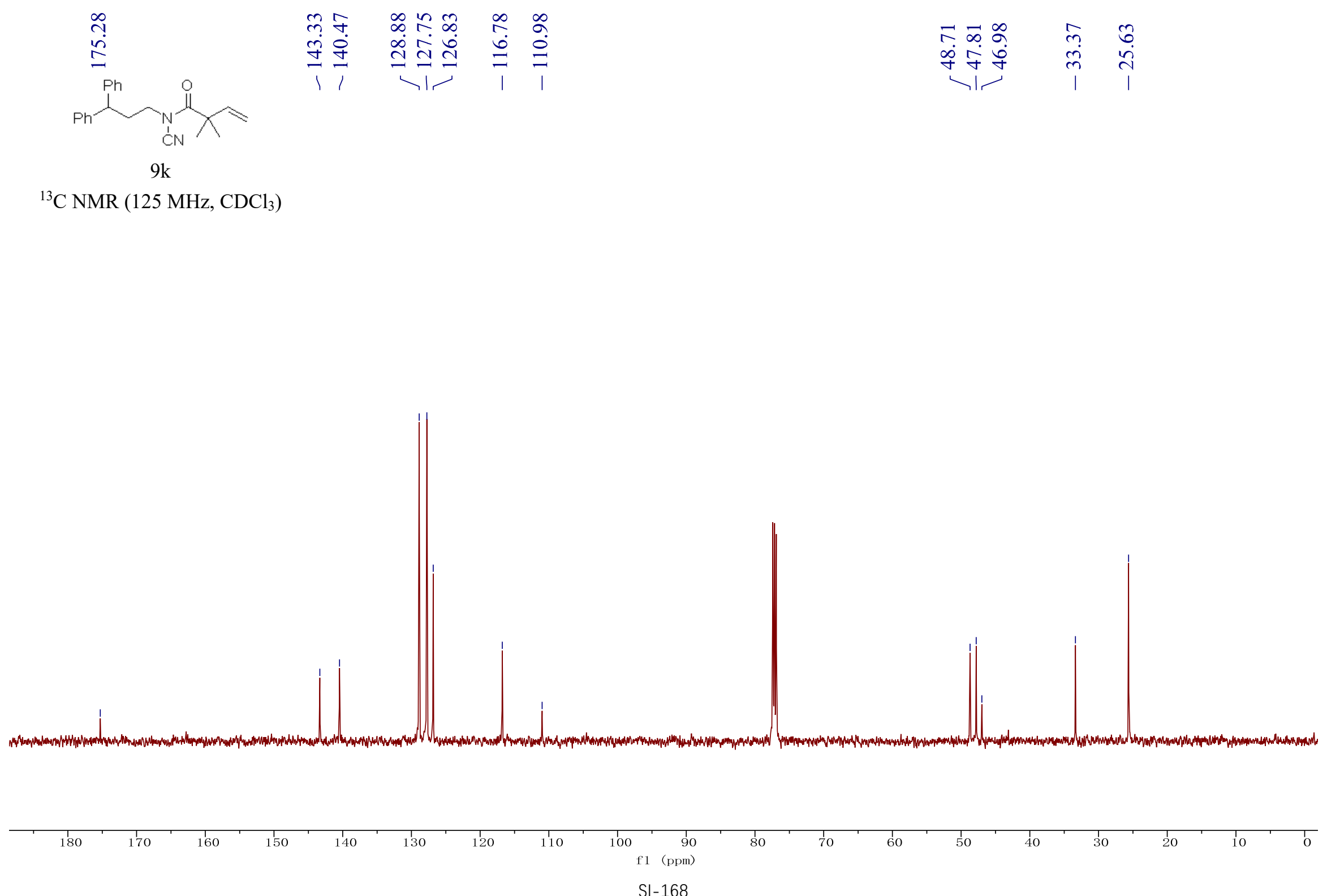


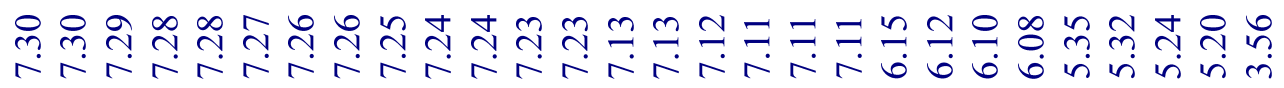

$\longrightarrow$

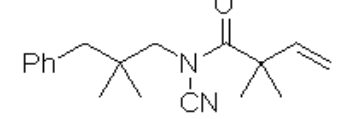

91

${ }^{1} \mathrm{H} \mathrm{NMR}\left(400 \mathrm{MHz}, \mathrm{CDCl}_{3}\right)$ $\int$

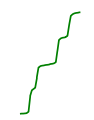

กิ

$\begin{array}{ll}\infty & a \\ \text { j } & 0\end{array}$
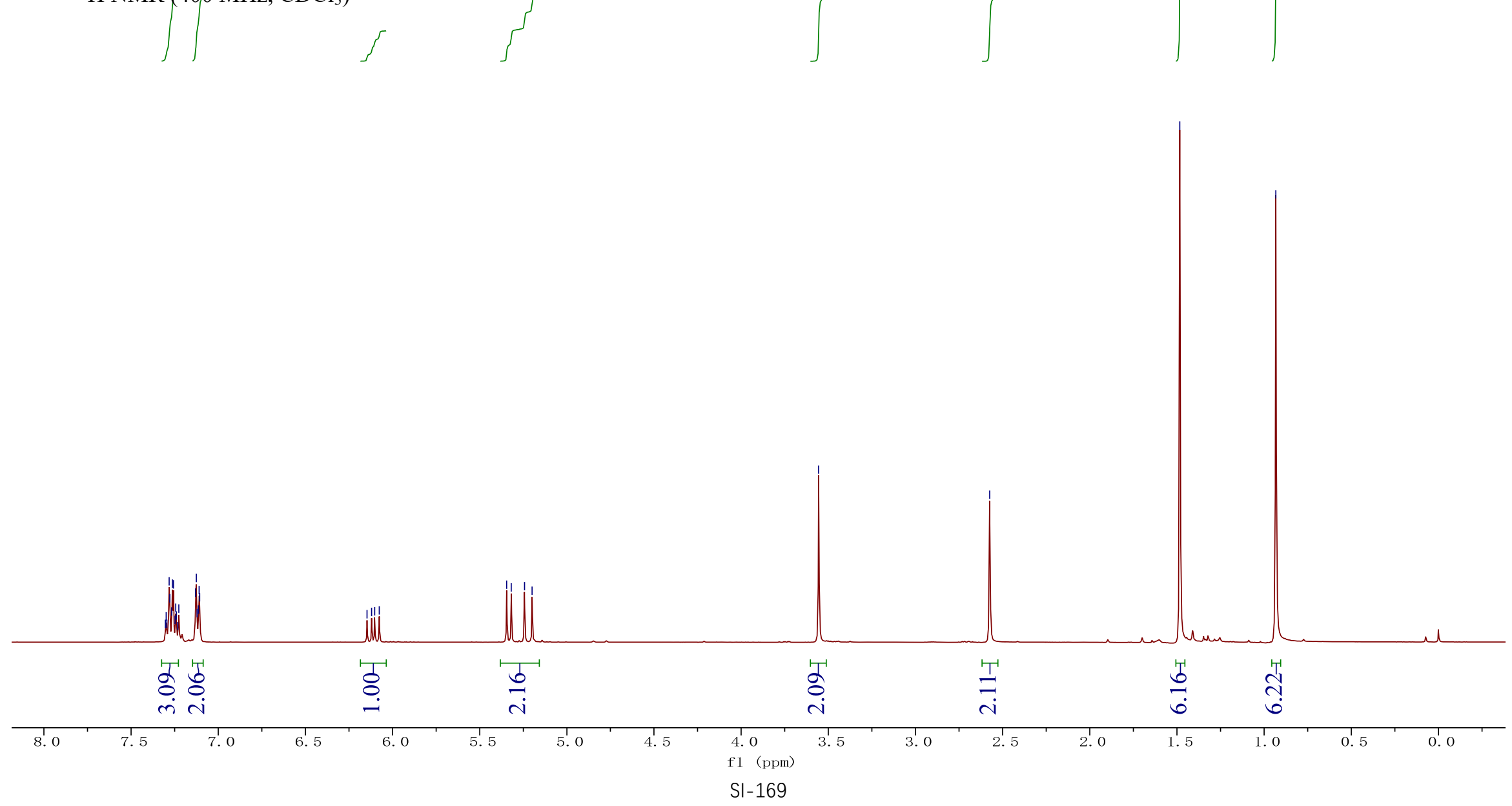


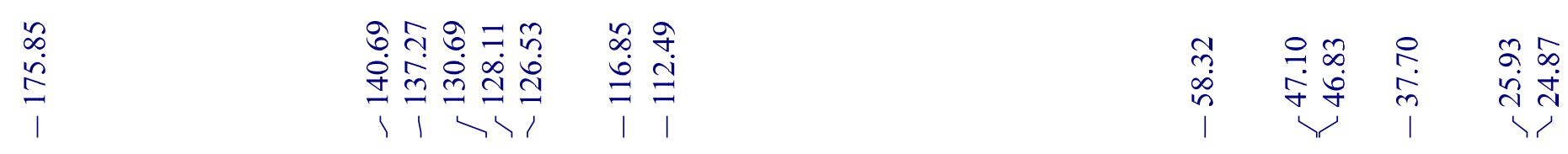

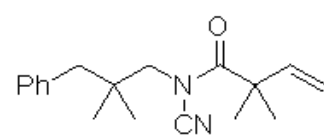

91

${ }^{13} \mathrm{C} \mathrm{NMR}\left(125 \mathrm{MHz}, \mathrm{CDCl}_{3}\right)$

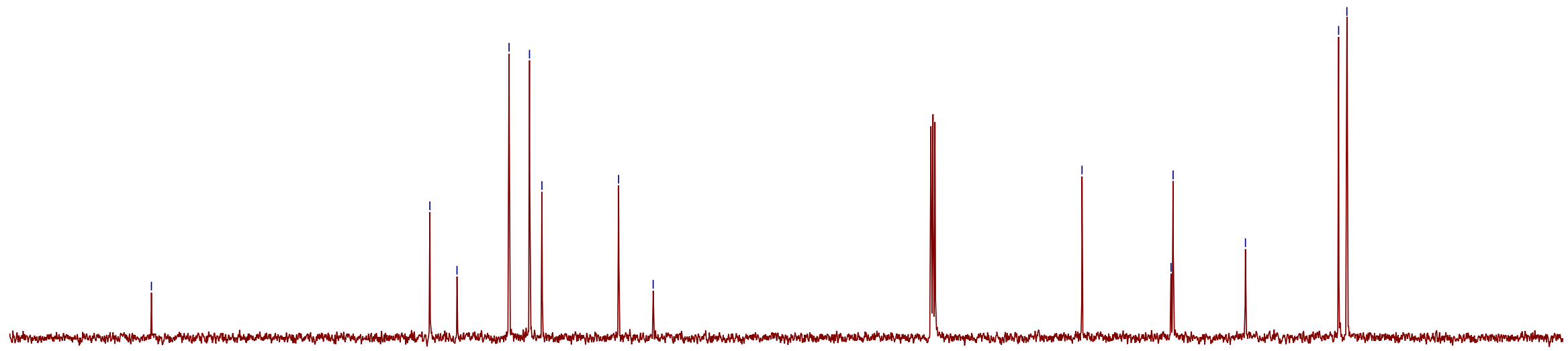

$\begin{array}{rrrrrrrrrrrrrrrrrr}1 & 1\end{array}$




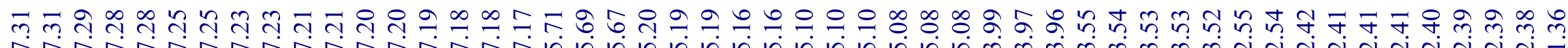
icicicicici

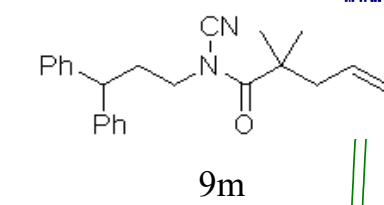

${ }^{1} \mathrm{H}$ NMR (500 MHz, $\mathrm{CDCl}_{3}$ )
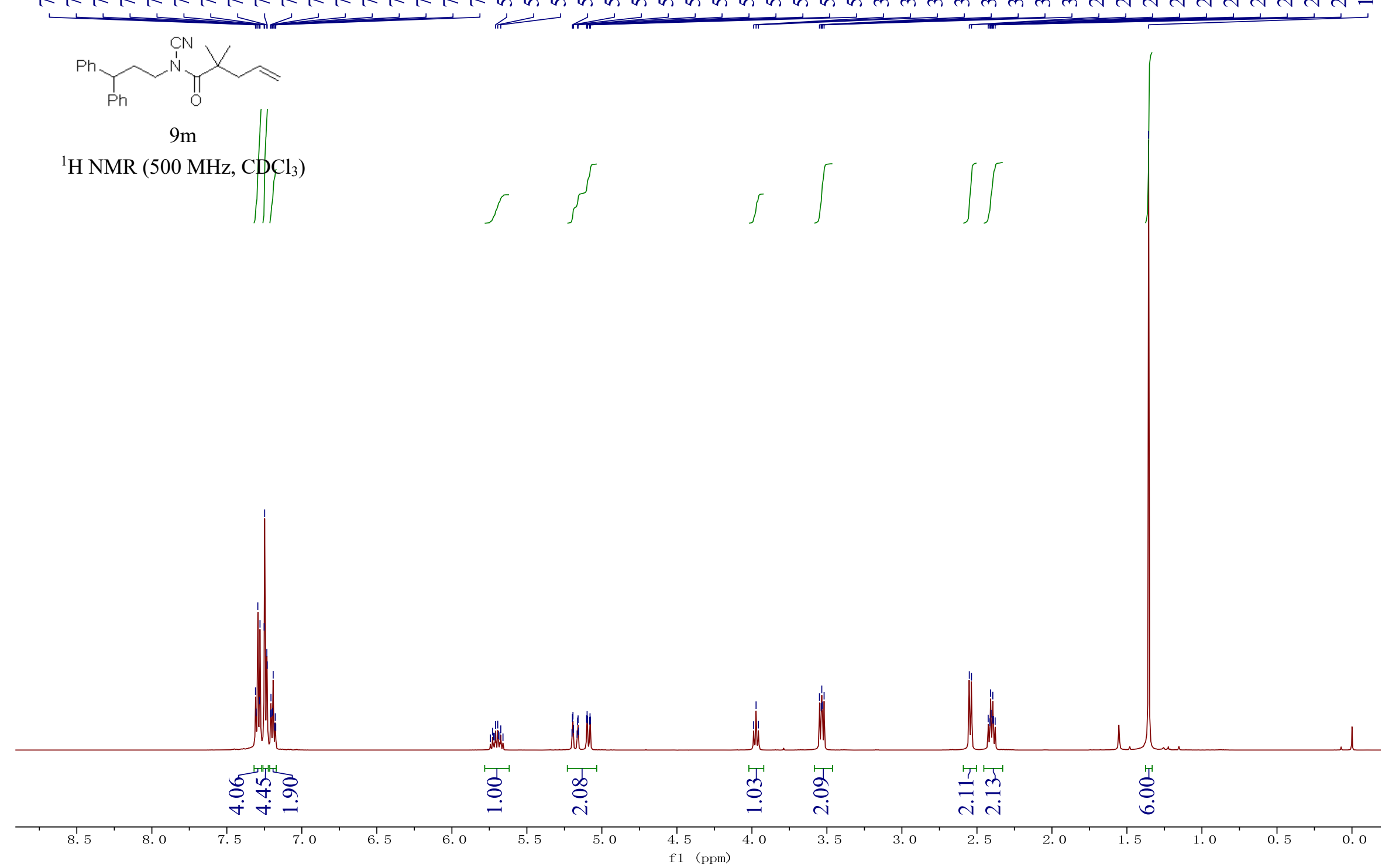


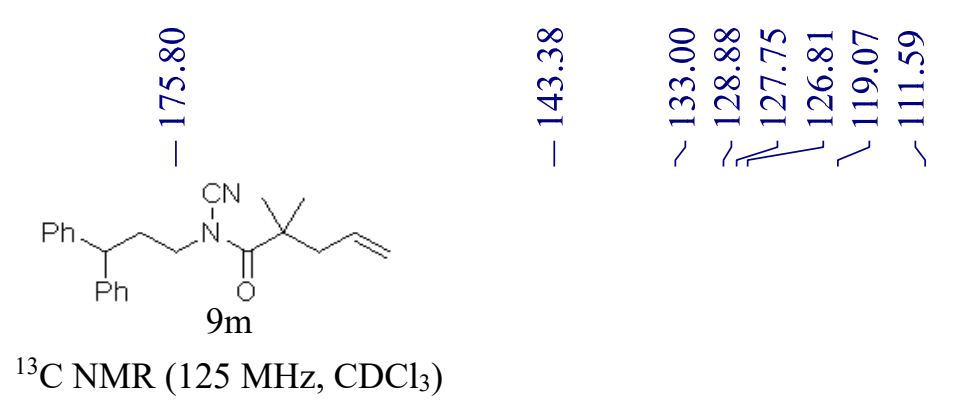

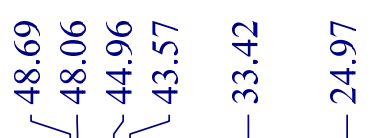

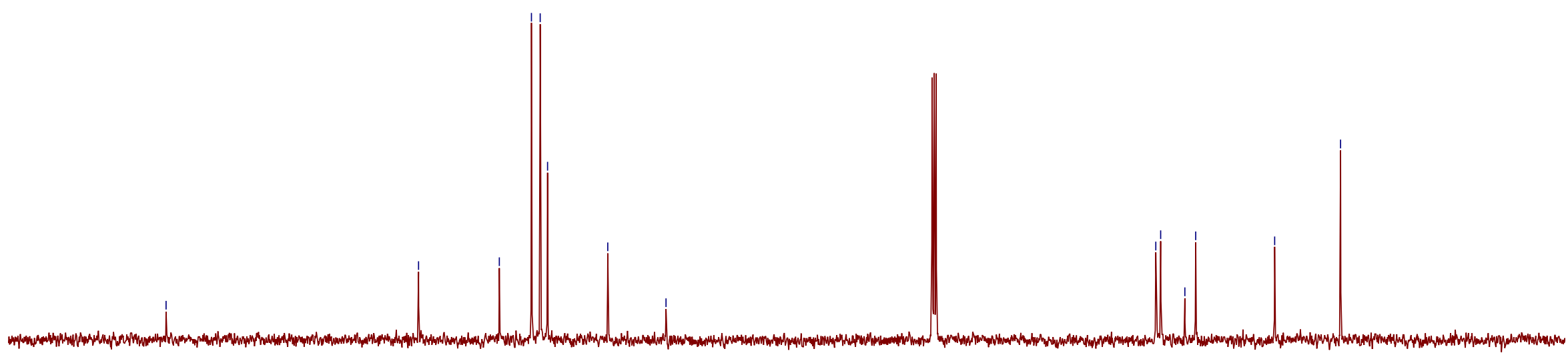

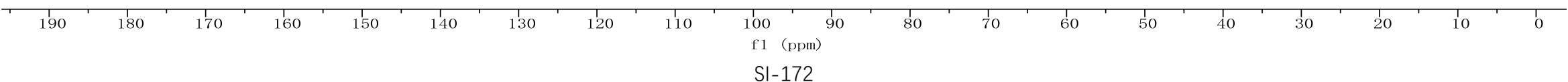




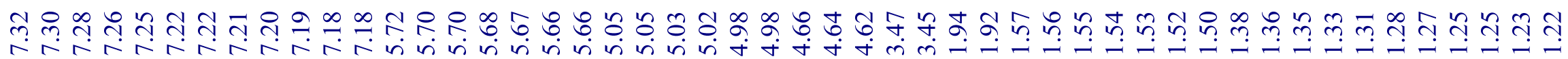

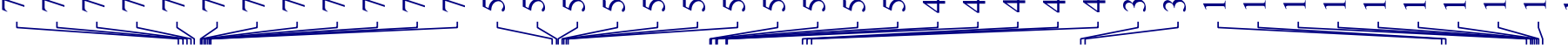

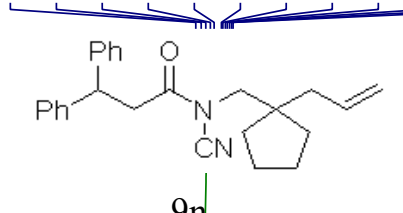

$9 \eta$

${ }^{1} \mathrm{H} \mathrm{NMR}\left(400 \mathrm{MHz}, \mathrm{CDCl}_{3}\right)$
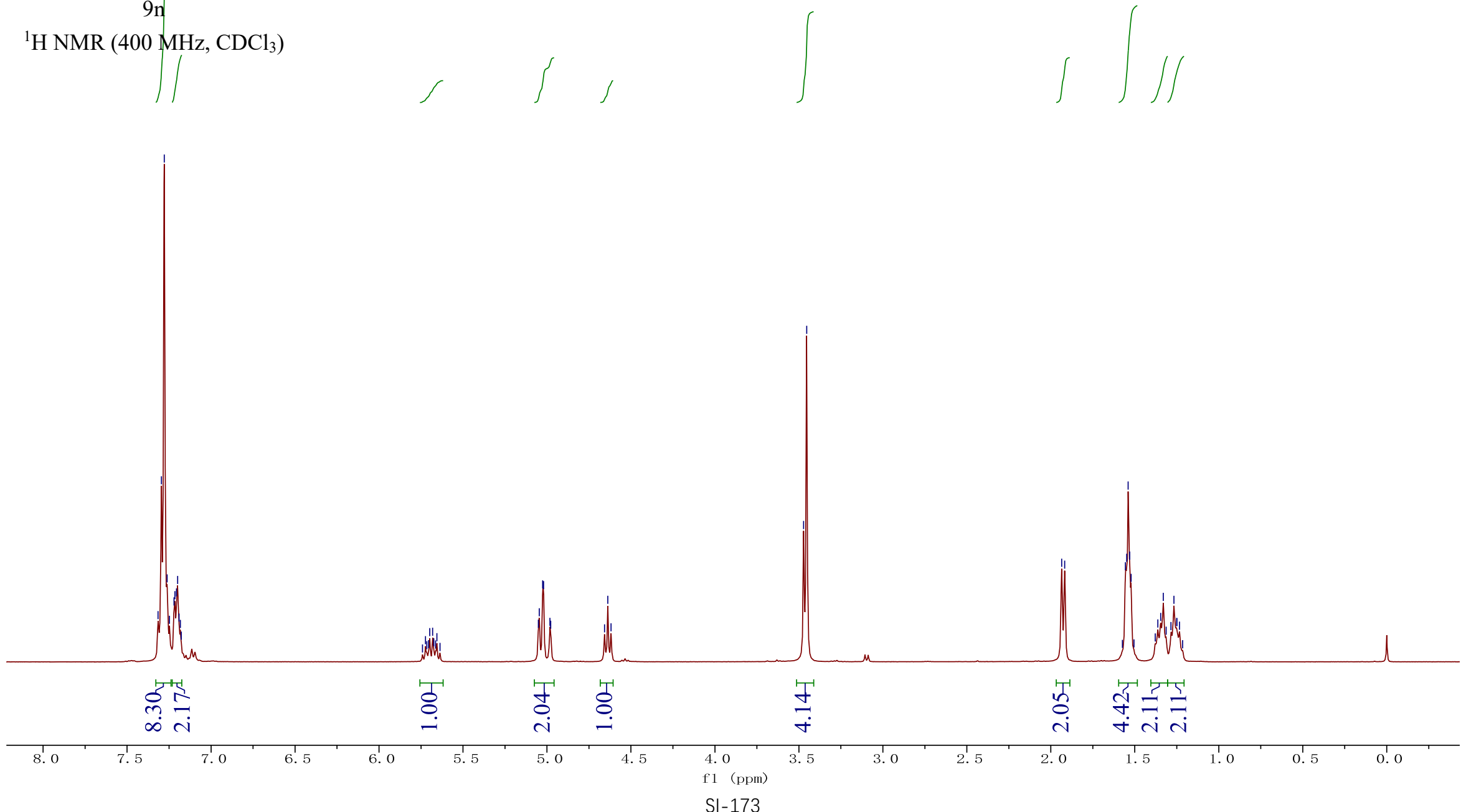


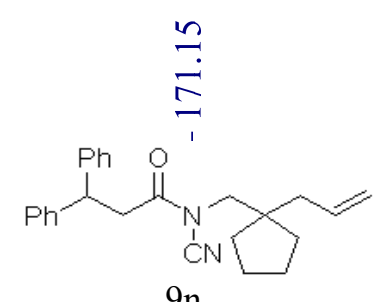

${ }^{13} \mathrm{C}$ NMR $\left(100 \mathrm{MHz}, \mathrm{CDCl}_{3}\right)$
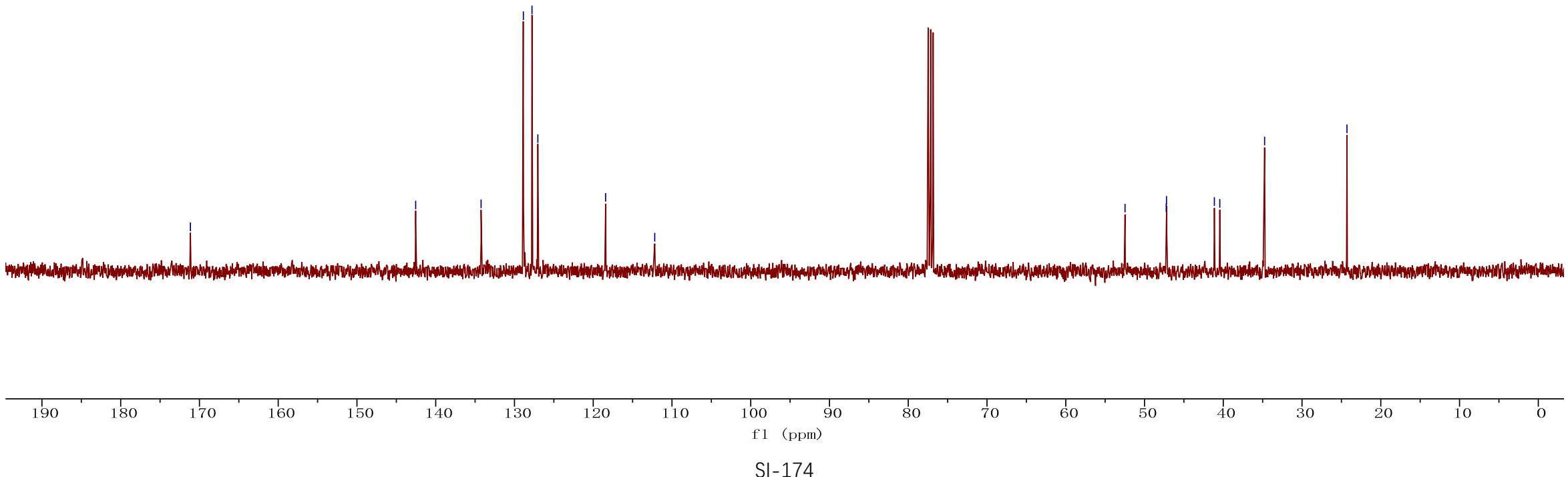


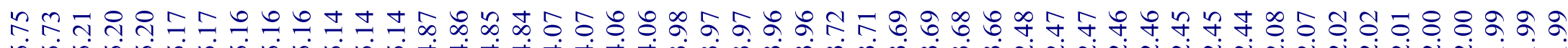

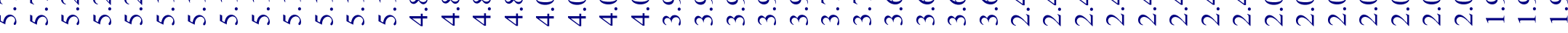

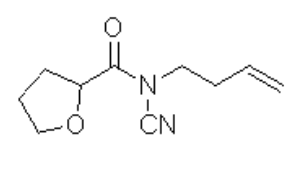

S11

${ }^{1} \mathrm{H}$ NMR $\left(500 \mathrm{MHz}, \mathrm{CDCl}_{3}\right)$
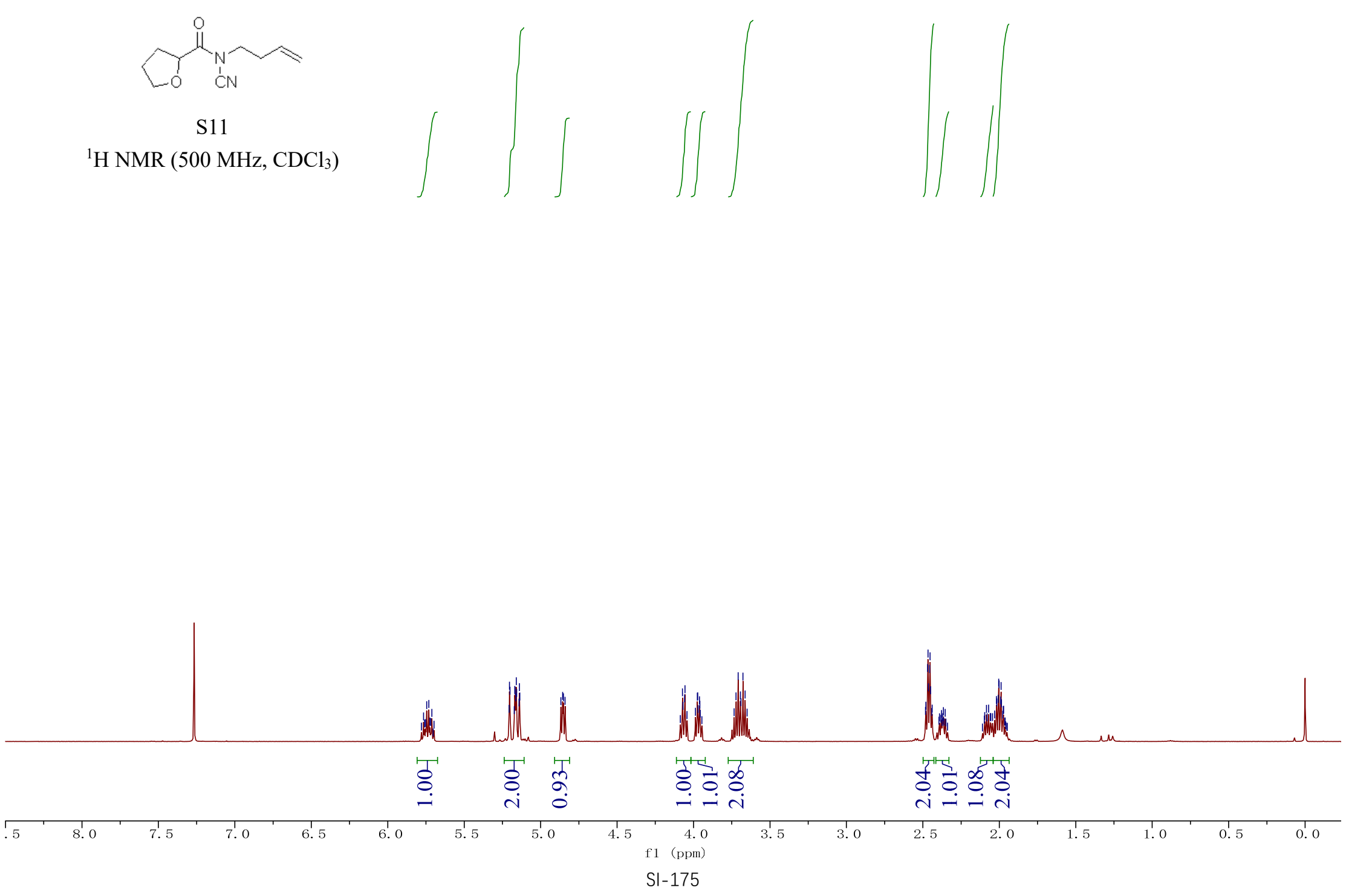


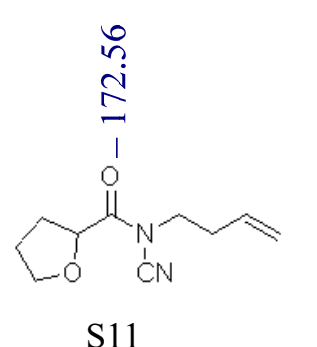

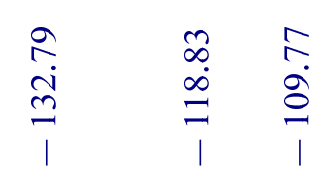

ㅇํㅇ

$\infty$

ज लंñ

S11

${ }^{13} \mathrm{C}$ NMR (125 MHz, $\mathrm{CDCl}_{3}$ )
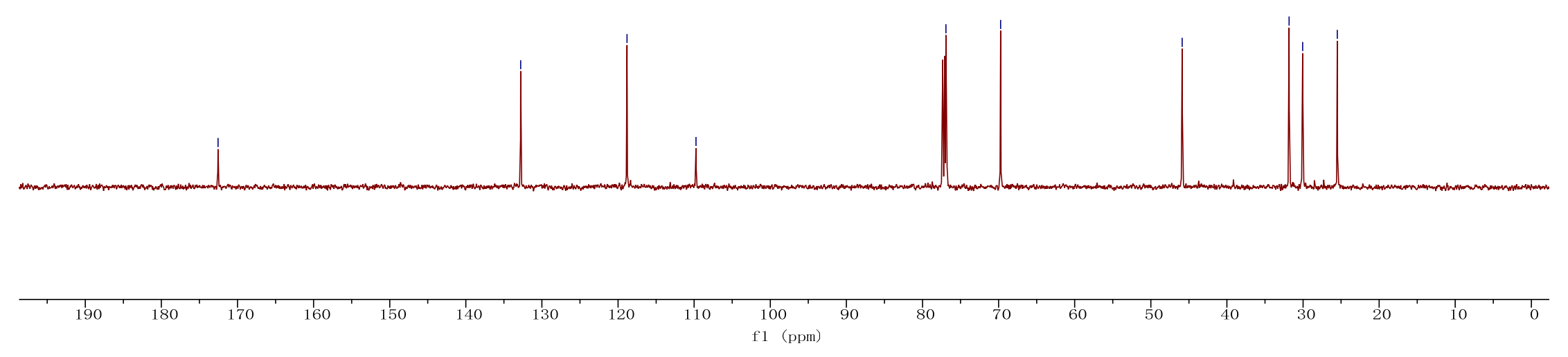

SI-176 


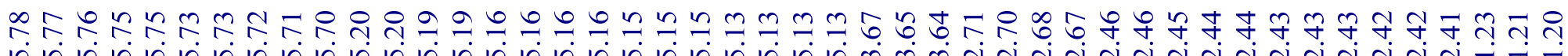

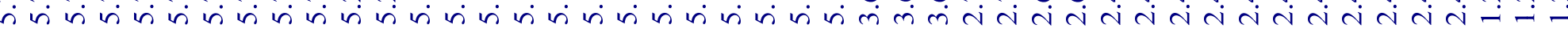
Me. $\int_{\substack{\mathrm{C} \\ \mathrm{C}}}^{O} \sim$

$\mathrm{S} 12$

${ }^{1} \mathrm{H}$ NMR (500 MHz, $\mathrm{CDCl}_{3}$ )
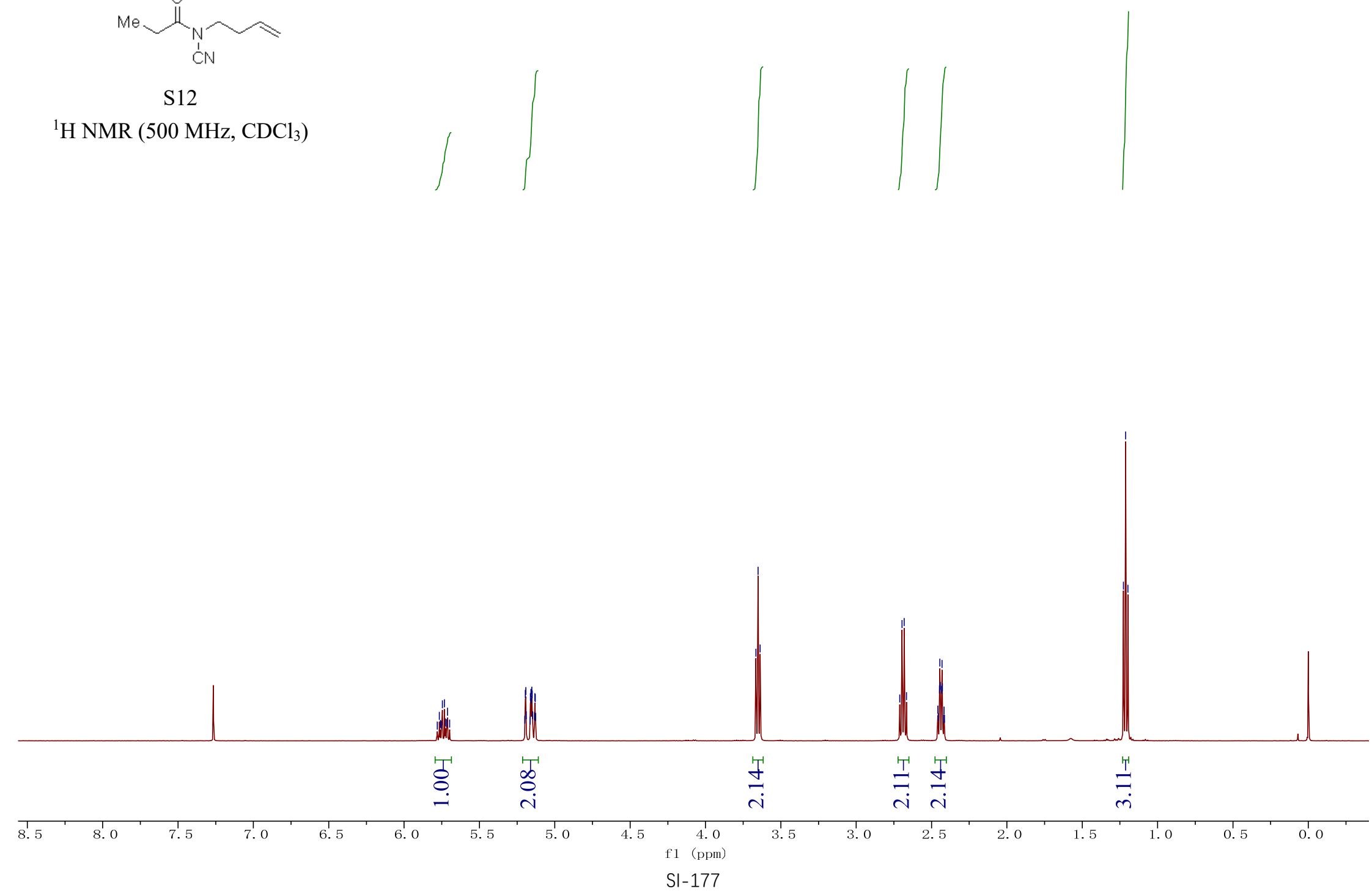


$$
{ }^{13} \mathrm{C} \mathrm{NMR}\left(125 \mathrm{MHz}, \mathrm{CDCl}_{3}\right)
$$

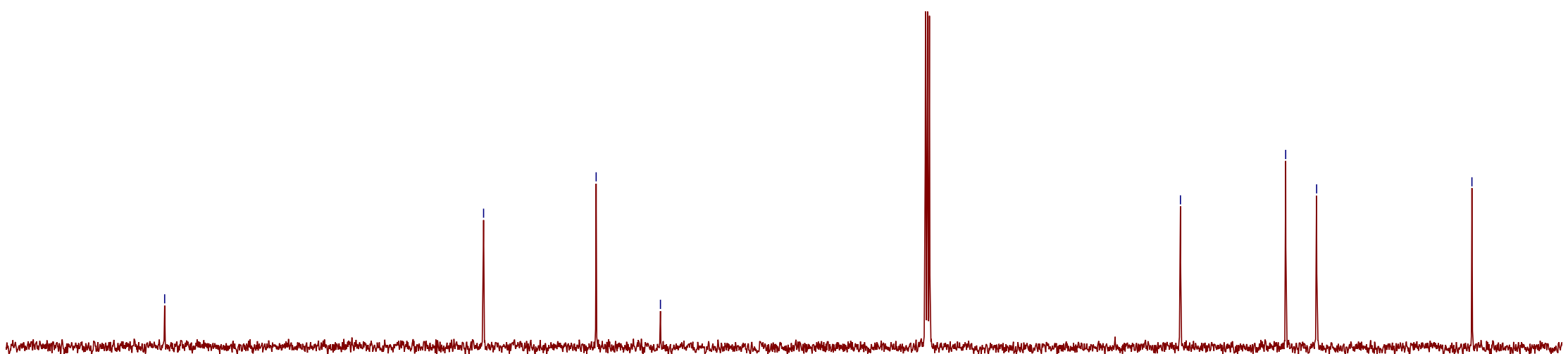

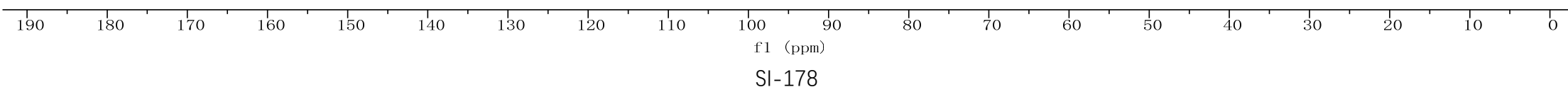




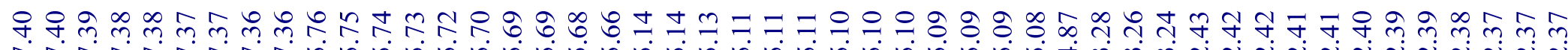
míniciciciciciar

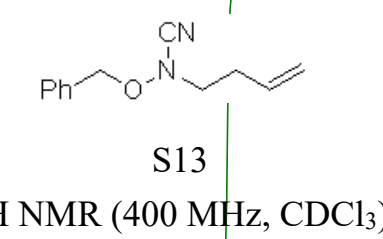

${ }^{1} \mathrm{H}$ NMR (400 MHz, $\mathrm{CDCl}_{3}$ )
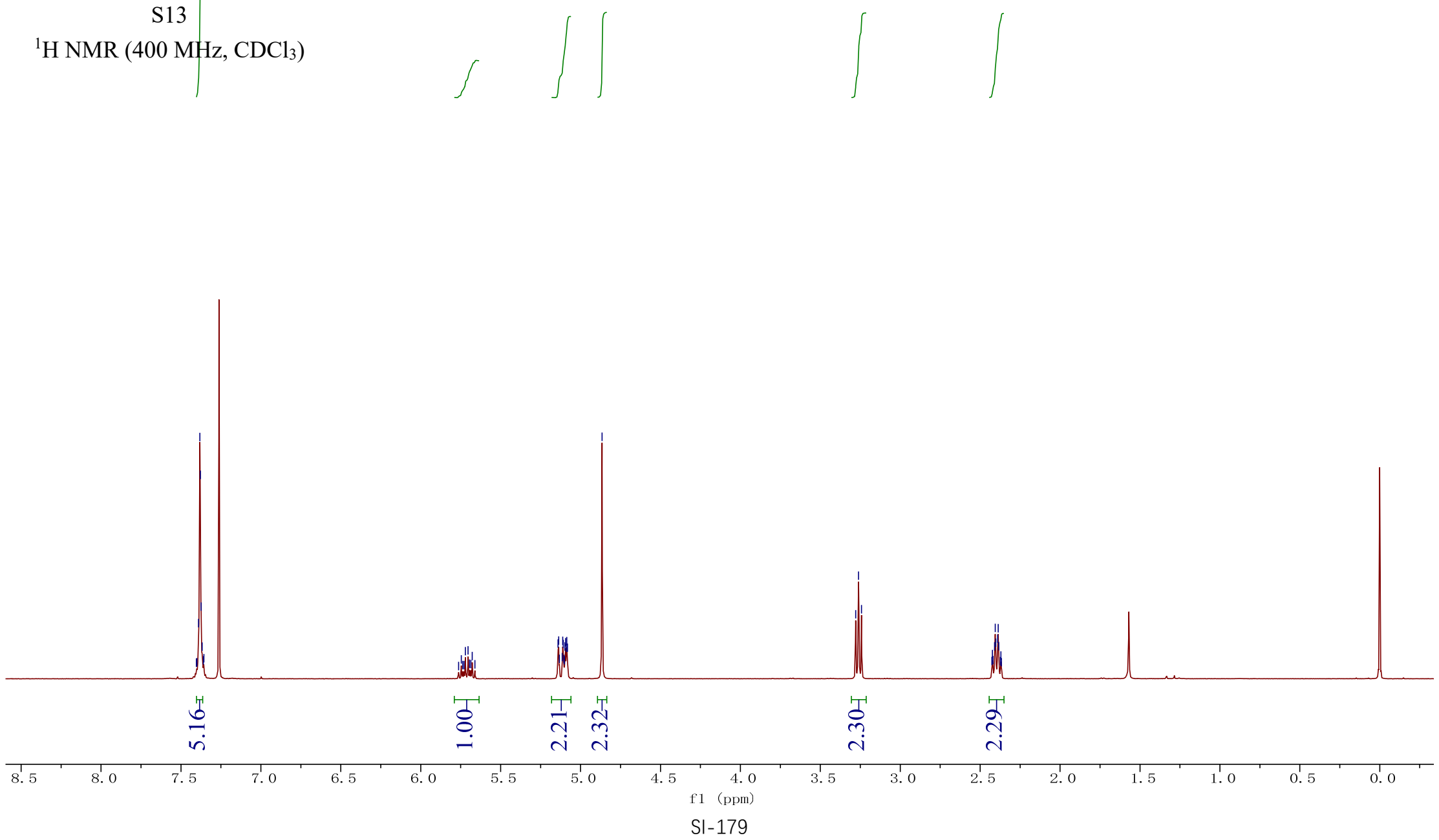


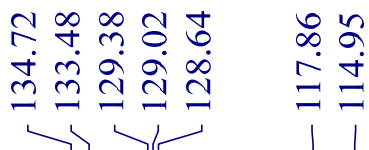

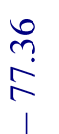

$n$
$n$
$n$
$n$
1

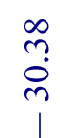

$$
{ }_{\mathrm{S} 13} \mathrm{Nh}^{\mathrm{N}}
$$

${ }^{13} \mathrm{C}$ NMR (125 MHz, $\left.\mathrm{CDCl}_{3}\right)$

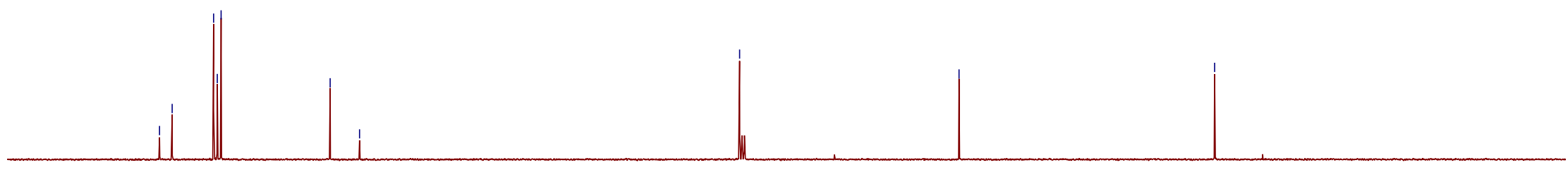

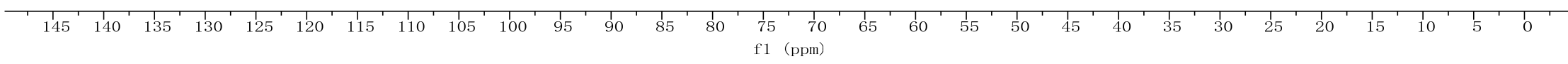
SI-180 


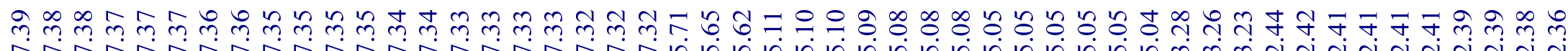

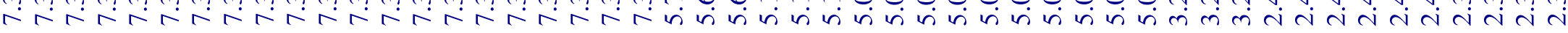

$$
{ }_{\mathrm{S} 14}^{\mathrm{Ph}}
$$

${ }^{1} \mathrm{H}$ NMR $\left(300 \mathrm{MHz}, \mathrm{CDCl}_{3}\right)$
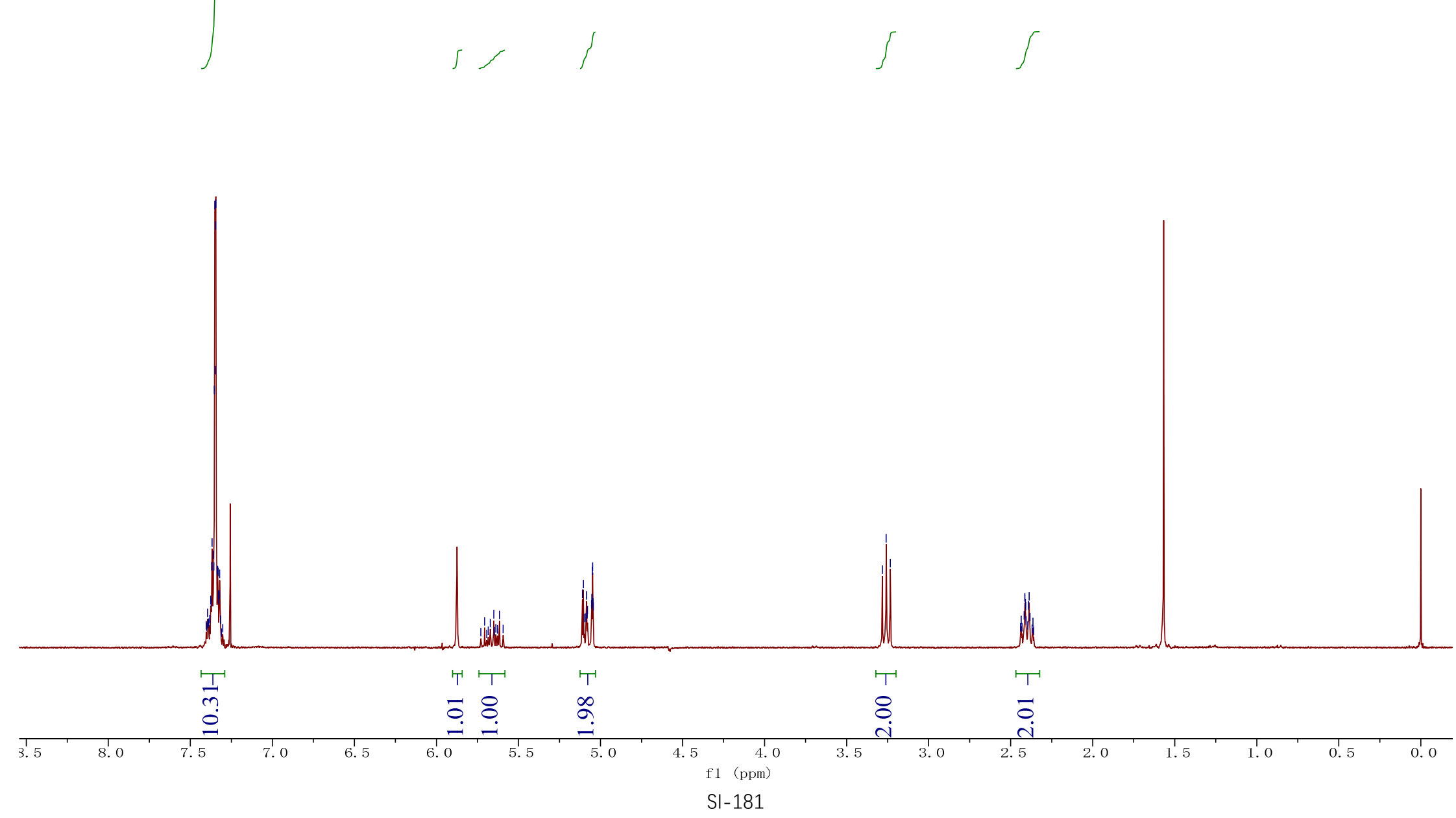


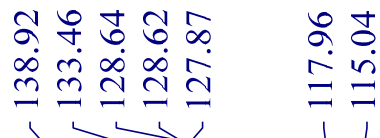

$\overrightarrow{\mathrm{Ph}} \mathrm{CN}$

$\mathrm{Ph}^{\mathrm{N}} \mathrm{O}$

S14

${ }^{13} \mathrm{C}$ NMR (125 MHz, $\left.\mathrm{CDCl}_{3}\right)$

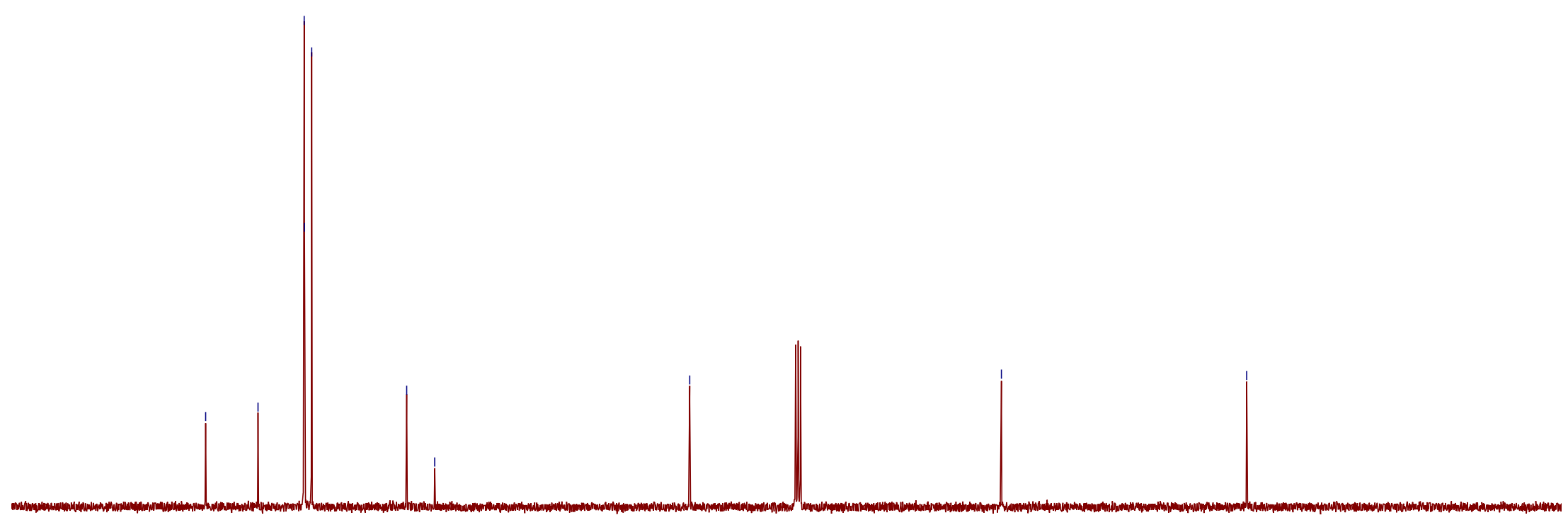

150

140

130

120

110

90

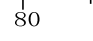

70

50

SI-182 


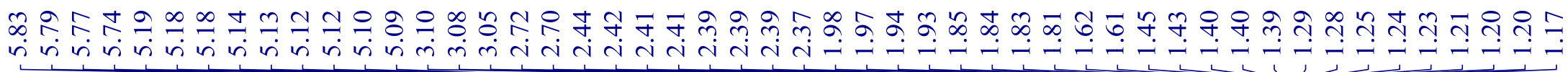

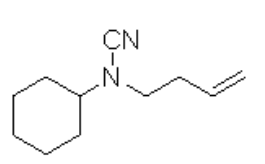

S15

${ }^{1} \mathrm{H} \mathrm{NMR}\left(300 \mathrm{MHz}, \mathrm{CDCl}_{3}\right)$
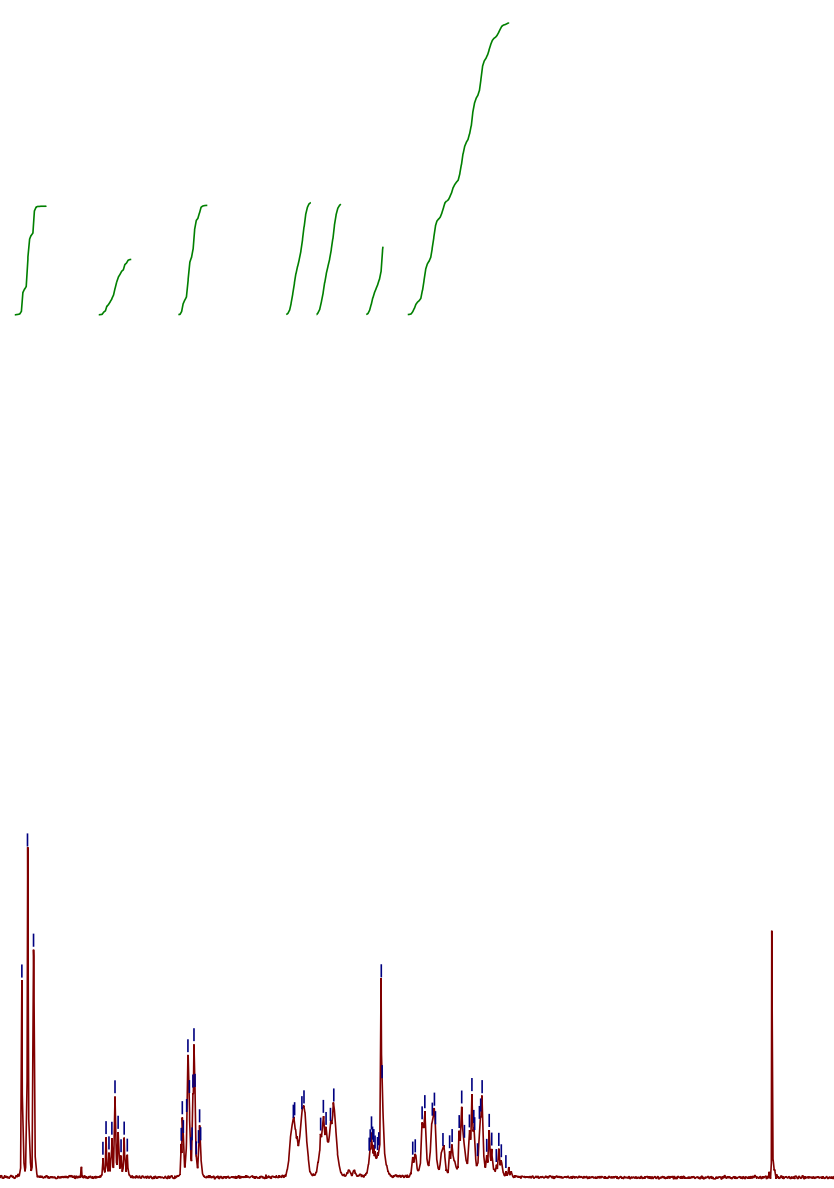

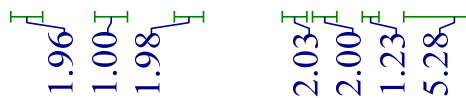

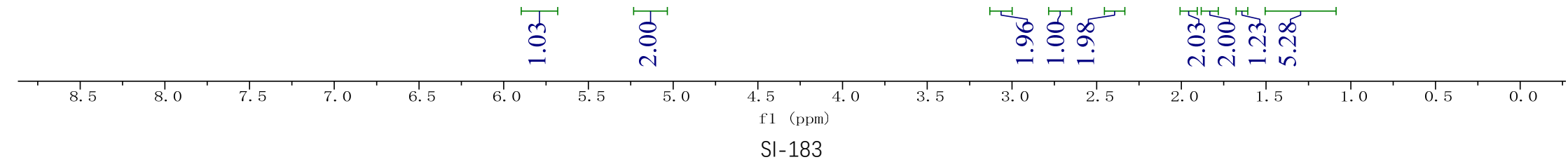




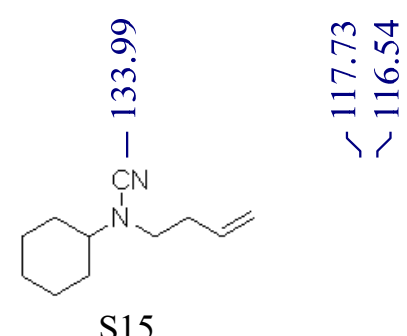

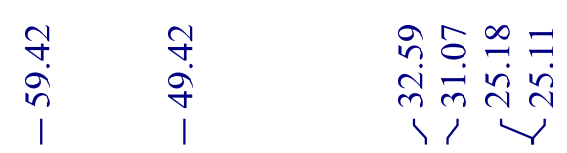

${ }^{13} \mathrm{C} \mathrm{NMR}\left(125 \mathrm{MHz}, \mathrm{CDCl}_{3}\right)$

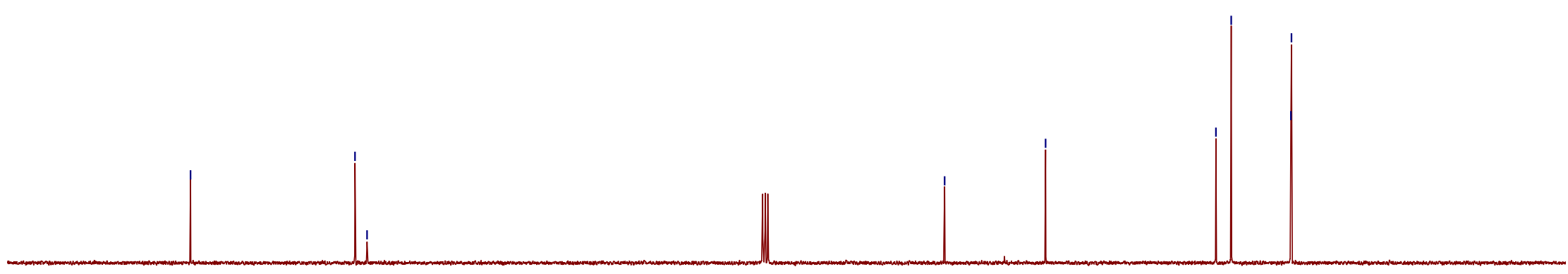

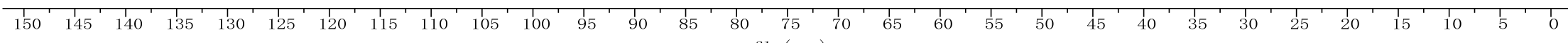
f1 ( $\mathrm{ppm})$ 


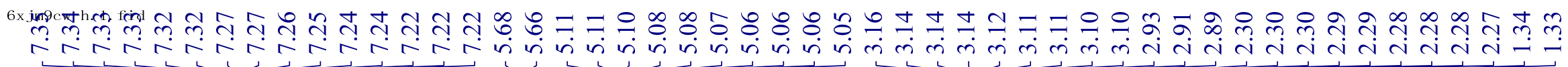
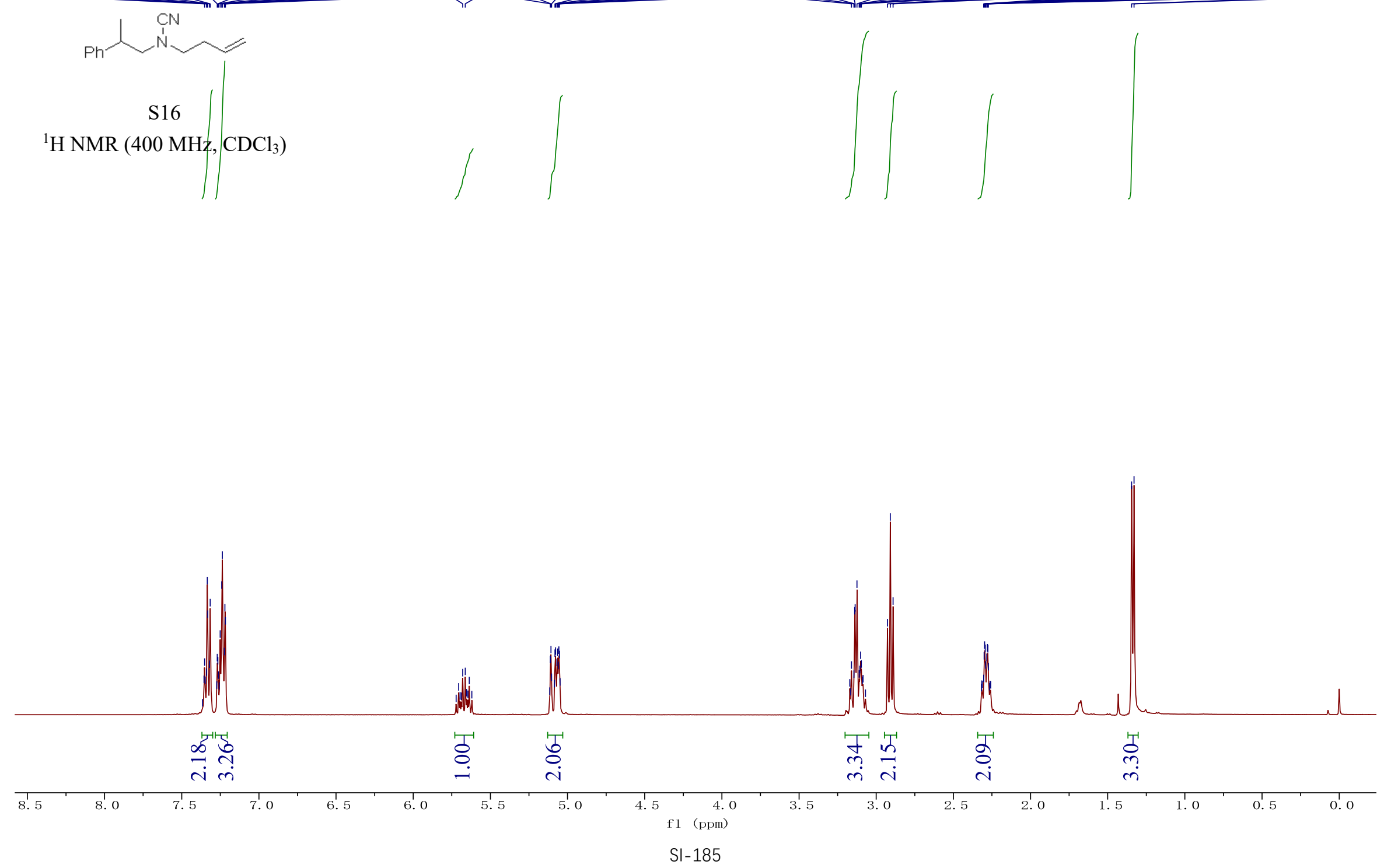


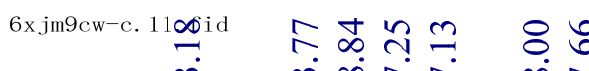

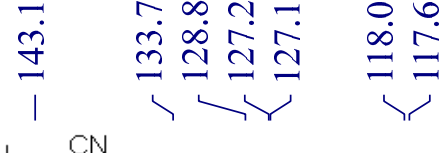

$$
\begin{aligned}
& \text { 策 }
\end{aligned}
$$

${ }^{13} \mathrm{C}$ NMR $\left(125 \mathrm{MHz}, \mathrm{CDCl}_{3}\right)$
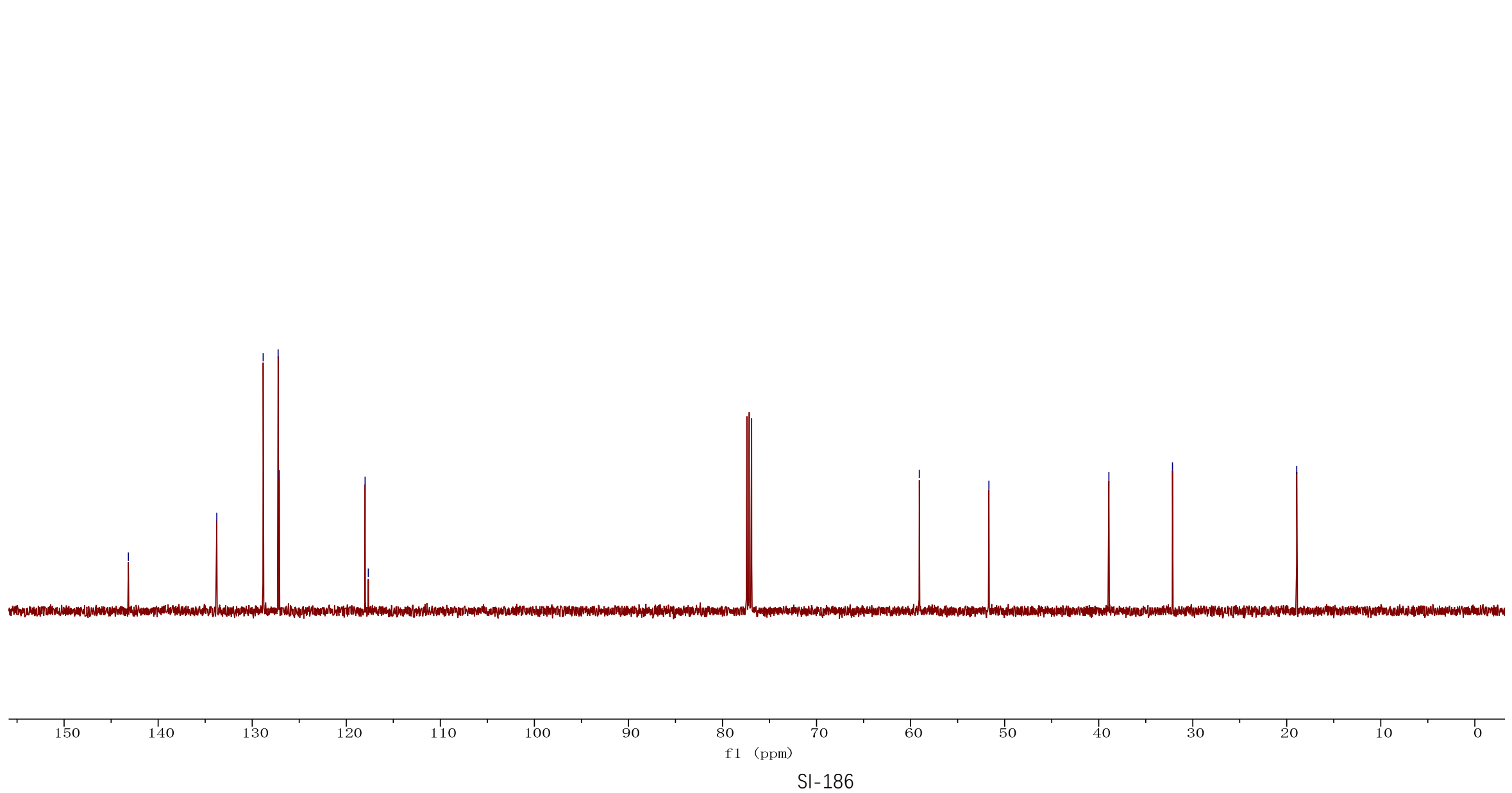


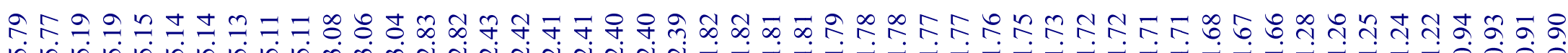

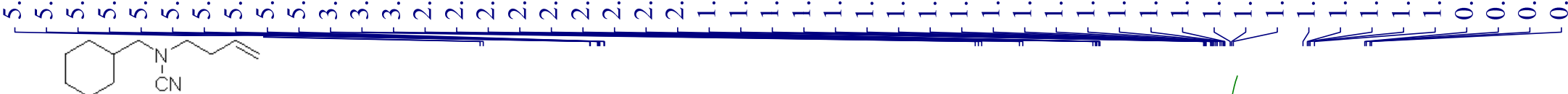

S17

${ }^{1} \mathrm{H}$ NMR (400 MHz, $\left.\mathrm{CDCl}_{3}\right)$
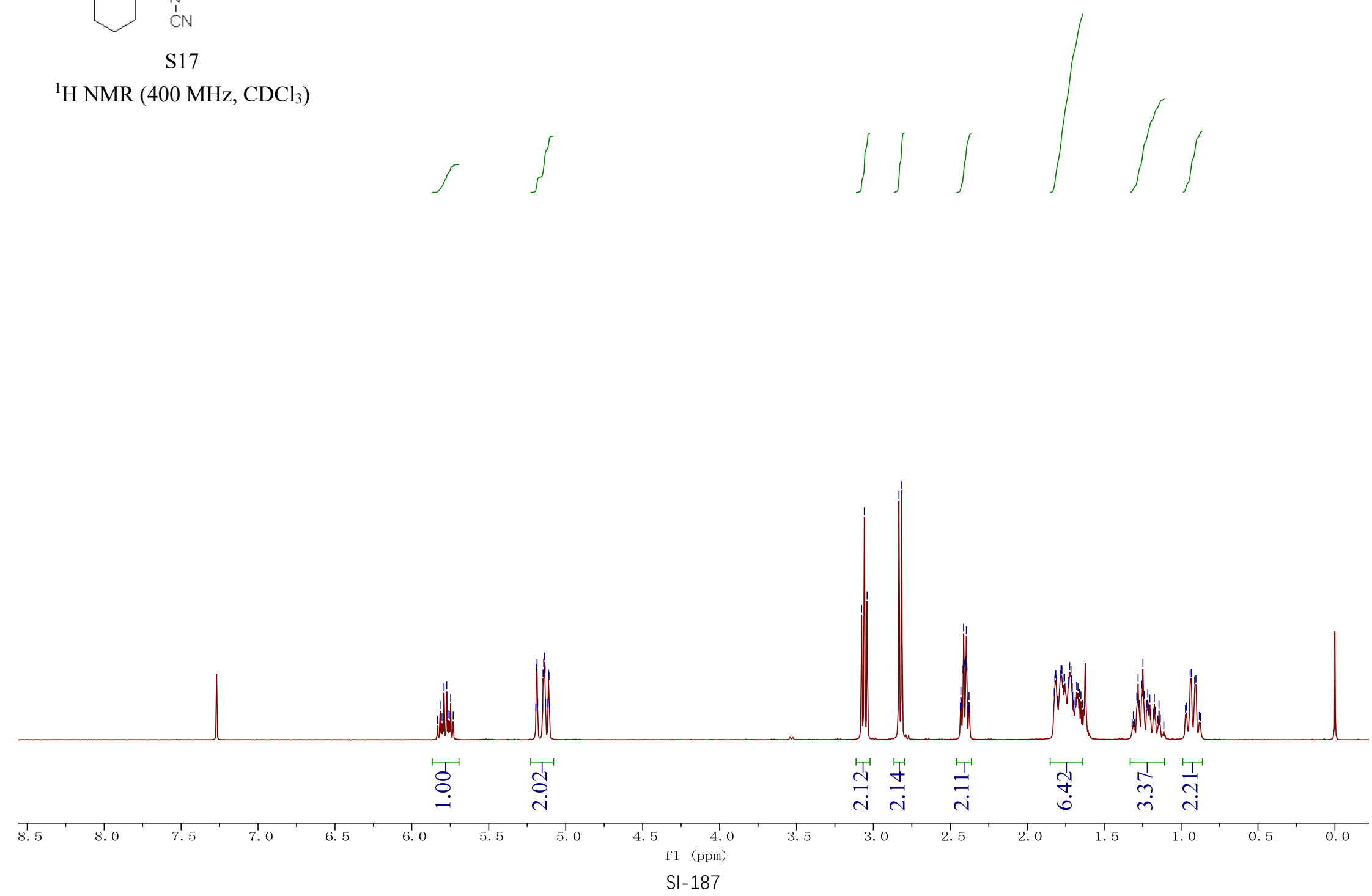

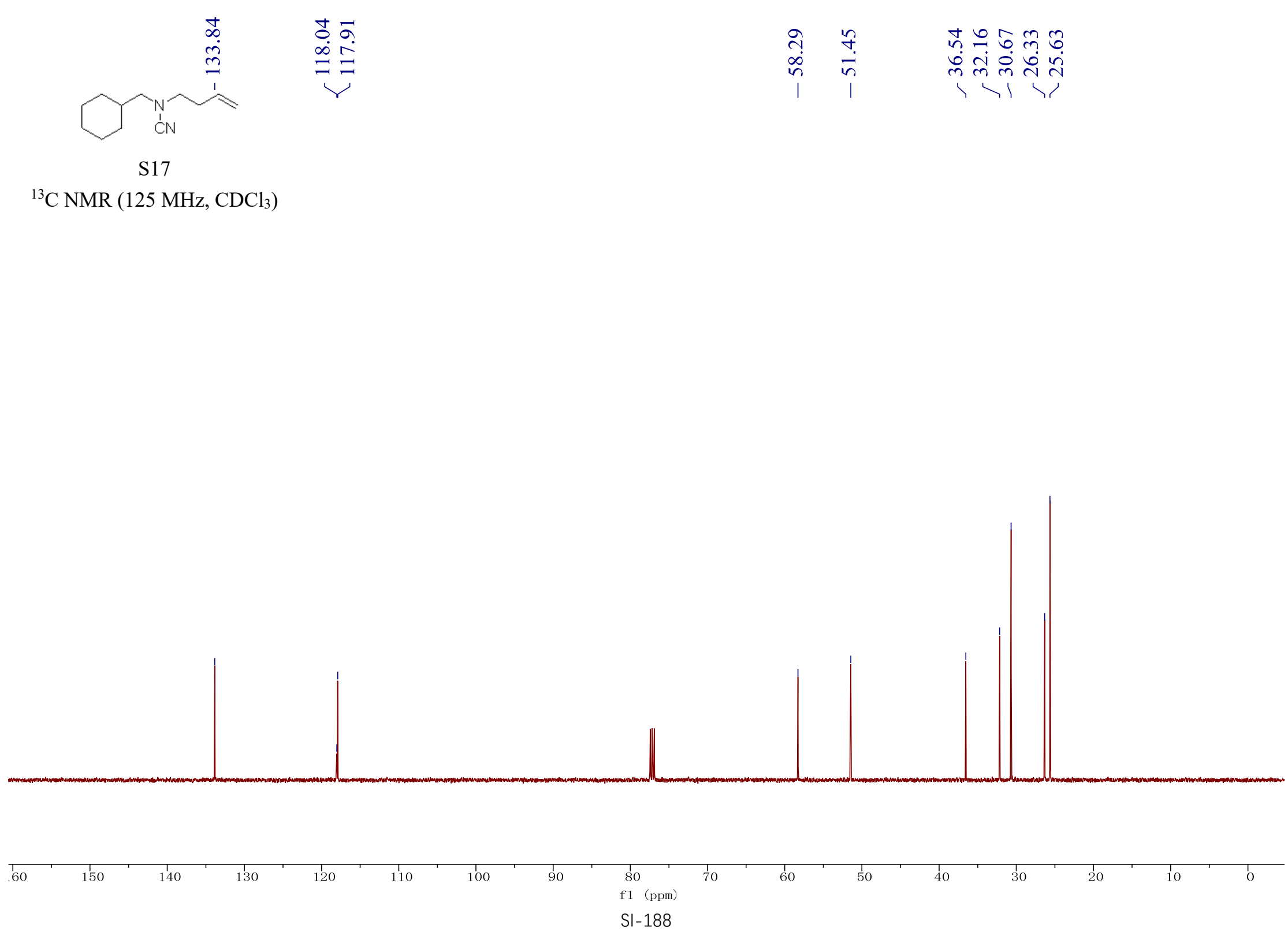


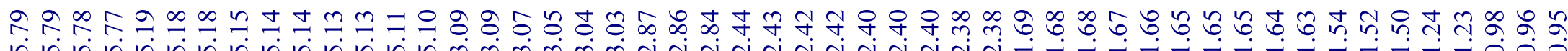

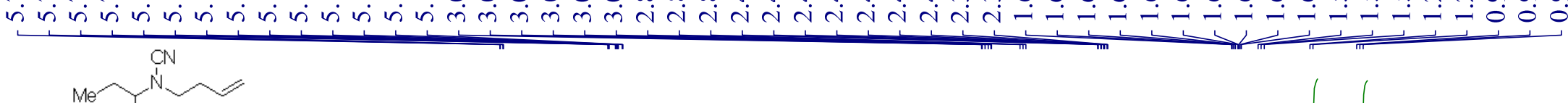

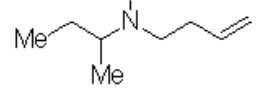

S18

${ }^{1} \mathrm{H}$ NMR $\left(400 \mathrm{MHz}, \mathrm{CDCl}_{3}\right)$
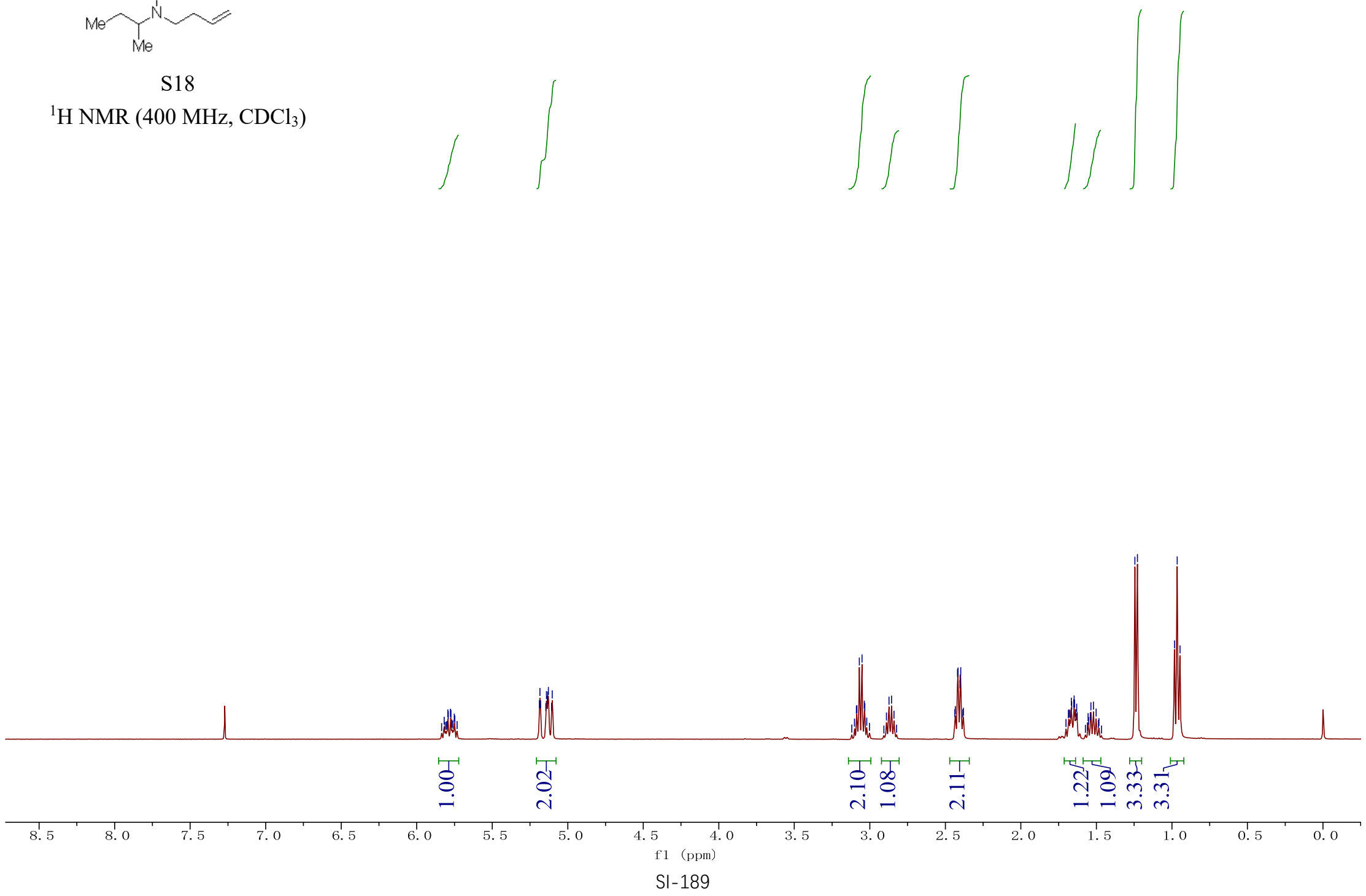

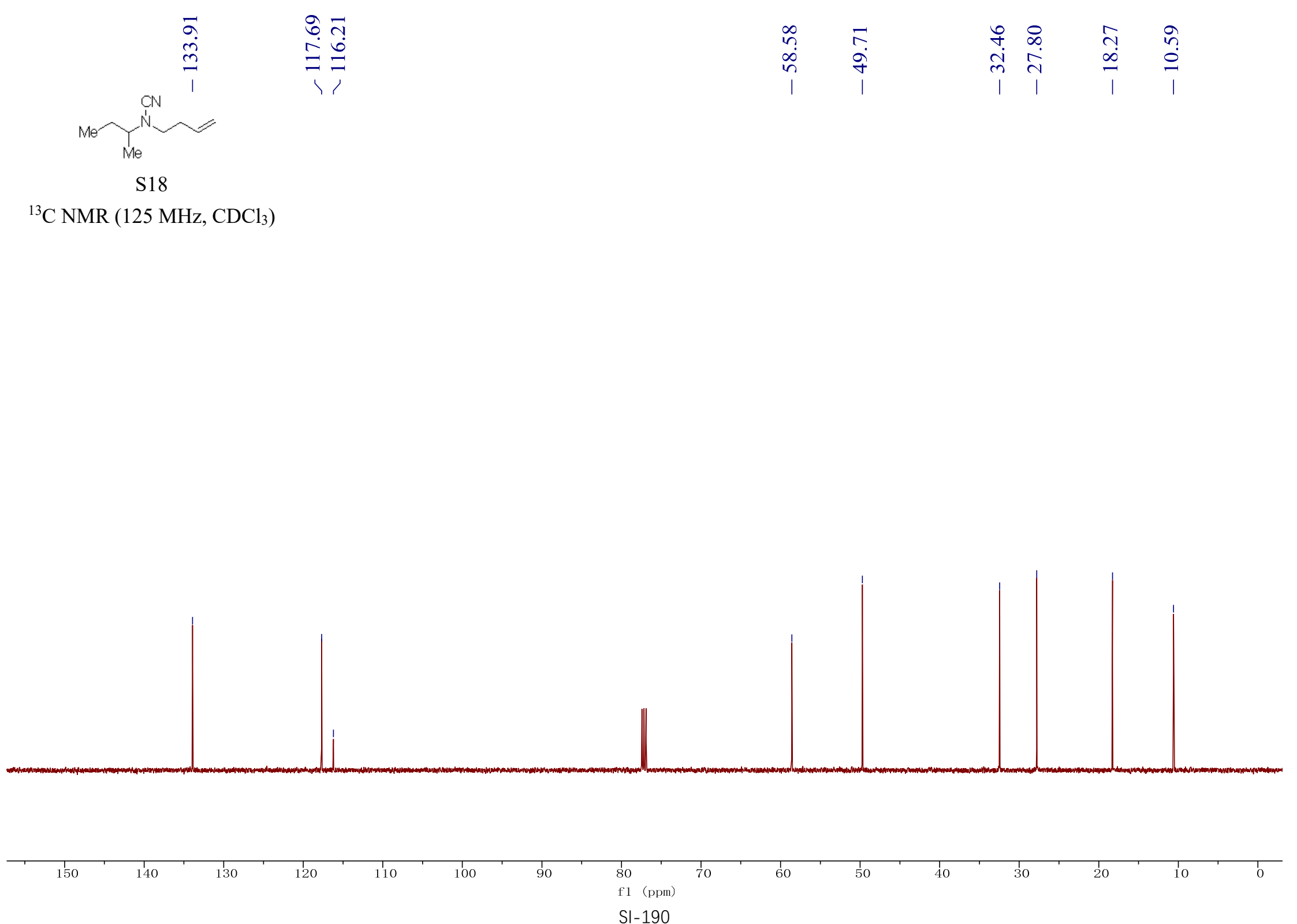


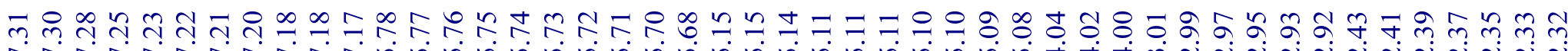
-
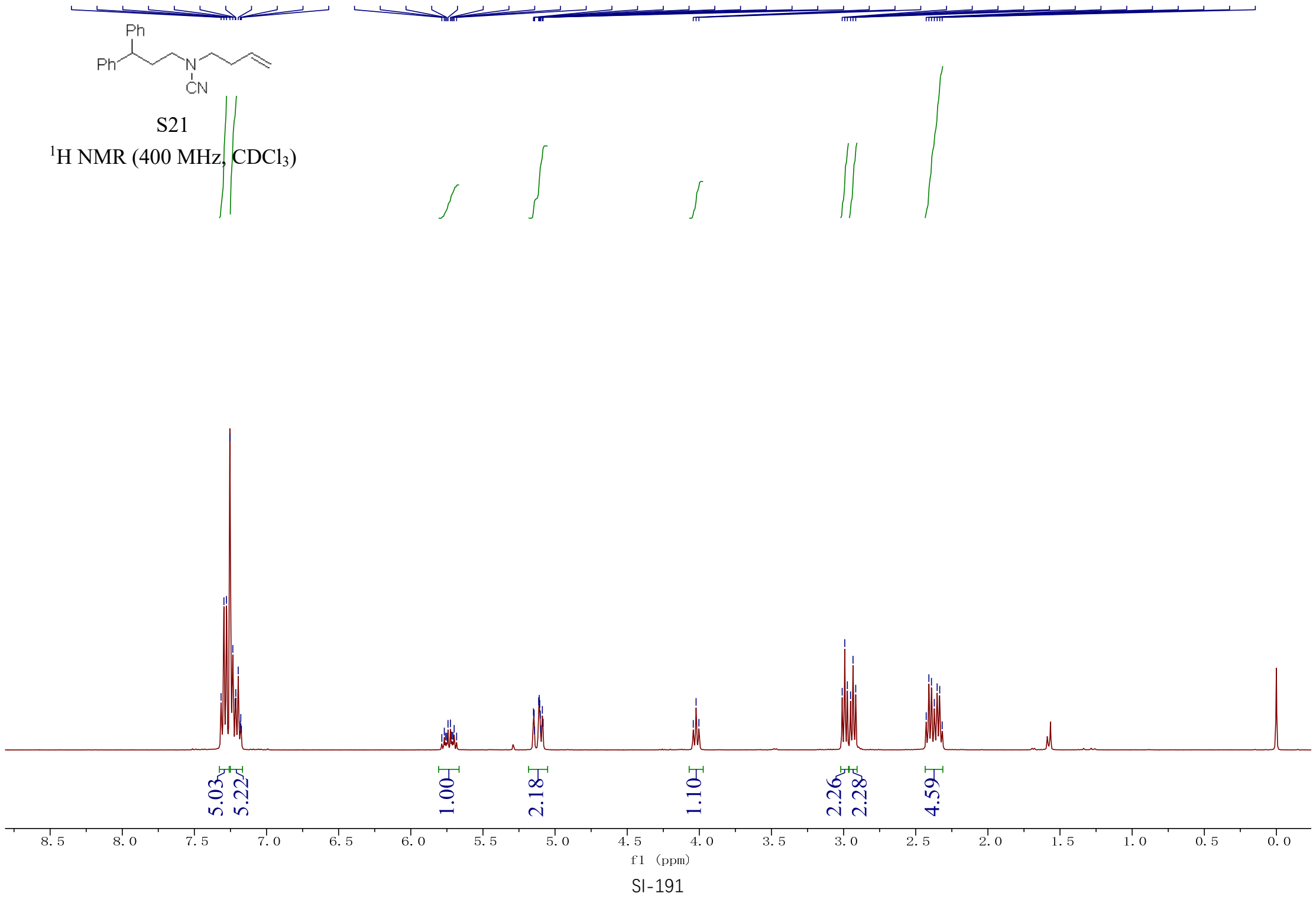


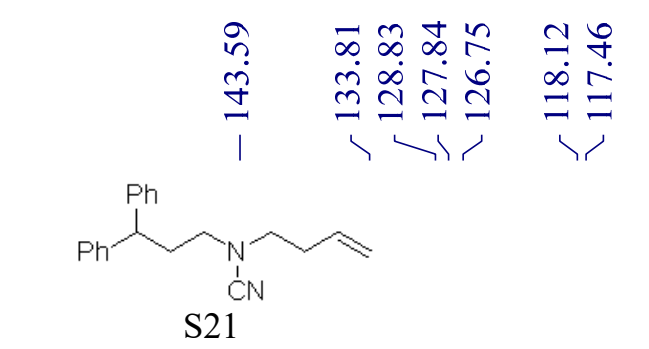

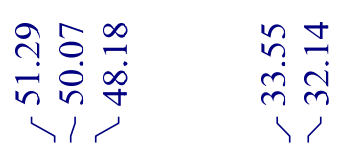

${ }^{13} \mathrm{C}$ NMR (125 MHz, $\mathrm{CDCl}_{3}$ )

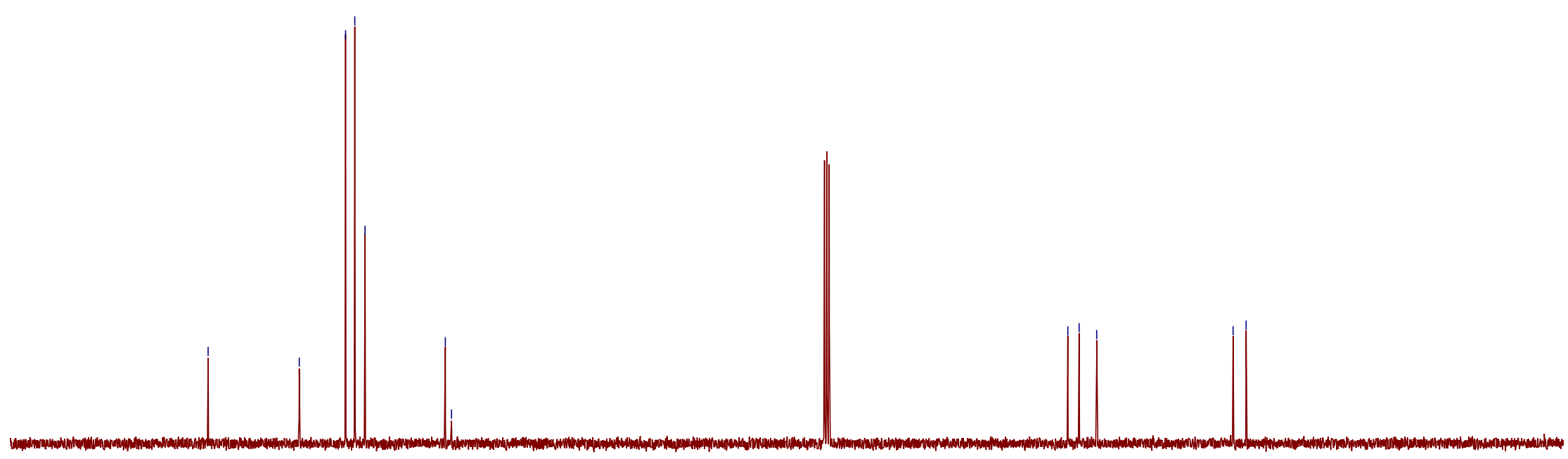

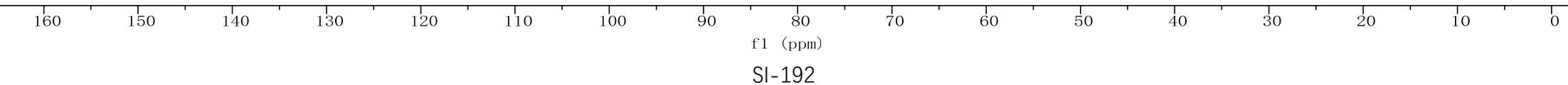




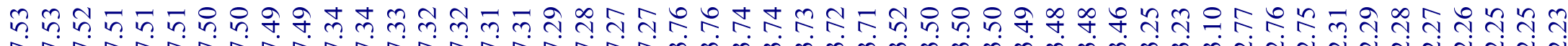

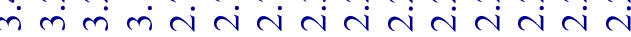
11
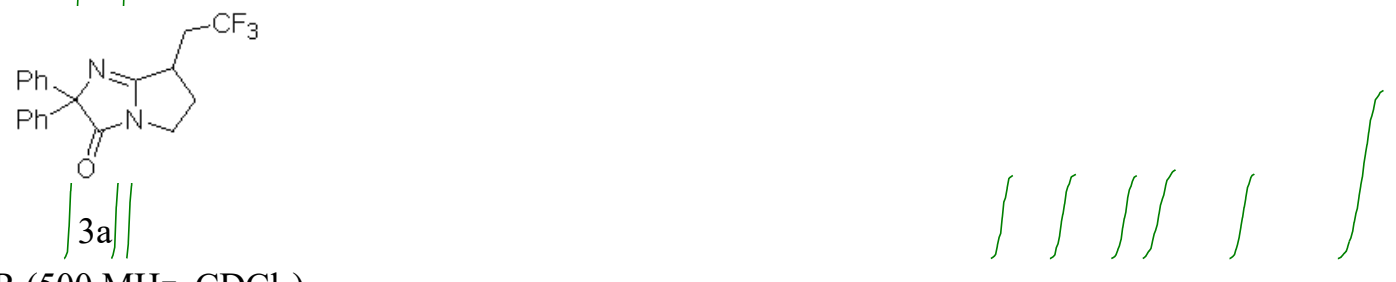

${ }^{1} \mathrm{H}$ NMR $\left(500 \mathrm{MHz}, \mathrm{CDCl}_{3}\right)$

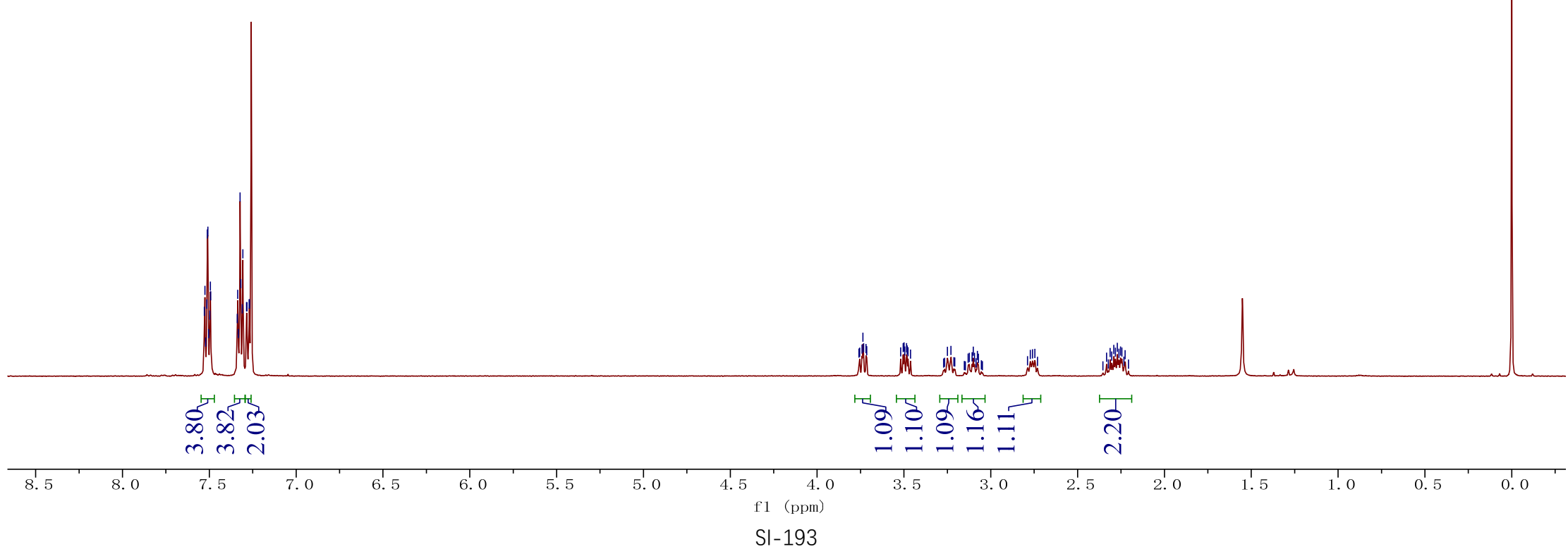




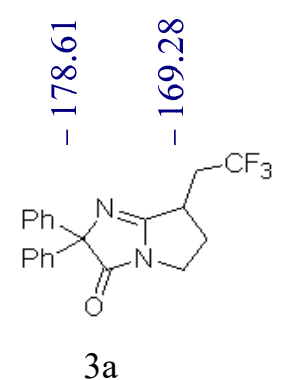

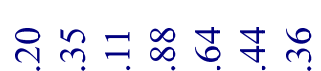

लेंलिलमल

${ }^{13} \mathrm{C}$ NMR (125 MHz, $\left.\mathrm{CDCl}_{3}\right)$

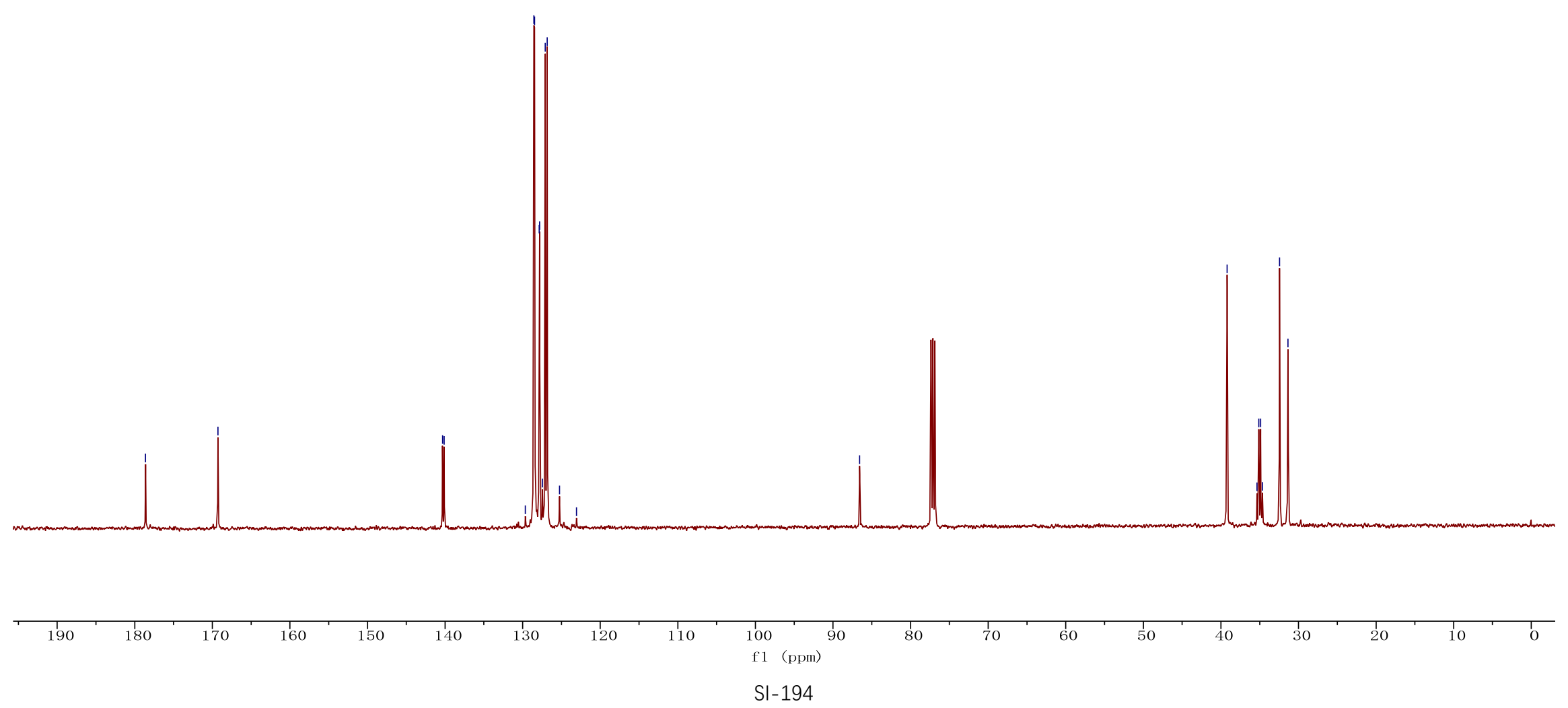




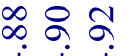

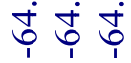

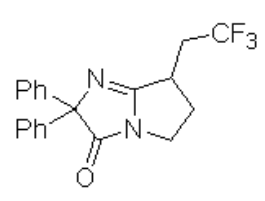

$3 a$

${ }^{19} \mathrm{~F}$ NMR $\left(470 \mathrm{MHz}, \mathrm{CDCl}_{3}\right)$

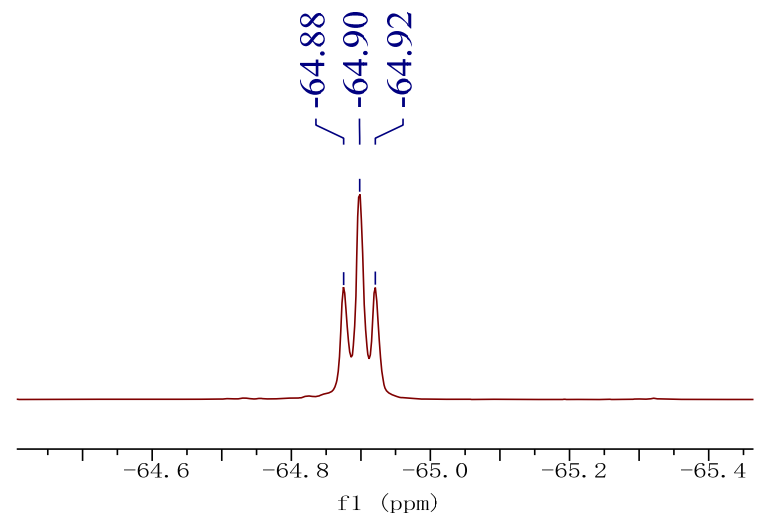

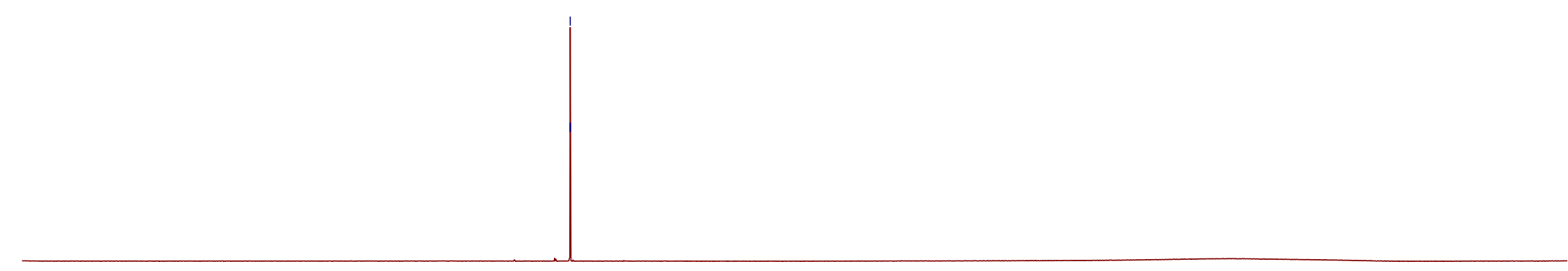

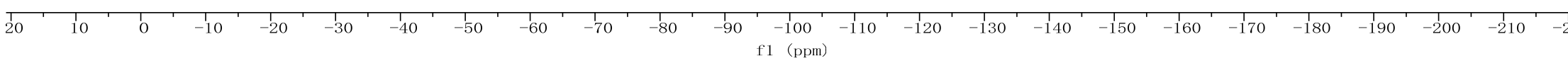
SI-195 


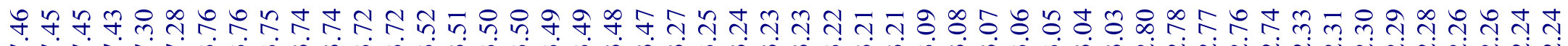

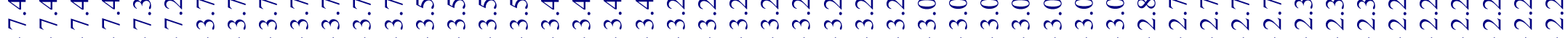

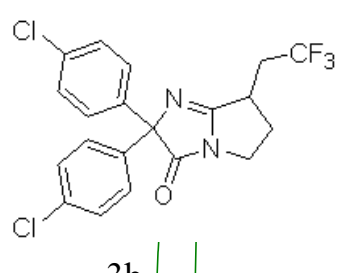

$3 \mathrm{~b}$

${ }^{1} \mathrm{H} \mathrm{NMR}\left(500 \mathrm{MHz}, \mathrm{CDCl}_{3}\right)$
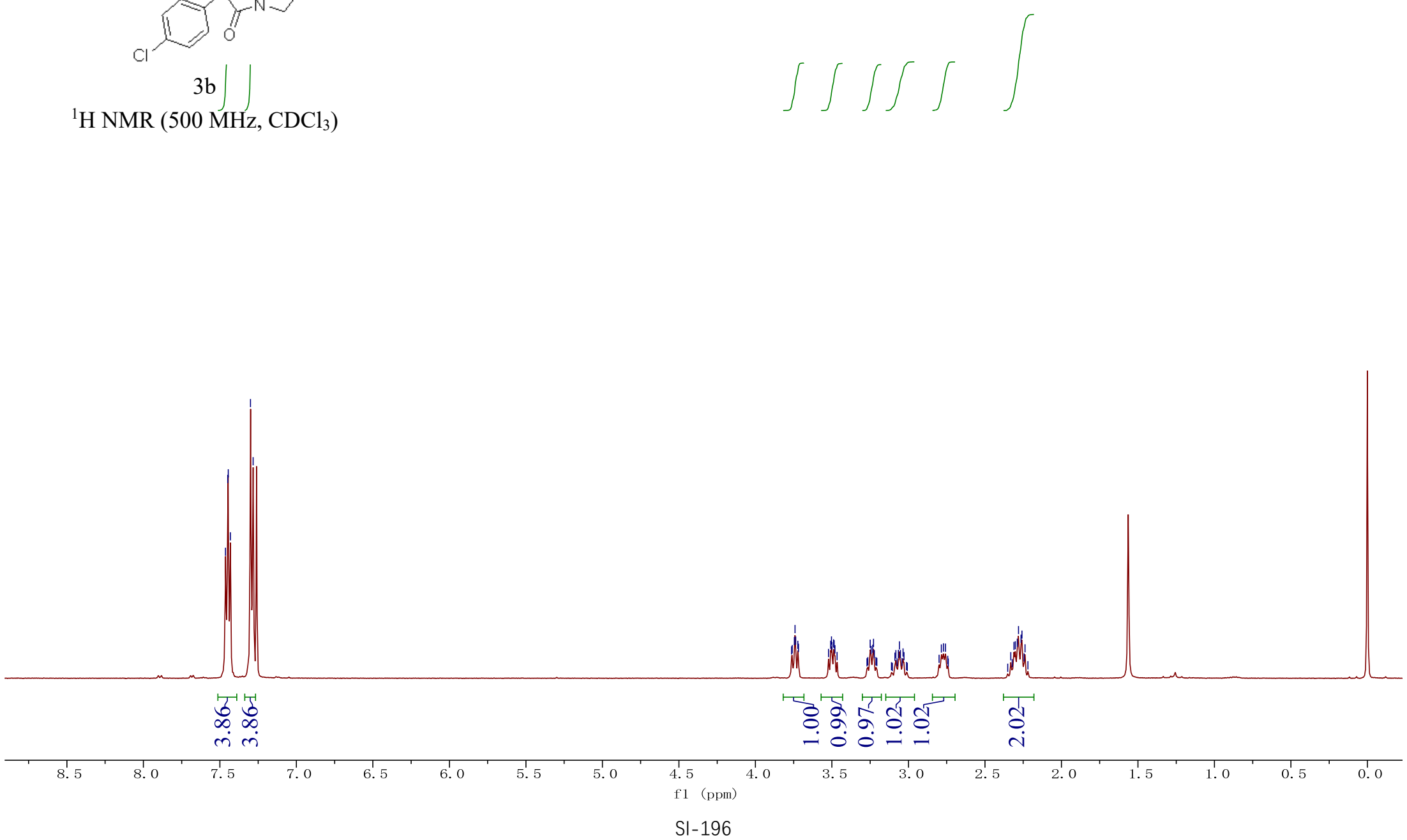


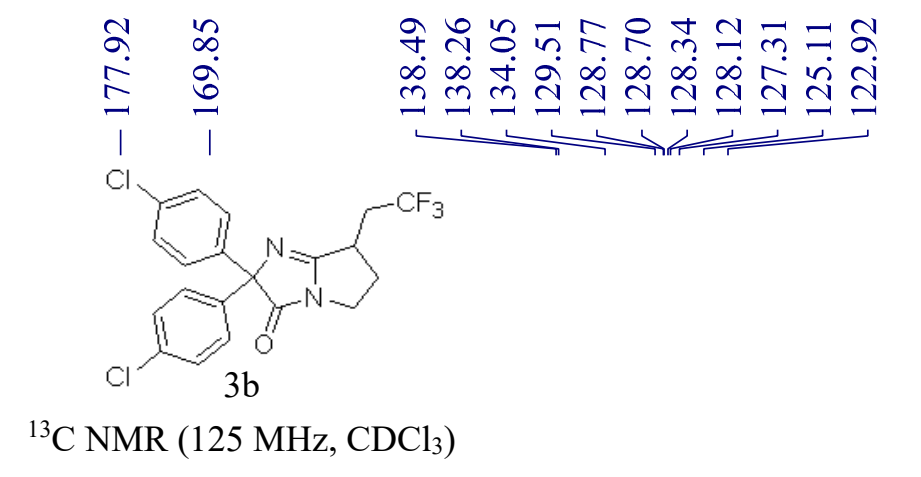

\begin{tabular}{c}
\multirow{3}{*}{} \\
$\infty$ \\
1 \\
1
\end{tabular}

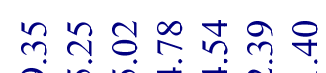

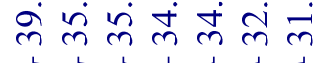

(1)

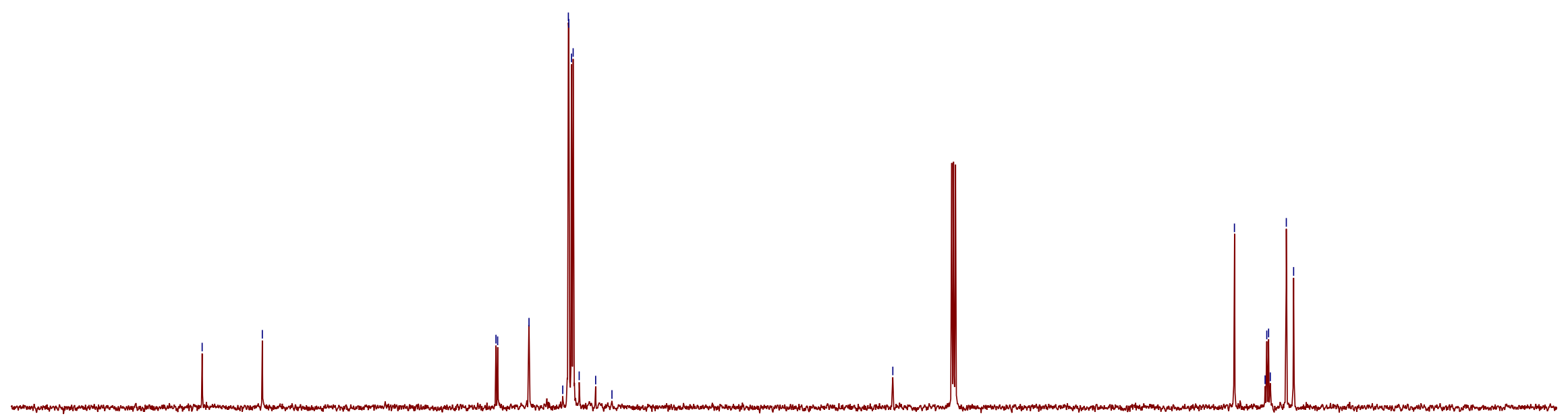


$\infty \infty$

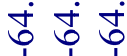

$\checkmark$

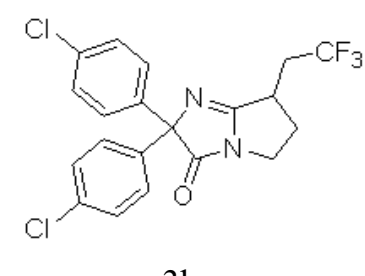

$3 b$

${ }^{19} \mathrm{~F}$ NMR $\left(470 \mathrm{MHz}, \mathrm{CDCl}_{3}\right)$

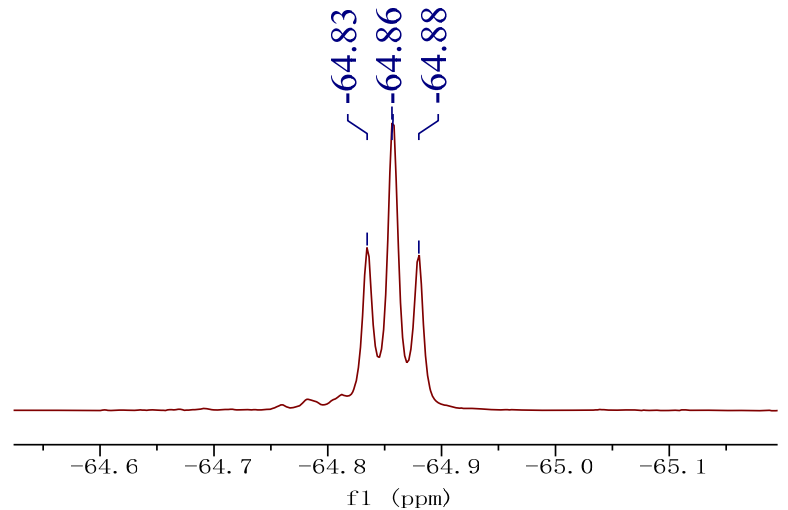

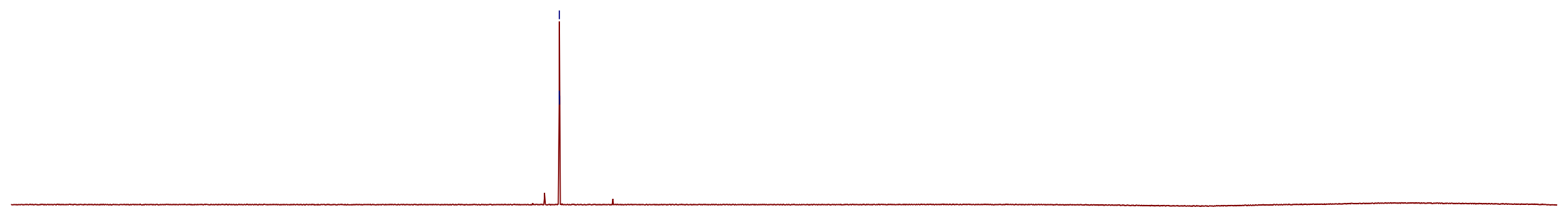

\begin{tabular}{lllllllllllllllllllllllllllllll}
1 & 10 & 10 & 0 & -10 & -20 & -30 & -40 & -50 & -60 & -70 & -80 & -90 & -100 & -110 & -120 & -130 & -140 & -150 & -160 & -170 & -180 & -190 & -200 & -210 & -2 \\
\hline
\end{tabular} SI-198 


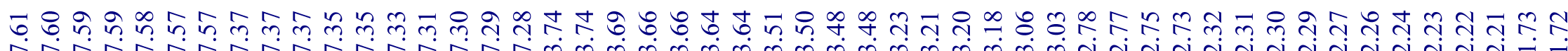
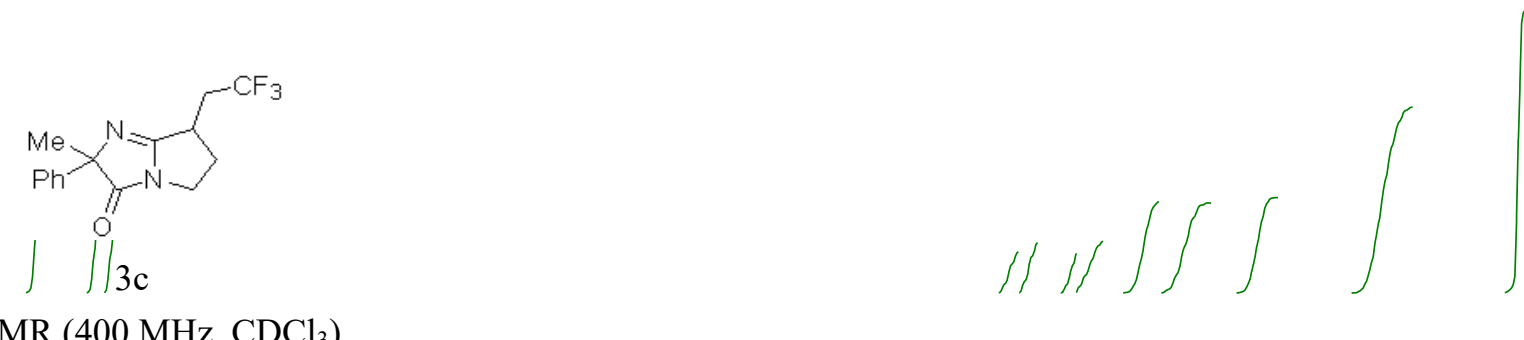

${ }^{1} \mathrm{H}$ NMR $\left(400 \mathrm{MHz}, \mathrm{CDCl}_{3}\right)$

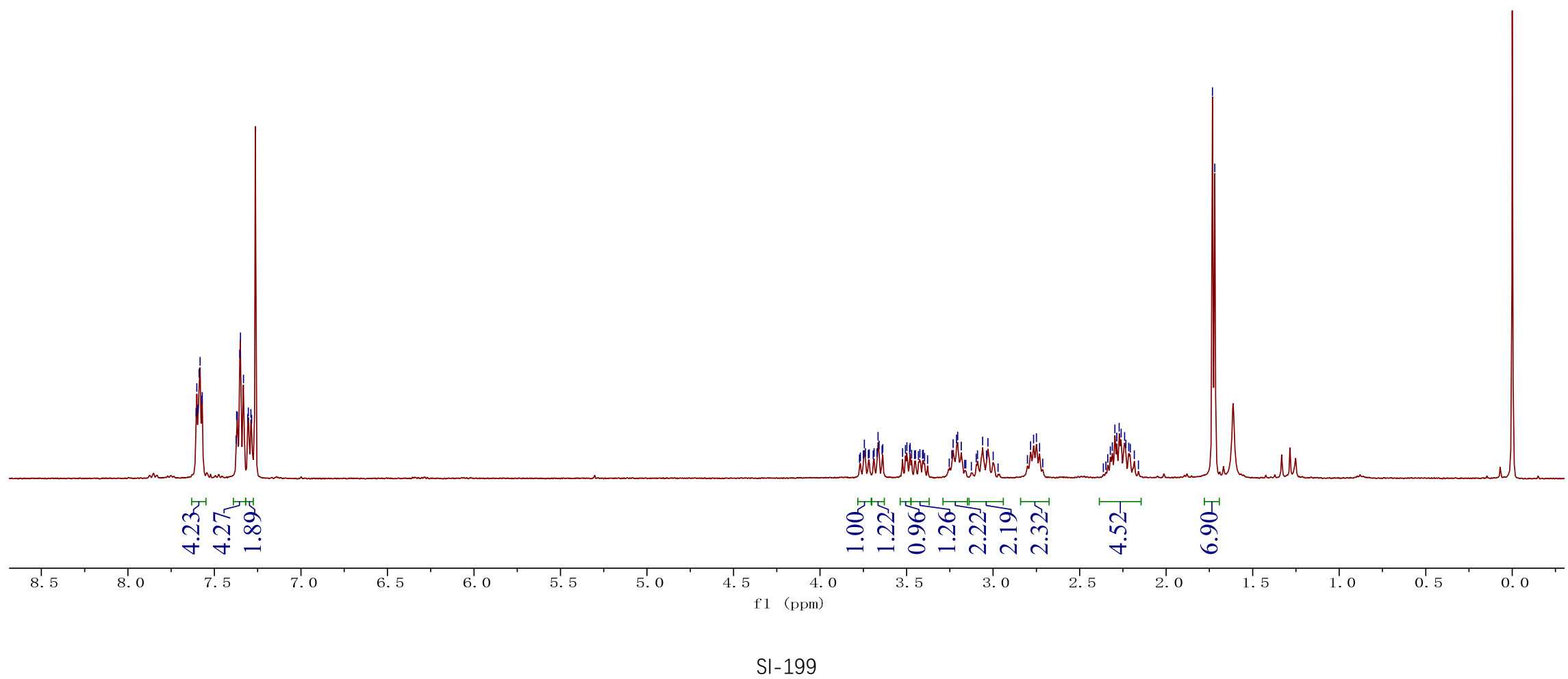




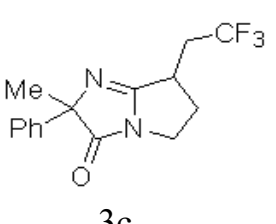

${ }^{13} \mathrm{C}$ NMR $\left(125 \mathrm{MHz}, \mathrm{CDCl}_{3}\right)$

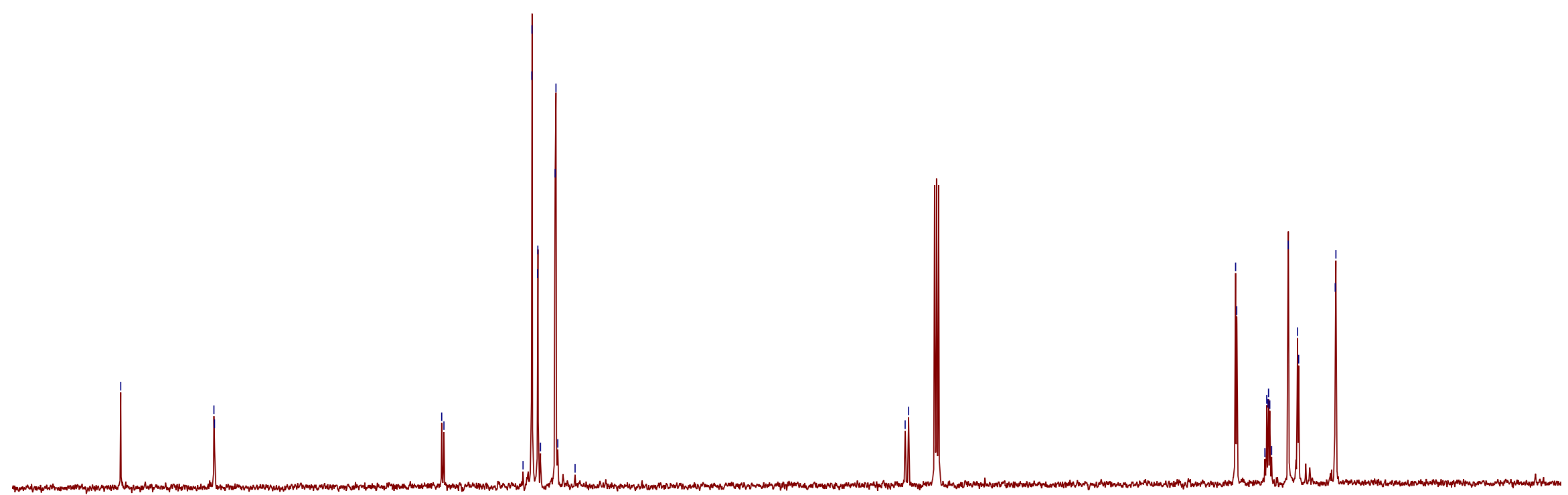

$190 \quad 180$

160

150

140

130

120 

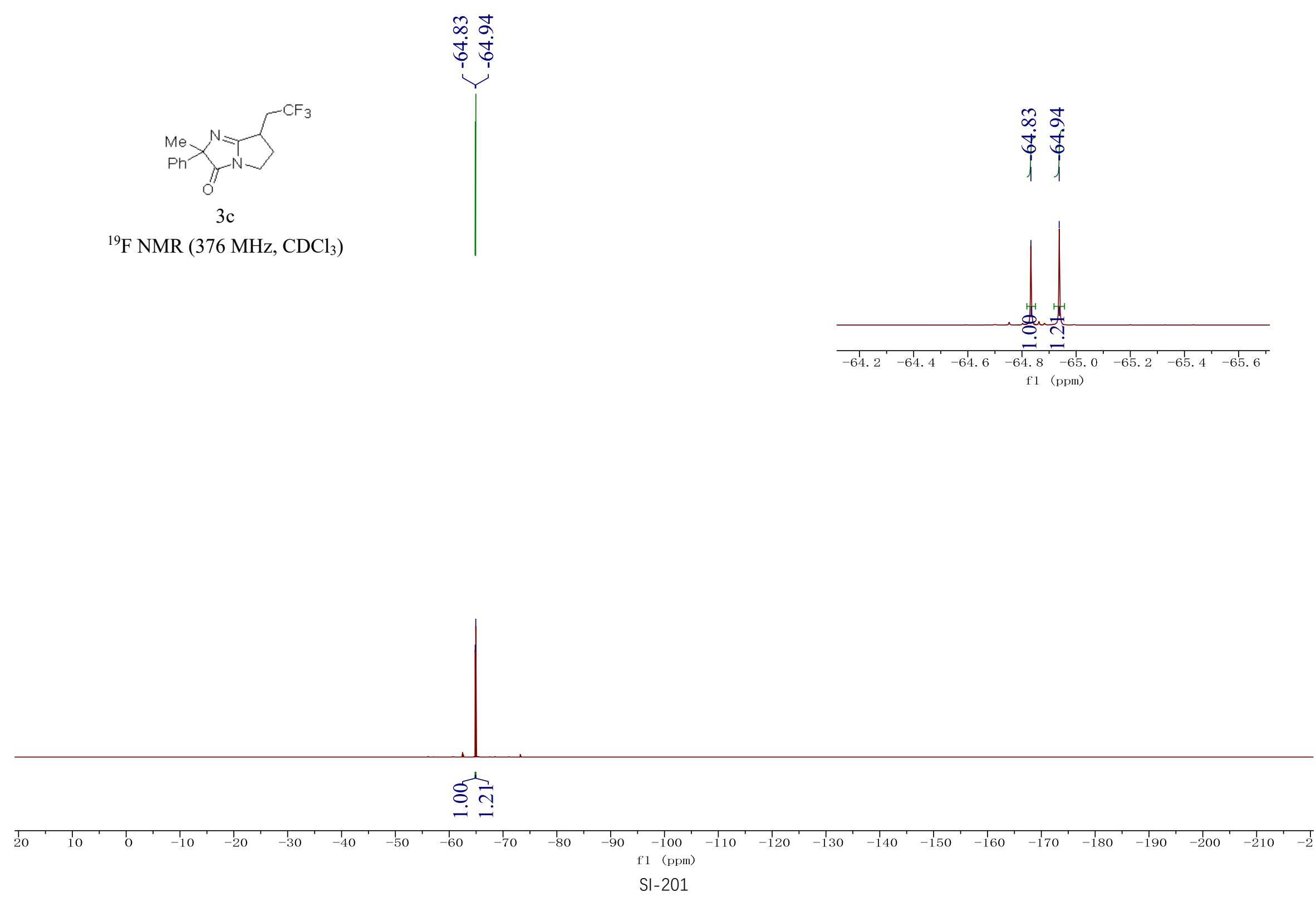


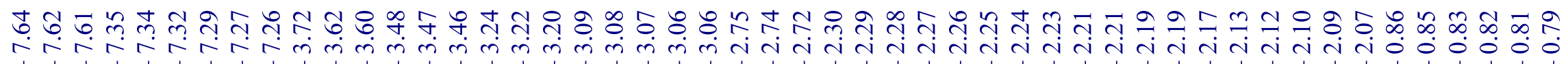

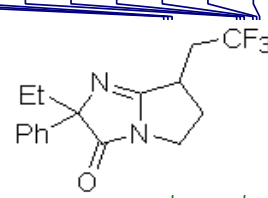

$3 \mathrm{~d}$

${ }^{1} \mathrm{H}$ NMR $\left(500 \mathrm{MHz}, \mathrm{CDCl}_{3}\right)$

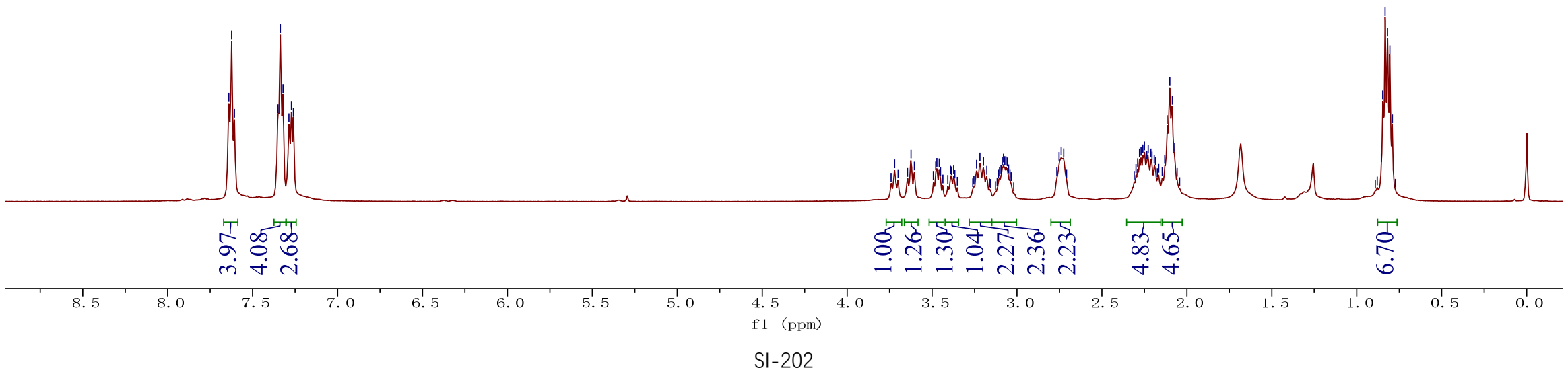



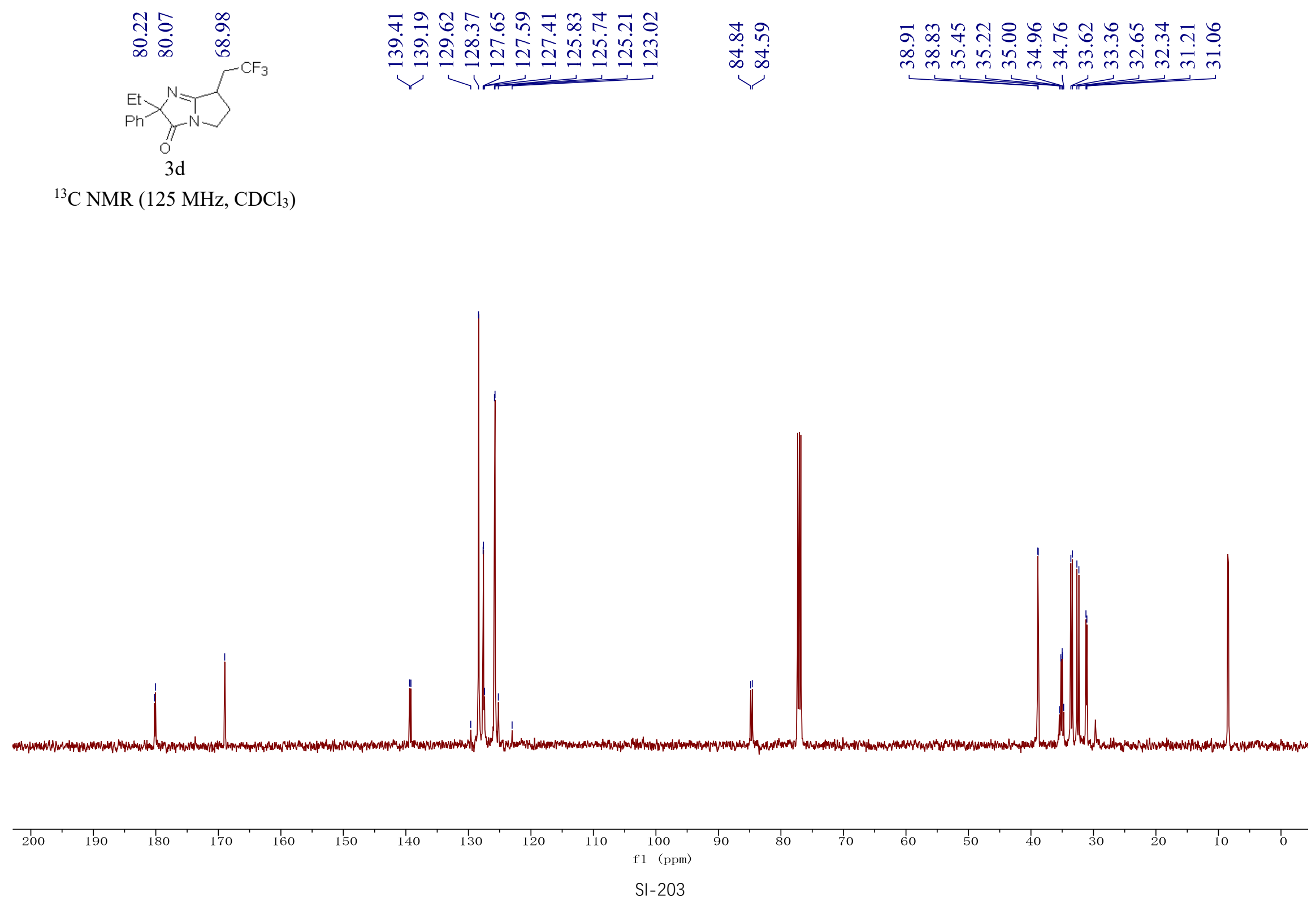


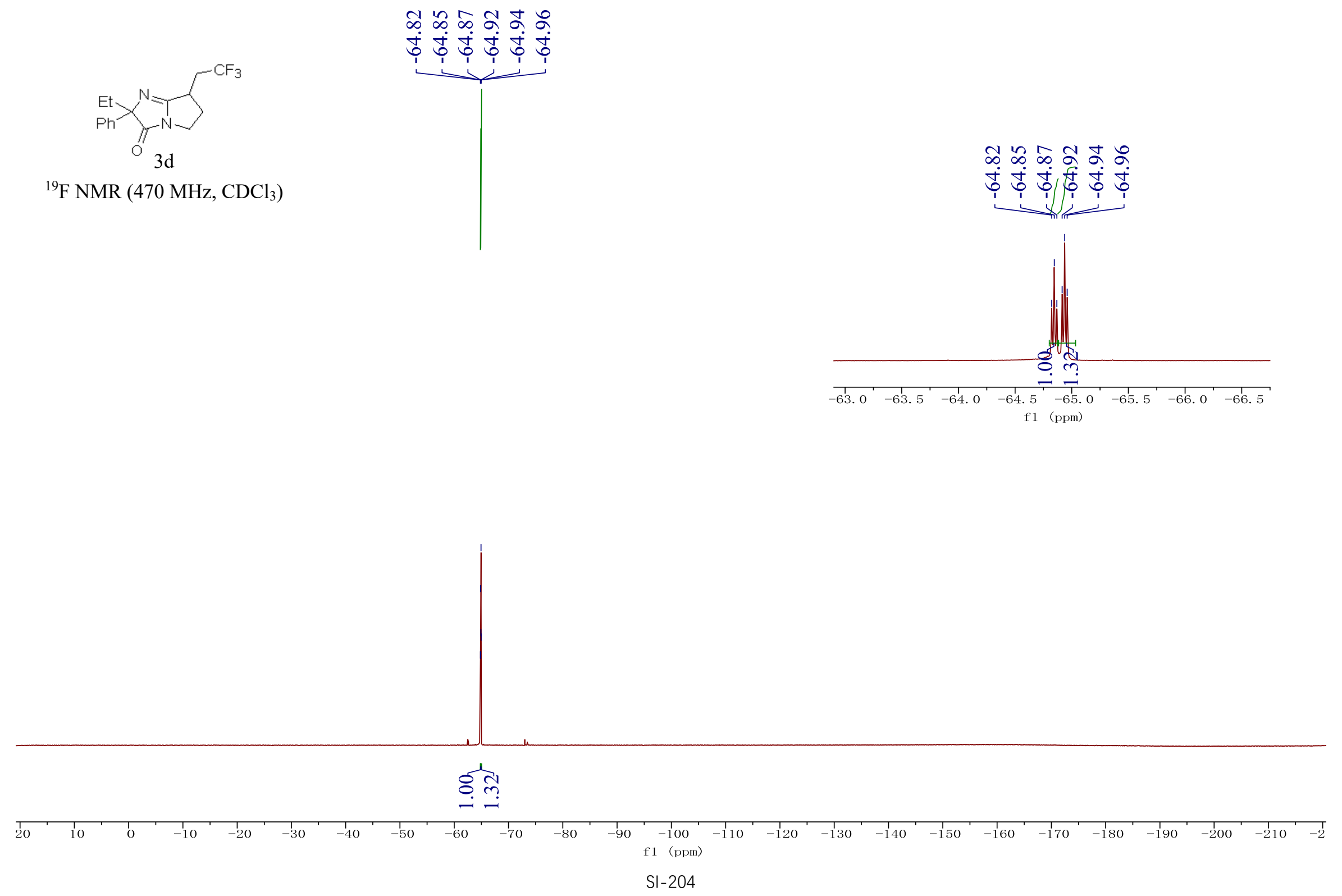




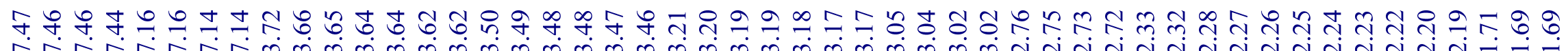
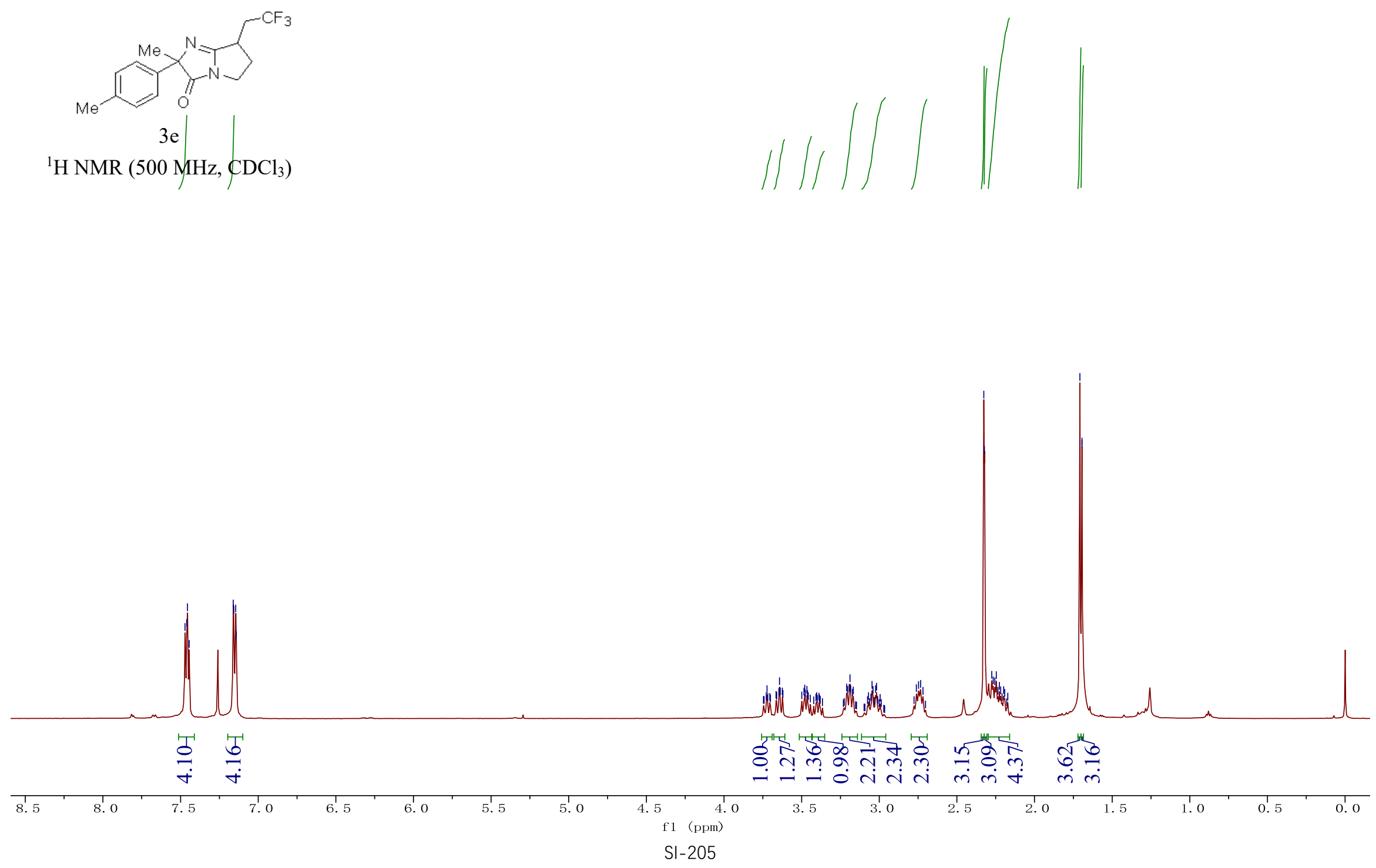


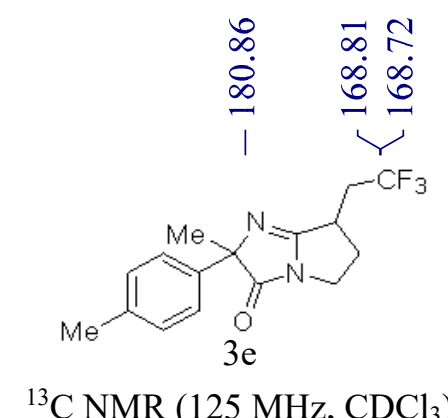

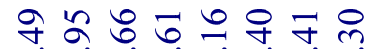

的穴主主主主

ㅎำ

ì

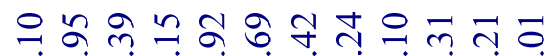

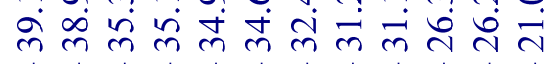

${ }^{13} \mathrm{C}$ NMR $\left(125 \mathrm{MHz}, \mathrm{CDCl}_{3}\right)$

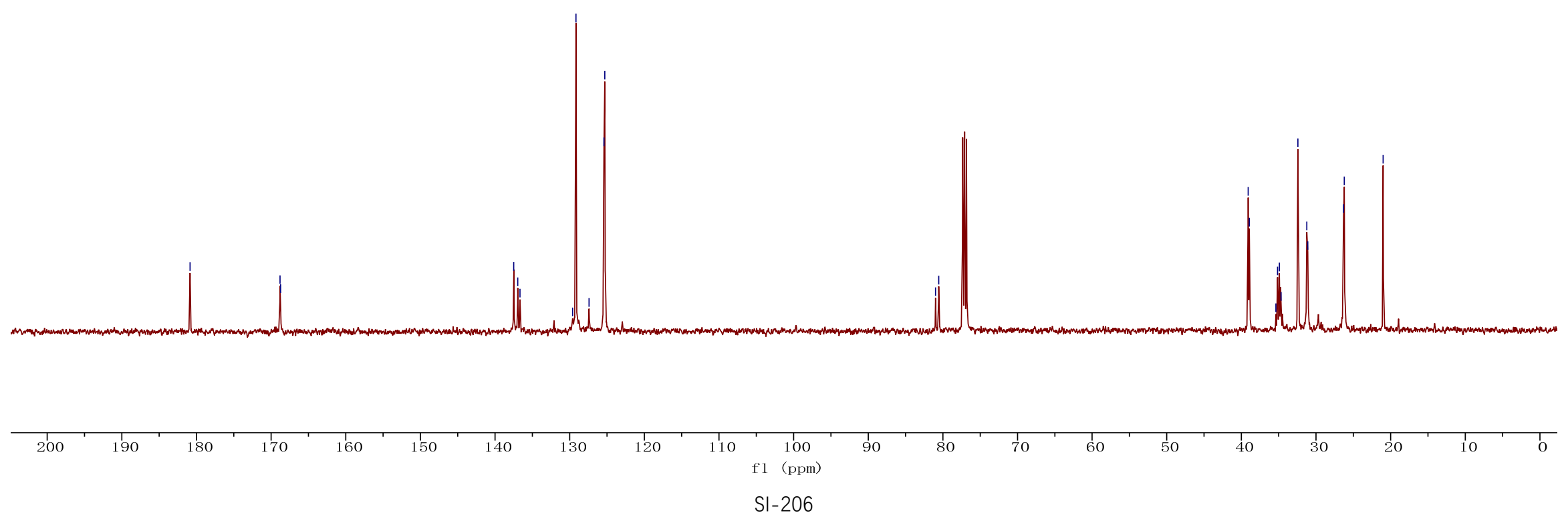


시료

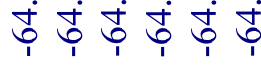

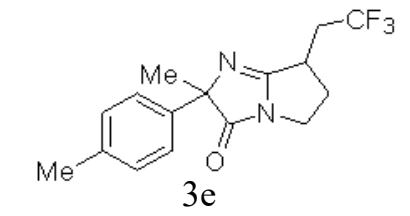

${ }^{19} \mathrm{~F}$ NMR (470 MHz, $\mathrm{CDCl}_{3}$ )

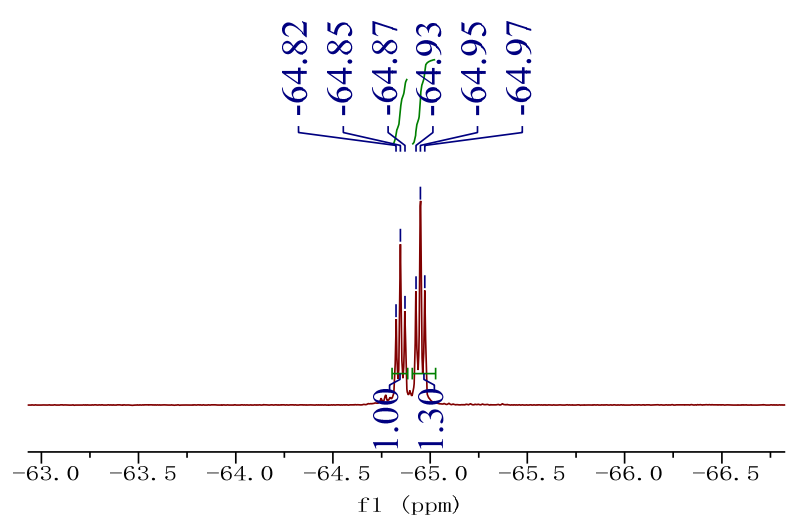

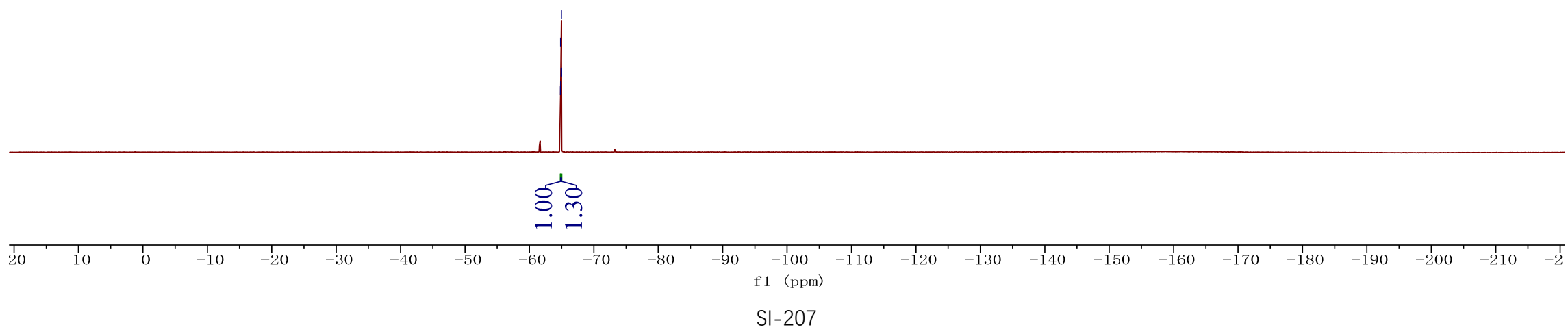




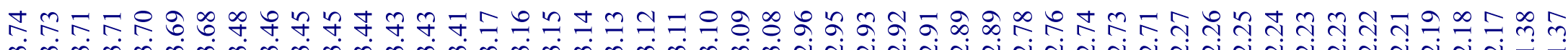

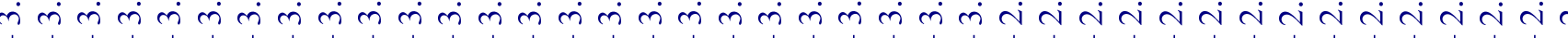
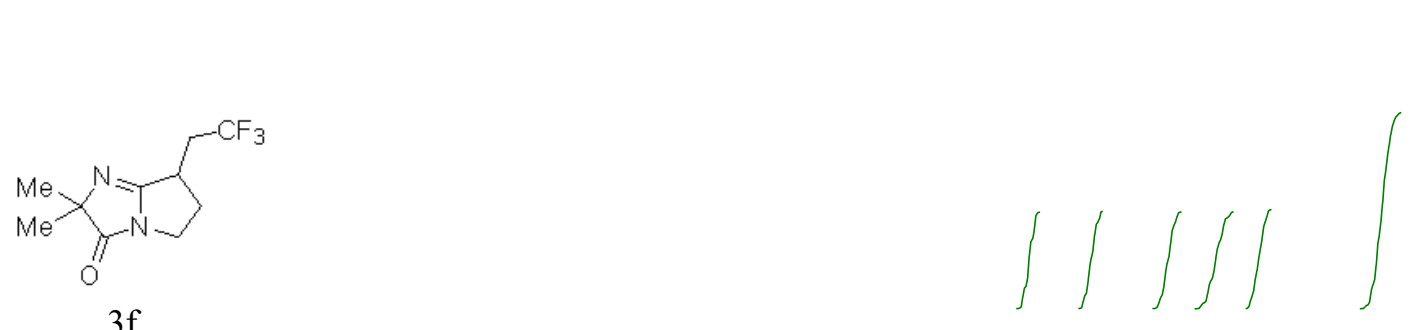

${ }^{1} \mathrm{H}$ NMR (400 MHz, $\left.\mathrm{CDCl}_{3}\right)$

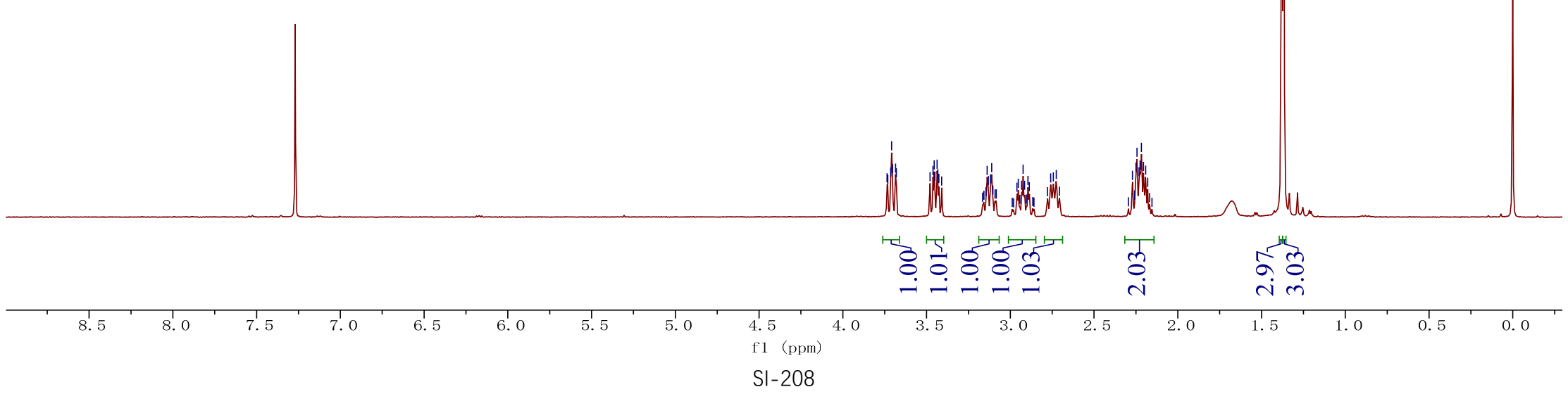




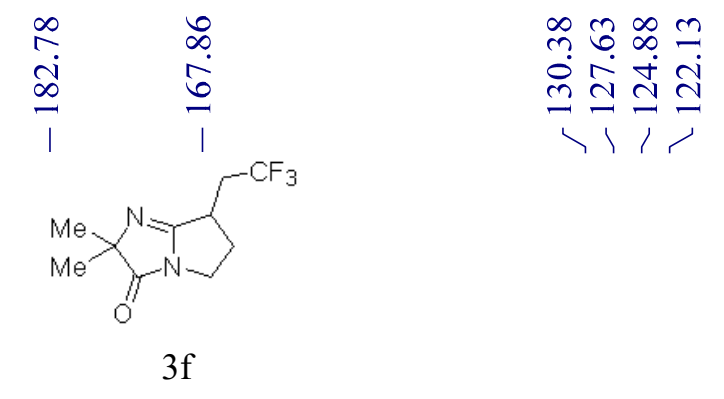

${ }^{13} \mathrm{C}$ NMR (100 MHz, $\left.\mathrm{CDCl}_{3}\right)$

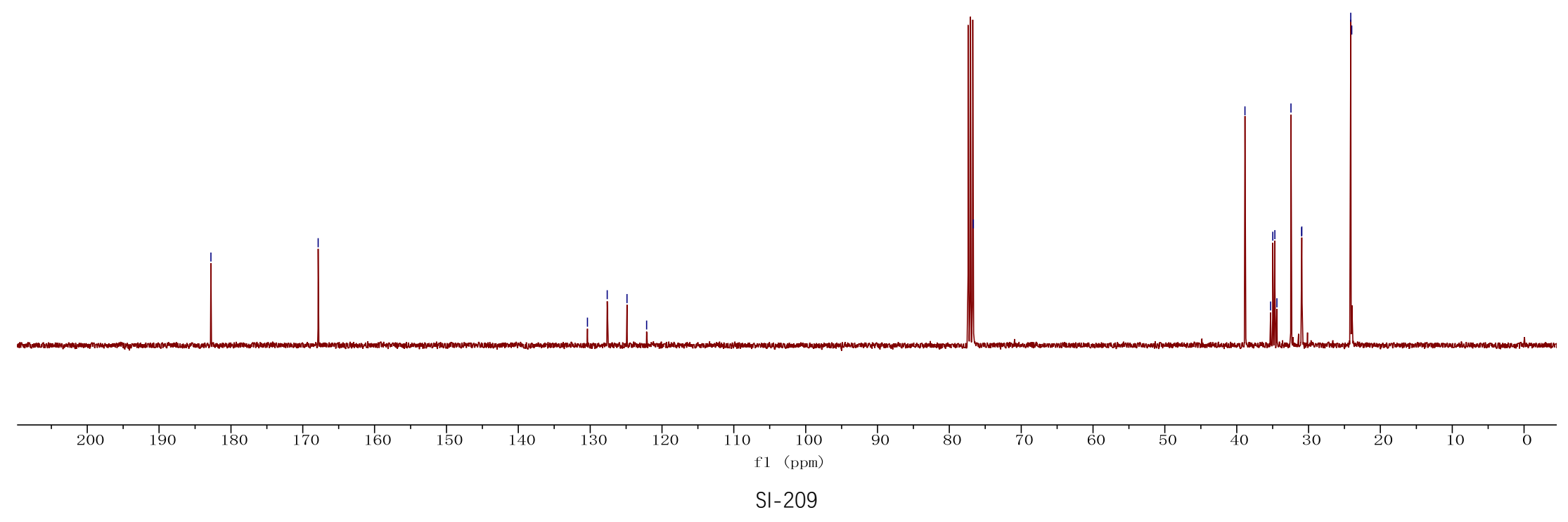




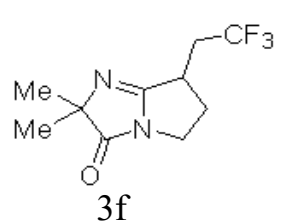

${ }^{19} \mathrm{~F}$ NMR (376 MHz, $\mathrm{CDCl}_{3}$ )

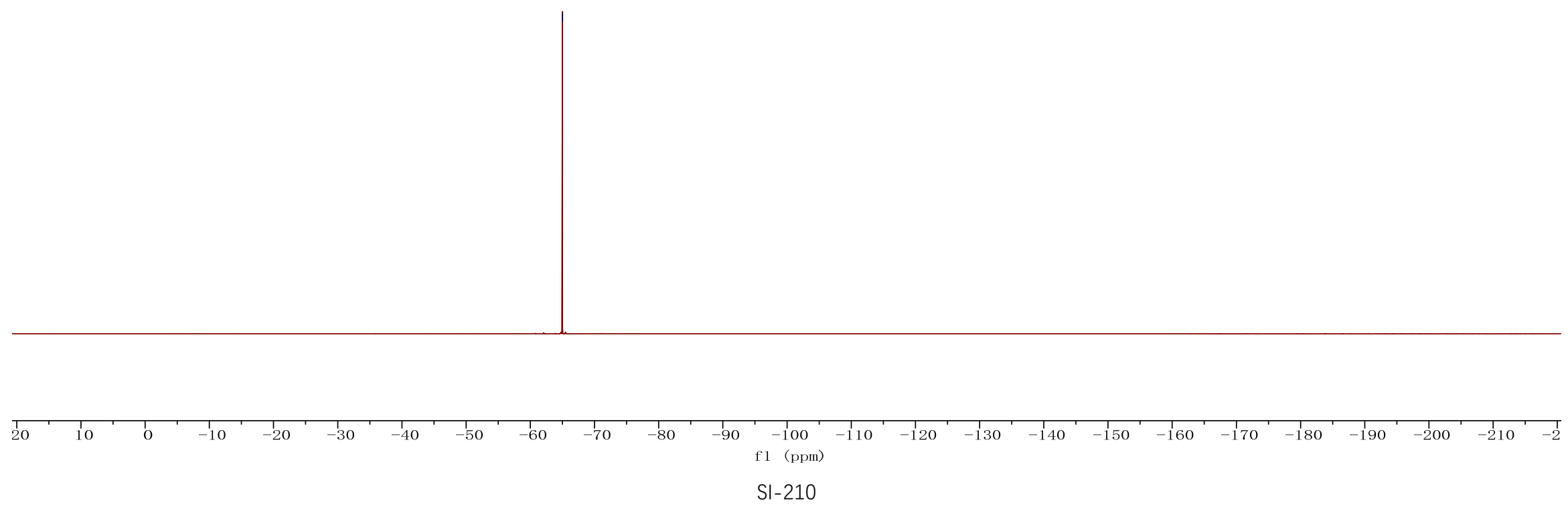




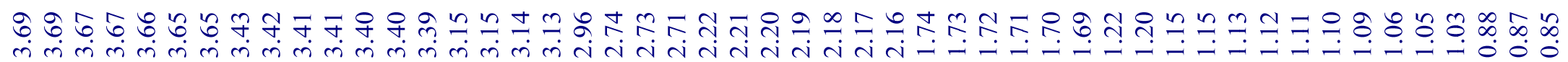

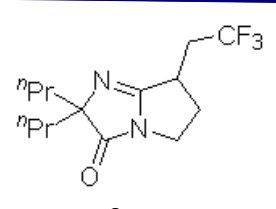

$3 \mathrm{~g}$

${ }^{1} \mathrm{H} \mathrm{NMR}\left(500 \mathrm{MHz}, \mathrm{CDCl}_{3}\right)$
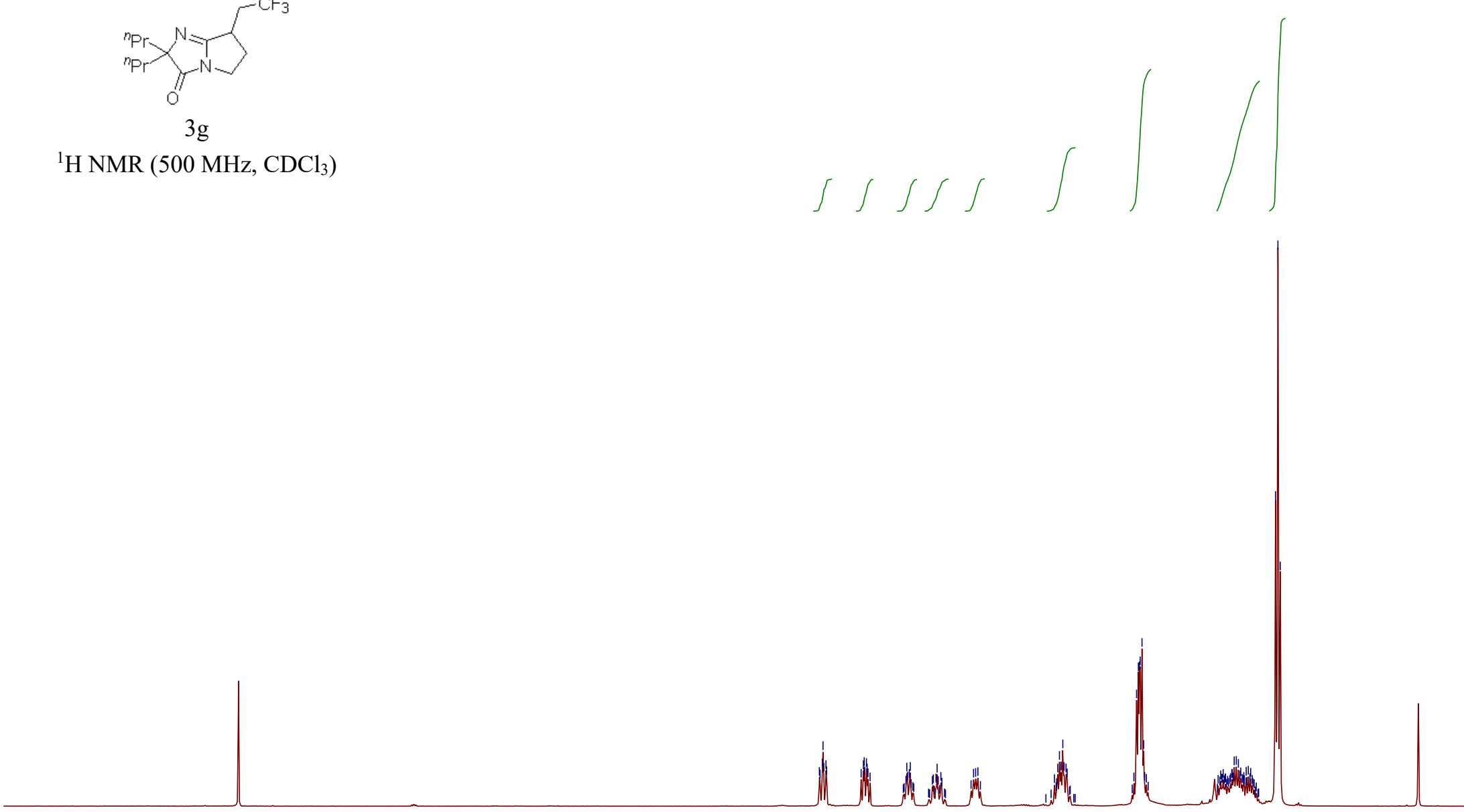

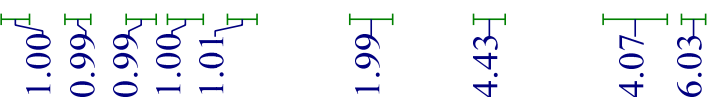

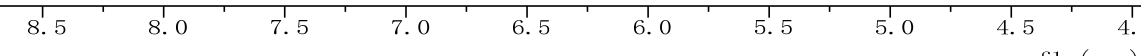



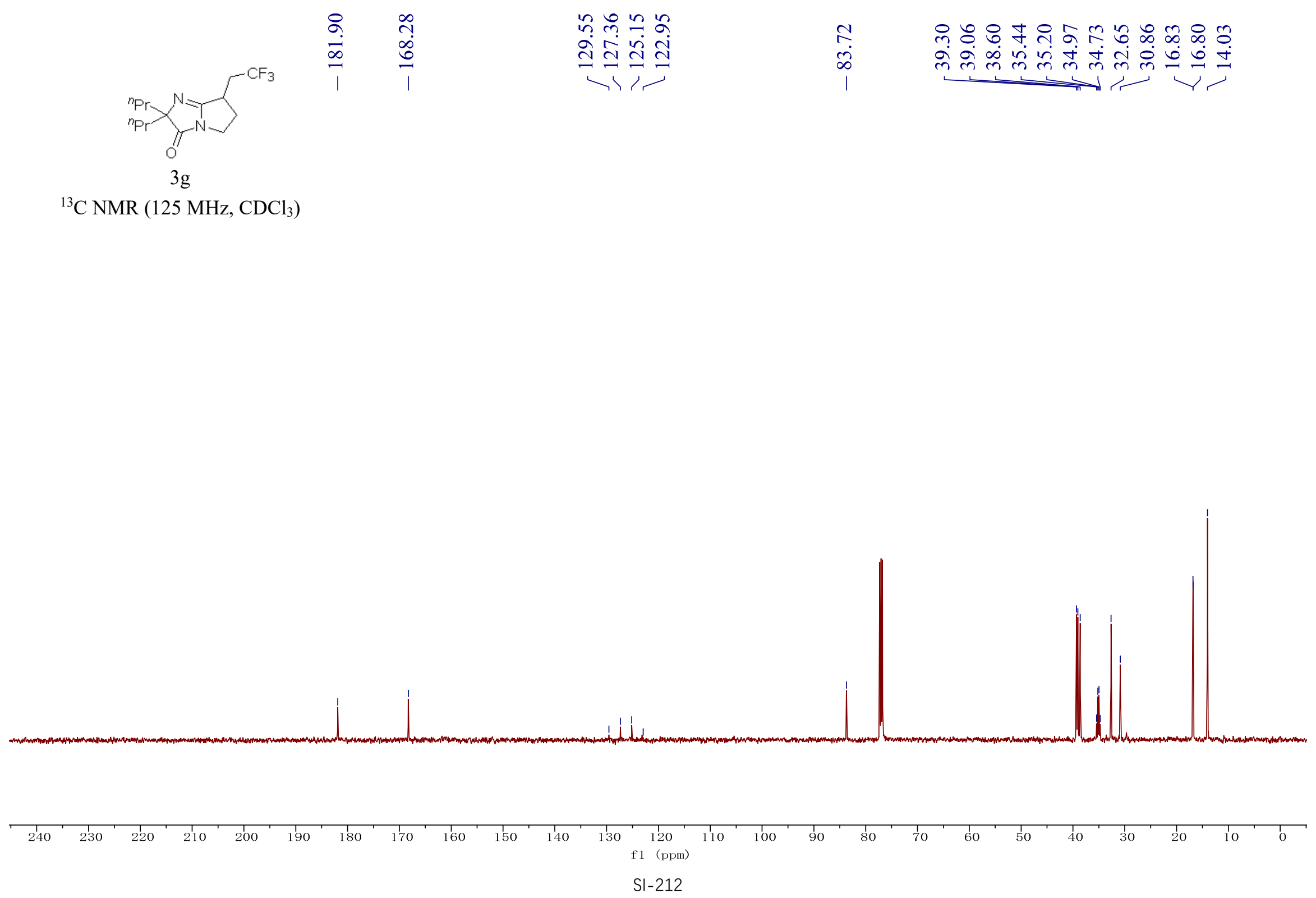
ㅎํㅇ

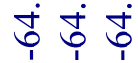

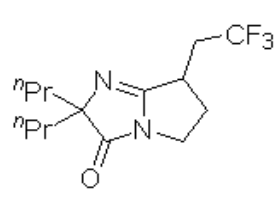

$3 \mathrm{~g}$

${ }^{19} \mathrm{~F}$ NMR $\left(470 \mathrm{MHz}, \mathrm{CDCl}_{3}\right)$

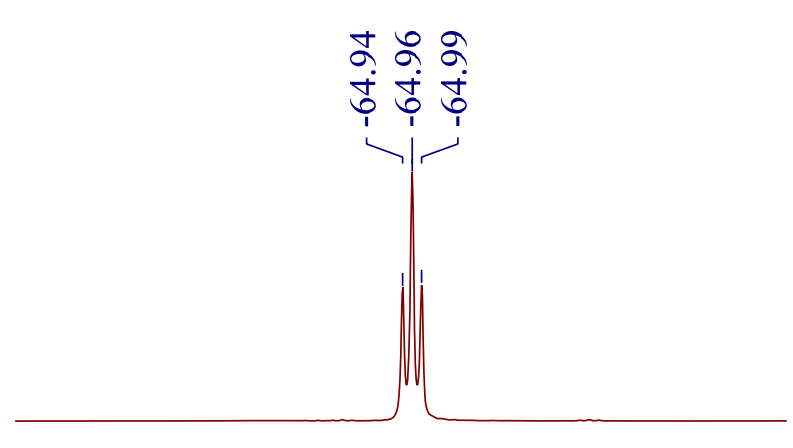

$-64.2-64.4-64.6-64.8-65.0-65.2-65.4-65.6 \quad-65.8$ f1 (ppm)

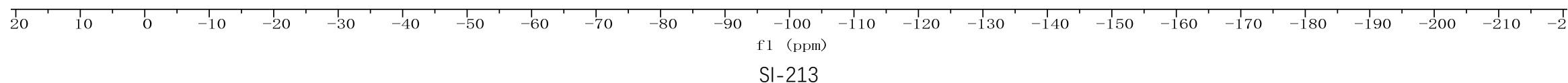




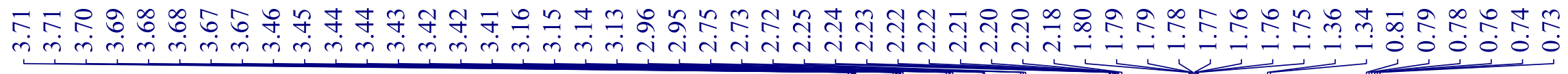

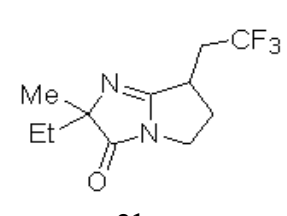

$3 \mathrm{~h}$

${ }^{1} \mathrm{H}$ NMR (500 MHz, $\mathrm{CDCl}_{3}$ )
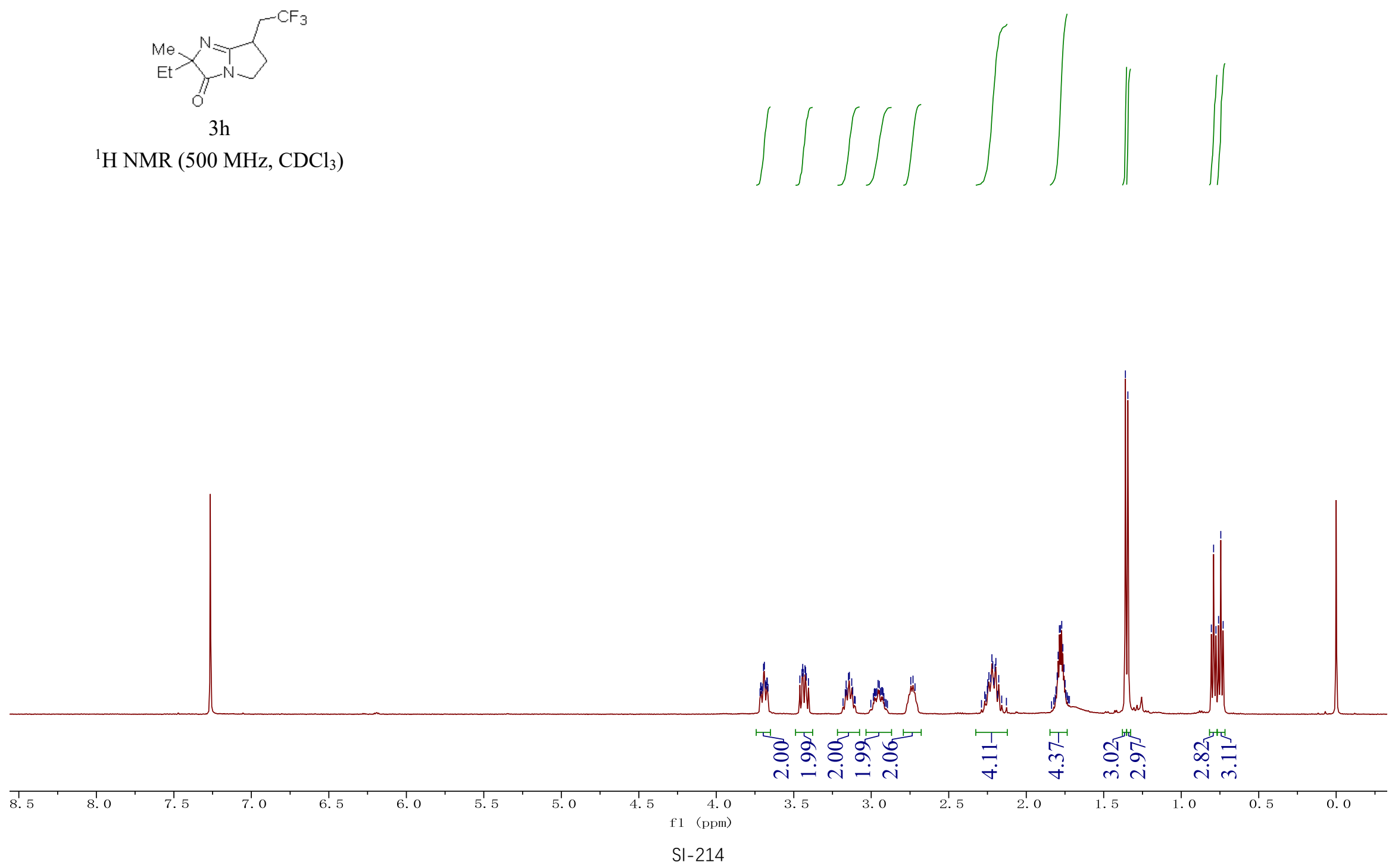


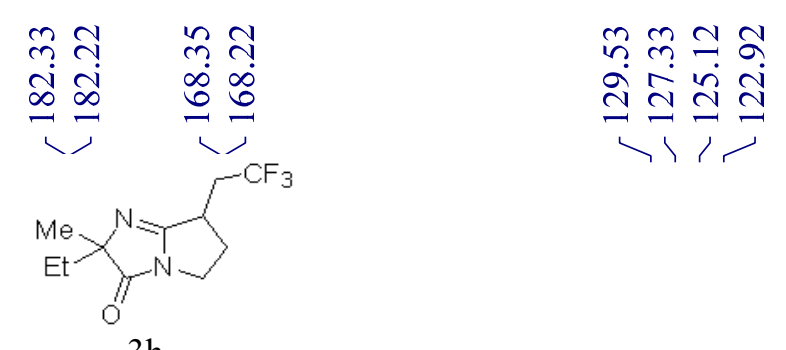

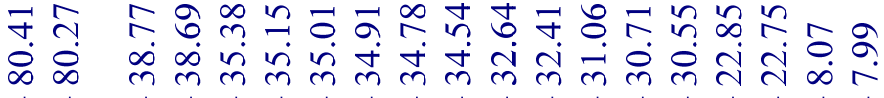

$3 \mathrm{~h}$

${ }^{13} \mathrm{C}$ NMR $\left(125 \mathrm{MHz}, \mathrm{CDCl}_{3}\right)$
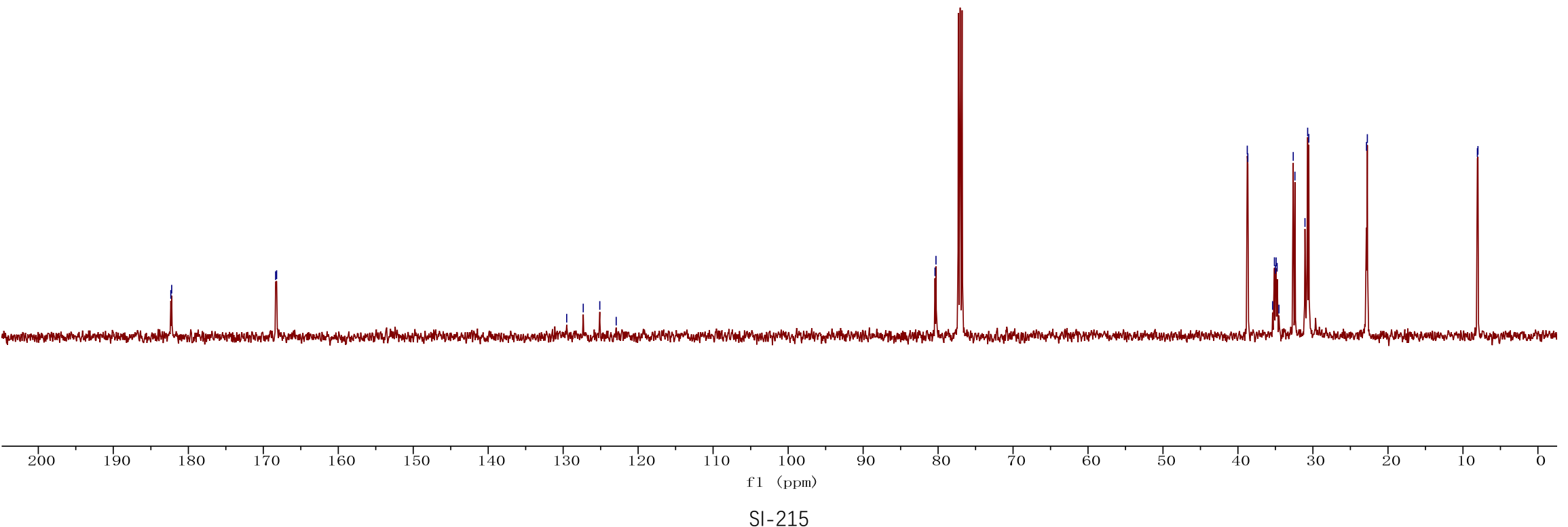


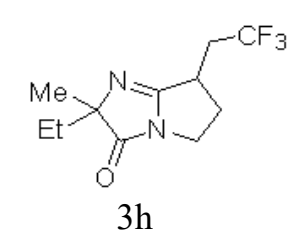

${ }^{19} \mathrm{~F}$ NMR (470 MHz, $\left.\mathrm{CDCl}_{3}\right)$

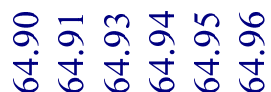

రి

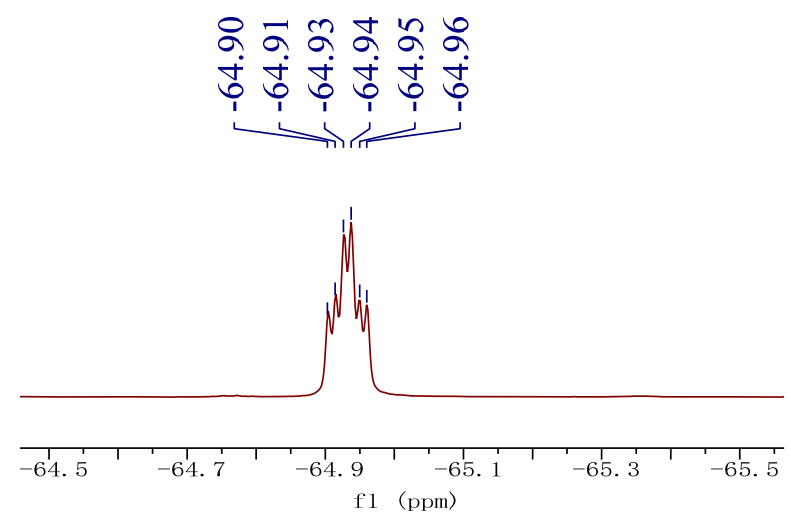

20

$20 \quad 10$

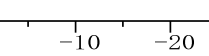

$-\frac{1}{1}+30$

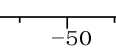

$-60$

$-80$

$-100-110$

$-120$

$-130 \quad-140$

$\begin{array}{llllllll}-150 & -160 & -170 & -180 & -190 & -200 & -210 & -2\end{array}$

$\mathrm{SI}-216$ 

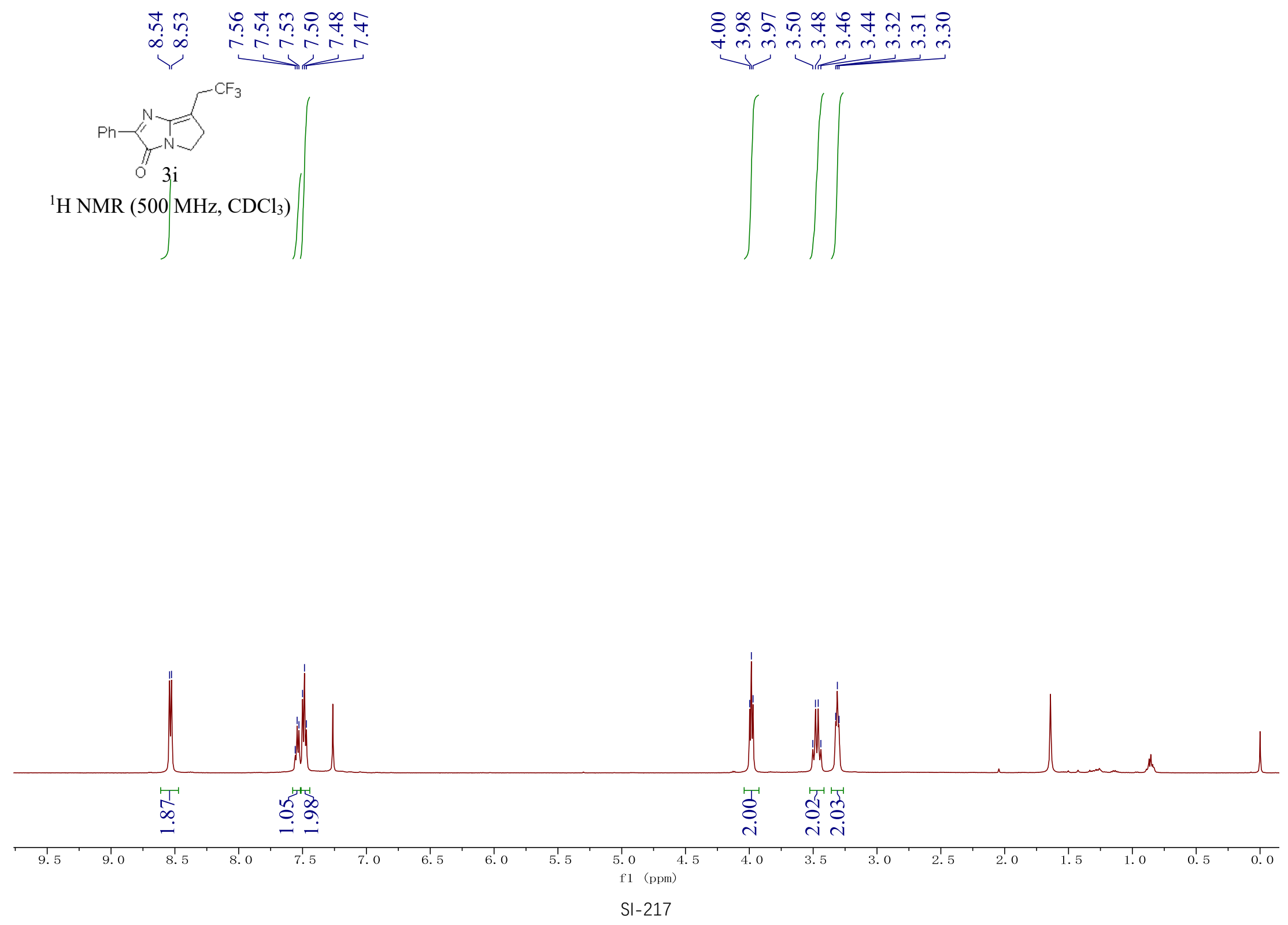


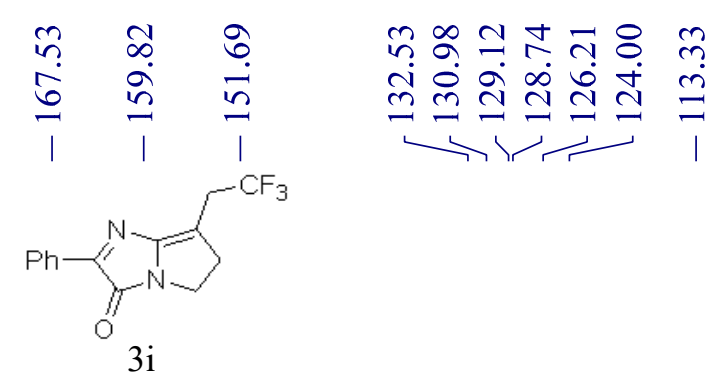

${ }^{13} \mathrm{C}$ NMR (125 MHz, $\left.\mathrm{CDCl}_{3}\right)$

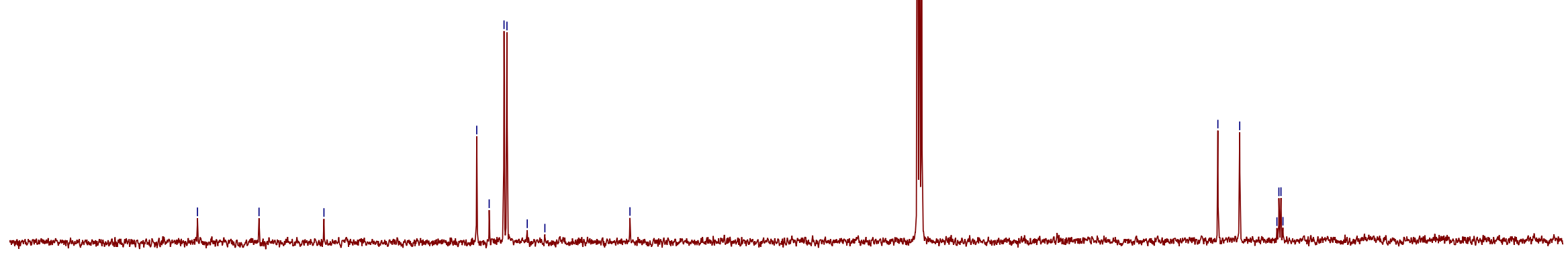


둥 ț

గ్ర

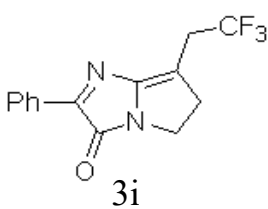

${ }^{19} \mathrm{~F}$ NMR $\left(470 \mathrm{MHz}, \mathrm{CDCl}_{3}\right)$
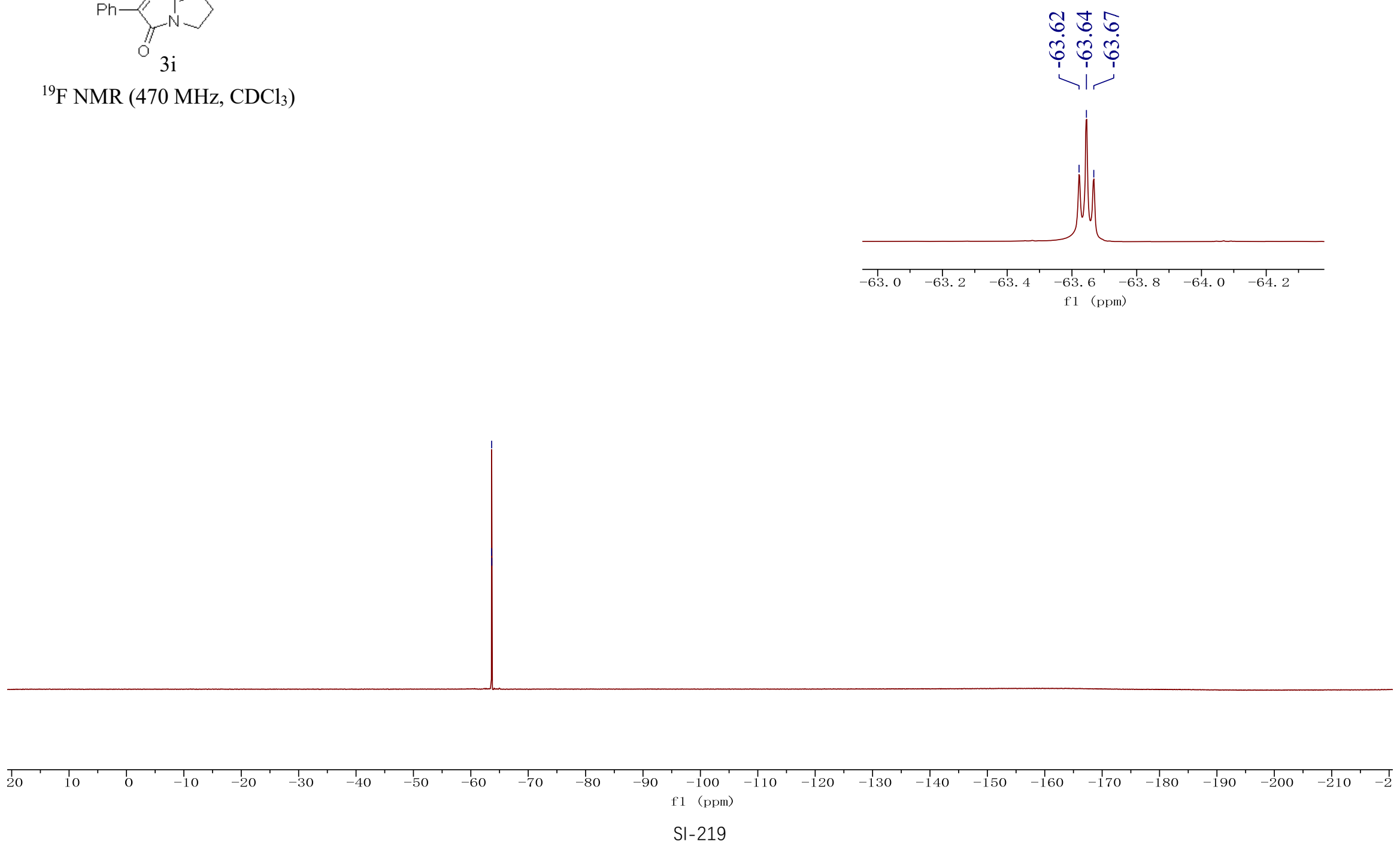


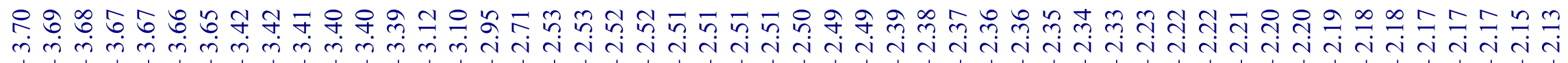

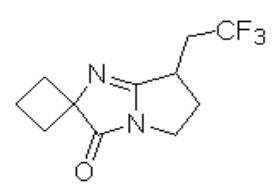

$3 \mathrm{j}$

${ }^{1} \mathrm{H} \mathrm{NMR}\left(500 \mathrm{MHz}, \mathrm{CDCl}_{3}\right)$
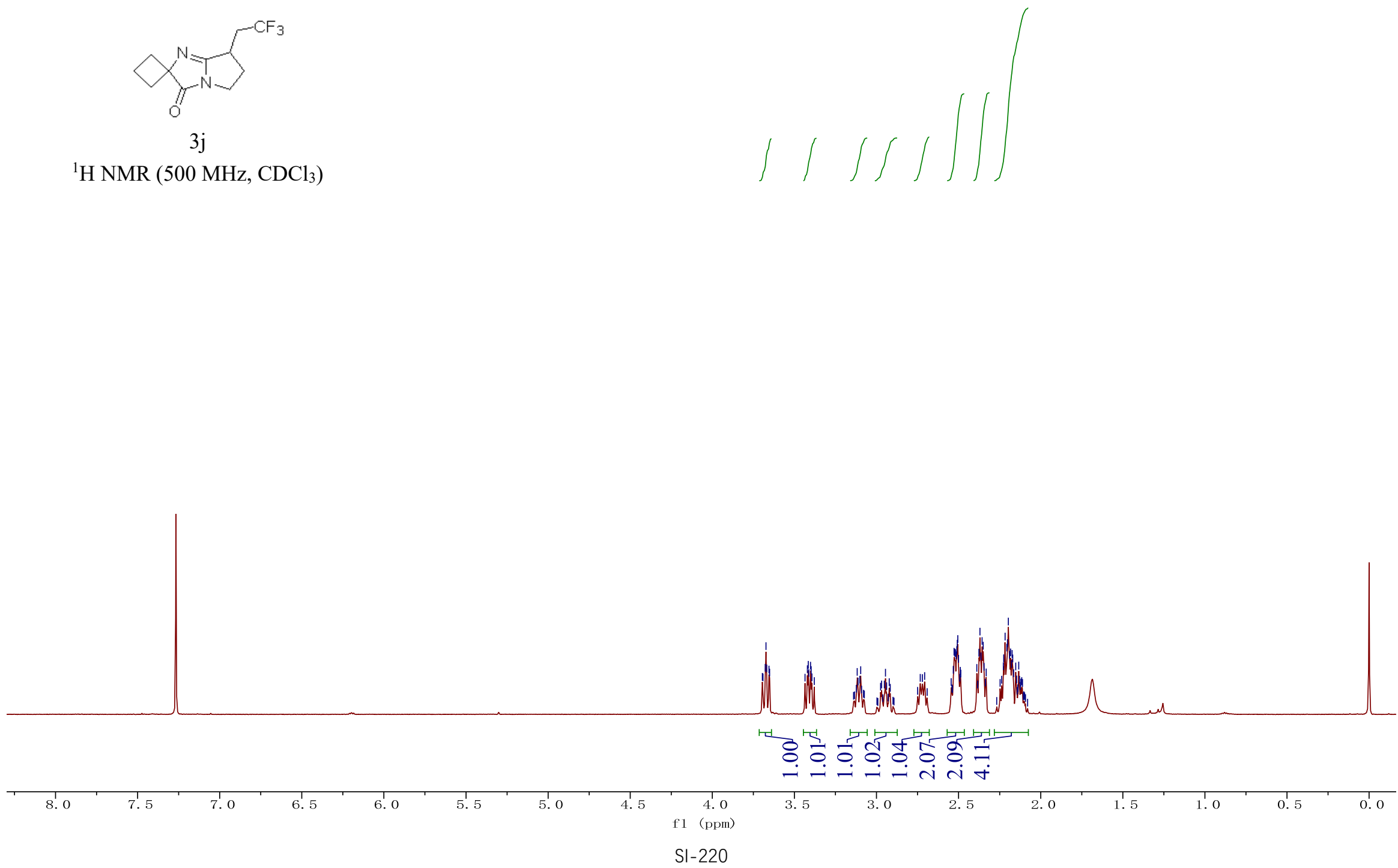


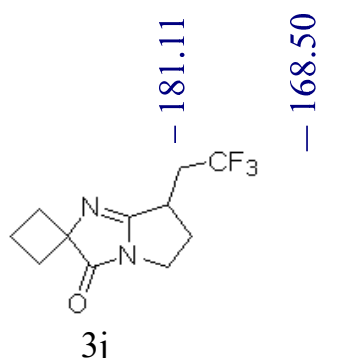

$3 \mathrm{j}$

${ }^{13} \mathrm{C}$ NMR $\left(125 \mathrm{MHz}, \mathrm{CDCl}_{3}\right.$ )

ํำ 눙ำ

ิㅚㄷㅚำ

$\stackrel{\substack{\infty \\ \infty}}{\infty}$

웃ำ

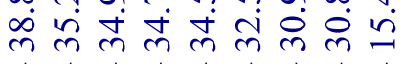
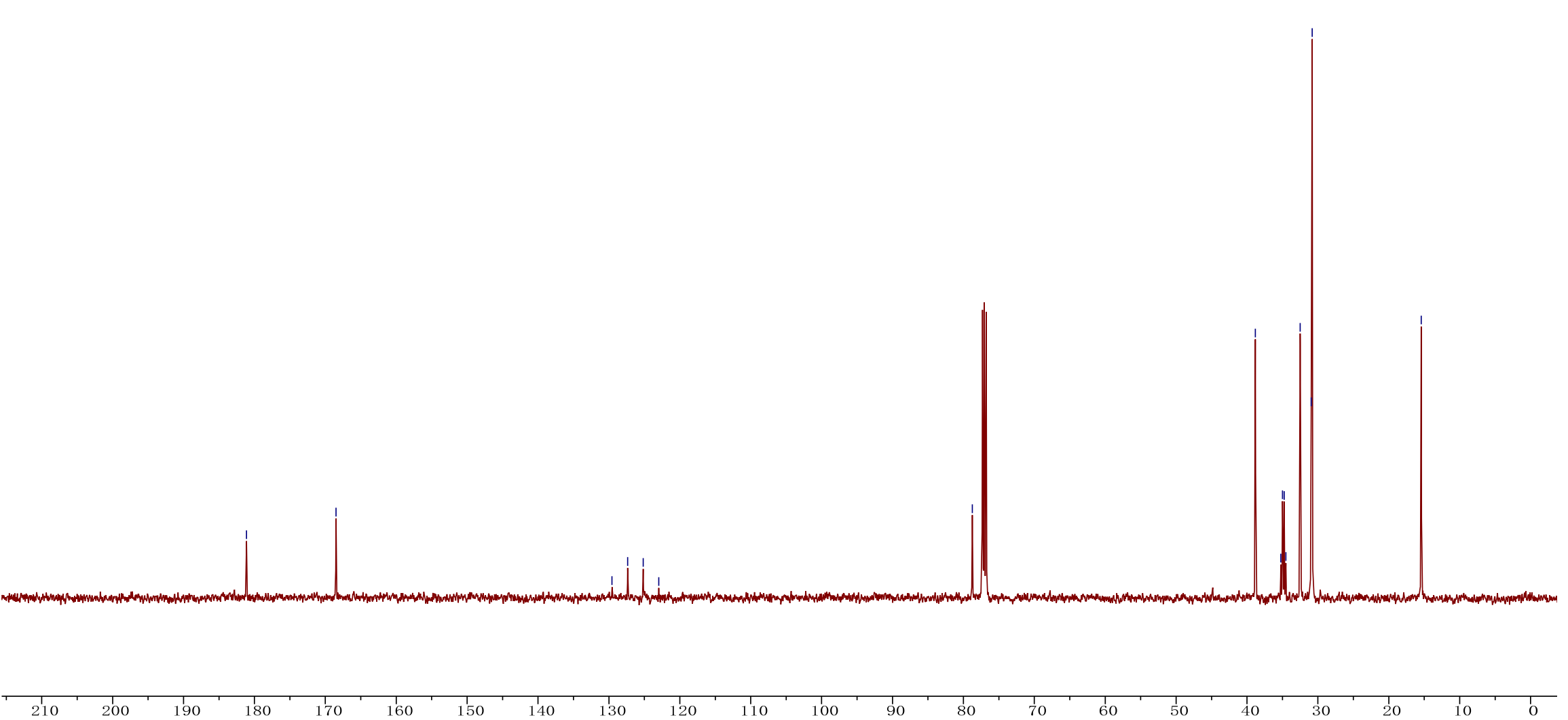

(60)

150

130

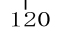

$110 \quad 100$

90

60

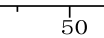

30

20 


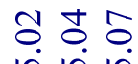

जिं

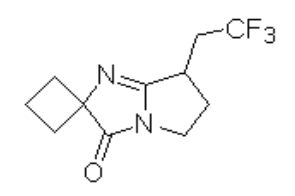

$3 \mathrm{j}$

${ }^{19} \mathrm{~F}$ NMR $\left(470 \mathrm{MHz}, \mathrm{CDCl}_{3}\right)$

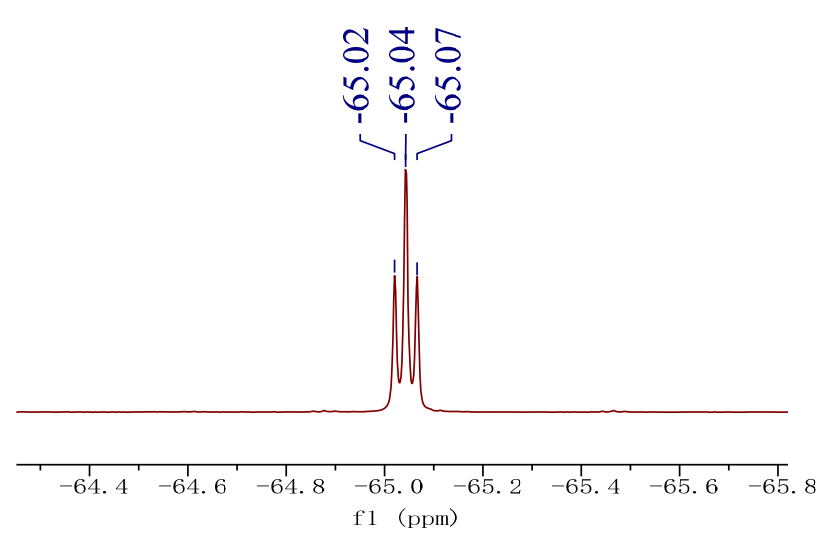

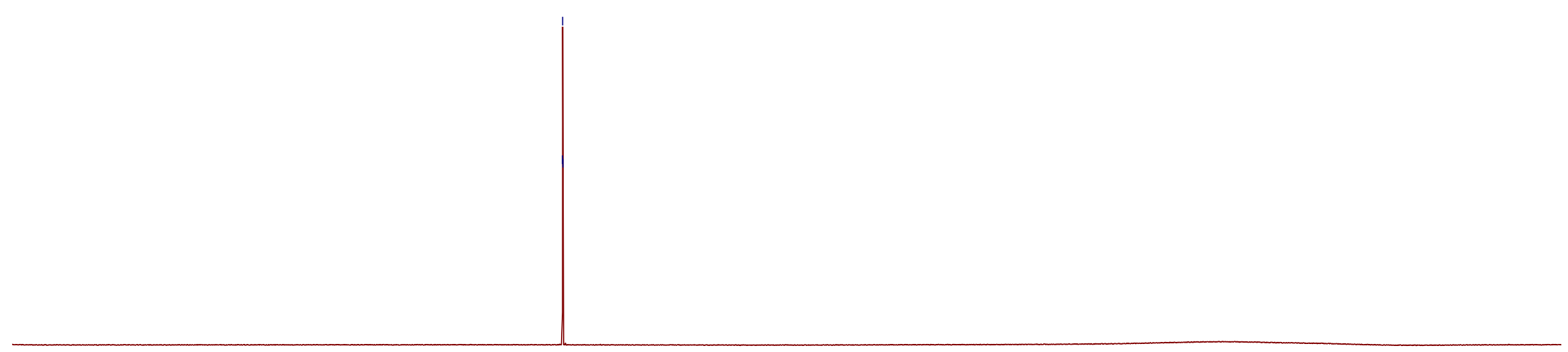




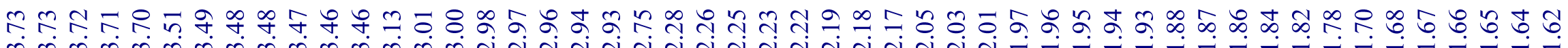

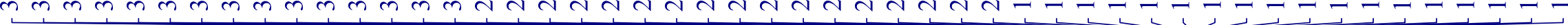

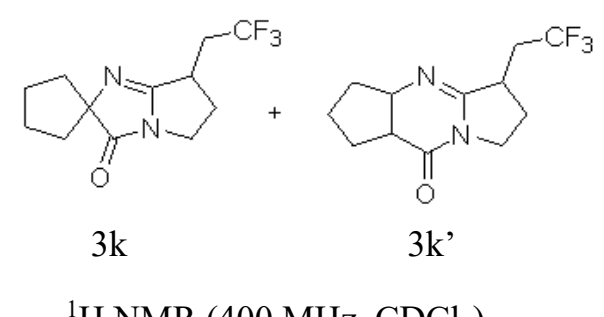

${ }^{1} \mathrm{H}$ NMR (400 MHz, $\mathrm{CDCl}_{3}$ )
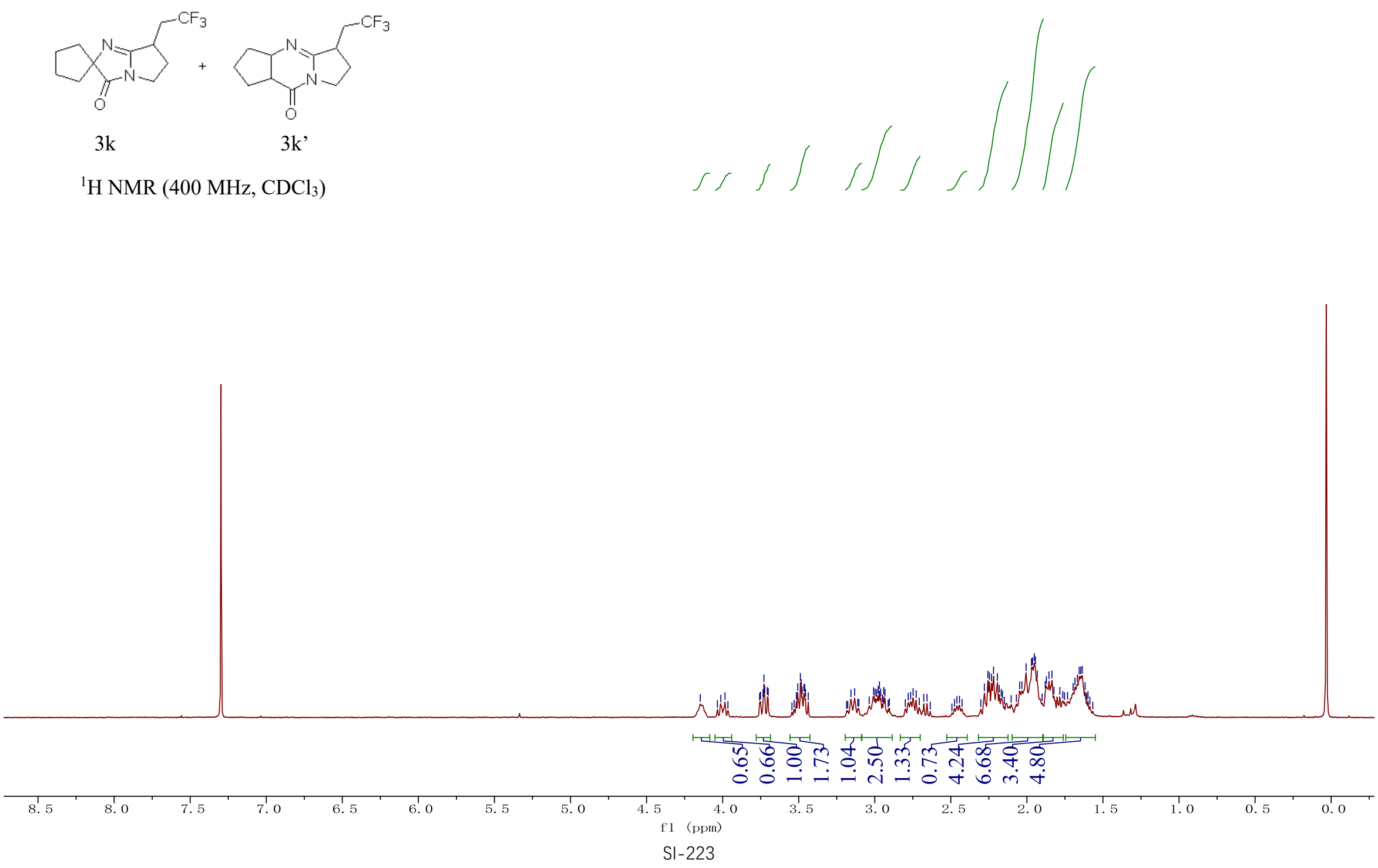


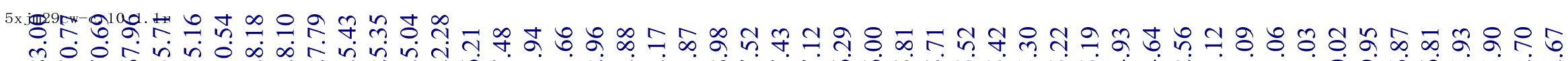

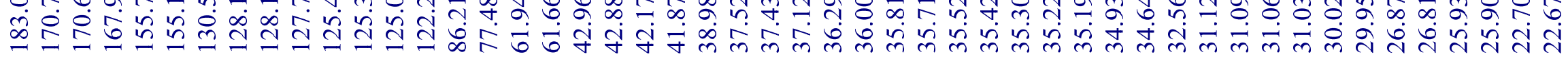

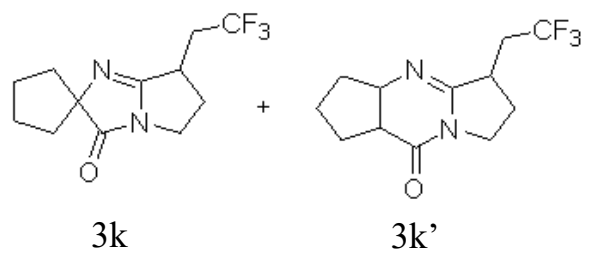

${ }^{13} \mathrm{C}$ NMR $\left(100 \mathrm{MHz}, \mathrm{CDCl}_{3}\right)$

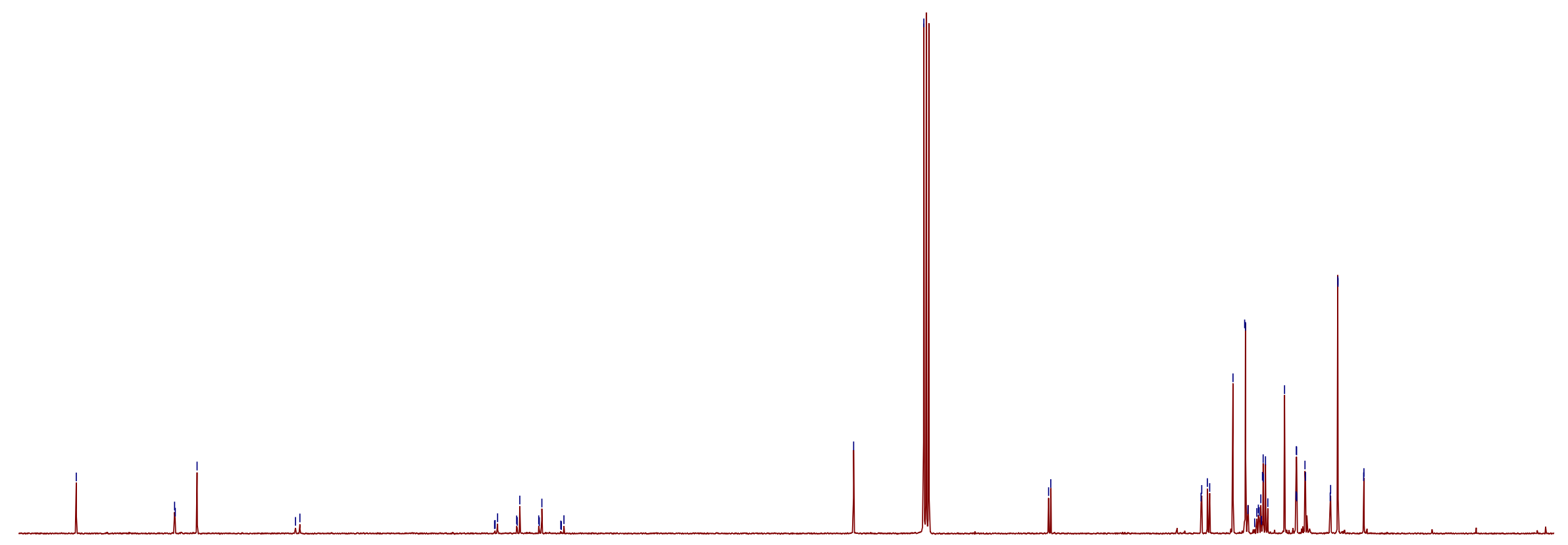



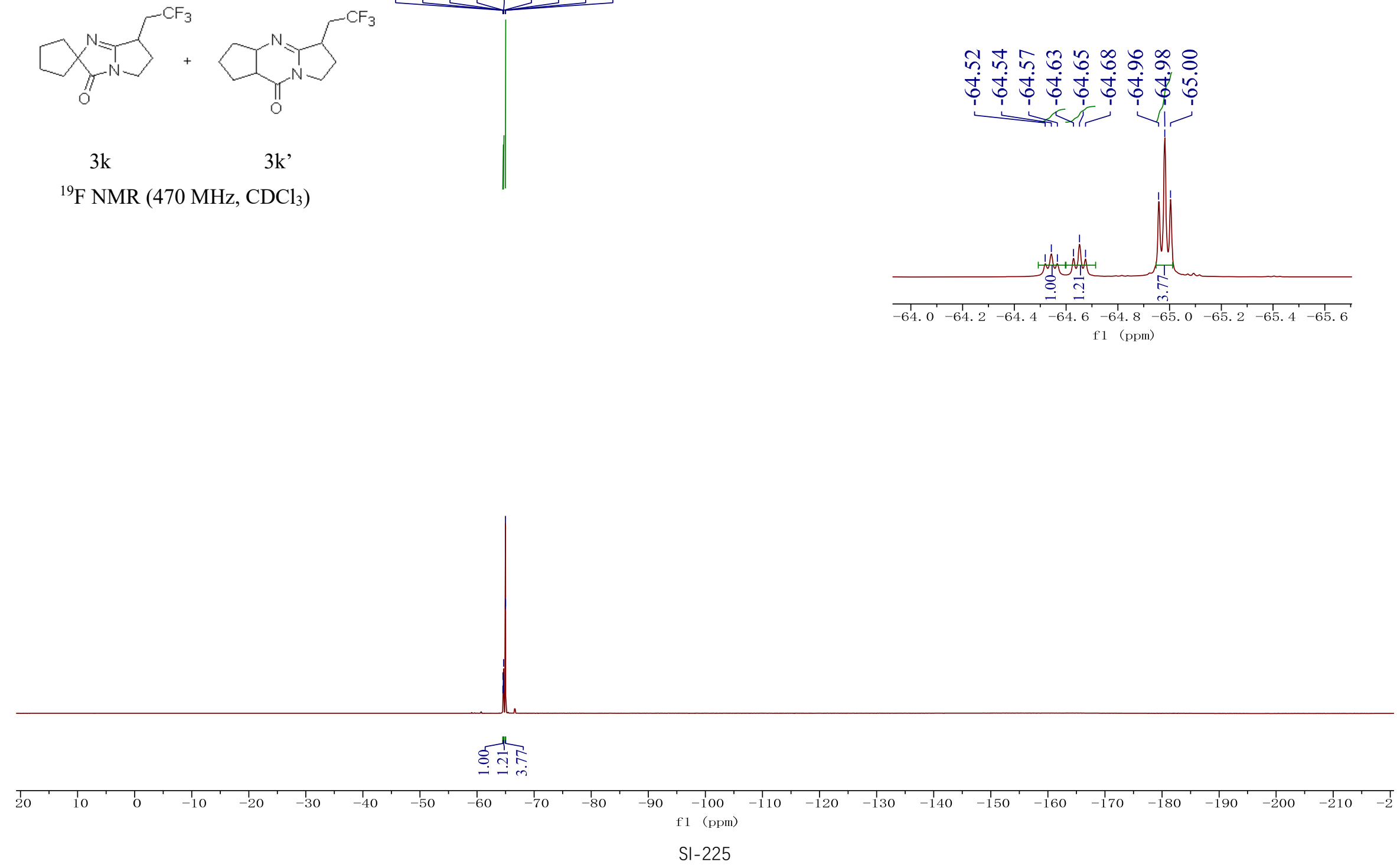


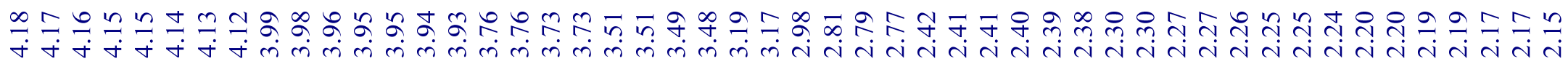

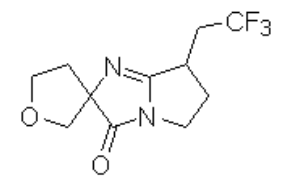

31

${ }^{1} \mathrm{H}$ NMR $\left(400 \mathrm{MHz}, \mathrm{CDCl}_{3}\right)$
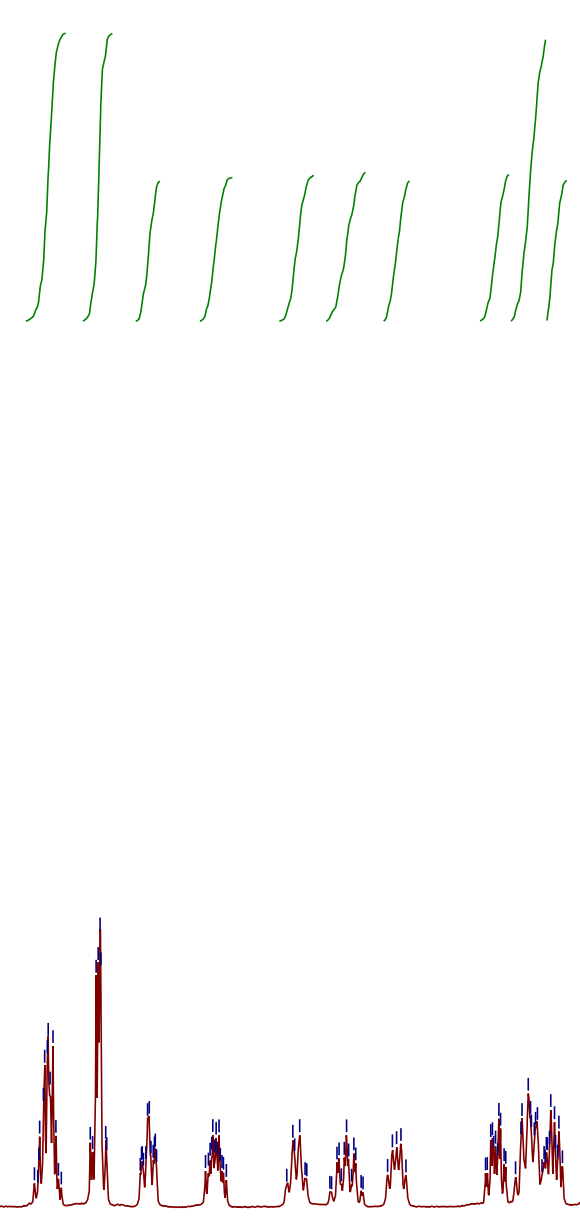

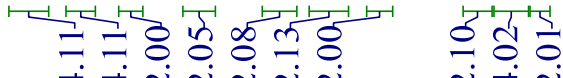




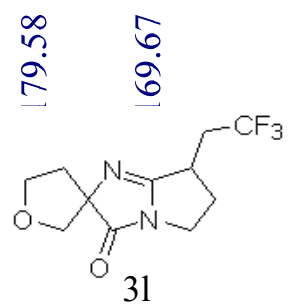

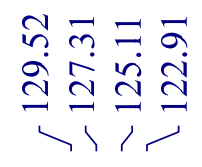

$=8$

क

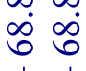

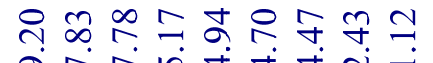

$\checkmark$

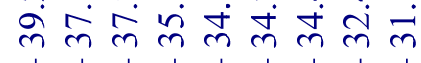

31

${ }^{13} \mathrm{C}$ NMR (125 MHz, $\mathrm{CDCl}_{3}$ )

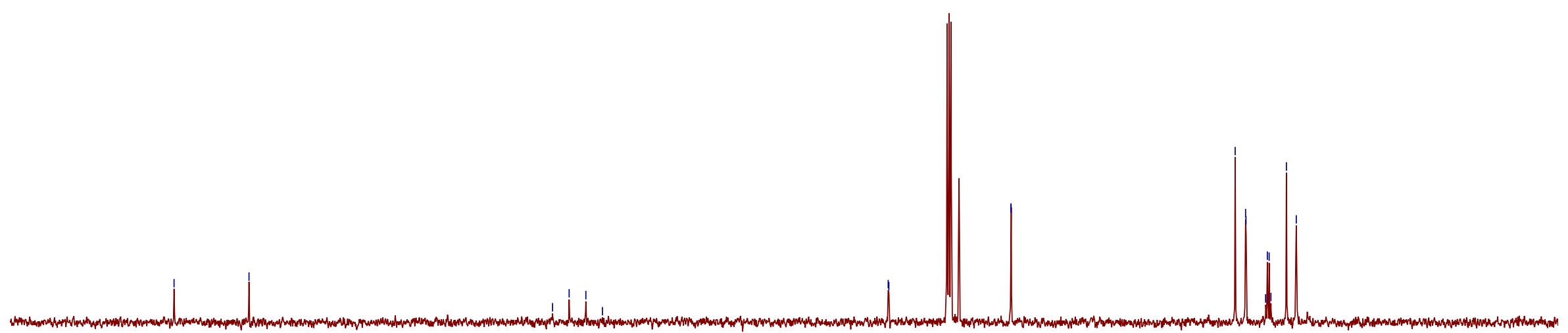




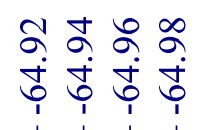

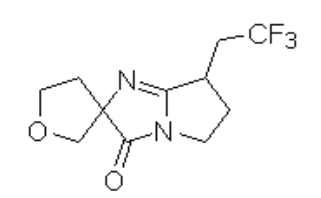

31

${ }^{19} \mathrm{~F}$ NMR $\left(470 \mathrm{MHz}, \mathrm{CDCl}_{3}\right)$

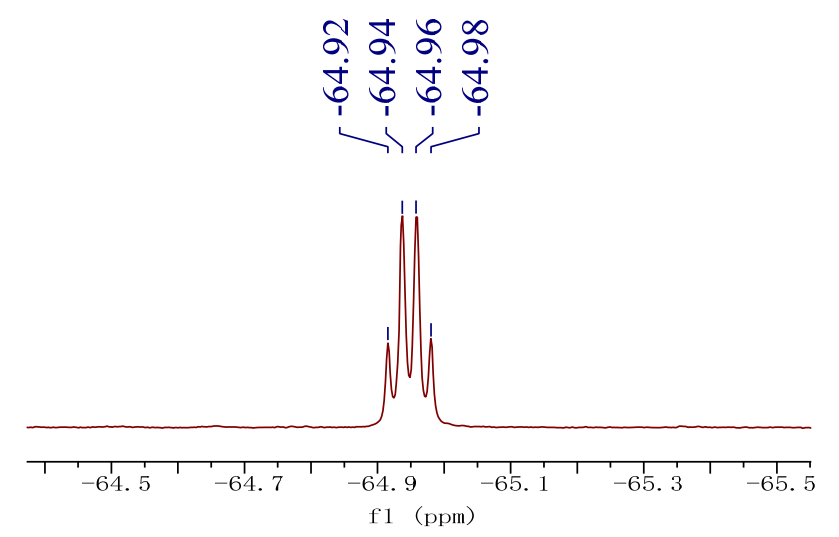

섷형워

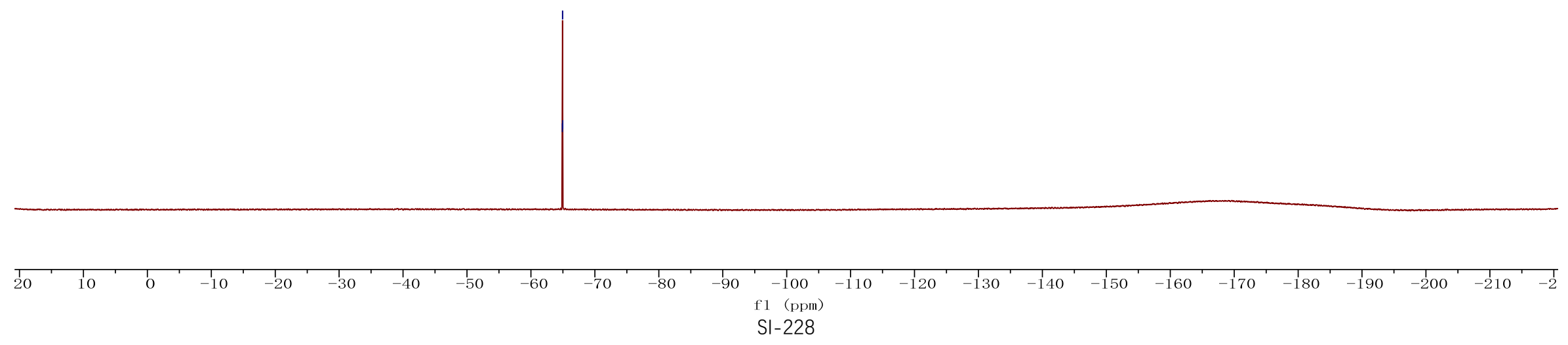




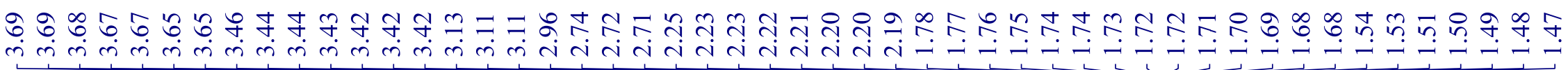
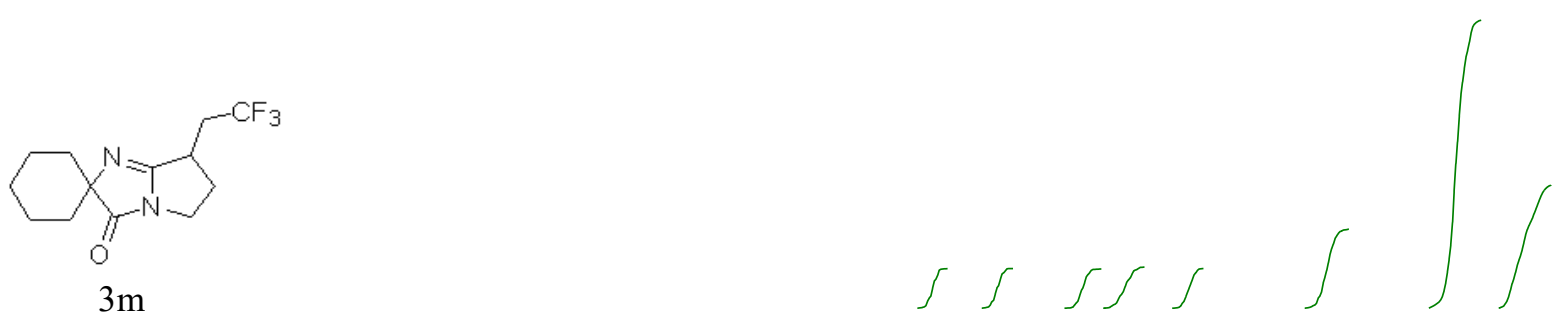

${ }^{1} \mathrm{H}$ NMR $\left(500 \mathrm{MHz}, \mathrm{CDCl}_{3}\right.$ )

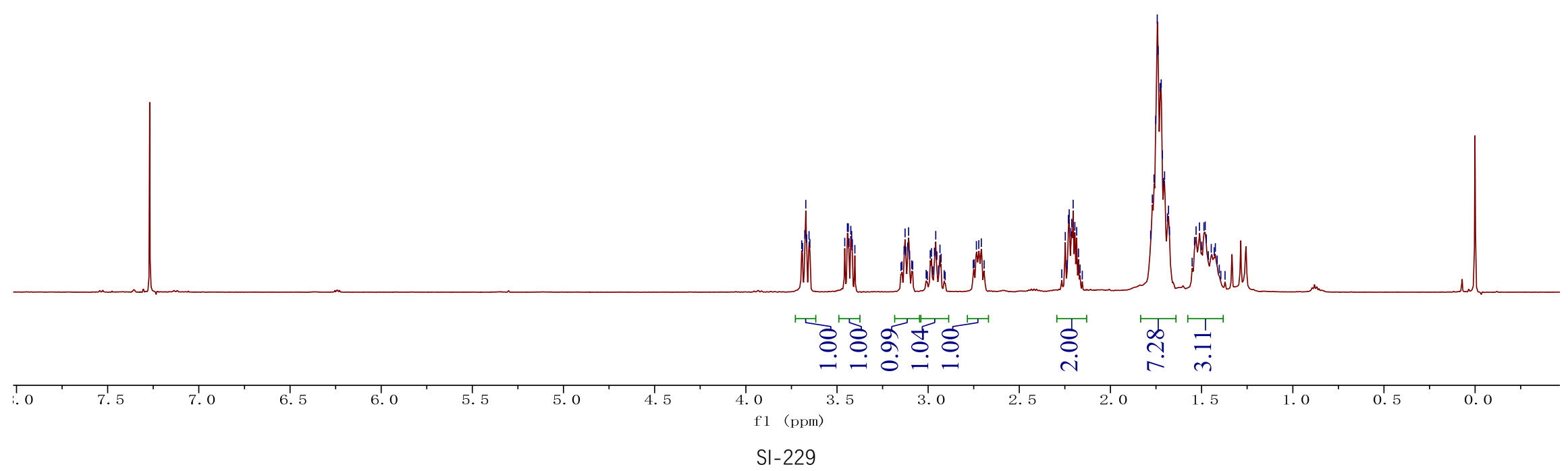



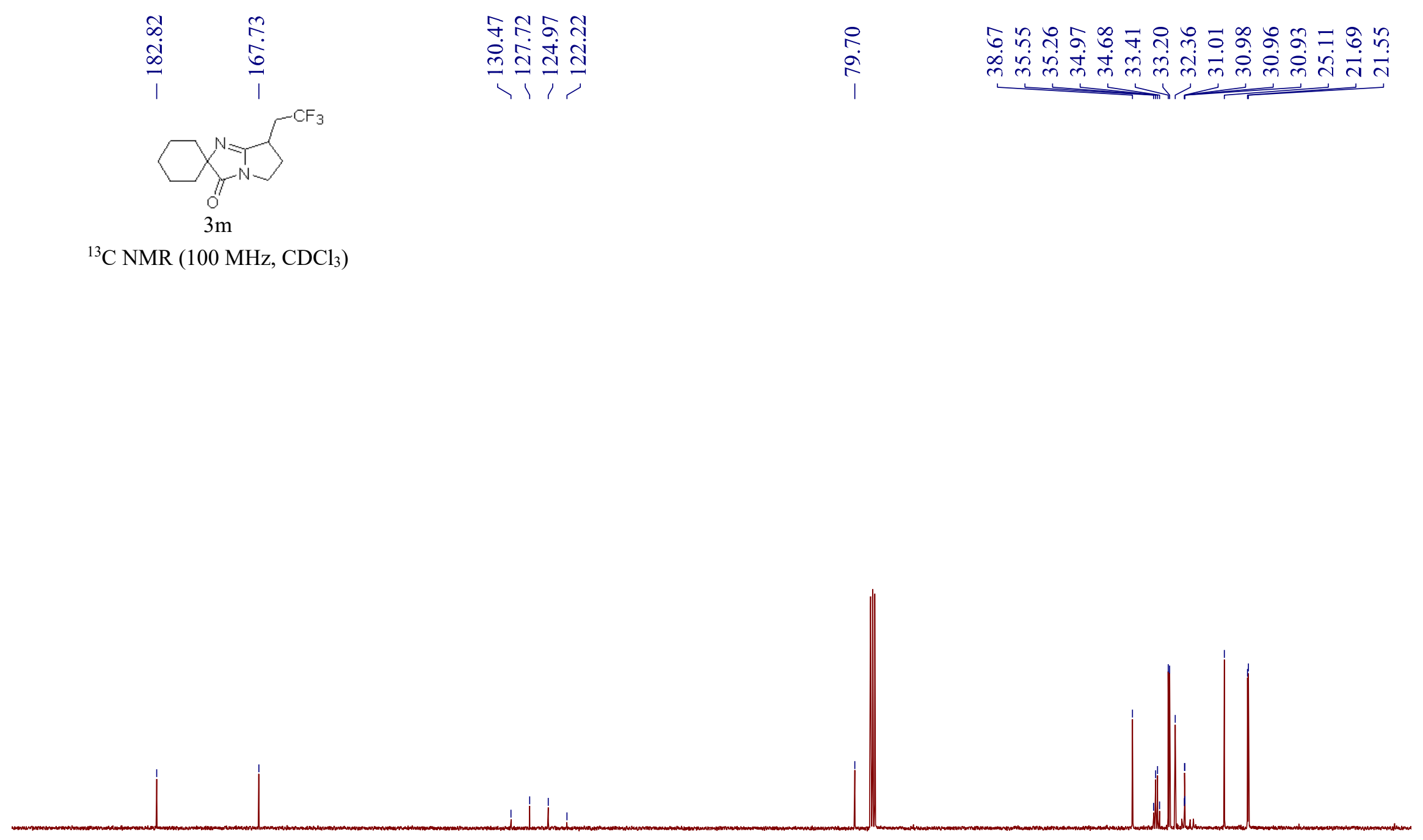


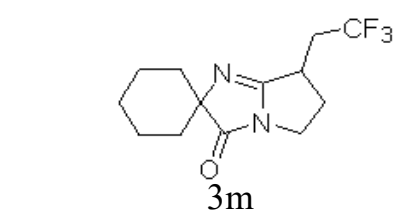

${ }^{19} \mathrm{~F}$ NMR (376 MHz, $\mathrm{CDCl}_{3}$ )

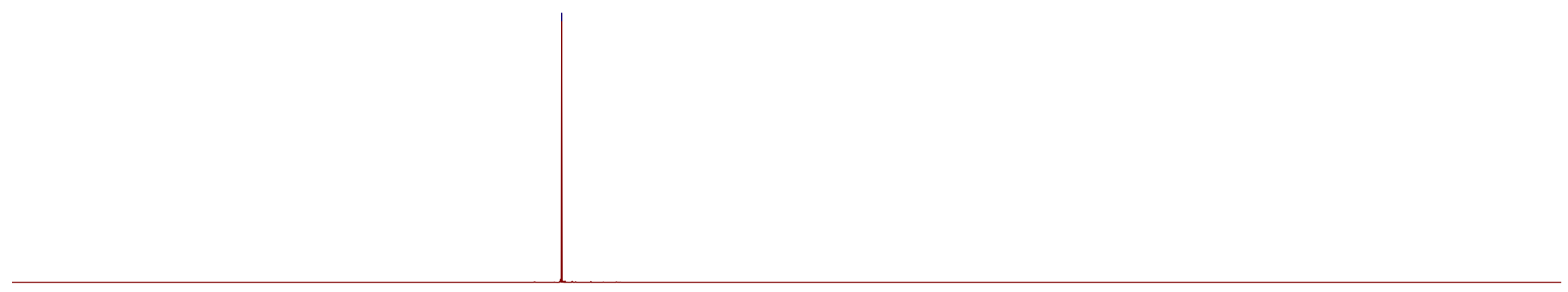




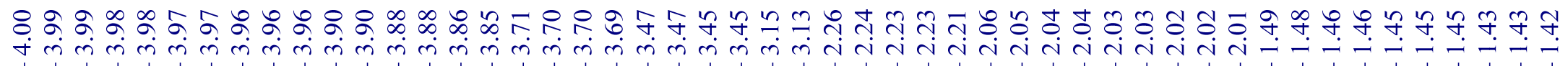

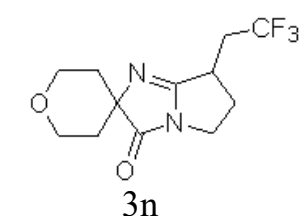

${ }^{1} \mathrm{H}$ NMR (500 MHz, $\left.\mathrm{CDCl}_{3}\right)$
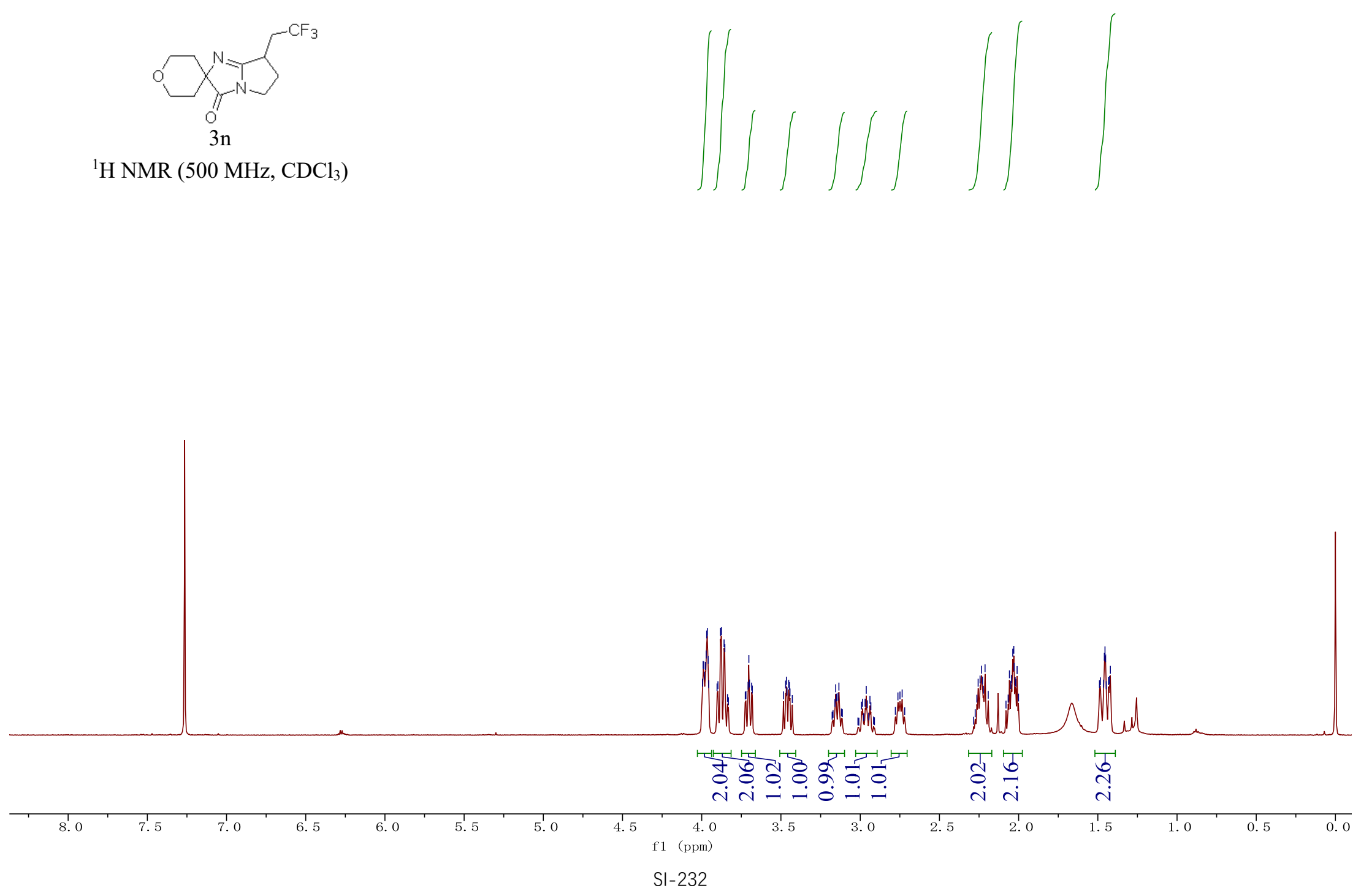


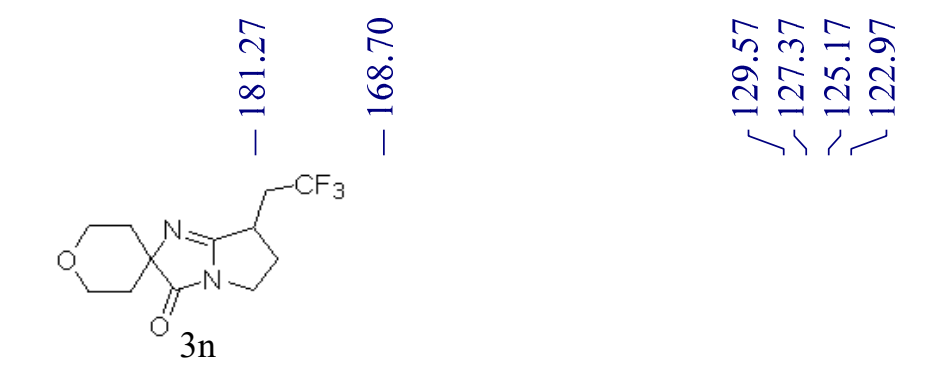

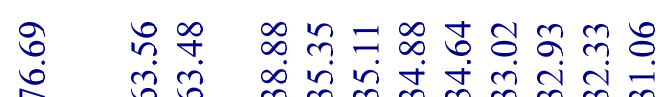

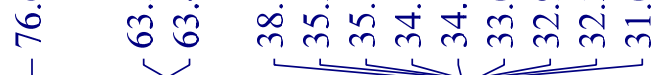

${ }^{13} \mathrm{C}$ NMR $\left(125 \mathrm{MHz}, \mathrm{CDCl}_{3}\right)$

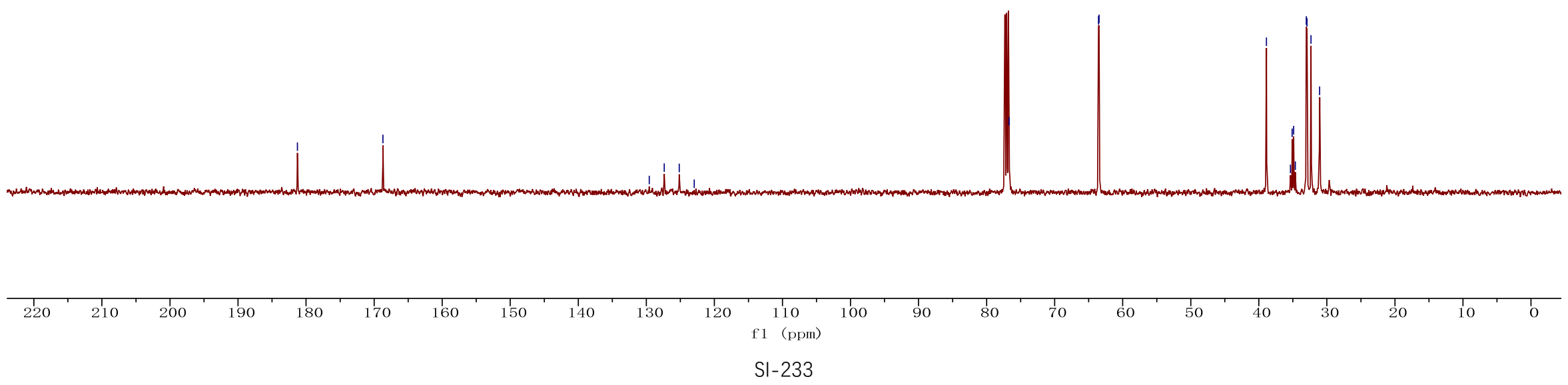


$\begin{array}{lll}\infty & \infty & 0 \\ \infty & \infty \\ \dot{0} & \mathbb{0} & \dot{f} \\ 1 & 1\end{array}$

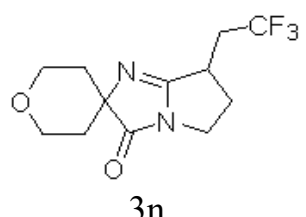

${ }^{19} \mathrm{~F}$ NMR $\left(470 \mathrm{MHz}, \mathrm{CDCl}_{3}\right)$

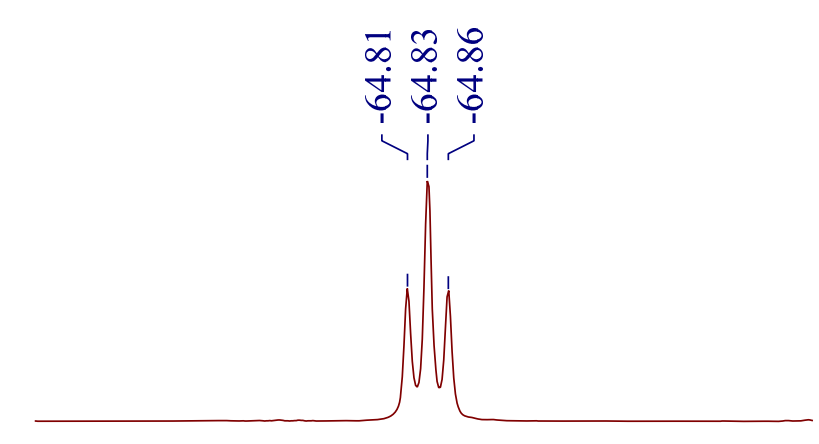

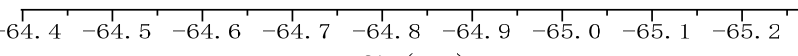

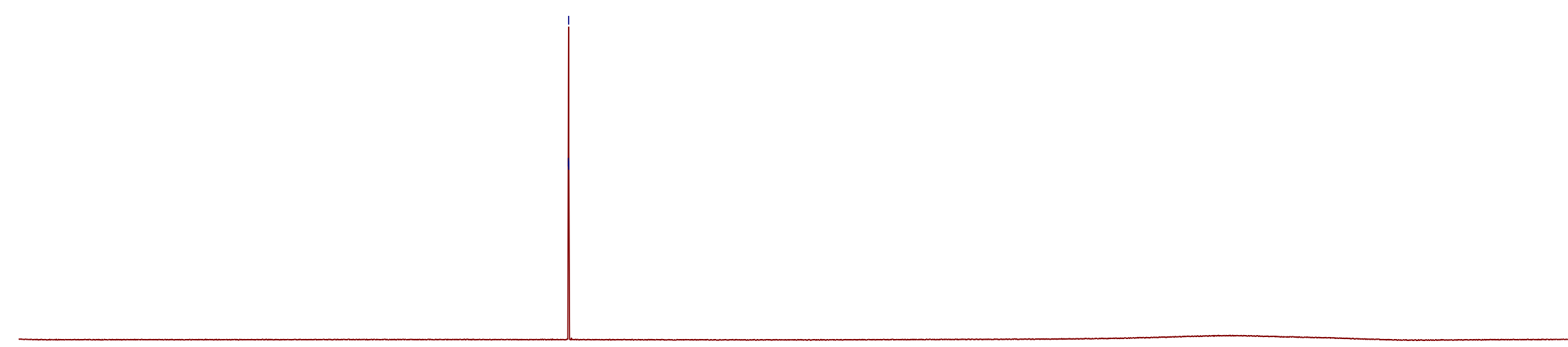

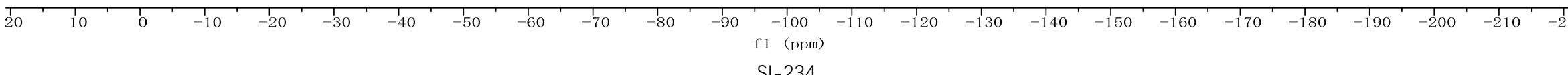




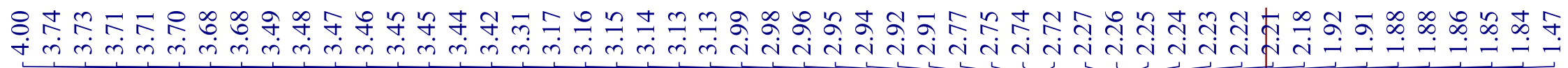

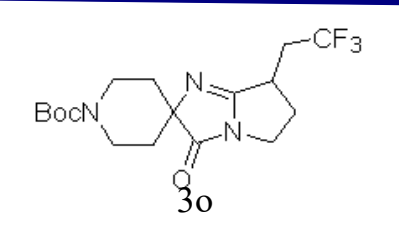

${ }^{1} \mathrm{H}$ NMR (400 MHz, $\mathrm{CDCl}_{3}$ )
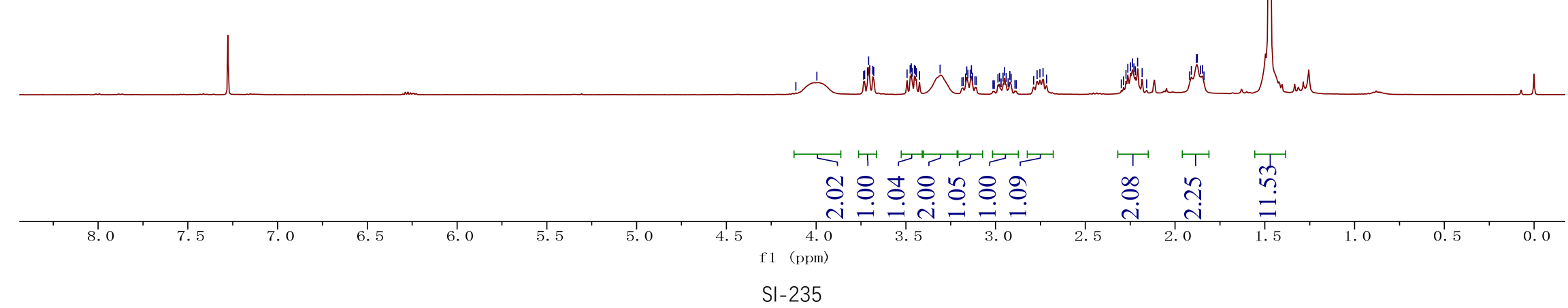

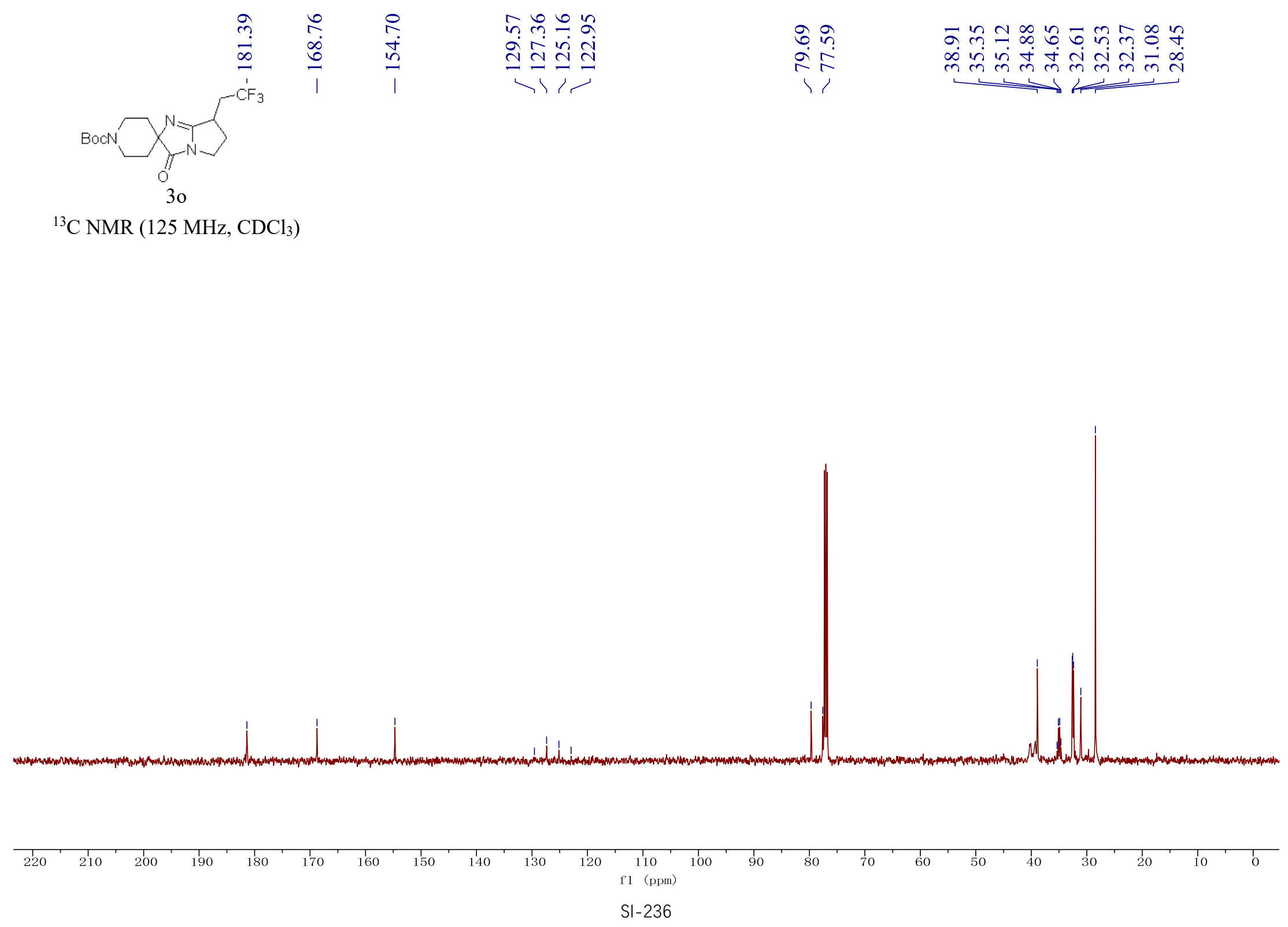


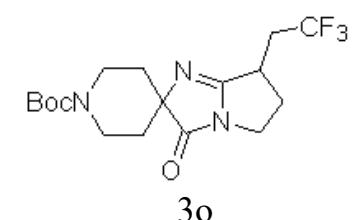

草

${ }^{19} \mathrm{~F} \mathrm{NMR}\left(470 \mathrm{MHz}, \mathrm{CDCl}_{3}\right)$

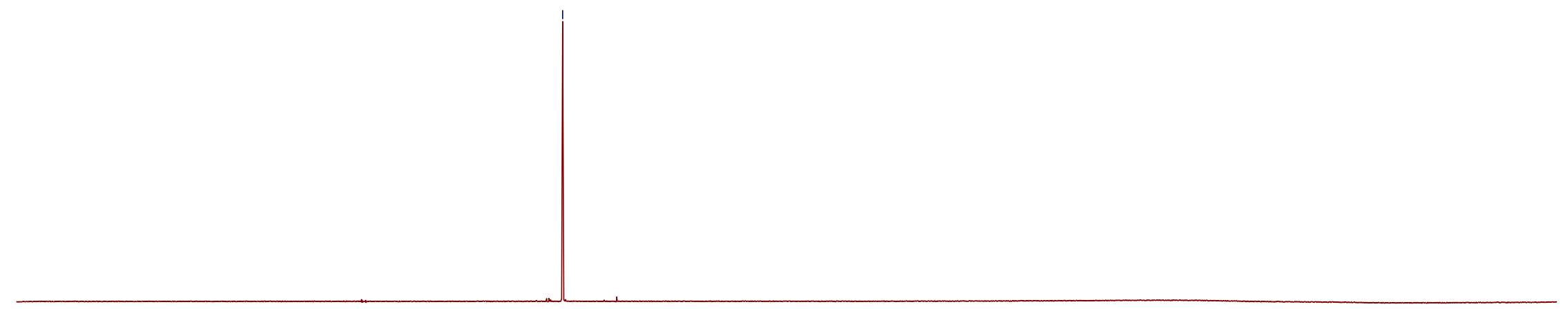

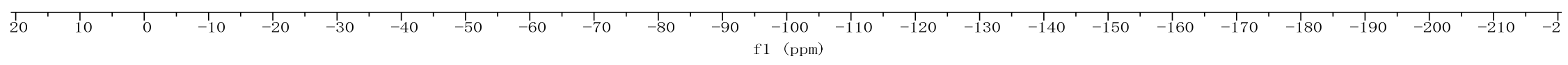
SI-237 


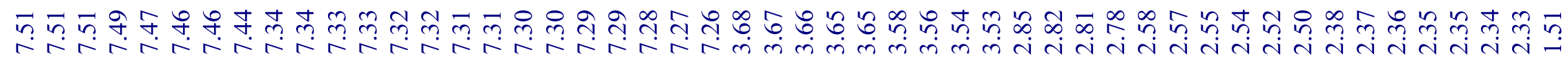

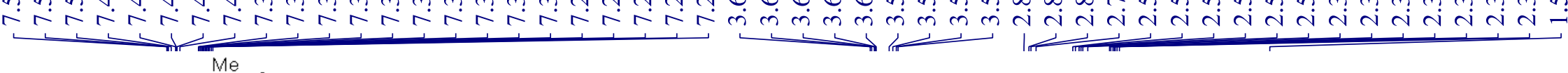
$\mathrm{Ph}_{0}^{\mathrm{N}=1} \mathrm{CF}^{\mathrm{CF}_{3}}$

$3 p$

${ }^{1} \mathrm{H}$ NMR (400 MHz, $\mathrm{CDCl}_{3}$ )

$\iint$
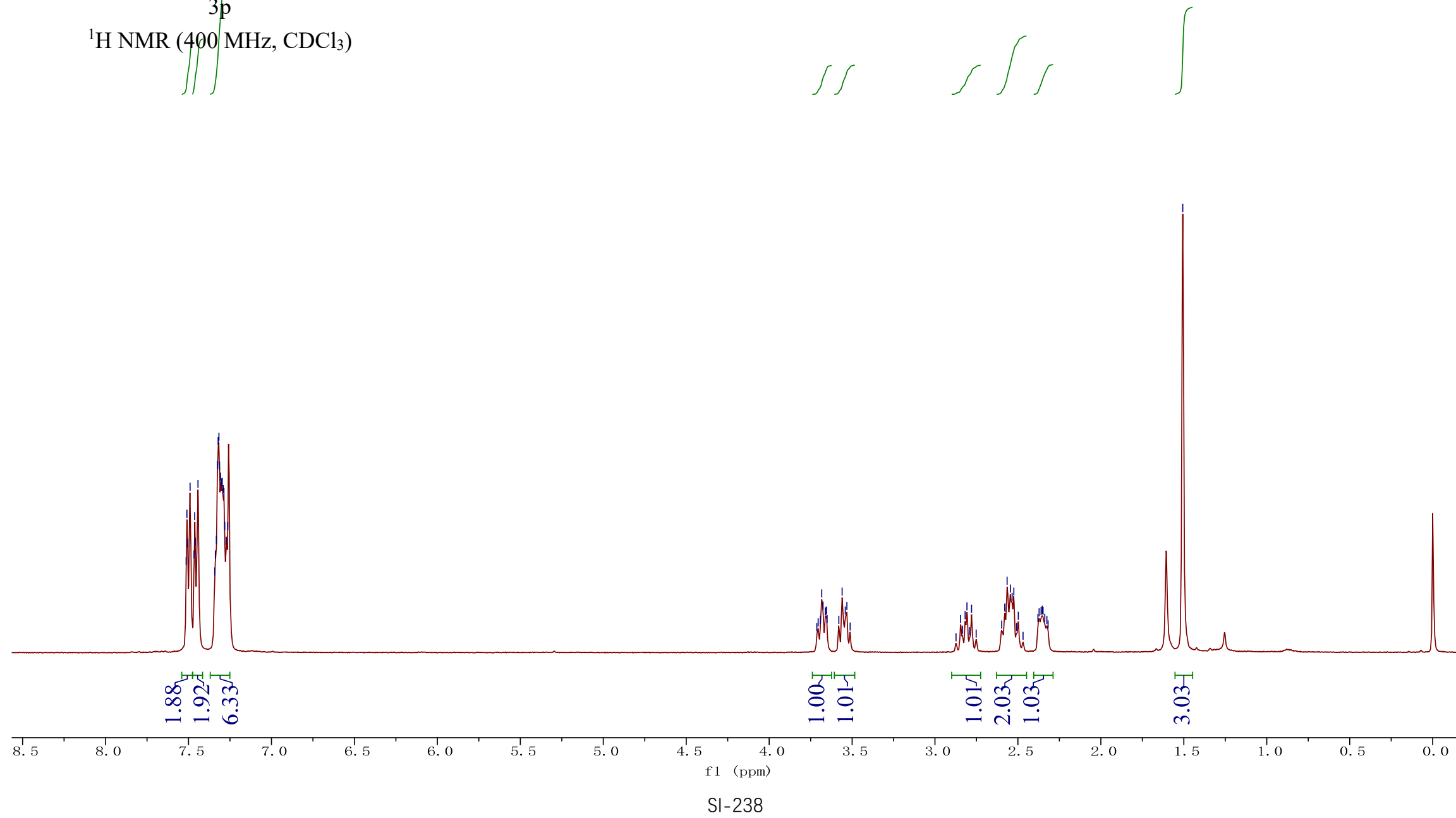

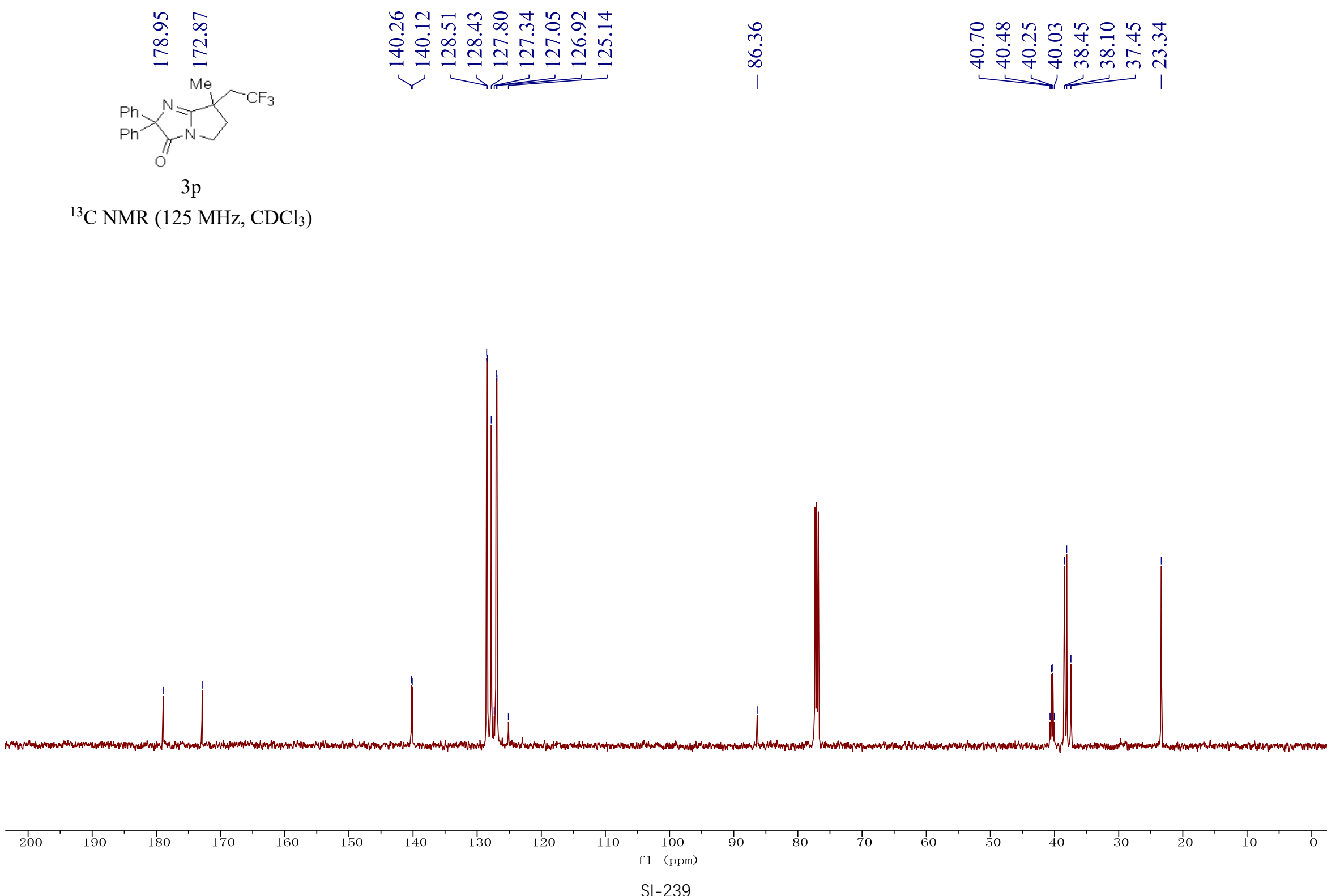
$\mathrm{Ph}^{\mathrm{Nh}} \mathrm{T}^{\mathrm{Me}}$

$3 p$

${ }^{19} \mathrm{~F}$ NMR (470 MHz, $\left.\mathrm{CDCl}_{3}\right)$

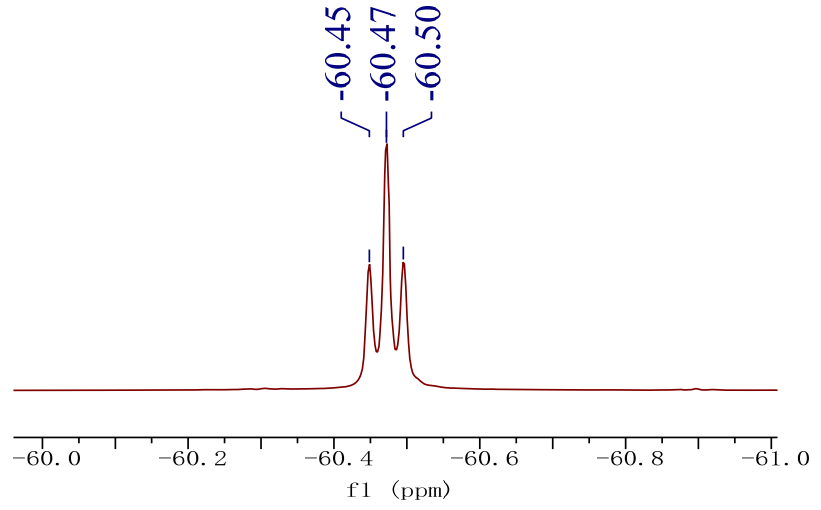




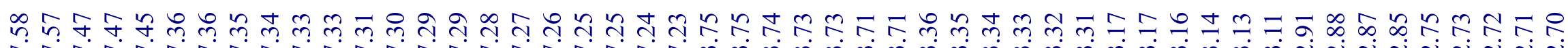

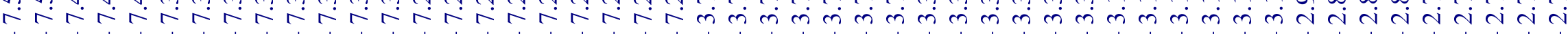
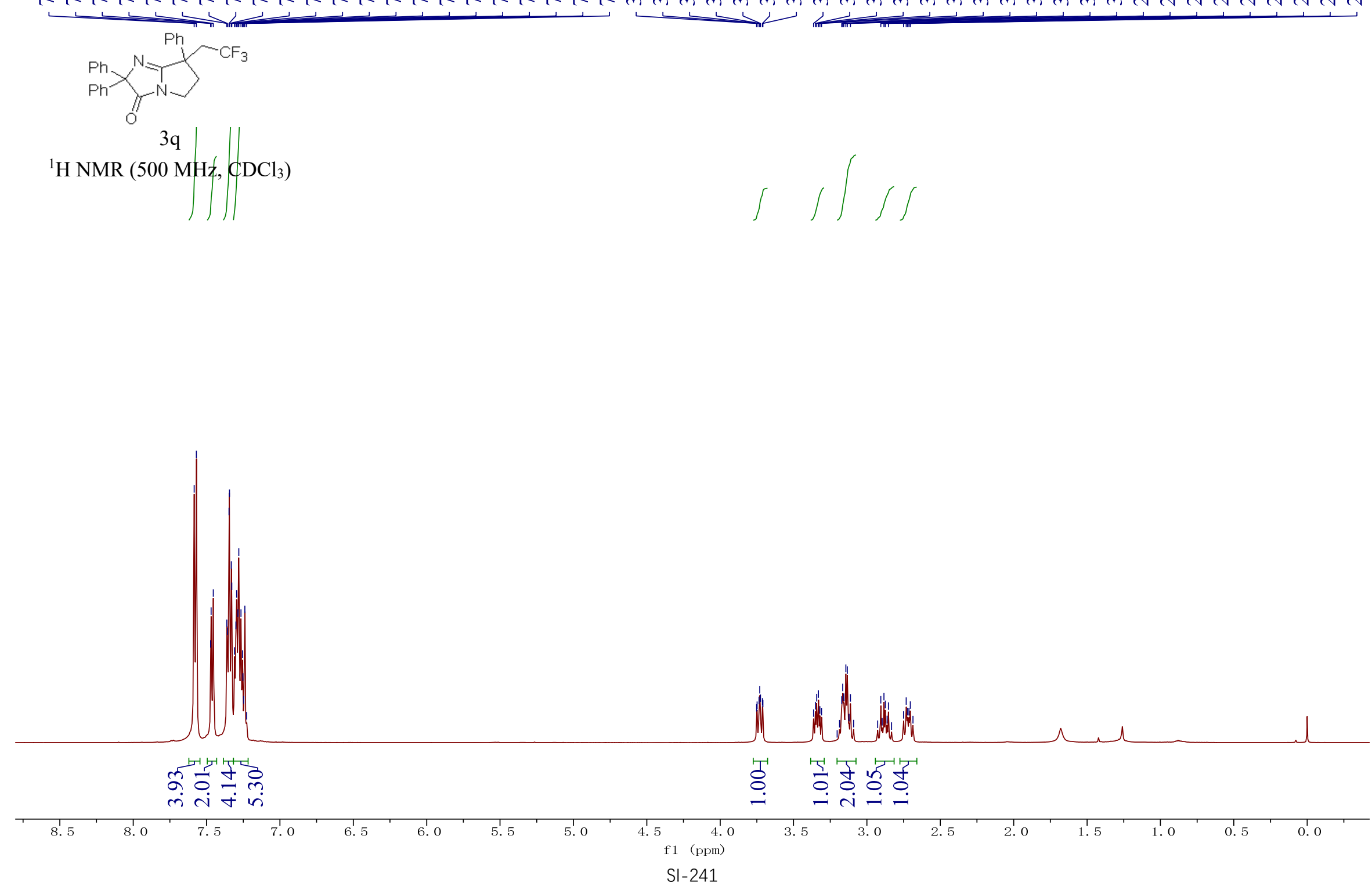

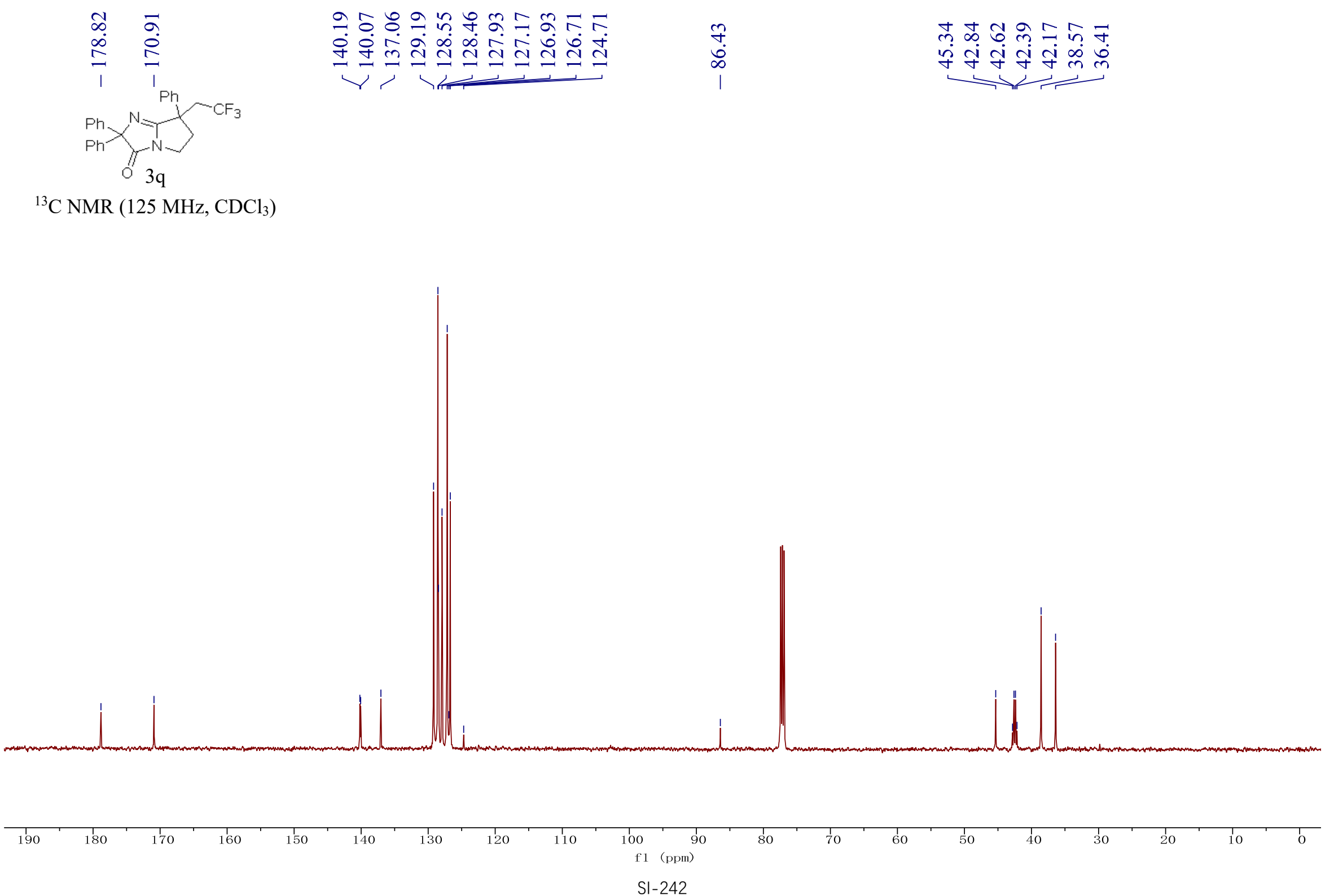
$\begin{array}{lll}m & n & \infty \\ m & m & m \\ 0 & 0 \\ 0 & 0 \\ 1 & 1 \\ 1\end{array}$

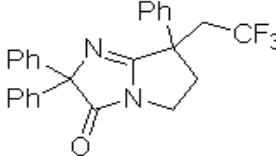

$3 q$

${ }^{19} \mathrm{~F}$ NMR $\left(470 \mathrm{MHz}, \mathrm{CDCl}_{3}\right)$
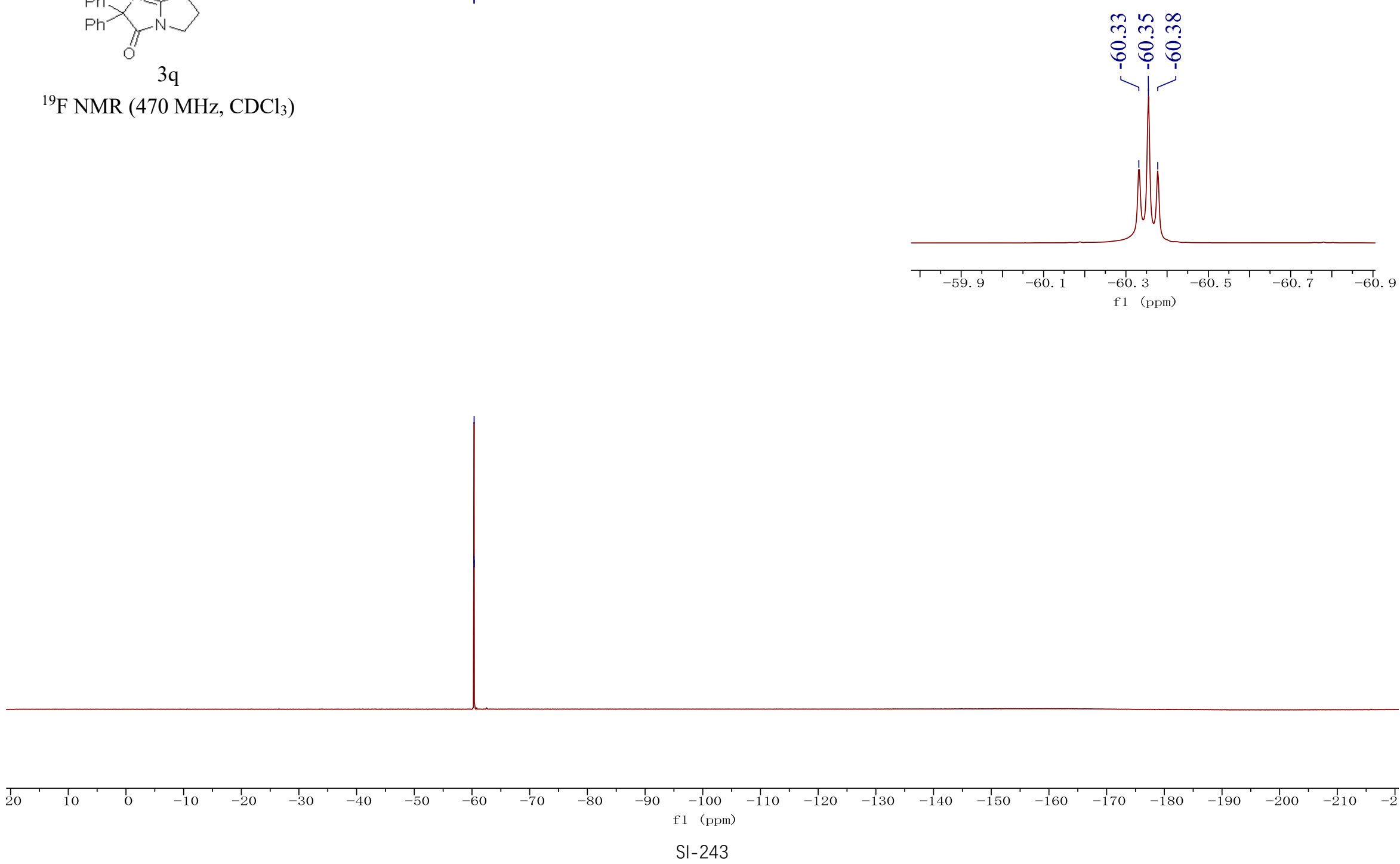


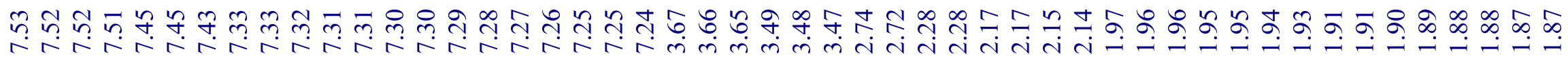
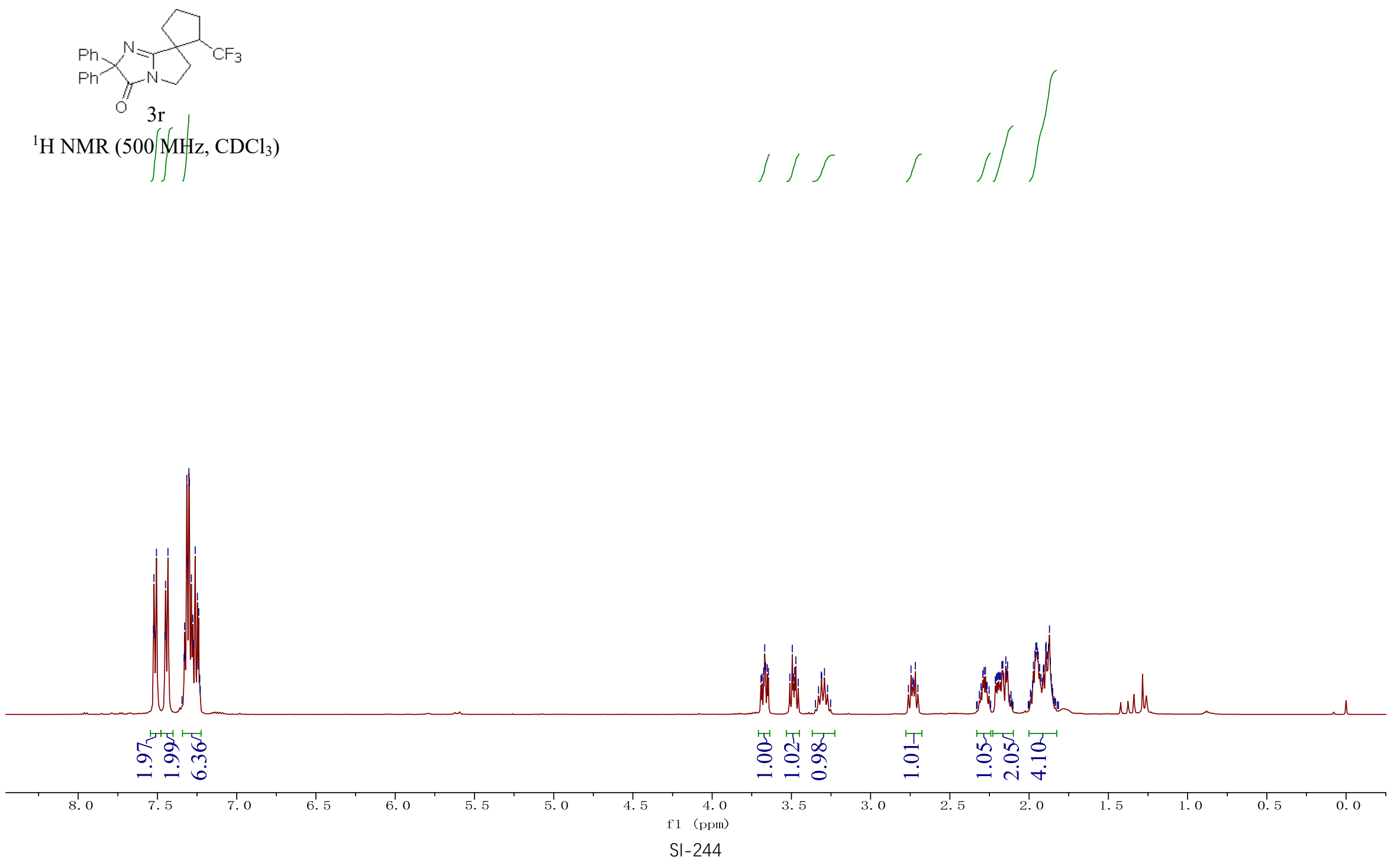

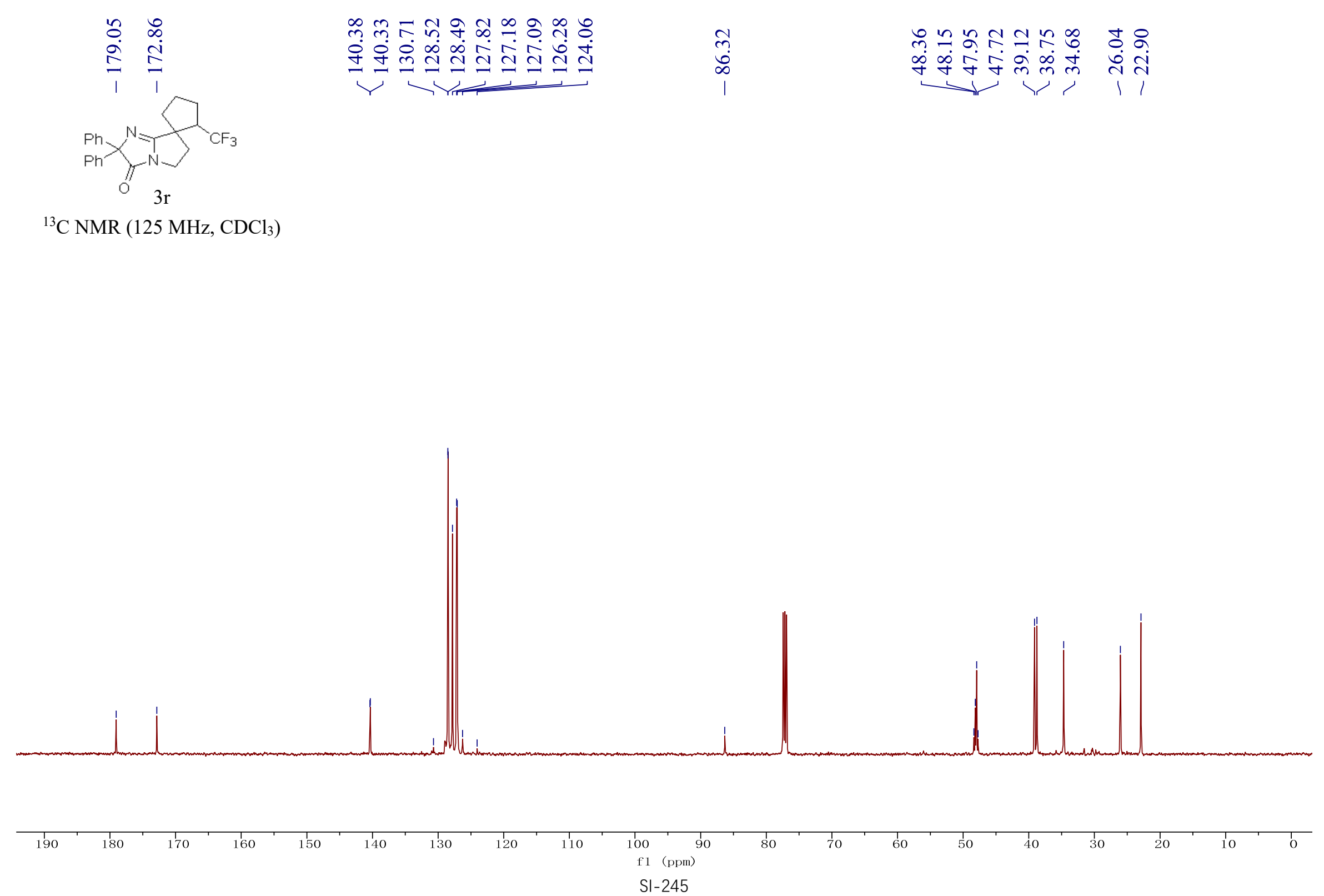
$\stackrel{+}{\sim} \stackrel{0}{\sim}$

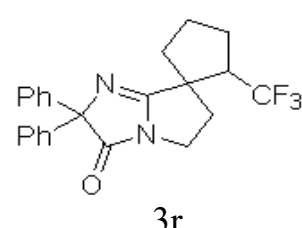

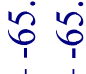

$$
3 \mathrm{r}
$$

${ }^{19} \mathrm{~F}$ NMR $\left(470 \mathrm{MHz}, \mathrm{CDCl}_{3}\right)$

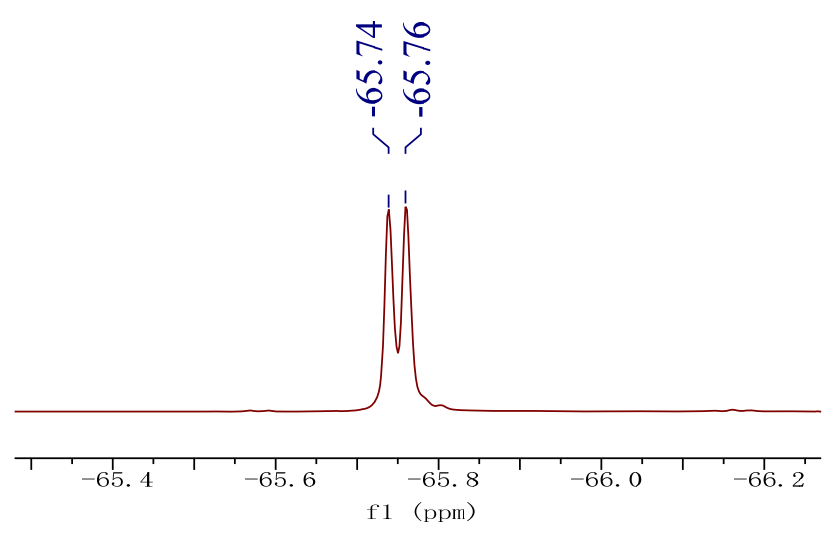

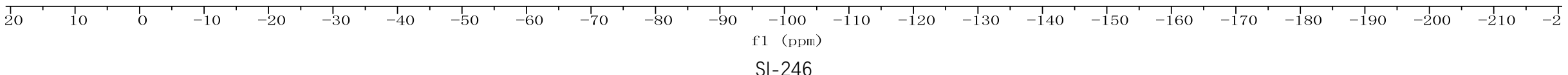




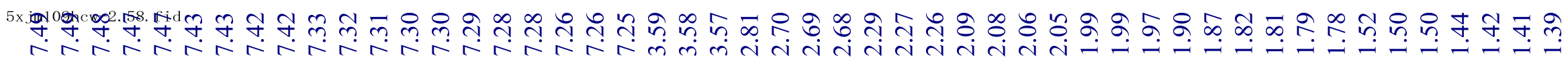

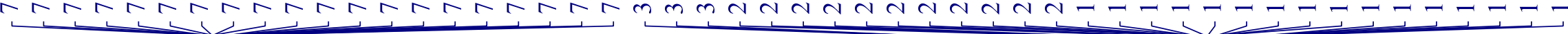

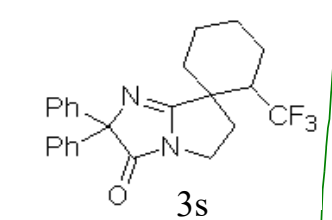

${ }^{1} \mathrm{H} \mathrm{NMR} \mathrm{(500} \mathrm{MHz,}\left(\mathrm{CDCl}_{3}\right)$

$\iint$
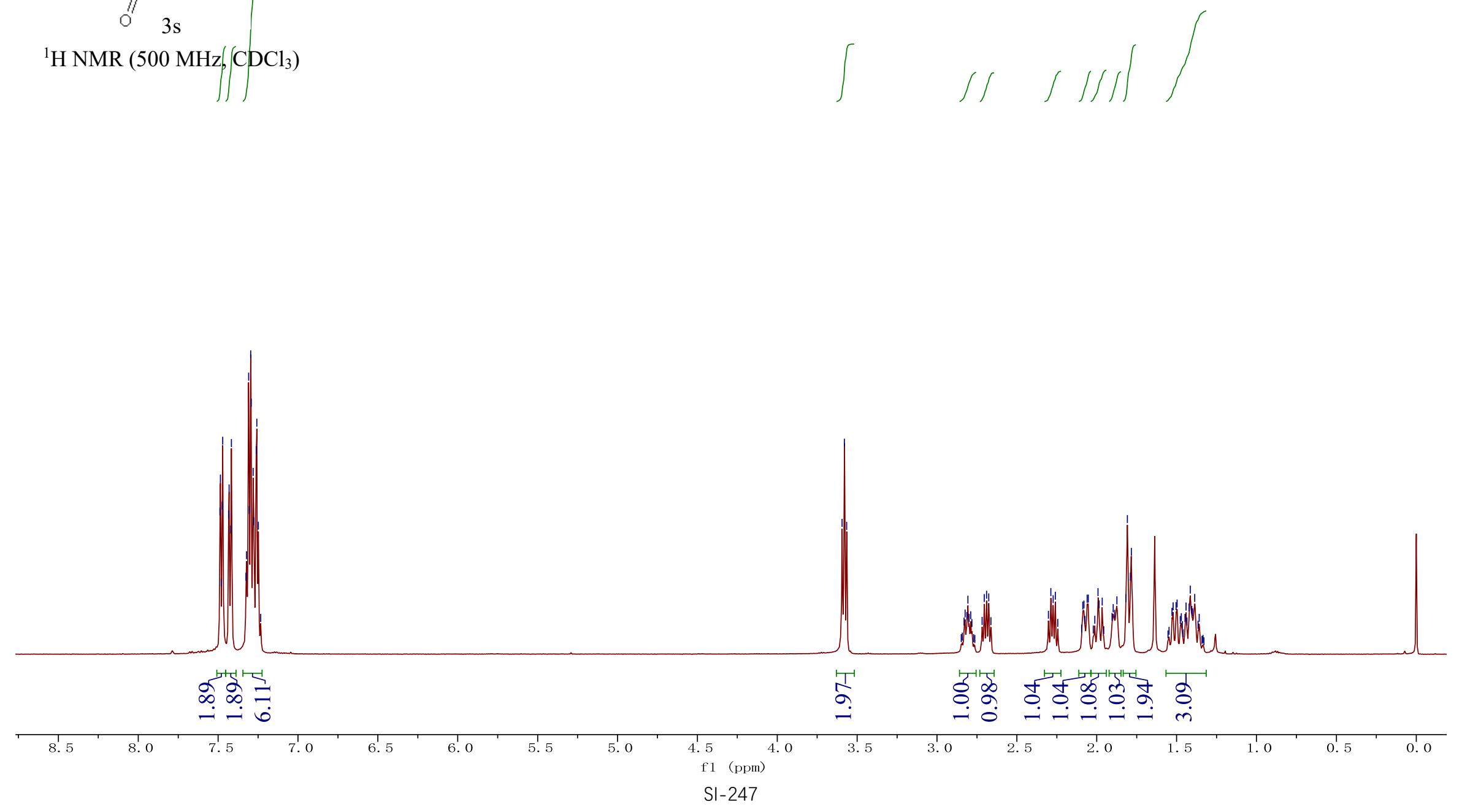
5xjm109c. 15. fid

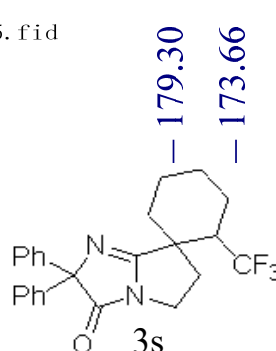

${ }^{13} \mathrm{C}$ NMR (125 MHz, $\mathrm{CDCl}_{3}$ )

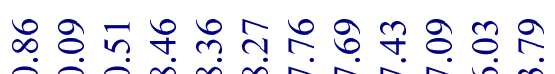

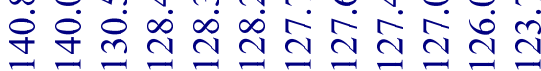

ปป⿻

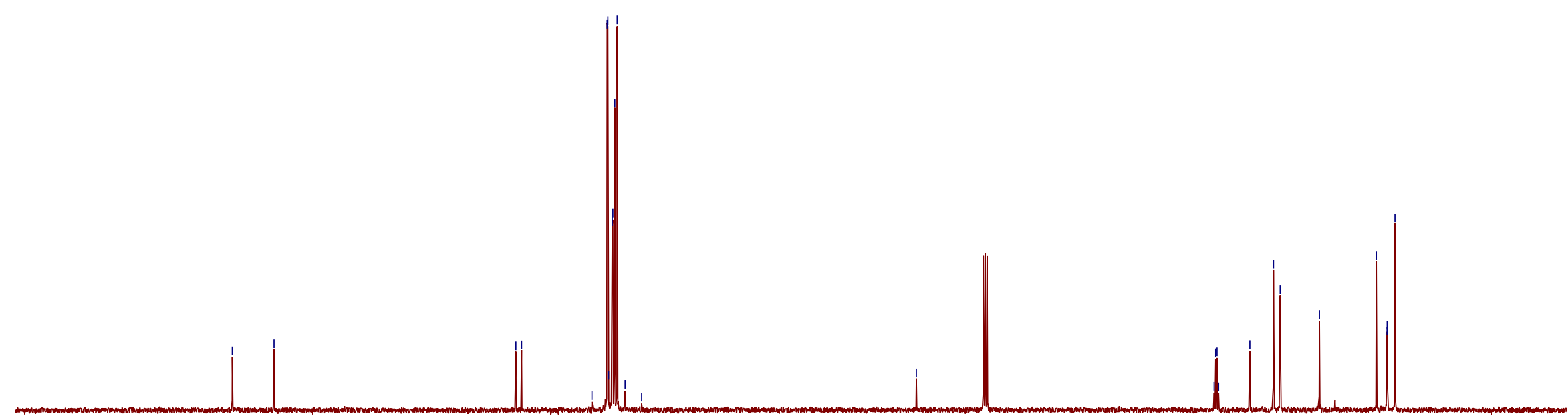

$200 \quad 190$

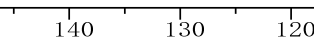


5xjm109cw-f. 55. fid

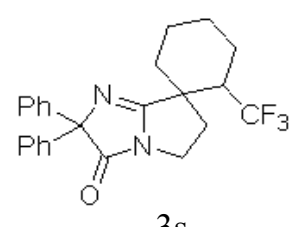

$3 \mathrm{~s}$

${ }^{19} \mathrm{~F}_{\mathrm{NMR}}\left(470 \mathrm{MHz}, \mathrm{CDCl}_{3}\right.$ ) 


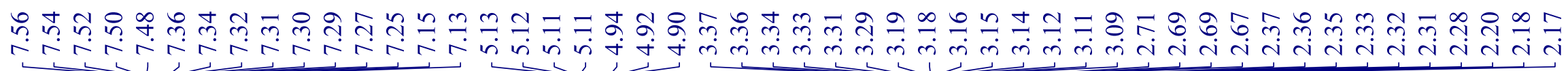
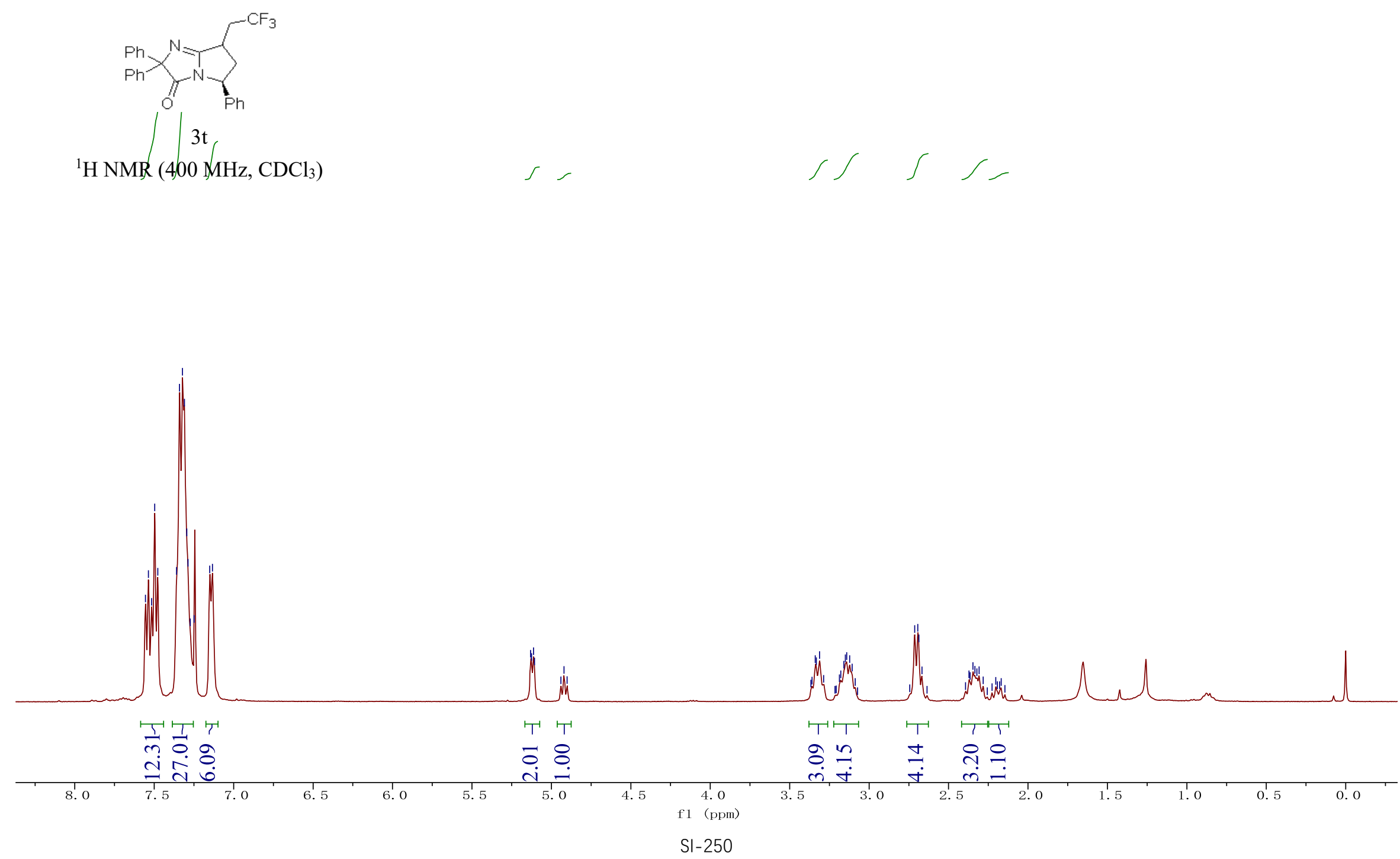


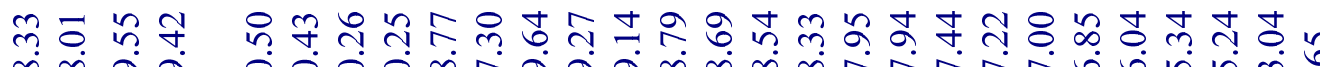

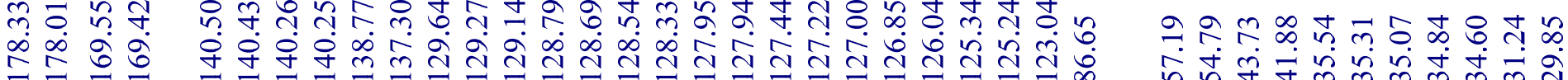

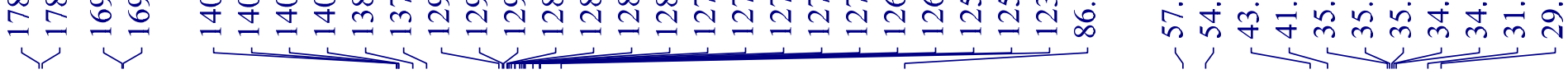

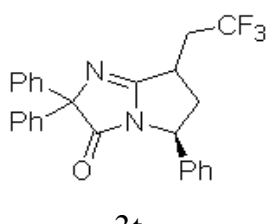

${ }^{13} \mathrm{C}$ NMR $\left(125 \mathrm{MHz}, \mathrm{CDCl}_{3}\right)$

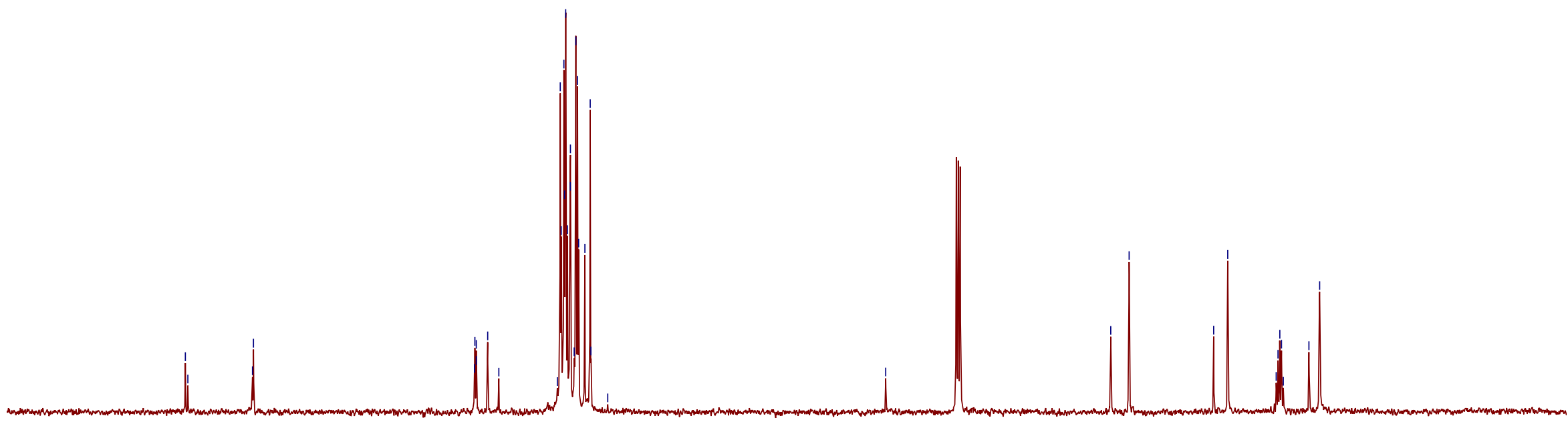

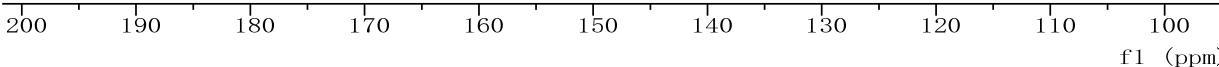

SI-251 
$\bar{\infty} \propto \infty \approx \sigma$

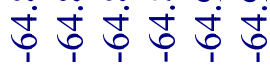

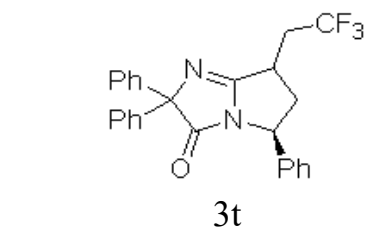

${ }^{19} \mathrm{~F}$ NMR (470 MHz, $\mathrm{CDCl}_{3}$ )
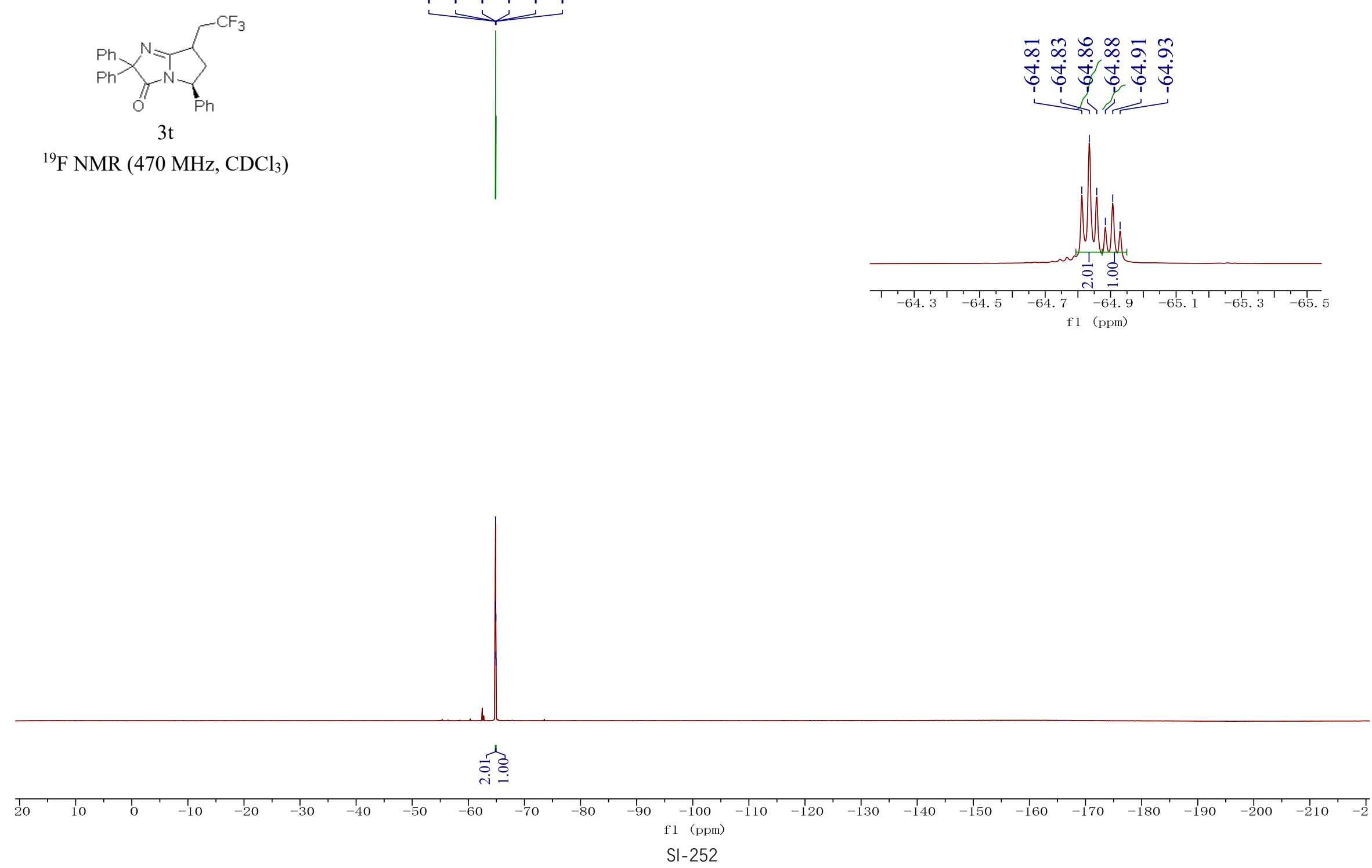


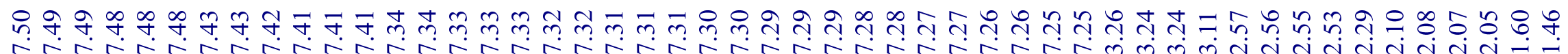

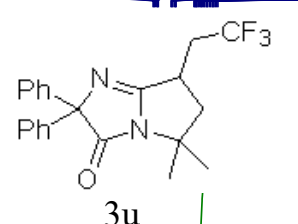

${ }^{1} \mathrm{H}$ NMR (500 MHz, $\left.\mathrm{CDCl}_{3}\right)$
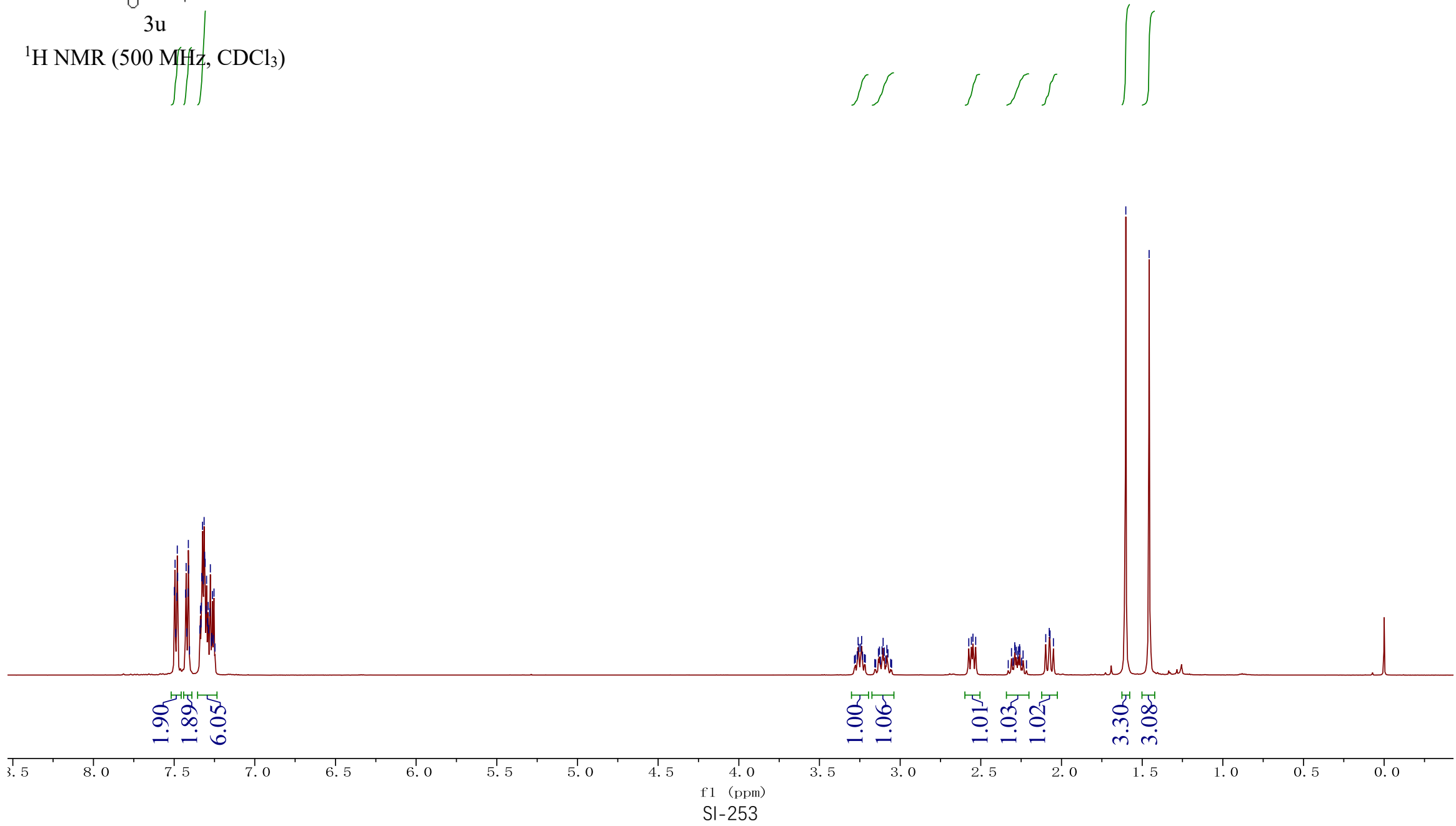


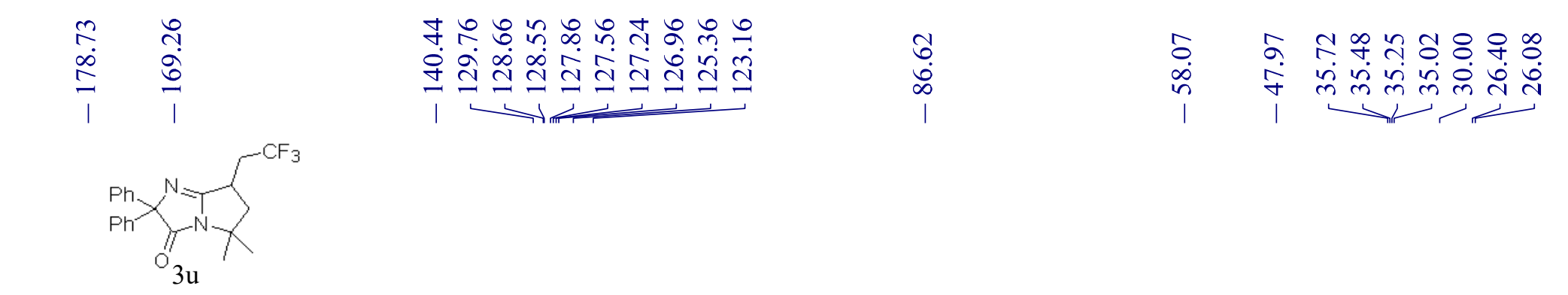

${ }^{13} \mathrm{C}$ NMR (125 MHz, $\mathrm{CDCl}_{3}$ )

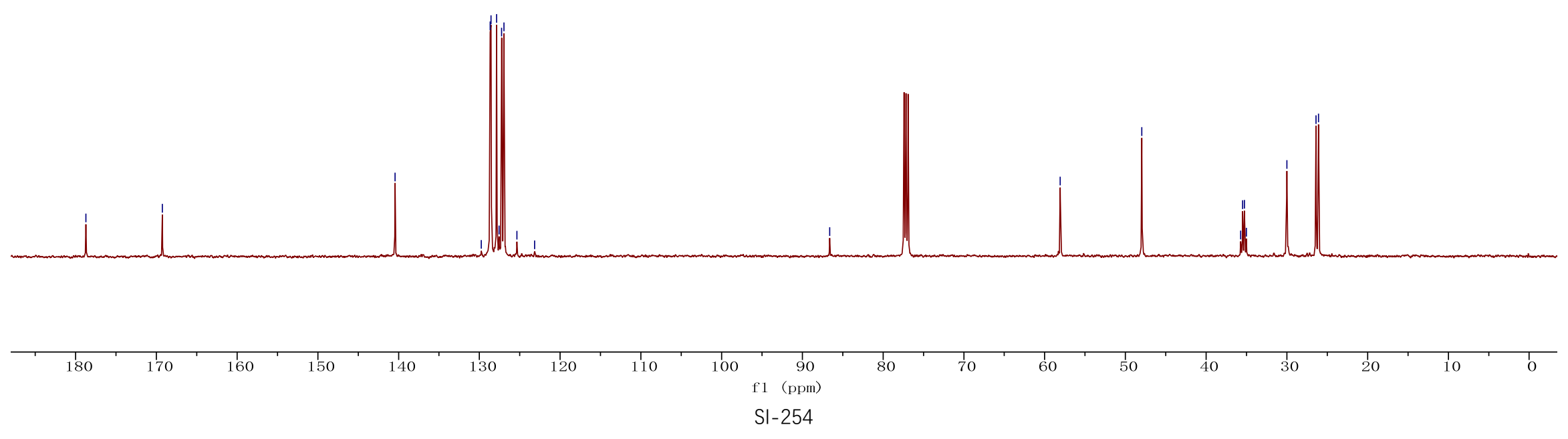


สุ

守 守 守

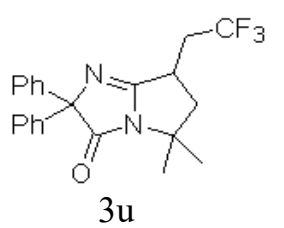

i

$3 \mathrm{u}$

${ }^{19} \mathrm{~F}$ NMR (470 MHz, $\left.\mathrm{CDCl}_{3}\right)$
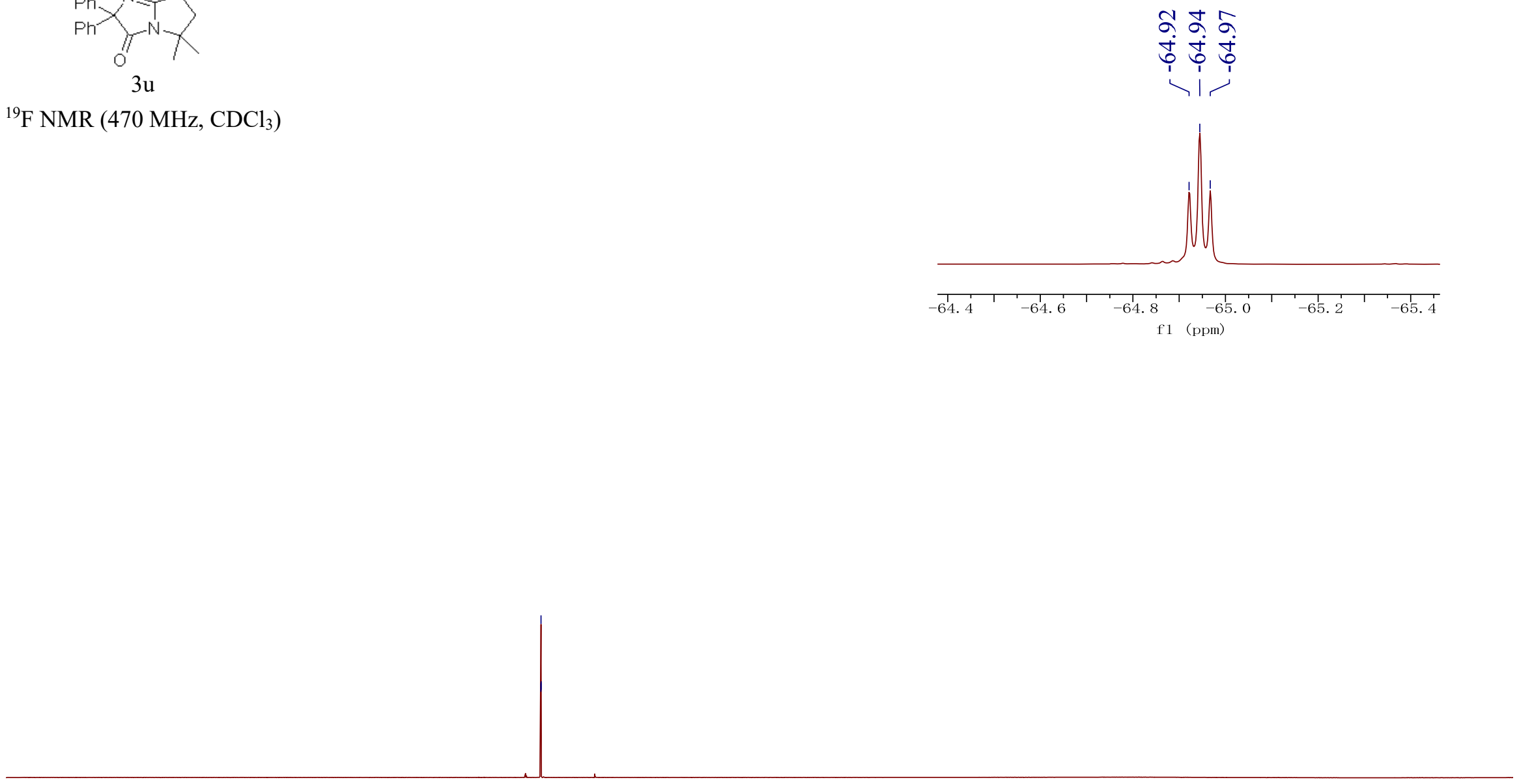


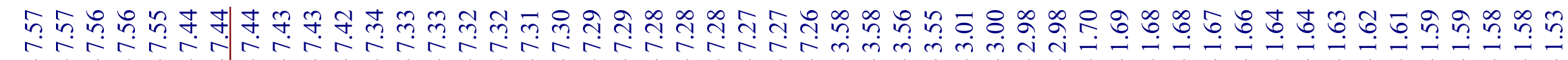

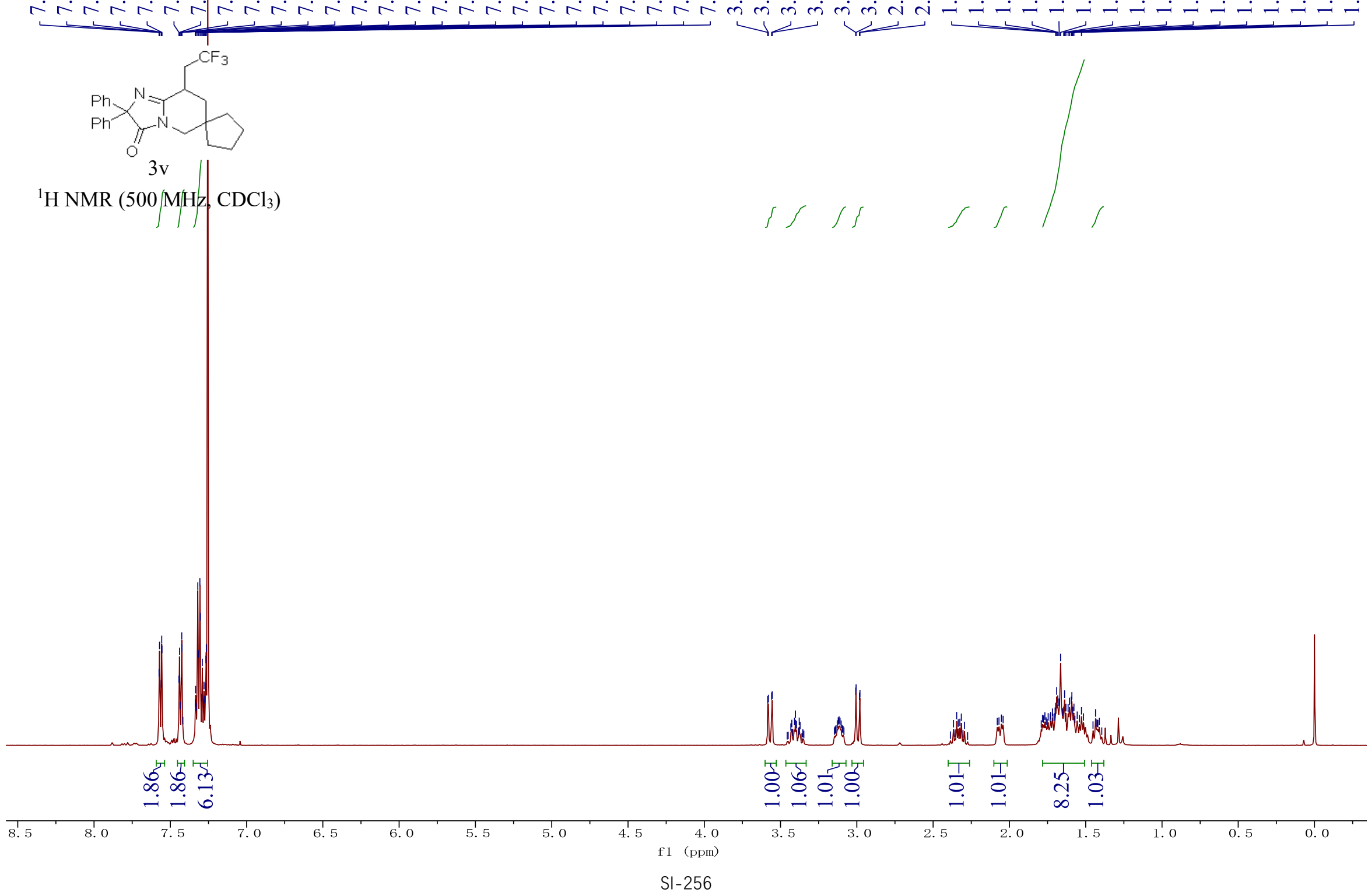



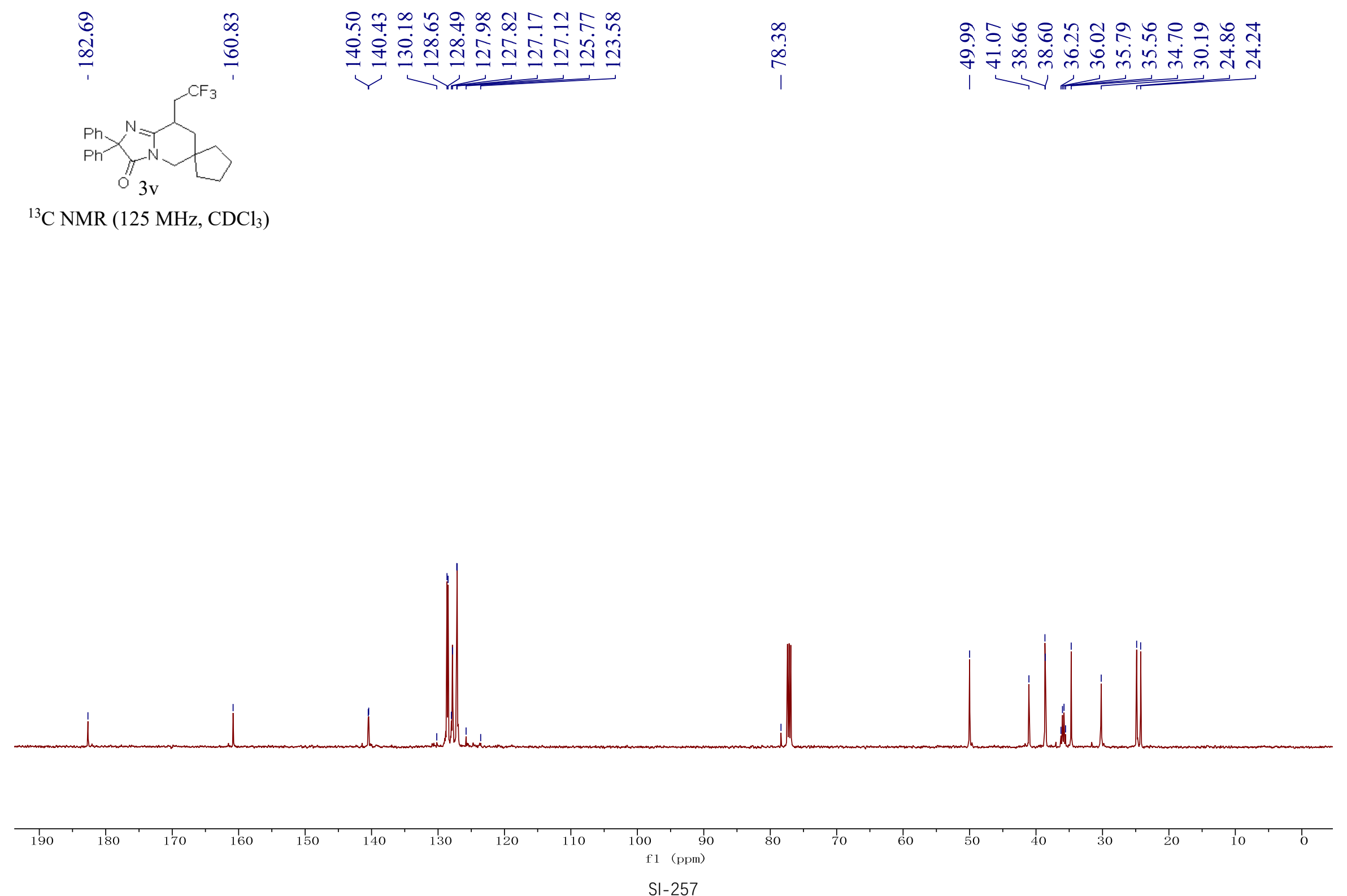
贷

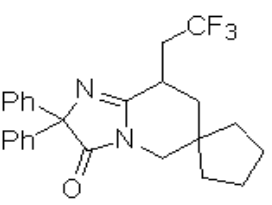

$3 \mathrm{v}$

${ }^{19} \mathrm{~F}$ NMR $\left(470 \mathrm{MHz}, \mathrm{CDCl}_{3}\right)$

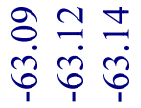

111

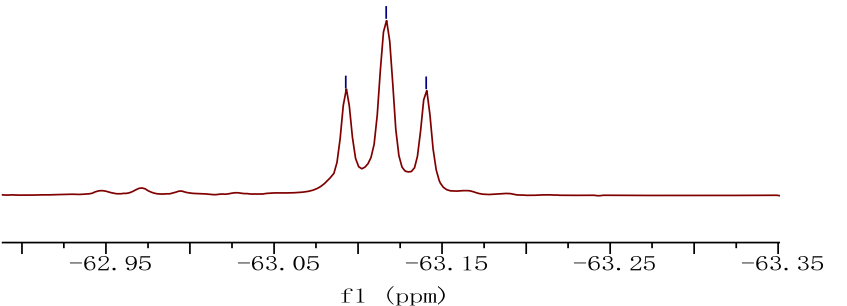

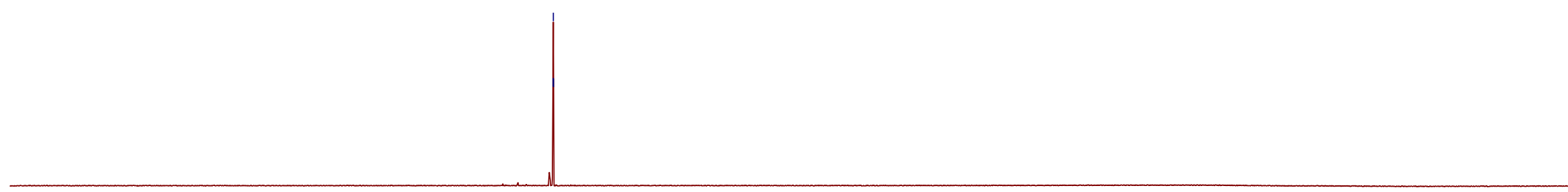




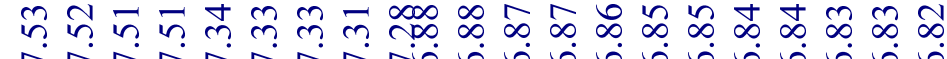

तN

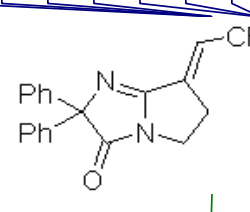

4

${ }^{1} \mathrm{H} \mathrm{NMR}\left(500 \mathrm{MHz}, \mathrm{CDCl}_{3}\right)$ f $f$

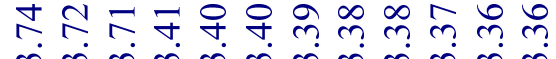

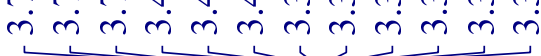

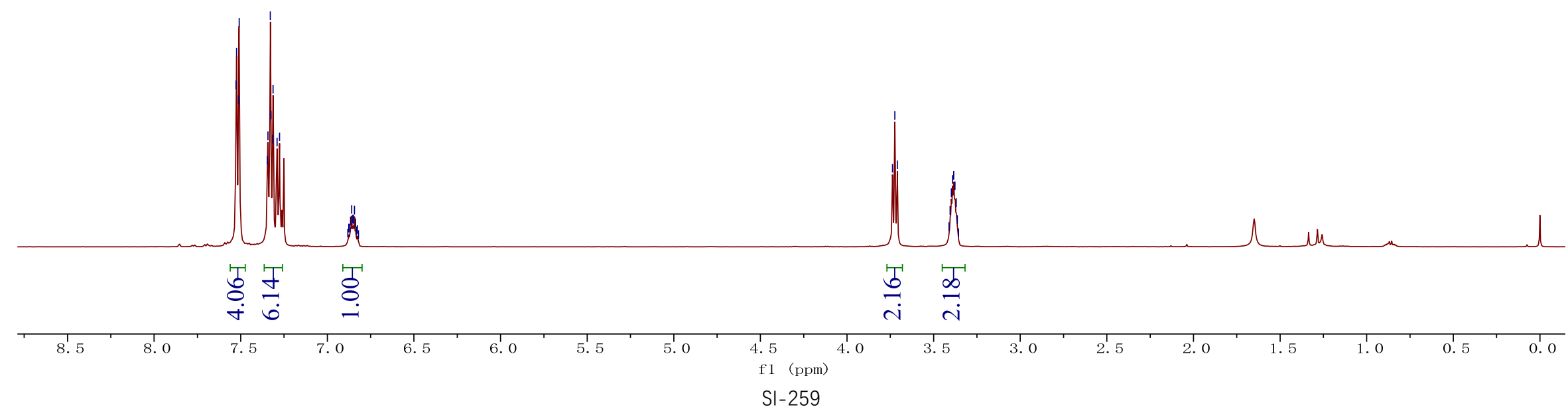



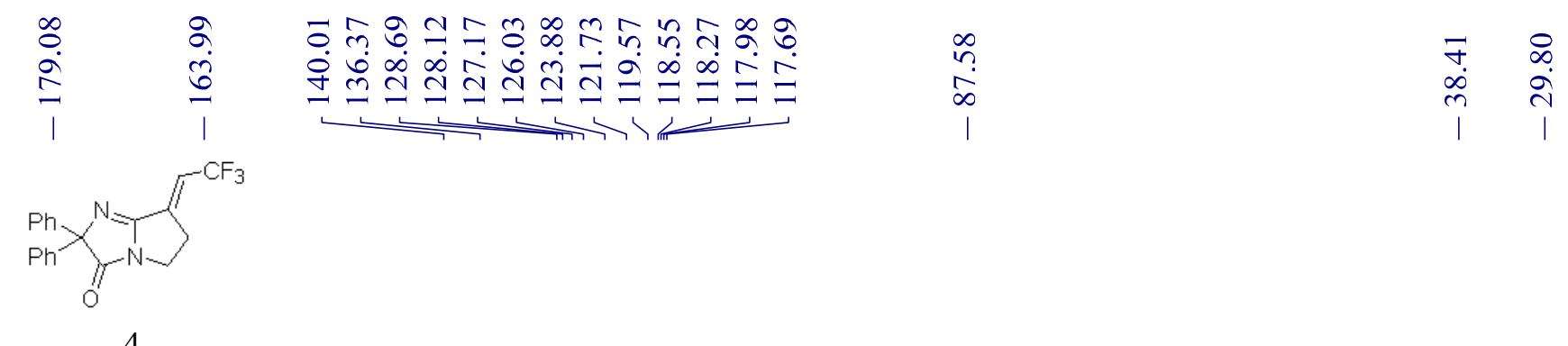

${ }^{13} \mathrm{C}$ NMR (125 MHz, $\mathrm{CDCl}_{3}$ )

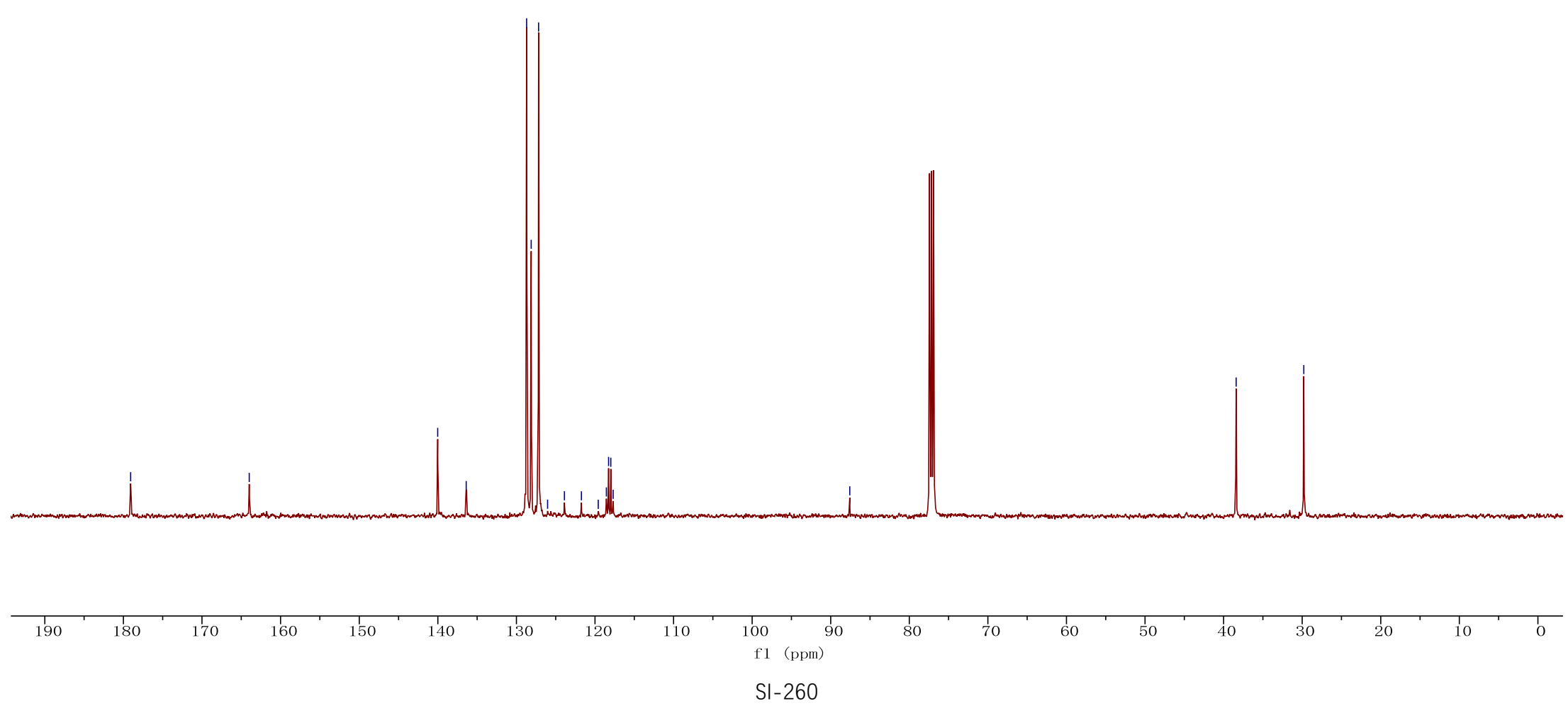




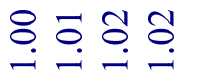

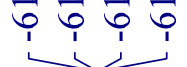

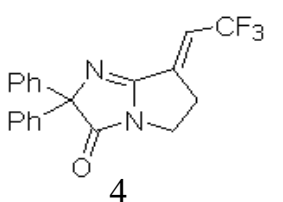

${ }^{19} \mathrm{~F}$ NMR (470 MHz, $\left.\mathrm{CDCl}_{3}\right)$

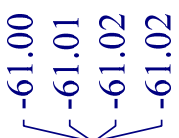

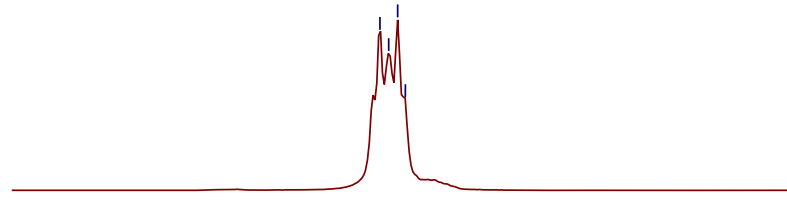

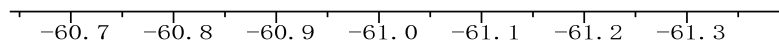
f1 (ppm)

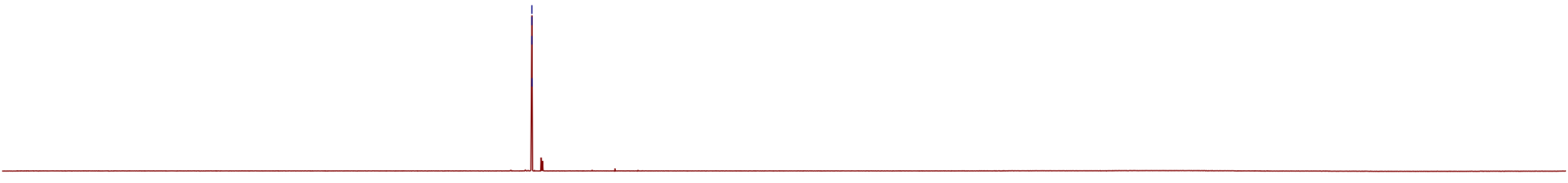




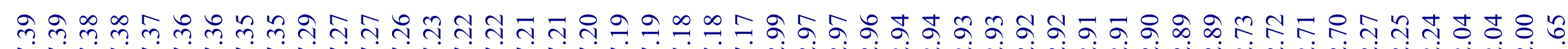

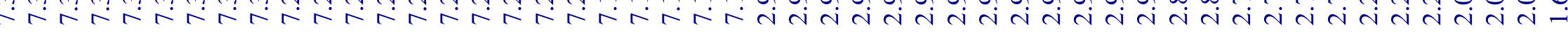

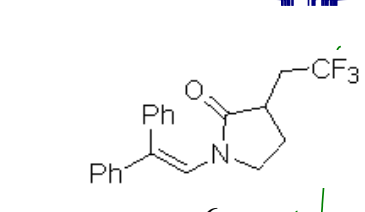

$6 \mathrm{a}$

${ }^{1} \mathrm{H}$ NMR (400 MHz, $\left.\mathrm{CDCl}_{3}\right)$

T.
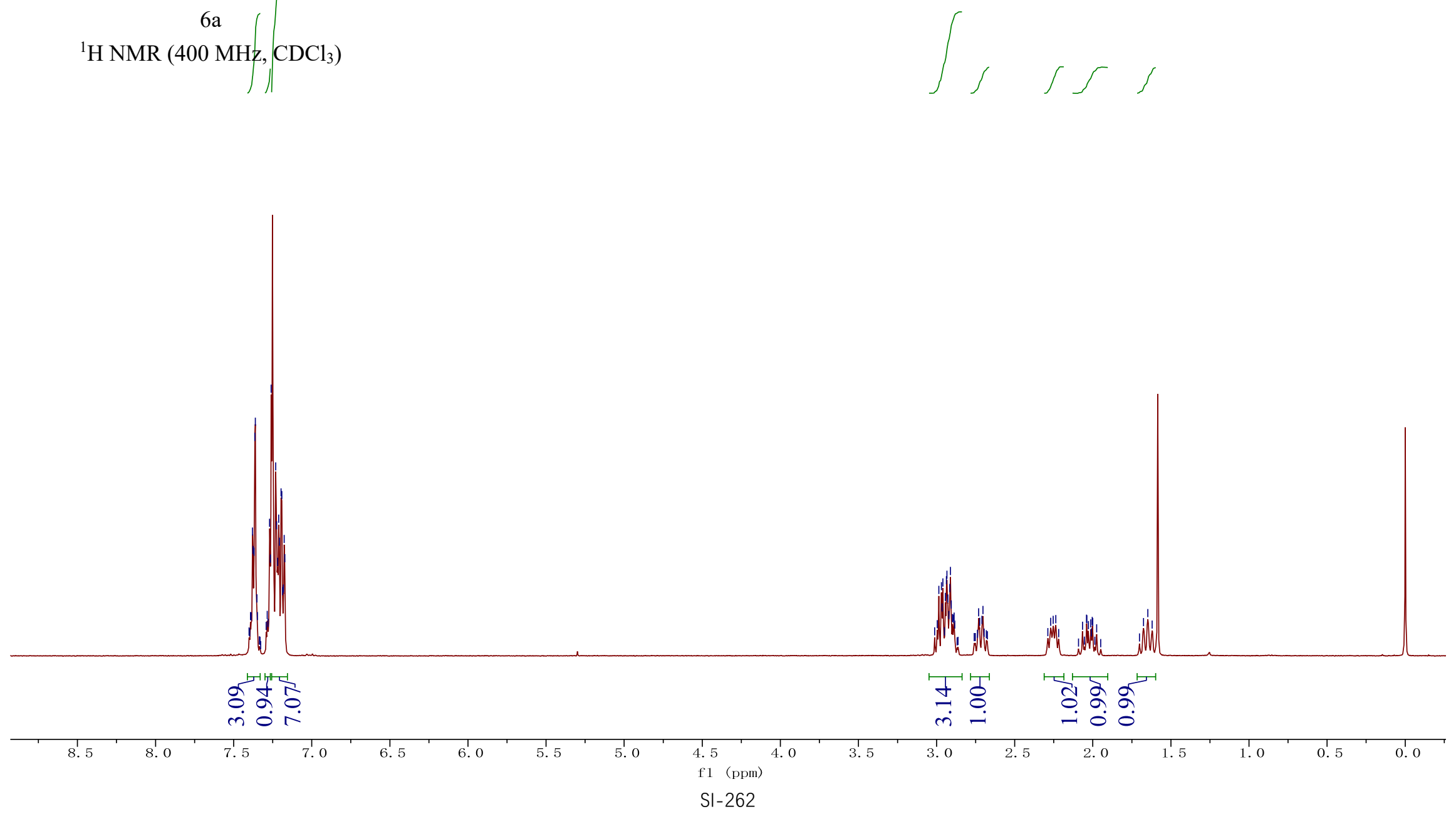


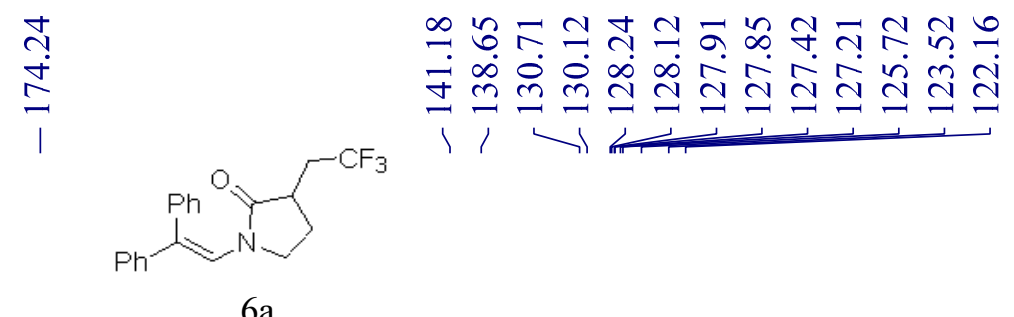

ำ mำ

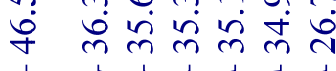

${ }^{13} \mathrm{C}$ NMR $\left(125 \mathrm{MHz}, \mathrm{CDCl}_{3}\right)$

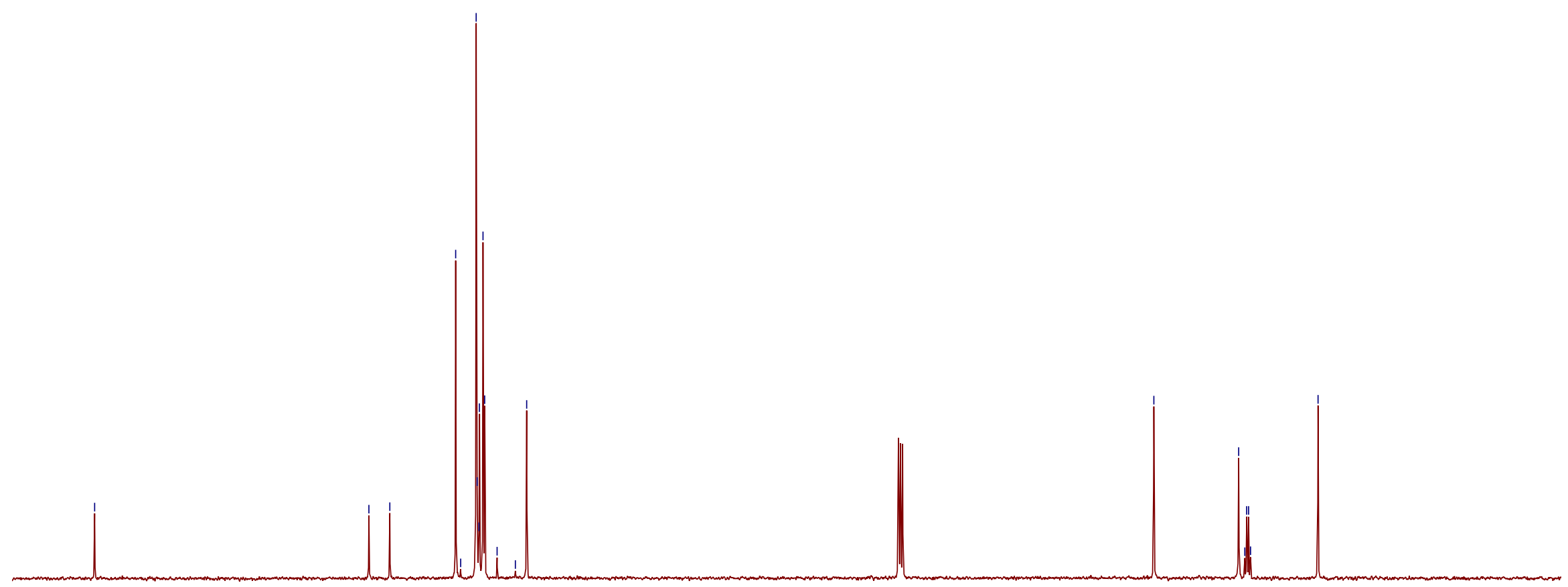




\section{\& \\ 帒}

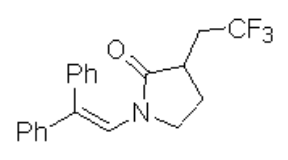

$6 \mathrm{a}$

${ }^{19} \mathrm{~F}$ NMR $\left(470 \mathrm{MHz}, \mathrm{CDCl}_{3}\right)$

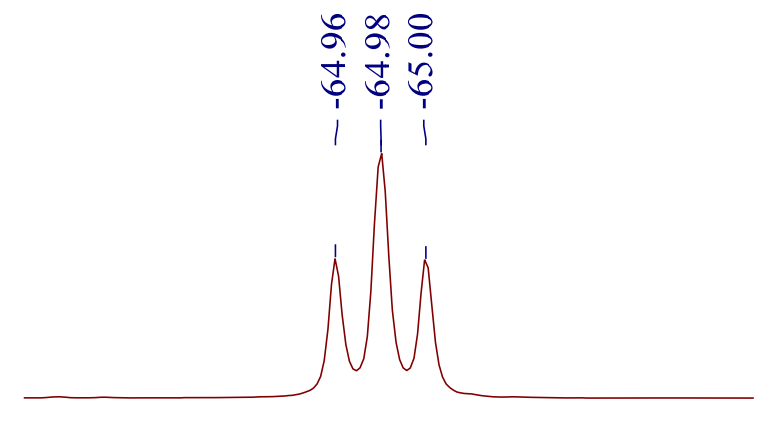

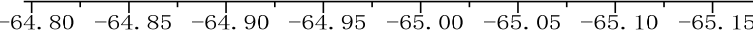
f1 (ppm)

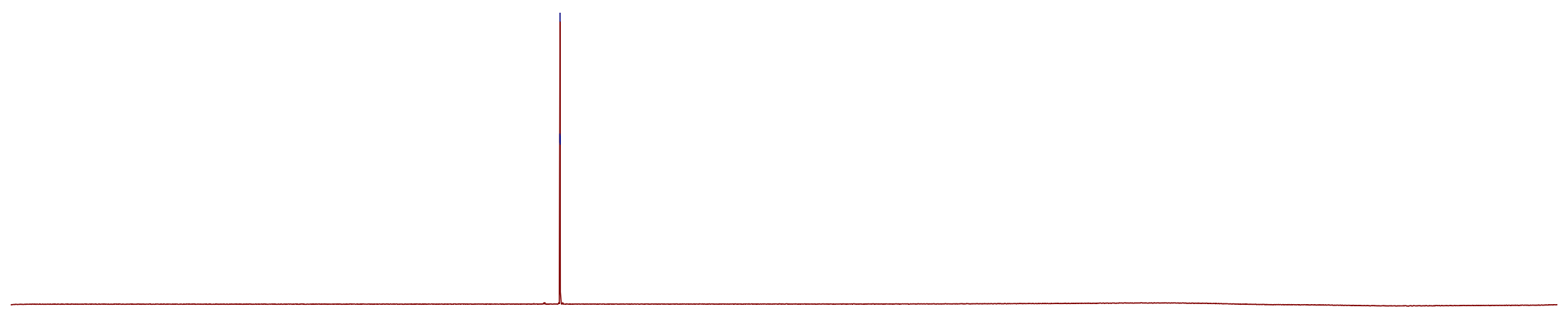




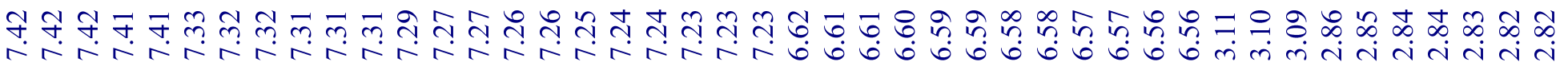
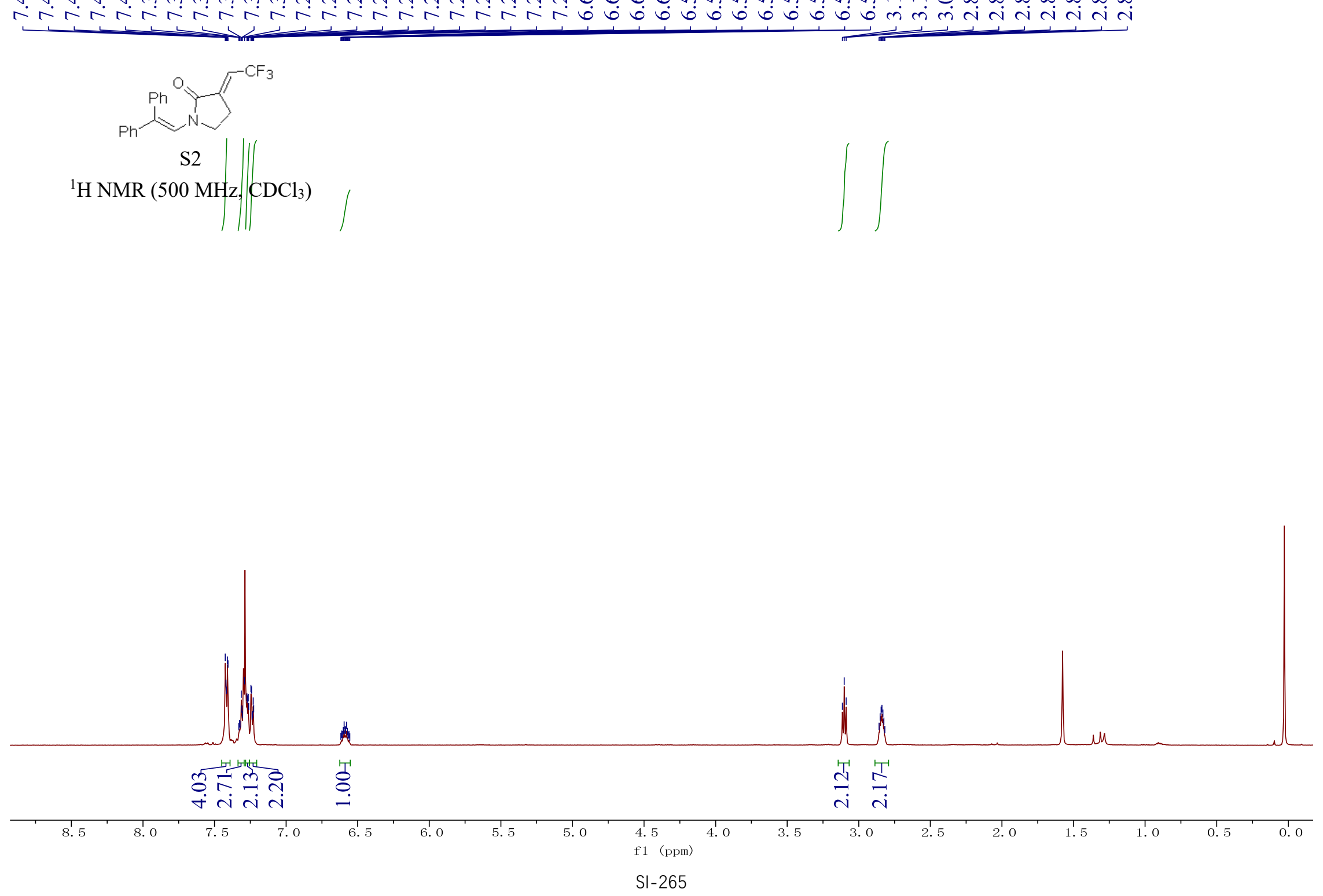
$\stackrel{ }{0}$ \&

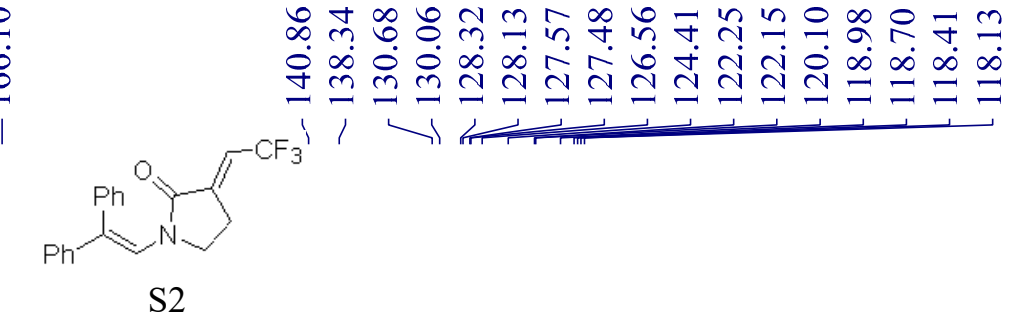

${ }^{3} \mathrm{C}$ NMR (125 MHz, $\mathrm{CDCl}_{3}$ )

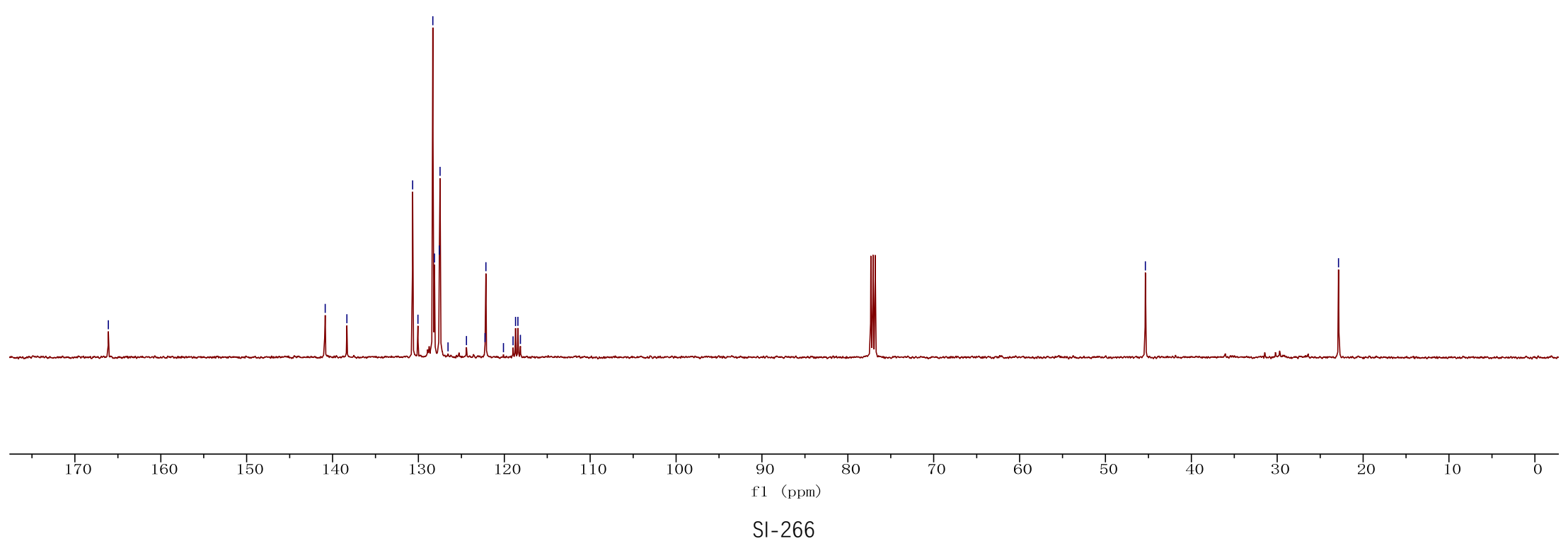




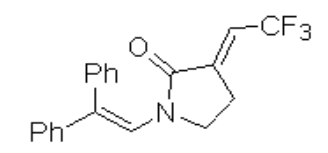

S2

${ }^{19} \mathrm{~F}$ NMR (376 MHz, $\mathrm{CDCl}_{3}$ )

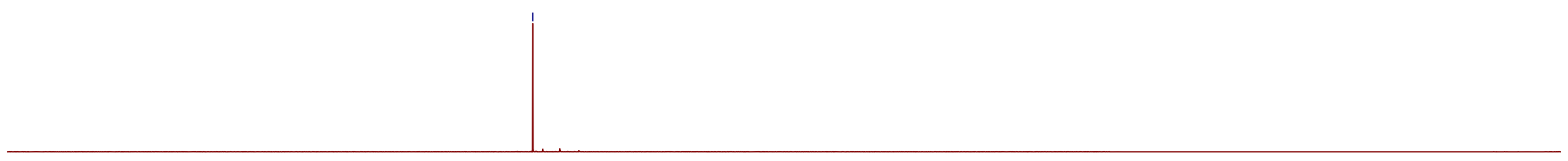

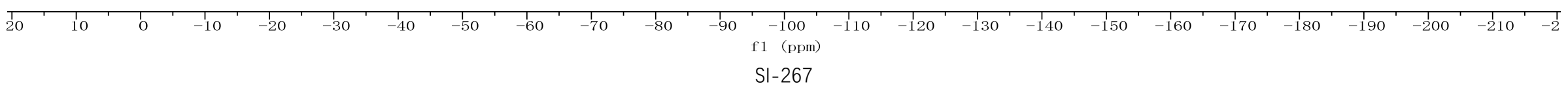




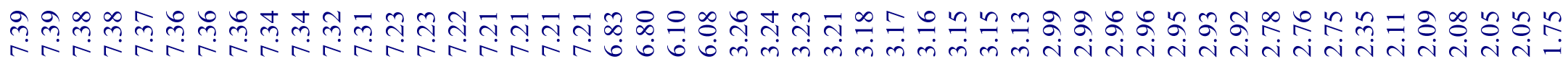

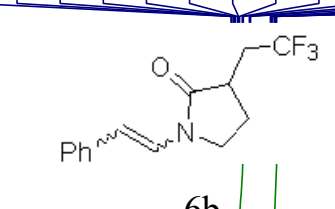

${ }^{1} \mathrm{H} \mathrm{NMR}\left(400 \mathrm{MHz}, \mathrm{CDCl}_{3}\right)$
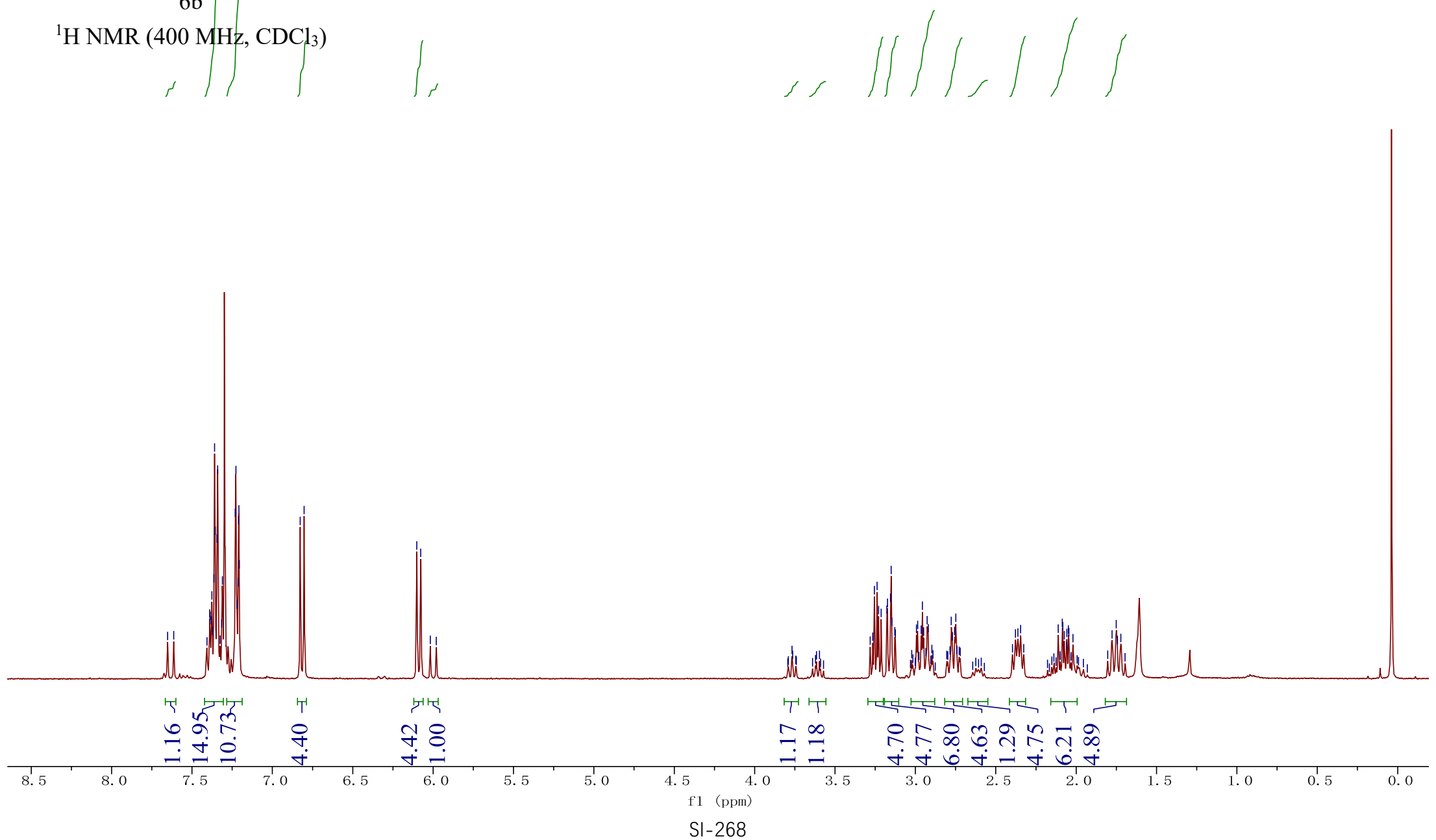


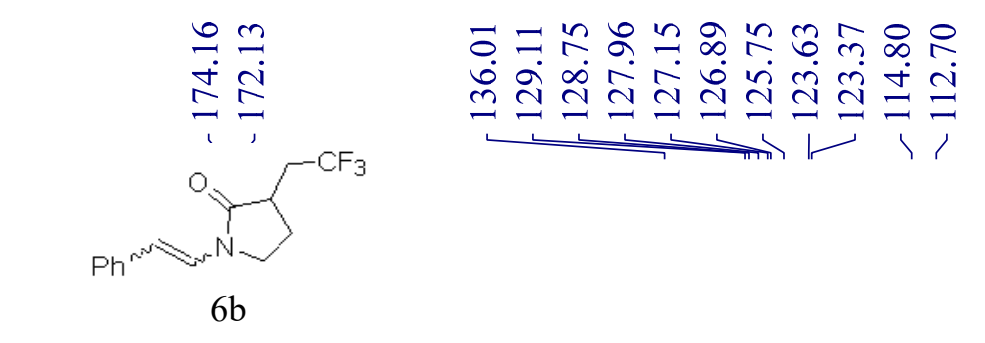

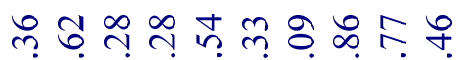

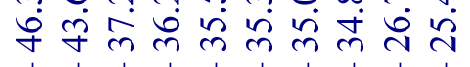

${ }^{13} \mathrm{C}$ NMR (125 MHz, $\left.\mathrm{CDCl}_{3}\right)$

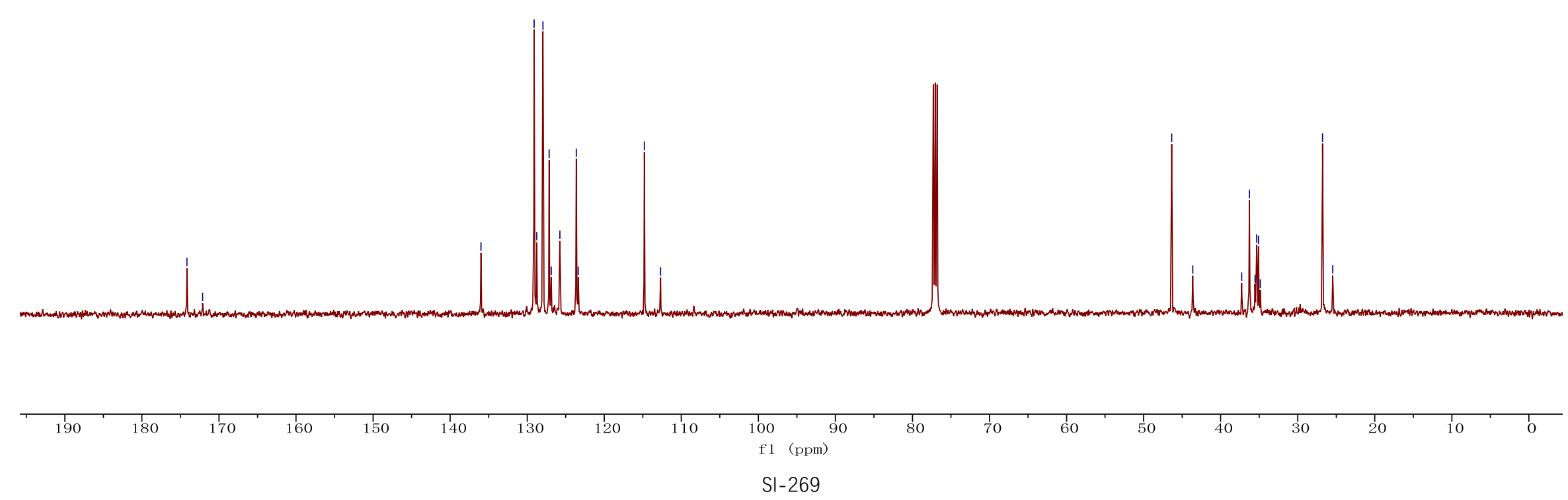


สูลิวิ

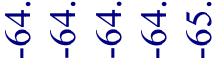

$\mathrm{Ph}^{2}$

$6 \mathrm{~b}$

${ }^{19} \mathrm{~F}$ NMR $\left(470 \mathrm{MHz}, \mathrm{CDCl}_{3}\right)$
읭

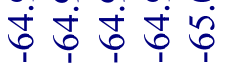

$\longrightarrow$

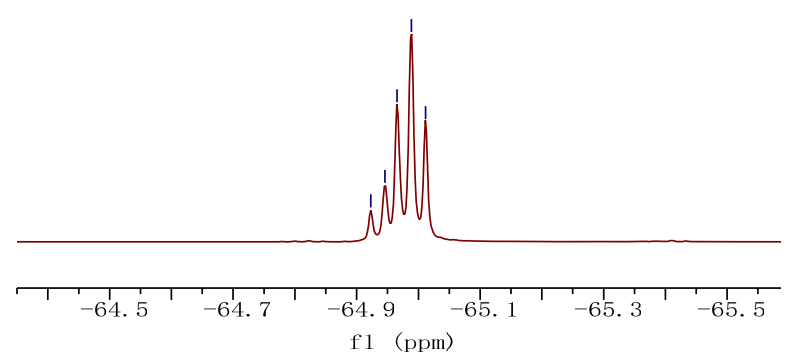

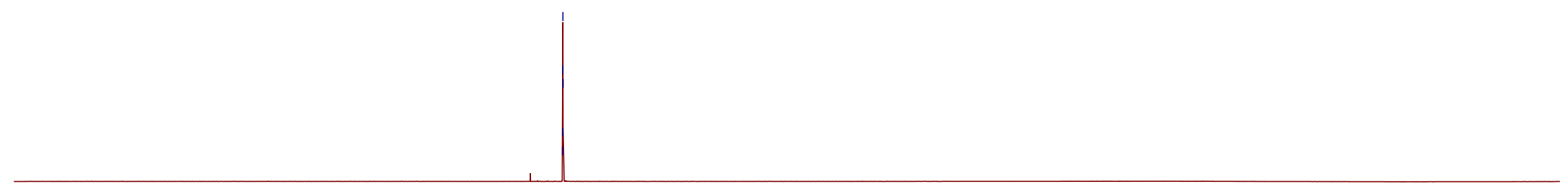

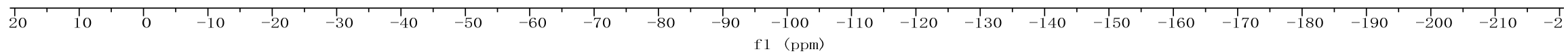
SI-270 


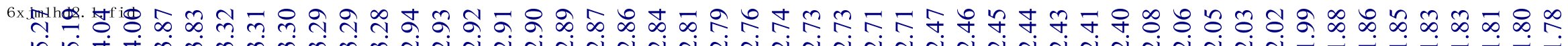

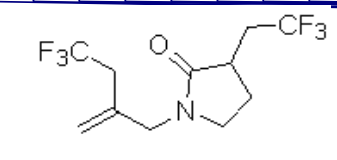

$6 \mathrm{~d}$

${ }^{1} \mathrm{H}$ NMR (400 MHz, $\mathrm{CDCl}_{3}$ )
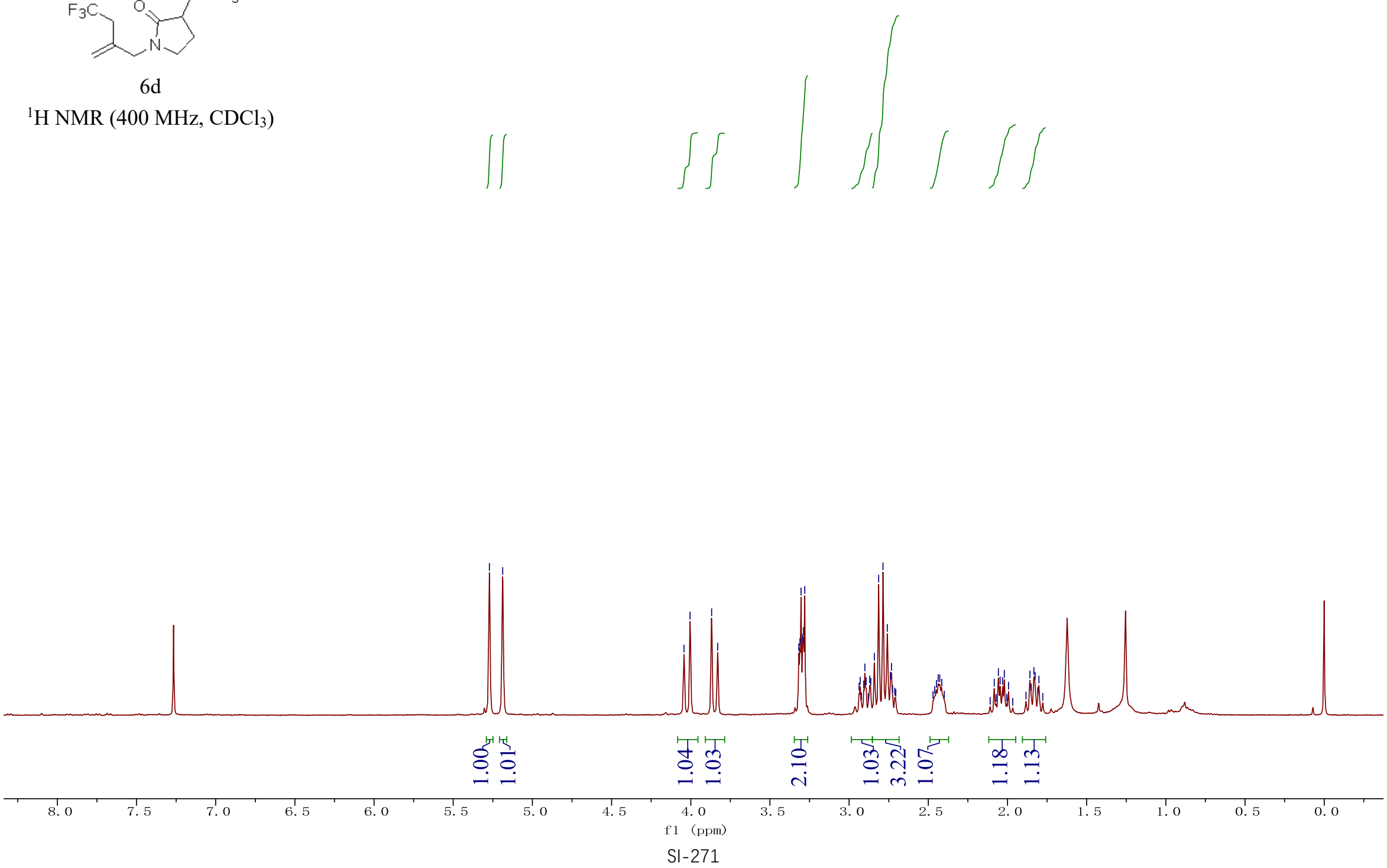


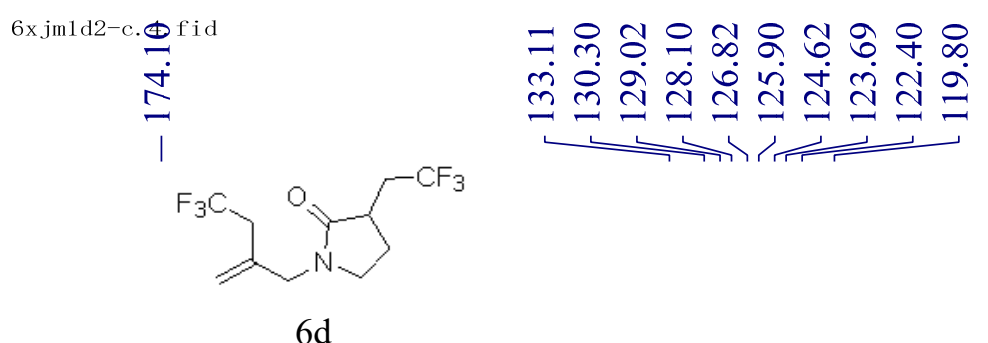

${ }^{13} \mathrm{C}$ NMR (125 MHz, $\left.\mathrm{CDCl}_{3}\right)$

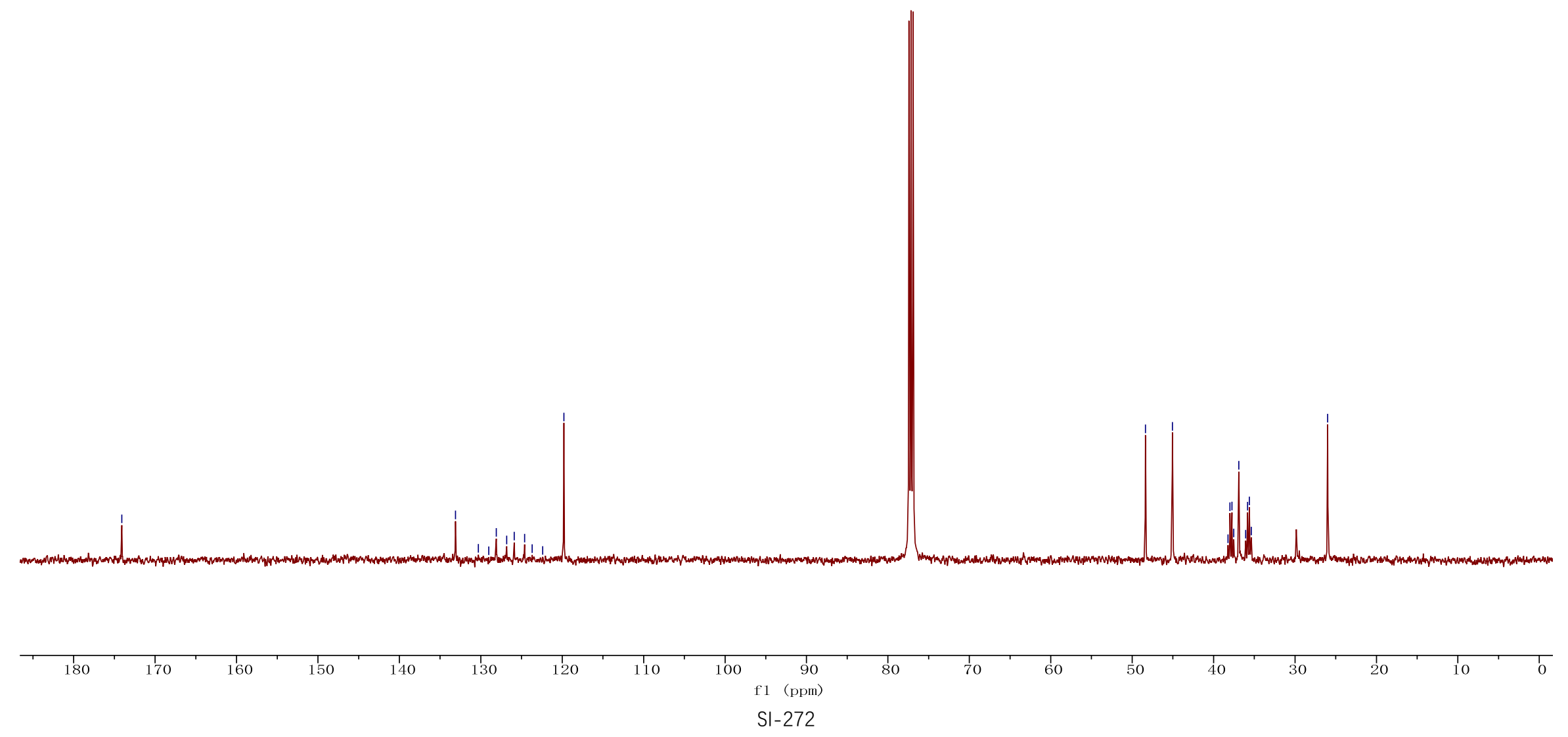


6xjm1d2-f. 8. fid

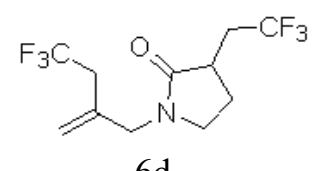

$6 \mathrm{~d}$

${ }^{19} \mathrm{~F}$ NMR (470 MHz, $\mathrm{CDCl}_{3}$ )

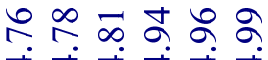

莳 它

1

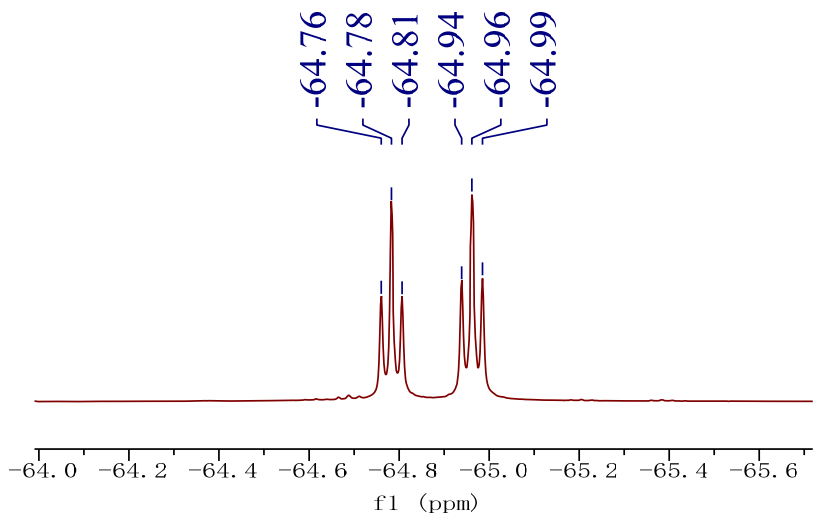

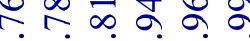

\begin{tabular}{l}
1 \\
\hline
\end{tabular}

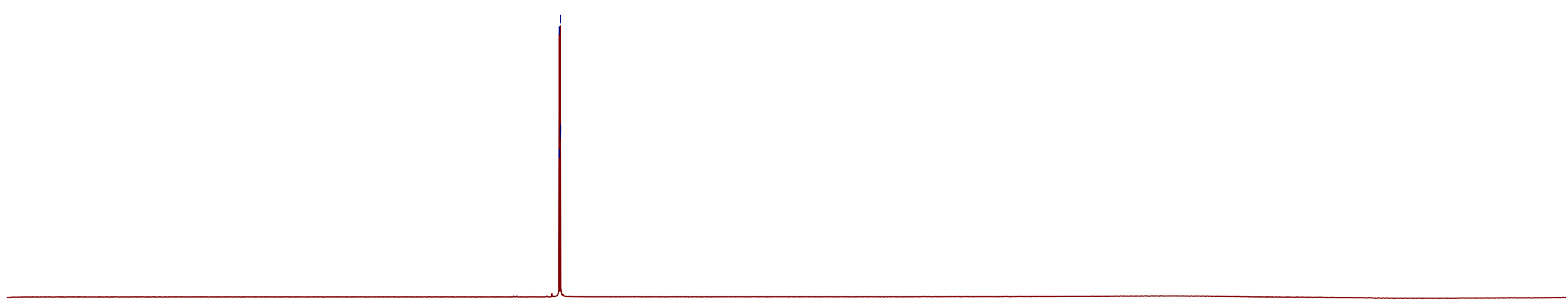

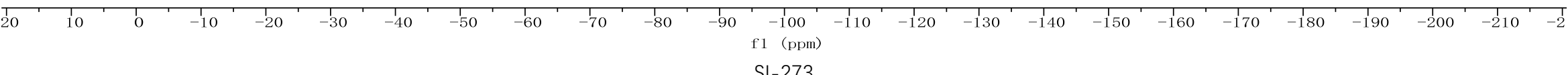




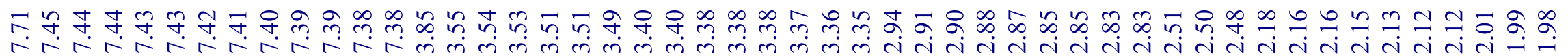

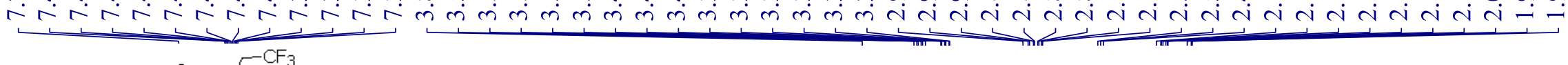
$\mathrm{Ph} \sim \mathrm{CO}_{2} \mathrm{Me}$

${ }^{1} \mathrm{H}$ NMR $\left(400 \mathrm{MHz}, \mathrm{CDCl}_{3}\right)$

$\int$
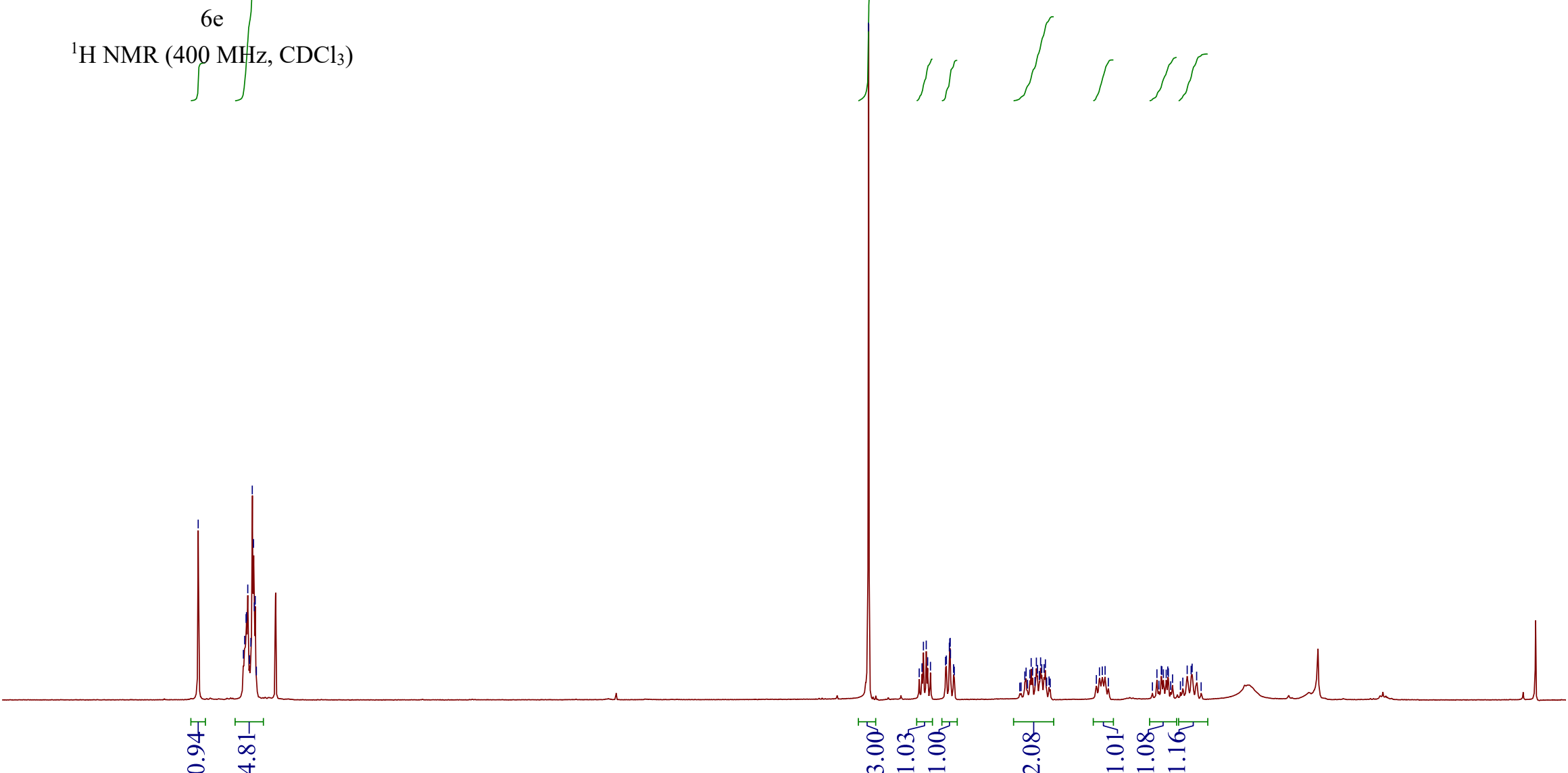

$8.5+8.0$ 


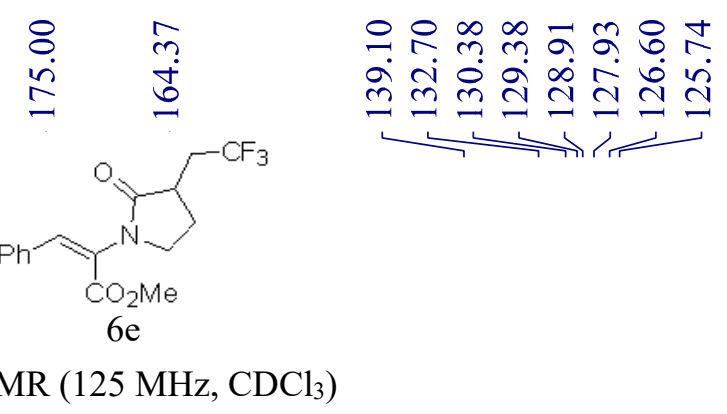

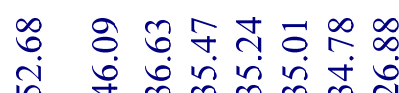

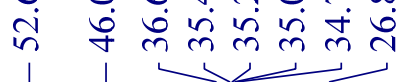
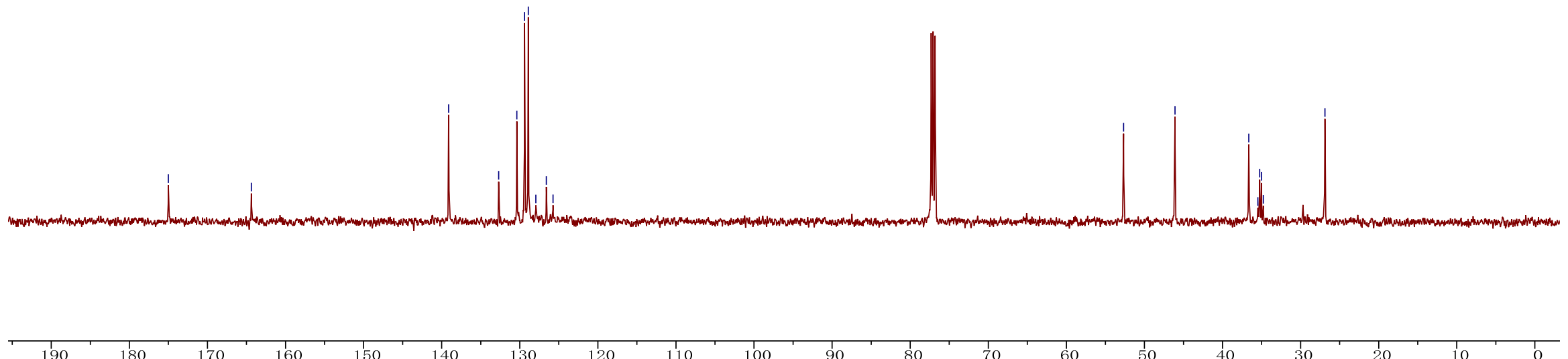

f1 (ppm)

80

70

50

30

20

10

SI-275 
क

草

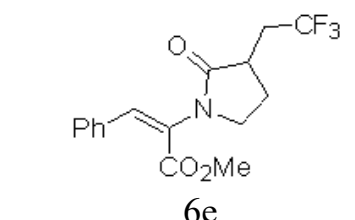

${ }^{19} \mathrm{~F}$ NMR $\left(470 \mathrm{MHz}, \mathrm{CDCl}_{3}\right)$

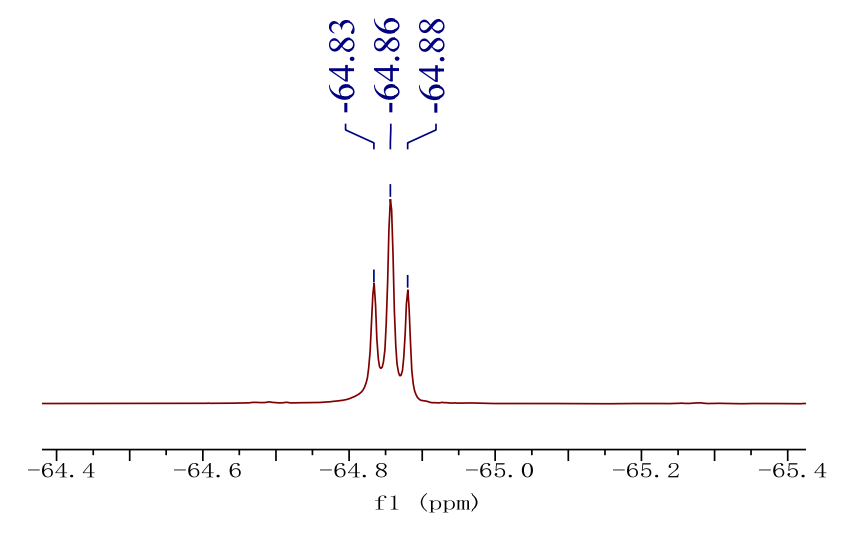

$\infty$

î

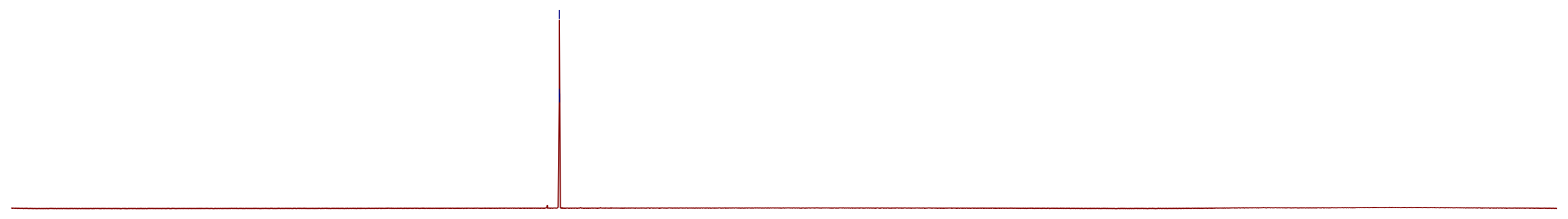




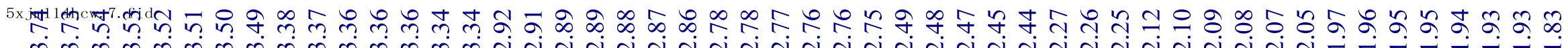

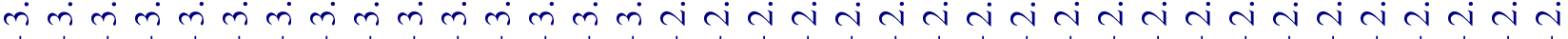

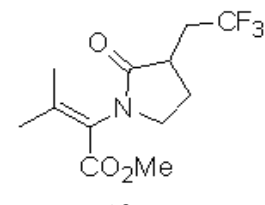

$6 f$

${ }^{1} \mathrm{H}$ NMR $\left(500 \mathrm{MHz}, \mathrm{CDCl}_{3}\right)$
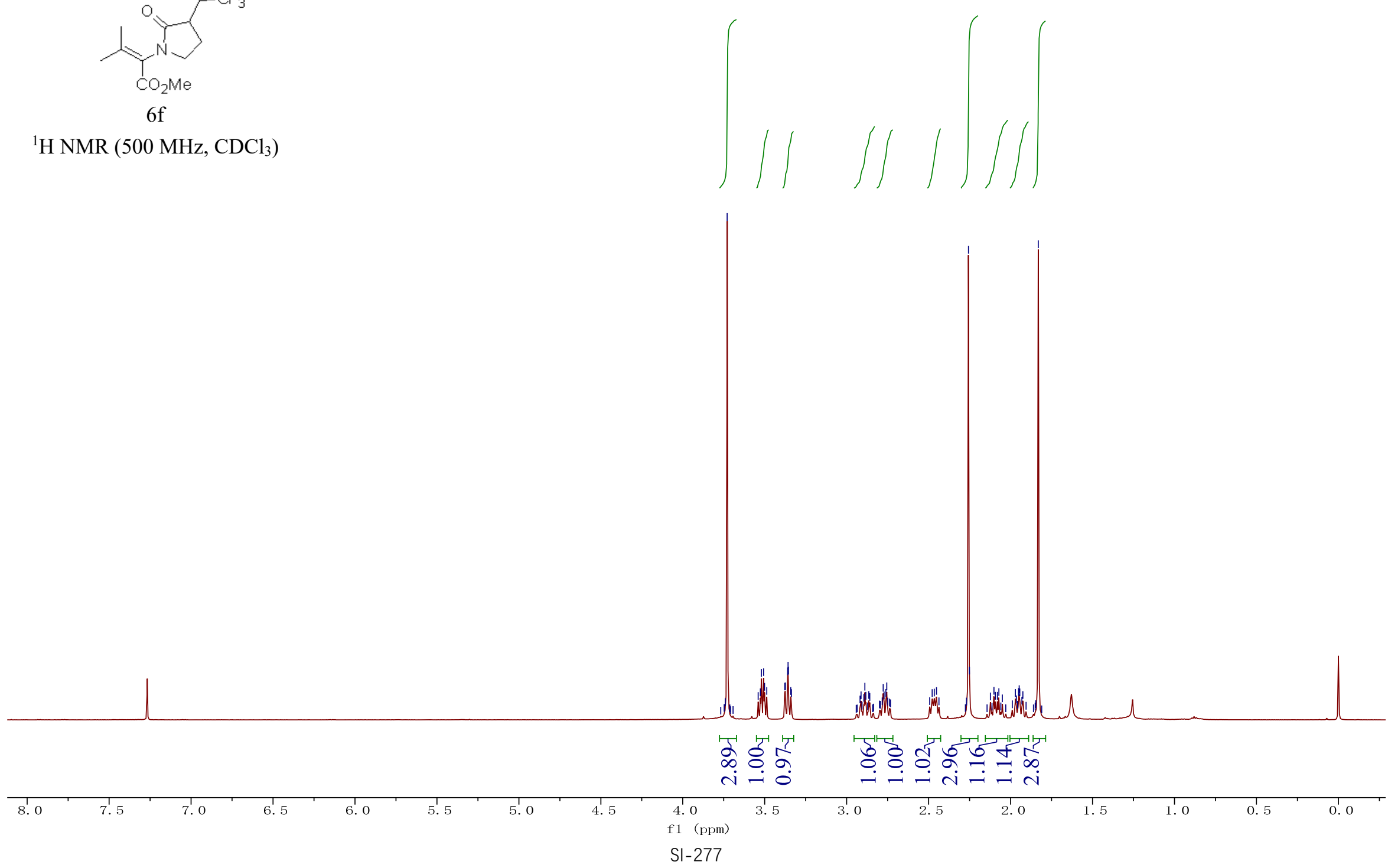


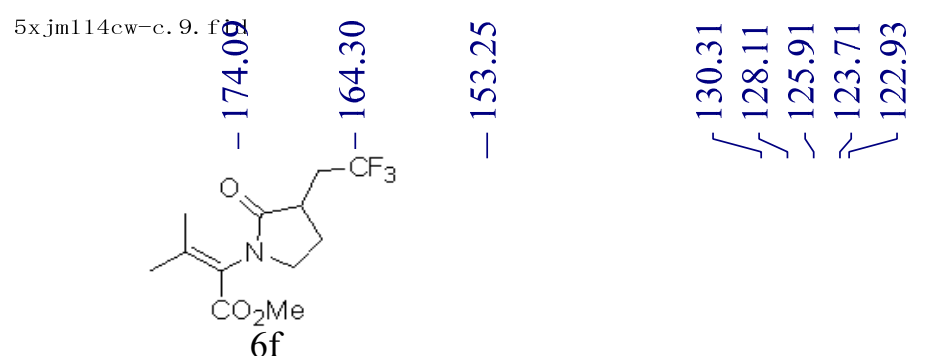

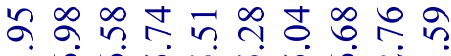

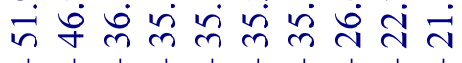

${ }^{13} \mathrm{C}$ NMR (125 MHz, $\left.\mathrm{CDCl}_{3}\right)$

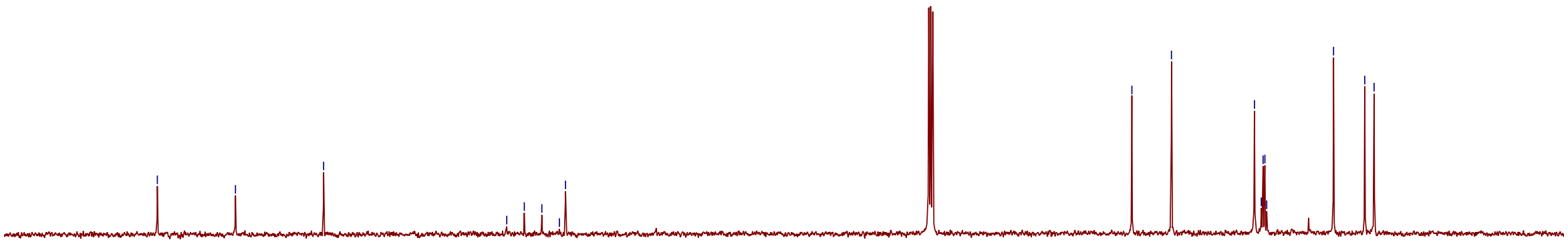


$5 x j m 114 c w-f .8$. fid

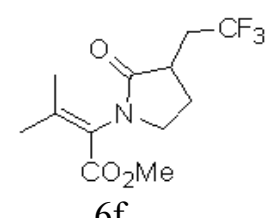

${ }^{19} \mathrm{~F}$ NMR (470 MHz, $\mathrm{CDCl}_{3}$ )

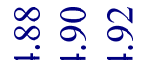

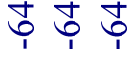

1

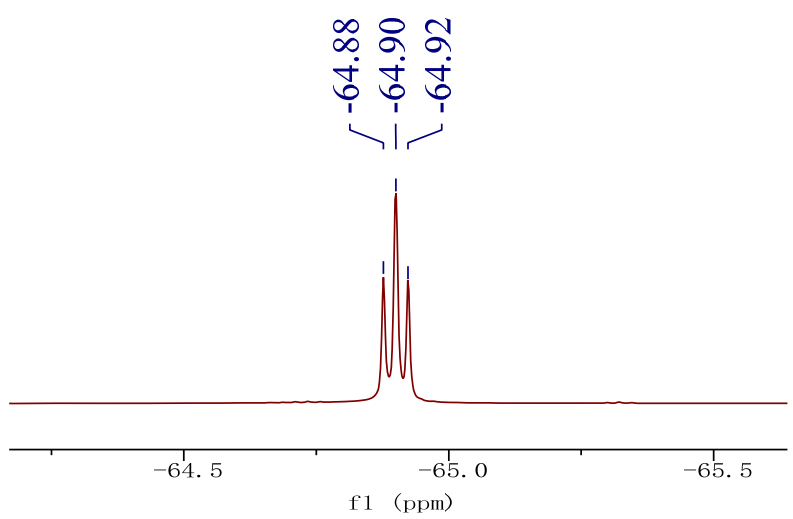

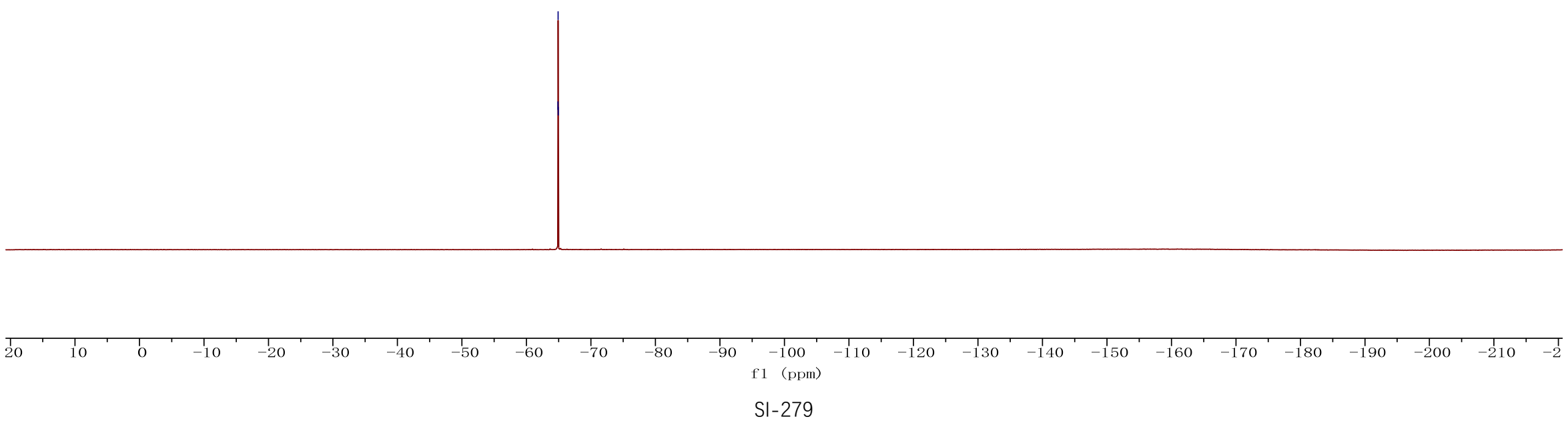


Ô.

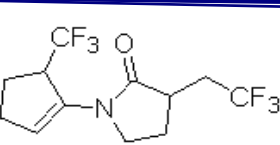

$6 \mathrm{~g}$

${ }^{1} \mathrm{H} \mathrm{NMR}\left(500 \mathrm{MHz}, \mathrm{CDCl}_{3}\right)$
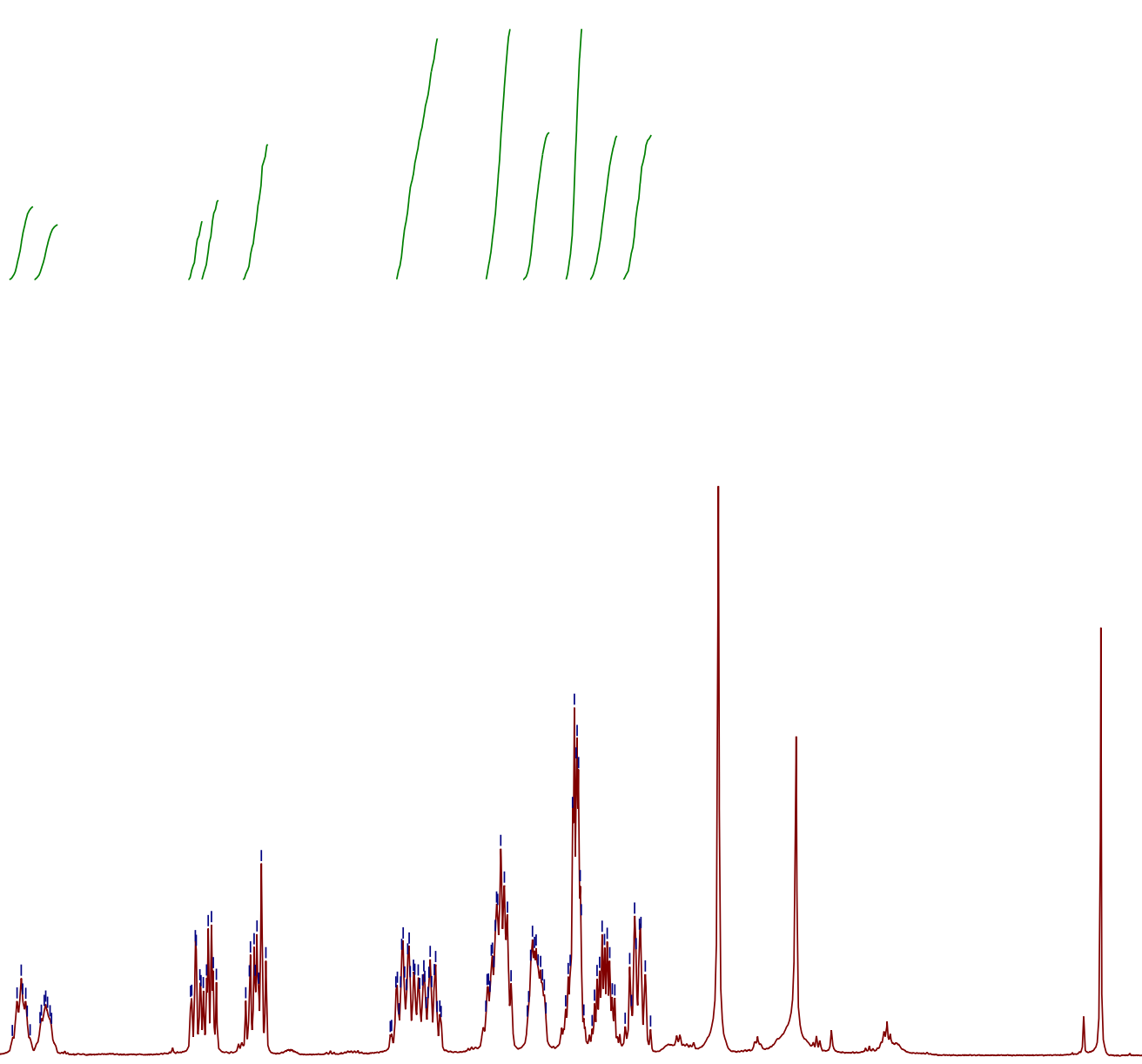

○े

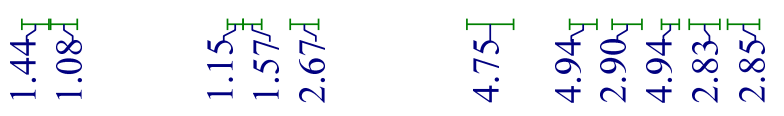

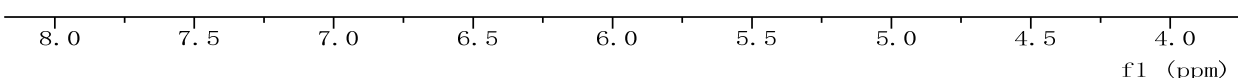




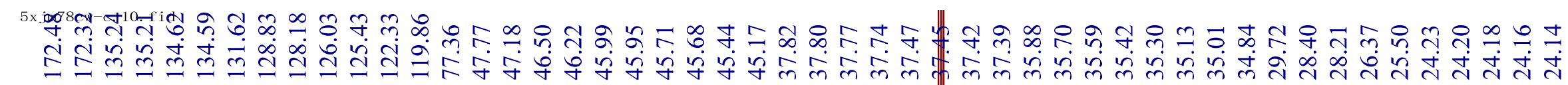

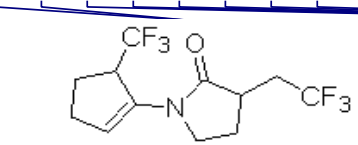

$6 \mathrm{~g}$

${ }^{13} \mathrm{C}$ NMR $\left(100 \mathrm{MHz}, \mathrm{CDCl}_{3}\right)$

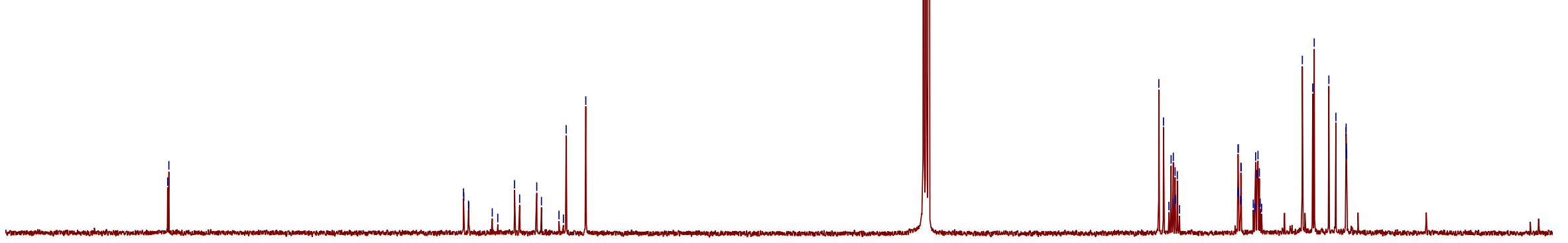



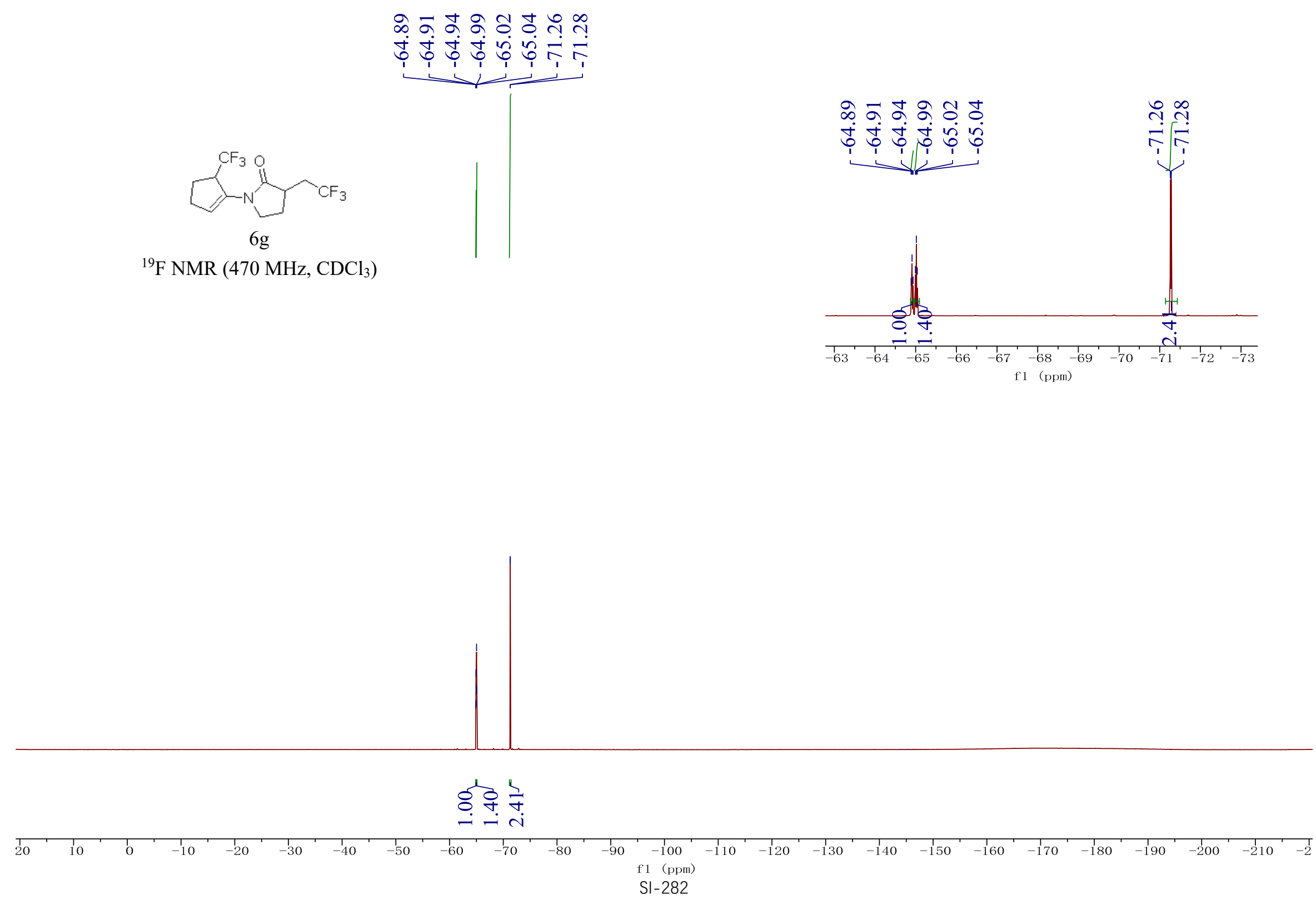


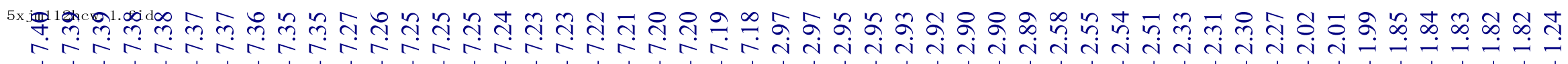
$\mathrm{Ph}+\mathrm{CF} 3$ Ph $6 \mathrm{~h}$

${ }^{1} \mathrm{H}$ NMR (400 MHz, $\mathrm{CDCl}_{3}$ )<smiles></smiles>
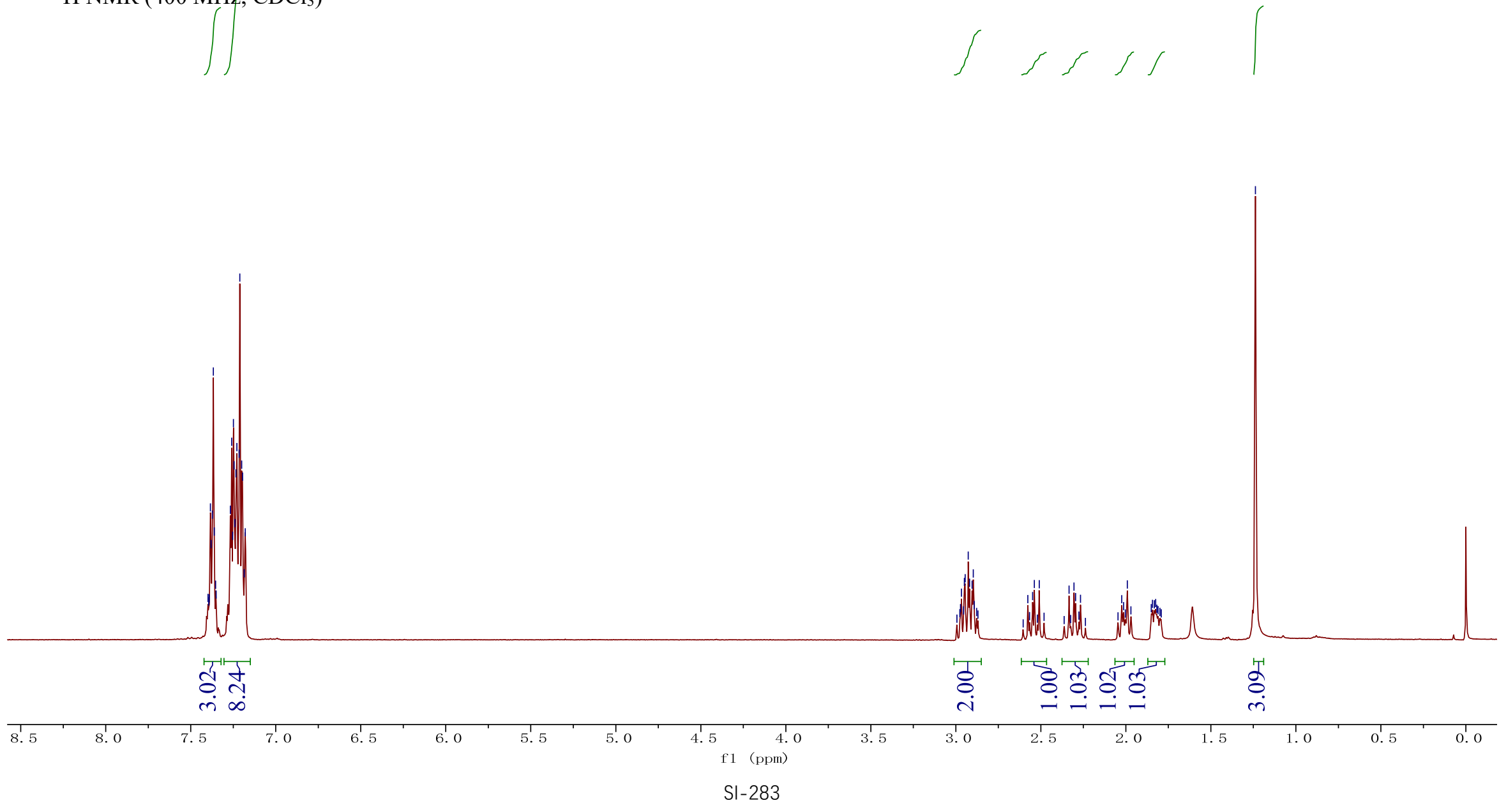


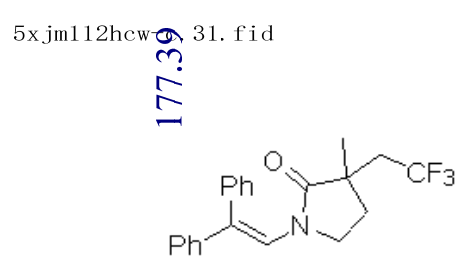

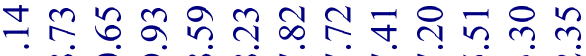

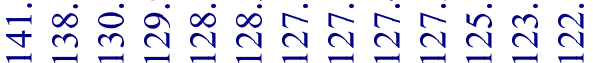

I

$6 \mathrm{~h}$

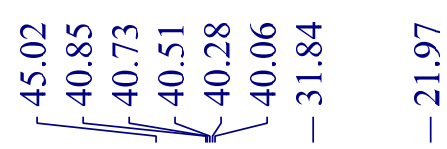

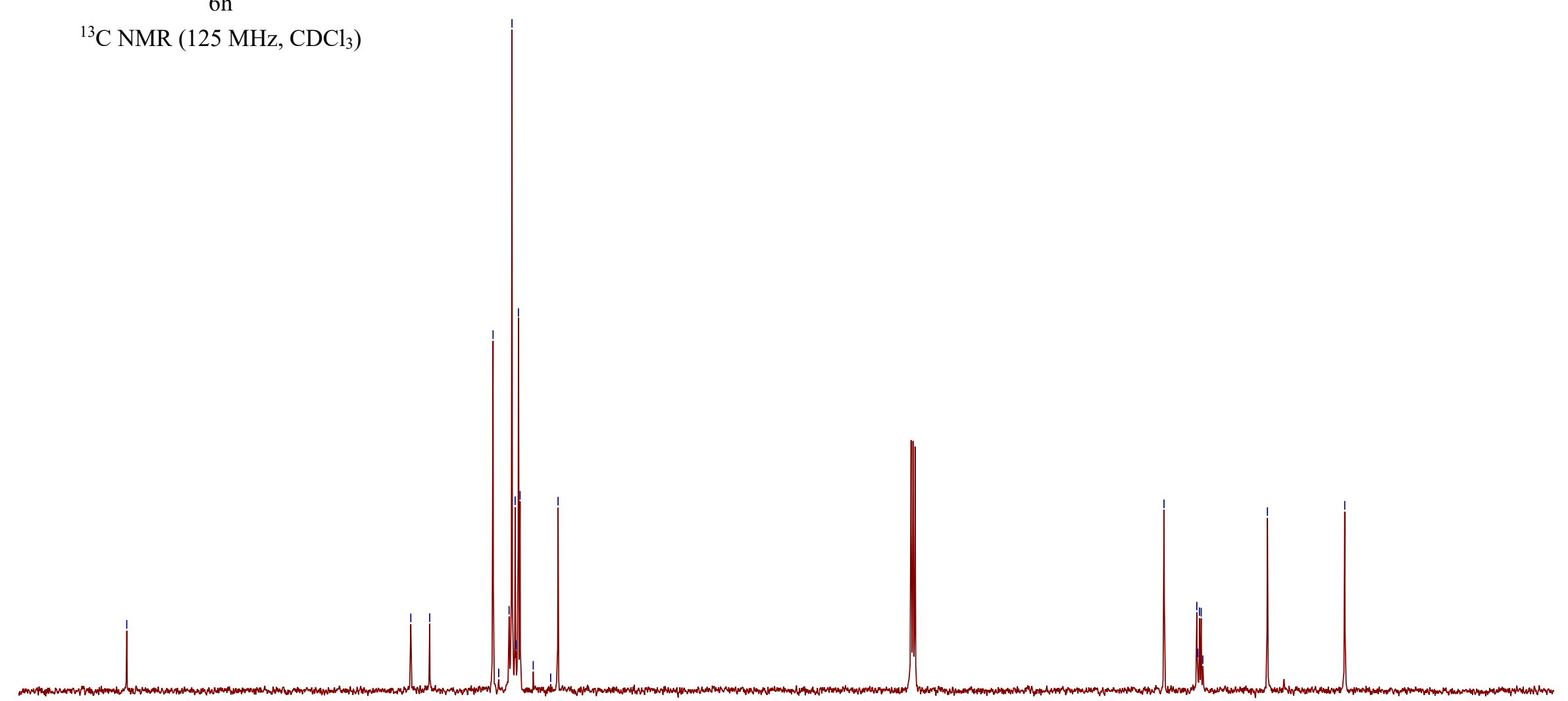


5xjm112hcw-f. 30. fid

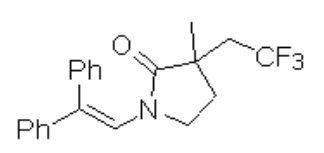

$6 \mathrm{~h}$

${ }^{19} \mathrm{~F}$ NMR (470 MHz, $\mathrm{CDCl}_{3}$ )

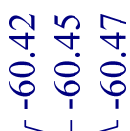

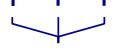

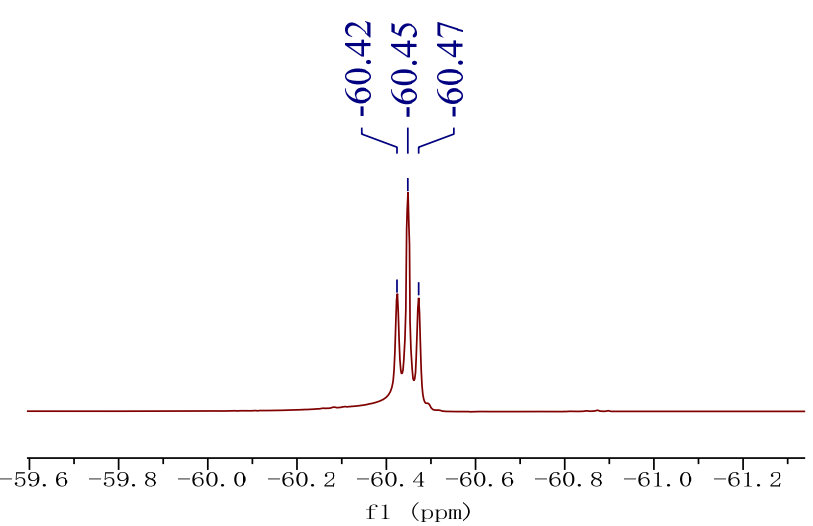

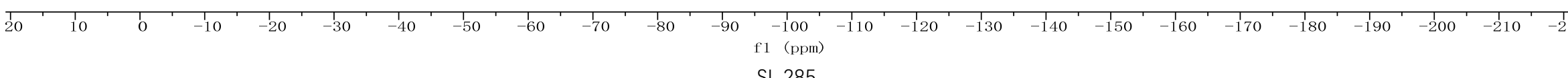




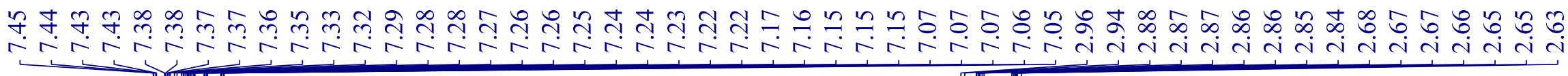

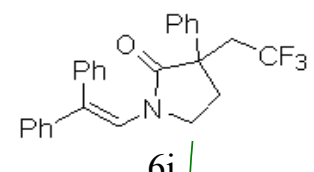

$6 \mathrm{i}$

${ }^{1} \mathrm{H} \mathrm{NMR}\left(500 \mathrm{MHz}, \mathrm{CDCl}_{3}\right)$

$\iiint \int$

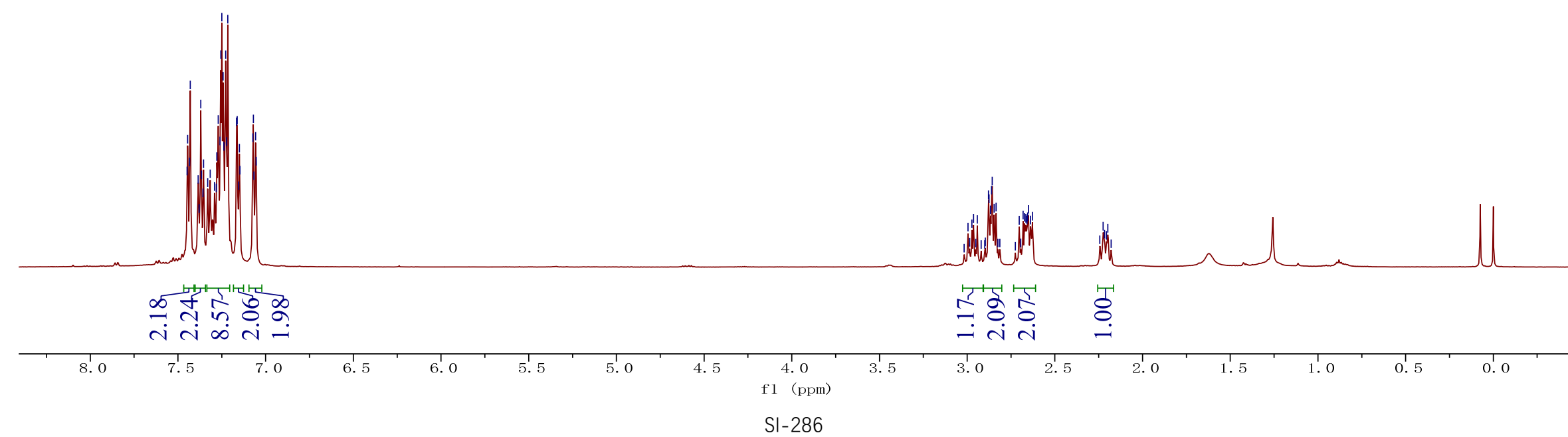




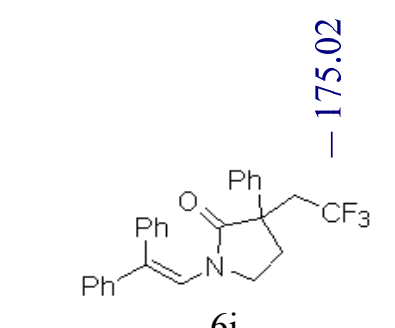

61

${ }^{13} \mathrm{C}$ NMR (125 MHz, $\left.\mathrm{CDCl}_{3}\right)$
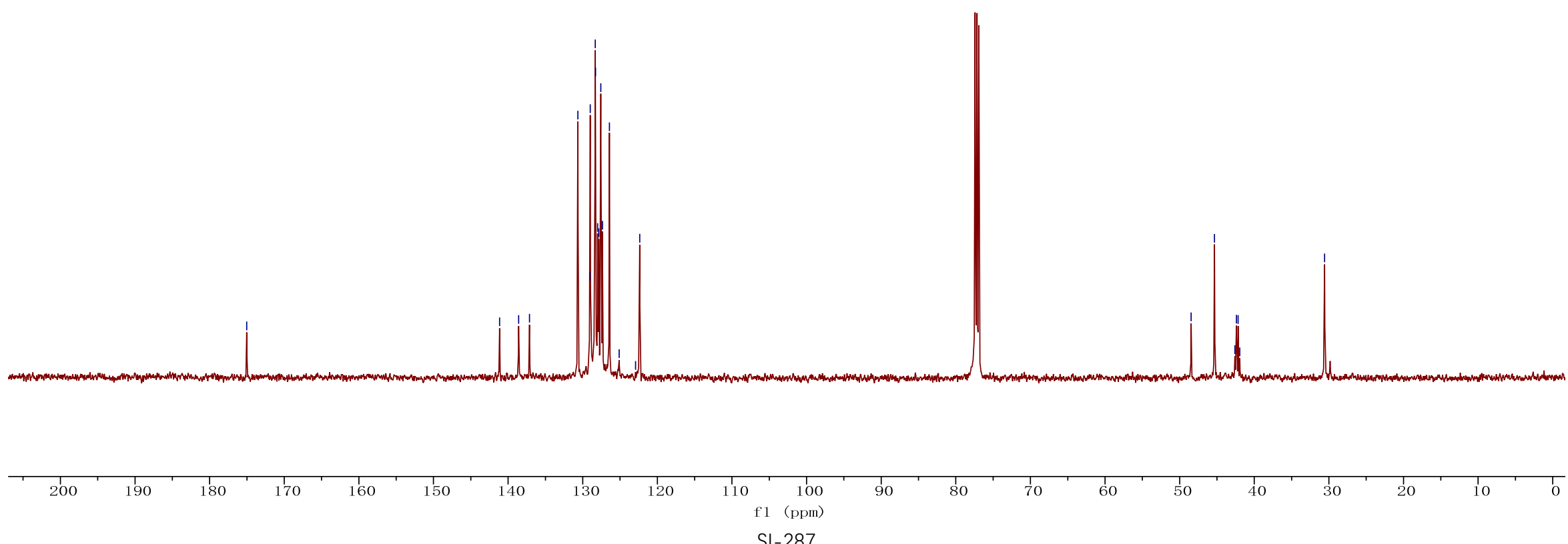
गे ले न

1
1
1

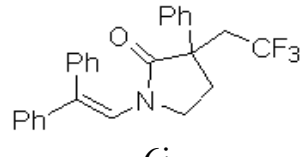

$6 \mathrm{i}$

${ }^{19} \mathrm{~F}$ NMR $\left(470 \mathrm{MHz}, \mathrm{CDCl}_{3}\right)$
गे ले F

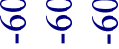

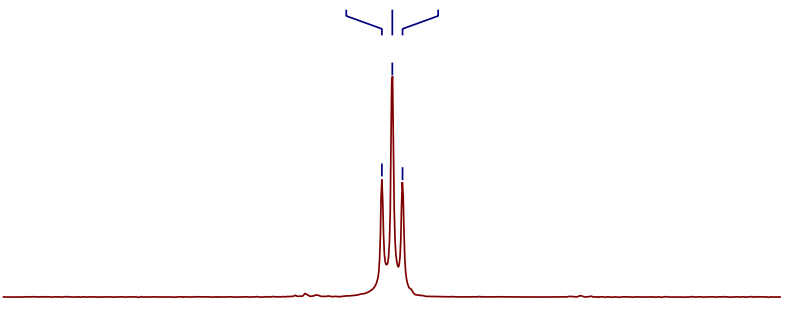

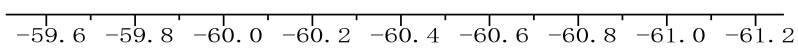
f1 (ppm) 


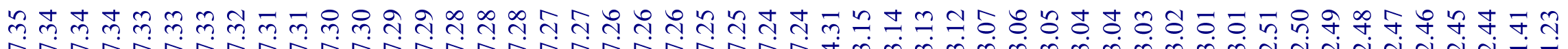

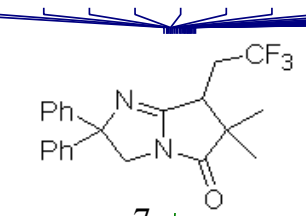

${ }^{1} \mathrm{H}$ NMR $\left(500 \mathrm{MHz}, \mathrm{CDCl}_{3}\right)$
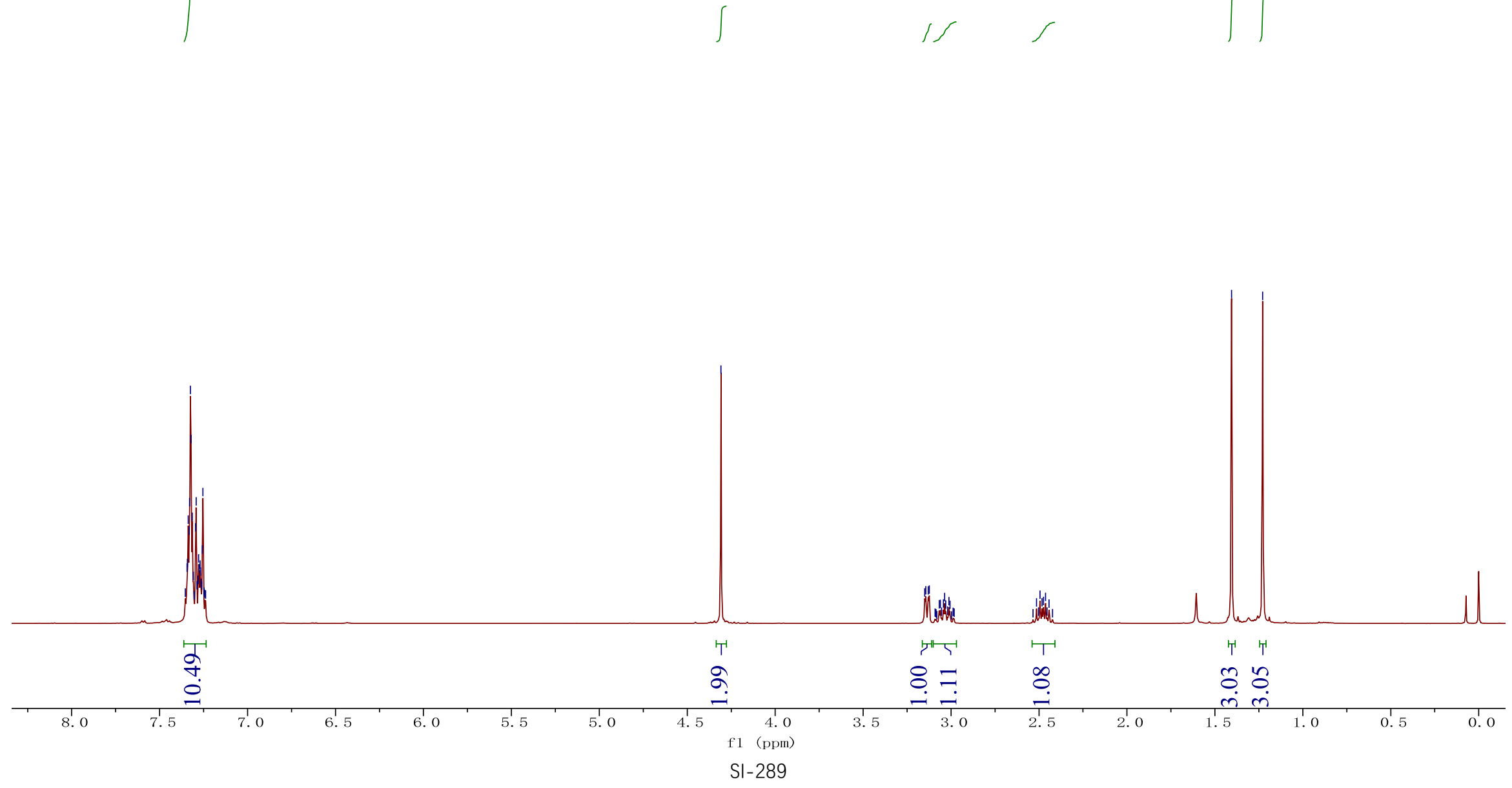

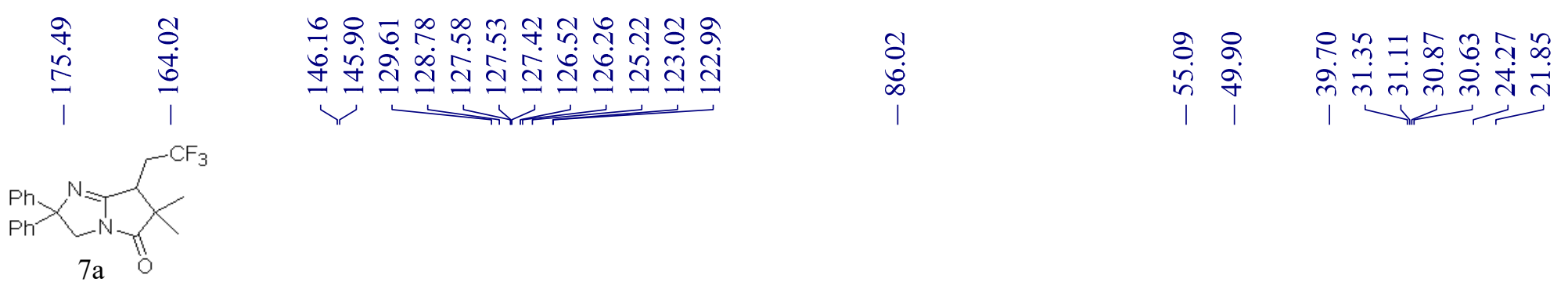

${ }^{13} \mathrm{C}$ NMR (125 MHz, $\mathrm{CDCl}_{3}$ )

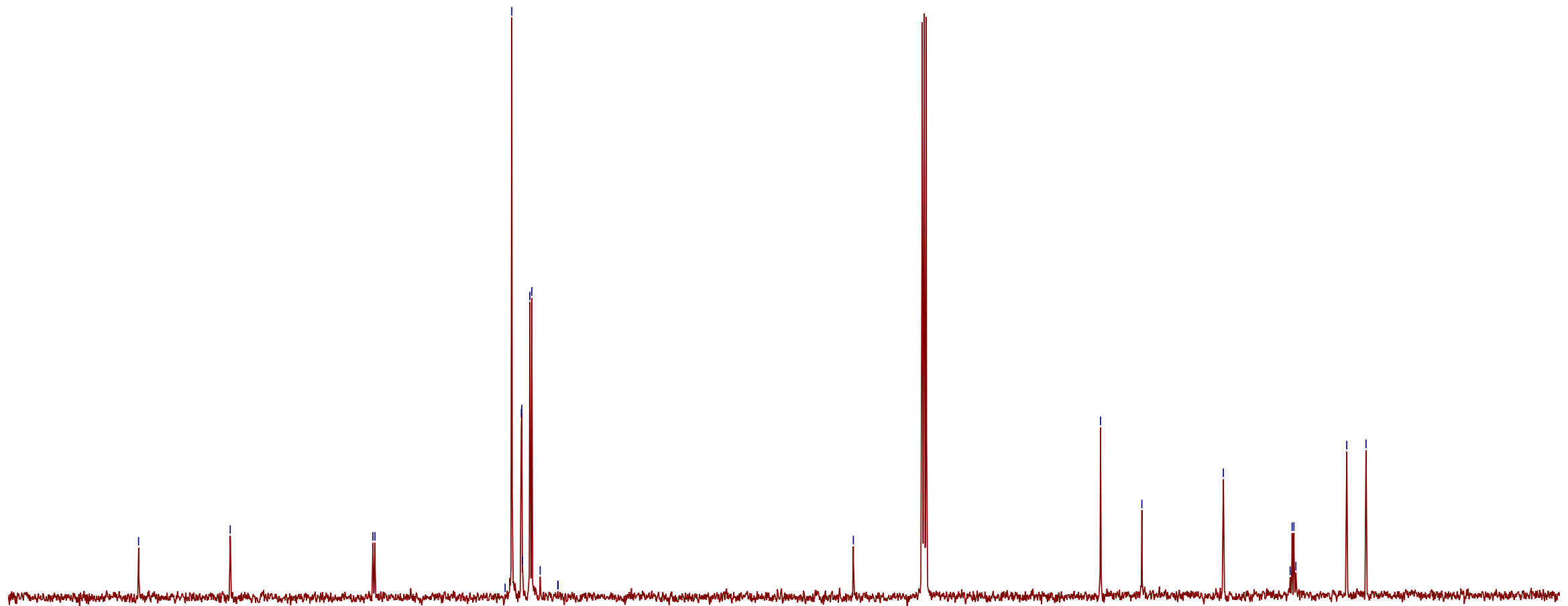




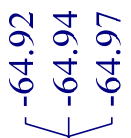

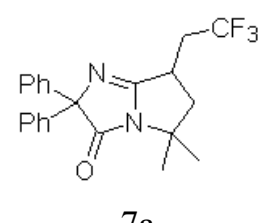

$7 \mathrm{a}$

${ }^{19} \mathrm{~F}$ NMR $\left(470 \mathrm{MHz}, \mathrm{CDCl}_{3}\right)$

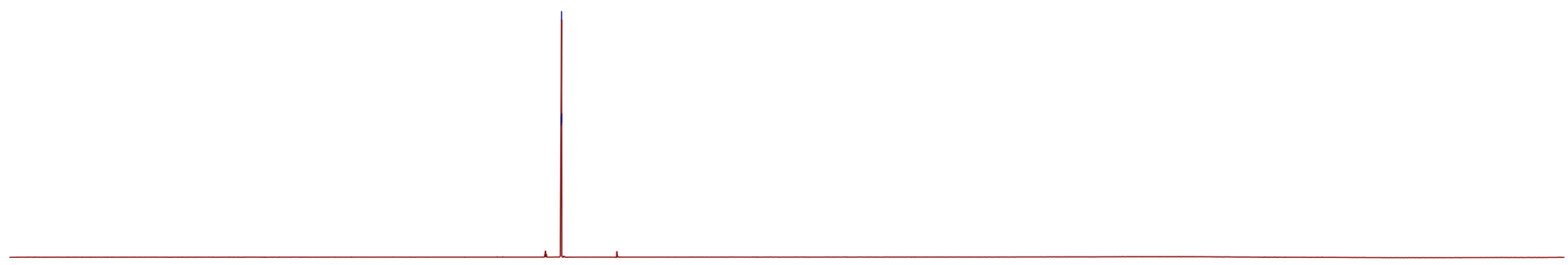

20

$20 \quad 10$
종ํㅇ

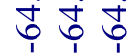

iा

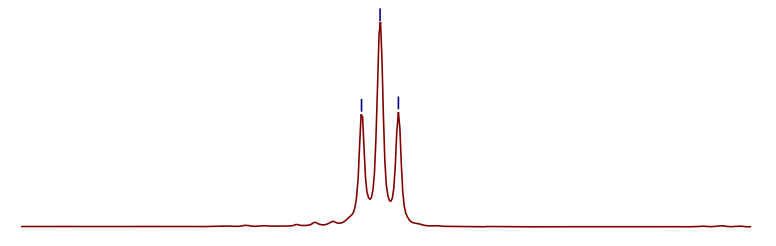

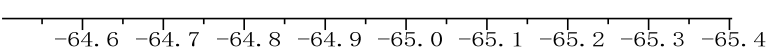
f1 (ppm) 


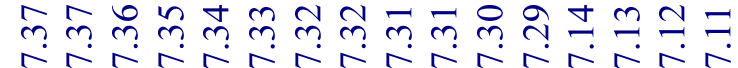
$-\mathrm{CF}_{3}$

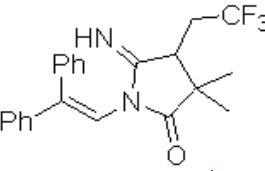

$6 \mathrm{j}$

${ }^{1} \mathrm{H} \mathrm{NMR}\left(400 \mathrm{MHz}, \mathrm{CDCl}_{3}\right)$

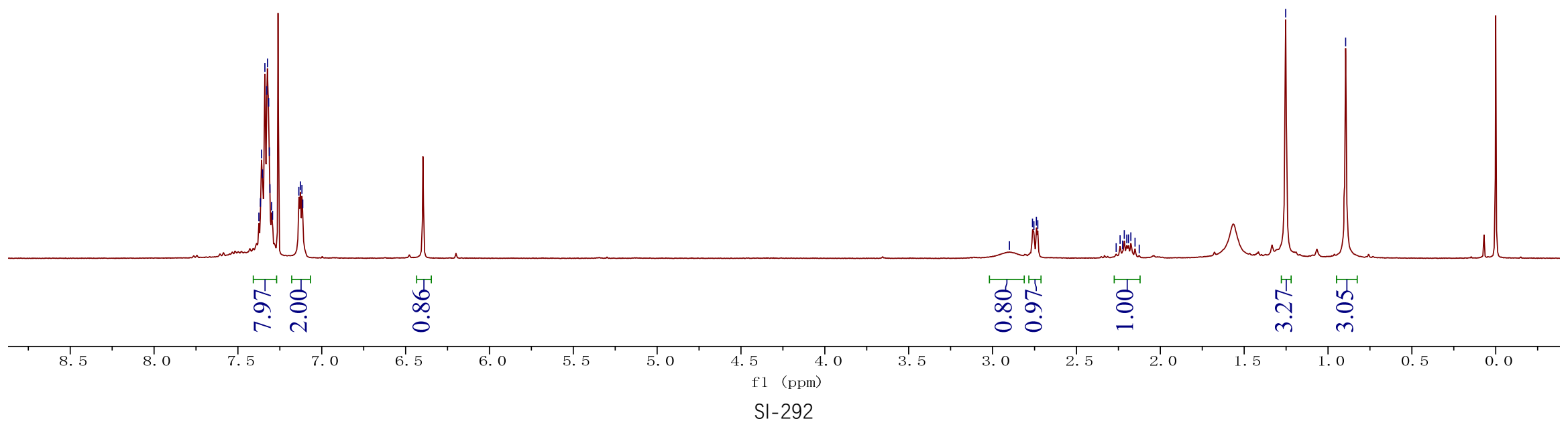

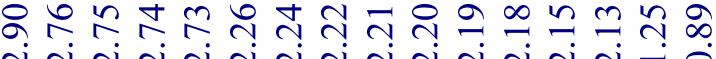

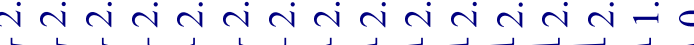

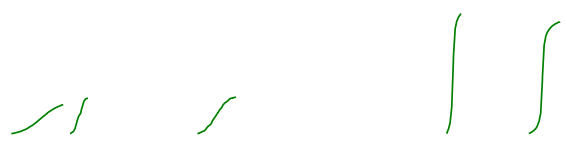




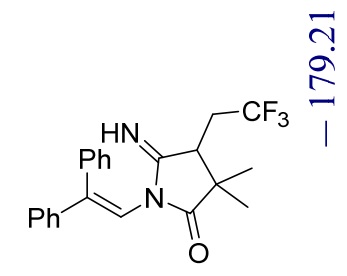

$6 \mathrm{j}$
정 त्र

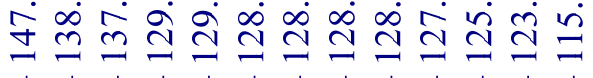

च fo

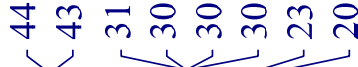

${ }^{13} \mathrm{C} \mathrm{NMR}\left(125 \mathrm{MHz}, \mathrm{CDCl}_{3}\right)$

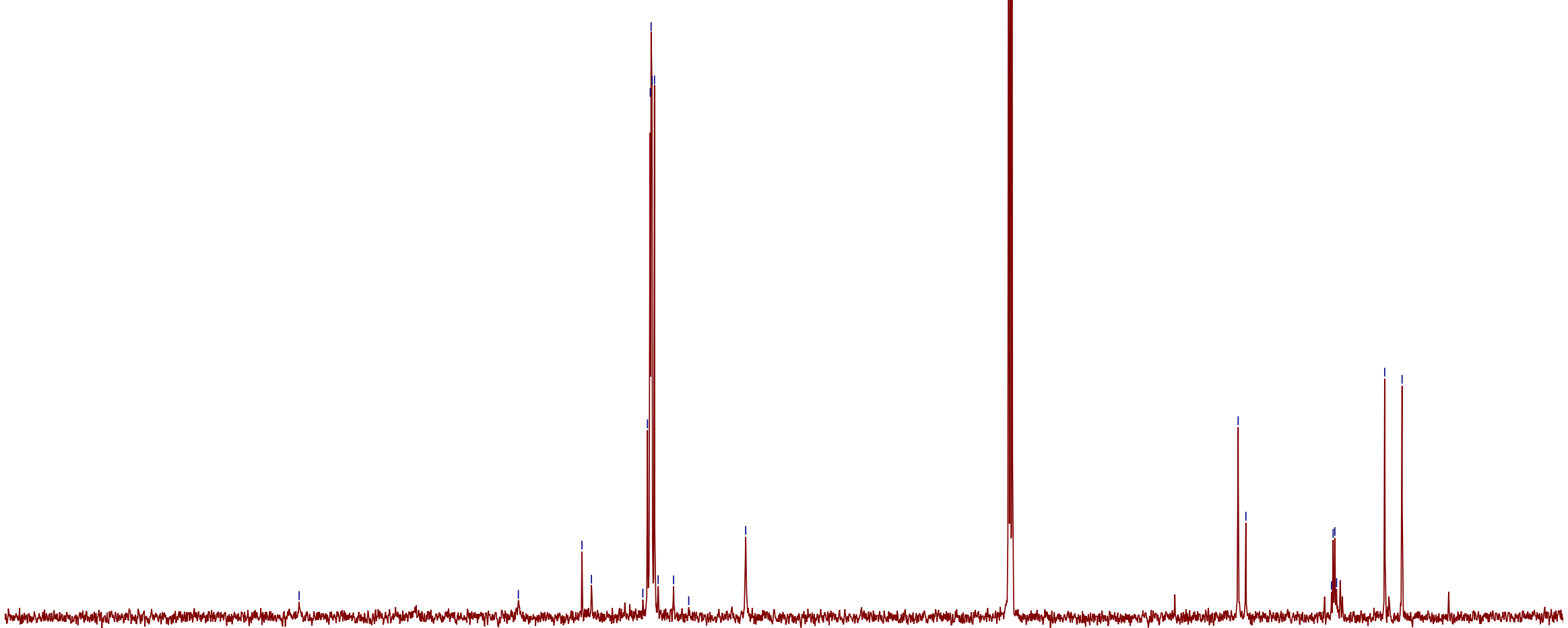

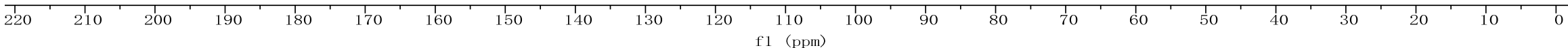
SI-293 


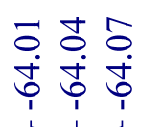

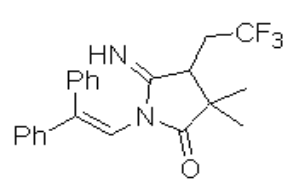

$6 \mathrm{j}$

${ }^{19} \mathrm{~F}$ NMR $\left(376 \mathrm{MHz}, \mathrm{CDCl}_{3}\right)$

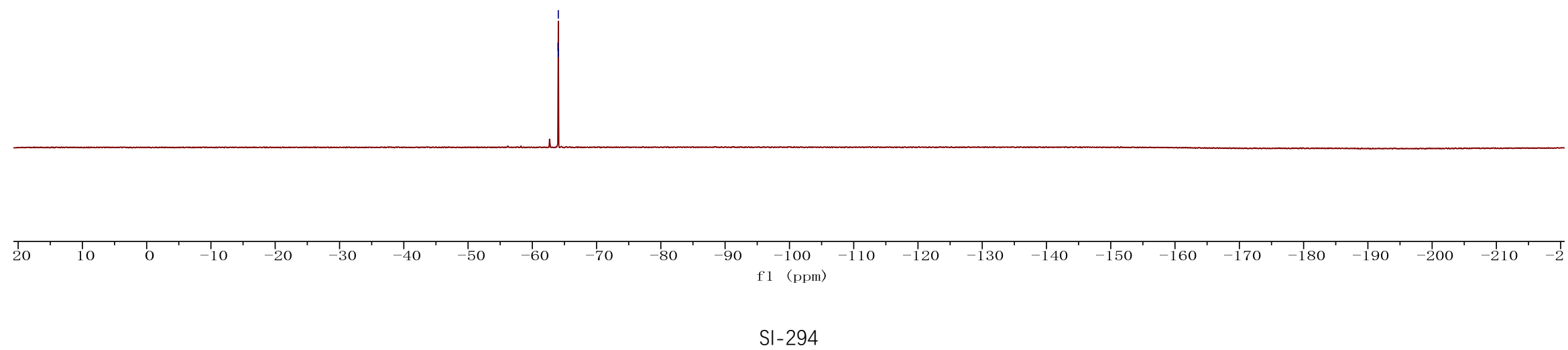

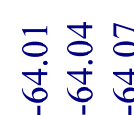

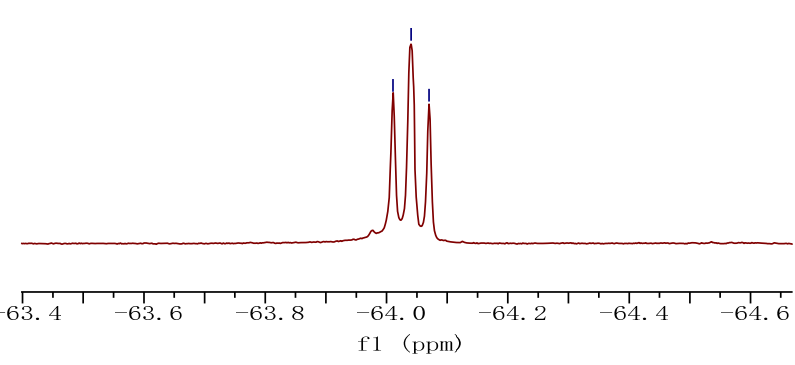




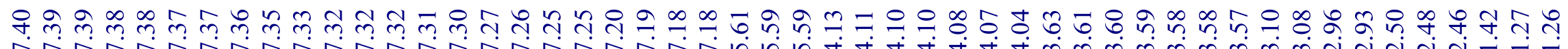

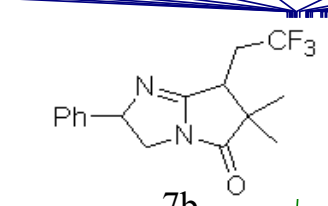

${ }^{1} \mathrm{H}$ NMR $\left(400 \mathrm{MHz}, \mathrm{CDCl}_{3}\right)$
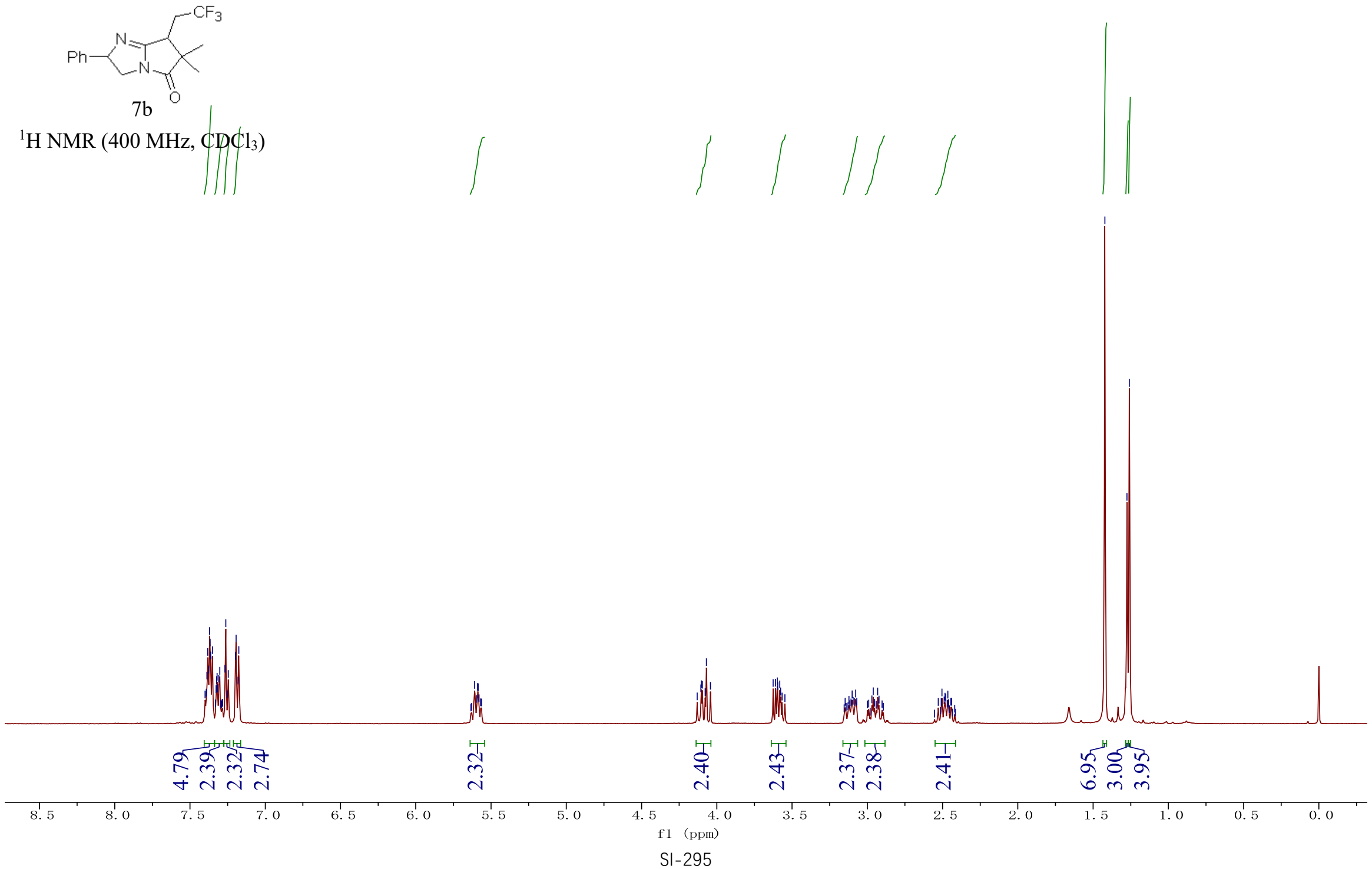


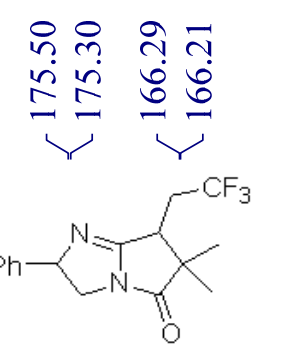

${ }^{13} \mathrm{C}$ NMR $\left(125 \mathrm{MHz}, \mathrm{CDCl}_{3}\right)$

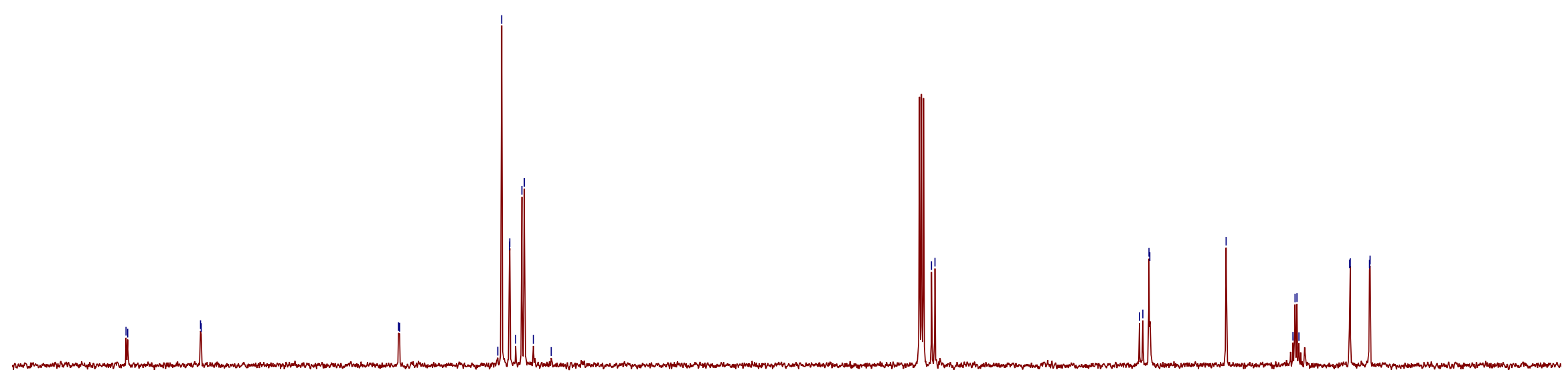

${ }_{180}^{1} 170 \quad \frac{1}{160} \quad \frac{150}{140}$ 


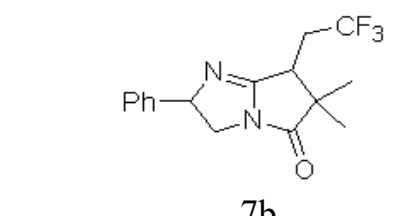

$7 \mathrm{~b}$

${ }^{19} \mathrm{~F}$ NMR (470 MHz, $\mathrm{CDCl}_{3}$ )

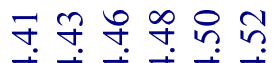

它 ț

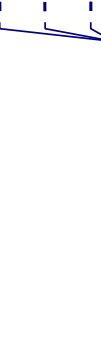

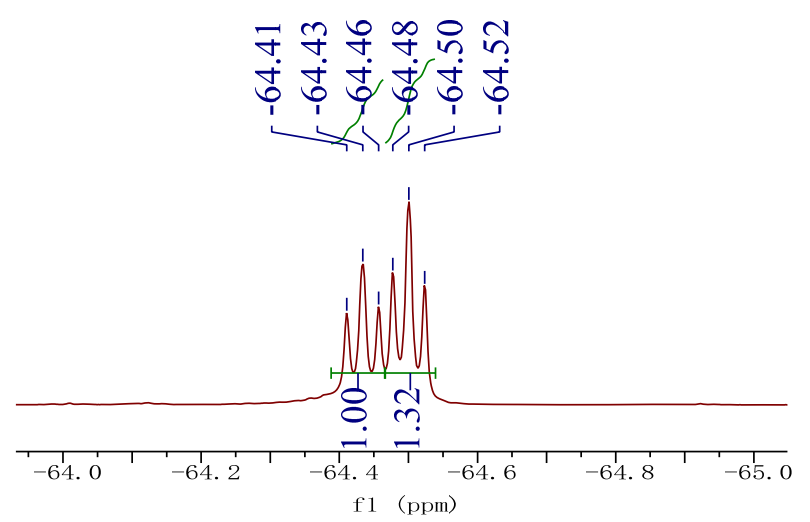

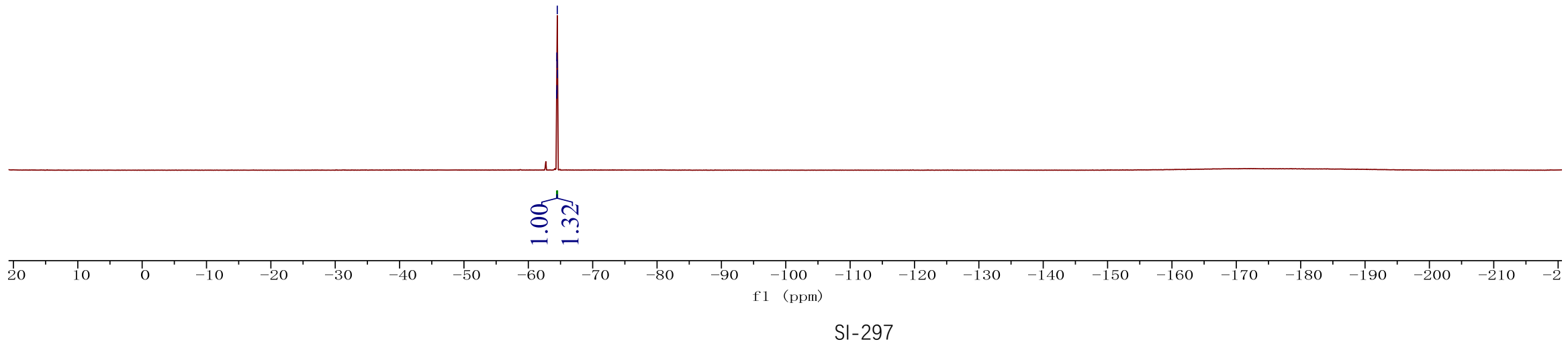




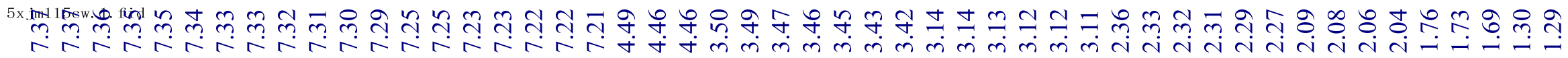

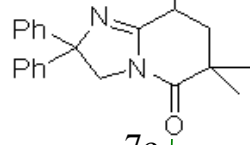

${ }^{1} \mathrm{H} \mathrm{NMR}\left(400 \mathrm{MHz}, \mathrm{CDCl}_{3}\right)$

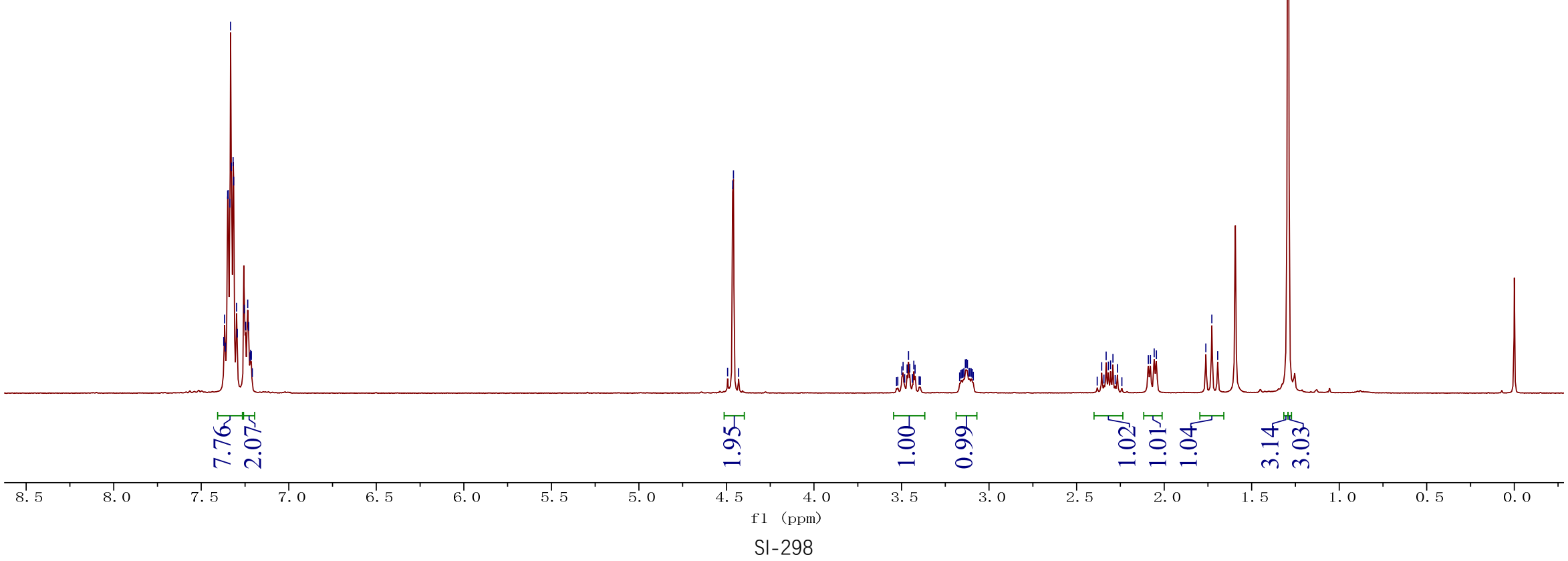




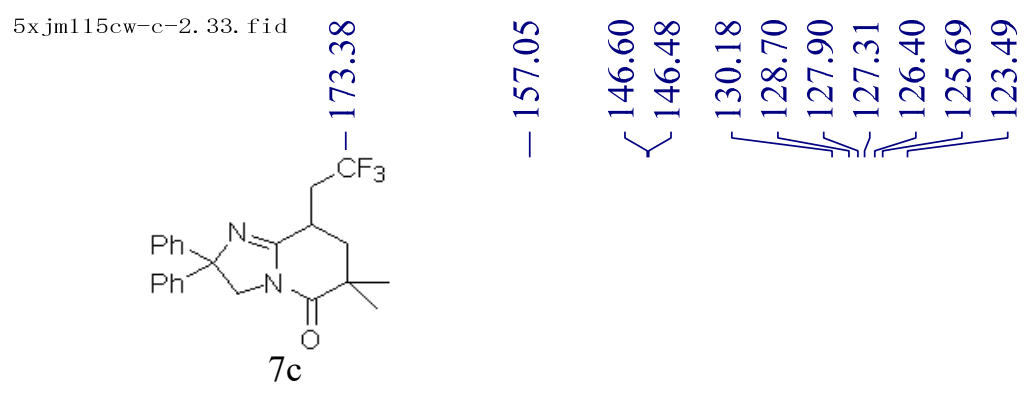

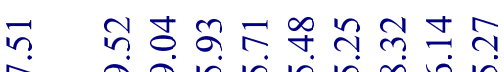

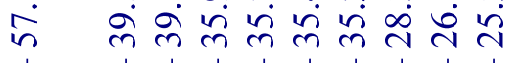

${ }^{13} \mathrm{C}$ NMR (125 MHz, $\left.\mathrm{CDCl}_{3}\right)$

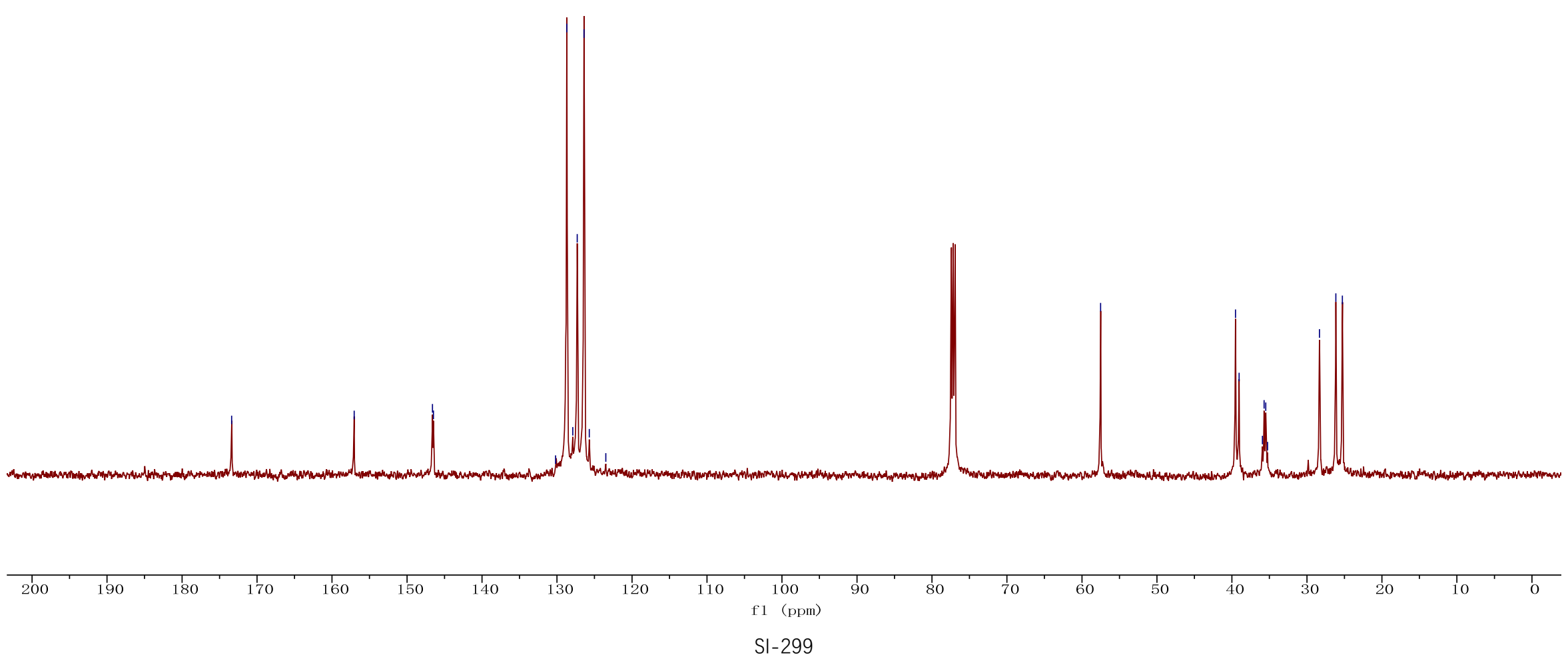


5x jm115cw-f. 31. fid

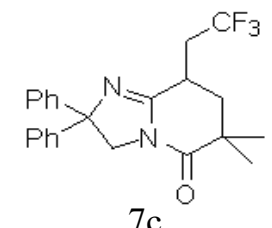

${ }^{19} \mathrm{~F}$ NMR (470 MHz, $\mathrm{CDCl}_{3}$ )
흐와

फ़्र

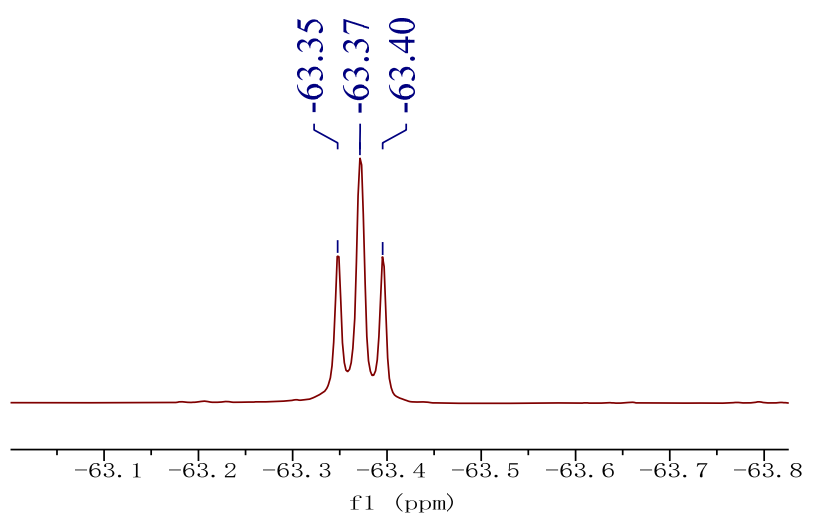

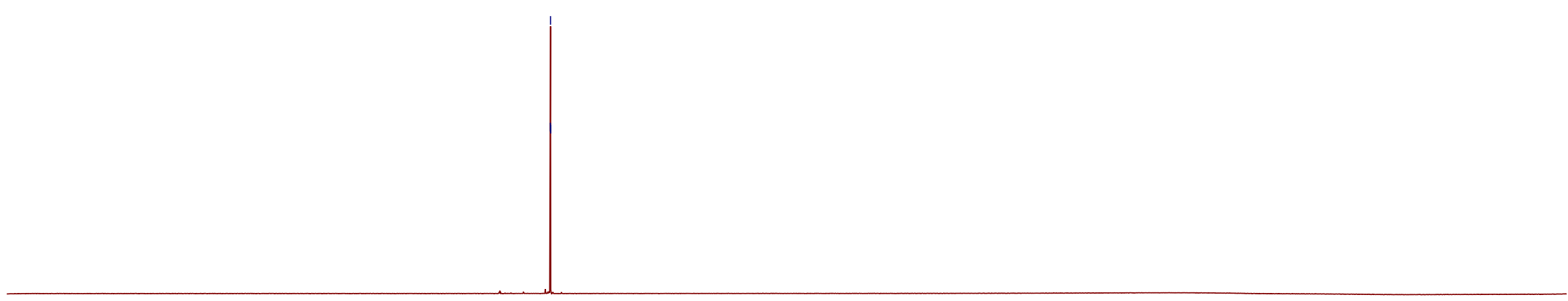




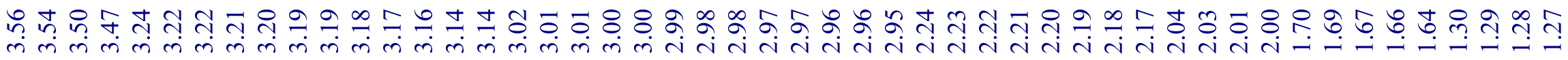
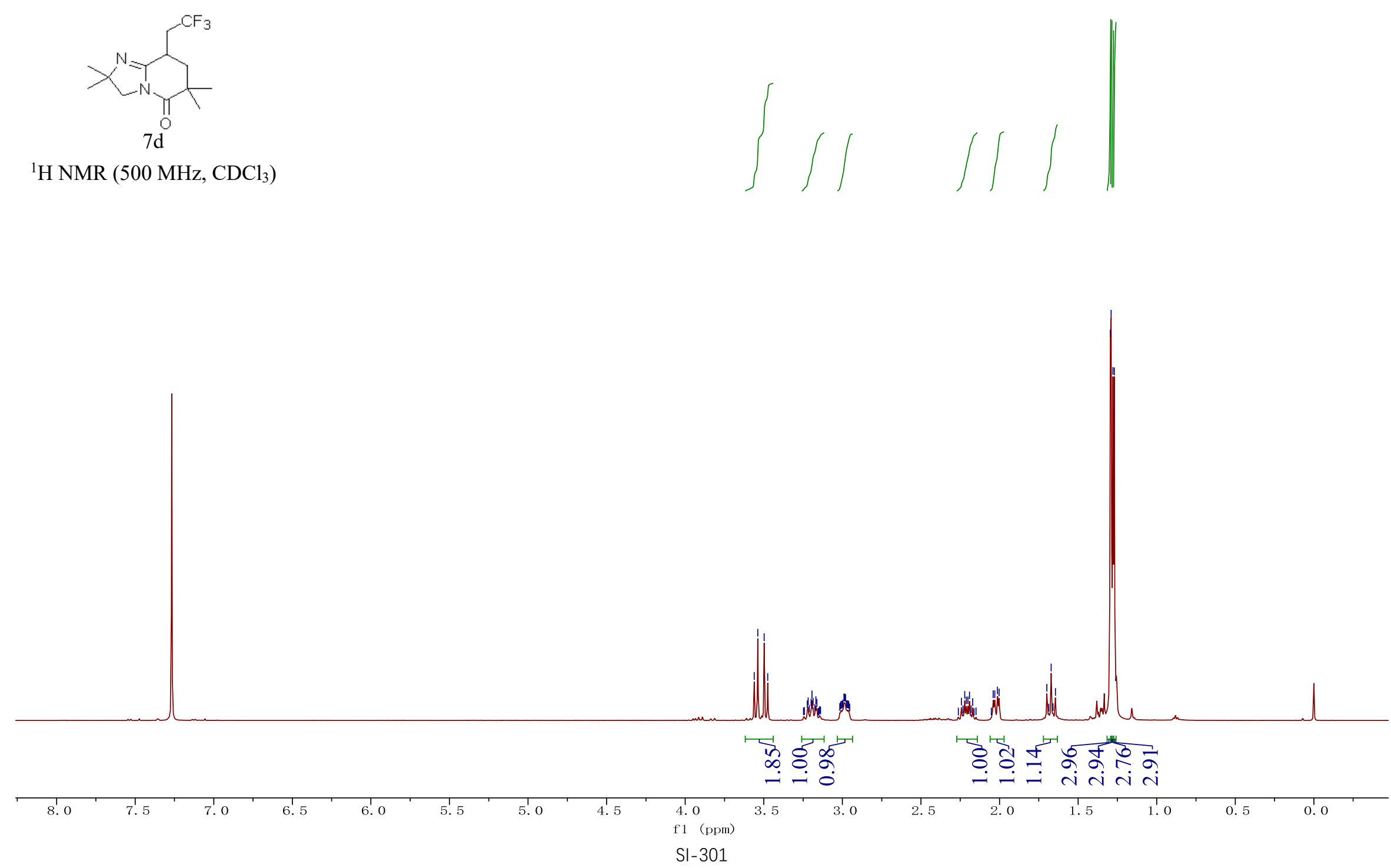


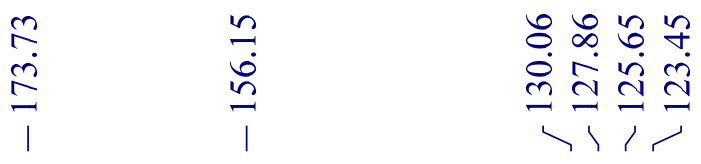

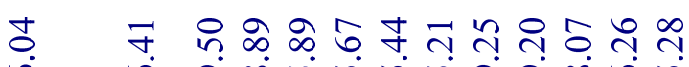

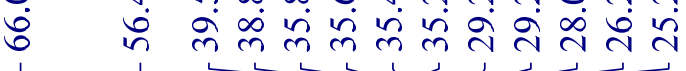

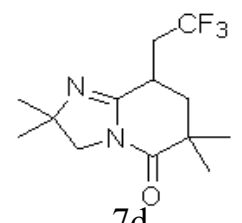

${ }^{13} \mathrm{C}$ NMR (125 MHz, $\left.\mathrm{CDCl}_{3}\right)$

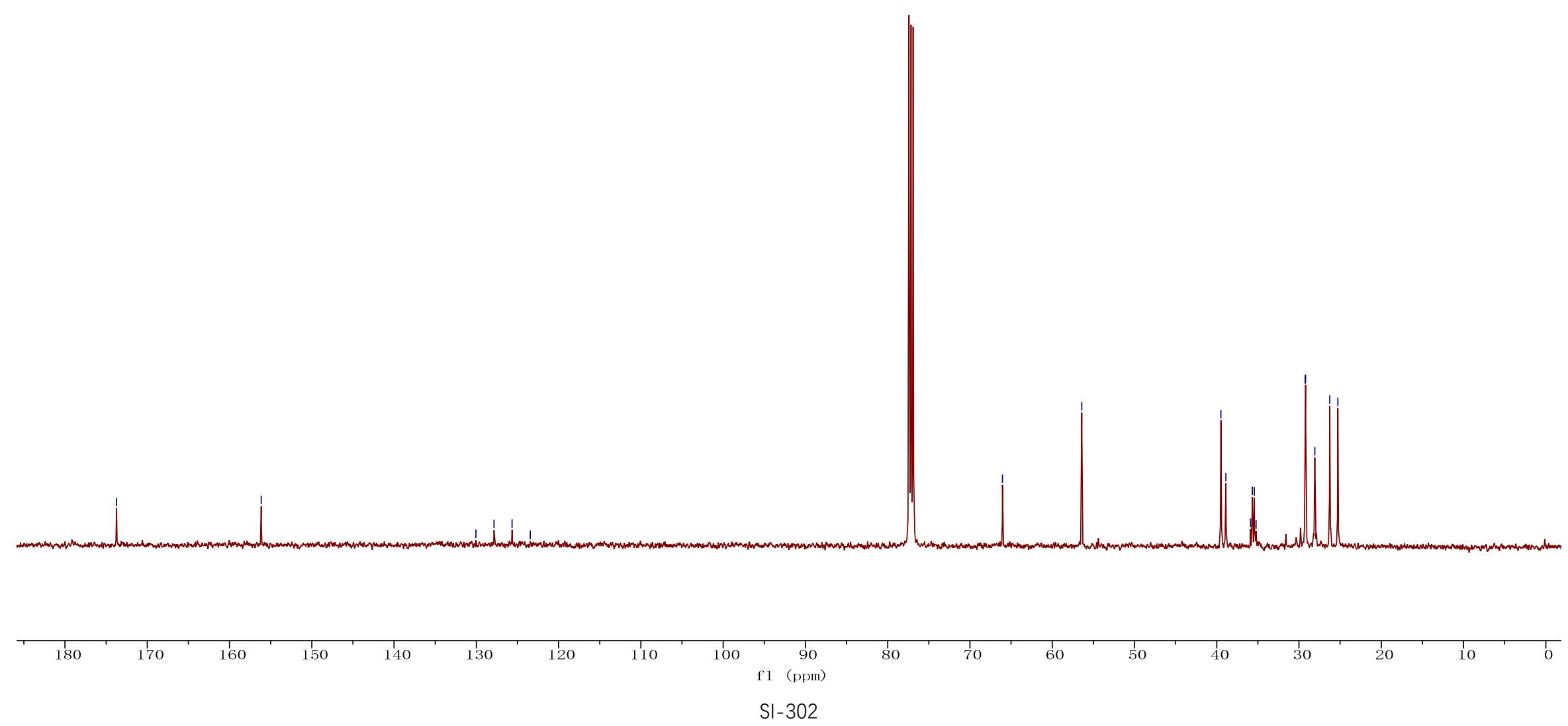


गे भ. 寸

ڤ్రి

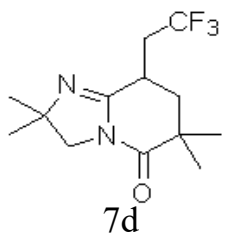

${ }^{19} \mathrm{~F}$ NMR $\left(470 \mathrm{MHz}, \mathrm{CDCl}_{3}\right.$ )

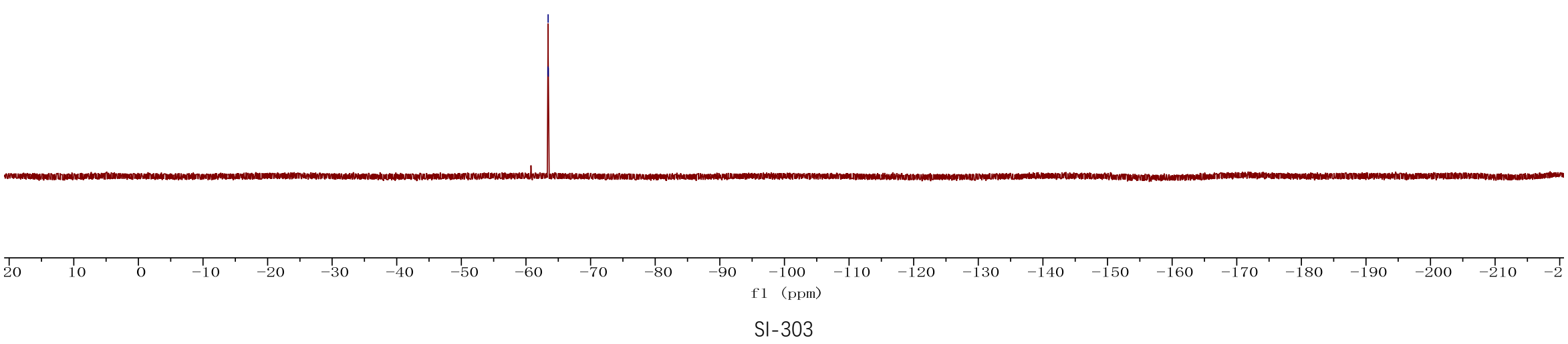

ले 광

बुं

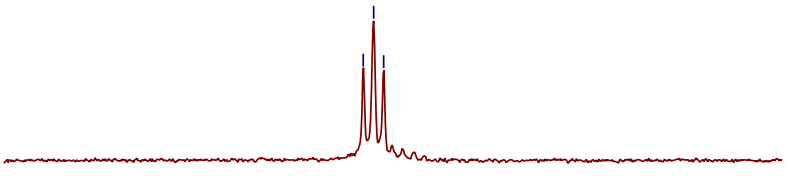

$-62.6^{\prime}-62.8^{\prime}-63.0-63.2^{\prime}-63.4-63.6^{\prime}-63.8^{\prime}-64.0^{\prime}-6^{\prime} .2^{\prime}$ f1 (ppm) 


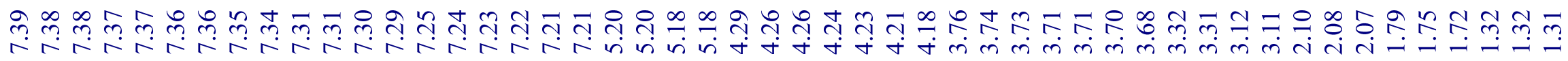

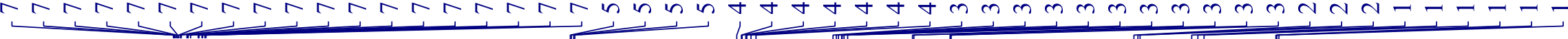
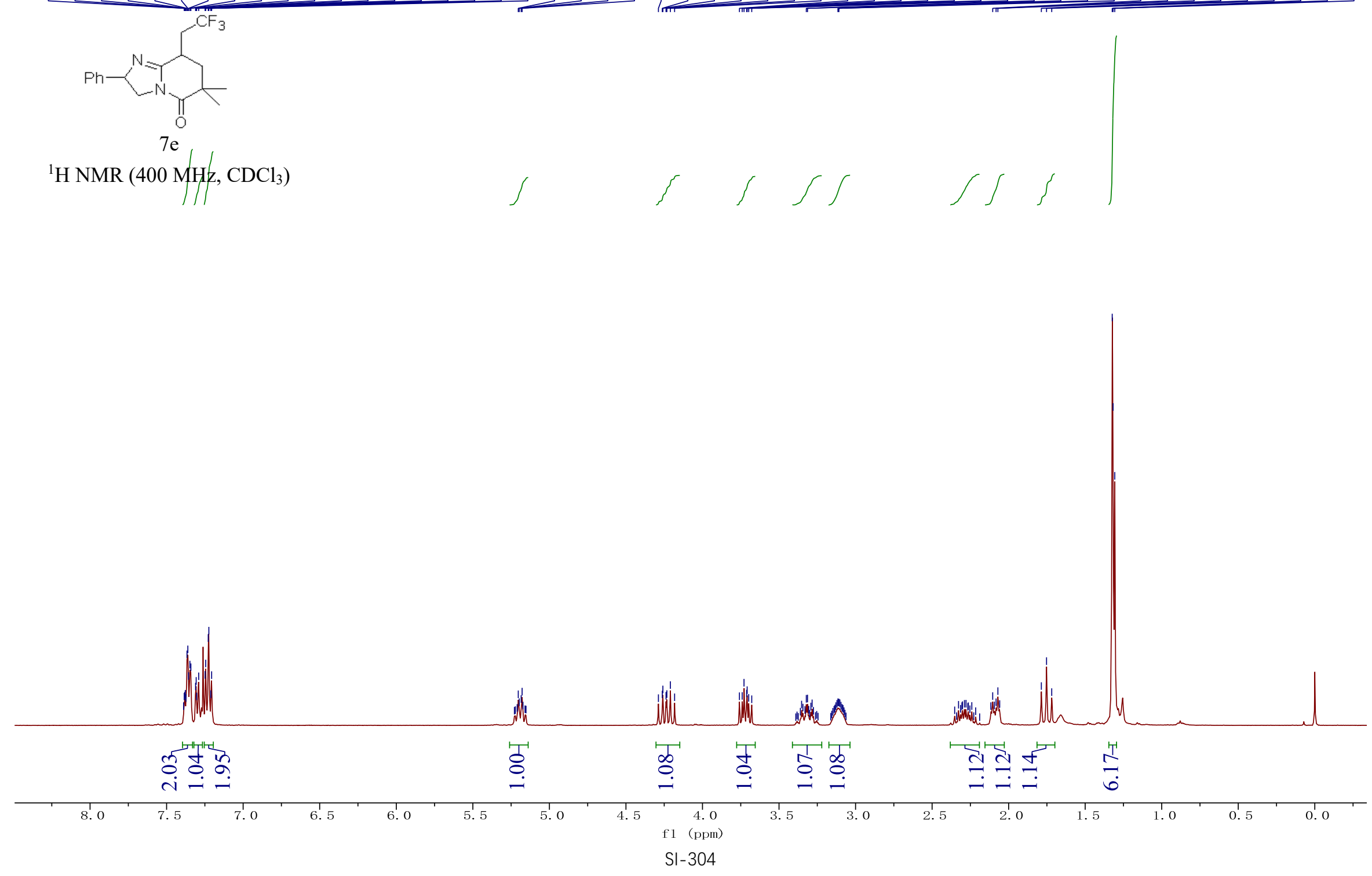


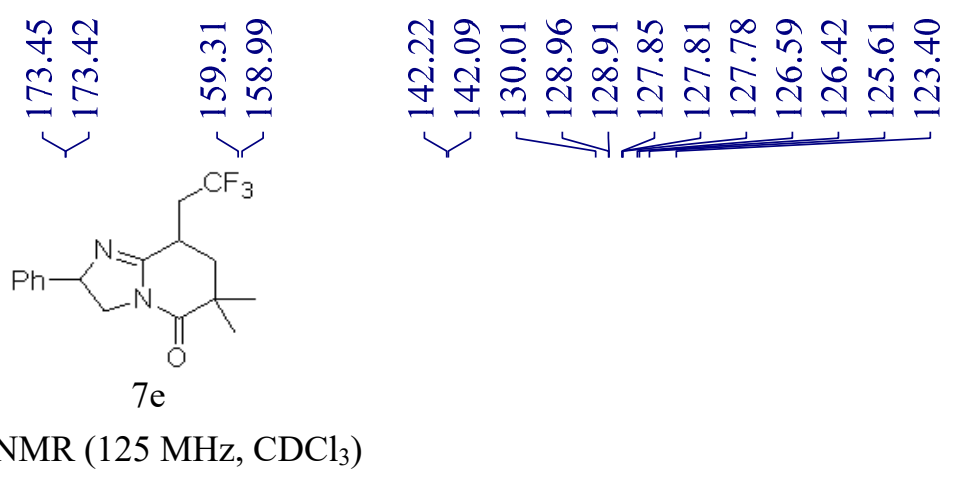

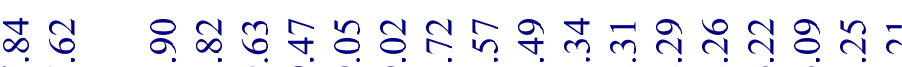

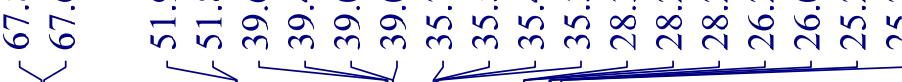

${ }^{13} \mathrm{C}$ NMR $\left(125 \mathrm{MHz}, \mathrm{CDCl}_{3}\right)$

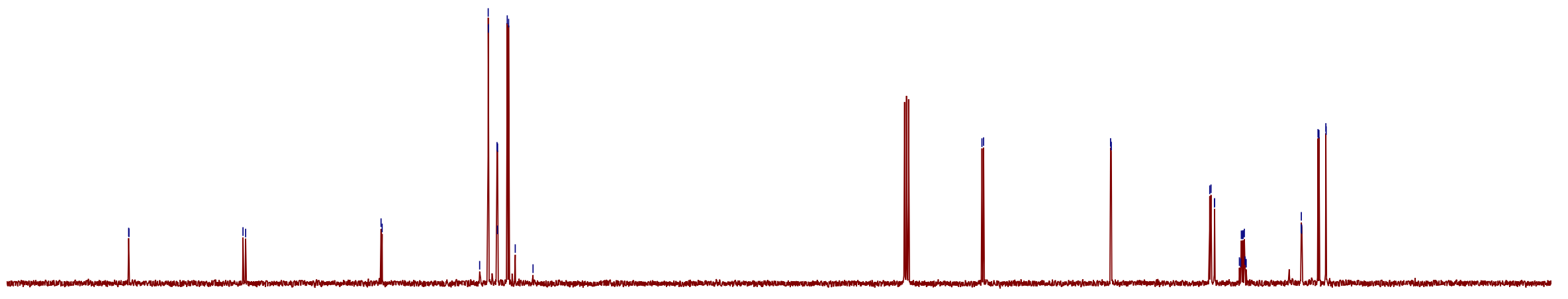

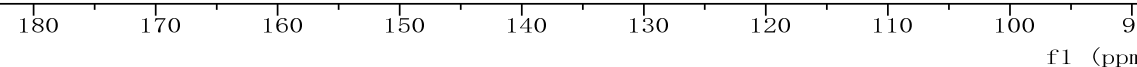
SI-305 


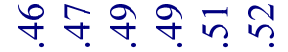

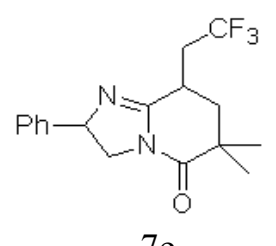

$7 \mathrm{e}$

${ }^{19} \mathrm{~F}$ NMR $\left(470 \mathrm{MHz}, \mathrm{CDCl}_{3}\right)$

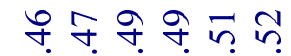

仓ें

1

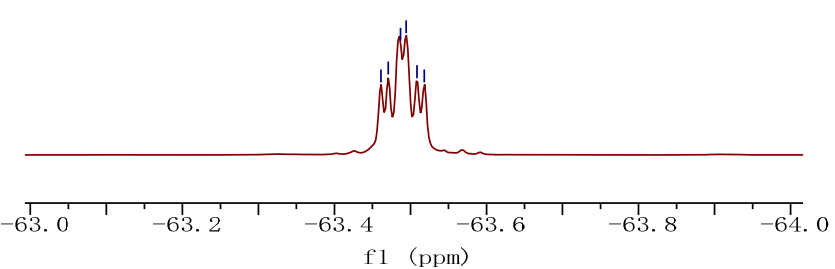

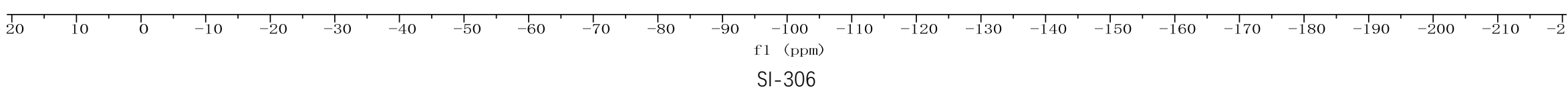




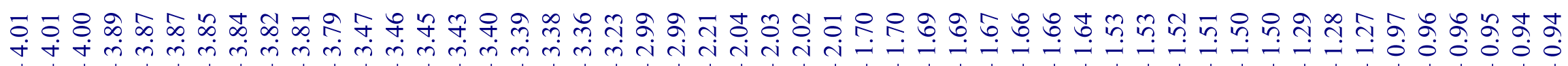
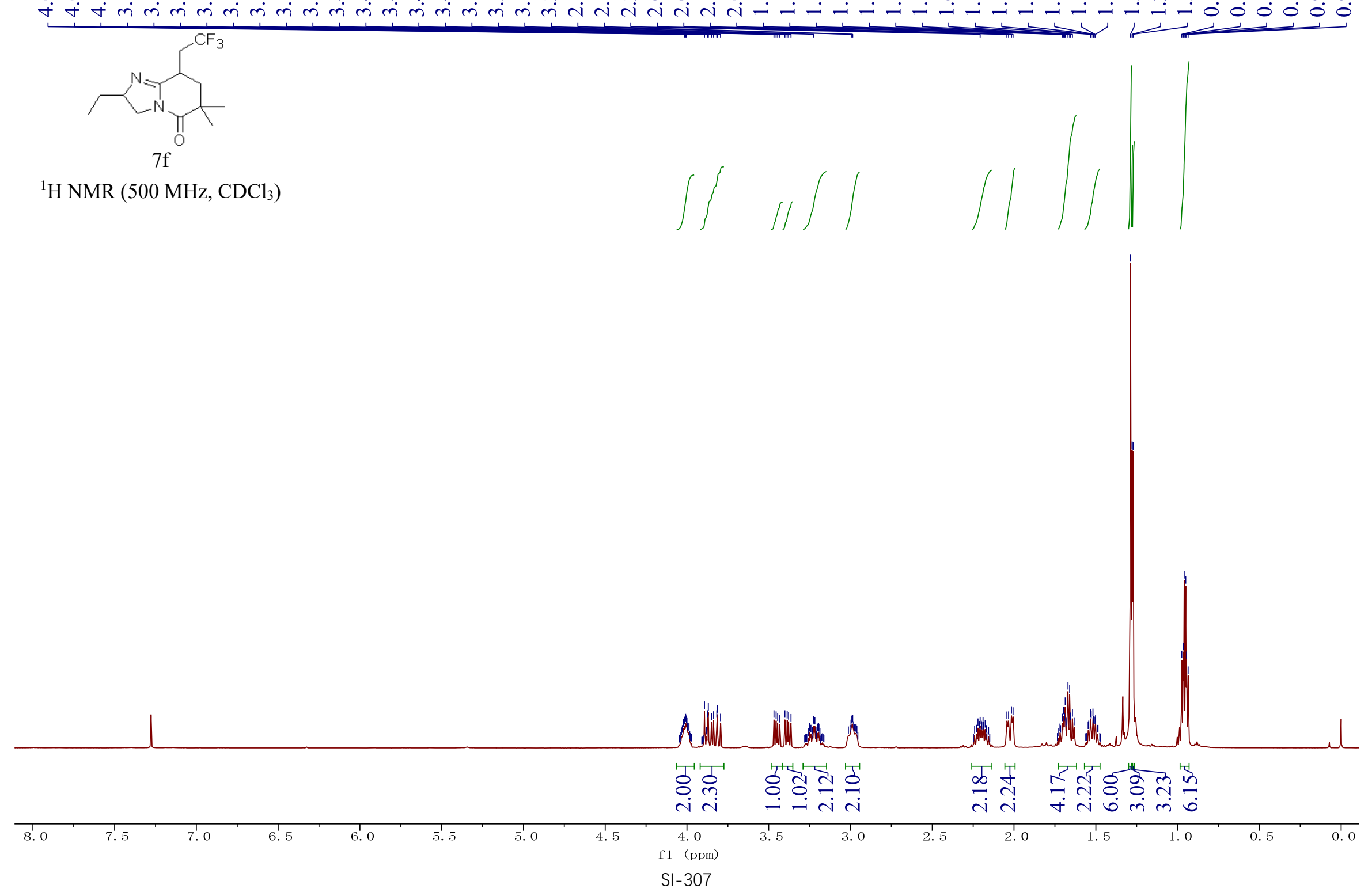


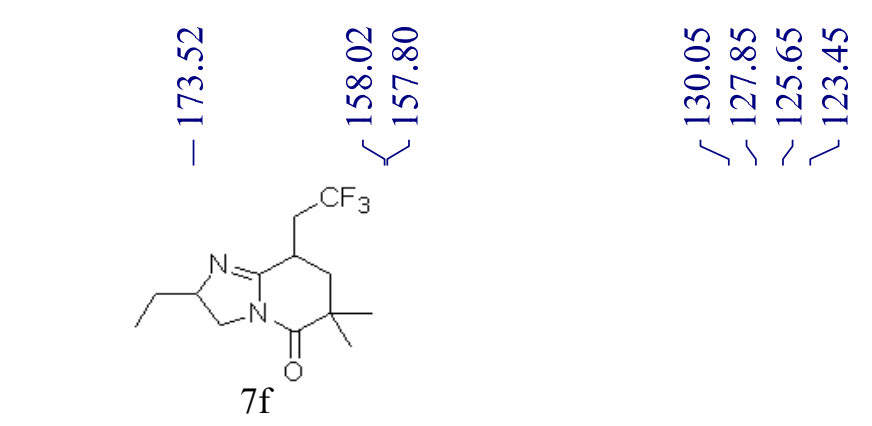

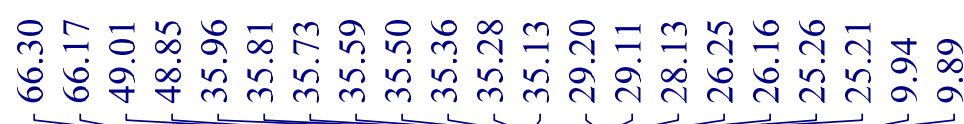

${ }^{13} \mathrm{C}$ NMR (125 MHz, $\left.\mathrm{CDCl}_{3}\right)$
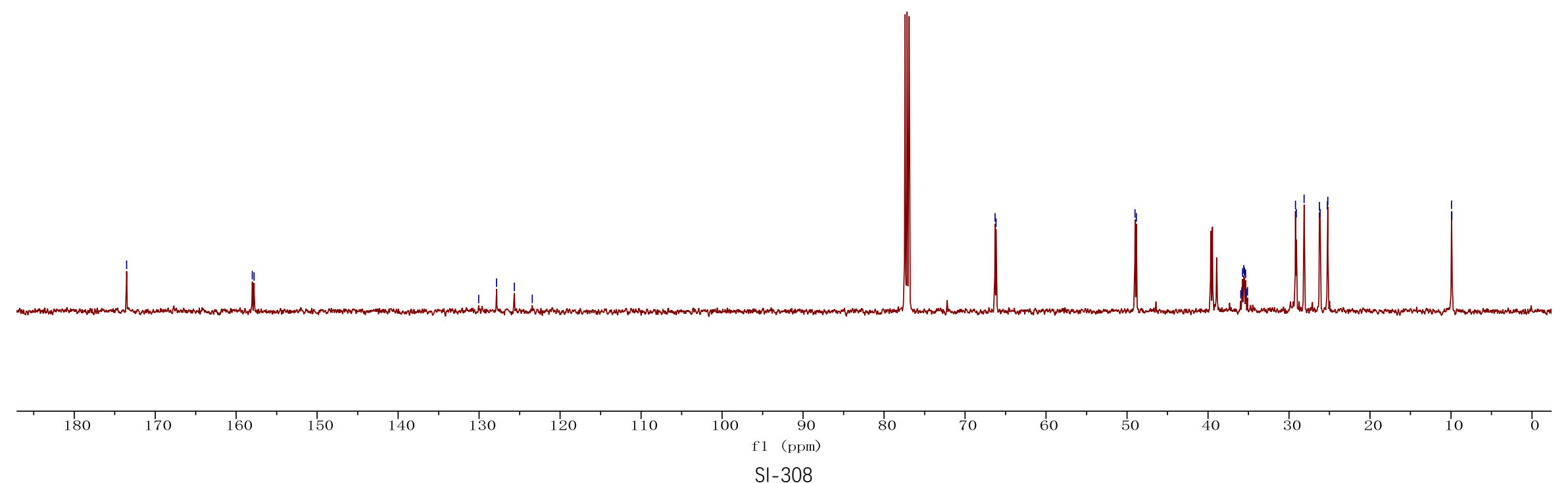


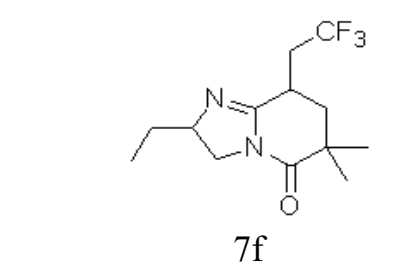

${ }^{19} \mathrm{~F}$ NMR (470 MHz, $\left.\mathrm{CDCl}_{3}\right)$

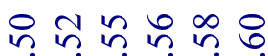

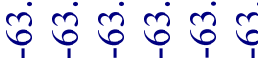

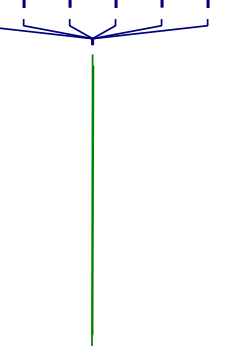

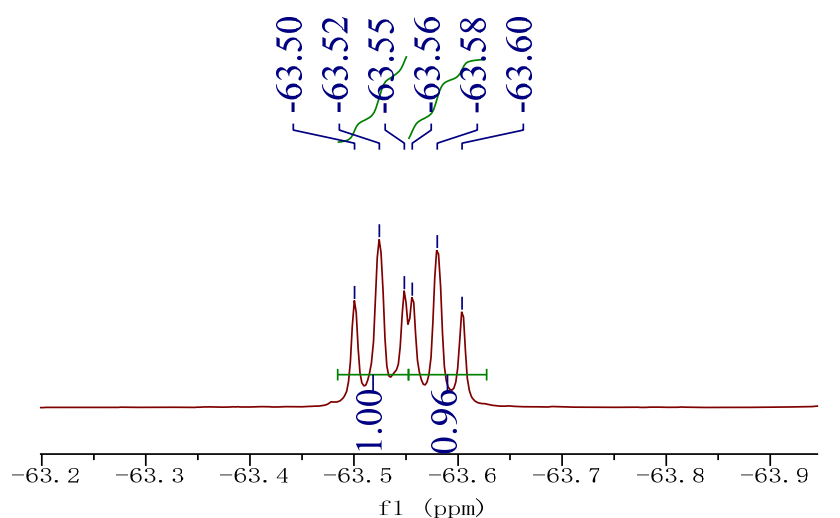

우요

1

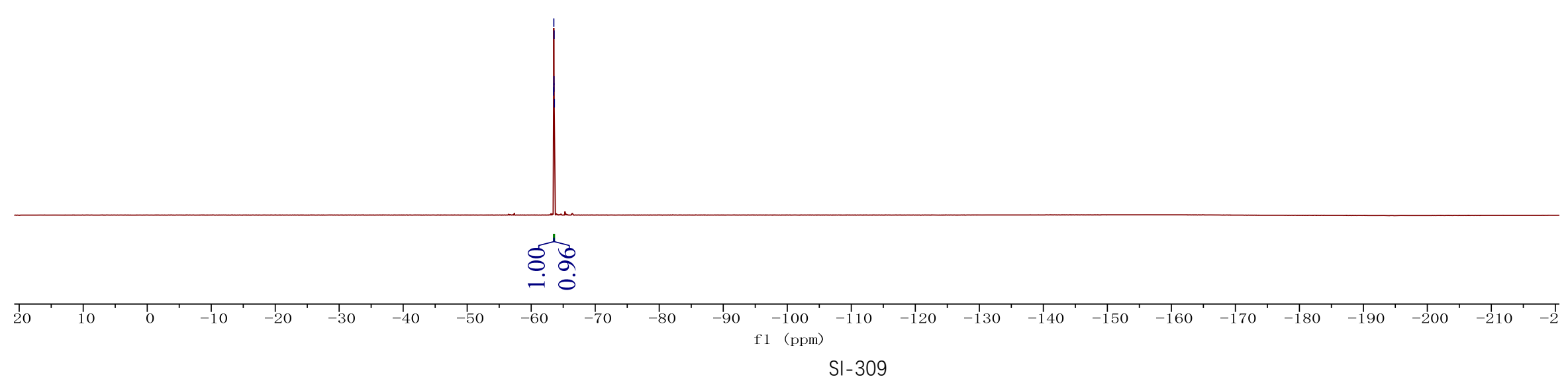




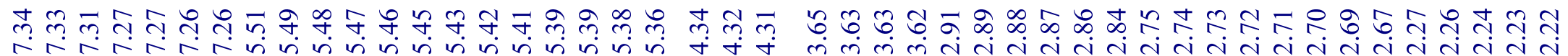

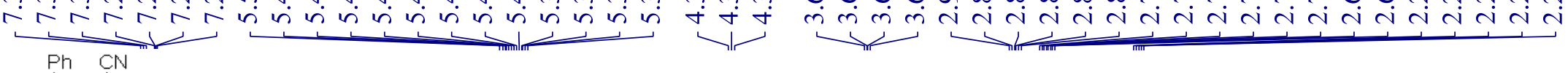
Pro $2 \mathrm{CF}_{3}$ 8

${ }^{1} \mathrm{H}$ NMR $\left(500\left(\mathrm{MHz}, \mathrm{CDCl}_{3}\right)\right.$

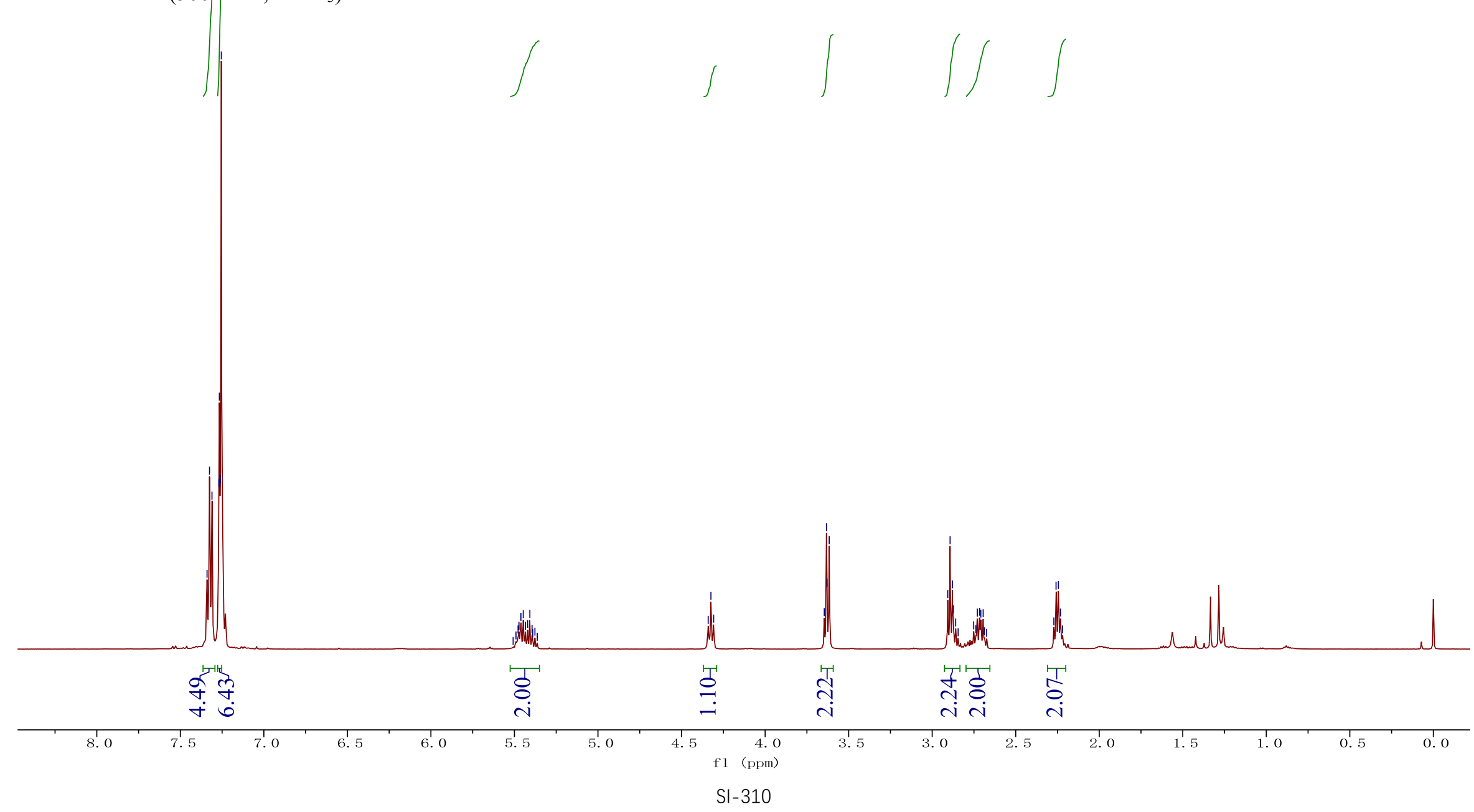




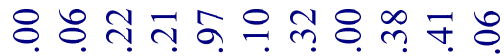

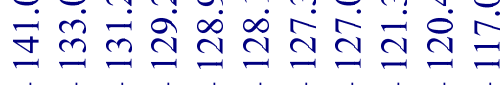

$\longrightarrow$

Ph CN

Ph

${ }^{13} \mathrm{C}$ NMR (125 MHz, $\left.\mathrm{CDCl}_{3}\right)$

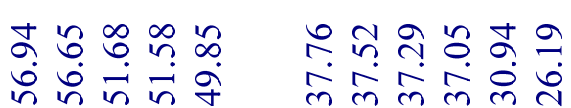

mmm

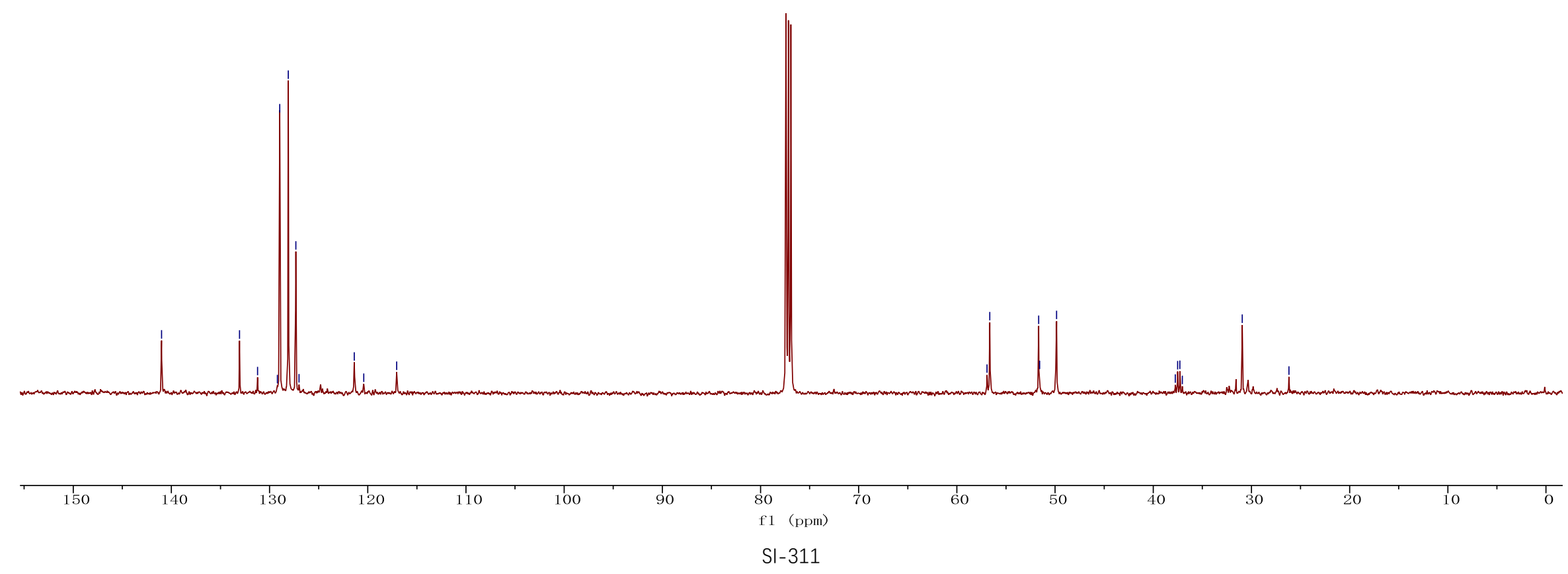




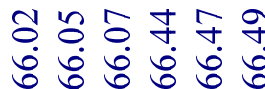

Ph CN

نا

8

${ }^{19} \mathrm{~F}$ NMR $\left(470 \mathrm{MHz}, \mathrm{CDCl}_{3}\right)$

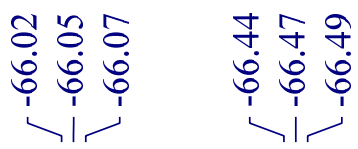
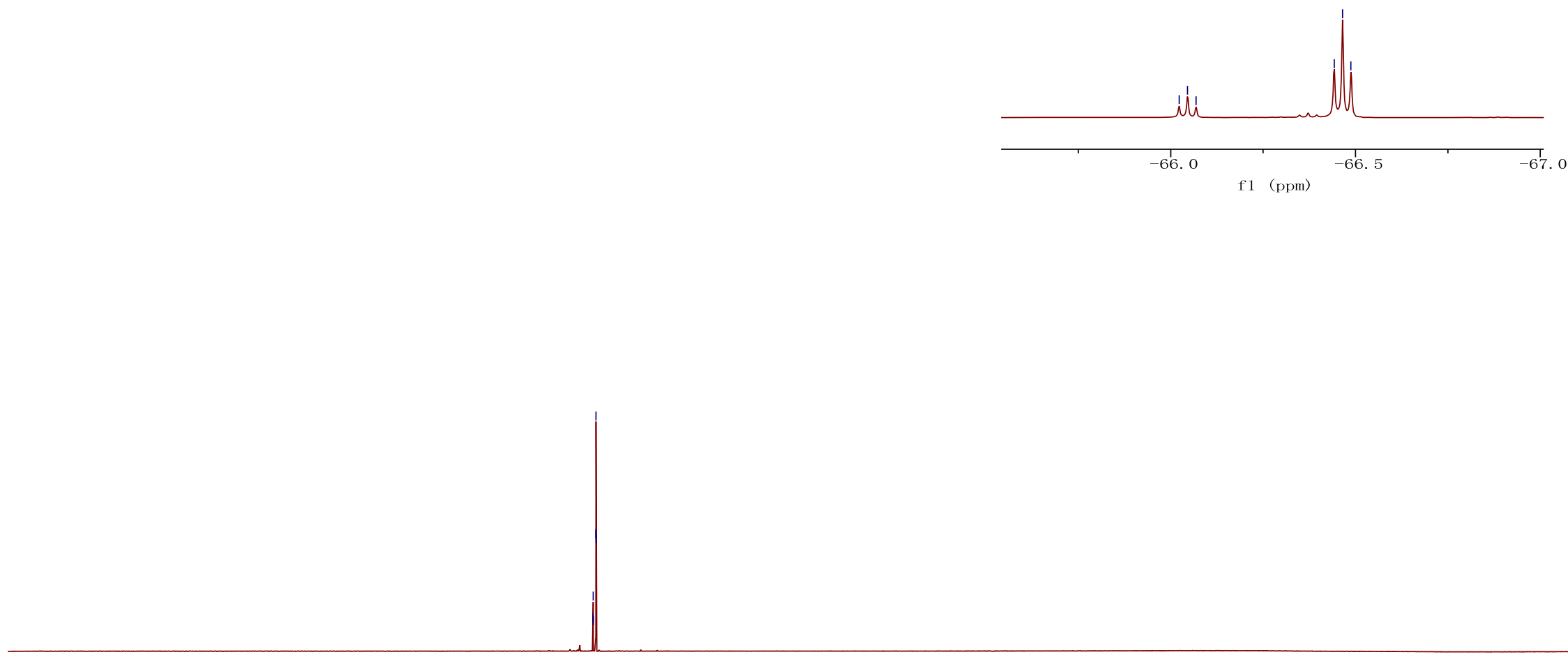

20

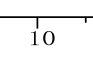

o
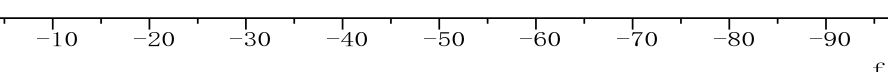
$\mathrm{f} 1 \stackrel{-100}{(\mathrm{ppm})}$

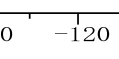

$-130$
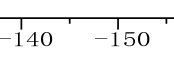


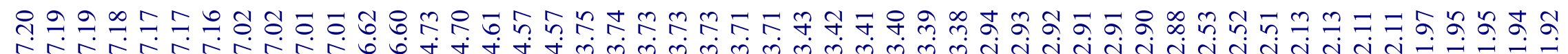

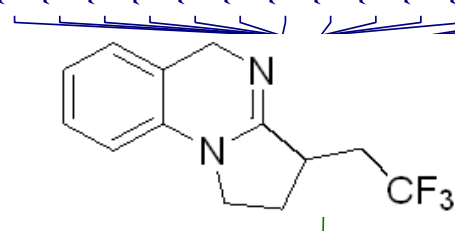

$10 \mathrm{a}$

${ }^{1} \mathrm{H} \mathrm{NMR}\left(500 \mathrm{MHz}, \mathrm{CDCl}_{3}\right)$
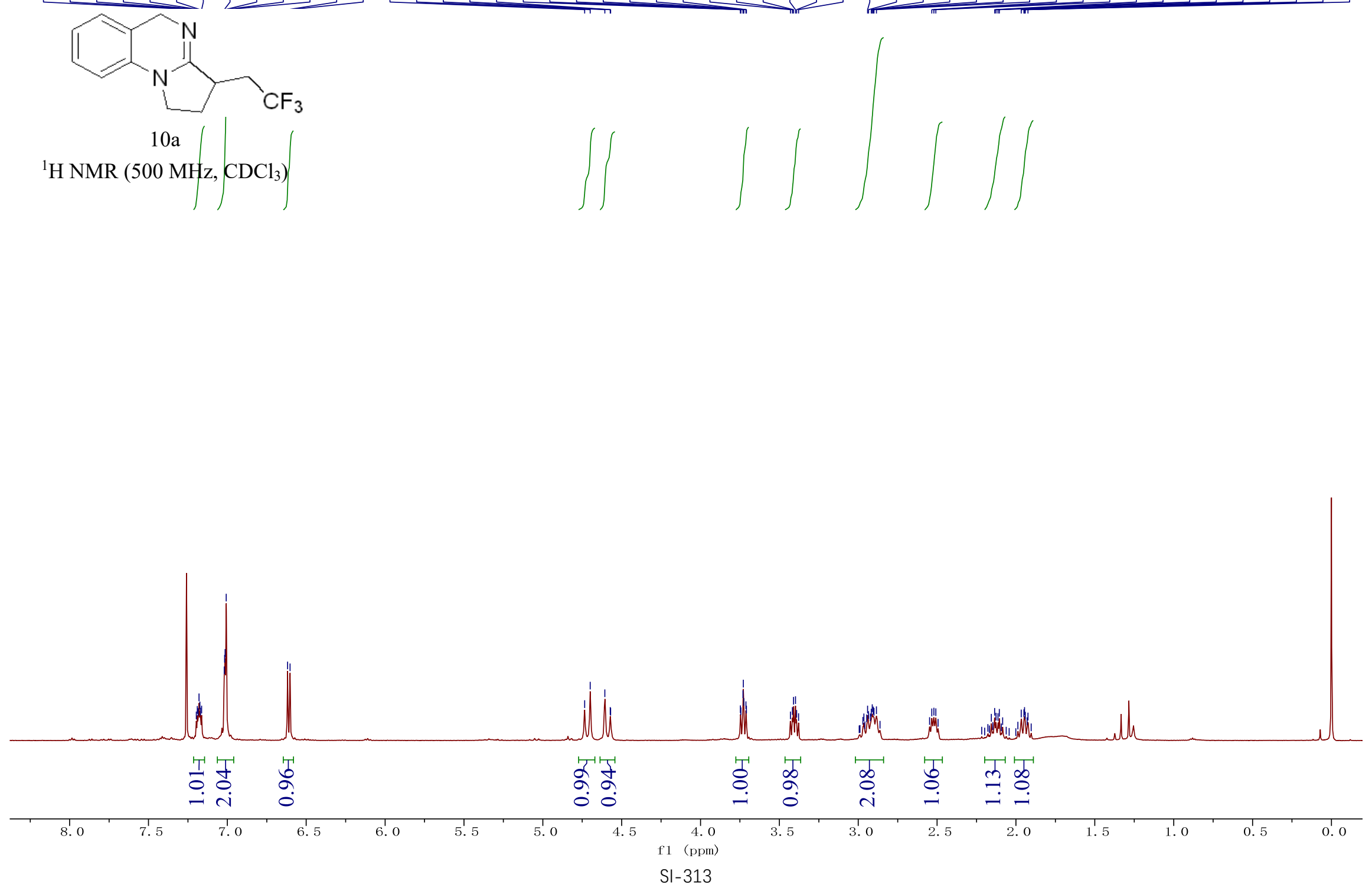


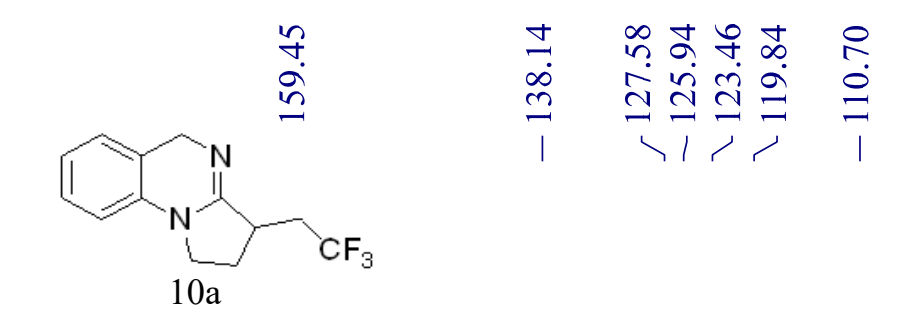

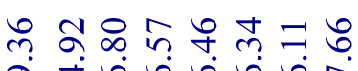

子े

${ }^{13} \mathrm{C}$ NMR (125 MHz, $\left.\mathrm{CDCl}_{3}\right)$

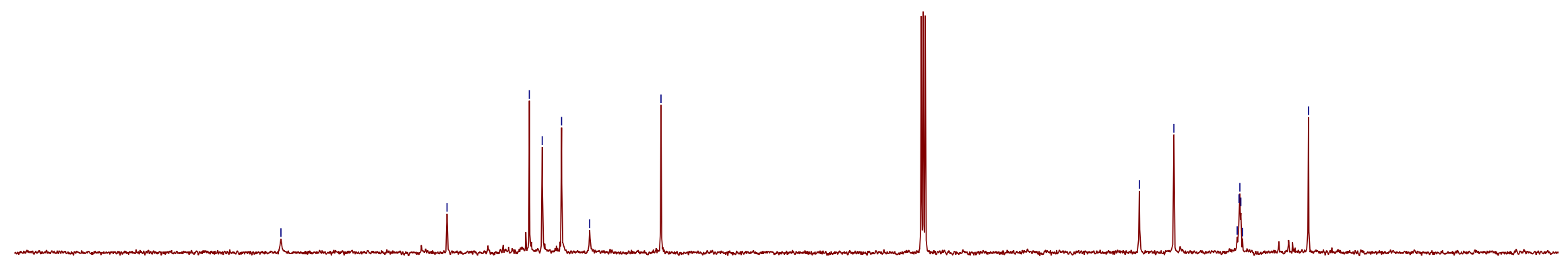


곤

守 守 守

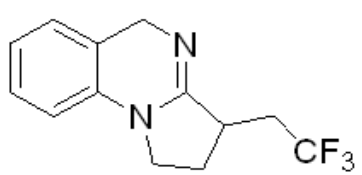

$10 \mathrm{a}$

${ }^{19} \mathrm{~F}$ NMR $\left(470 \mathrm{MHz}, \mathrm{CDCl}_{3}\right)$

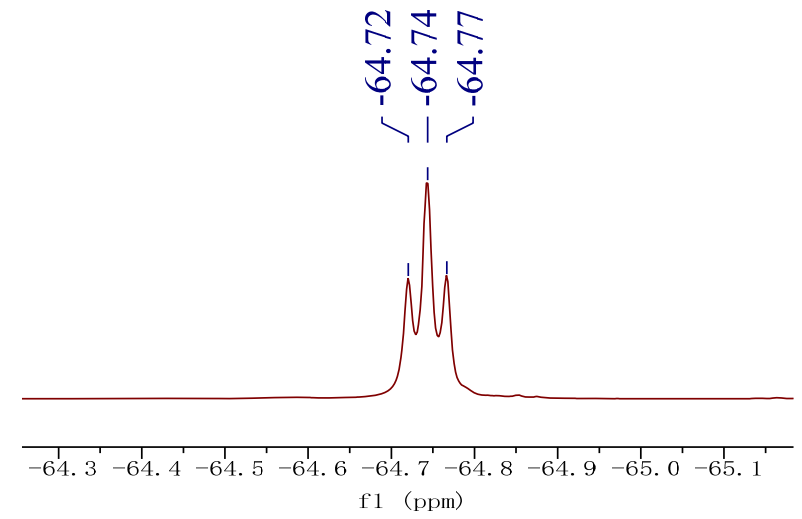

f1 (ppm)

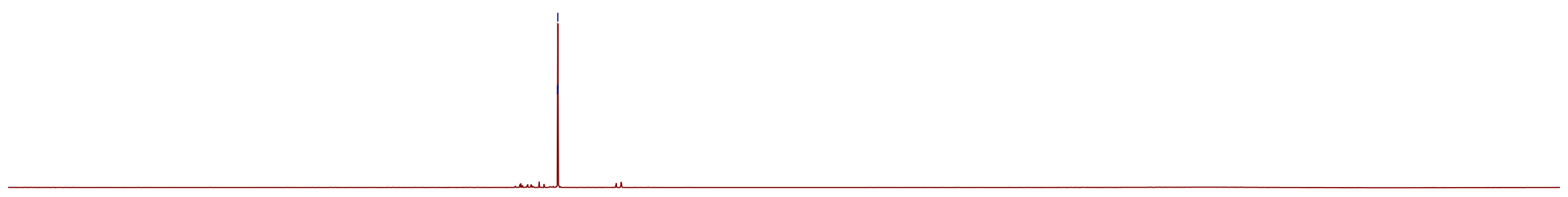




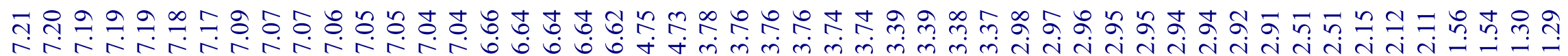
rrarrar
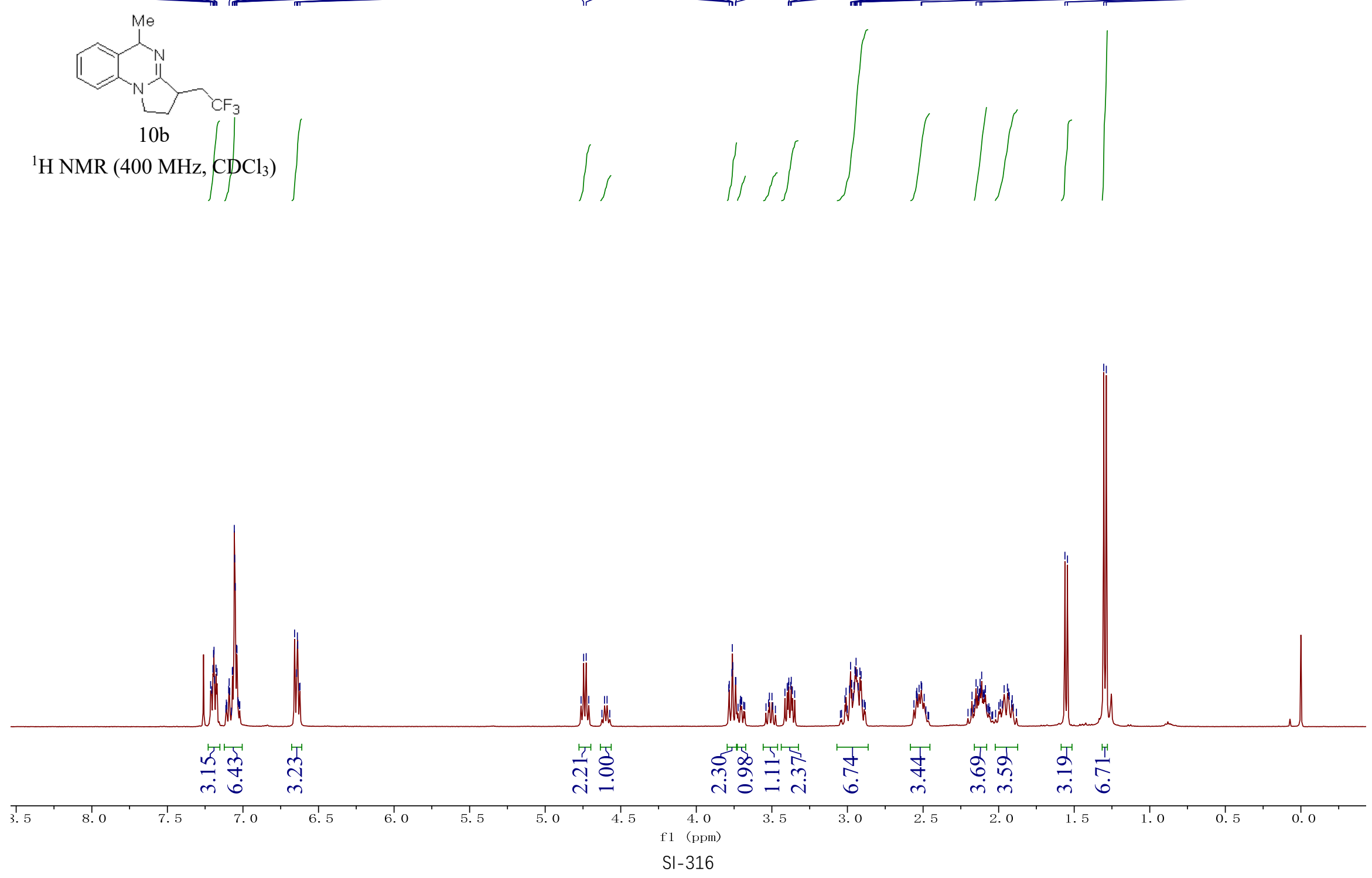


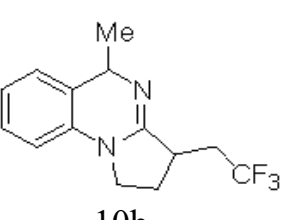

$10 \mathrm{~b}$

${ }^{13} \mathrm{C} \mathrm{NMR}\left(125 \mathrm{MHz}, \mathrm{CDCl}_{3}\right)$

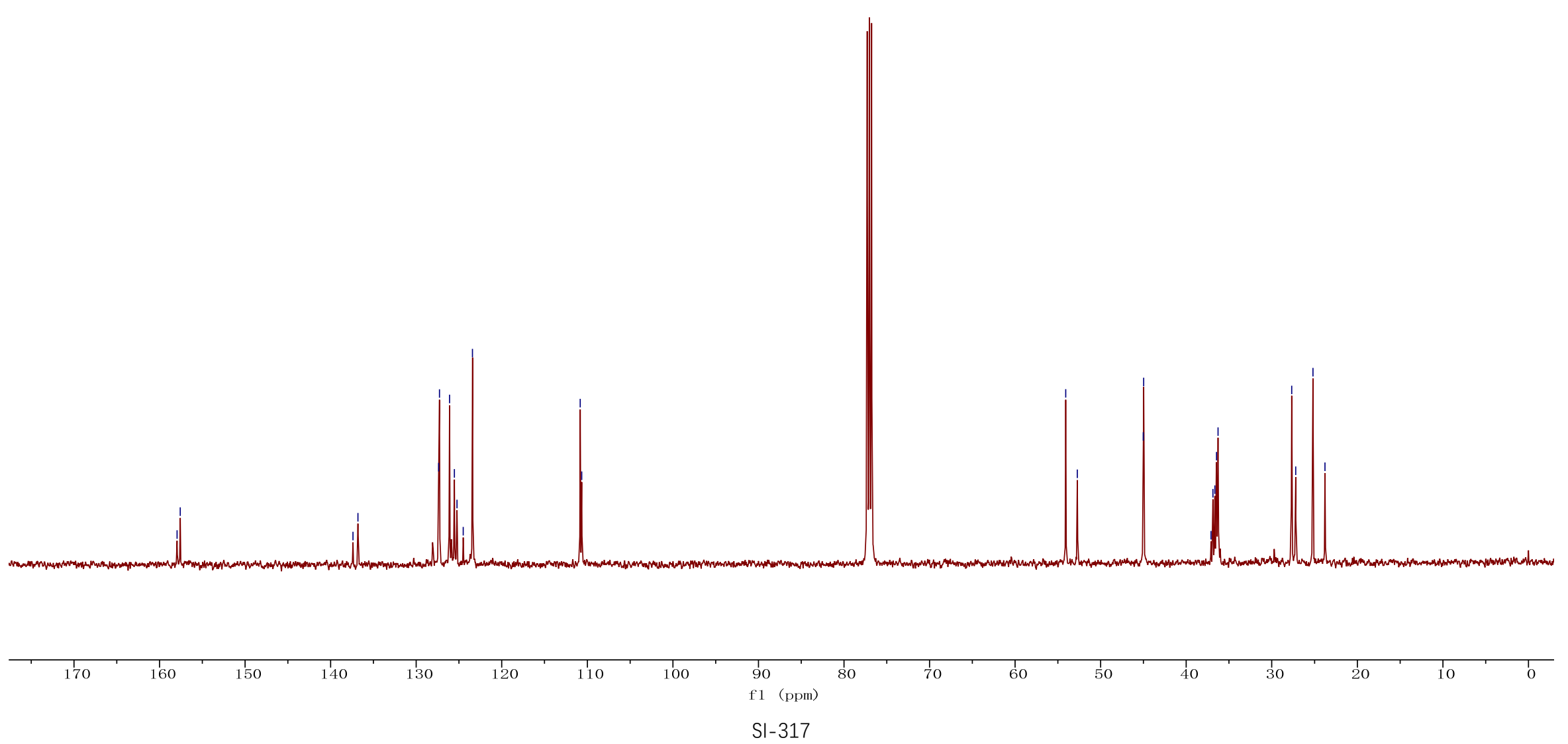




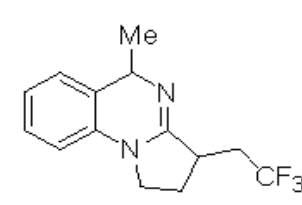

$10 \mathrm{~b}$

${ }^{19} \mathrm{~F}$ NMR (470 MHz, $\mathrm{CDCl}_{3}$ )

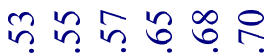

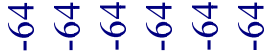

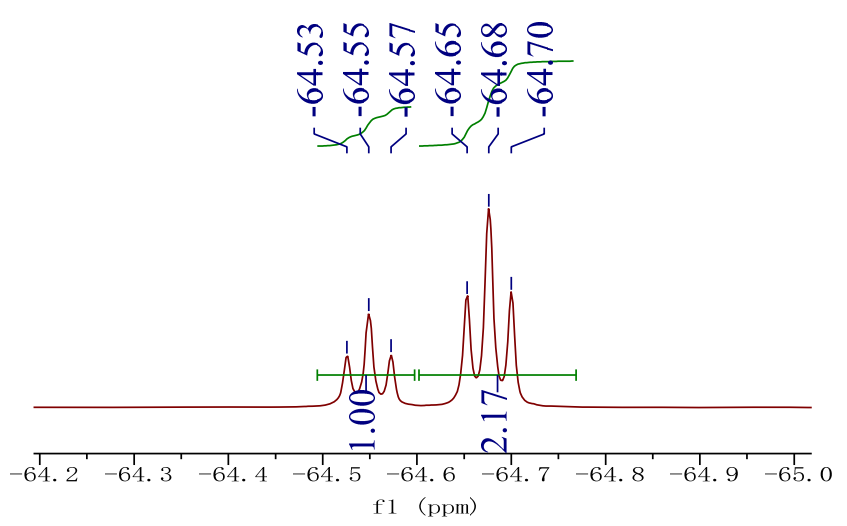

응 n

i

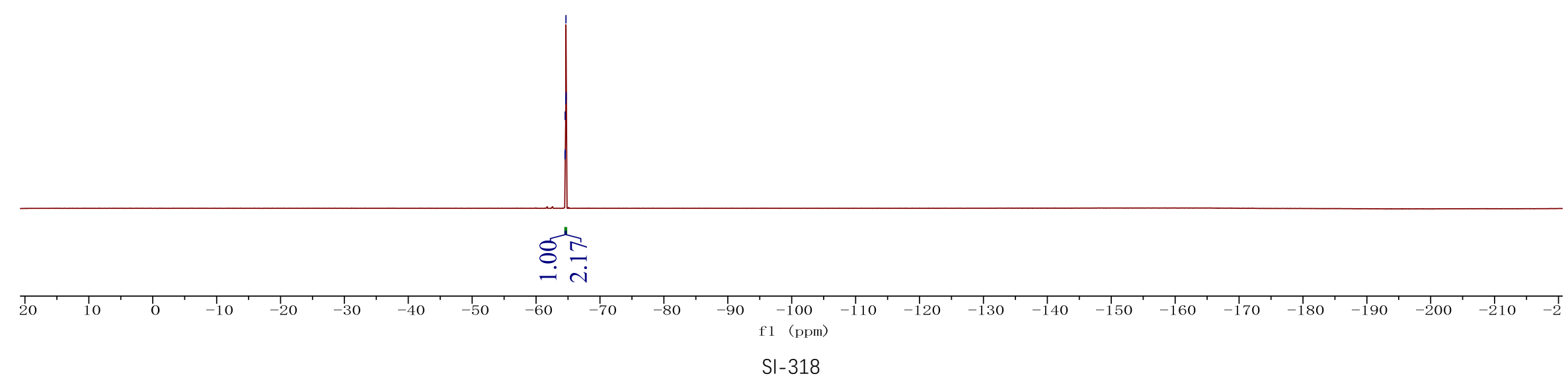




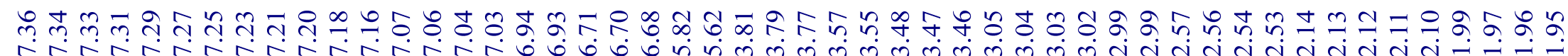
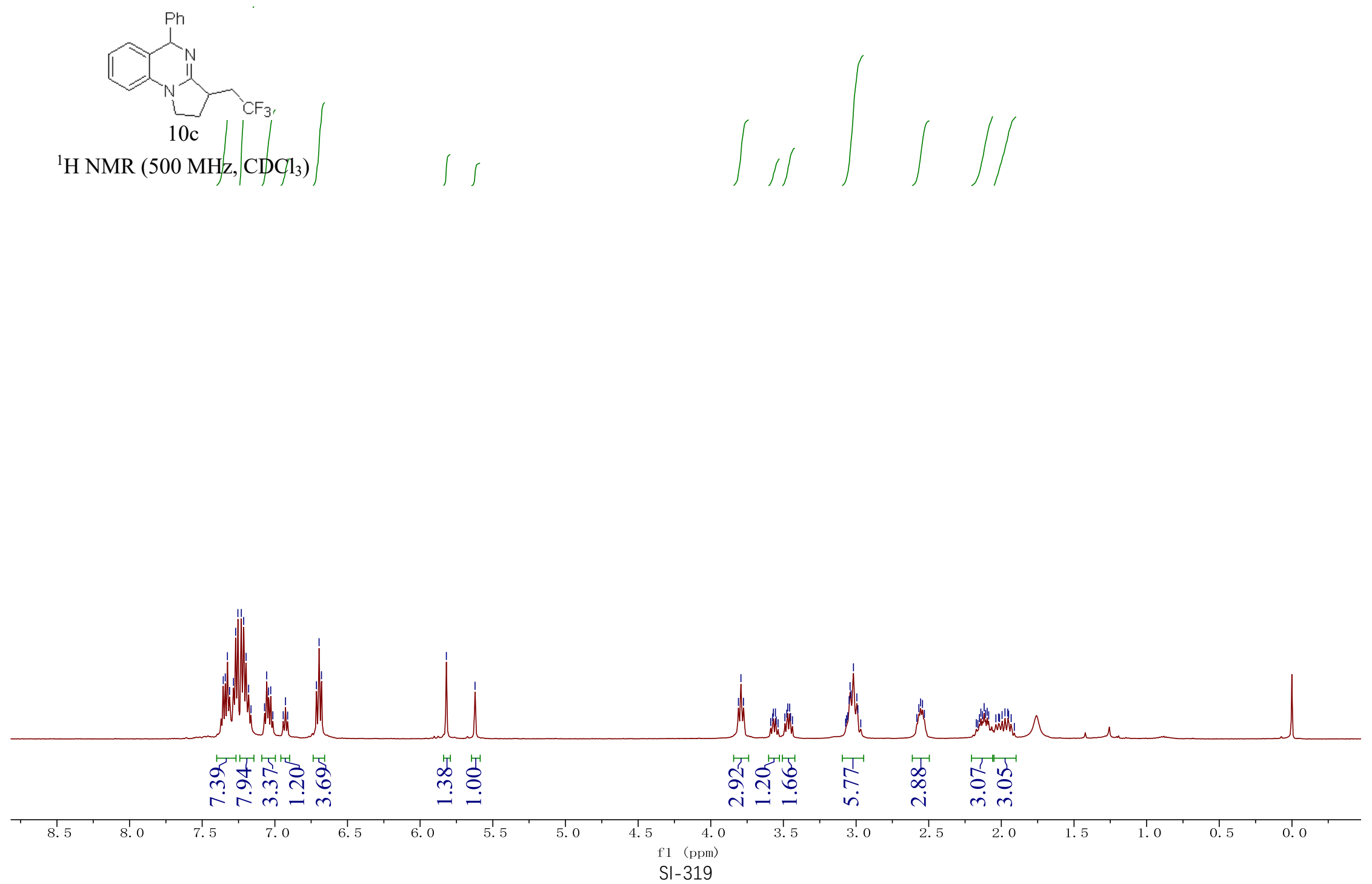


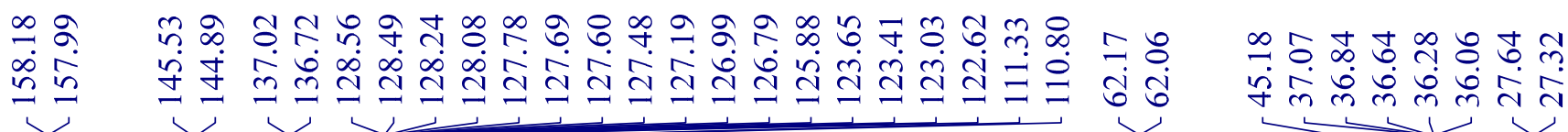

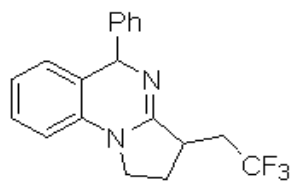

$10 \mathrm{c}$

${ }^{13} \mathrm{C}$ NMR $\left(125 \mathrm{MHz}, \mathrm{CDCl}_{3}\right)$
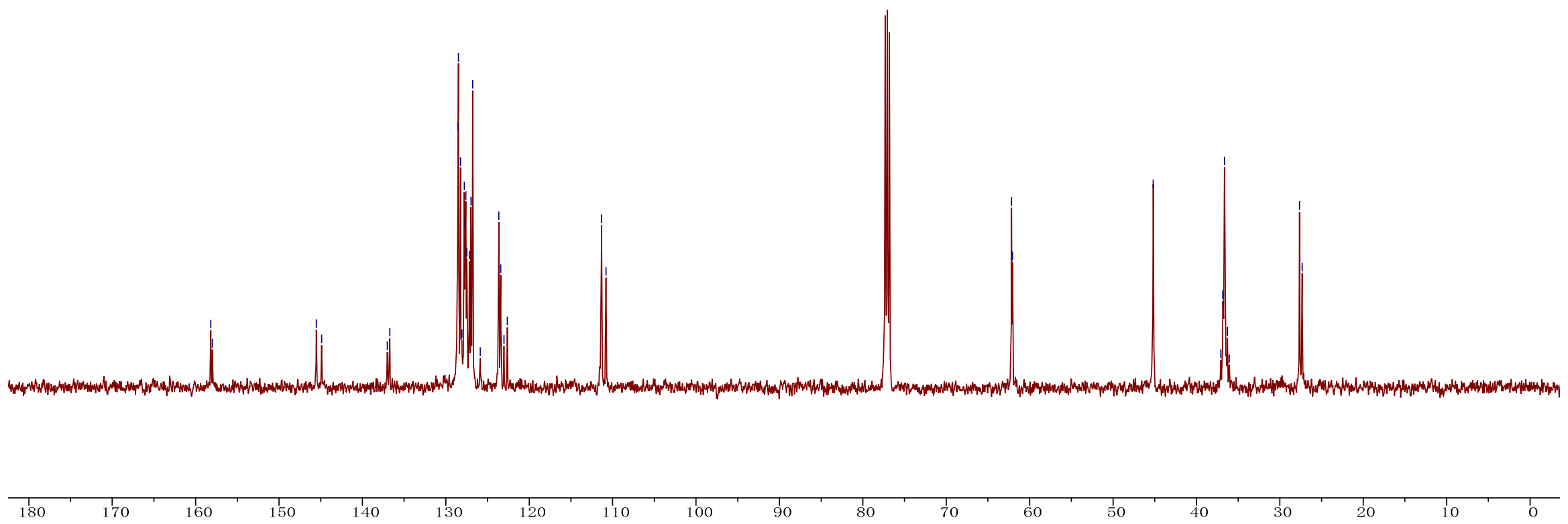


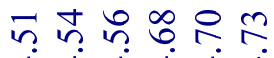

ن

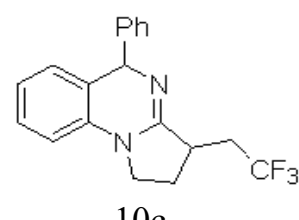

${ }^{19} \mathrm{~F}$ NMR $\left(470 \mathrm{MHz}, \mathrm{CDCl}_{3}\right)$

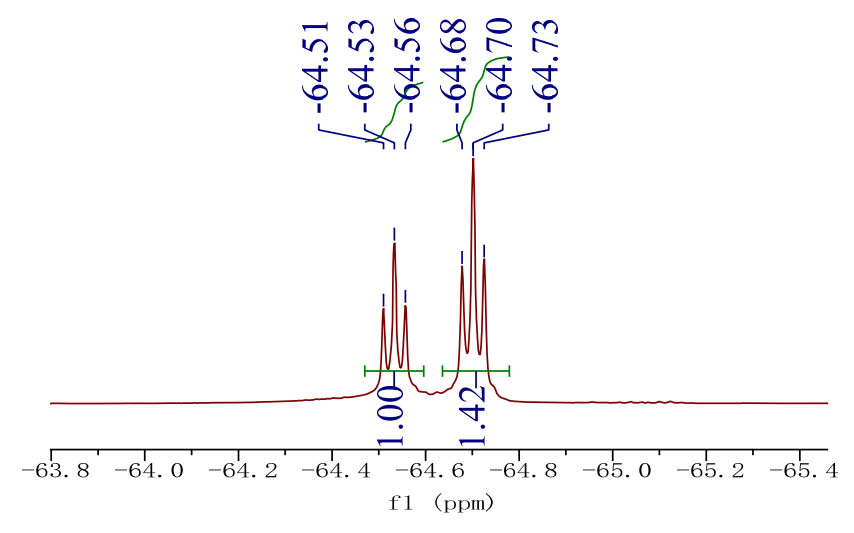

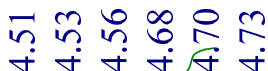

1 1

(र)

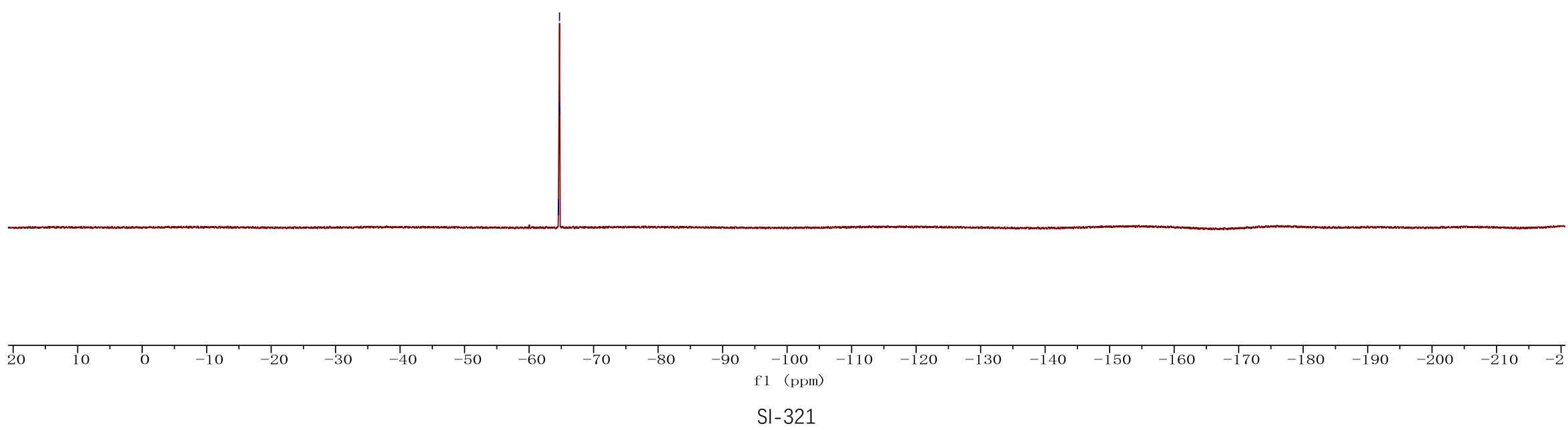




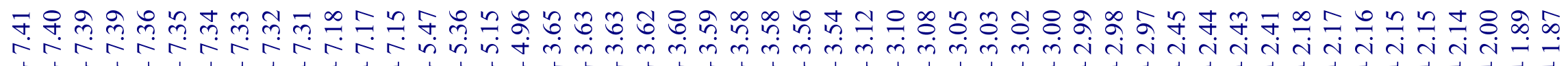

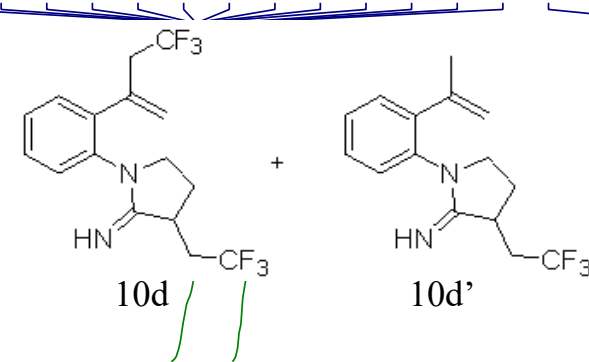

${ }^{1} \mathrm{H}$ NMR (500 MHz, $\mathrm{CDCl}_{3}$ )

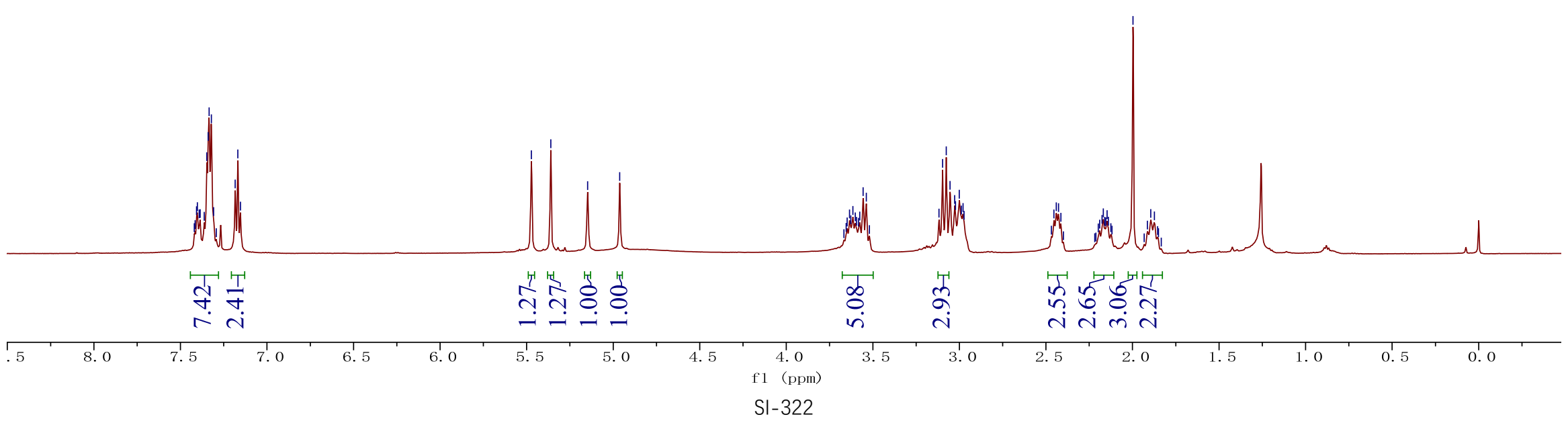




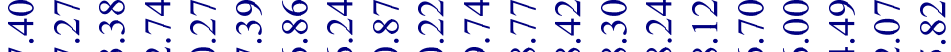

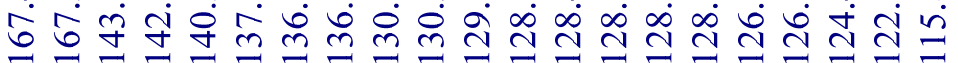

r
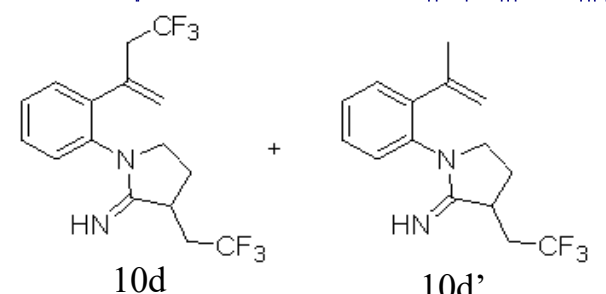

${ }^{13} \mathrm{C}$ NMR $\left(125 \mathrm{MHz}, \mathrm{CDCl}_{3}\right)$

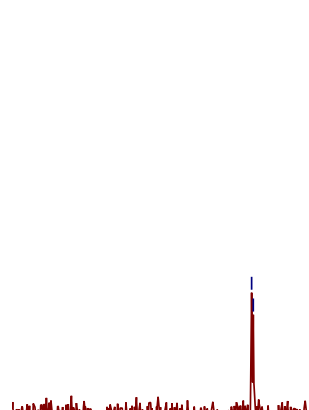

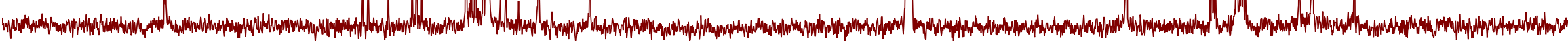

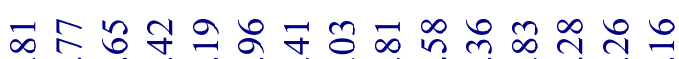

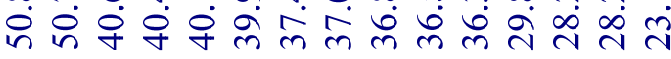

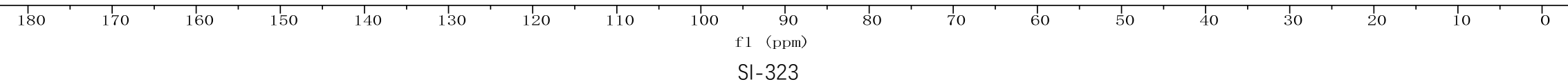



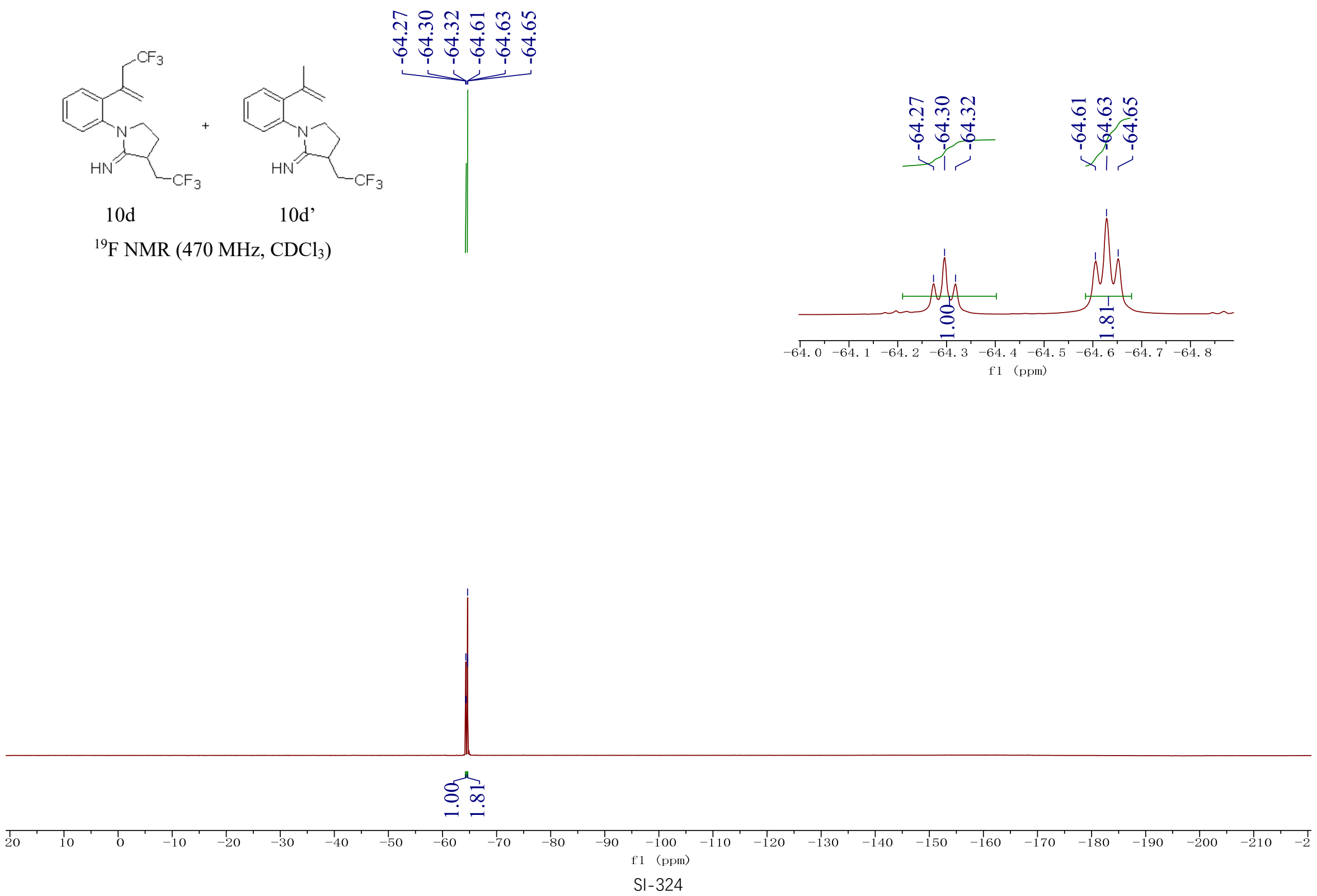


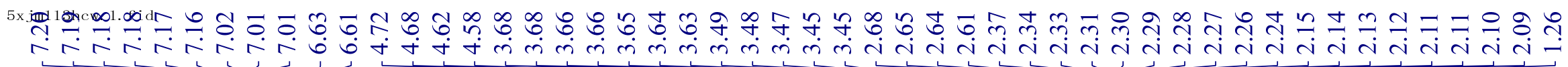

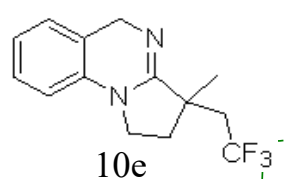

${ }^{1} \mathrm{H} \mathrm{NMR}\left(400 \mathrm{MHz}, \mathrm{CDCl}_{3}\right)$
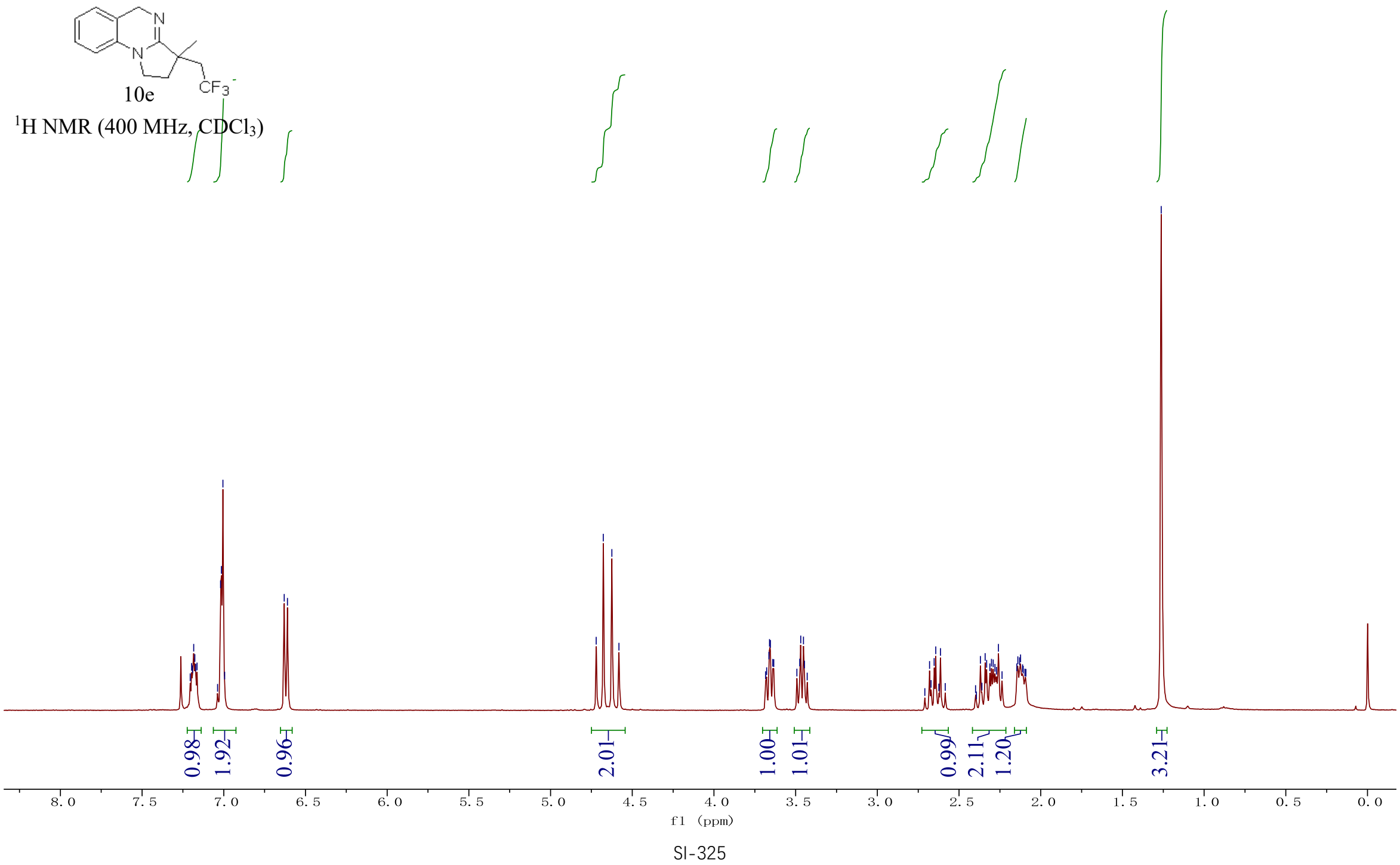
5xjm113cw-c. 420

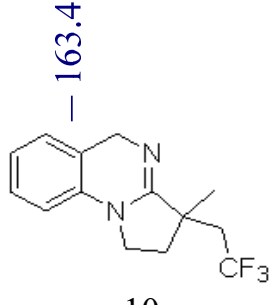

$10 \mathrm{e}$

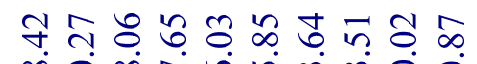

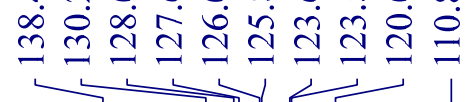

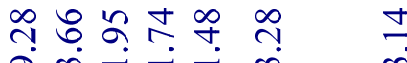

守守守宇

${ }^{13} \mathrm{C}$ NMR (125 MHz, $\mathrm{CDCl}_{3}$ )

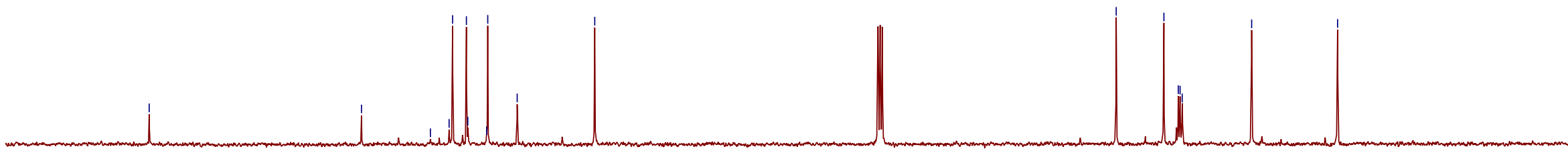


5x jm113cw-f. 41. fid

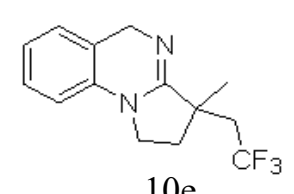

${ }^{19} \mathrm{~F}$ NMR (470 MHz, $\mathrm{CDCl}_{3}$ )
ले ले

每

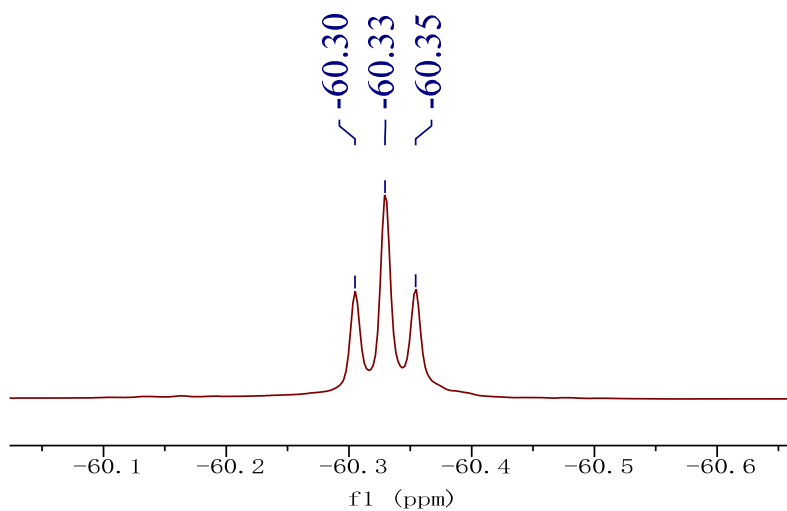

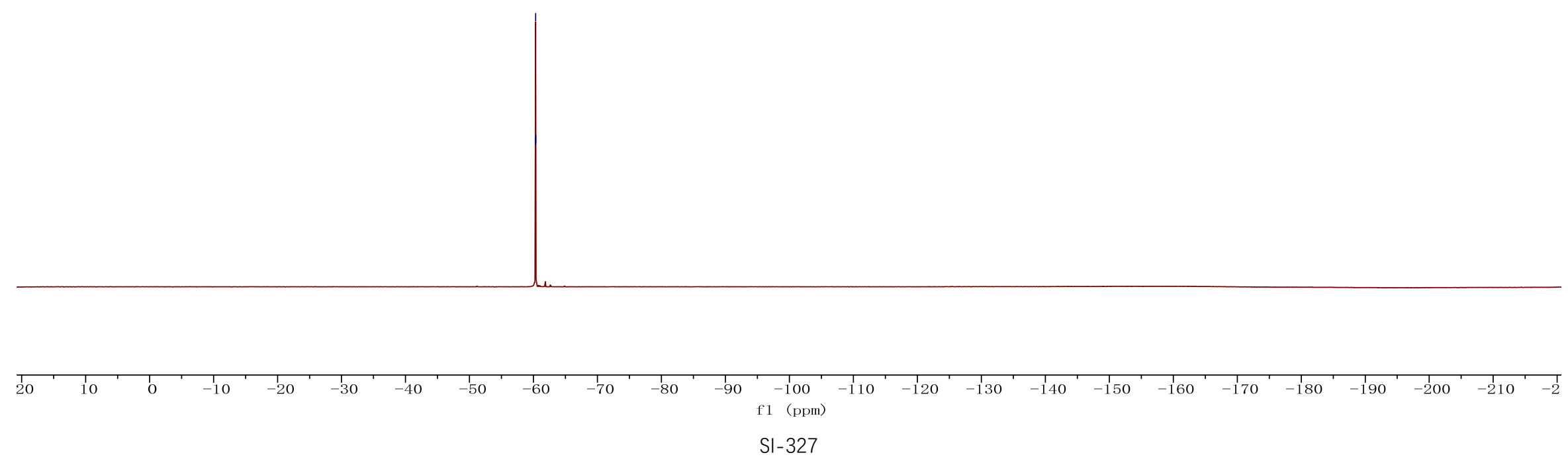




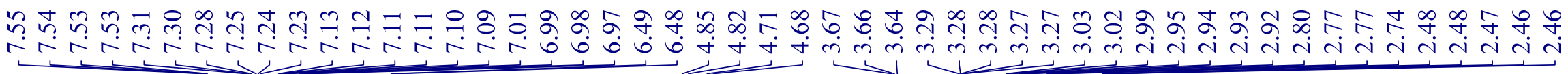

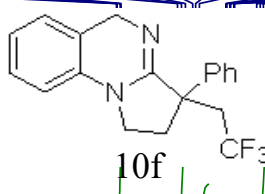

${ }^{1} \mathrm{H}$ NMR (500 MHz, $\left(\mathrm{CDCl}_{3}\right)$

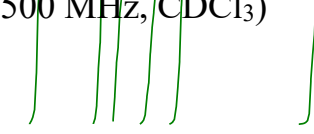

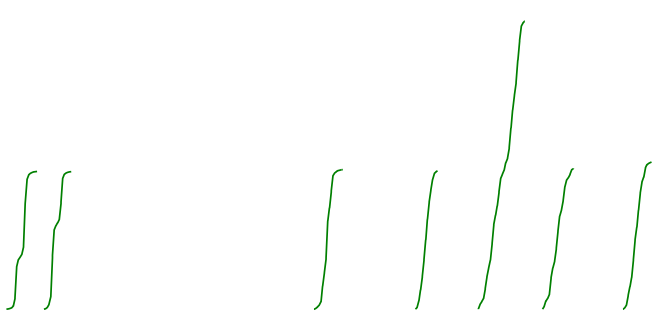

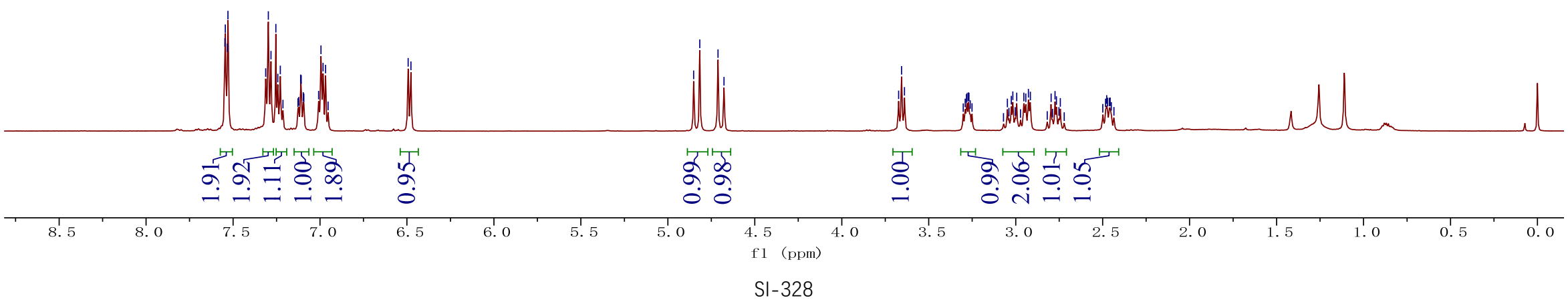




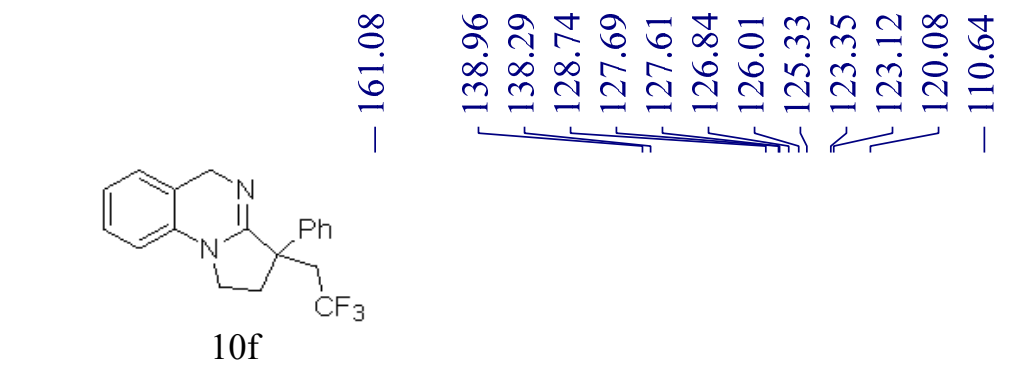

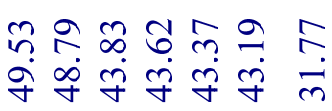

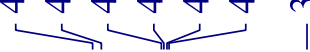

${ }^{13} \mathrm{C}$ NMR $\left(125 \mathrm{MHz}, \mathrm{CDCl}_{3}\right)$
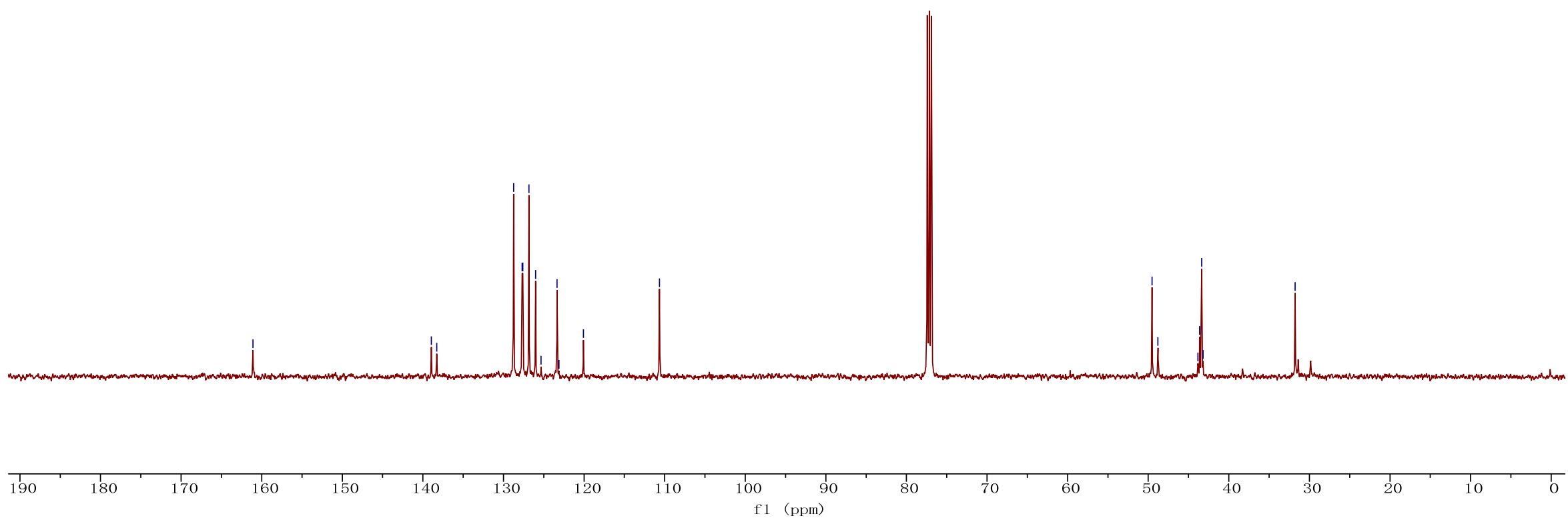

SI-329 
일

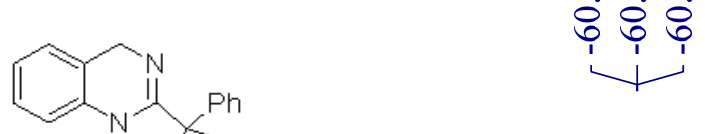

of

$\mathrm{CF}_{3}$

${ }^{19} \mathrm{~F}$ NMR $\left(470 \mathrm{MHz}, \mathrm{CDCl}_{3}\right)$

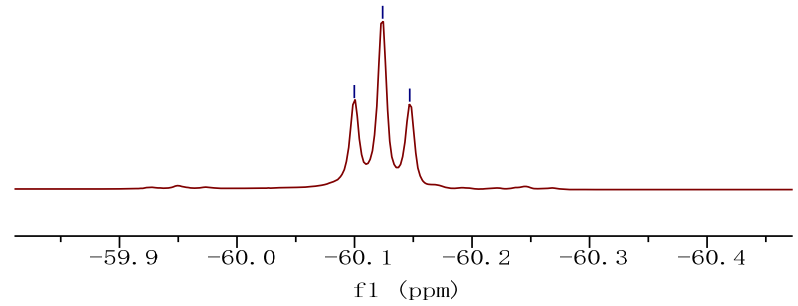

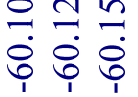

$>1$

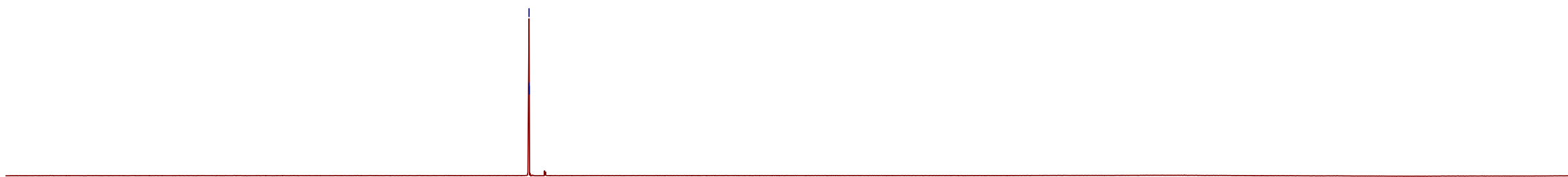




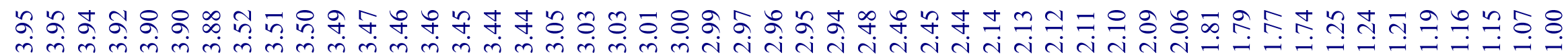

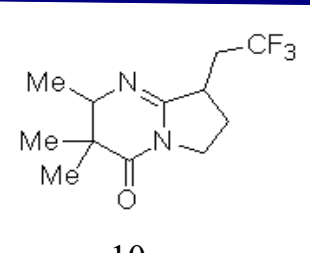

$10 \mathrm{~g}$

${ }^{1} \mathrm{H}$ NMR $\left(500 \mathrm{MHz}, \mathrm{CDCl}_{3}\right)$
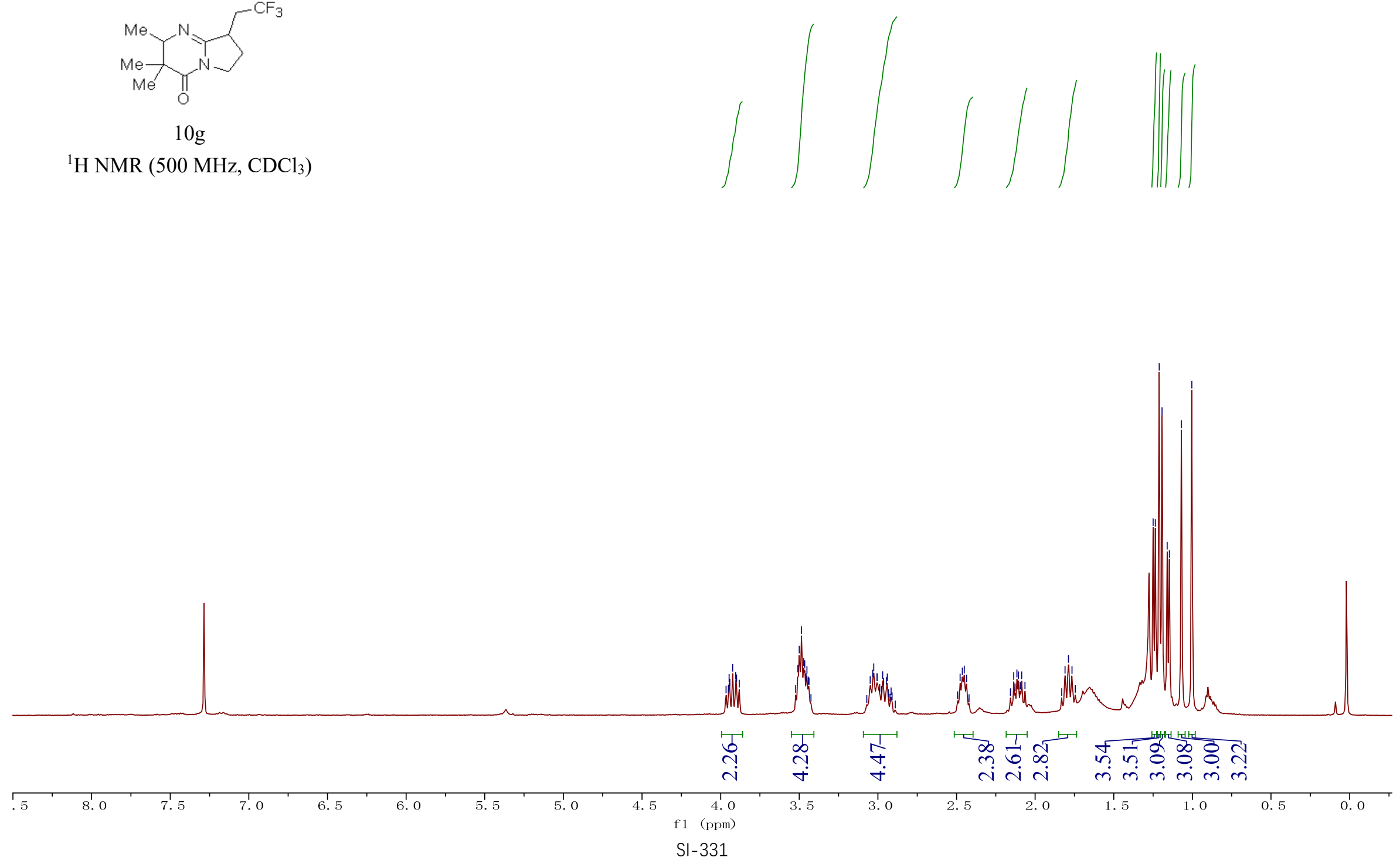


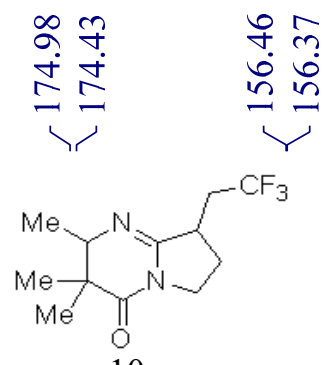

$10 \mathrm{~g}$

${ }^{13} \mathrm{C}$ NMR (125 MHz, $\left.\mathrm{CDCl}_{3}\right)$

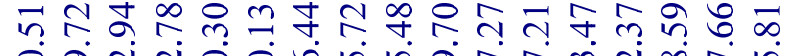

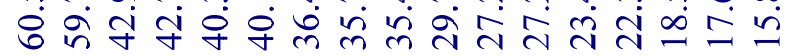




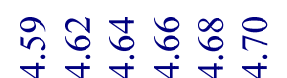

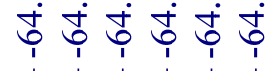

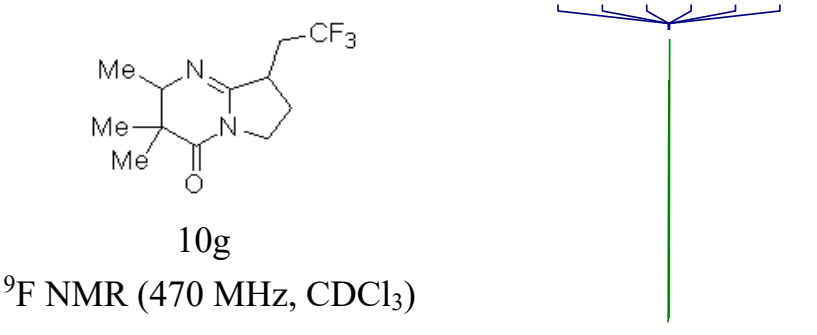

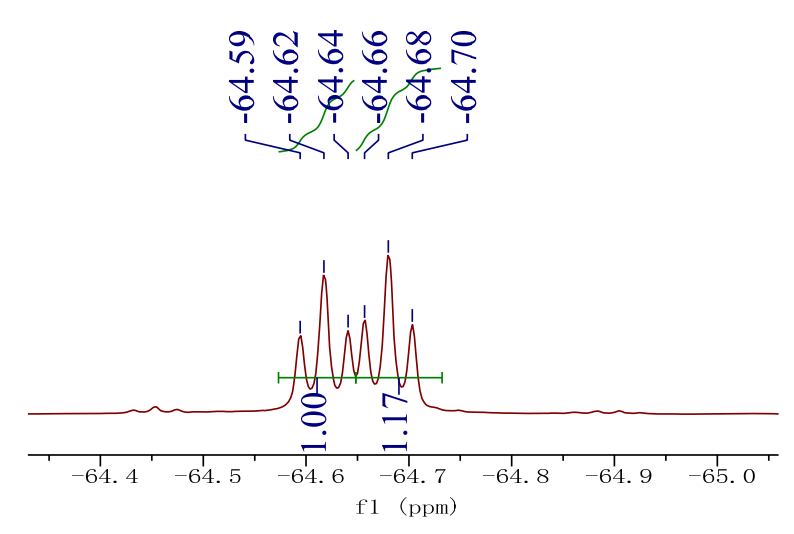

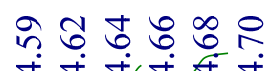

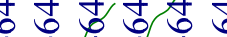

全11?

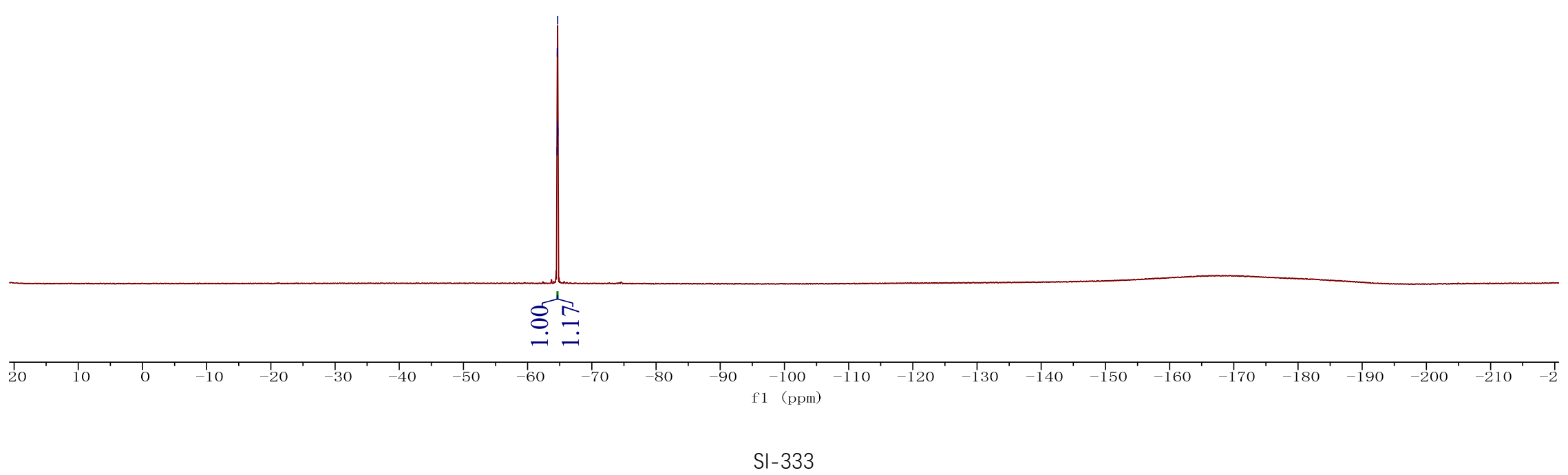




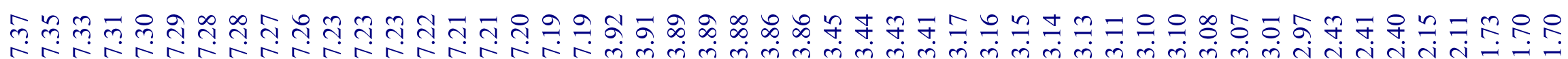

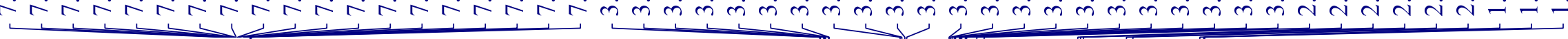
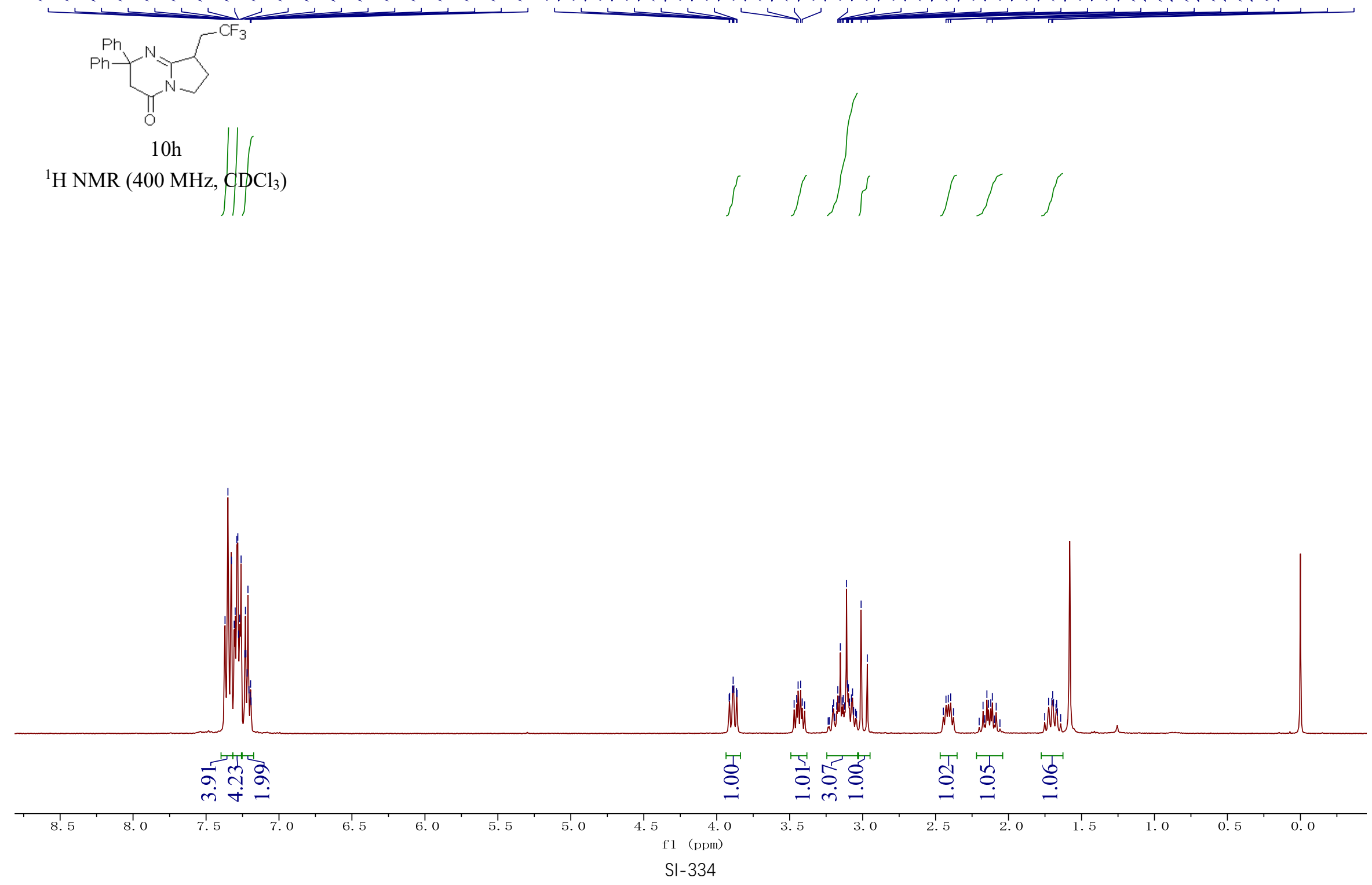


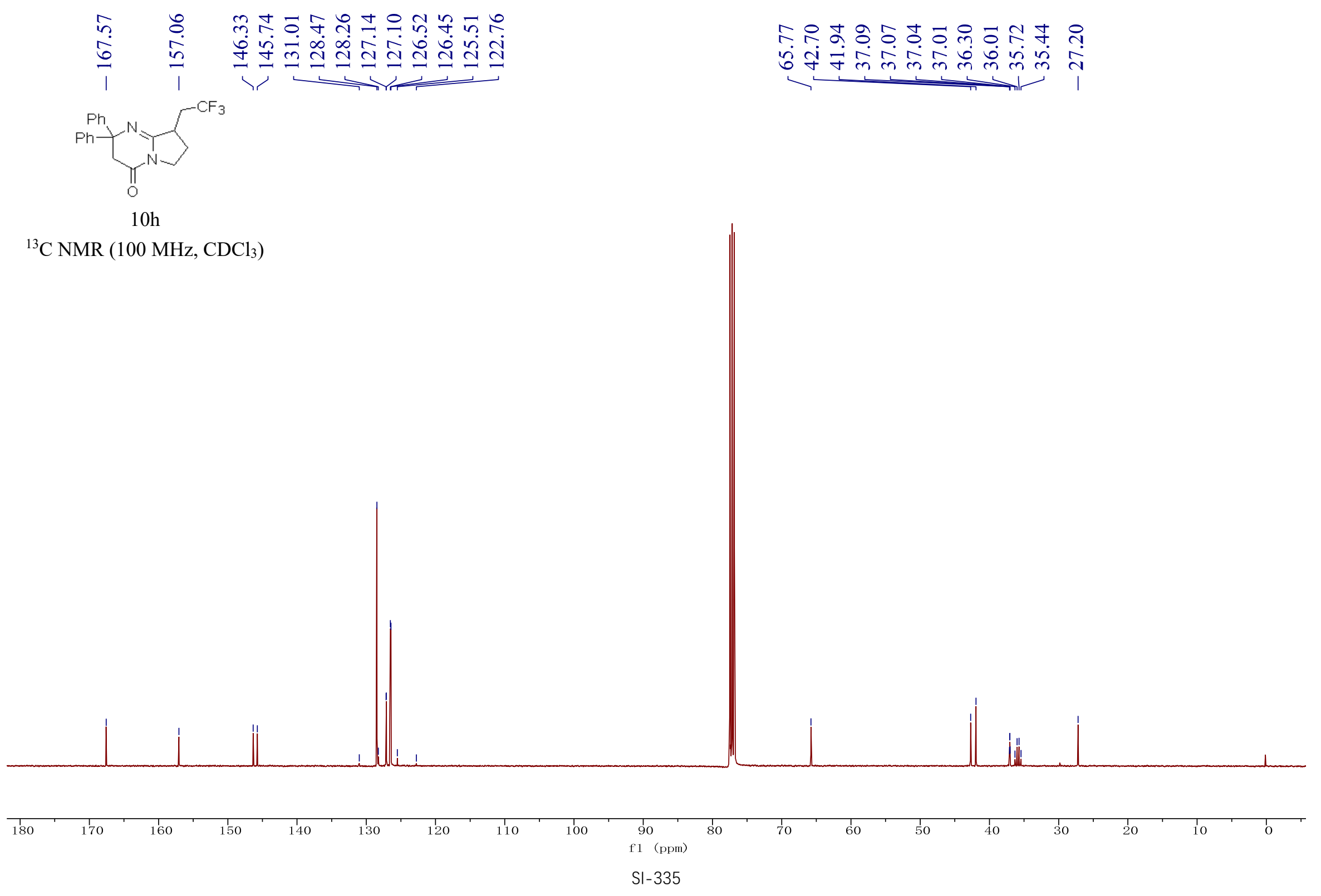




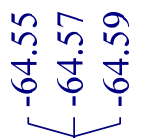

诤 的

î

${ }^{19} \mathrm{~F}$ NMR $\left(470 \mathrm{MHz}, \mathrm{CDCl}_{3}\right)$

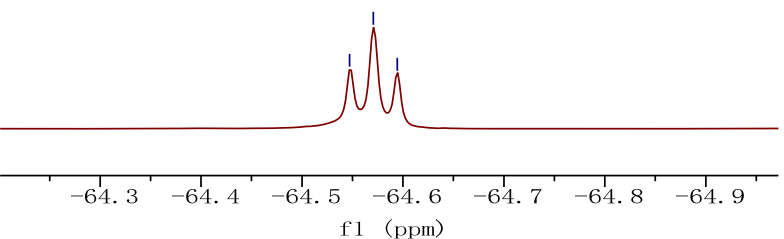




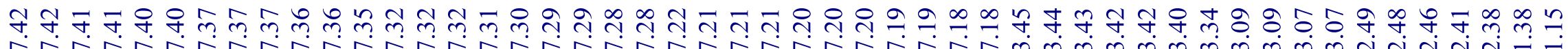
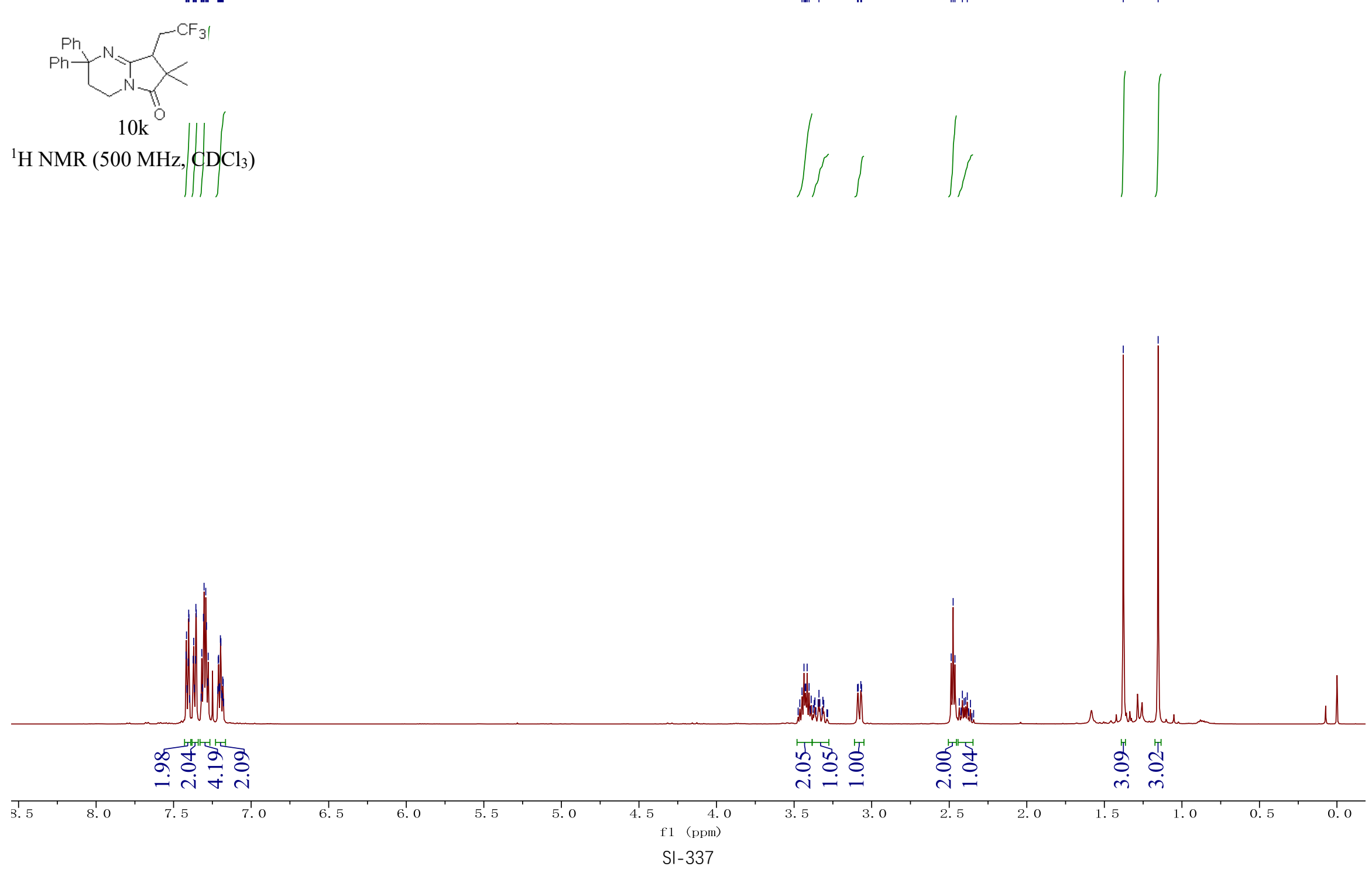

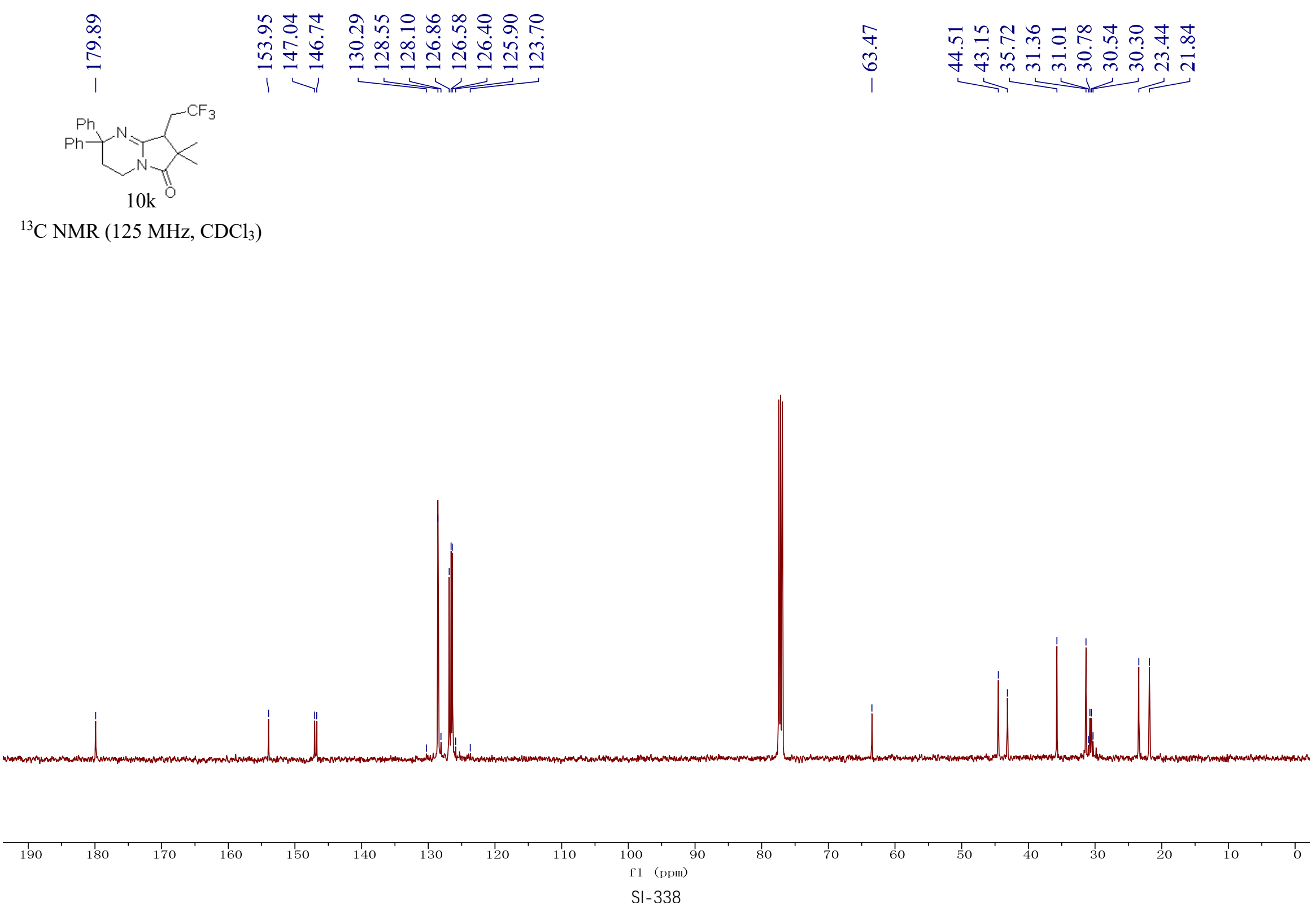
초ำ

ஸुં

Y

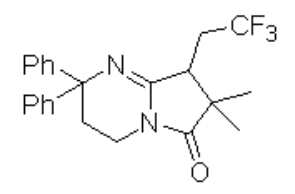

$10 \mathrm{k}$

${ }^{19} \mathrm{~F}$ NMR $\left(470 \mathrm{MHz}, \mathrm{CDCl}_{3}\right)$

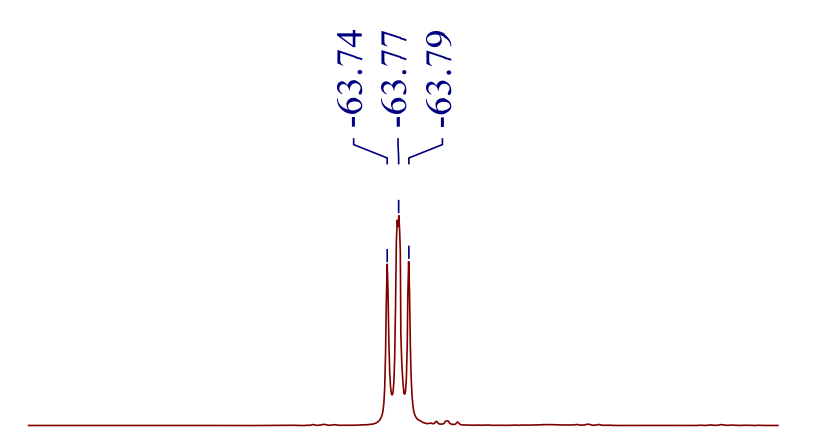

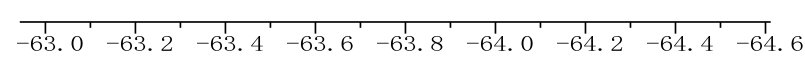

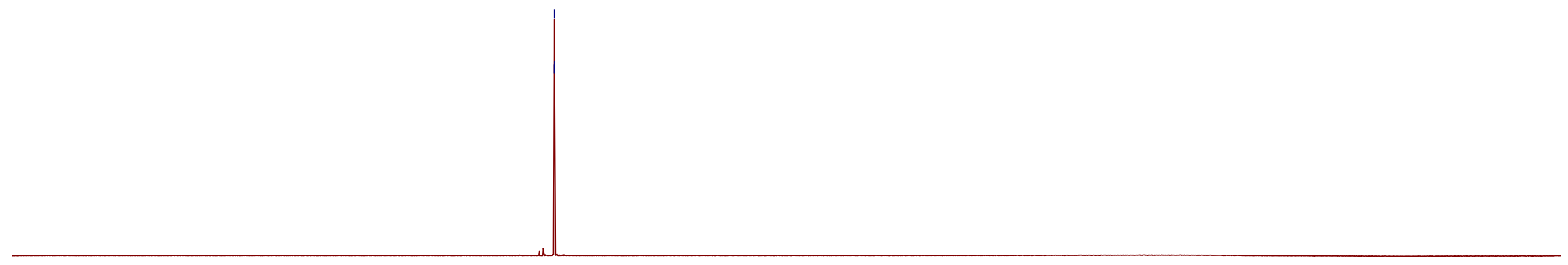




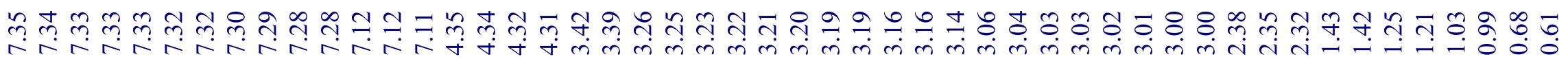

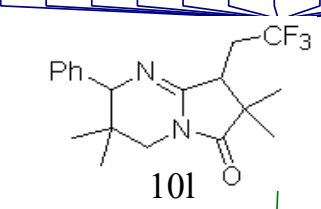

${ }^{1} \mathrm{H} \mathrm{NMR}\left(500 \mathrm{MHz}, \mathrm{CDCl}_{3}\right)$
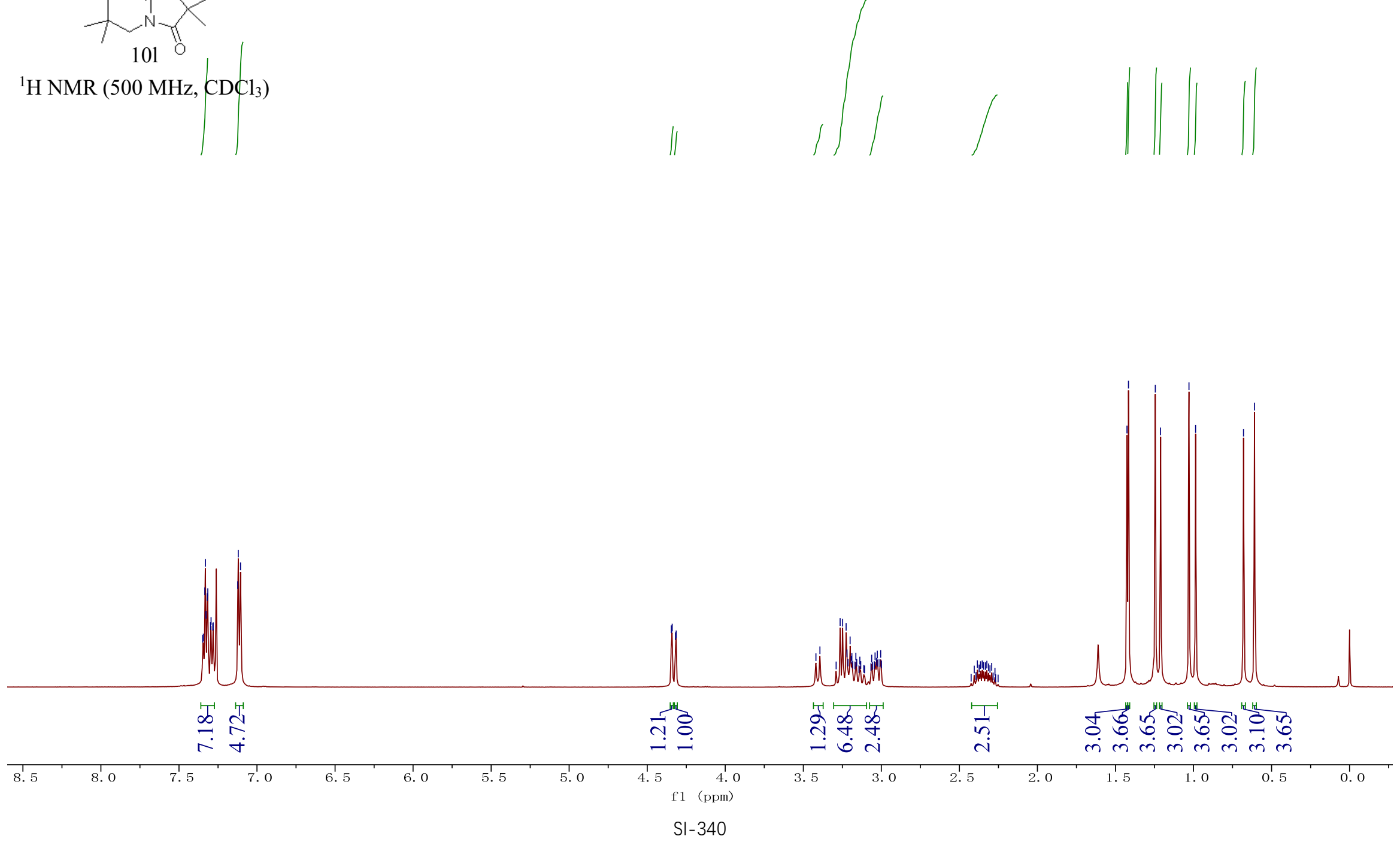


\section{웅
$\stackrel{0}{0}$
$\infty$}

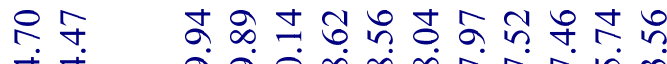

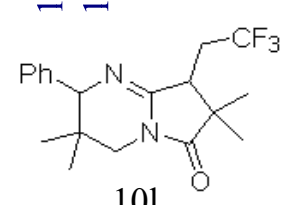

101
윰

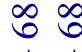

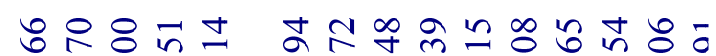

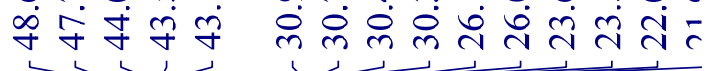

${ }^{13} \mathrm{C}$ NMR (125 MHz, $\left.\mathrm{CDCl}_{3}\right)$
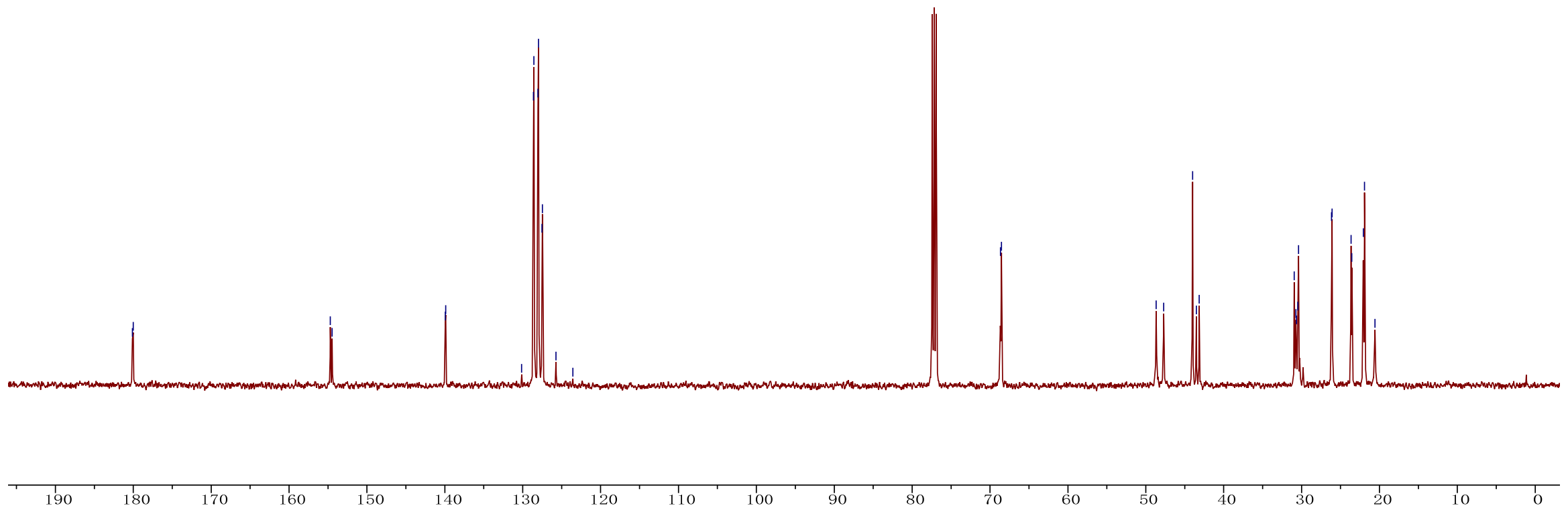
$\infty$ क \&

भुં

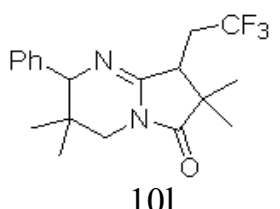

${ }^{19} \mathrm{~F}$ NMR $\left(470 \mathrm{MHz}, \mathrm{CDCl}_{3}\right)$

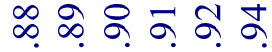
तुं

11

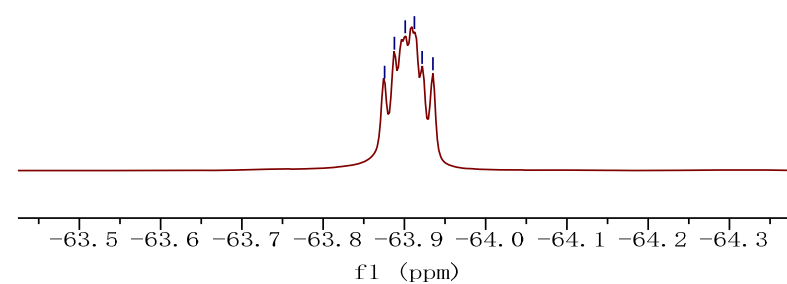

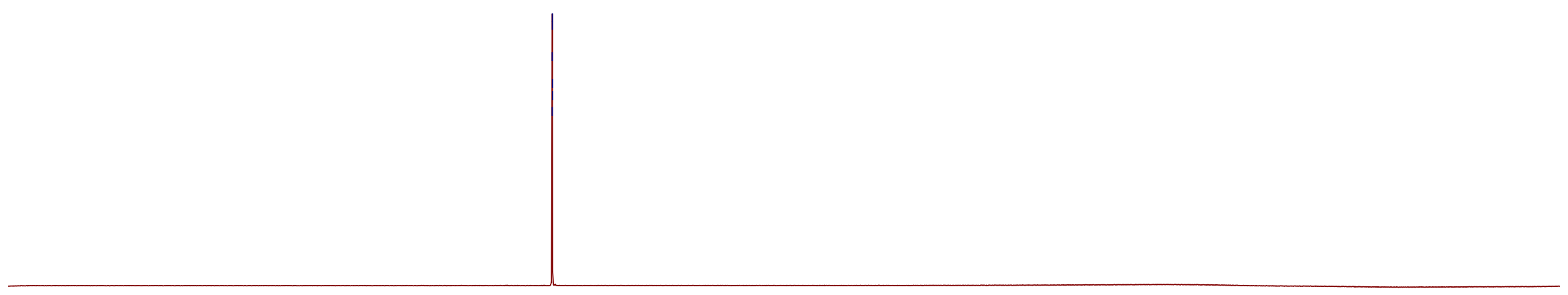




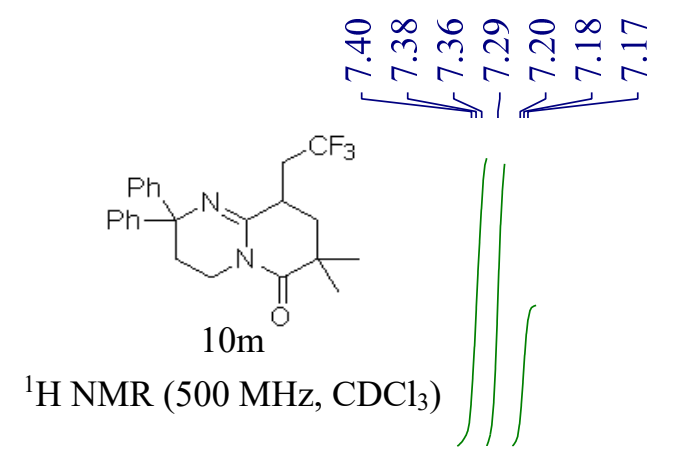

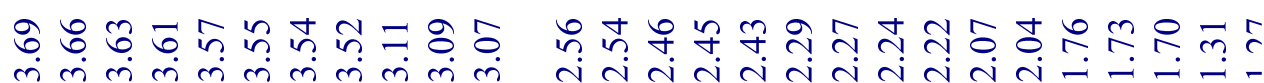

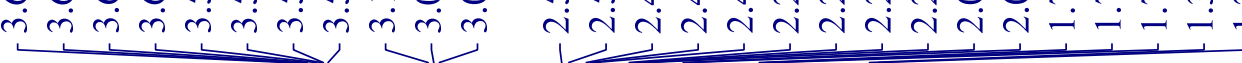
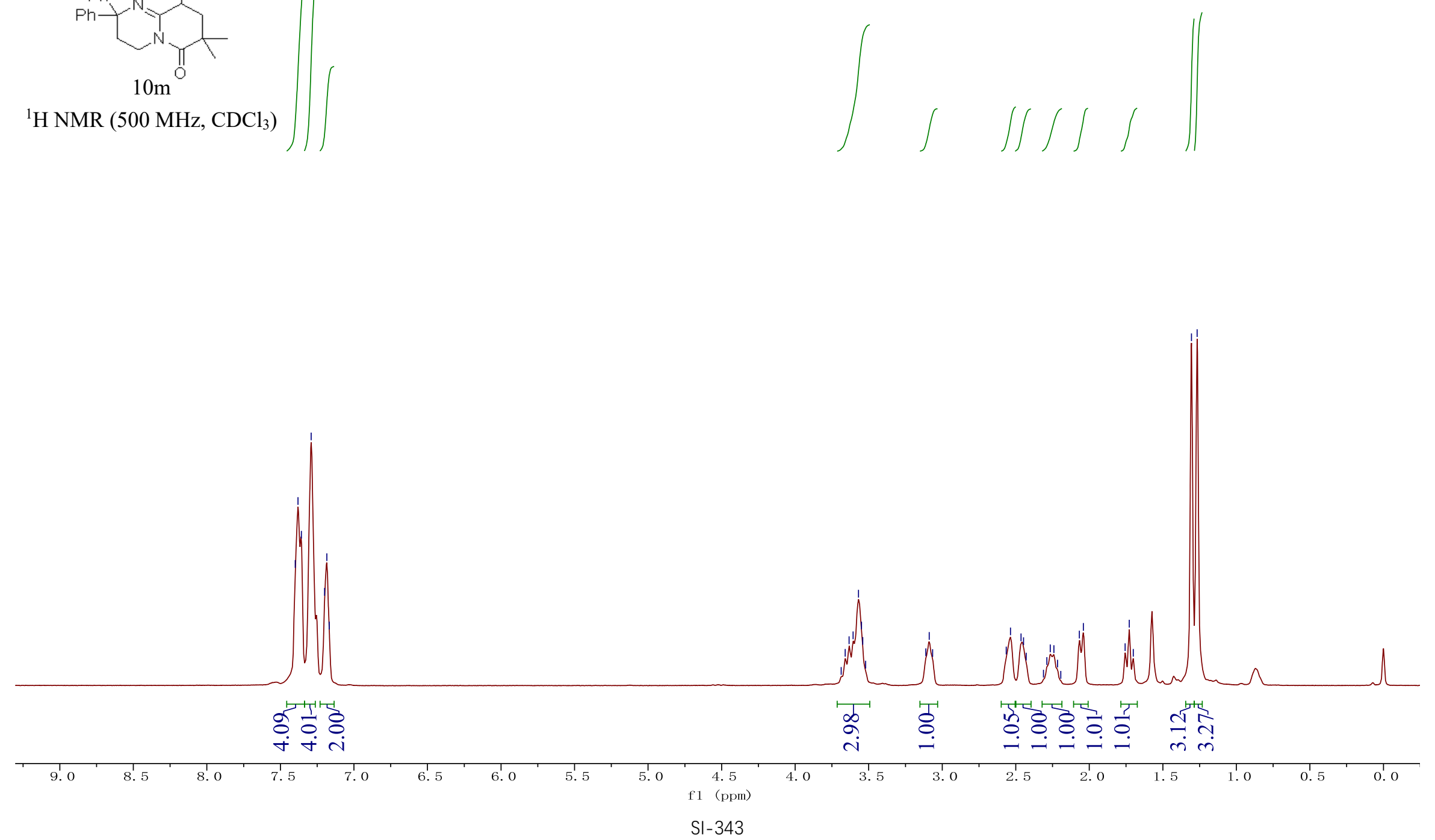


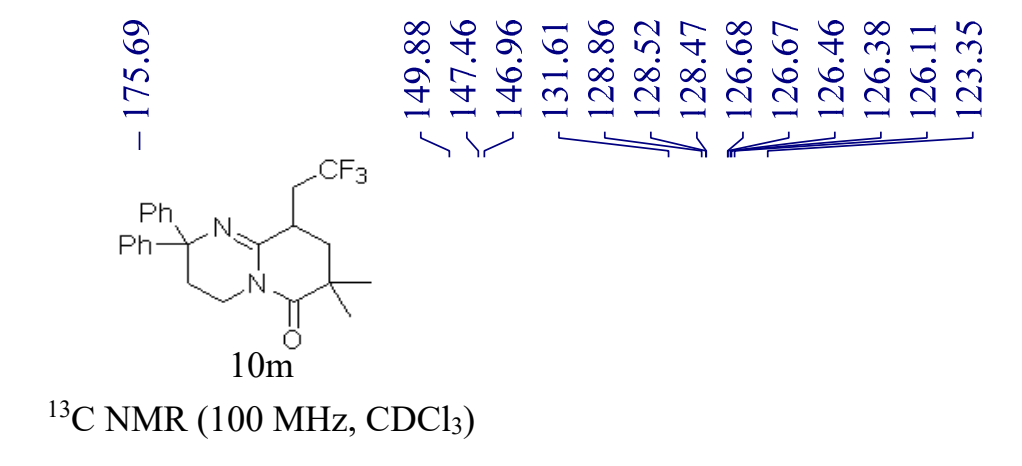

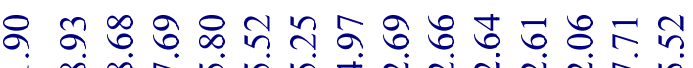

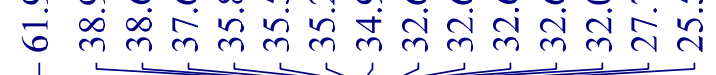

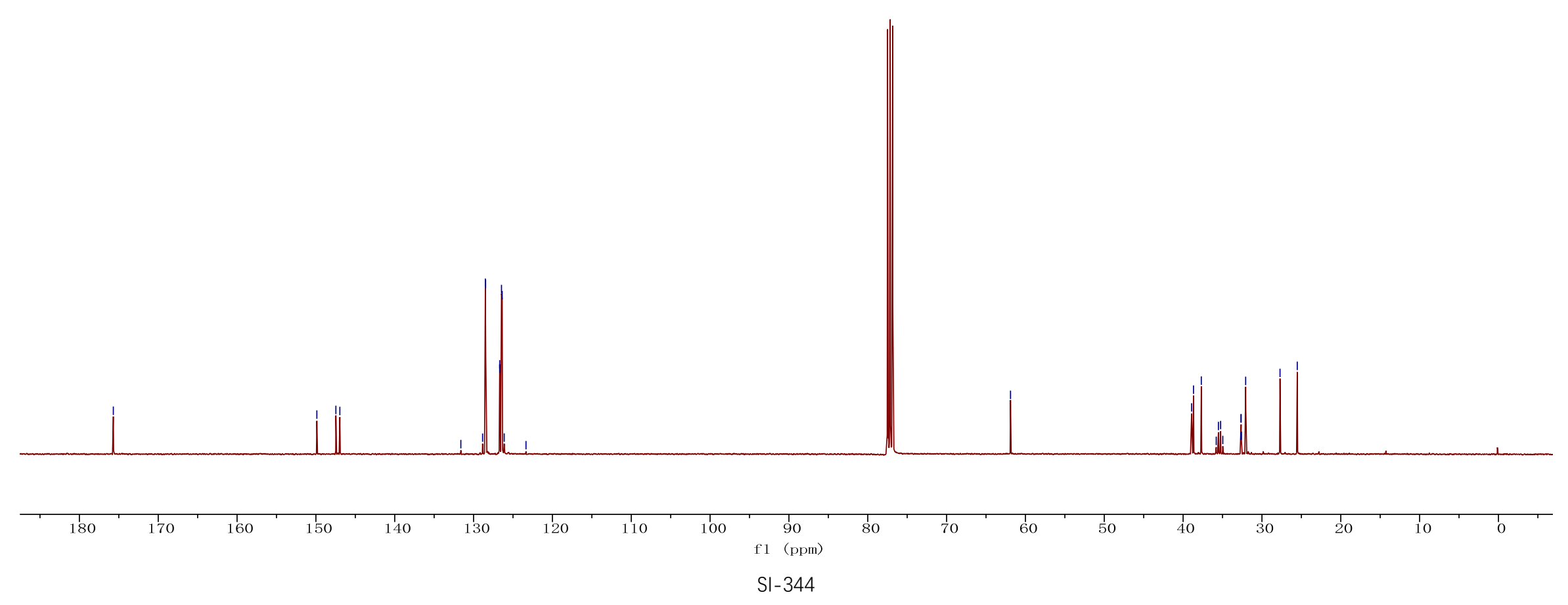




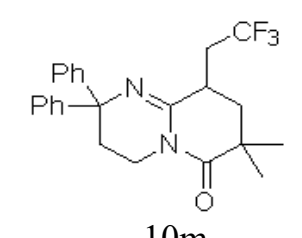

$10 \mathrm{~m}$

${ }^{19} \mathrm{~F}$ NMR $\left(470 \mathrm{MHz}, \mathrm{CDCl}_{3}\right)$

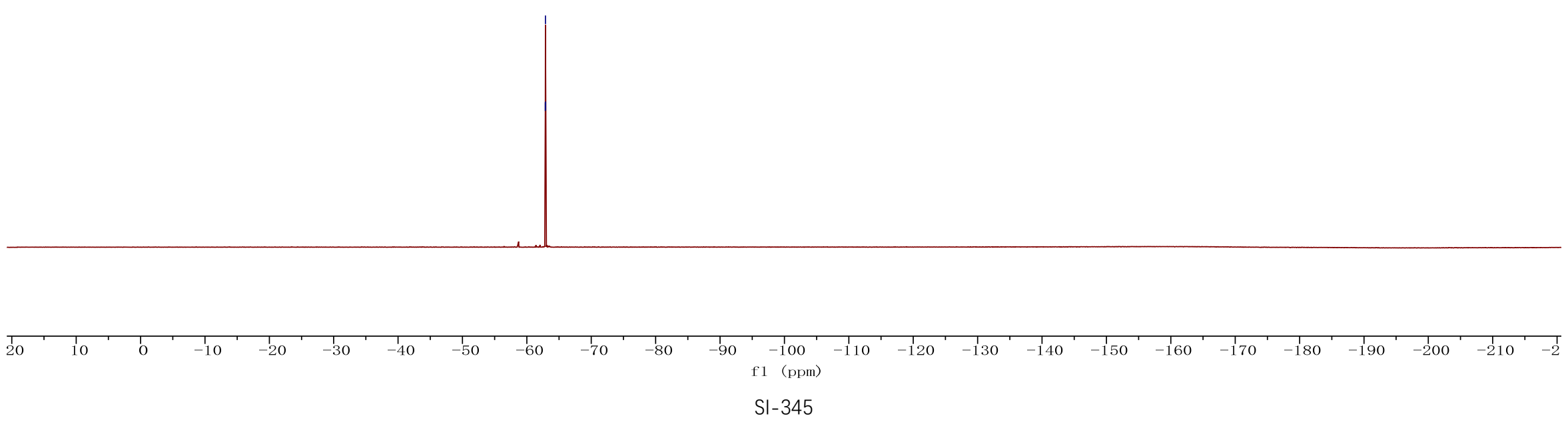

$\infty$ के

(i
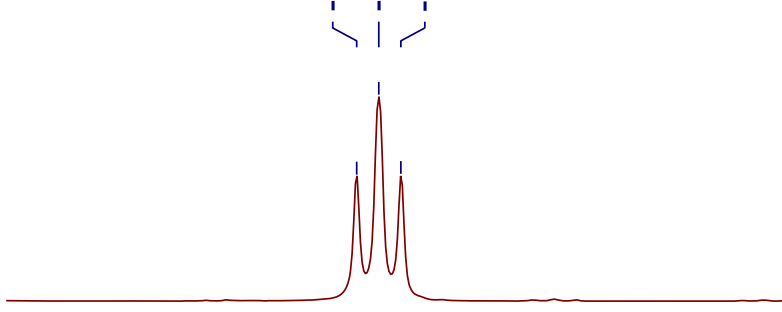

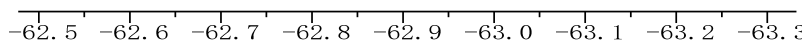
f1 (ppm) 


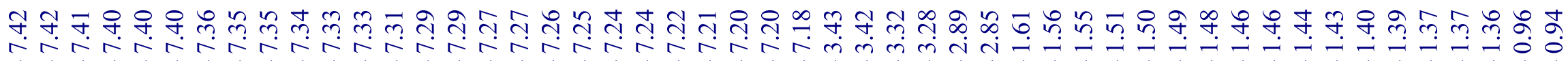

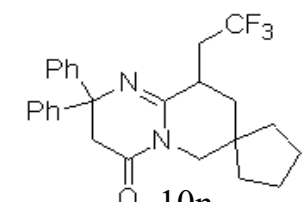

${ }^{1} \mathrm{H}$ NMR (400 MHz. $\left.\mathrm{CDCl}_{3}\right)$
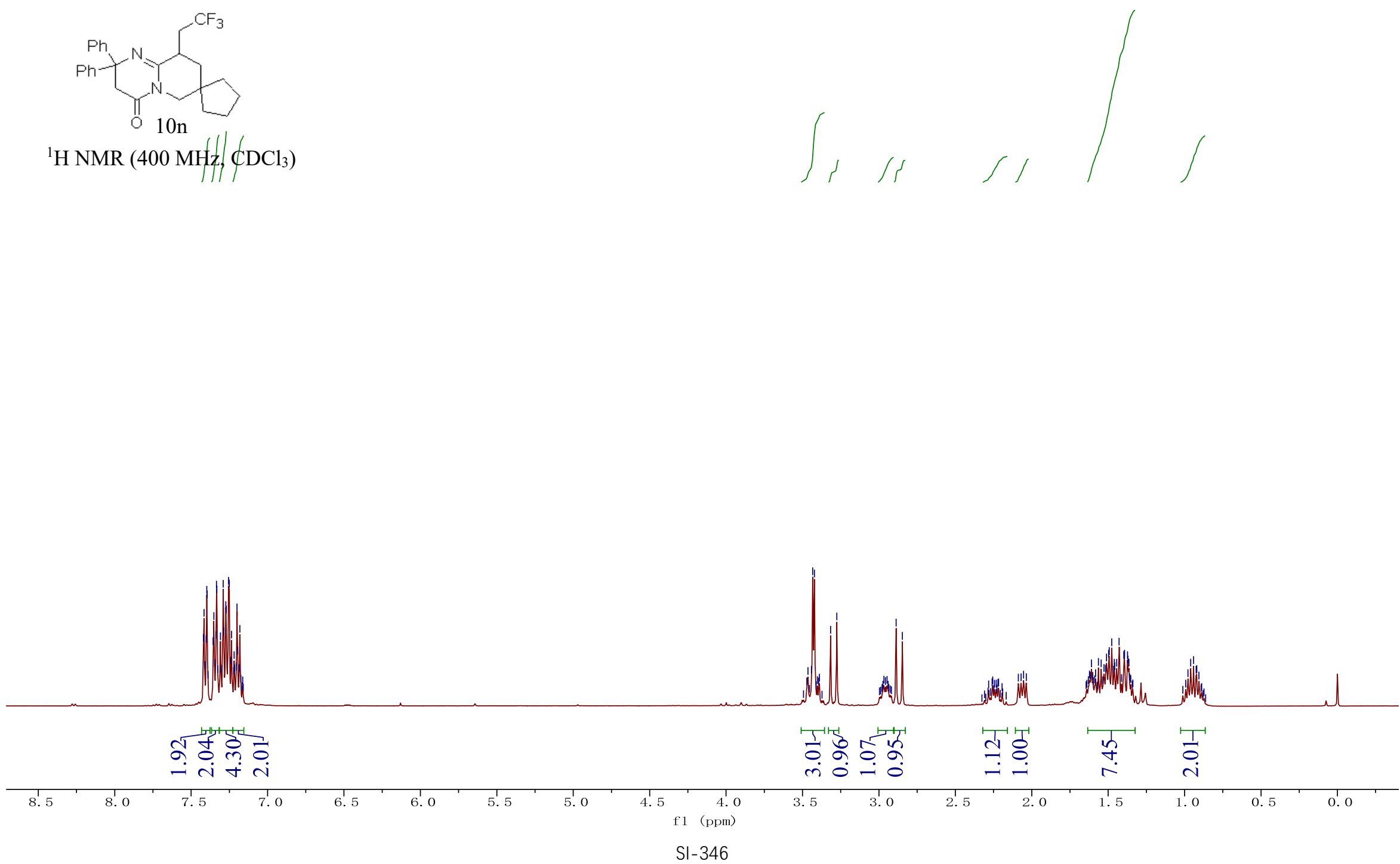


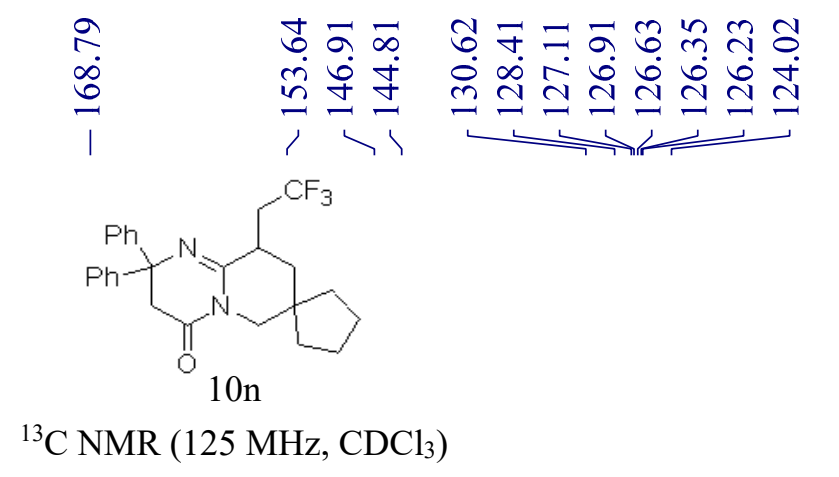

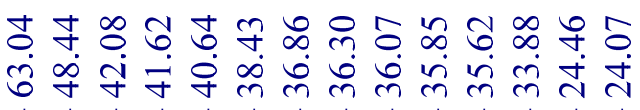

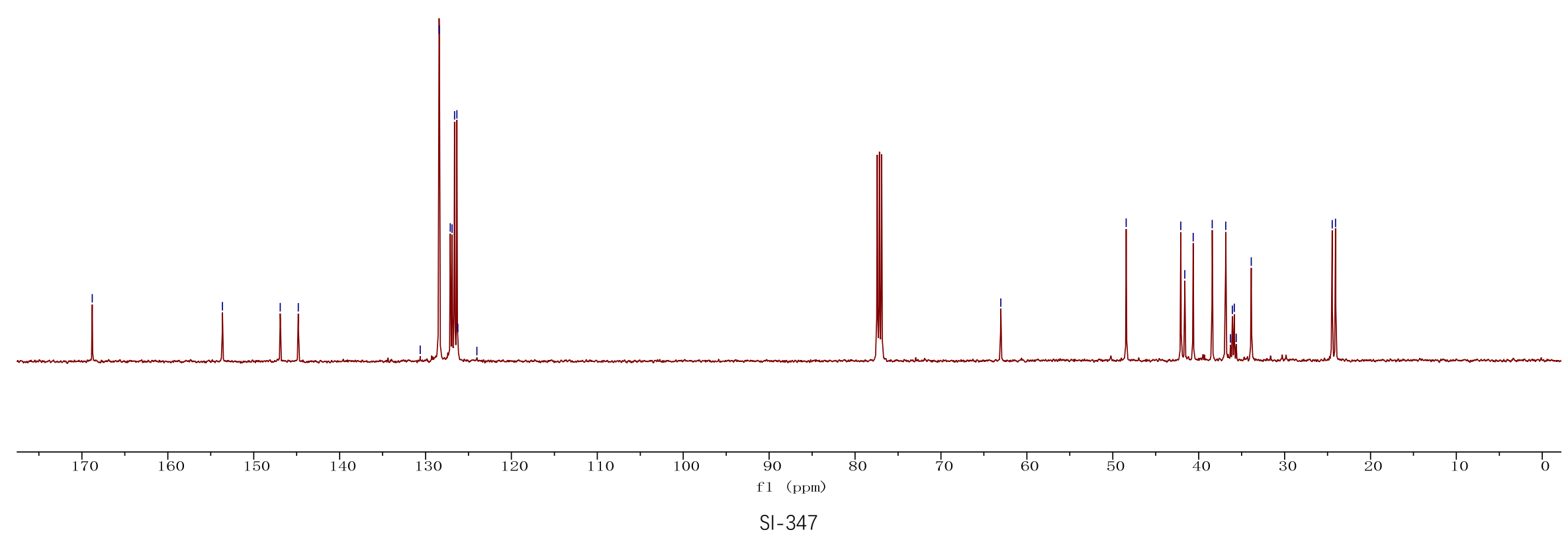



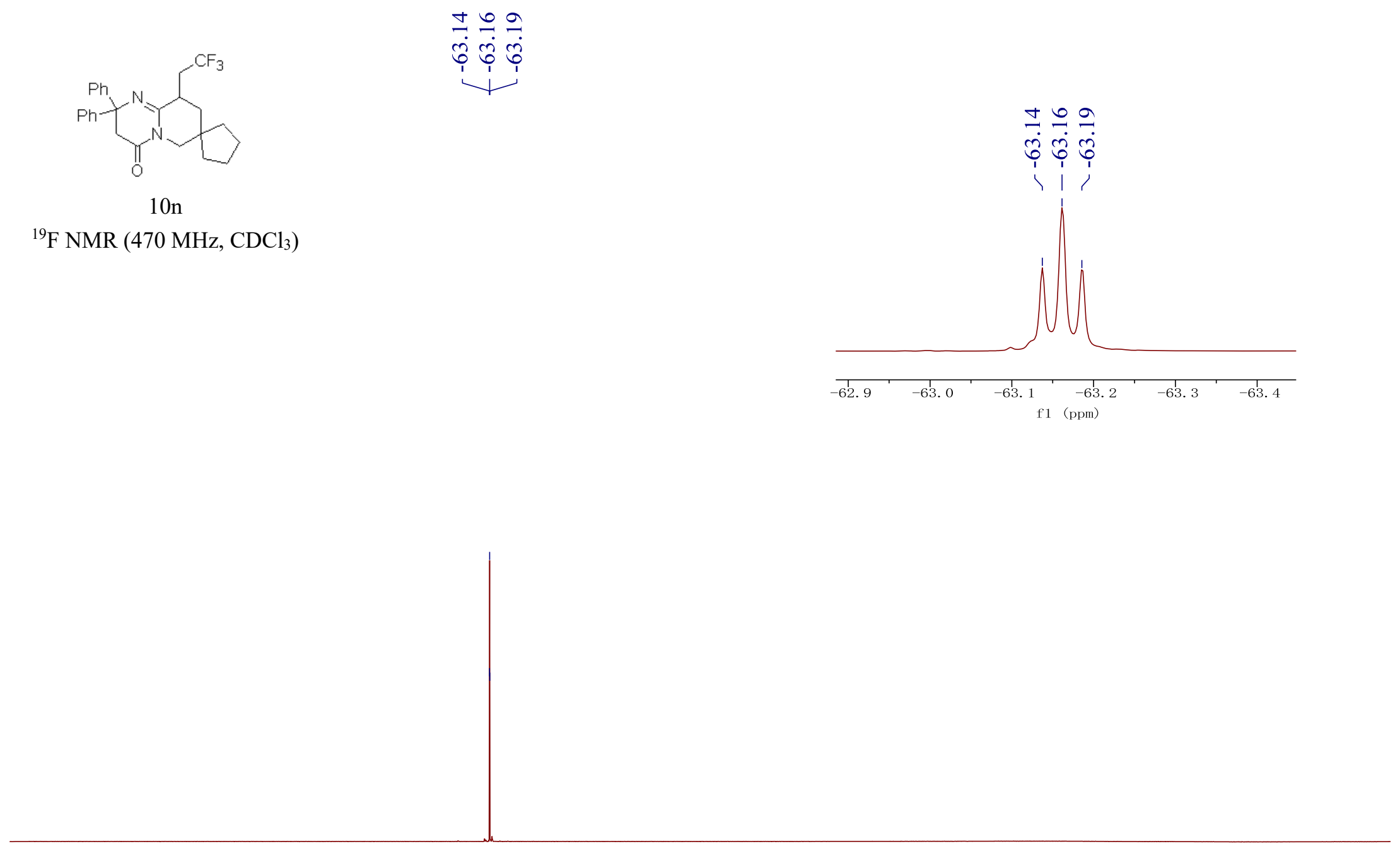

$20 \quad 10$ 


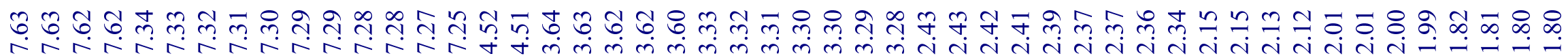

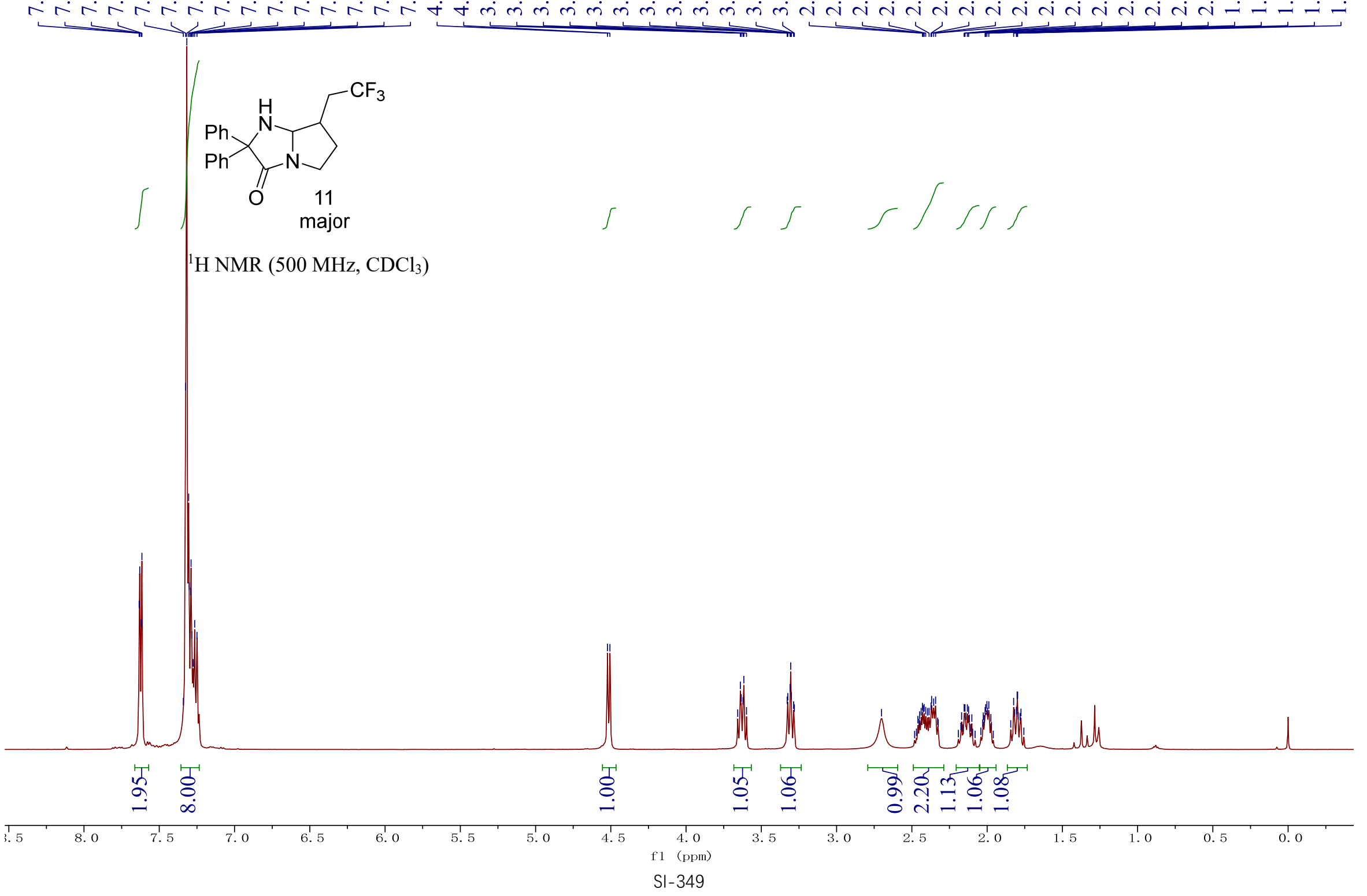



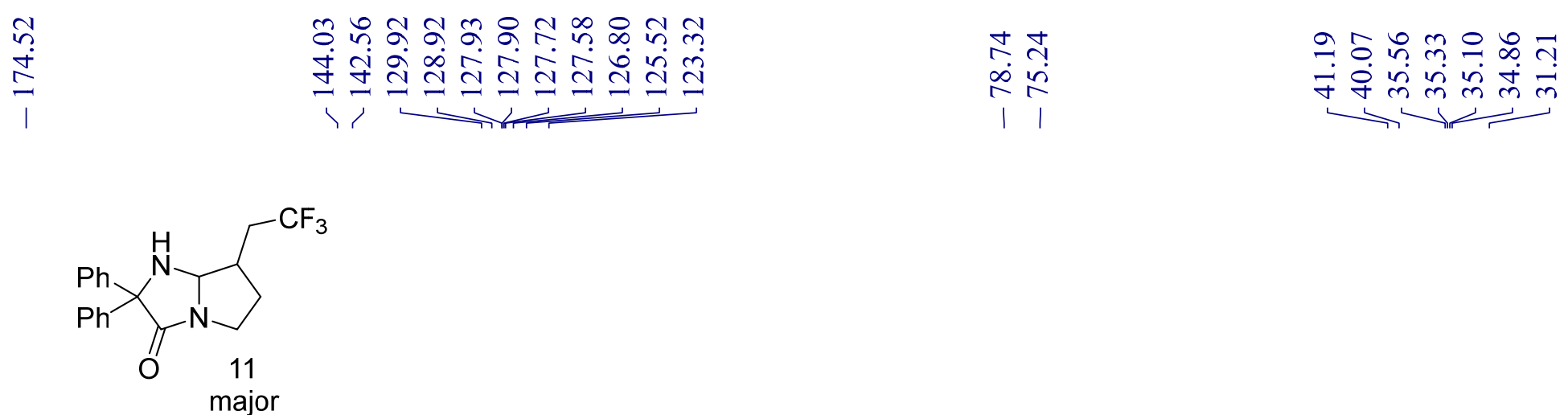

${ }^{13} \mathrm{C}$ NMR (125 MHz, $\mathrm{CDCl}_{3}$ )
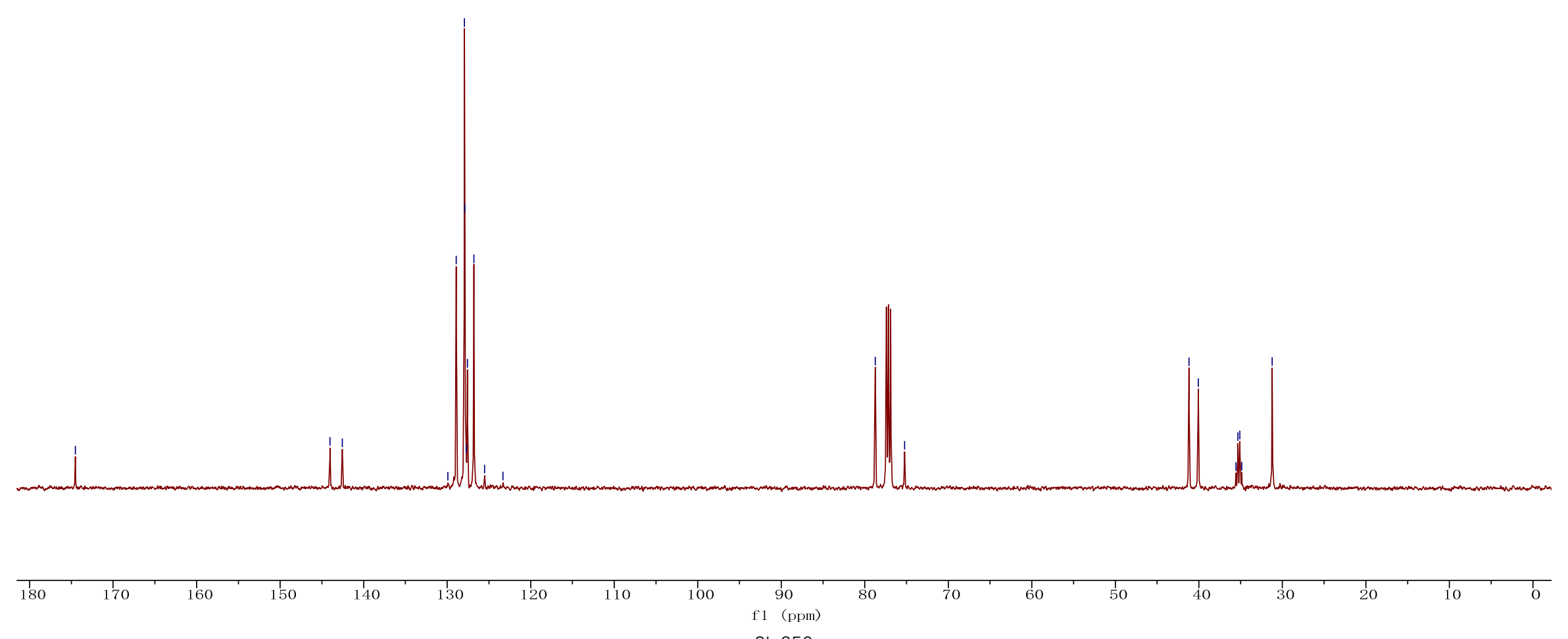
每

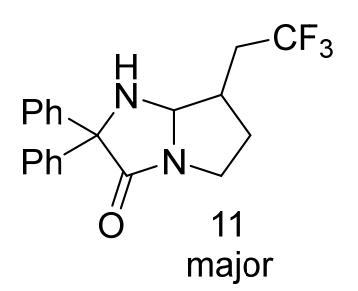

${ }^{19} \mathrm{~F}$ NMR (470 MHz, $\mathrm{CDCl}_{3}$ )

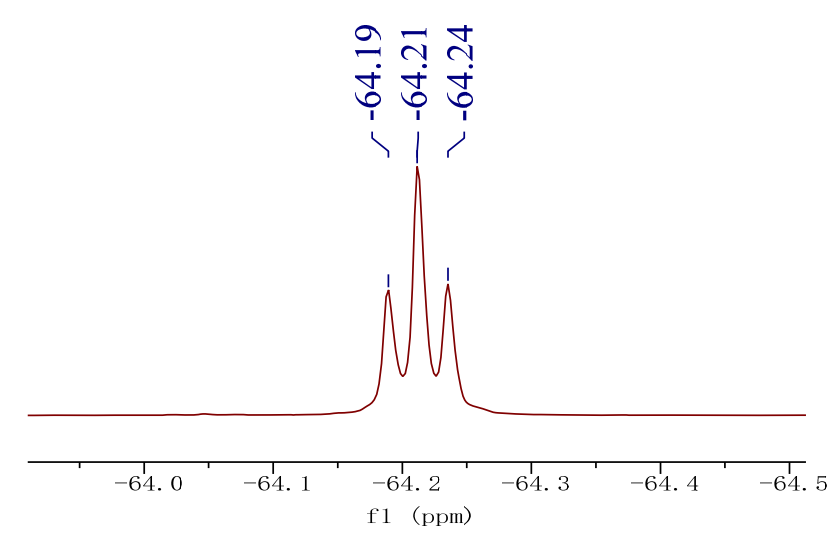




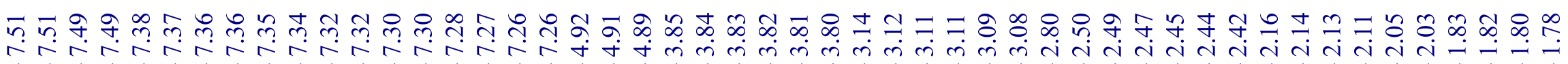

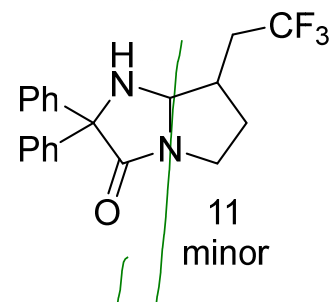

${ }^{1} \mathrm{H} \mathrm{NMR}\left(400 \mathrm{MHz}, \mathrm{CDCl}_{3}\right)$

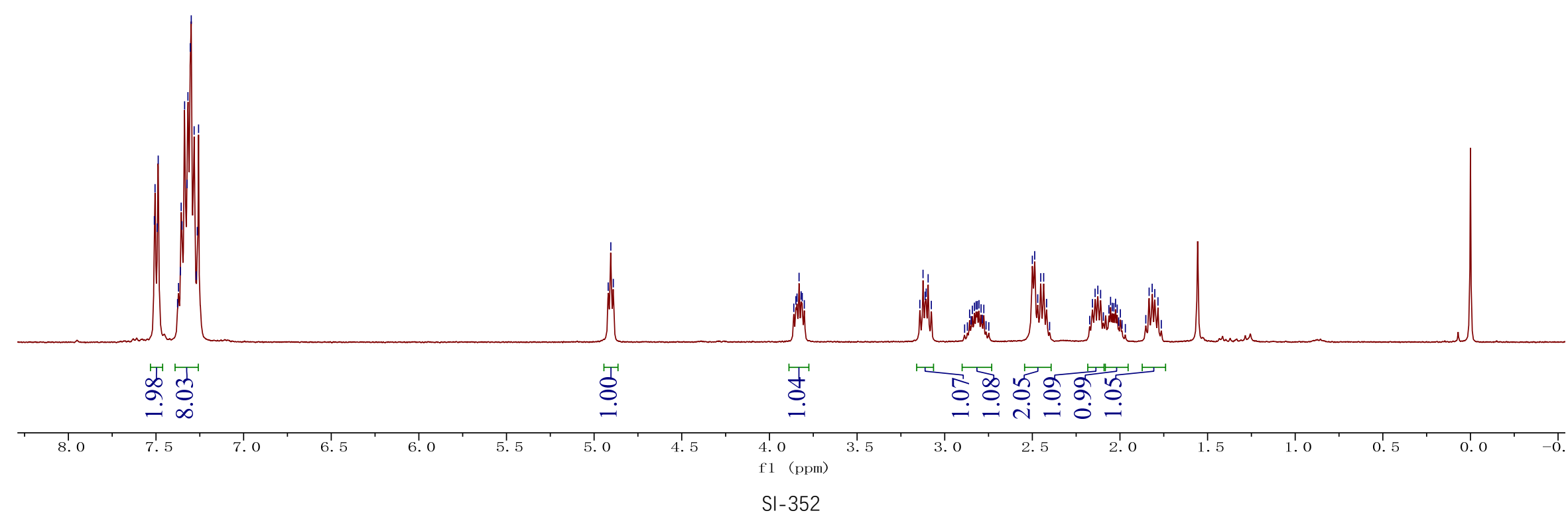




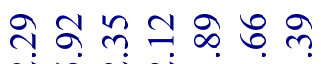

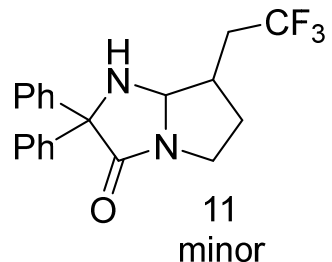

${ }^{13} \mathrm{C}$ NMR (125 MHz, $\left.\mathrm{CDCl}_{3}\right)$

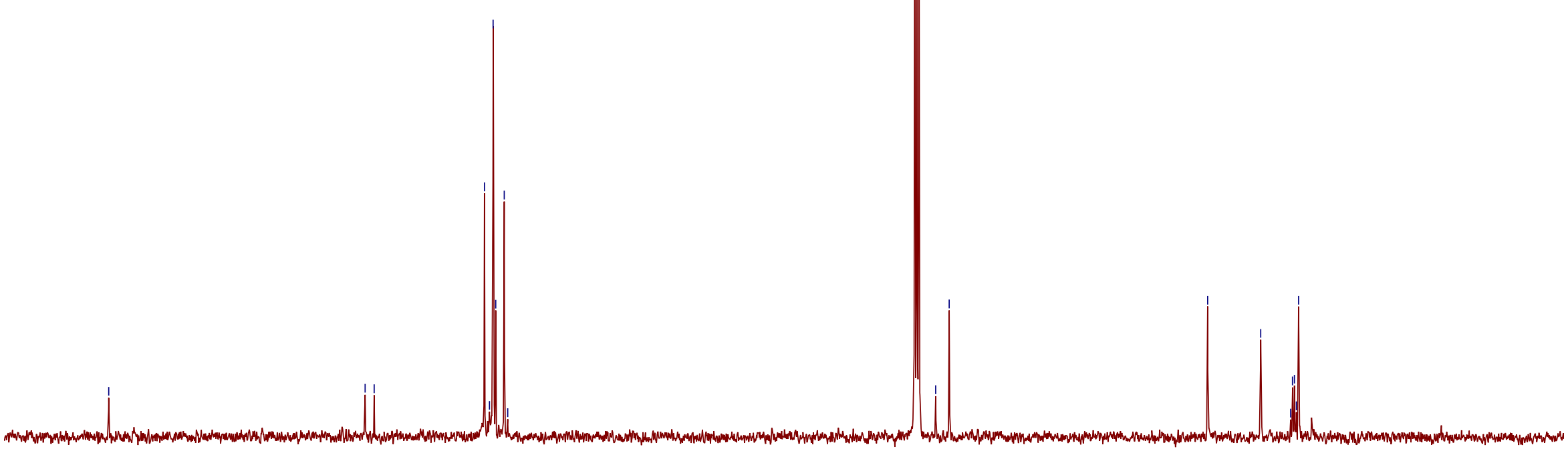

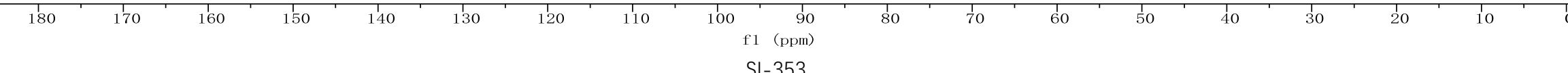


î.

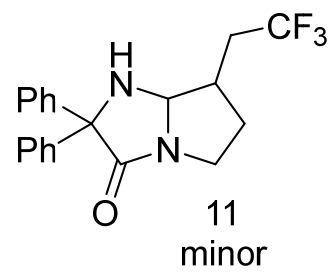

${ }^{19} \mathrm{~F}$ NMR $\left(470 \mathrm{MHz}, \mathrm{CDCl}_{3}\right)$

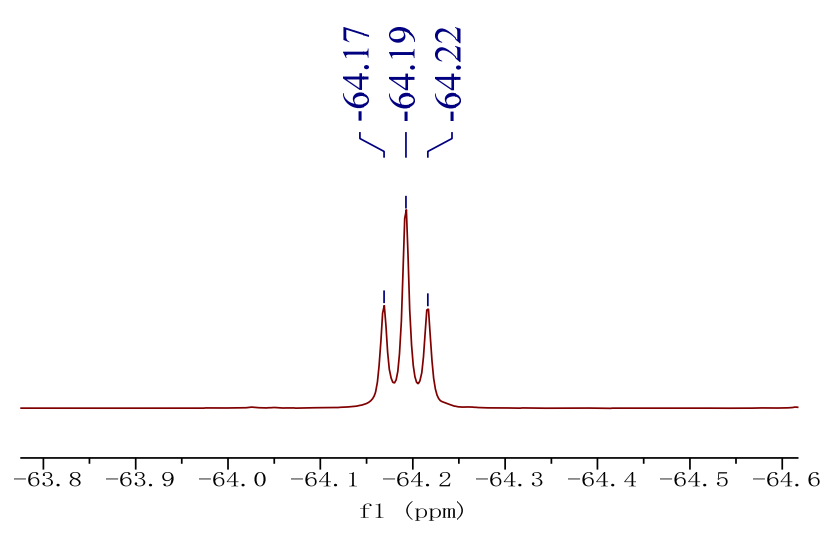

f1 (ppm)

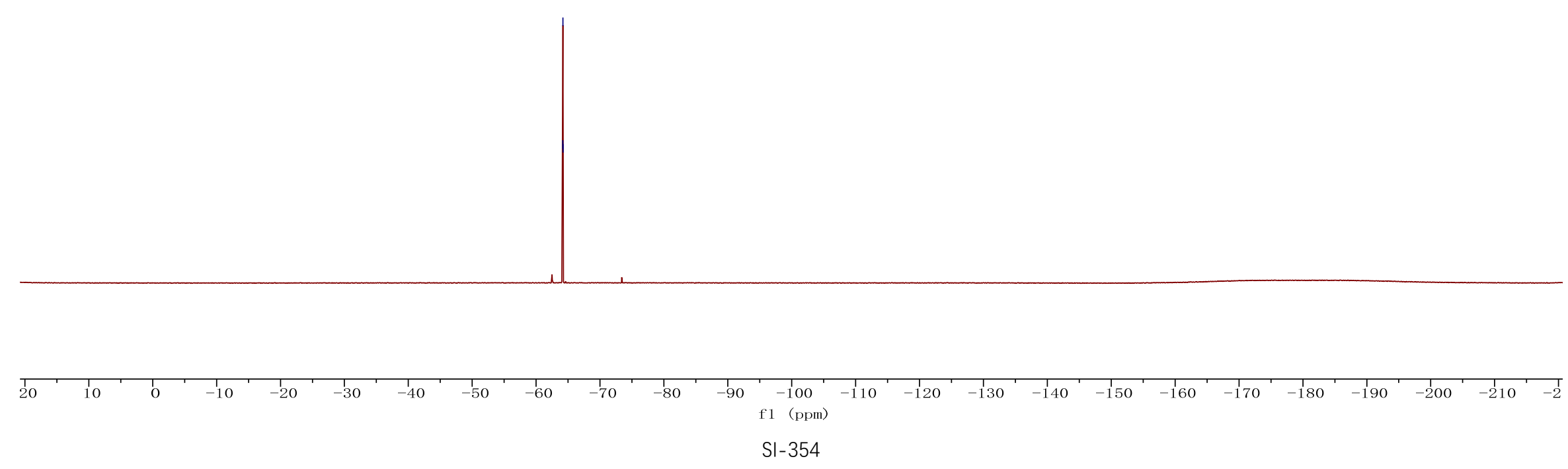




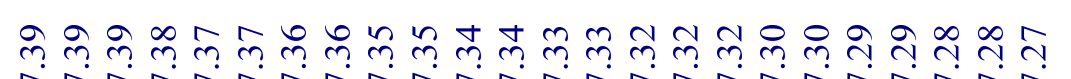

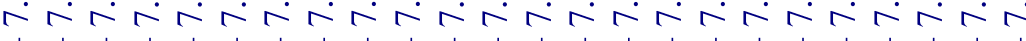

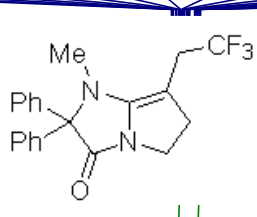

12

${ }^{1} \mathrm{H}$ NMR (500 MHz, $\left.\mathrm{CDCl}_{3}\right)$

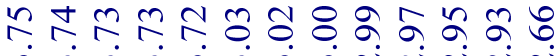

लें்

$\longrightarrow$

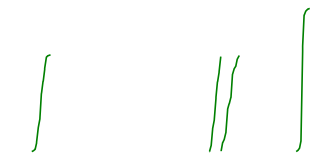

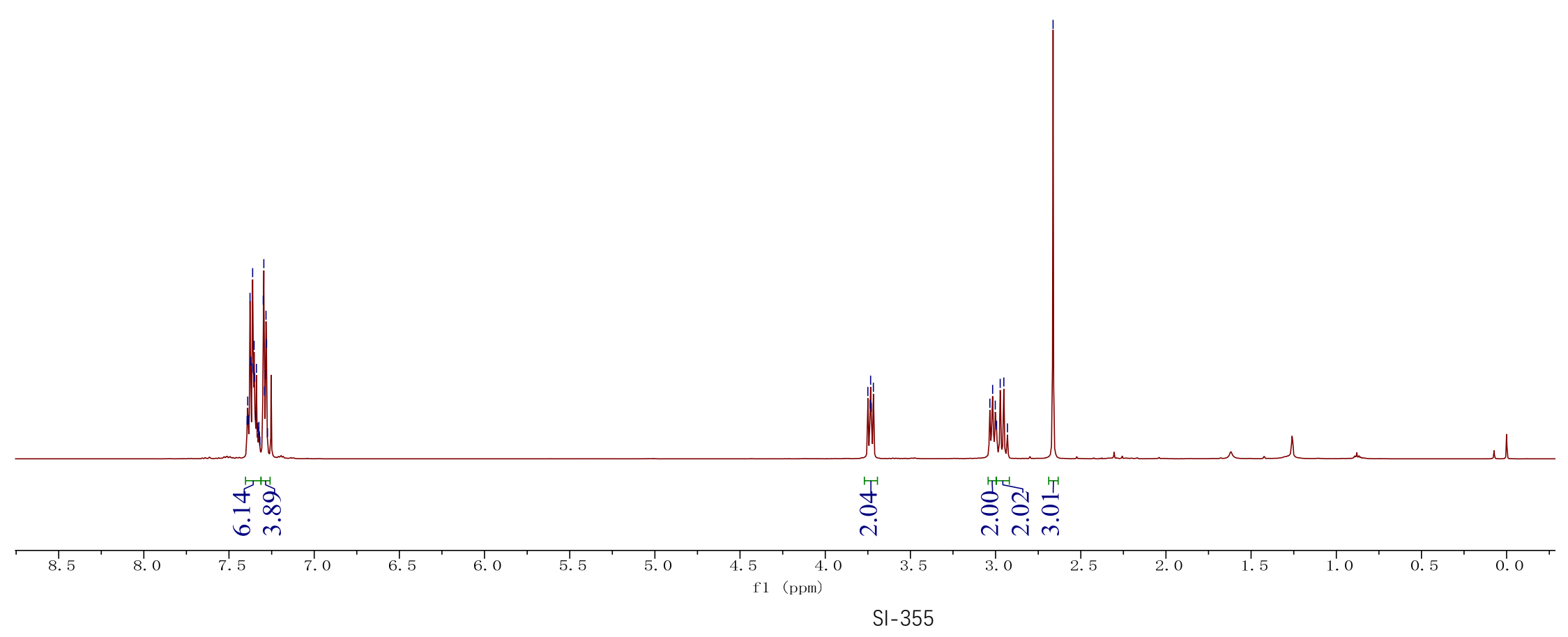



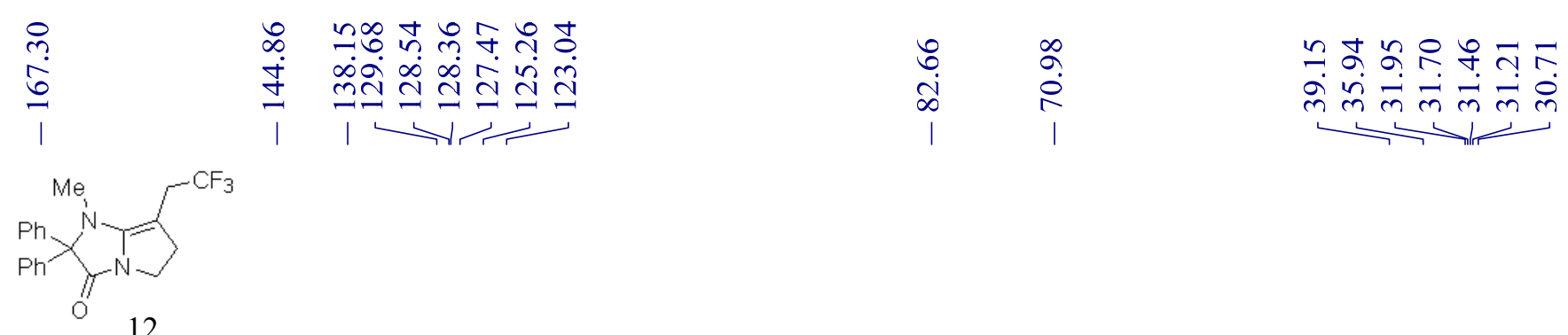

${ }^{13} \mathrm{C} \mathrm{NMR}\left(125 \mathrm{MHz}, \mathrm{CDCl}_{3}\right)$
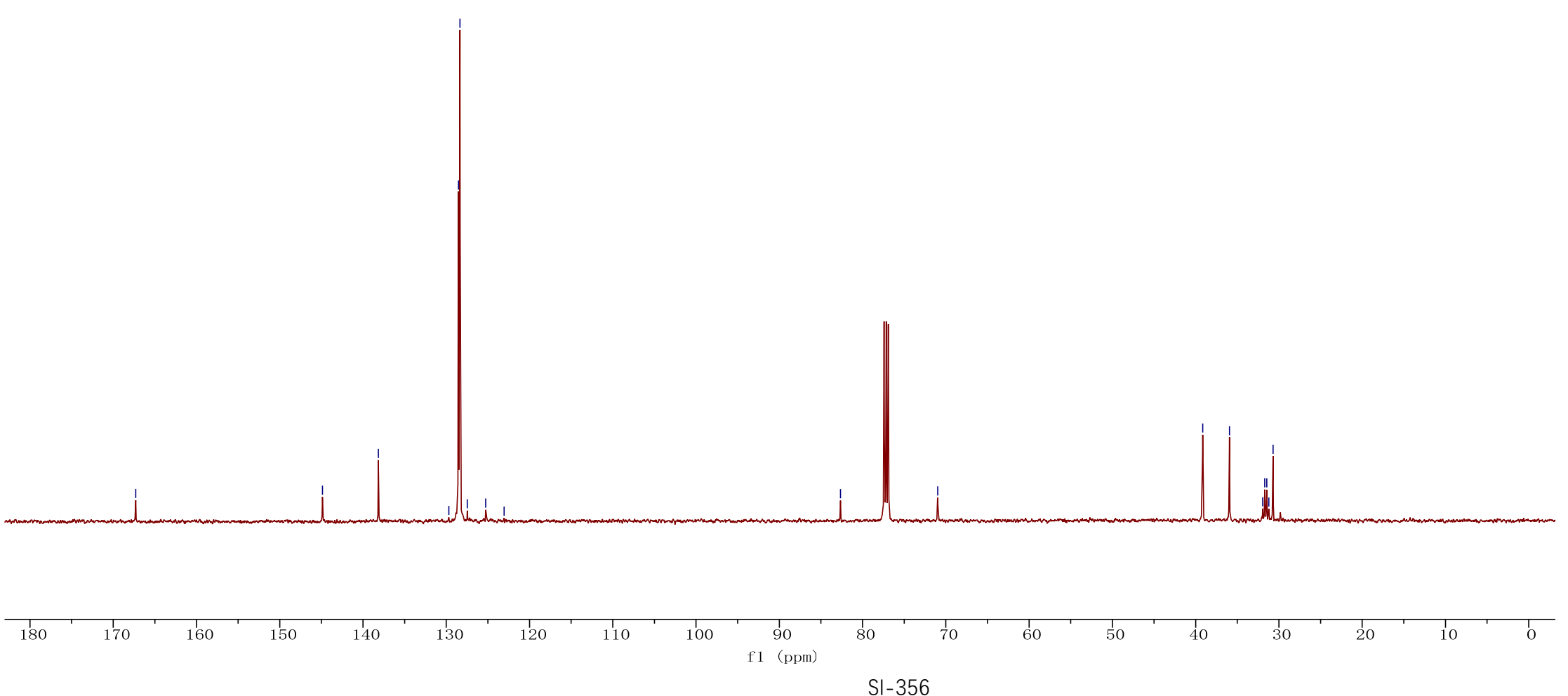
뉴고ำ

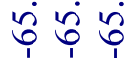

$\mathrm{Ph}_{\mathrm{O}}^{\mathrm{Me}}$

12

${ }^{19} \mathrm{~F}$ NMR (470 MHz, $\left.\mathrm{CDCl}_{3}\right)$ $\stackrel{1}{\wedge}$

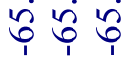

111

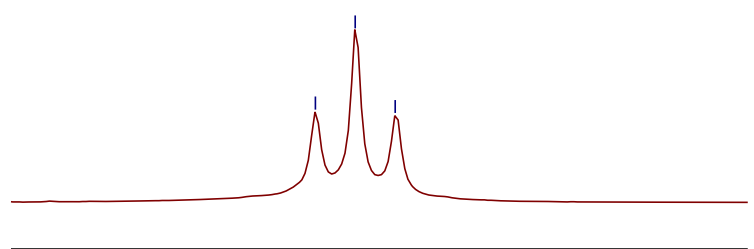

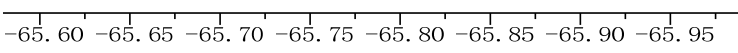
(1) (ppm)

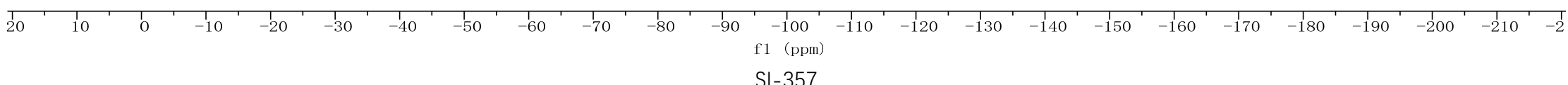




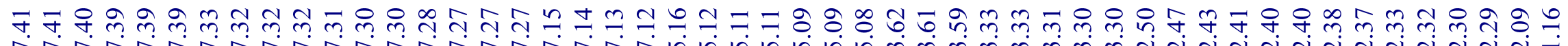

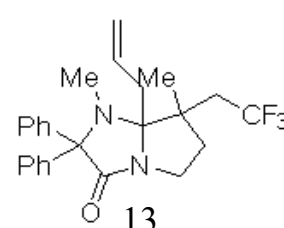

${ }^{1} \mathrm{H}$ NMR (400 MHz, $\mathrm{CDC}_{3}$ )
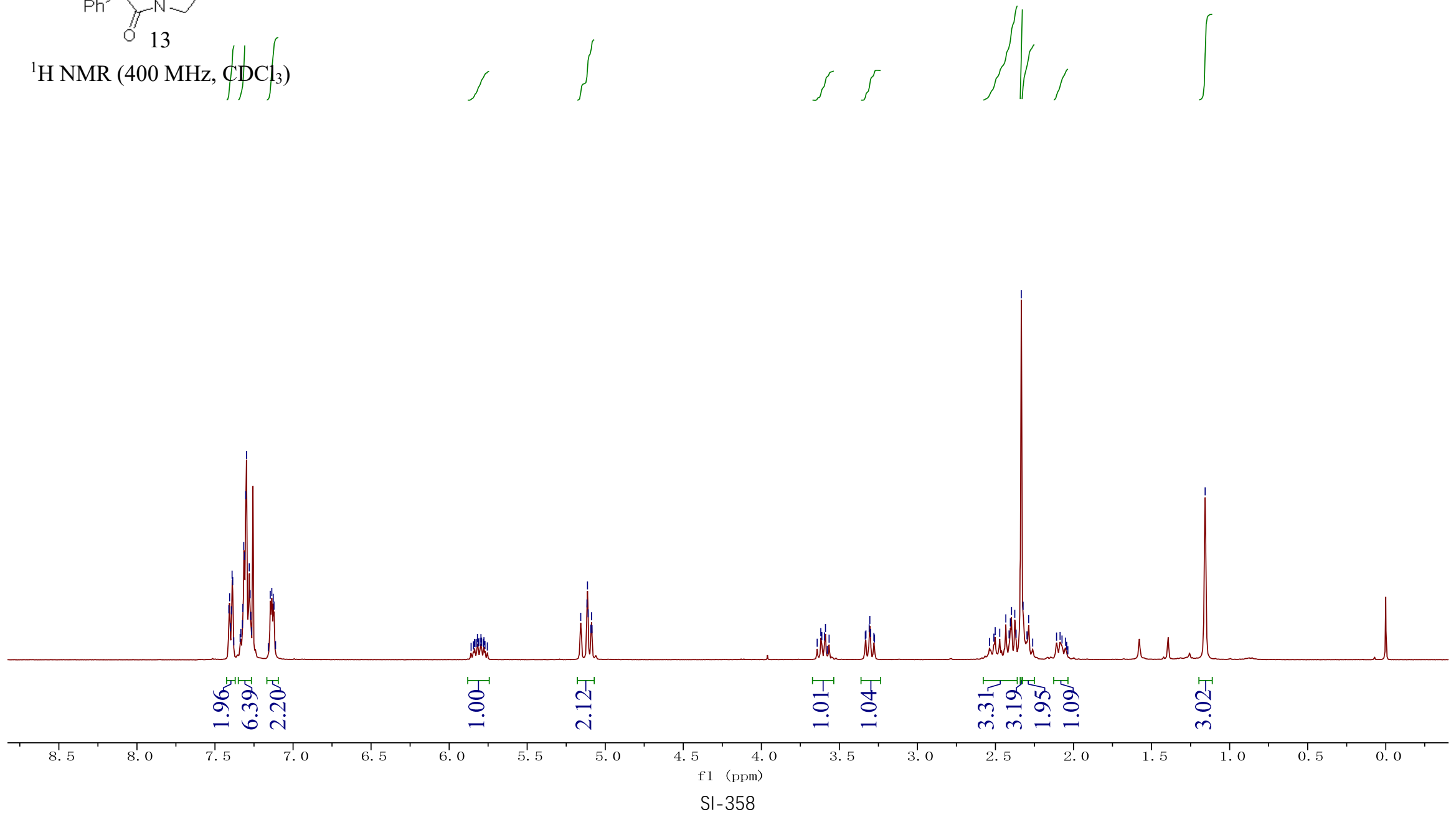


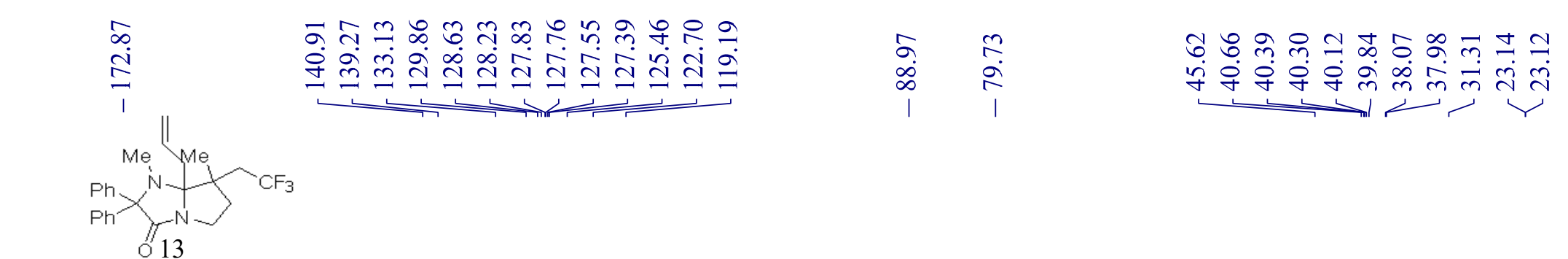

${ }^{13} \mathrm{C}$ NMR (100 MHz, $\mathrm{CDCl}_{3}$ )
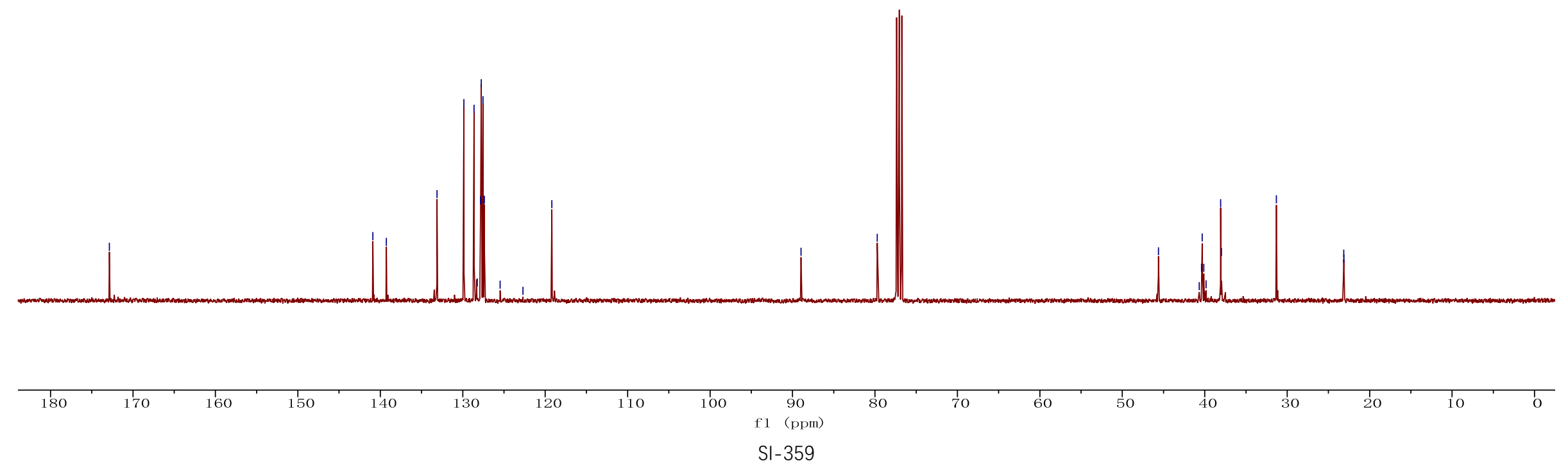


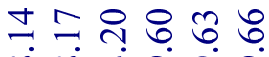

की

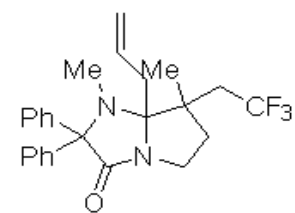

13

${ }^{19} \mathrm{~F}$ NMR $\left(376 \mathrm{MHz}, \mathrm{CDCl}_{3}\right)$

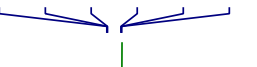

궁

$\begin{array}{lll}\infty & \infty & \infty \\ n & n & 0\end{array}$

it

우 ชูํํㅇ

กें

in

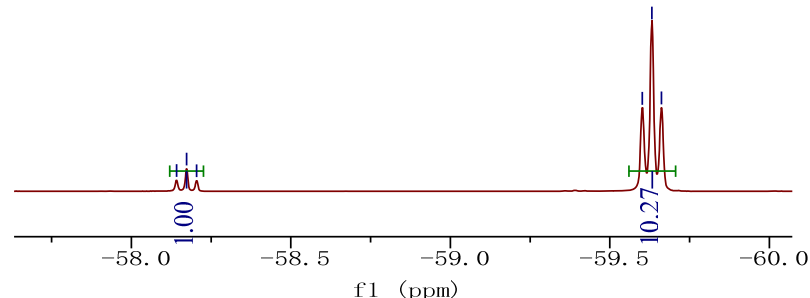

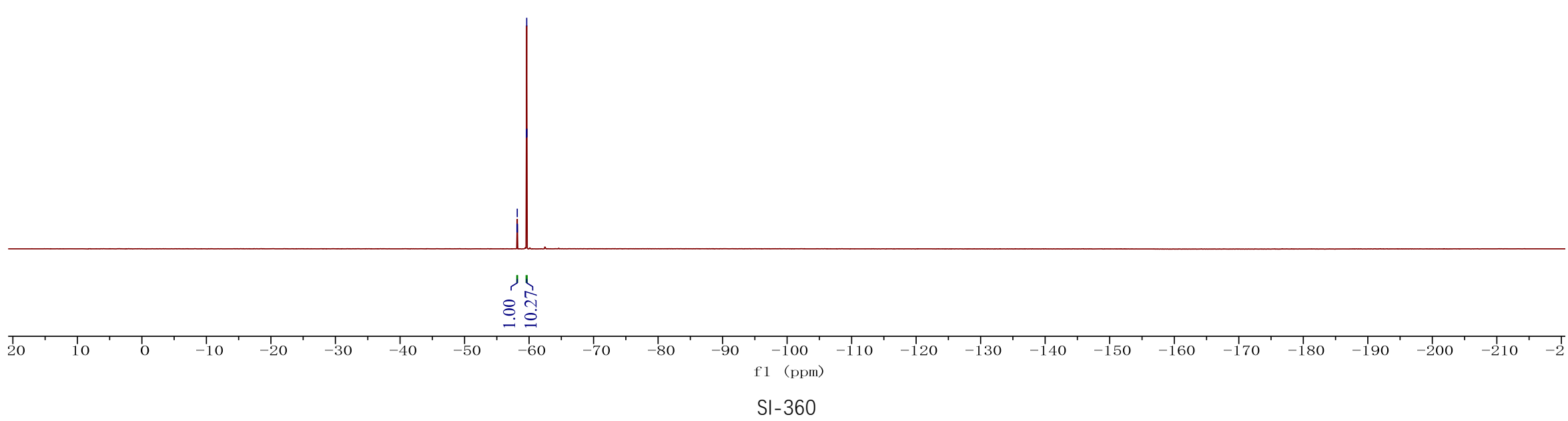




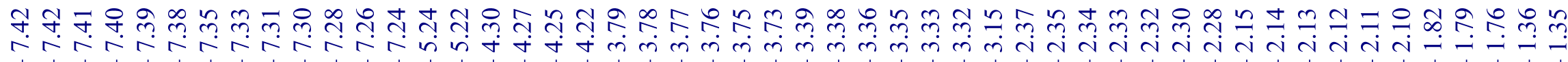
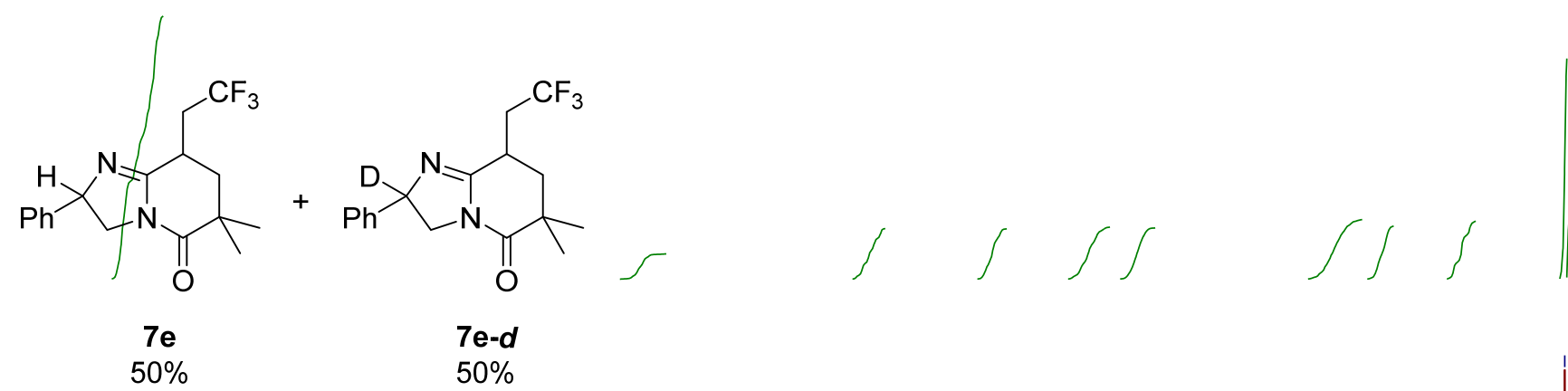

One pot KIE: ${ }^{1} \mathrm{H}$ NMR $\left(400 \mathrm{MHz}, \mathrm{CDCl}_{3}\right)$

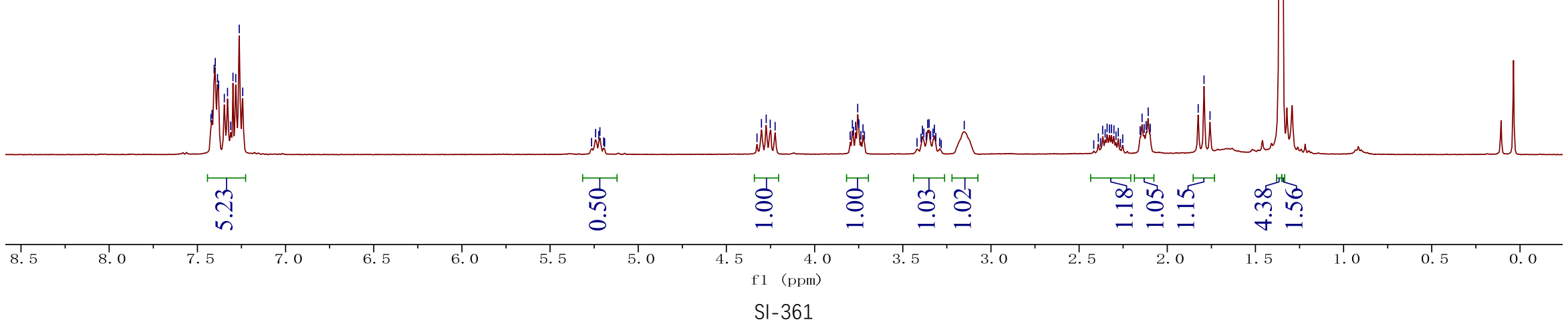




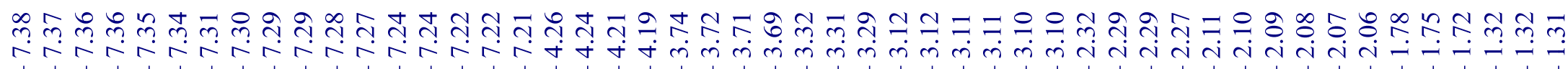

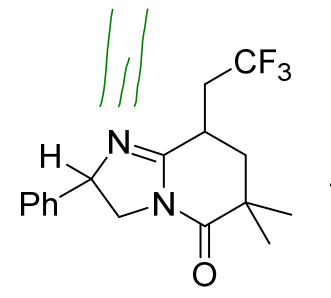

$7 e$

$29 \%$

Intramolecular KIE: ${ }^{1} \mathrm{H} \mathrm{NMR}\left(500 \mathrm{MHz}, \mathrm{CDCl}_{3}\right)$

7e-d

$71 \%$

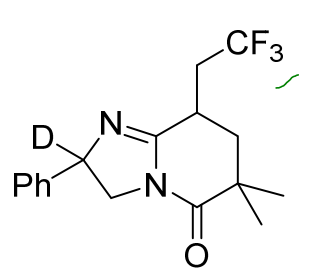

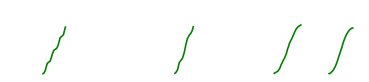
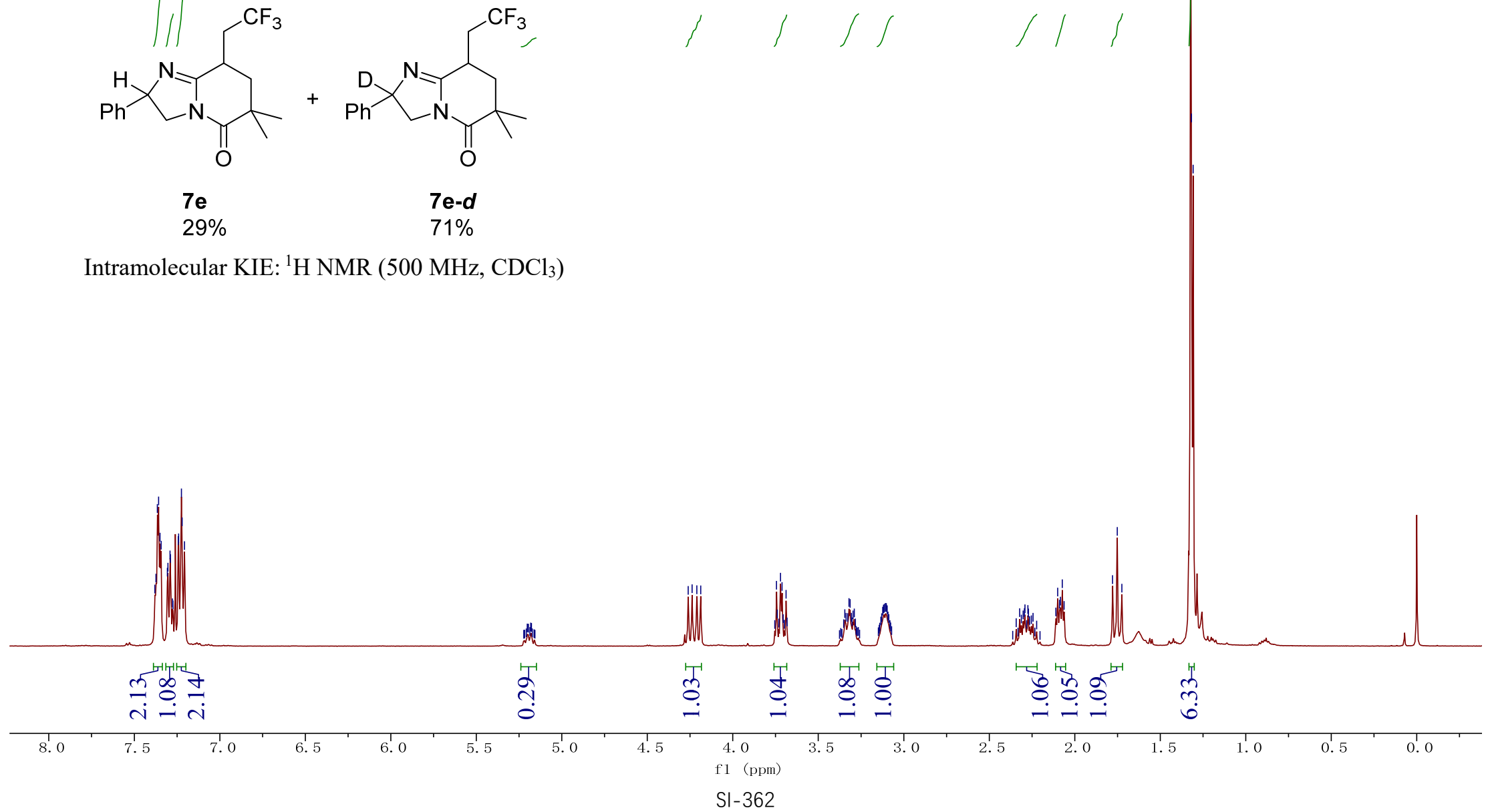


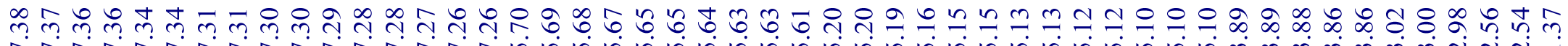

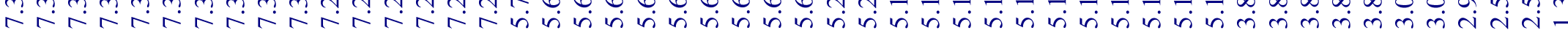
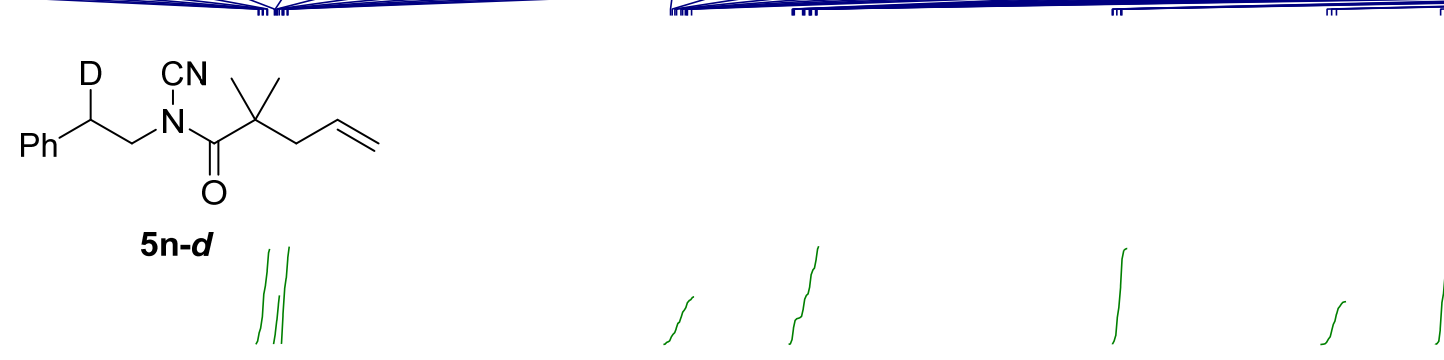

${ }^{1} \mathrm{H}$ NMR $\left(400 \mathrm{MHz}, \mathrm{CDCl}_{3}\right)$

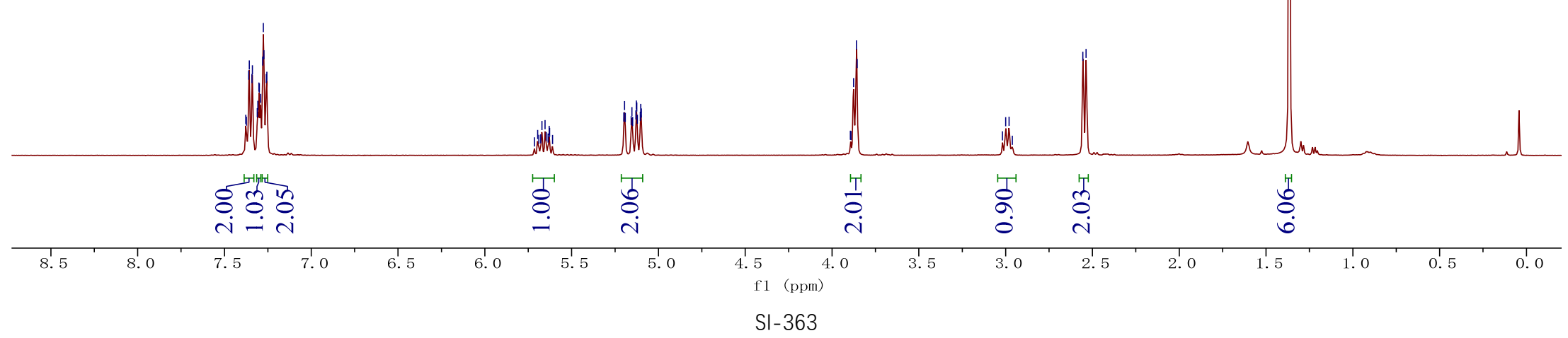




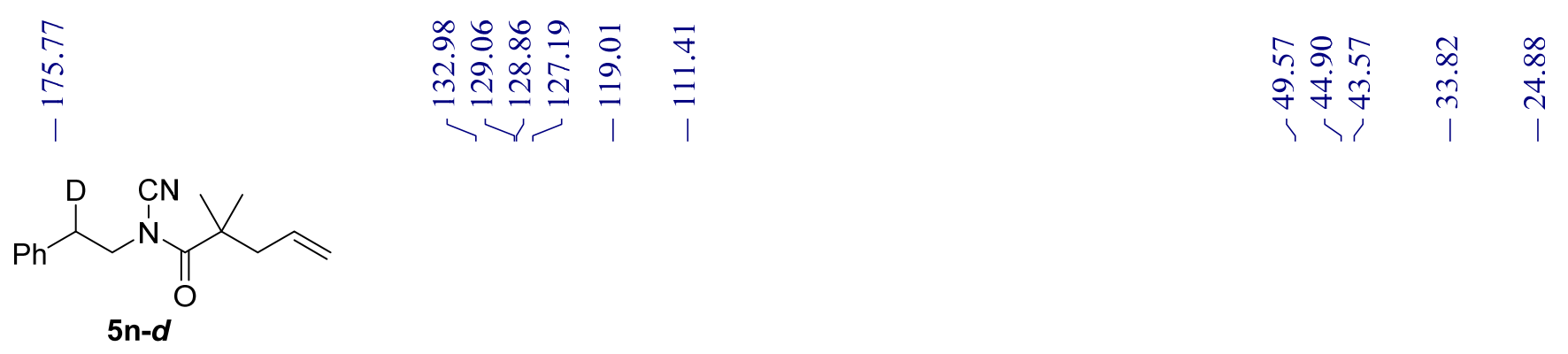

${ }^{13} \mathrm{C}$ NMR $\left(100 \mathrm{MHz}, \mathrm{CDCl}_{3}\right)$

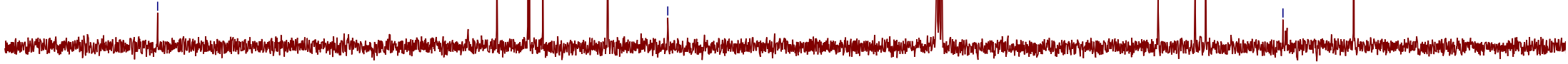
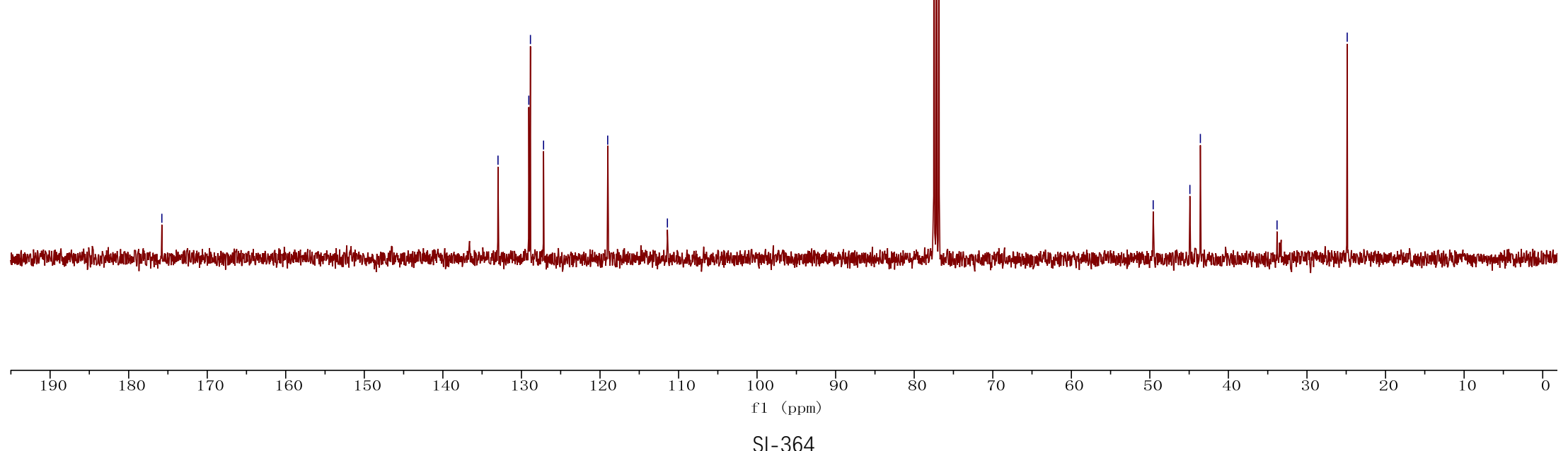


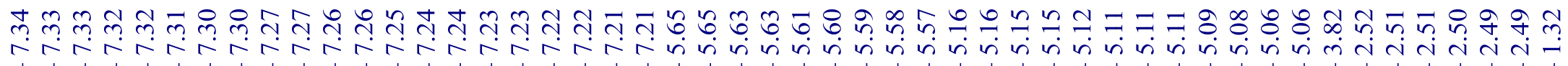
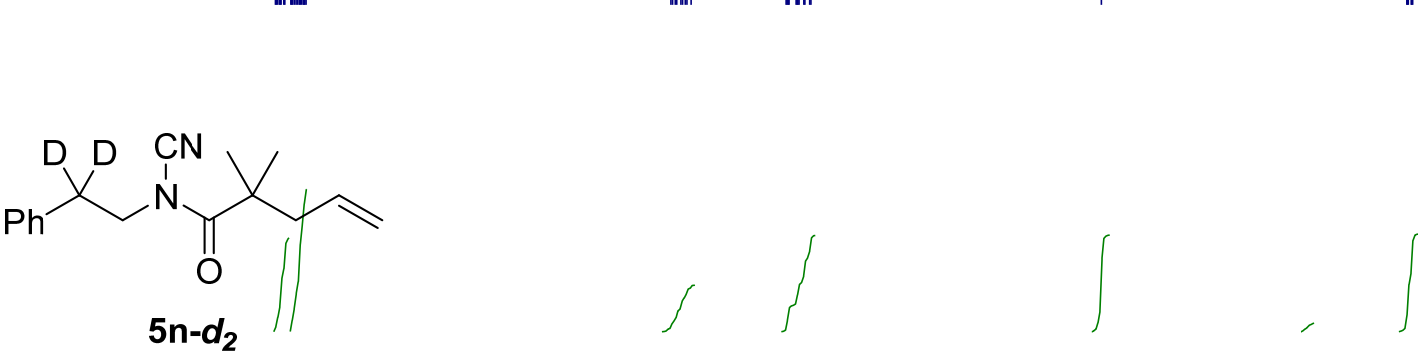

${ }^{1} \mathrm{H}$ NMR (400 MHz, $\mathrm{CDCl}_{3}$ )
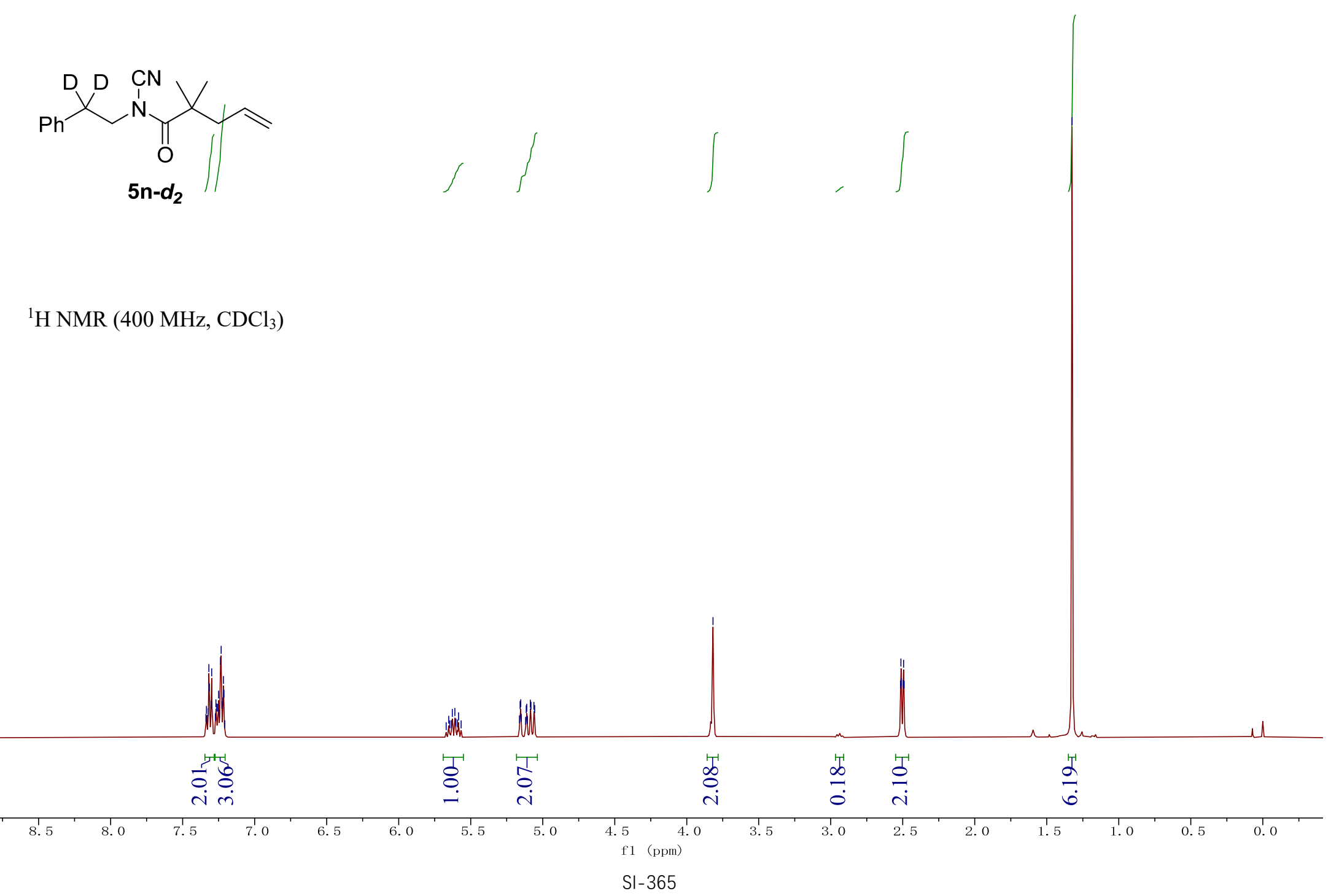

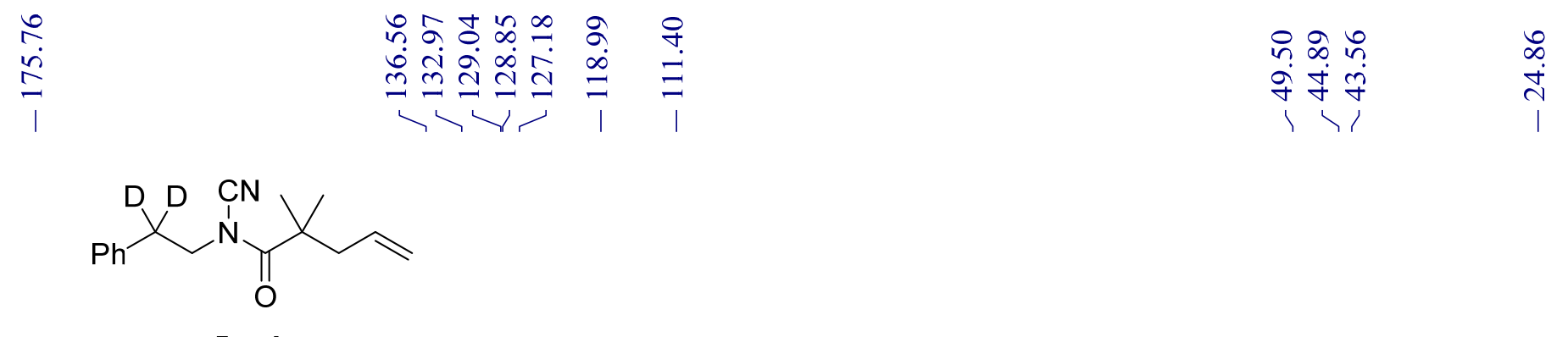

${ }^{13} \mathrm{C}$ NMR (125 MHz, $\mathrm{CDCl}_{3}$ )

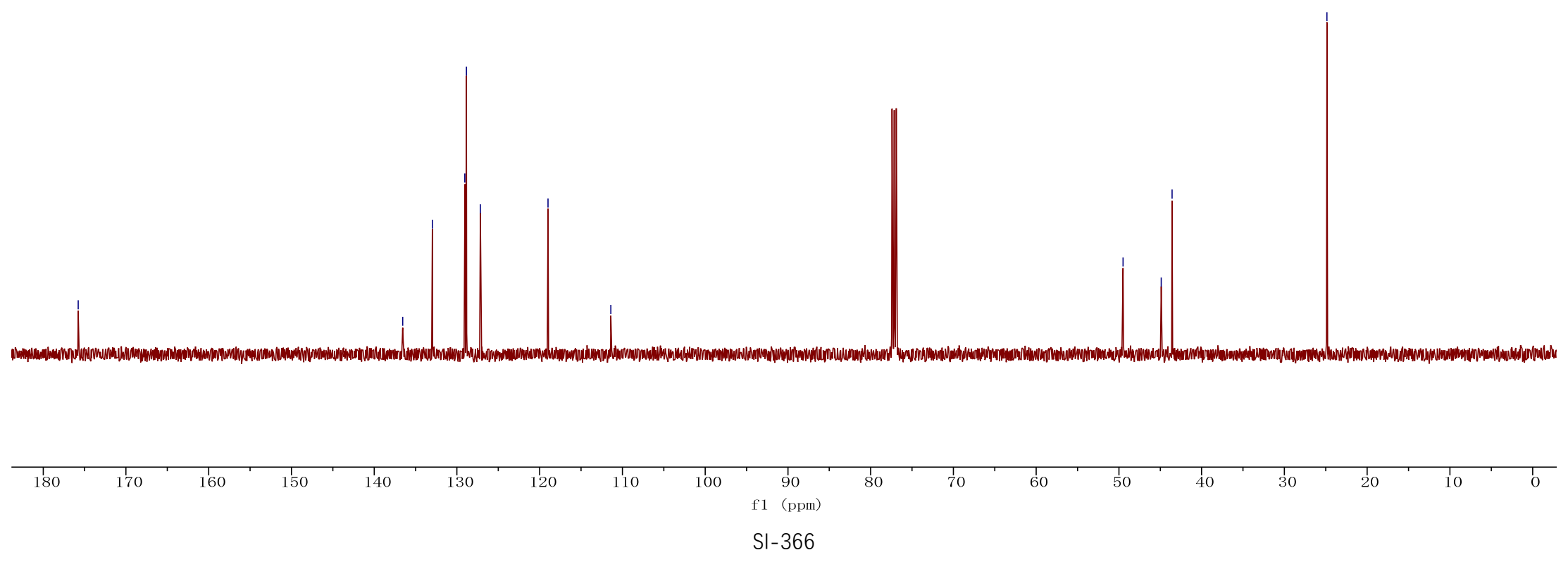


m rañ
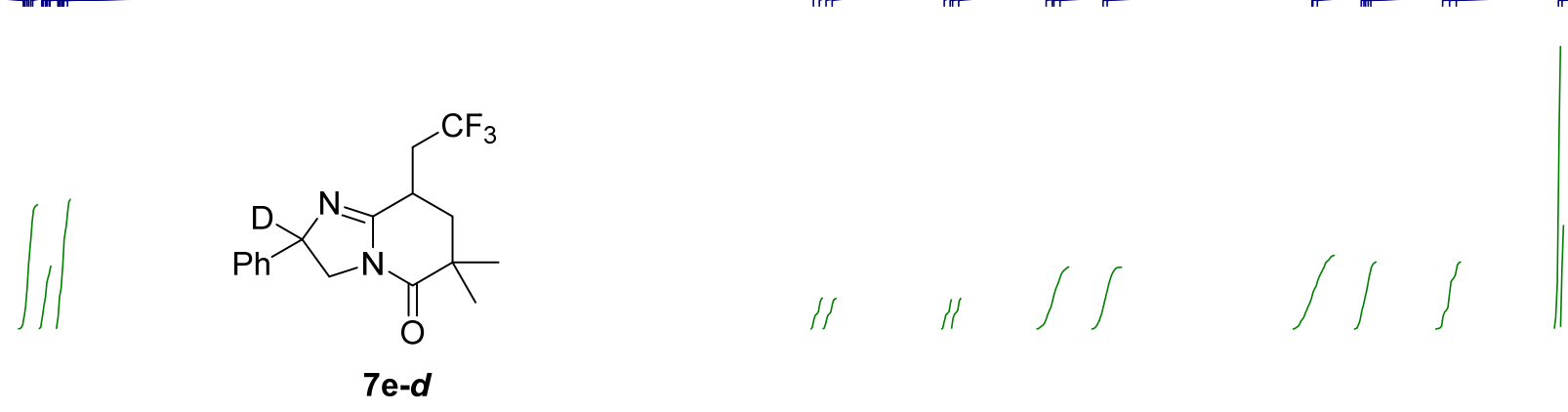

${ }^{1} \mathrm{H} \mathrm{NMR}\left(400 \mathrm{MHz}, \mathrm{CDCl}_{3}\right)$

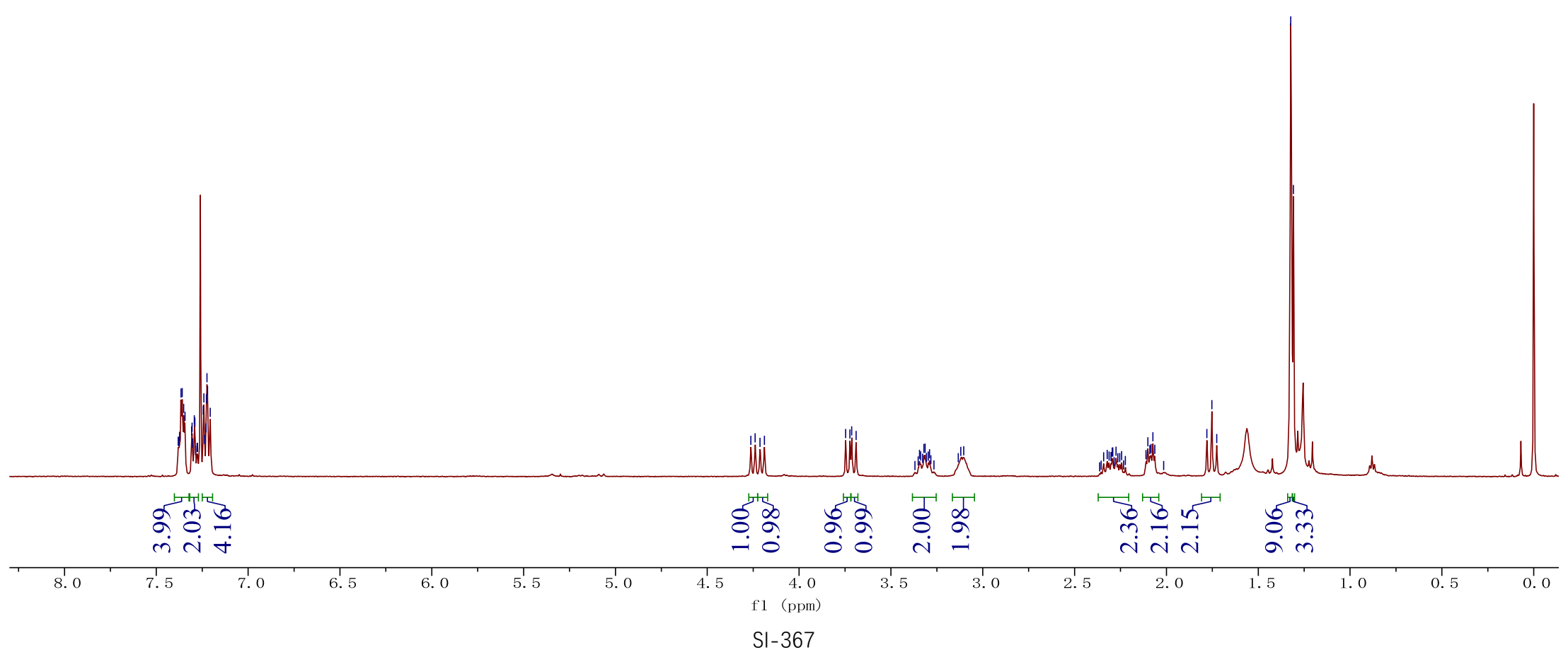




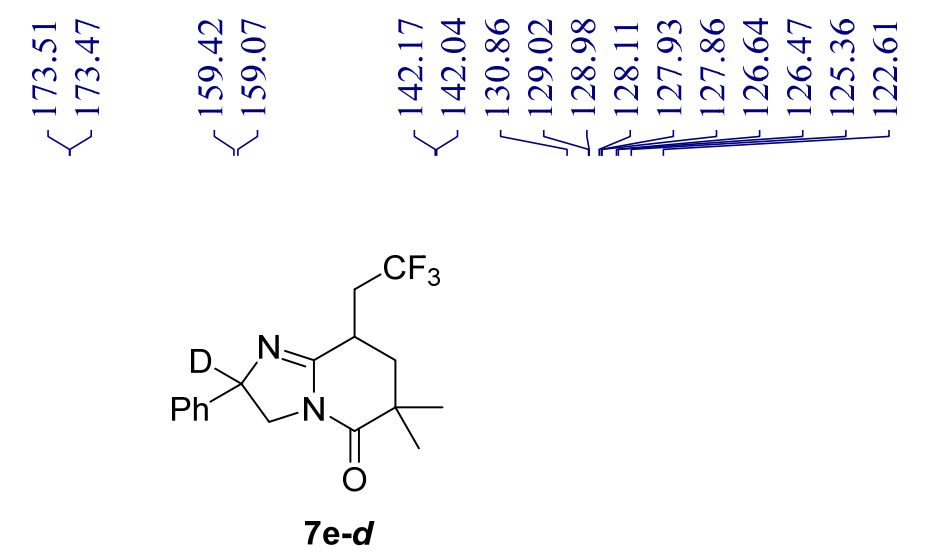

t)

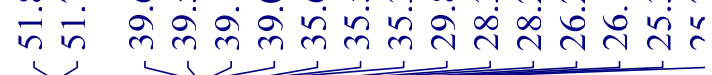

${ }^{13} \mathrm{C}$ NMR $\left(100 \mathrm{MHz}, \mathrm{CDCl}_{3}\right)$
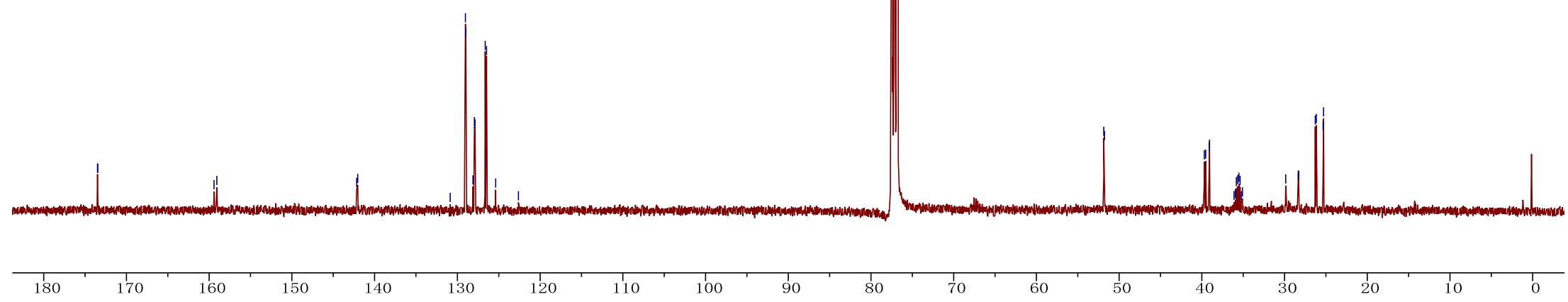

$180+170+160$

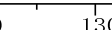

120 
者守䠉

क़िं

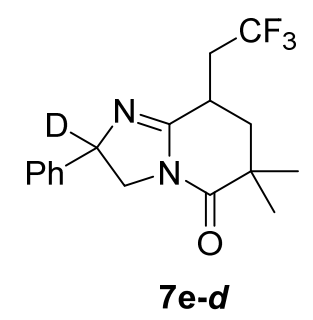

${ }^{19} \mathrm{~F}$ NMR (376 MHz, $\mathrm{CDCl}_{3}$ )

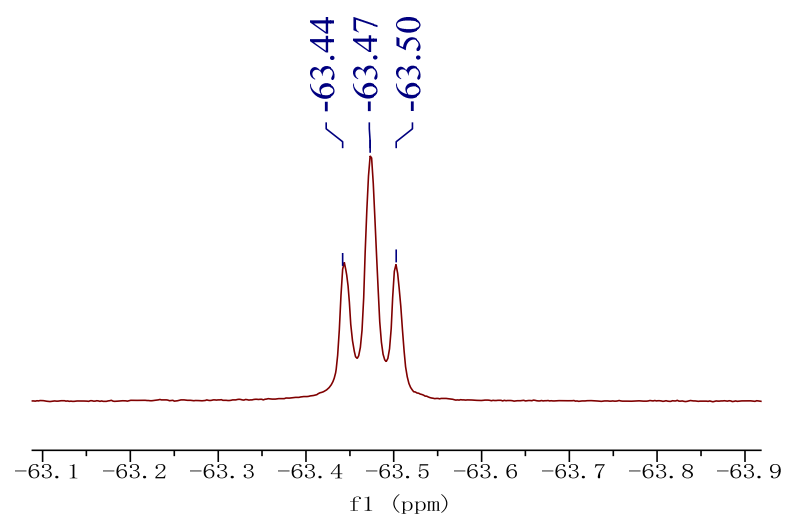

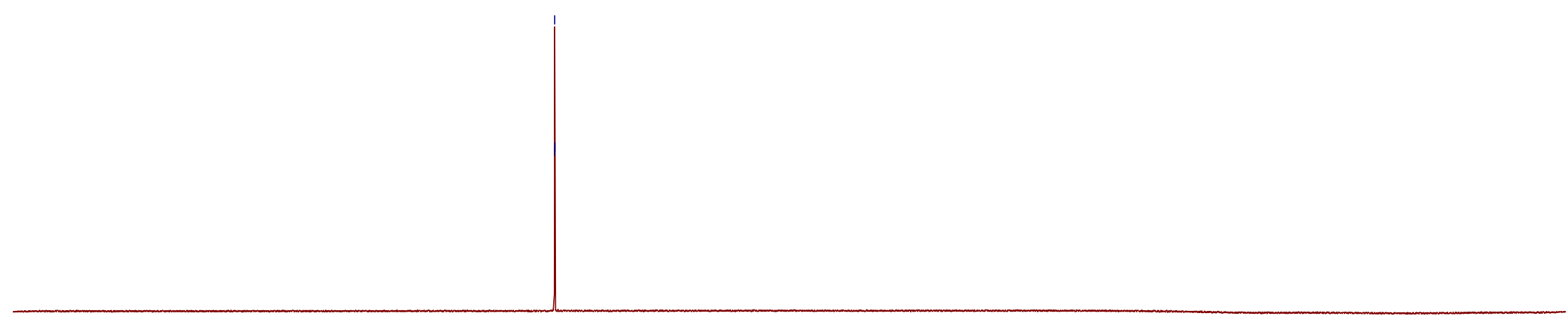

